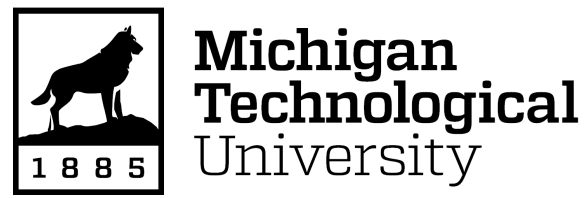

Michigan Technological University Digital Commons @ Michigan Tech

Dissertations, Master's Theses and Master's Reports

2019

\title{
ACOUSTIC LOCALIZATION TECHNIQUES FOR APPLICATION IN NEAR-SHORE ARCTIC ENVIRONMENTS
}

Miles B. Penhale

Michigan Technological University, mbpenhal@mtu.edu

Copyright 2019 Miles B. Penhale

\section{Recommended Citation}

Penhale, Miles B., "ACOUSTIC LOCALIZATION TECHNIQUES FOR APPLICATION IN NEAR-SHORE ARCTIC ENVIRONMENTS", Open Access Dissertation, Michigan Technological University, 2019.

https://doi.org/10.37099/mtu.dc.etdr/900

Follow this and additional works at: https://digitalcommons.mtu.edu/etdr

Part of the Acoustics, Dynamics, and Controls Commons 
ACOUSTIC LOCALIZATION TECHNIQUES FOR APPLICATION IN NEARSHORE ARCTIC ENVIRONMENTS

By

Miles B. Penhale

\begin{abstract}
A DISSERTATION
Submitted in partial fulfillment of the requirements for the degree of DOCTOR OF PHILOSOPHY

In Mechanical Engineering - Engineering Mechanics
\end{abstract}

MICHIGAN TECHNOLOGICAL UNIVERSITY

2019

(C) 2019 Miles B. Penhale 
This dissertation has been approved in partial fulfillment of the requirements for the Degree of DOCTOR OF PHILOSOPHY in Mechanical Engineering - Engineering Mechanics.

Department of Mechanical Engineering - Engineering Mechanics

Dissertation Advisor: Andrew Barnard.

Committee Member: Charles Van Karsen.

Committee Member: Jason Blough.

Committee Member: Roger Turpening.

Department Chair: William Predebon. 


\section{Table of Contents}

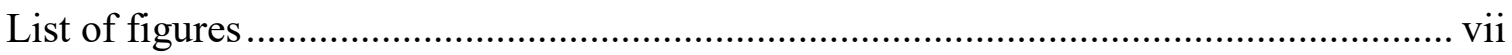

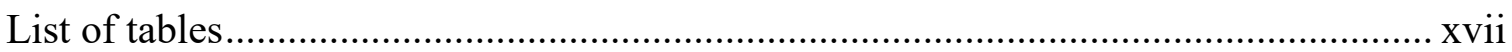

Author contribution statement ........................................................................... xviii

Acknowledgements ................................................................................................. xix

Terms, Abbreviations, Nomenclature, and Symbols .................................................... xxii

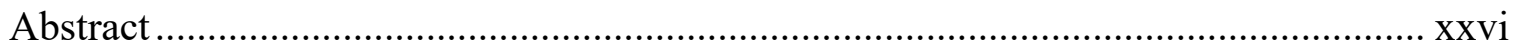

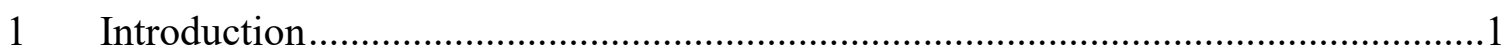

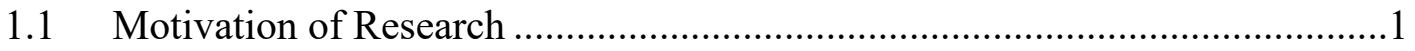

1.2 Explanation of Chapters ...........................................................................

2 Multi-Modal and Short-Range Transmission Loss in Thin, Ice-Covered, Near-Shore

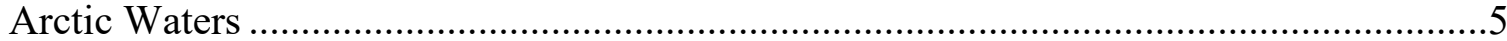

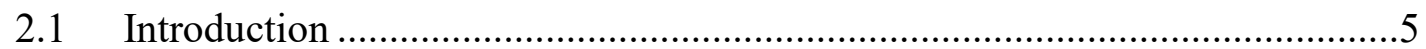

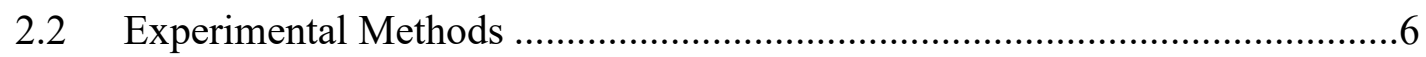

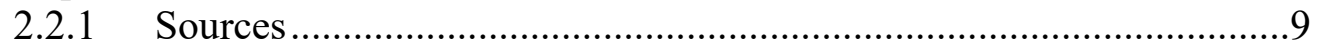

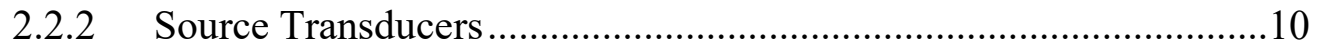

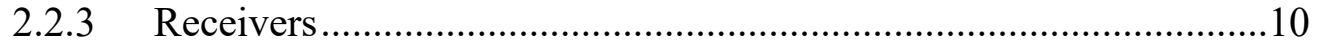

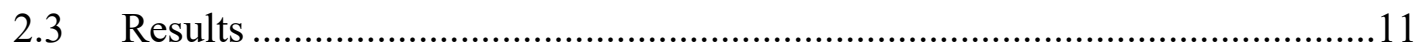

2.3.1 Temporal Propagation and Sound Speed........................................11

2.3.2 Frequency Response Functions.......................................................16

2.3.3 Hammer Experiment FRFs .........................................................17

2.3.4 Cannon Experiment FRFs...........................................................21

2.3.5 Transmission Loss ...........................................................................24

2.3.5.1 Tonal Underwater Transmission Loss ...........................24

2.3.5.2 Atmospheric Transmission Loss ....................................25

2.3.5.3 Air - Ice - Water Transmission Loss ………………......25

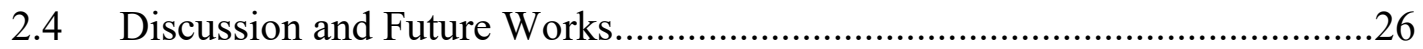

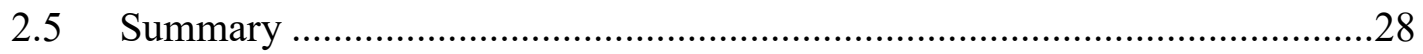

3 Acoustic Studies on an Arctic-Like Surrogate: Lake Superior...................................30

3.1 Relation to Previous Work ………………………......................................

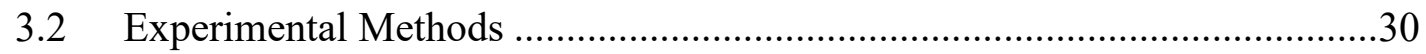

3.2.1 Test Environment......................................................................

3.2.2 Sources and Receivers ............................................................... 


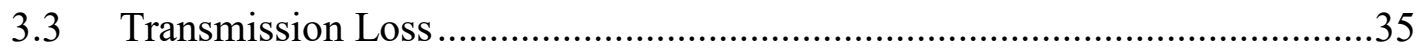

3.3.1 Additional Attenuation Mechanisms ………..................................36

3.3.1.1 Implementation of the MLS signal for RL measurement 37

3.3.1.2 Portage Lake RL ...........................................................

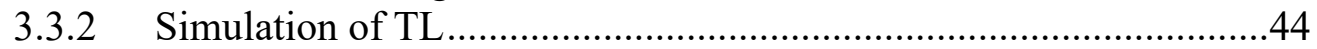

3.3.2.1 Ray Tracing Algorithms ……………………................46

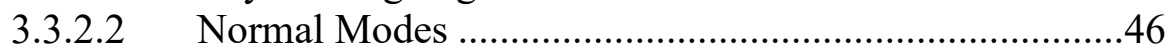

3.3.2.3 Fast Field Program ...............................................................

3.3.2.4 Parabolic Equation Solving with Finite Difference

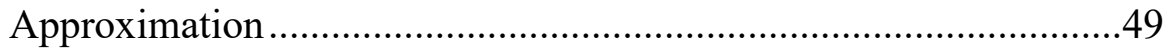

3.3.2.5 Comparison and Implementation .....................................50

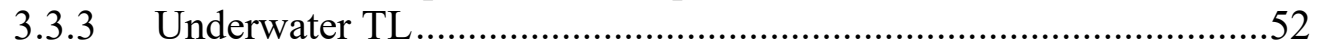

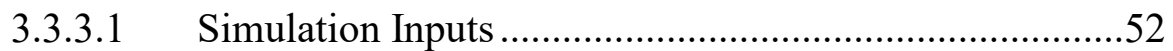

3.3.3.2 TL vs. Range and Depth ................................................54

3.3.3.3 Data/Model Comparison.................................................56

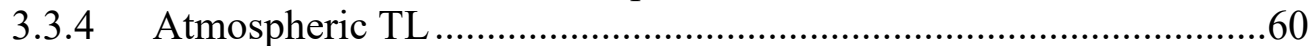

3.3.4.1 Cannon Blast Signal........................................................60

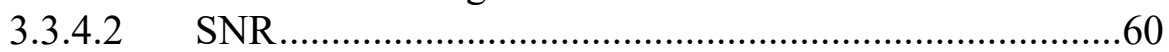

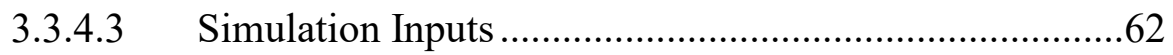

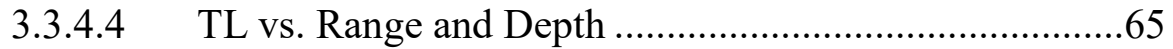

3.3.4.5 Data/Model Comparison.................................................68

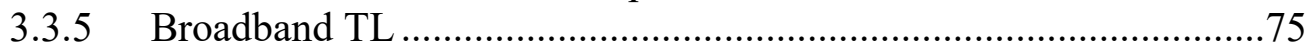

3.4 Effects of Ice Boreholes on FRFs between Air and Water .............................76

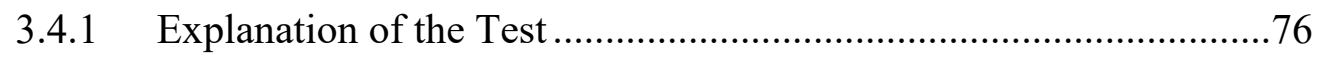

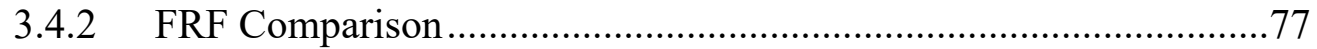

3.5 Impedance Relationship between Ice and Water ..........................................78

3.5.1 Elastic Properties and Sound Speeds of the Ice .................................79

3.5.2 Solving the Wave Equation with Impedance Matched Boundary

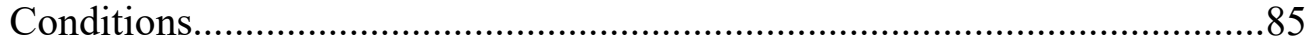

3.5.3 Ice layer drivepoint FRF (dynamic mobility) ...................................8

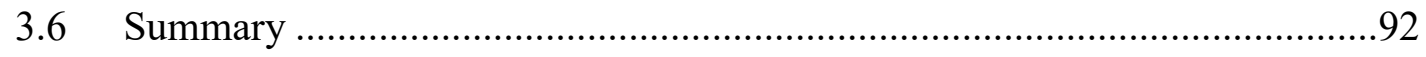

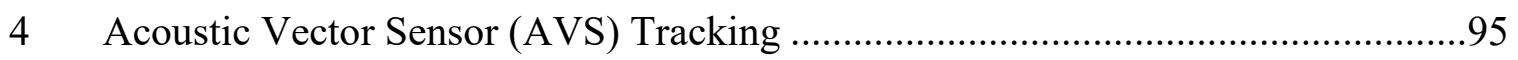

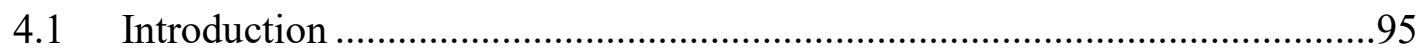

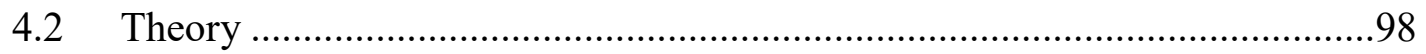

4.2.1 Derivation of the acoustic intensity vector .....................................99

4.2.2 Intensity from Finite Difference Methods .....................................101

4.2.3 Phase Calibration for $p p$ Methods.....................................................102

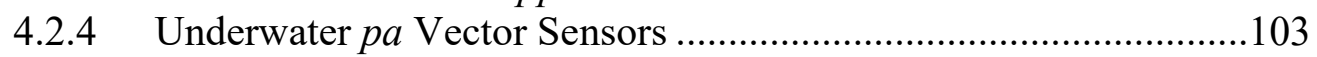

4.2.5 Atmospheric $p p$ Vector Sensors....................................................104

4.2.6 Moving Standard Deviation (MSD) Processing .............................106

4.2.7 GPS Ground Truth DOA and Error ................................................108 


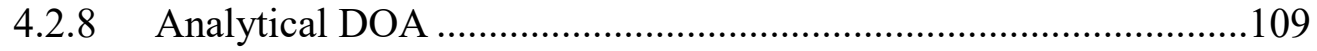

4.2.9 Multi-Sensor Localization .......................................................111

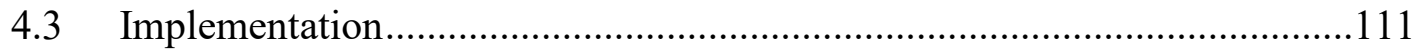

4.3.1 Underwater $p a$ Vector Sensors ......................................................112

4.3.1.1 Stationary Source Experiment ........................................112

4.3.1.2 Non-Stationary Source Experiment ..............................112

4.3.2 Atmospheric $p p$ Vector Sensors......................................................114

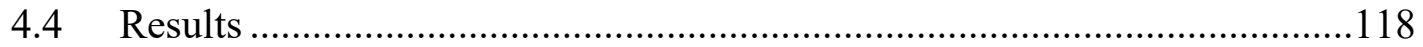

4.4.1 Underwater $p a$ Vector Sensors .....................................................118

4.4.1.1 Pitch, Roll, and Heading Variation of pa AVS .............118

4.4.1.2 DOA of the Stationary Source .......................................119

4.4.1.3 DOA of the Non-Stationary Source ...............................123

4.4.1.4 Multi-Sensor Localization of the Non-Stationary Source 129

4.4.2 Atmospheric $p p$ Vector Sensors......................................................134

4.4.2.1 DOA Spectral Variation.................................................134

4.4.2.2 DOA of the Non-Stationary Source ................................141

4.4.2.3 MSD vs. Integration DOA Techniques..........................144

4.4.2.4 Azimuthal Error of pp AVS ..........................................145

4.4.2.5 Analytical DOA comparison.........................................149

4.4.2.6 Multi-Sensor Localization ............................................154

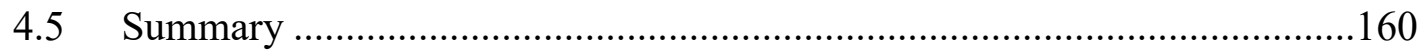

$5 \quad$ Conclusions and Future Work …………………….......................................162

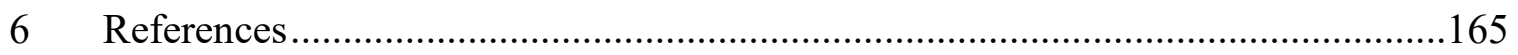

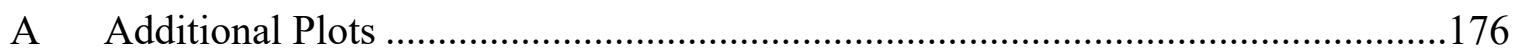

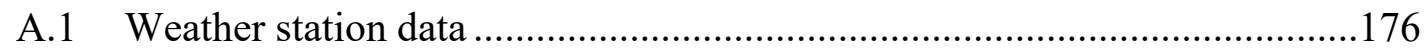

A.2 Portage Lake Bathymetry ………………………….................................177

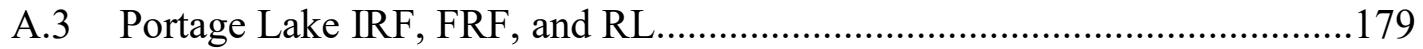

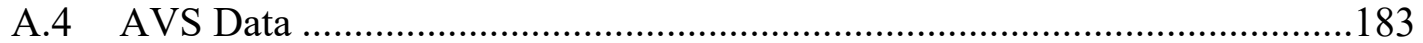

A.4.1 Acoustic Hardware Phase Mismatch ...............................................183

A.4.2 Target Vehicle Path (GPS Position) ……………............................183

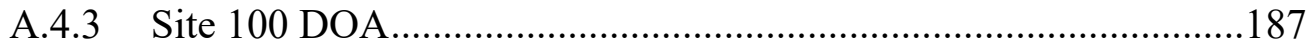

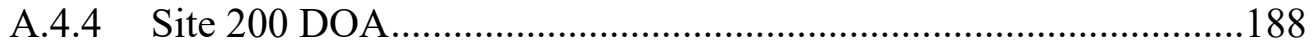

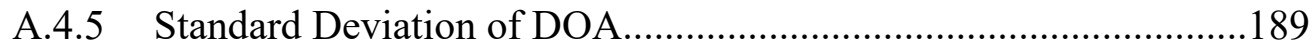

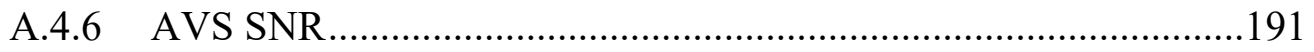

A.4.7 Vehicle Speed ............................................................................. 192

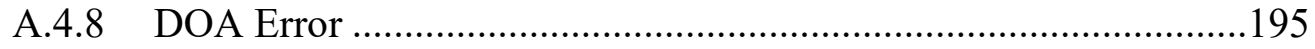




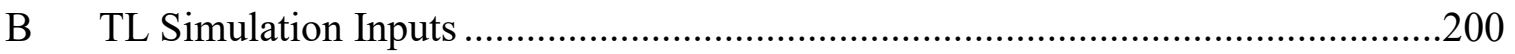

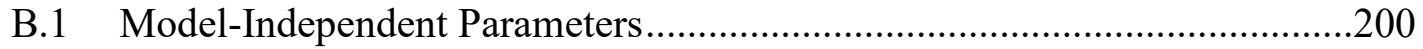

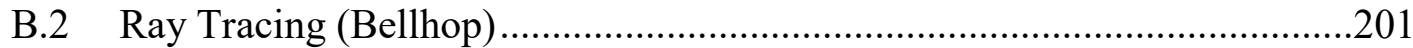

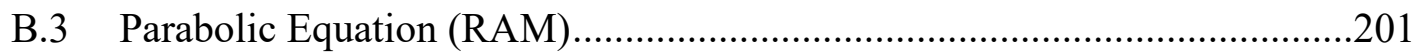

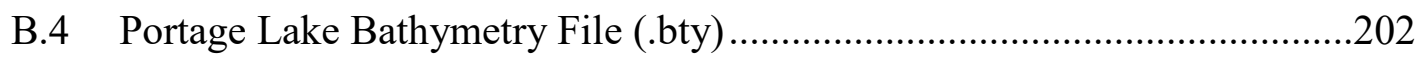

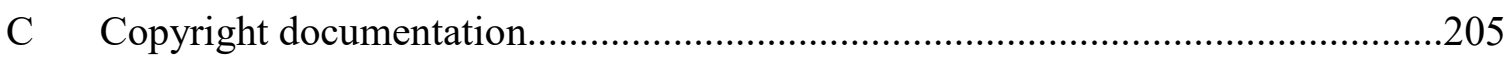

C.1 Permission 1 ........................................................................................205

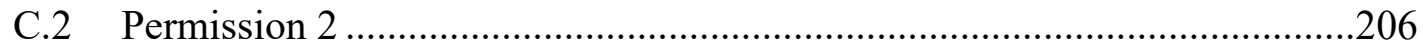

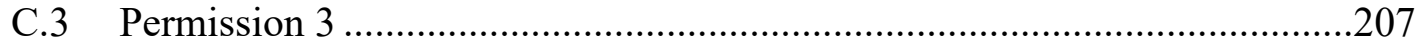




\section{List of figures}

Figure 1.1 Quantity and age of ice in the Arctic ocean in January 1984. Courtesy:

NASA's Scientific Visualization Studio ${ }^{1}$

Figure 1.2 Quantity and age of ice in the Arctic ocean in January 2015. Courtesy:

NASA's Scientific Visualization Studio ${ }^{1}$ 2

Figure 2.1 Site layout approximately $2 \mathrm{~km}$ off the coast of Barrow, AK........................8

Figure 2.2 Shore-fast sea ice conditions near the test sites: (A) Site 1 looking seaward.

(B) Photograph from cluster of sites 2, 3, and 6 looking east towards sites 1, 4, and 5. (C) Large ice ridge near site 3. (D) Scale of large ice ridges compared to a person. Large ice ridges were approximately $5 \mathrm{~m}$ high. (E) Site 2 looking towards site 3

Figure 2.3 Source site schematic of various acoustic sources and receivers. Arrows indicate drive point measurements between source and receiver with adequate signal-to-noise ratio. Units of output signal over input signal are indicated.

Figure 2.4 Receiver site transducer layout. Site 1 shown.

Figure 2.5 Ice-to-ice and ice-to-water temporal propagation. Source (site 4) to receiver (site 1) distance approximately $50 \mathrm{~m}$. (A) Hammer force input ( $\mathrm{dB}$ re $1 \mathrm{~N} / \mathrm{rt}(\mathrm{Hz})$ ) at site 4 source location. (B) Geophone response $(\mathrm{dB}$ re $1 \mathrm{~m} / \mathrm{s} / \mathrm{rt}(\mathrm{Hz}))$ at site 4 source location. (C) Hydrophone response $(\mathrm{dB}$ re $1 \mathrm{~Pa} / \mathrm{rt}(\mathrm{Hz}))$ at site 4 source location. (D) Geophone response $(\mathrm{dB}$ re $1 \mathrm{~m} / \mathrm{s} / \mathrm{rt}(\mathrm{Hz}))$ at site 1 receiver location.

(E) Hydrophone response $(\mathrm{dB}$ re $1 \mathrm{~Pa} / \mathrm{rt}(\mathrm{Hz}))$ at site 1 receiver location. .12

Figure 2.6 Air-to-air temporal propagation from the cannon source to the microphones at various receiver distances. (A) atmospheric sound level at source location (site 5) normalized to $1 \mathrm{~m}$. (B) atmospheric sound level at site 1 approximately $164 \mathrm{~m}$ from source. (C) atmospheric sound level at site 3 approximately $853 \mathrm{~m}$ from source. (D) atmospheric sound level at site 2 approximately $1035 \mathrm{~m}$ from source. All levels: $\mathrm{dB}$ re $1 \mathrm{~Pa} / \mathrm{Hz}$.

Figure 2.7 Air-to-ice-to-water temporal propagation from the cannon source to the hydrophones at various receiver distances. (A) Underwater sound level at site 5. (B) Underwater sound level at site 1 approximately $164 \mathrm{~m}$ from source. (C) Underwater sound level at site 3 approximately $853 \mathrm{~m}$ from source. All levels: $\mathrm{dB}$ re $1 \mathrm{~Pa} / \mathrm{Hz}$.

Figure 2.8 Sound speed profiles measured with conductivity, temperature, and depth (CTD) probe for sites 1 and 2. Measurements taken on 12/04/16 at UTC 15:53:32 and UTC 17:07:47 for sites 1 and 2 respectively. 
Figure 2.9 Source input levels from instrumented force hammer (left-hand scale) and propane cannon (right-hand scale).

Figure 2.10 (A) Drive point FRFs and (B) $\mathrm{COH}$ measured between the hammer and the geophone (left-hand scale) or hydrophone (right-hand scale) at the source location. Theoretical infinite plate mobility for sea ice conditions in Barrow (-66 $\mathrm{dB}$ re $1 \mathrm{~m} / \mathrm{s} / \mathrm{N})$ is indicated.

Figure 2.11 (a) Drive point FRFs and (b) $\mathrm{COH}$ between the cannon source and the geophone (left-hand scale) or hydrophone (right-hand scale) at the source location

Figure 2.12 (A) Propane cannon blast measured at the source location microphone. (B) Microphone measurements at $50 \mathrm{~m}$ and $100 \mathrm{~m}$ receiver locations. (C) Geophone measurements at $50 \mathrm{~m}$ and $100 \mathrm{~m}$ locations. The flexural wave amplitude in the ice (C) is significantly reduced upon arrival of the atmospheric acoustic wavefront (B) indicating the detection of an air-coupled flexural wave at $29 \mathrm{~Hz}$. .23

Figure 2.13 Measured underwater acoustic transmission loss data shown with theoretical cylindrical and spherical spreading curves for reference.

Figure 2.14 Measured atmospheric transmission loss data and theoretical spherical spreading curve shown for reference.

Figure 2.15 Measured transmission loss data through the combined air-ice-water path...26

Figure 3.1 Acoustic experiment sites in the Keweenaw Waterway. (A) Keweenaw Waterway (Portage Lake). (B) Zoomed in view of test sites.. .31

Figure 3.2 (A) Bathymetry and (B) underwater sound speed profile of Portage Lake in the measurement area.... 32

Figure 3.3 Experiment site setup on Portage Lake (Keweenaw Waterway) (A) Site 1 (source site). Propane cannon is not pictured. (B) Data system box. Data system and underwater speaker amplifier powered by $12 \mathrm{~V}$ car battery. (C) Site 2 looking north toward site 1 .

Figure 3.4 Modeled reflection loss for generic bottom types. Valid for $10-100 \mathrm{kHz}$. (Reproduced with permission from APL-UW High-Frequency Ocean Environmental Acoustic Models Handbook ${ }^{54}$ ).

Figure 3.5 Theoretical (A) arrival times and (B) grazing angles for ice and bottom reflections at the measurement sites on Portage Lake. 
Figure 3.6 Impulse response function $1 \mathrm{~m}$ from the underwater source. (A) Full IRF from MLS signal. (B) Incident arrival. (C) Windowed ice reflection (D) Windowed bottom reflection.

Figure 3.7 Example window functions used on the IRF to separate the incident, ice reflection, and bottom reflection components.

Figure 3.8 (A) Frequency response functions and (B) Reflection loss for $\phi I=89^{\circ}$ and $\phi B=86^{\circ}$. Source to receiver distance equal to $1 \mathrm{~m}$.

Figure 3.9 Impulse response function $110 \mathrm{~m}$ from the underwater source. (A) Full IRF from MLS signal. (B) Incident arrival. (C) Windowed ice reflection (D) Windowed bottom reflection.

Figure 3.10 (A) Frequency response functions and (B) Reflection loss for $\phi I=3.8^{\circ}$ and $\phi B=7^{\circ}$. Source to receiver distance equal to $110 \mathrm{~m}$.

Figure 3.11 Domains of applicability of underwater acoustic propagation models. (Reproduced with permission from P.C. Etter, Underwater Acoustic Modeling and Simulation ${ }^{48}$ )

Figure 3.12 Acoustic Properties of the bottom half-space for simulating underwater TL.

Figure 3.13 Simulated bottom RL from ACTUP environmental models.

Figure 3.14 Simulated (Bellhop) underwater TL vs. range and depth at $1000 \mathrm{~Hz}$. Acoustic source depth at $3 \mathrm{~m}$. Portage Lake bathymetry shown in black. .55

Figure 3.15 Simulated (RAM) underwater TL vs. range and depth at $1000 \mathrm{~Hz}$. Acoustic source depth at $3 \mathrm{~m}$. Portage Lake bathymetry shown in black.

Figure 3.16 Underwater TL comparison for Bellhop and RAM simulations at $1000 \mathrm{~Hz}$, receiver depth $3 \mathrm{~m}$. Range detail shown with (A) Log and (B) Linear scales.......56

Figure 3.17 Underwater TL data and comparison to Bellhop and RAM simulations.......59

Figure 3.18 Cannon blast sound pressure level $(1 \mathrm{~m})$ averaged for all experiments........60

Figure 3.19 Signal-to-Noise Ratio for multiple cannon blasts at various ranges. ............61

Figure 3.20 Atmospheric environment model in ACTUP.........................................62

Figure 3.21 Atmospheric sound speed profile from wind speed and temperature data. For inputs: $T=-16.4^{\circ} \mathrm{C}, V w=4.48 \mathrm{~m} / \mathrm{s}, h=10 \mathrm{~m}, b=1$. .64

Figure 3.22 Air column properties within the atmospheric ACTUP model. .64 
Figure 3.23 Simulated Bellhop atmospheric TL vs. range and depth at $500 \mathrm{~Hz}$. Acoustic source depth at $99 \mathrm{~m}$ (one meter above the ice)....................................................66

Figure 3.24 Simulated RAM atmospheric TL vs. range and depth at $500 \mathrm{~Hz}$. Acoustic source depth at $99 \mathrm{~m}$ (one meter above the ice).

Figure 3.25 Atmospheric TL comparison for Bellhop and RAM simulations at $500 \mathrm{~Hz}$. Range detail shown with (A) Log and (B) Linear scales.......................................67

Figure 3.26 Atmospheric TL for $1 / 3$ - Octave bands $20 \mathrm{~Hz}$ to $63 \mathrm{~Hz}$............................70

Figure 3.27 Atmospheric TL for $1 / 3$ - Octave bands $80 \mathrm{~Hz}$ to $250 \mathrm{~Hz}$.............................71

Figure 3.28 Atmospheric TL for $1 / 3$ - Octave bands $315 \mathrm{~Hz}$ to $1000 \mathrm{~Hz}$.........................72

Figure 3.29 Atmospheric TL for $1 / 3$ - Octave bands $1250 \mathrm{~Hz}$ to $4000 \mathrm{~Hz}$......................73

Figure 3.30 Atmospheric TL for $1 / 3$ - Octave bands $5000 \mathrm{~Hz}$ to $8000 \mathrm{~Hz}$......................74

Figure 3.31 Underwater and atmospheric broadband averaged TL comparison................75

Figure 3.32 Ice cylinder with frozen-in PVC for ice borehole experiment. ......................77

Figure 3.33 Air to Water FRF comparison with open and closed ice borehole..................78

Figure 3.34 Ice layer wave-speed diagram. Wave types considered for the ice layer are compressional, shear, Rayleigh, and flexural. Air and water boundary conditions effect the through-thickness resonant frequency in drivepoint mobility. Force input $F$ and geophone responses $u i$ are measured quantities.

Figure 3.35 Hammer impulse and geophone response of ice layer. (A) Hammer impact. (B) Geophone traces. (C) Hammer impact zoomed in. (D) Geophone traces zoomed in with higher gain.

Figure 3.36 Theoretical compressional and shear wave speed. Ice density $(\rho)$ assumed $915 \mathrm{~kg} / \mathrm{m}^{3}$. A) Compressional speed for multiple $E$. Measured compressional wave speed $2657 \mathrm{~m} / \mathrm{s}$ shown as horizontal line. B) Shear wave speed.

Figure 3.37 Flexural wave dispersion curve from geophone data $48 \mathrm{~m}$ from source. Flexural waves begin at approximately $100 \mathrm{~ms}$. Wave arrivals preceding $100 \mathrm{~ms}$ are compressional, shear, or Rayleigh waves.

Figure 3.38 Theoretical flexural wave speed for thin plates per Equation 3.43. Measured flexural speed and frequency indicated with red data points. .85

Figure 3.39 Transcendental equation for determining modal frequencies. Throughthickness modal frequencies in the ice layer are determined at the intersections.. 90 
Figure 3.40 Drivepoint FRF (mobility) for hammer input and geophone response. (A) Hammer input autopower spectra (B) Drivepoint mobility (C) Phase of FRF (D) Coherence

Figure 4.1 Spatial filter width for classic beamforming in ULA with various number of sensors.

Figure 4.2 Simulated MUSIC DOA estimation for various number of sensors in ULA.

Two sources of $2000 \mathrm{~Hz}$ at $-18^{\circ}$ and $52^{\circ}, \mathrm{N}=1024$ samples, $\mathrm{d}=0.0508 \mathrm{~m}$.

Figure 4.3 Microphone configuration and coordinate system for atmospheric $(p p)$ vector sensor.

Figure 4.4 Data processing flow for atmospheric pp AVS.

Figure 4.5 Moving Standard Deviation (MSD) processing routine block diagram.........108

Figure 4.6 Image Source-Path-Receiver diagram for atmospheric AVS.

Figure 4.7 Vector sensor (Meggitt VS-209) with local Cartesian axes identified. Pitch, Roll, and Heading are defined as rotation about the $\mathrm{Y}, \mathrm{Z}$, and $\mathrm{X}$-axes respectfully. 133

Figure 4.8 Snowmobile used for underwater vector sensor tracking experiment shown at site 6 near the start of the snowmobile track.

Figure 4.9 Snowmobile position measured over 120 seconds by handheld GPS onboard the snowmobile. Underwater vector sensor measurements were made at sites 2 and 3. GPS coordinates of sites 2 and 3 are: N 71.36630, W 156.64004 and $\mathrm{N}$ 71.36557, W 156.63444 respectively.

Figure 4.10 Map of approximate vector sensor test range location (red box) at KRC...114

Figure 4.11 (A) $p p$ vector sensor setup at KRC test site. (B) AVS orientation with respect to north and west. (C) SQobold data system and Motorola two way-radio for triggering data collects. (D) Microphones in the 3D printed fixture without the windscreen.

Figure 4.12 (A) Polaris Ranger driven around the test sites at KRC. (B) GPS/GLONASS antenna and differential correction antenna (C) VBOX GPS System in Ranger cabin.

Figure 4.13 Underwater AVS measurement for stationary pings. Signal and background noise level in selected frequency bands. Low-frequency data (left) and highfrequency data (right) are shown for the vector sensor hydrophone (upper) and accelerometer (lower). 
Figure 4.14 Underwater AVS data at site 1 for stationary underwater sound source at site 4 Low-frequency ping range. Mean azimuth and elevation angles are shown with red dashed lines.

Figure 4.15 Underwater AVS data at site 1 for stationary underwater sound source at site 4 High-frequency ping range. Mean azimuth and elevation angles are shown with red dashed lines.

Figure 4.16 (A) Azimuth and (B) elevation spectra measured at site 2. Mean and standard deviation indicated for the selected time instant $\mathrm{T}=38$ seconds. .123

Figure 4.17 Mean azimuth angle computed from the underwater AVS(A) site 2, and (B) site 3, compared to ground truth GPS DOA. AVS DOA averaged between 10 to $5000 \mathrm{~Hz}$ at each time step. (C) Standard deviation of the DOA computed at each time step. .125

Figure 4.18 Mean elevation angle computed from the underwater AVS(A) site 2, and (B) site 3, compared to ground truth GPS elevation. AVS DOA averaged between 10 to $5000 \mathrm{~Hz}$ at each time step. (C) Standard deviation of the elevation computed at each time step

Figure 4.19 Mean range from measurement site to the snowmobile computed from the underwater vector sensor(A) site 2 and (B) site 3 compared to ground truth GPS range.

Figure 4.20 Underwater AVS signal to noise ratio during the 120 -second measurement period (A) hydrophone and (B) accelerometer.

Figure 4.21 Selected example time instances and localization areas. Dashed lines indicate the standard deviation beams for sites 2 (blue) and 3 (red).

Figure 4.22 Selected example time instances when it was not possible to localize the snowmobile.

Figure 4.23 Selected time instance $(\mathrm{T}=32 \mathrm{~s})$ with localization area computed (A) without elevation circle and (B) with elevation circle.

Figure 4.24 Localized area as a function of time. All localization instances are represented using azimuth data alone as well as azimuth plus elevation data when possible

Figure 4.25 Localized area as a function of azimuth angle. All localization instances are represented for both site 2 and site 3 using azimuth data alone as well as azimuth plus elevation data when possible. 133

Figure 4.26 GPS track of the Polaris vehicle maneuvers for experiment 90. .136 
Figure 4.27 (A) SPL and (B) DOA for site 100 experiment 90 at $\mathrm{t}=25$ seconds. Mean and standard deviation computed across the entire spectra. Actual DOA $=90^{\circ}$.

Figure 4.28 DOA spectra and MSD for time instant $t=25$ seconds. MSD computed across 50 frequency lines. Frequencies included for averaging when standard deviation less than $20^{\circ}$. Actual DOA $=90^{\circ}$ .138

Figure 4.29 Site 100 AVS DOA error for various number of frequency lines in $\sigma$ processing. Experiment 90. $\mathrm{N}=25600$ samples, $\sigma c=20^{\circ}$

Figure 4.30 Site 100 AVS DOA error for various standard deviation cutoffs. Experiment $90, \mathrm{~N}=25600$ samples, $n \Delta f=10$ frequency lines.

Figure 4.31 Site 100 AVS DOA error for various blocksizes. Experiment 90, $n \Delta f=10$ frequency lines, and $\sigma c=20^{\circ}$.

Figure 4.32 pp vector sensor data for experiment 90. (A) Site 100 DOA. (B) Site 200 DOA. (C) Absolute angular error between DOA measured with AVS and ground truth. (D) Standard deviation of site 100 and site 200 DOA. (E) SNR at measurement locations. (F) Vehicle speed. DOA data in this figure are computed with MSD processing.

Figure 4.33 Zoomed in view of Figure 4.32 from 20 to 60 seconds. .143

Figure 4.34 Comparison of integration vs MSD method for DOA estimation, experiment 80 .

Figure 4.35 Azimuthal AVS error site 200, experiment 80. $\mathrm{N}=25600, n \Delta f=10, \sigma c=20^{\circ}$. (A) FEA error for stationary source with infinite SNR from Wajid et al. (B) Measured DOA error, (C) SNR, (D) Vehicle speed.

Figure 4.36 Azimuthal AVS error site 200, experiment 83. $\mathrm{N}=25600, n \Delta f=10$, $\sigma c=20^{\circ}$.(A) FEA error for stationary source with infinite SNR from Wajid et al. (B) Measured DOA error, (C) SNR, (D) Vehicle speed.

Figure 4.37 Vehicle speed characteristics for experiments 79 to 83 relative to site 200 . (A) Vehicle Speed. (B) Distance between site 200 and vehicle position. (C) Angular Speed around site 200.

Figure 4.38 Analytical AVS DOA error considering direct path propagation only. $h s=$ $1 \mathrm{~m}, f=1000 \mathrm{~Hz}$. 150

Figure 4.39 Analytical AVS DOA error considering direct and reflected paths. $h s=1 \mathrm{~m}$, $f=1000 \mathrm{~Hz}$. .150 
Figure 4.40 Source height to wavelength ratio for various frequencies.

Figure 4.41 DOA error for various $h s / \lambda$ with increasing source height and constant frequency $f=1000 \mathrm{~Hz}$.

Figure 4.42 DOA error for various $h s / \lambda$ with increasing frequency and constant source

height $h s=1 \mathrm{~m}$.

Figure 4.43 Example localization estimation utilizing DOA $\pm \sigma$ lines for $\mathrm{t}=9.5$ seconds.

Figure 4.44 Square root localization area for experiments with vehicle traveling in circular pattern.

Figure 4.45 Square root localization area for experiments 90 and 91 (irregular pattern).

Figure 4.46 Square root localization area for experiments in between AVS sties.

Figure 4.47 Percent localization for all experiments.

Figure A.1 Weather station data for February $4^{\text {th }} 2018$. Measured at Great Lakes Research Center in Houghton, MI. Experiments on Portage Lake took place during times between the vertical dashed lines.

Figure A.2 Weather station data for February $8^{\text {th }}$ 2018. Measured at Great Lakes Research Center in Houghton, MI. Experiments on Portage Lake took place during times between the vertical dashed lines.

Figure A.3 Portage Lake bathymetry map for test site area.

Figure A.4 Portage Lake relative bottom hardness for test site area. Absolute units are not reported.

Figure A.5 Impulse response function $48 \mathrm{~m}$ from the underwater source. (A) Full IRF from MLS signal. (B) Incident arrival. (C) Windowed ice reflection (D) Windowed bottom reflection.

Figure A.6 (A) Frequency response functions and (B) Reflection loss for $\phi I=8.5^{\circ}$ and $\phi B=16^{\circ}$. Source to receiver distance equal to $48 \mathrm{~m}$.

Figure A.7 Impulse response function $210 \mathrm{~m}$ from the underwater source. (A) Full IRF from MLS signal. (B) Incident arrival. (C) Windowed ice reflection (D) Windowed bottom reflection. 
Figure A.8 (A) Frequency response functions and (B) Reflection loss for $\phi I=2^{\circ}$ and $\phi B=4^{\circ}$. Source to receiver distance equal to $210 \mathrm{~m}$.

Figure A.9 AVS measurement channel phase mismatch for site 100 and site 200 .........183

Figure A.10 Vehcile GPS tracks for AVS experiments 62 through 73 ..........................184

Figure A.11 Vehcile GPS tracks for AVS experiments 74 through 85 ..........................185

Figure A.12 Vehcile GPS tracks for AVS experiments 86 through 92 ……...................186

Figure A.13 DOA from site 100 (red dot) and GPS ground truth (black). AVS experiments 62 to 73 ......

Figure A.14 DOA from site 100 (red dot) and GPS ground truth (black). AVS experiments 74 to 85 .

Figure A.15 DOA from site 100 (red dot) and GPS ground truth (black). AVS experiments 86 to 92 .

Figure A.16 DOA from site 200 (blue dash) and GPS ground truth (black). AVS experiments 62 to 73 .

Figure A.17 DOA from site 200 (blue dash) and GPS ground truth (black). AVS experiments 74 to 85 .

Figure A.18 DOA from site 200 (blue dash) and GPS ground truth (black). AVS experiments 86 to 92 .

Figure A.19 DOA standard deviation for sites 100 (red dot) and 200 (blue dash). AVS experiments 62 to 73 .

Figure A.20 DOA standard deviation for sites 100 (red dot) and 200 (blue dash). AVS experiments 74 to 85 .

Figure A.21 DOA standard deviation for sites 100 (red dot) and 200 (blue dash). AVS experiments 86 to 92

Figure A.22 SNR for sites 100 and 200. AVS experiments 62 to 73.

Figure A.23 SNR for sites 100 and 200. AVS experiments 74 to 85 .

Figure A.24 SNR for sites 100 and 200. AVS experiments 86 to 92 .

Figure A.25 Target Vehicle Speed. AVS experiments 62 to 73. 193

Figure A.26 Target Vehicle Speed. AVS experiments 74 to 85 . 
Figure A.27 Target Vehicle Speed. AVS experiments 86 to 92.

Figure A.28 Vehicle speed characteristics for experiments 67 to 72 relative to site 100 . (A) Vehicle Speed. (B) Distance between site 100 and vehicle position. (C) Angular Speed around site 100.

Figure A.29 pp AVS error at site 100, experiment 67. (A) DOA error and (B) SNR (C) Vehicle speed.

Figure A.30 pp AVS error at site 100, experiment 68. (A) DOA error and (B) SNR (C) Vehicle speed. 196

Figure A.31 pp AVS at site 100, experiment 69. (A) DOA error and (B) SNR (C) Vehicle speed.

Figure A.32 pp AVS error at site 100, experiment 70. (A) DOA error and (B) SNR (C) Vehicle speed.

Figure A.33 pp AVS error at site 100, experiment 72. (A) DOA error and (B) SNR (C) Vehicle speed.

Figure A.34 pp AVS error at site 100, experiment 72. (A) DOA error and (B) SNR (C)

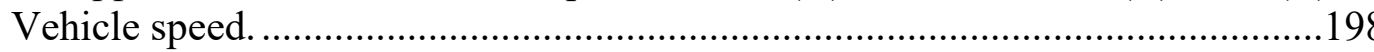

Figure A.35 pp AVS error at site 200, experiment 79. (A) DOA error and (B) SNR (C) Vehicle speed.

Figure A.36 pp AVS error at site 200, experiment 81. (A) DOA error and (B) SNR (C) Vehicle speed.

Figure A.37 pp AVS error at site 200, experiment 82. (A) DOA error and (B) SNR (C) Vehicle speed. 


\section{List of tables}

Table 2.1 Distance between source and receiver sites in meters. .....................................

Table 3.1 Acoustic experiment site GPS position and distances on Portage Lake............32

Table 3.2 Transducer model numbers and sensitivities used on Portage Lake .................33

Table 3.3 Underwater Transmission Loss Data. Data measured on Portage Lake under

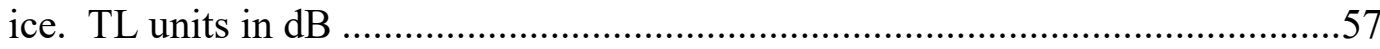

Table 3.4 Atmospheric Transmission Loss Data. ........................................................68

Table 3.5 Ice layer sound speeds and elastic properties. Comparison of theoretical and measured speeds of in the ice and estimation of ice layer elastic properties. Theoretical sound speeds computed with the elastic properties shown

Table 3.6 Input properties for theoretical FRF .89

Table 4.1 Atmospheric pp AVS experiments description of target vehicle maneuvers ..117

Table 4.2 Gravity and magnetic field sensor data over the course of one 120-second data collect.

Table 4.3 Underwater AVS localized area statistics for selected time intervals. Time intervals represent instances when there was low SNR (row 2) or high SNR (row 3 ) at the vector sensor measurement location.

Table 4.4 Square root localization area statistics for all vehicle tracking experiments. The values are represented as the square root of the area for improved intuition. .....159 


\section{Author contribution statement}

Chapter 2 of this dissertation is reproduced from:

Miles Penhale, Andrew Barnard and Robert Shuchman, "Multi-Modal and Short-Range Transmission Loss in Thin, Ice-Covered, Near-Shore Arctic Waters", J. Acoust. Soc. Am. 143(5), 3126-3137 (2018),

with permission of the Acoustical Society of America.

Copyright documentation may be seen in Appendix C. 


\section{Acknowledgments}

First and foremost, I would like to thank my parents. I would never have been able to make it through my many years of education without their unwavering love and support.

To Joy, my loving girlfriend. Thank you so much for supporting me throughout this process. Working on a Ph.D. has demanded a majority of my focus for many years now. You have been very patient and understanding despite it being stressful at times. Thank you :)

I would like to thank my advisor Andrew Barnard for his guidance throughout my graduate studies. Andrew, I don't believe that I would have been successful in grad school had it not been for having such an excellent advisor. You have pushed me to understand concepts I never would have pursued on my own. Thank you for always being an advocate and a mentor for me along the way.

Thanks to my committee members Chuck Van Karsen, Jason Blough, and Roger Turpening.

Chuck, I wouldn't be where I am today without your teaching. It was your mechanical vibrations class where I first found interest in all things NVH and acoustics. I'll never forget, "Thinking is not optional!" Truer words haven't been spoken. Your encouragement, stories, and subtle humor have never gone unappreciated. Thanks for being a great mentor to me.

Jason, I hear that Cincinnati folks can't get their Ph.D. until they are 30 years old. Hopefully the same doesn't apply to Tech students or else I have about three more Houghton winters ahead of me (not that I would mind that, but I think I'm ready to graduate). Thanks for providing your insight on my experiments and helping me understand many complex data processing techniques.

Roger, your guidance on seismology concepts and the underlying mathematics was very much needed and appreciated. My knowledge has been significantly broadened by our discussions and your suggested readings. I will miss stopping by your office to discuss an interesting idea or hear one of your stories. Thanks for everything.

Thanks to Bob Shuchman, Joe Burns, Christopher Roussi, Brian Wilson, and Benjamin Hart at Michigan Technological Research Institute for facilitating data collection in Barrow and allowing me to join their expedition in the first place. Our trip to Barrow kicked off my endeavors in Arctic acoustics. A trip that I will frequently and fondly remember.

Thanks to Dayton Pax and Jamey Anderson for help with ArcMap GIS and extracting my bathymetry data on Portage Lake. I would have been lost on my own. 
Guy Meadows, Mike Abbot, and Chris Pinnow at the Great Lakes Research Center. You all have helped me very much along the way to facilitate my data collection on Portage Lake. Thank you!

Geoff Gwaltney and Jim Waineo at Keweenaw Research Center for help with vector sensor testing. You both provided several suggestions which significantly simplified my testing procedures and data processing. Not to mention providing the test range and much of the hardware that I needed. Thanks for all your help!

Thanks to Nancy Barr for teaching me how to write and present my research more clearly. STEM research is meaningless if it can't be communicated. I'm still not an expert communicator, but I think I am much better after your classes, Nancy, thank you!

Marty Toth and Steve Lehman, thanks for your design suggestions, help with machining, and hardware development. I'll miss coming up to the $6^{\text {th }}$ floor MEEM to have a chat. Stand by!

To all my friends at Michigan Tech, Jon, Will, Eric, Ben, Joe, Josh, and so many others...my time here has been significantly enriched by our experiences and adventures. As we have been studying hard in grad school, perhaps the grass has seemed greener elsewhere, but I now truly think that the grass really is greenest at Tech (or, should I say the snow is the whitest? ... Anyway, perhaps Josh can confirm). Thanks to all of you, I have a vast collection of memories which will forever highlight my time in Houghton and Michigan's Upper Peninsula.

The work in Chapter 2 was funded by DARPA under contract number W15QKN-16-C0018. The DARPA Program Manager for this project was Dr. John Kamp, Strategic Technology Office (STO). Upon his retirement from DARPA during the project, Dr. Kamp was replaced by Dr. Lisa Zurk (STO).

Much of my research has been funded by the Office of Naval Research. I am truly grateful for the multiple fellowships that have been awarded to me through ONR to perform my research. Thanks to Bob Headrick of ONR for helping me to secure several of these.

Finally, thanks to anyone that I have missed. So many people have helped me along the way. 
To sailors of the Big Lakes.

\author{
Lake Huron rolls, Superior Sings \\ In the ruins of her ice water mansion. \\ Old Michigan steams like a young man's dreams, \\ The islands and bays are for sportsmen. \\ And farther below Lake Ontario \\ Takes in what Lake Erie can send her \\ And the iron boats go as the mariners all know \\ With the gales of November remembered.
}

Gordon Lightfoot, The wreck of the Edmund Fitzgerald 


\section{Terms, Abbreviations, Nomenclature, and Symbols}

A: amplitude, 46

$A_{n}: n^{\text {th }}$ residue of a system, 88

$A_{\text {poly }}$ : area of polygon, 111

$A_{\text {segment: }}$ area of segment of circle, 111

ACTUP: acoustic toolbox user-interface and post-processor, 52

AVS: acoustic vector sensor, 95

Bellhop: ray tracing acoustic simulation, 52

c: speed of sound (air), 14

$\mathrm{c}(\mathrm{r}, \mathrm{z})$ : sound speed profile, 50

$\mathrm{c}_{0}$ : nominal sound speed, 63

$\mathrm{c}_{\text {eff }}(\mathrm{z})$ : effective sound speed, 63

$\mathrm{c}_{\mathrm{f}}$ : flexural wave speed, 80

$\mathrm{C}_{\mathrm{I}}$ : ice sound speed (compressional), 89

$\mathrm{c}_{\mathrm{p}}$ : compressional wave speed in a plate, 79

$\mathrm{C}_{\mathrm{R}}$ : Rayleigh wave speed, 80

$\mathrm{c}_{\mathrm{s}}$ : shear wave speed, 79

$\mathrm{c}_{\mathrm{w}}$ : water sound speed, 89

$\mathrm{COH}$ : coherence, 17

CORS: continuously operating reference stations, 115

CTD: conductivity, temperature, and depth (probe), 16

d: environment depth, 46; sensor spacing in an array, 96

D: flexural rigidity, 18; upper boundary of the depth dimension, 47

$\mathrm{D}_{\mathrm{S}}$ : source depth, 123

$\mathrm{D}_{\mathrm{VS}}$ : vector sensor depth, 104

dB: decibel, 25

DOA: direction of arrival, 95

E: elastic modulus, 18; error function, 109

f: frequency in cycles per second $(\mathrm{Hz}), 18$

F: force, 80, 99

$\mathrm{f}_{\mathrm{n}}$ : natural frequency $(\mathrm{Hz}), 88$

$\mathrm{F}_{\mathrm{s}}$ : sample rate, 107

FFP: fast field program, 45

FFT: fast Fourier transform, 16

FHT: fast Hadamard transform, 38

FRF: frequency response function, 6

g: gravitational field sensor vector, 103; wavenumber kernel, 48

G: shear modulus, 79

$\mathrm{G}_{\mathrm{xx}}$ : Auto-power spectrum of $\mathrm{x}, 16$

$\mathrm{G}_{\mathrm{yx}}$ : Cross-power spectrum between $\mathrm{x}$ and $\mathrm{y}, 16$ 
GPS: global positioning system, 7

GUI: graphical user interface, 51

h: sensor height for measuring wind speed, 63

$\mathrm{H}_{0}^{1}$ : zeroth order Hankel function of the first kind, 47

$\mathrm{h}_{\mathrm{r}}$ : receiver height, 110

$\mathrm{h}_{\mathrm{s}}$ : source height, 110

I: acoustic intensity, 98

$\mathrm{I}_{\mathrm{a}}$ : active intensity, 100

$\mathrm{I}_{\mathrm{mn}}$ : intensity between microphones $\mathrm{m}$ and $\mathrm{n}, 105$

$\mathrm{I}_{\mathrm{r}}$ : reactive intensity, 100 ; sound intensity at the receiver, 35

$\mathrm{I}_{\mathrm{s}}$ : sound intensity at the source, 35

IMU: inertial measurement unit, 115

IRF: impulse response function, 38

$j$ : imaginary unit, 104

$\mathrm{J}_{0}$ : zeroth-order Bessel function, 48

$\mathrm{k}$ : wavenumber, 45

Kracken: normal modes acoustic simulation, 52

KRC: Keweenaw research center, 114

$L$ : ice thickness, 18; length of pseudo-random signal, 38; plate thickness, 80

LA: localization area, 154

m: magnetic field sensor vector, 103; number of modes of a system, 88

M: modal mass, 89; number of sensors in an array, 96

MDOT: Michigan department of transportation, 115

MLS: maximal length sequence, 37

MSD: moving standard deviation, 108

$\mathrm{N}$ : blocksize (ie. number of samples), 107

$\mathrm{n}(\mathrm{r}, \mathrm{z})$ : index of refraction, 50

$\mathrm{n}_{\mathrm{ac}}$ : magnetic north rotational vector, 103

NAH: near-field acoustic holography, 95

NI: national instruments, 7

NM: normal modes, 45

$\mathrm{n} \Delta \mathrm{f}$ : number of frequency lines, 108

p: acoustic pressure, $47,86,98$

P: phase, 46

pa: pressure-particle acceleration, 95

PE: parabolic equation, 45

pp: pressure-pressure, 95

PSD: power spectral density, 11

pu: pressure-particle velocity, 95

PVC: polyvinal chloride, 76

$\mathrm{Q}$ : volume velocity, 110

$\mathrm{Q}_{\text {fixed }}^{\mathrm{T}}$ : vector sensor transformation matrix, 103

r: range dimension, 45

R: radius of vehicle path around AVS site, 149; specific gas constant, 13 
$\mathrm{R}_{\mathrm{GPS}}$ : ground truth range from source to receiver from GPS, 109

$\mathrm{R}_{\mathrm{VS}}$ : range from source to receiver determined by vector sensor, 104

RAM: parabolic equation acoustic simulation, 52

RL: reflection loss, 36

RT: ray tracing, 45

RTK: real-time kinematic, 115

$\mathrm{S}_{\mathrm{x}}$ : linear spectra of $\mathrm{x}, 16$

Scooter: fast field program and Green's function acoustic simulation, 52

SL: Sturm-Liouville, 47

SLA: square root of localization area, 154

SNR: signal to noise ratio, 7

SONAR: sound navigation and ranging, 95

SPL: sound pressure level, 10

$\mathrm{t}$ : time variable, 45

T: block time interval, 107

$\mathrm{T}_{\mathrm{k}}$ : temperature in Kelvin, 13

TL: transmission loss, 35

$\mathrm{TL}_{\mathrm{AA}}$ : atmospheric transmission loss, 25

$\mathrm{TL}_{\mathrm{AIW}}$ : air-ice-water transmission loss, 26

$\mathrm{TL}_{\mathrm{UW}}$ : underwater transmission loss, 24

$\mathrm{u}$ : general function of $\mathrm{x}$ and $\mathrm{y}, 49$; particle velocity, 98

$\mathrm{u}_{\mathrm{ac}}$ : upward rotational vector, 103

$\mathrm{u}_{\mathrm{i}}$ : particle velocity of ice, 80

ULA: uniform linear array, 96

UTC: coordinated universal time, 112

UTV: utility task vehicle, 115

uu: particle velocity - particle velocity, 95

$\mathrm{v}$ : linear vehicle speed, 149

$\mathrm{V}_{\mathrm{b}}$ : volume of brine in ice, 18

$\mathrm{v}_{\mathrm{p}}$ : compressional wave velocity, 18

$\mathrm{V}_{\mathrm{w}}$ : wind velocity, 63

W: mechanical work, 99; sound power, 22

$\mathrm{w}_{\mathrm{ac}}$ : magnetic west rotational vector, 103

z: depth dimension, 45; thickness dimension of ice, 86

Z: acoustic impedance, 86

$\alpha$ : absorption coefficient, 27; cross-sectional area, 46

$\gamma$ : air coupled flexural wave dimensionless parameter, 22; ratio of specific heats, 13

$\Delta \mathrm{f}$ : frequency resolution, 107

$\triangle \mathrm{LAT}$ : difference in latitude between source and receiver, 108

$\triangle \mathrm{LON}$ : difference in longitude between source and receiver, 109

$\delta$ S: differential area of fluid, 99

$\Delta \mathrm{t}$ : time between samples, 107

$\Delta \phi:$ phase difference, 102

$\zeta$ : damping ratio, 89 
$\dot{\theta}:$ angular speed of vehicle around AVS site, 149

$\bar{\theta}$ : mean direction of arrival, 107

$\theta_{\mathrm{GPS}}$ : ground truth azimuth direction of arrival from GPS, 109

$\theta_{\mathrm{i}}$ : incident angle, 110

$\theta_{\mathrm{r}}:$ reflected angle, 110

$\theta_{\mathrm{Vs}}$ : direction of arrival or azimuth of vector sensor, 104

$\lambda$ : wavelength, 45

$\Lambda$ : seperation constant, 47

$\Lambda_{\mathrm{m}}$ : eigenvalue, 47

$\lambda_{\mathrm{n}}: \mathrm{n}^{\text {th }}$ pole of a system, 88

$v$ : Poisson's ratio, 18

$\rho$ : density, 18

$\rho_{\mathrm{I}}$ : ice density, 89

$\rho_{\mathrm{w}}$ : water density, 89

$\sigma$ : standard deviation, 129,154

$\sigma(\theta)$ : standard deviation of direction of arrival, 107

$\sigma_{c}:$ standard deviation cutoff, 108

$\Phi$ : function of spatial and time dimensions, 45

$\phi$ : function of spatial variables in helmholtz equation, 45

$\phi_{\mathrm{B}}$ : bottom reflection grazing angle, 41

$\Phi_{\mathrm{GPS}}$ : ground truth elevation direction of arrival from GPS, 109

$\phi_{\mathrm{I}}$ : ice reflection grazing angle, 41

$\phi_{\mathrm{p}}$ : phase of acoustic pressure, 100

$\phi_{\mathrm{u}}$ : phase of acoustic particle velocity, 100

$\Phi_{\mathrm{VS}}$ : elevation direction of arrival of vector sensor, 104

$\psi$ : function of depth and range in Helmholtz equation, 50

$\psi_{\mathrm{B}}$ : with of array spatial filter response, 96

$\psi_{1}^{\mathrm{m}}$ : field variable at a given point $(\mathrm{m}, 1), 50$

$\Psi_{\mathrm{m}}(\mathrm{z})$ : eigenfunction, 47

$\omega:$ circular frequency $(\mathrm{rad} / \mathrm{s}), 80$

$\omega_{\mathrm{d}}:$ damped natural frequency $(\mathrm{rad} / \mathrm{s}), 89$

$\omega_{\mathrm{n}}:$ natural frequency $(\mathrm{rad} / \mathrm{s}), 89$ 


\section{Abstract}

The Arctic environment has undergone significant change in recent years. Multi-year ice is no longer prevalent in the Arctic. Instead, Arctic ice melts during summer months and re-freezes each winter. First-year ice, in comparison to multi-year ice, is different in terms of its acoustic properties. Therefore, acoustic propagation models of the Arctic may no longer be valid. The open water in the Arctic for longer time periods during the year invites anthropogenic traffic such as civilian tourism, industrial shipping, natural resource exploration, and military exercises. It is important to understand sound propagation in the first-year ice environment, especially in near-shore and shallow-water regions, where anthropogenic sources may be prevalent. It is also important to understand how to detect, identify, and track the anthropogenic sources in these environments in the absence of large acoustic sensory arrays.

The goals of this dissertation are twofold: 1) Provide experimental transmission loss (TL) data for the Arctic environment as it now exists, that it may be used to validate new propagation models, and 2) Develop improved understanding of acoustic vector sensor (AVS) performance in real-world applications such as the first-year Arctic environment.

Underwater and atmospheric acoustic TL have been measured in the Arctic environment. Ray tracing and parabolic equation simulations have been used for comparison to the TL data. Generally, good agreement is observed between the experimental data and simulations, with some discrepancies. These discrepancies may be eliminated in the future with the development of improved models.

Experiments have been conducted with underwater $p a$ and atmospheric $p p$ AVS to track mechanical noise sources in real-world environments with various frequency content and signal to noise ratio (SNR). A moving standard deviation (MSD) processing routine has been developed for use with AVS. The MSD processing routine is shown to be superior to direct integration or averaging of intensity spectra for direction of arrival (DOA) estimation. DOA error has been shown to be dependent on ground-reflected paths for $p p$ AVS with analytical models. Underwater AVS have been shown to be feasible to track on-ice sources and atmospheric AVS have been shown feasible to track ground vehicle sources. 


\section{Introduction}

\subsection{Motivation of Research}

In recent years, primarily the last two decades, the ice in the Arctic is thinning and melting altogether during the summer months. This leads to ice which re-forms during each new winter season; i.e. first-year ice. This is contrary to the multi-year ice environment which has dominated the Arctic for the past several centuries. In January 1984, roughly $30 \%$ of the Arctic ice was first-year ice, $40 \%$ of the ice was between 2 to 4 years in age, and $30 \%$ of the ice was greater than 4 years old ${ }^{1}$. By comparison, in January 2015 , greater than $60 \%$ of the ice makeup was first-year ice, $35 \%$ of the ice was between 2 to 4 years old, and less than $5 \%$ of the ice was greater than 4 years old ${ }^{1}$ (See Figure 1.1 and Figure 1.2).

First-year ice is very different in terms of its acoustic properties when compared to multiyear ice. The first-year ice tends to create complex acoustic environments in the nearshore zones of the Arctic due to ice ridging. Acoustic propagation is not well understood in this environment. Additionally, the increasing absence of ice during the summer months and the reduction of ice in the spring, fall, and winter is inviting for human exploration, shipping, tourism, military exercises, and other anthropogenic activities.

In this relatively new Arctic environment, it is of interest: 1) to understand how sound propagates and decays throughout the environment; and 2) to understand how to acoustically detect and track anthropogenic sources in the air, on the ice, and underwater using small sensor packages.

These interests are important because they directly influence military defense capabilities in the Arctic realm. Having sensor packages which are capable to detect and track sources allows for greater awareness, strategic planning, and responsiveness. Typically, the sensor technology which provide these capabilities consist of large sensor arrays and require intensive data processing. Reduction of numbers of sensors and miniaturization of sensor packages will allow for improved rapid deployment and versatility for applications in the near-shore Arctic. Additionally, knowledge regarding propagation and decay of acoustic energy throughout the environment is necessary to understand how relative position between source and sensor effect detection accuracy.

This dissertation will focus on providing data and analysis which will advance the knowledge with regard to these research interests. Sound propagation data for the firstyear Arctic environment will be reported and compared to current propagation models. Tracking and localization experiments will be carried out using small acoustic sensor packages with realistic acoustic sources. 


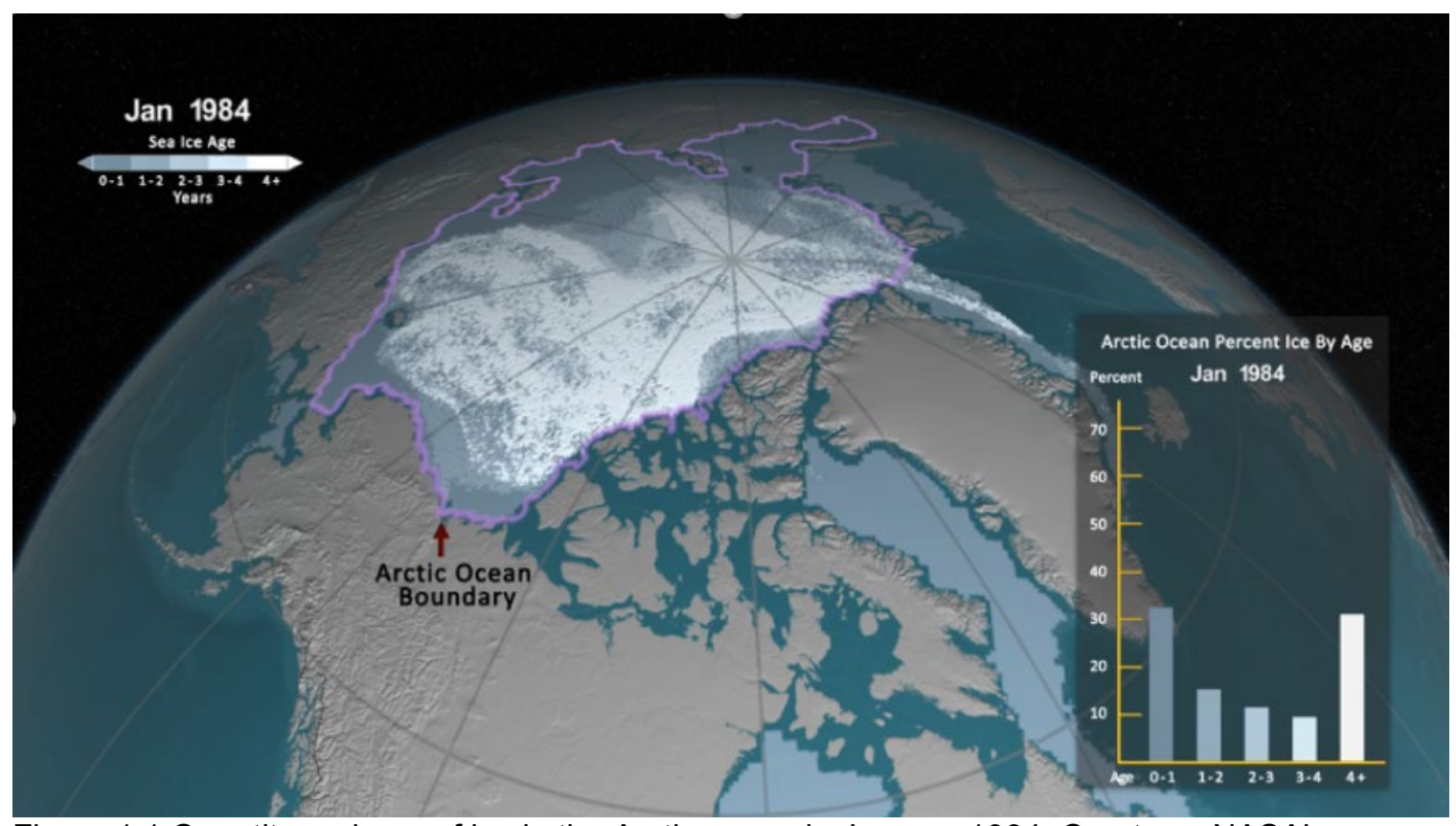

Figure 1.1 Quantity and age of ice in the Arctic ocean in January 1984. Courtesy: NASA's Scientific Visualization Studio ${ }^{1}$

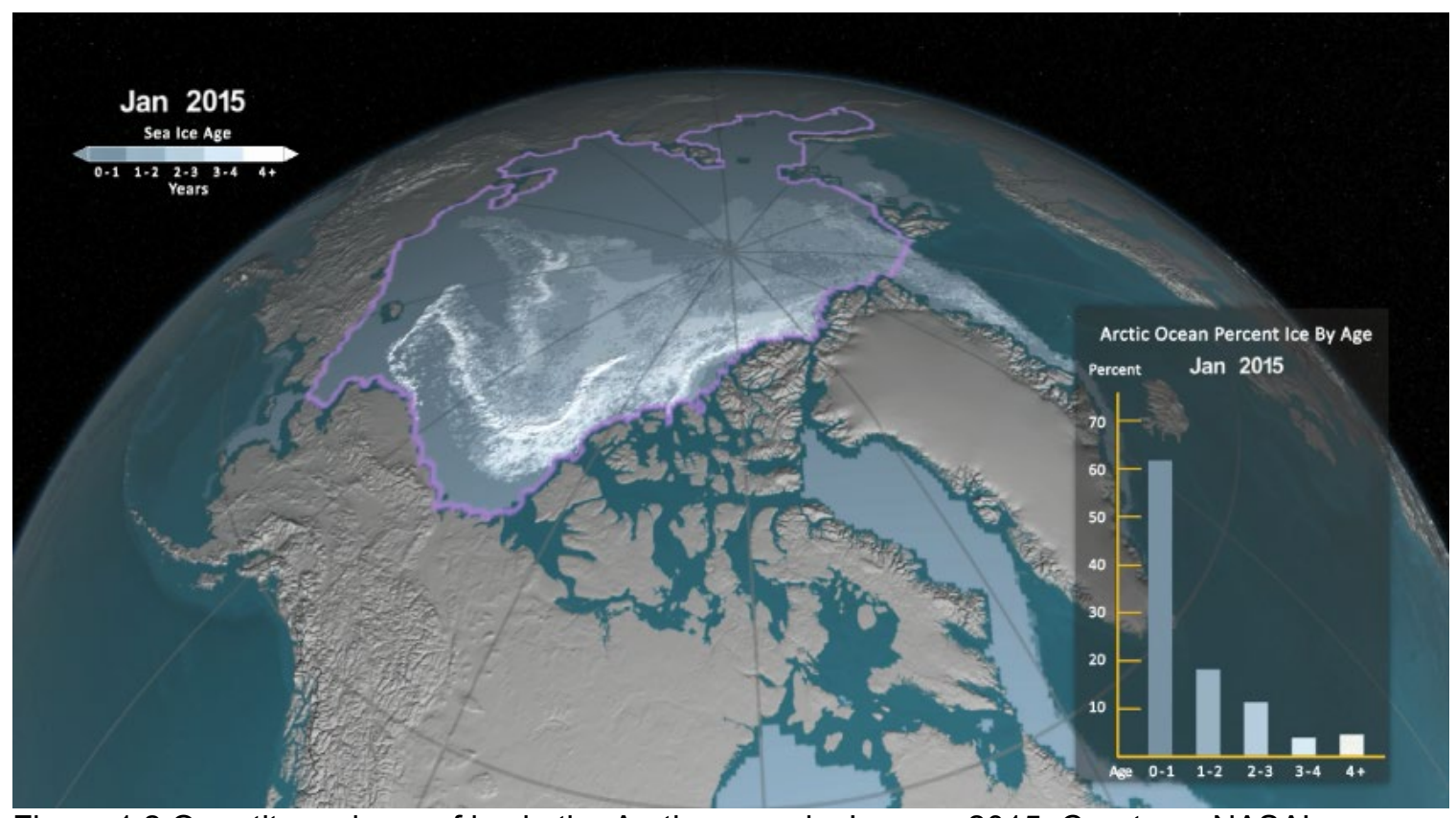

Figure 1.2 Quantity and age of ice in the Arctic ocean in January 2015. Courtesy: NASA's Scientific Visualization Studio ${ }^{1}$ 


\subsection{Explanation of Chapters}

Chapter 2 is a reproduction of a Journal of the Acoustical Society of America publication titled Multi-Modal and Short-Range Transmission Loss in Thin, Ice-Covered, Near-Shore Arctic Waters ${ }^{2}$. This chapter describes acoustic propagation experiments which were carried out during April 2016 in Barrow, Alaska in the first-year, near-shore Arctic ice zone. The collected data are presented and analyzed in terms of transmission loss and frequency response functions. Due to inclement weather conditions in Barrow during the designated test period, the number of days allowed for data collection were cut short and the amount of data collected was far less than originally anticipated. Additionally, information regarding the bottom type, bathymetry, and temporal wind speed at the test area were not able to be collected. Therefore, the collected data lacked spatial resolution and it was not possible to compare the collected data to TL simulations based on environmental factors. Follow-up experimentation was necessary to further understand the acoustic characteristics in this environment.

Chapter 3 describes follow-up experiments conducted in February 2018 in Michigan's Keweenaw Waterway, which is part of Lake Superior. Lake Superior provides a nearshore Arctic-like surrogate environment during the winter months, encompassing similar ice thickness, environmental temperatures and wind speeds, water depths, and sound speed profiles. The caveat being that Lake Superior is entirely freshwater and therefore salinity effects on acoustic propagation cannot be analyzed.

Section 3.3 reports the underwater and atmospheric TL data collected in Lake Superior. The TL data are compared to ray tracing and parabolic equation simulations based on measured environmental parameters such as wind speed, air temperature, bathymetry, reflection loss, and sound speed profiles. Reflection loss of the bottom and under-ice surface are shown to have significant influence on the measured TL. The measurement of reflection loss using a maximal length sequence (pseudorandom signal) is described in section 3.3.1. The simulation techniques used for TL comparison are described in section 3.3.2.

Section 3.4 investigates the effects of ice boreholes on the transmission of acoustic energy between the air and the water. This effect is of interest for sensor packages which are deployed through the ice layer. In such cases, the transmission of acoustic energy directly between the air and the water is shown to be a negligible factor for sensor accuracy.

Section 3.5 investigates the through-thickness resonance in the ice layer in response to impacts on the ice surface. In this section, the elastic properties and sound speeds of the ice layer are determined experimentally. It is shown that knowledge of the acoustic impedance of the ice and water allows for estimation of the ice thickness using dynamic mobility measurements. This insight is useful for rapidly deployed sensor packages in which ice thickness must be determined in situ. 
Chapter 4 describes acoustic tracking experiments utilizing acoustic vector sensors (AVS) of two types; underwater pressure - particle acceleration $(\mathrm{pa})$ and atmospheric pressure - pressure ( $p p)$. AVS were chosen for study due to their compact size and ability to provide direction or arrival (DOA) estimation with few quantities of sensors. The data from the AVS experiments are presented and analyzed in this chapter.

Often, the sources which must be sensed in real-world practical applications - especially in Arctic environments - are non-stationary and emit complex frequency content with various source amplitude in noisy environments. The sources considered in this chapter are specifically selected with respect to these difficult tracking conditions.

Sections 4.3.1 and 4.4.1 describe experiments in which underwater $p a$ AVS were deployed through the ice during testing in Barrow, AK. Data were collected to track a snowmobile traveling on the ice. Sections 4.3.2 and 4.4.2 describe atmospheric $p p$ AVS experiments which were conducted at the Keweenaw Research Center during August 2018 in Calumet, MI. Data were collected to track a ground-based utility vehicle.

A spectral moving standard deviation (MSD) processing routine is developed (Section 4.2.6) to provide improved DOA estimation for these practical tracking applications. In section 4.4.2.3, the MSD processing routine is shown to be superior to traditional integration (or averaging) over the entire frequency band.

Section 4.2.8 describes an analytical simulation for $p p$ AVS. The results of the simulation are compared to the AVS experimental data in sections 4.4.2.4 and 4.4.2.5 in terms of DOA error. The $p p$ AVS error is shown to be influenced by the groundreflected path between the acoustic source and the AVS.

Combining the DOA estimation from multiple sites is shown to provide localization estimation in sections 4.2.9, 4.4.1.4, and 4.4.2.6. Underwater $p a$ AVS are shown to be feasible for DOA estimation and localization of on-ice acoustic sources. Atmospheric $p p$ AVS are shown to be feasible for DOA estimation and localization of ground-based acoustic sources. 


\section{Multi-Modal and Short-Range Transmission Loss in Thin, Ice-Covered, Near-Shore Arctic Waters}

\subsection{Introduction}

In the past century, there has been a great deal of research conducted regarding acoustic propagation in multilayered media such as air, ice, and water. In floating ice sheets, the theory of wave propagation is well developed ${ }^{3-12}$ and has been corroborated by several experiments ${ }^{13-20}$. With the exception of a few studies ${ }^{12,17}$, the majority of this research focuses on low frequency (approximately less than $100 \mathrm{~Hz}$ ) propagation over long-ranges (generally greater than $1 \mathrm{~km}$ ).

It is well known that the global climate change is affecting the Arctic ice layers ${ }^{21-24}$. In general, the ice layer formations are much different than those which were studied in early acoustic experiments. The majority of multi-year pack ice, which has been extensively studied, is now melting between seasons giving rise to an increase of annually formed first-year ice ${ }^{21,22}$. The shore-fast ice sheet has previously been composed of multi-year ice that travels to shore on currents and gets trapped in the first year ice. Due to the overwhelming loss of multi-year ice in the Arctic as a whole, the near-shore environment is now composed of predominantly first-year ice. First-year, shore-fast ice is thinner, more saline, and of different density and strength than multi-year ice $^{25}$ and is deserving of specific study into its acoustic properties.

In addition, this changing Arctic environment warrants new investigation into the acoustic detection, identification, and tracking of anthropogenic sources. Because there is less ice in the Arctic environment for longer time periods during the year, there is expected to be increased near-shore anthropogenic activity ${ }^{24,26-28}$. This activity may come in the form of Arctic shipping through the Northwest Passage, natural resource exploration, and tourism. It is of interest to determine the location and type of these anthropogenic sources. Sensing of sources in the first-year shore-fast ice environment is non-trivial due to ice ridging and ever-changing ice movements. Furthermore, first-year, near-shore ice is not well understood in terms of acoustic properties. Therefore, new data are required to understand the acoustic transmission paths in the first-year, near-shore ice environment and to validate algorithms for detection, identification, and tracking of anthropogenic sources in shallow water (less than $50 \mathrm{~m}$ ) with thin, irregular ice sheets.

Typically, acoustic transmission loss (TL) data have been measured using arrays of geophones on the ice surface, arrays of hydrophones underwater, or microphones in the air. Sometimes combinations of either geophones and hydrophones, or geophones and microphones have been used to better understand acoustic propagation ${ }^{14,15}$. However, the combination of all three sensor modalities is uncommon. Combining a small number of sensors of all three modalities may enhance source detection, identification, and tracking using lower cost sensor nodes. For this reason, this study investigates the simultaneous acquisition of data from all three sensing modalities. 
Early experiments often excited layered media with explosives $3,4,14,17,19$. This excitation method is now less common due to environmental regulation on explosive acoustic sources ${ }^{29}$. Due to these regulations, new excitation methods are necessary. Recent techniques for ice excitation include freezing a wooden or steel post into the ice and hitting the post with a sledgehammer ${ }^{18,30}$. For underwater excitation, lightbulbs, or other crushable containers, have been placed at depth and imploded to create an acoustic source ${ }^{29,30}$. While some studies take care to control the source excitation levels in some manner ${ }^{12,30}$, source levels have not been systematically quantified or measured.

This set of experiments is conducted in Arctic environments regarding the multimodal, short range, and shallow water response to calibrated and measured sources. In the industrial noise and vibration field, a common method for determining acoustic path characteristics in response to a known source level is the calculation of Frequency Response Functions (FRFs) ${ }^{31-33}$. The FRF is a spectral frequency domain ratio of a response measurement to a source measurement. This method is not commonly used in the seismo-acoustic or Arctic-acoustic fields. In this chapter, FRFs are used to quantify the multipath characteristics in the Arctic environment. Using FRF calculations, spectral frequency domain ratios are determined between microphone, geophone, and hydrophone responses to various measured source inputs, namely, a small propane cannon, an instrumented impact hammer, and an underwater speaker.

The primary focus of this chapter is the data which were collected in Barrow, AK, in April 2016. The data are analyzed in terms of temporal propagation, TL, and FRFs over short ranges in the first-year ice, near-shore Arctic environment.

\subsection{Experimental Methods}

Acoustic propagation measurements were recorded during April 8-15, 2016. All measurements were conducted approximately $2 \mathrm{~km}$ offshore of Barrow, Alaska, on firstyear, shore-fast ice that was between $1.05-1.15 \mathrm{~m}$ thick at the test sites. Water depth below the ice was 8-12 m deep. A total of 6 sites were identified: three were used to collect data and three were used for acoustic excitation into the air, ice, or water. Sites 1 , 2, and 3 were receiver locations and sites 4, 5, and 6 were source locations (Figure 2.1). Acoustic propagation distances between source and receiver sites are seen in Table 2.1.

The sea ice conditions varied significantly throughout the test area (Figure 2.2). At the cluster of sites 1, 4, and 5, the ice was relatively flat, without any large ridges. At the cluster of sites 2,3 , and 6 , the ice was also flat but was surrounded on all sides by large ice ridges. The largest of these ridges was near site 3 and was approximately $5 \mathrm{~m}$ high. It was suspected that some of the large ice ridges may have been bottom-fast because the water was only 8-12 $\mathrm{m}$ deep at all sites, however, this was not confirmed. The space between the two site clusters was moderately covered with smaller ice ridges 2-3 $\mathrm{m}$ high. Test sites were chosen where there was flat ice to facilitate deployment of equipment and where they were sufficiently close to shore to commute via snowmobile on a daily basis. Ice ridges were avoided as much as possible; however, large open areas of ice, without 
ridges, were sparse in the shore-fast environment. The rugged ice conditions made it difficult to locate reasonable test locations and limited the number of test locations that could effectively be measured. In addition, the weather during the test window limited the data gathering to 3 days (April 9, 12, and 13) out of the 8-day window. Although this is expected for testing in the harsh environment of the Arctic, it severely limited the collected data set spatially and only priority locations were able to be collected.

Table 2.1 Distance between source and receiver sites in meters.

\begin{tabular}{|c|c|c|c|c|}
\hline & & \multicolumn{3}{|c|}{ Receiver sites } \\
\hline & & Site 1 & Site 2 & Site 3 \\
\hline \multirow[t]{3}{*}{ Source Sites } & Site 4 & 50.3 & 929.9 & 743.2 \\
\hline & Site 5 & 164.3 & 1034.7 & 853.2 \\
\hline & Site 6 & 703.5 & 117.5 & 105.3 \\
\hline
\end{tabular}

Generally speaking, each experiment measured the acoustic path characteristics between source sites and receiver sites. Each experiment used either a propane cannon, instrumented force hammer, or underwater speaker to create an acoustic excitation of the environment. The excitation level of the source was quantified at the source location by a microphone in the air, geophone on the ice, and a hydrophone underwater. The receiver sites measured the acoustic responses down range with microphones, hydrophones, and geophones. The variation in distances between source and receiver sites facilitated estimation of TL as a function of distance. In addition, spectral analysis of the drive point (source and receiver co-located) transducers allowed for calculations of FRFs. It was the intent to characterize the TL and FRFs between all source-receiver combinations. However, in the case of a few source-receiver combinations, there was not sufficient signal to noise ratio (SNR) to allow for analysis.

At source sites, data were collected with a headless, 4-slot, National Instruments (NI) cRIO-9024. The data acquisition modules used were NI-9234, NI-9269, NI-9467, and NI-9344. These modules were used for acoustic data collection, signal output (as necessary), GPS location and timing signal acquisition, and system control, respectively. At receiver sites, data were collected with a 4-slot, cRIO-9031. Three NI-9234 modules were used to collect acoustic data and one NI-9467 module was used for GPS location and timing signal acquisition. All acoustic data were sampled at $51200 \mathrm{~Hz}$ in blocks of 120 seconds (in subsequent sections, each 120 -second data collect is referred to as one experiment). GPS time and position data were sampled once at the beginning of every data collection. Source and receiver data collections were time synchronized in postprocessing via GPS timing signals. 


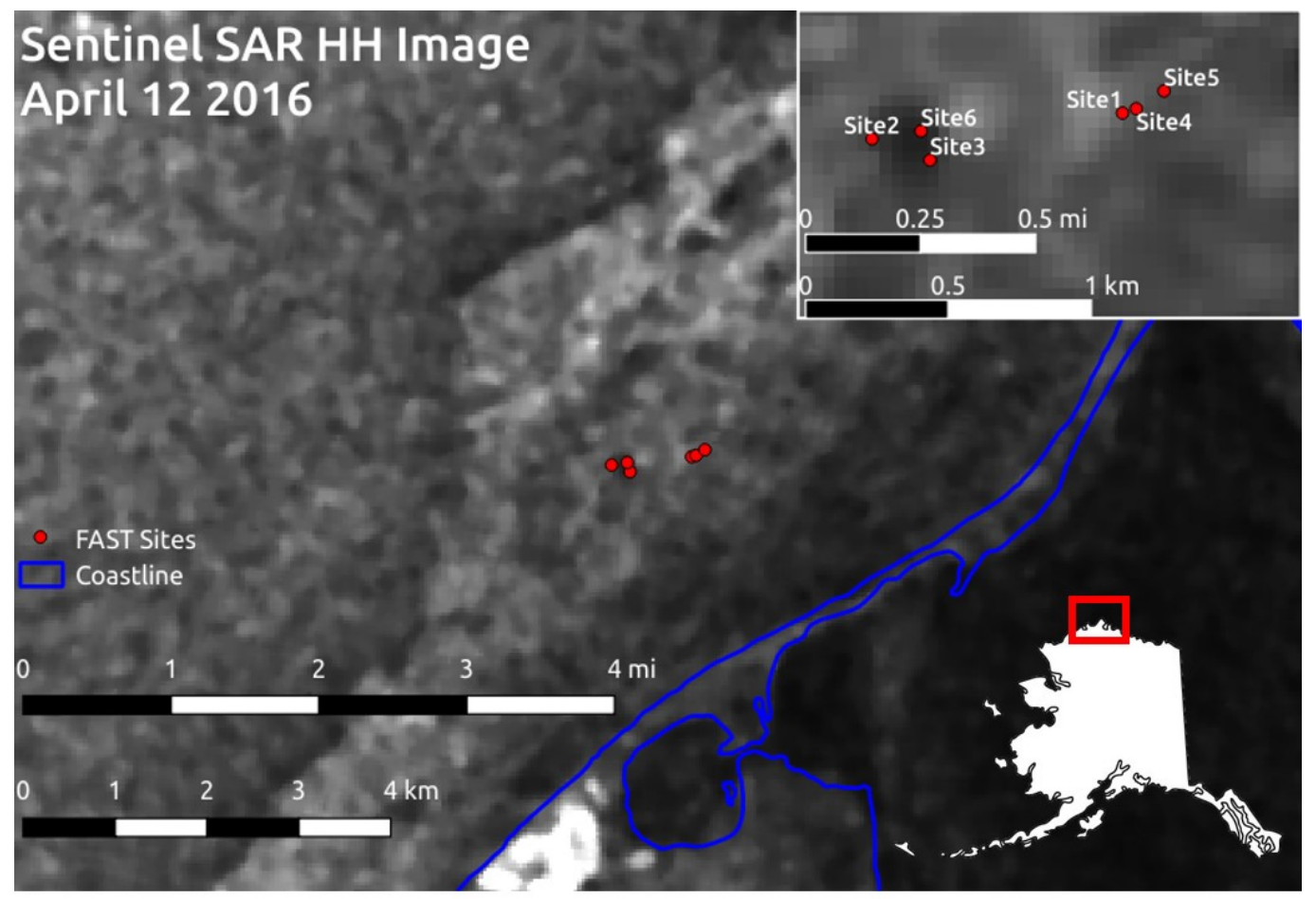

Figure 2.1 Site layout approximately $2 \mathrm{~km}$ off the coast of Barrow, AK.

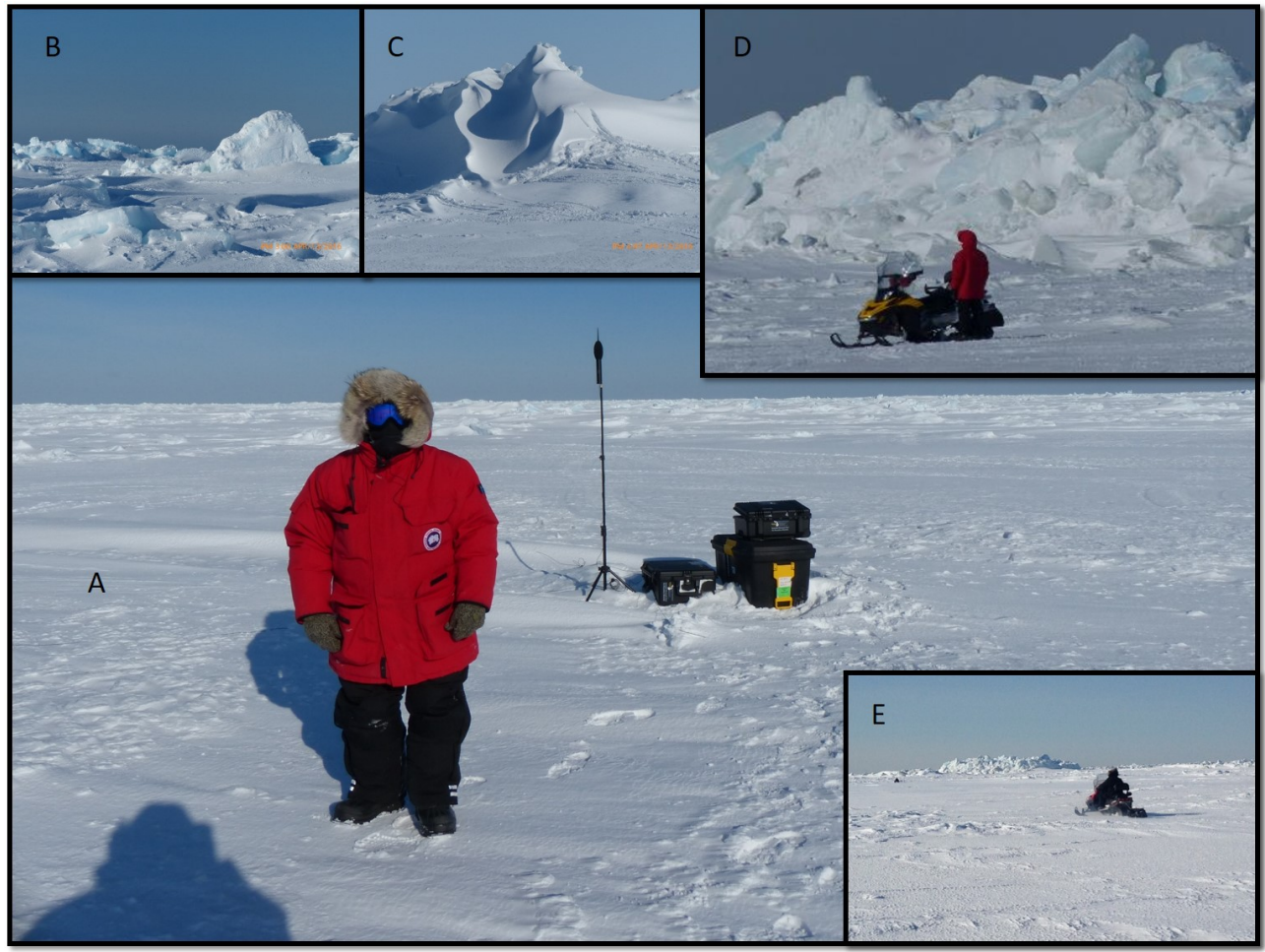

Figure 2.2 Shore-fast sea ice conditions near the test sites: (A) Site 1 looking seaward. (B) Photograph from cluster of sites 2, 3, and 6 looking east towards sites 1, 4, and 5. (C) Large ice ridge near site 3. (D) Scale of large ice ridges compared to a person. Large ice ridges were approximately $5 \mathrm{~m}$ high. (E) Site 2 looking towards site 3 . 


\subsubsection{Sources}

Three types of acoustic sources were used to excite the air, ice, or water for various experiments (Figure 2.3). The acoustic responses to each source were then measured down range at receiver sites. Several experiments were conducted using each source type to increase the number of potential averages that could be acquired during postprocessing. Only one type of source per experiment was used for excitation.

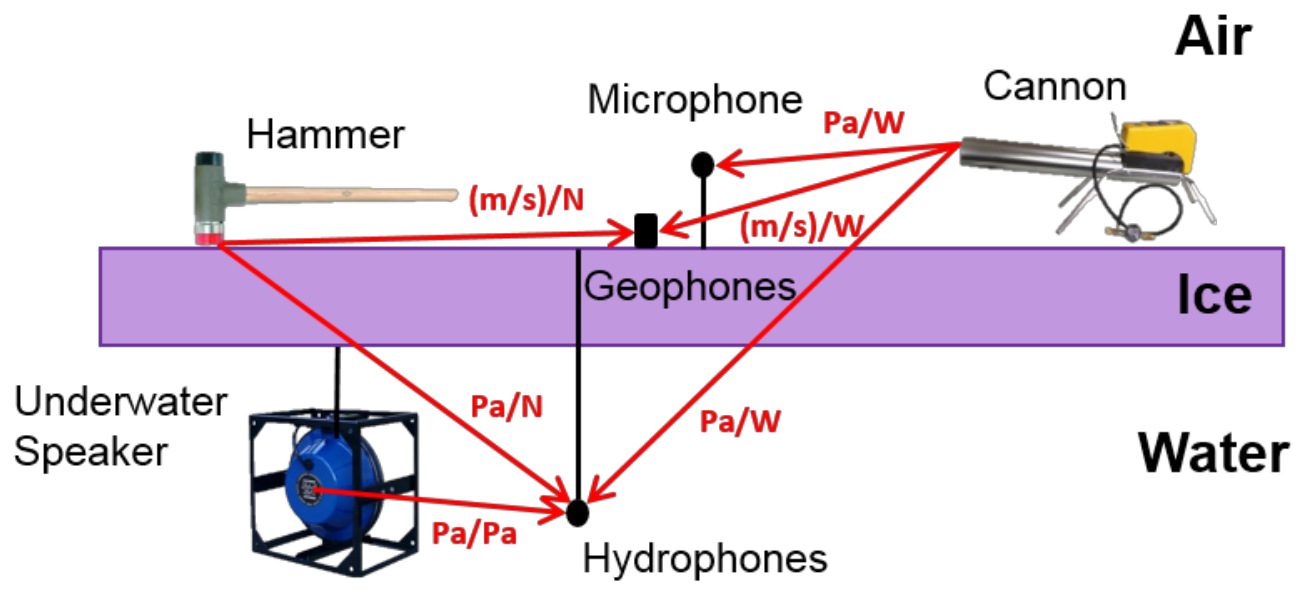

Figure 2.3 Source site schematic of various acoustic sources and receivers. Arrows indicate drive point measurements between source and receiver with adequate signal-to-noise ratio. Units of output signal over input signal are indicated.

A propane cannon (Zon Mark 4) was used as an atmospheric acoustic source. The detonation of propane created an impulsive pressure wave originating at the source location. The cannon did not shoot a projectile. A mechanical regulator was used to automatically fire the cannon one time approximately every 30 seconds. The regulator was set so that as many shots as possible could be recorded during the 120 second measurement period.

A $12 \mathrm{lb}$. instrumented force hammer $(P C B$ Model $086 D 50,0.23 \mathrm{mV} / \mathrm{N})$ was used to input energy to the ice sheet. The hammer was used to excite the ice sheet with an impact while it measured the force input to the ice sheet using an onboard force gage. The ice was struck directly since it was hard and flat at the source locations after removing a few inches of surface snow. The location of the hammer impact in relation to the source transducers changed slightly between experiments. This was necessary to find a relatively smooth and solid patch of ice to impact. At source sites where multiple hammer experiments were conducted, the ice would inevitably become chipped and nonuniform after one experiment. Therefore, it was necessary to move locations slightly (less than $1 \mathrm{~m}$ ) between experiments. During the 120 second measurement period, the ice was impacted with the force hammer as many times as was feasible (usually between 30 to 40 times). Any hits that were of poor quality (double hits and overloaded signals) were removed during post-processing. This resulted in 10-20 "good" hammer impacts per recording that could be used for spectral averaging per experiment. 
An underwater speaker (Lubell Labs Model LL916) was used as an underwater acoustic sound source. The speaker produced tones at various frequencies $(200,400,800,1600$, $6400 \mathrm{~Hz})$ and short chirps across frequency ranges $(40-2500 \mathrm{~Hz}$ and $4000-25000 \mathrm{~Hz})$. The tones and chirps were played over the 120 second measurement period. Each tone duration was 10 seconds and each chirp duration was 0.01 seconds. An amplifier (PylePro PZR 600) was used to drive the underwater speaker and maintain consistent sound levels between experiments. The underwater speaker was deployed through a drilled hole in the ice to a depth of $2 \mathrm{~m}$.

\subsubsection{Source Transducers}

In order to quantify the source levels, one microphone $(P C B 377 B 02,50 \mathrm{mV} / \mathrm{Pa})$, one single-axis, vertically oriented, geophone (Mark Products $19.7 \mathrm{~V} /(\mathrm{m} / \mathrm{s})$ ), and one hydrophone (Teledyne Reson TC 4032, $-170 \mathrm{~dB}$ re $1 \mathrm{~V} / \mu \mathrm{Pa}$ ) were placed near the source (Figure 3). At source sites, the hydrophone was placed at a depth of $2.5 \mathrm{~m}$ through a drilled hole in the ice. The distance between the hydrophone and the underwater speaker source (when used) was between $1 \mathrm{~m}$ and $2 \mathrm{~m}$. Precise distances between the underwater speaker and the hydrophone for each experiment were computed in post processing using time delays and measured sound speed in the water column. The microphone was placed on a tripod $70 \mathrm{~cm}$ above the ice and within $1 \mathrm{~m}$ of the hydrophone hole, opposite the underwater speaker hole. The microphone was $2.8 \mathrm{~m}$ from the muzzle of the propane cannon. The geophone was placed between the two holes which were drilled for the underwater source and the hydrophone. For all experiments, the hammer impact location was between approximately 1-3 m from the source geophone.

It should be noted for experiments when the cannon was used, the pressure wave overloaded the microphone at the source site. Because of limited spare equipment at the test site, a hydrophone (TC 4032) replaced the microphone in the air. The hydrophone was used at the source site only to record the atmospheric acoustic wave for the cannon experiments because its sensitivity was much lower than that of the microphones. For simplicity, and to avoid confusion, any atmospheric measurements will be referred to as microphone measurements.

\subsubsection{Receivers}

At each receiver site, several transducers were placed to simultaneously record the acoustic response from the source excitation (Figure 2.4). One microphone ( $P C B$ $378 B 02,50 \mathrm{mV} / \mathrm{Pa})$, one three-axis geophone $(G S$-One $3-C, 85.8 \mathrm{~V} /(\mathrm{m} / \mathrm{s}))$, and five hydrophones (Teledyne Reson TC 4013, $-211 \mathrm{~dB}$ re $1 \mathrm{~V} / \mu \mathrm{Pa}$ ) were used at each receiver location to measure the atmospheric sound pressure level (SPL), ice vibrational velocity, and underwater SPL respectively. The microphone, geophone, and one hydrophone were centrally located at the site. Four additional hydrophones were placed at cardinal directions of North, East, South, and West, $3 \mathrm{~m}$ from the center of the site. The microphones were placed $2 \mathrm{~m}$ above the ice and the hydrophones were placed at a depth 
of $5 \mathrm{~m}$ underwater through drilled holes in the ice. For purposes of this chapter, only the centrally located receiver hydrophone and vertical orientation of the receiver geophone are analyzed.

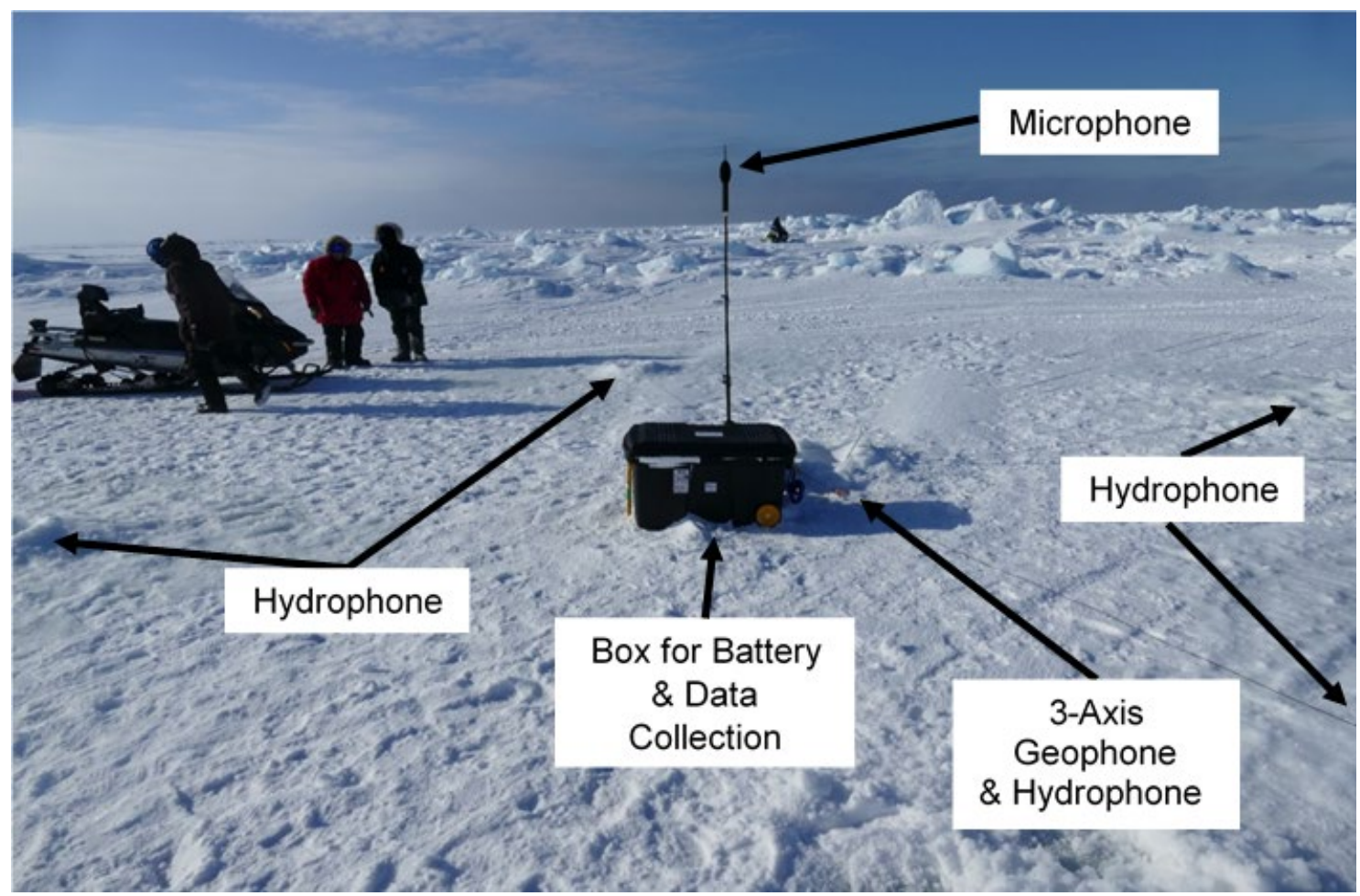

Figure 2.4 Receiver site transducer layout. Site 1 shown.

\subsection{Results}

\subsubsection{Temporal Propagation and Sound Speed}

At the outset of this analysis, it was necessary to determine the source-receiver combinations which had adequate signal-to-noise ratio (SNR) for any given experiment. Examining the spatial and temporal propagation, also gave an indication of which frequency ranges and distances could be used for future detection and tracking applications. Figure 2.5 to Figure 2.7 show the power spectral densities (PSD) as a function of both frequency and time measured at the source location and at selected receiver locations. The time scale on these plots is zoomed in to focus on only a few source inputs from the 120 second acquisition time. This was done for greater clarity of the temporal propagation.

For the hammer source experiment (Figure 2.5), the force input to the ice, as well as the geophone and hydrophone responses at the source location are investigated. Note that microphone data in response to hammer excitation was not included due to lack of SNR at receiver sites. At $50 \mathrm{~m}$ from the source (Figure 2.5d), the geophone has some 
detectable response especially at low frequencies (less than $200 \mathrm{~Hz}$ ). It comes as no surprise that low frequencies propagate well in the ice and mid-high frequencies are attenuated quite rapidly. Beyond $50 \mathrm{~m}$, there was no detectable signal in the geophone. By comparison, mid-high frequencies propagate better through the water and can be seen much more clearly in both the $50 \mathrm{~m}$ and $164 \mathrm{~m}$ sites. In general, it can be said that the water path transmits energy farther than the ice path for a direct force input to the ice.

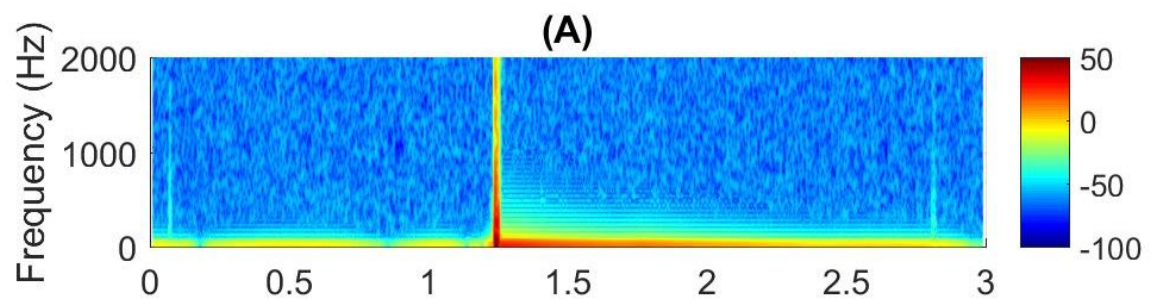

(B)

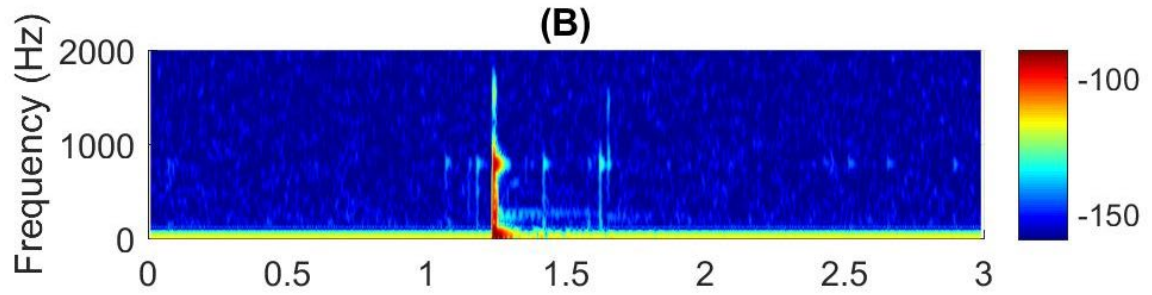

(C)

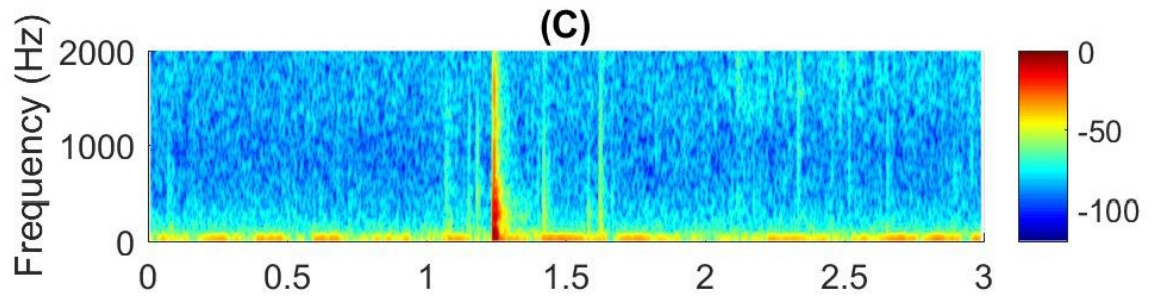

(D)

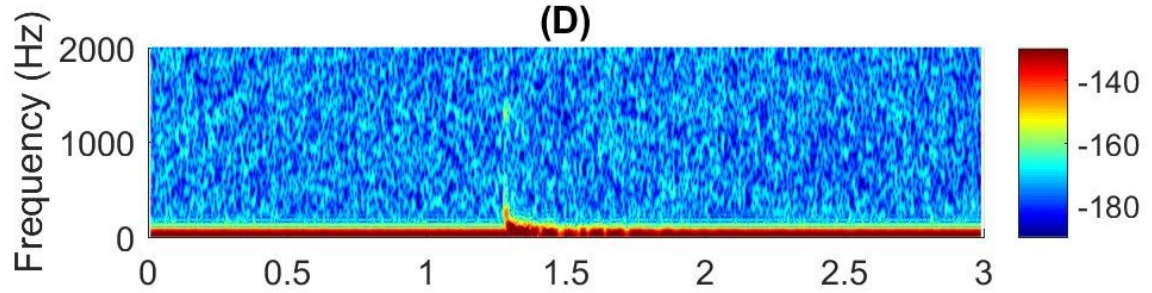

(E)

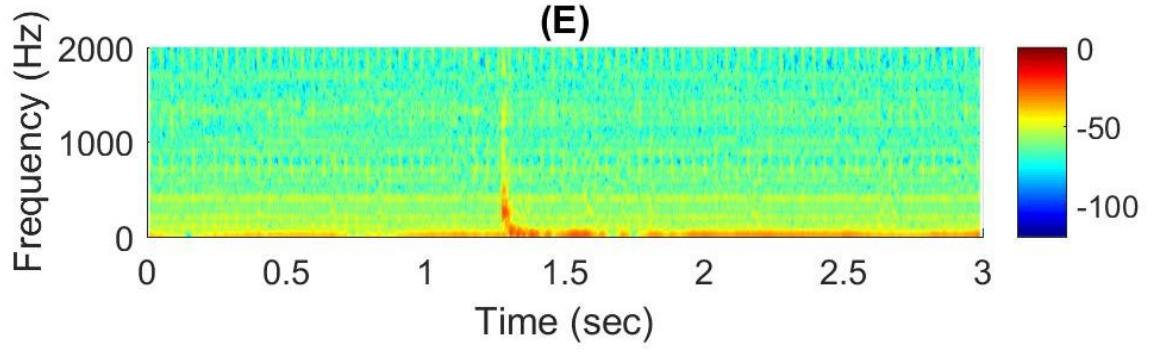

Figure 2.5 Ice-to-ice and ice-to-water temporal propagation. Source (site 4) to receiver (site 1) distance approximately $50 \mathrm{~m}$. (A) Hammer force input ( $\mathrm{dB}$ re $1 \mathrm{~N} / \mathrm{rt}(\mathrm{Hz})$ ) at site 4 source location. (B) Geophone response $(\mathrm{dB}$ re $1 \mathrm{~m} / \mathrm{s} / \mathrm{rt}(\mathrm{Hz}))$ at site 4 source location. (C) Hydrophone response $(\mathrm{dB}$ re $1 \mathrm{~Pa} / \mathrm{rt}(\mathrm{Hz}))$ at site 4 source location. (D) Geophone response $(\mathrm{dB}$ re $1 \mathrm{~m} / \mathrm{s} / \mathrm{rt}(\mathrm{Hz}))$ at site 1 receiver location. (E) Hydrophone response $(\mathrm{dB}$ re $1 \mathrm{~Pa} / \mathrm{rt}(\mathrm{Hz}))$ at site 1 receiver location. 


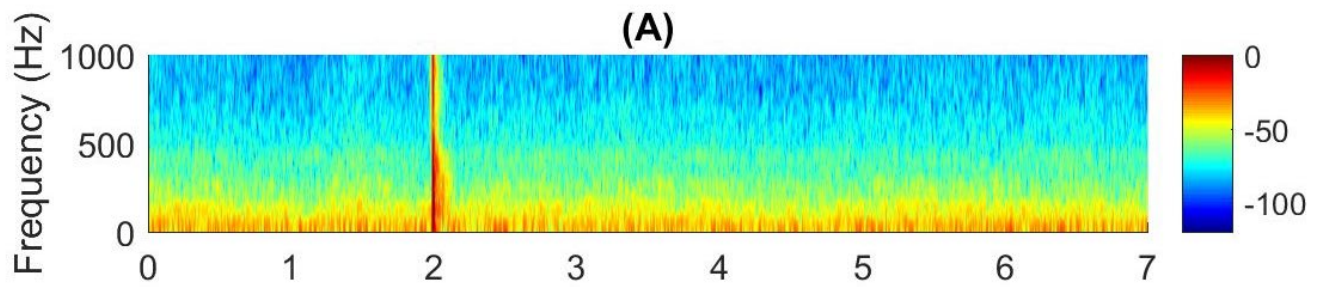

(B)

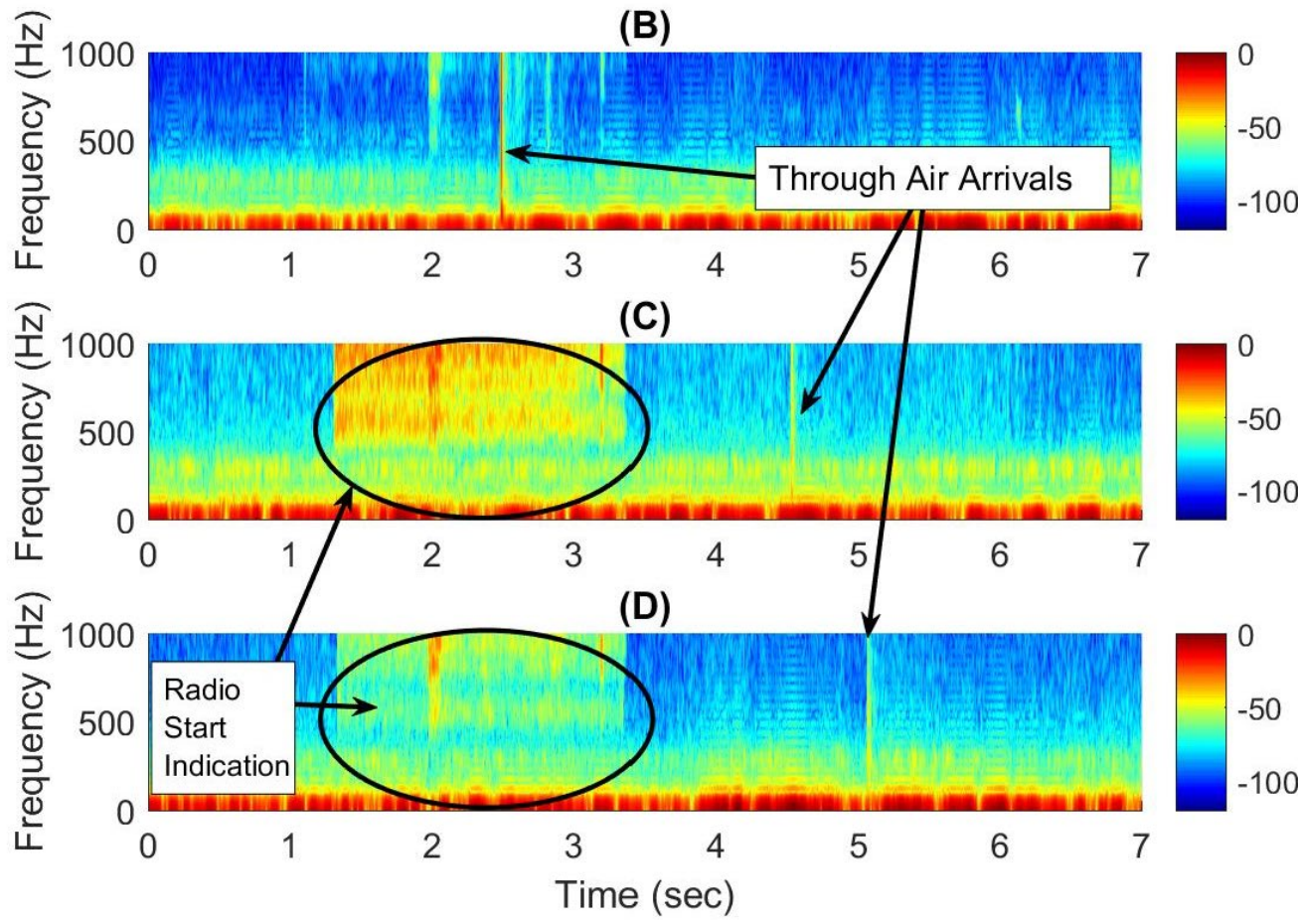

Figure 2.6 Air-to-air temporal propagation from the cannon source to the microphones at various receiver distances. (A) atmospheric sound level at source location (site 5) normalized to $1 \mathrm{~m}$. (B) atmospheric sound level at site 1 approximately $164 \mathrm{~m}$ from source. (C) atmospheric sound level at site 3 approximately $853 \mathrm{~m}$ from source. (D) atmospheric sound level at site 2 approximately $1035 \mathrm{~m}$ from source. All levels: $\mathrm{dB}$ re $1 \mathrm{~Pa} / \mathrm{Hz}$.

The atmospheric temporal propagation of the cannon blast is seen in Figure 2.6. In Figure 2.6 (c \& d), two large signal indications are visible between $1.4 \mathrm{sec}$ to $3.3 \mathrm{sec}$, before the arrival of the atmospheric acoustic pressure wave at approximately $4.5 \mathrm{sec}$ and $5.1 \mathrm{sec}$ respectively. During the cannon experiment, a hand-held two-way radio was held near the cannon and near the receiver microphones. As the cannon was about to fire, the transmitting (push-to-talk) feature of the source radio was activated. This transmitted the cannon sound via radio waves, which arrived much earlier than the acoustic pressure wave. Following the radio start indication, the atmospheric pressure wave can be seen at the receiver locations. Based on the time delay between the source and receiver sites, the air sound speed was determined to be $331 \mathrm{~m} / \mathrm{s}$. This is approximately $4 \mathrm{~m} / \mathrm{s}$ faster than the speed of sound determined from Equation 2.134, which is based on the ratio of specific heats $(\gamma=1.4)$, the specific gas constant $\left(R=287 \frac{\mathrm{J}}{\mathrm{kg} \mathrm{K}}\right)$, and the average air temperature $\left(T_{k}=266.7 \mathrm{~K}\right)$. The higher measured speed of sound is due to the receivers 
being down-wind from the cannon source, decreasing the time of flight (i.e. increasing sound speed). Wind speeds varied between $3.9 \mathrm{~m} / \mathrm{s}$ to $22.8 \mathrm{~m} / \mathrm{s}$ during this testing.

$$
c=\sqrt{\gamma R T_{k}}
$$

The low frequency $(40-2500 \mathrm{~Hz})$ chirps produced by the underwater source were detectable at the $164 \mathrm{~m}$ and the $853 \mathrm{~m}$ site hydrophones with $60 \mathrm{~dB}$ and $29 \mathrm{~dB}$ of SNR respectively. Similar results were seen for the high frequency $(4000-25000 \mathrm{~Hz})$ chirps and tonal frequencies. The signal from the underwater speaker was not detectable by the microphone or geophone receivers. Based on the time delay between the source and receiver sites for the underwater speaker chirps, the average underwater sound speed was determined to be approximately $1441 \mathrm{~m} / \mathrm{s}$. The hydrophone responses to the cannon blast in air (Figure 2.7) also confirm an average underwater sound speed of $1441 \mathrm{~m} / \mathrm{s}$. It is interesting to note in Figure 2.7c, that there are signals which are present before the arrival of the underwater sound wave. These indicate sound waves which traveled through the ice. Additionally, there are several signals after the arrival of the underwater sound wave which indicate reflections and scattering from the underwater environment. These reflections and scattering are expected due to the irregular ice ridges near sites 2 and 3 . 
(A)

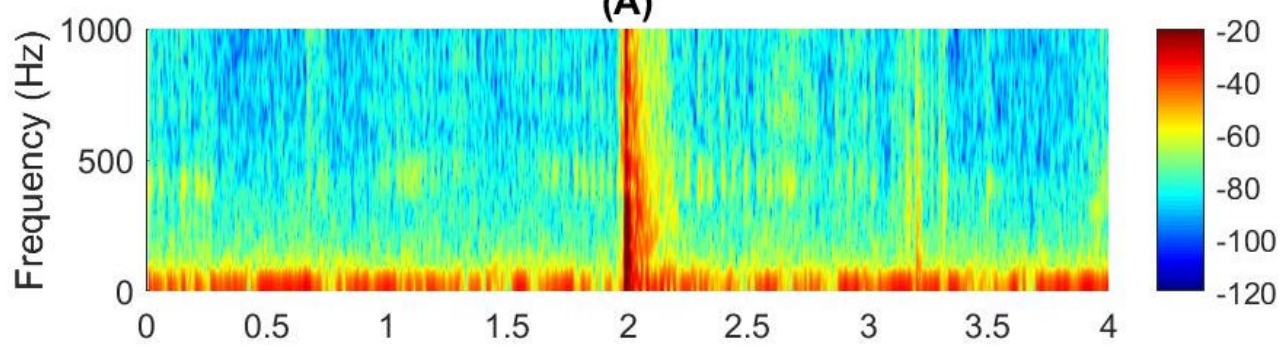

(B)

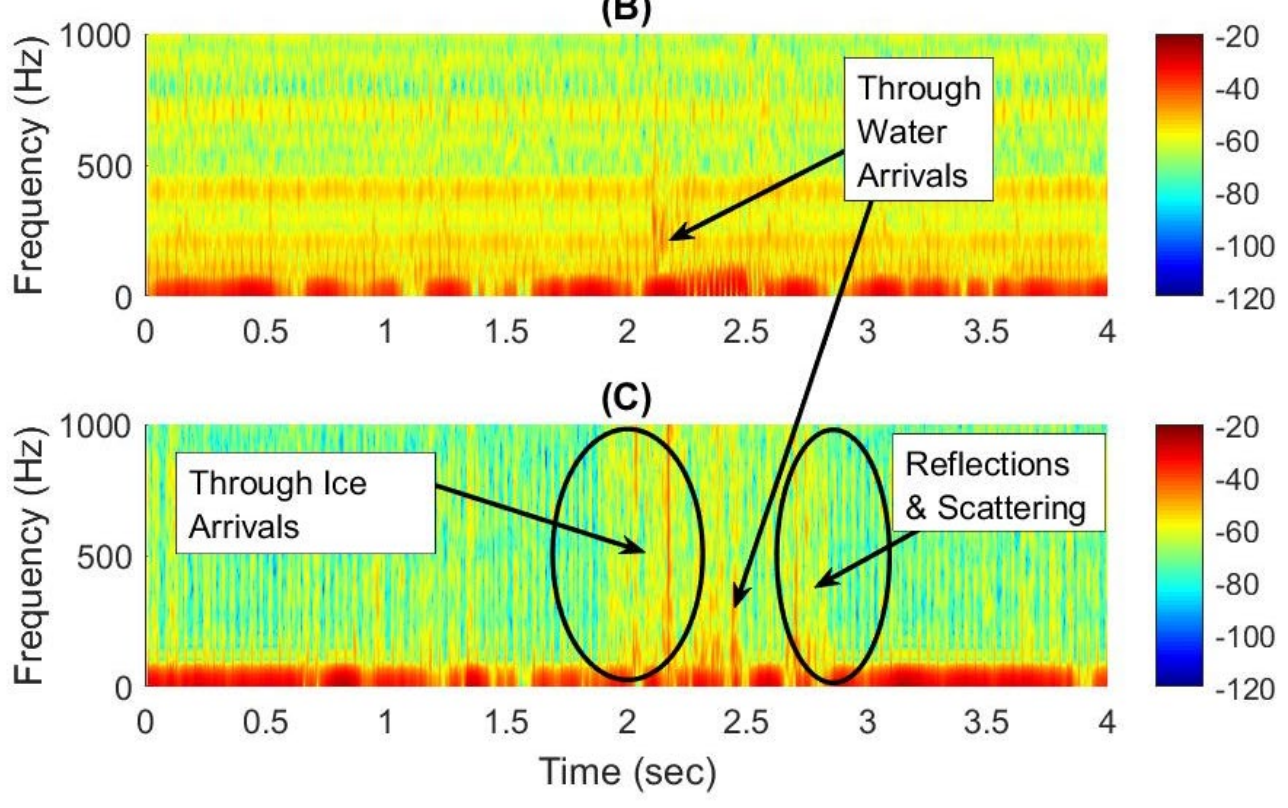

Figure 2.7 Air-to-ice-to-water temporal propagation from the cannon source to the hydrophones at various receiver distances. (A) Underwater sound level at site 5. (B) Underwater sound level at site 1 approximately $164 \mathrm{~m}$ from source. (C) Underwater sound level at site 3 approximately $853 \mathrm{~m}$ from source. All levels: $\mathrm{dB}$ re $1 \mathrm{~Pa} / \mathrm{Hz}$. 


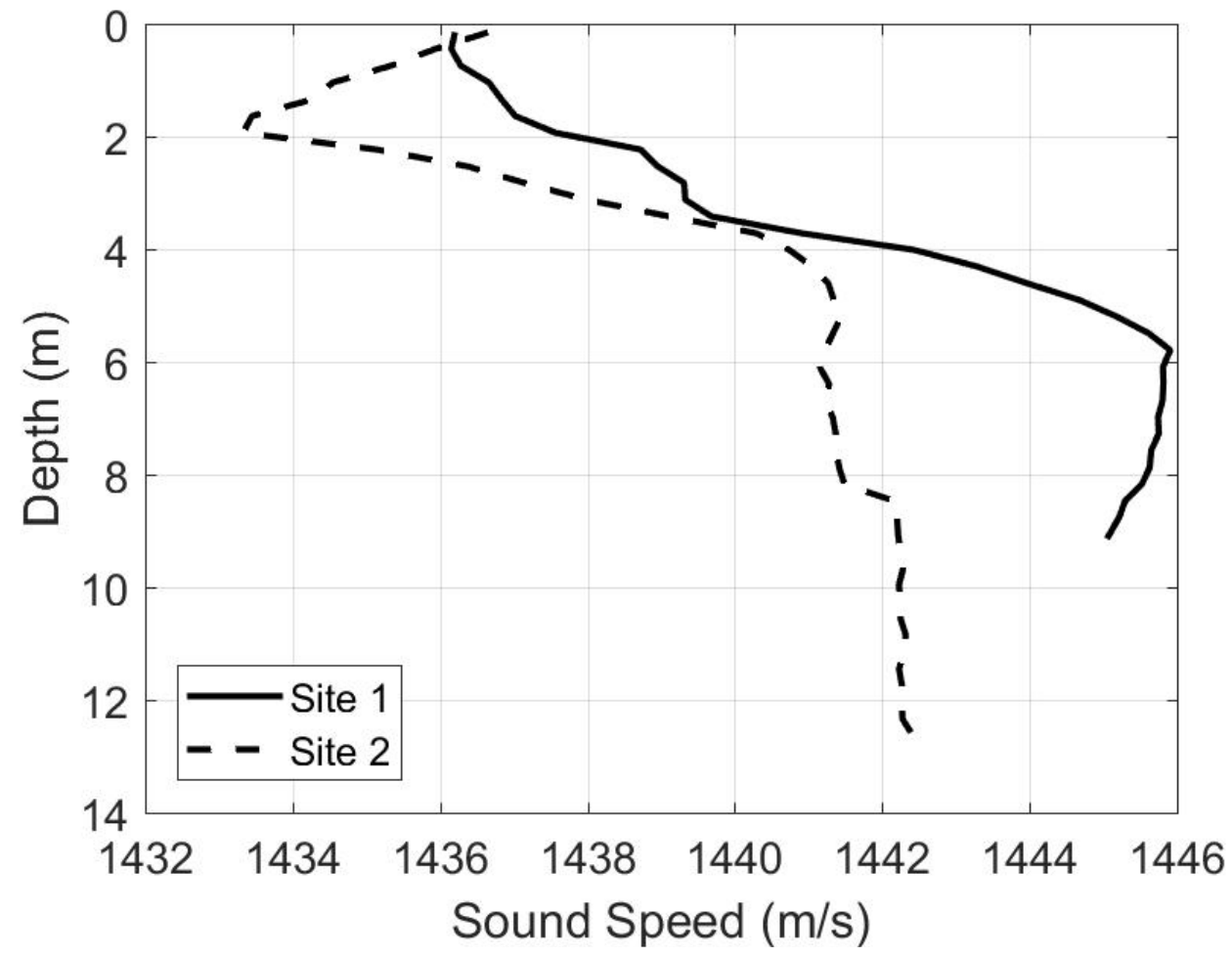

Figure 2.8 Sound speed profiles measured with conductivity, temperature, and depth (CTD) probe for sites 1 and 2. Measurements taken on 12/04/16 at UTC 15:53:32 and UTC 17:07:47 for sites 1 and 2 respectively.

In order to verify the time of flight sound speed measurement, the average sound speed was also measured directly with a SonTek CastAway conductivity, temperature, and depth (CTD) probe (Figure 2.8). From the CTD data, the sound speed underwater ranged from $1433 \mathrm{~m} / \mathrm{s}$ to $1446 \mathrm{~m} / \mathrm{s}$ for various depths. For the hydrophone depths used at source and receiver sites, the mean speed of sound is close to $1441 \mathrm{~m} / \mathrm{s}$. Therefore, the time of flight measurement of a sound speed of $1441 \mathrm{~m} / \mathrm{s}$ is reasonable and represents direct path propagation. The underwater sound speed profile was calculated by a CastAway CTD using the Chen-Millero method ${ }^{35,36}$.

For purposes of processing beyond this point, data which does not have at least $10 \mathrm{~dB}$ of signal-to-noise ratio based on the average ambient background noise is not included.

\subsubsection{Frequency Response Functions}

From the calibrated time series data for each experiment, the linear spectra of the source $\left(S_{x}\right)$ and the linear spectra of the receivers $\left(S_{y}\right)$ were determined by computing the Fast Fourier Transform (FFT) in Matlab. Using the linear spectra, the autopower spectra $\left(G_{x x}\right)$, crosspower spectra $\left(G_{y x}\right)$, and Frequency Response Functions (FRF) between source and receiver were determined (Equations 2.2 to 2.4$)^{32,37}$. 
The FRF is defined as the output signal (response) divided by the input signal (source) in the frequency domain ${ }^{32,37}$. Since the crosspower spectra averages out uncorrelated components, the noise on the response is minimized in the $\mathrm{H} 1$ formulation of FRF calculations ${ }^{32,37}$.

$$
\begin{gathered}
G_{x x}(\omega)=S_{x}^{*}(\omega) S_{x}(\omega) \\
G_{y x}(\omega)=S_{y}^{*}(\omega) S_{x}(\omega) \\
F R F(\omega)=\frac{G_{y x}(\omega)}{G_{y y}(\omega)}
\end{gathered}
$$

To determine the effectiveness of the FRF, an accompanying function, coherence $(\mathrm{COH})$, was calculated with Equation 2.5 $5^{32,37}$. Coherence represents the amount of the output signal that is linearly related to the input signal. The coherence function ranges from zero to one, where one represents $100 \%$ of the output signal being linearly related to the input signal and zero represents $0 \%$ of the output signal being linearly related to the input signal. In general, it is preferable to see coherence which is close to one at frequencies where the FRF is to be investigated. Coherence less than one can be caused by nonlinearity in the system, unmeasured inputs to the system (noise), an anti-resonance in the system, bias errors in the measurement, or some combination of all of these reasons ${ }^{32,37}$. The coherence for the measurements is investigated alongside the FRFs to provide validation that the received signal is linearly related to the source signal.

$$
\operatorname{COH}(\omega)=\frac{G_{x y}(\omega) G_{y x}(\omega)}{G_{x x}(\omega) G_{y y}(\omega)}
$$

\subsubsection{Hammer Experiment FRFs}

For hammer experiments, there were 30 to 40 hammer hits over the 120 second measurement period with 3 to 4 seconds between hits and various impact force with the hammer. Various impact force was used intentionally because data could not be inspected on-site due to the harsh environmental conditions. The designed variation in impact force provided the best likelihood of generating impacts with high SNR that was not overloaded. Only "good" hammer hits were used for post-processing (10 to 20 per experiment). Hammer hits were not included if the signal was overloaded or if there was a double hit. Note that a double hit was registered any time that the hammer struck the ice more than one time per swing. Double hits were not included because they can provide unequal excitation in the frequency range of interest. Blocks of data, 1 second in duration and containing a good hammer hit signal, were used to compute the autopower spectra. All of the autopower spectra for a given experiment were then averaged. The averaged autopower spectra for the hammer experiments can be seen in Figure 2.9. There is effective input energy between $1 \mathrm{~Hz}$ to $200 \mathrm{~Hz}$ because the autopower spectra is high enough to excite a system response and there is good coherence in this frequency 
range. At $200 \mathrm{~Hz}$ and above the autopower spectra begins to roll off at a rate of $-11.6 \mathrm{~dB}$ per octave. Beyond $2 \mathrm{kHz}$, the input autopower spectra is too low to excite any system responses. Additionally, coherence between the source and receiver begins to suffer due to low input energy above $2 \mathrm{kHz}$.

The drive point FRF and $\mathrm{COH}$ between the hammer input and the geophone response at the source site (drive point mobility) is shown in Figure 2.10. It can be seen that there is a large peak in the FRF at approximately $800 \mathrm{~Hz}$ and a corresponding harmonic at 1600 Hz. The $800 \mathrm{~Hz}$ peak and its harmonic are believed to correspond to the throughthickness compressional mode of the ice sheet.

It is well known for quarter-wavelength resonators, that frequency $(f)$ is related to the wave velocity $\left(v_{p}\right)$ and the wavelength by Equation $2.6^{38}$. The parameter $L$ can be set equal to the ice thickness which was $1.05 \mathrm{~m}$ at the drive point locations. Also, for the $800 \mathrm{~Hz}$ mode, $n$ can be set equal to 1 .

$$
f=\frac{(2 n-1) v_{p}}{4 L}
$$

Solving for the compressional wave velocity in the ice, $v_{p}$ is determined to be approximately equal to $3360 \mathrm{~m} / \mathrm{s}$. This is similar to the compressional wave speed reported by several other sources ${ }^{13,15,18}$.

To confirm that the measured drive point mobility is reasonable, the theoretical infinite plate mobility is plotted for comparison. To approximate the theoretical mobility, the ice elastic properties are needed. Assumptions for these properties were made based on referring to several papers ${ }^{25,39-43}$ in aggregate to determine reasonable values for firstyear ice in Barrow. Poisson's ratio $(v)$ was assumed to be 0.295 and the ice density $(\rho)$ was assumed to be $910 \mathrm{~kg} / \mathrm{m}^{25,39-41}$. Also, the volume of brine in the ice $\left(V_{b}\right)$ was assumed to be $20 \mathrm{ppt}$ which results in an elastic modulus $(E)$ of $2.98 \mathrm{GPa}$ from Equation $2.7^{25,42,43}$. From these elastic properties, the flexural rigidity $(D)$ and the infinite plate mobility can then be determined (Equations $2.8,2.9)^{44}$. It is seen in Figure 2.10a that the theoretical infinite plate mobility is approximately $-66 \mathrm{~dB}(\mathrm{re} 1 \mathrm{~m} / \mathrm{s} / \mathrm{N})$ based on Equation 2.9. This is very similar to the measured FRF level between 20 to $500 \mathrm{~Hz}$ where there is little modal response in the ice.

$$
\begin{gathered}
E=10-0.351 V_{b} \\
D=\frac{E L^{3}}{12\left(1-v^{2}\right)} \\
\frac{\ddot{x}}{F_{\text {inf }}}=\frac{1}{8 \sqrt{D \rho L}}
\end{gathered}
$$


By comparison, the drive point FRF between the hammer and the hydrophone (Figure 2.10) indicates that the hydrophone response is not affected by the modal properties of the ice.

The coherence for both the geophone and hydrophone are very close to one for the frequency ranges of 20 to $1000 \mathrm{~Hz}$. This indicates that for this frequency range the input signal is linearly related to the output signal. Below $10 \mathrm{~Hz}$, there is little response from the ice for the given input signal, causing coherence to be low. Above $1 \mathrm{kHz}$, the coherence also begins to drop off because the amount of energy input to the system (Figure 2.9) is decreasing. Low coherence could also be caused by non-linear ice stiffness and damping properties at these frequencies and variation in excitation amplitudes.

During all experiments, the wind speed varied between $3.9 \mathrm{~m} / \mathrm{s}$ to $22.8 \mathrm{~m} / \mathrm{s}$. The average wind speed was $15.0 \mathrm{~m} / \mathrm{s}(34 \mathrm{mph})$. This caused significant background noise at the microphone (despite using an environmental windscreen) that was not easily overcome by most of the sources (excluding the cannon). The atmospheric acoustic levels produced by the hammer hitting the ice were not loud enough to produce a coherent response at the microphone. Therefore, the drive point FRF between the hammer and the microphone are not reported.

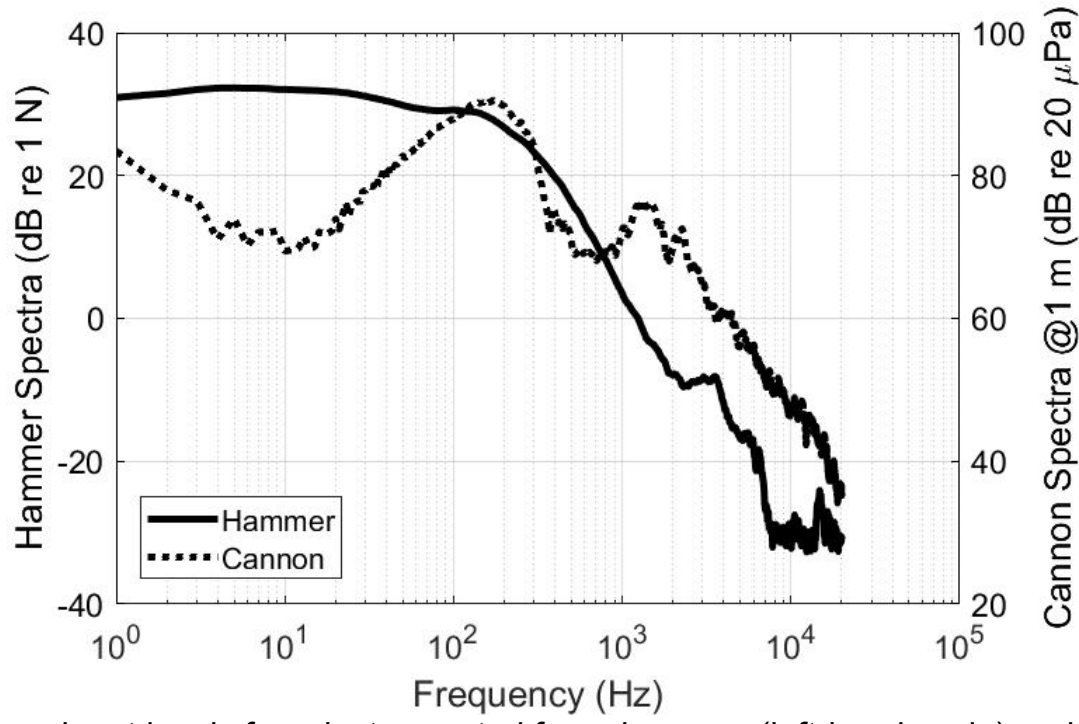

Figure 2.9 Source input levels from instrumented force hammer (left-hand scale) and propane cannon (right-hand scale). 
(A)

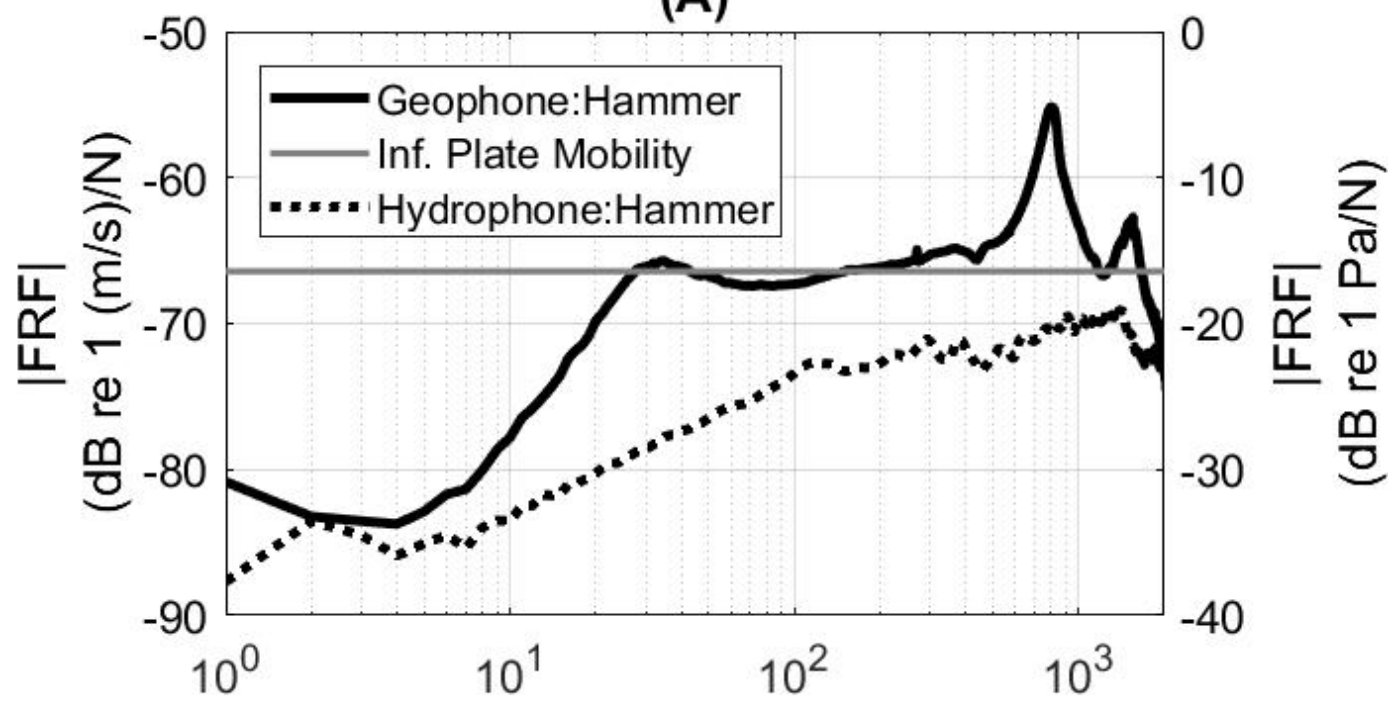

(B)

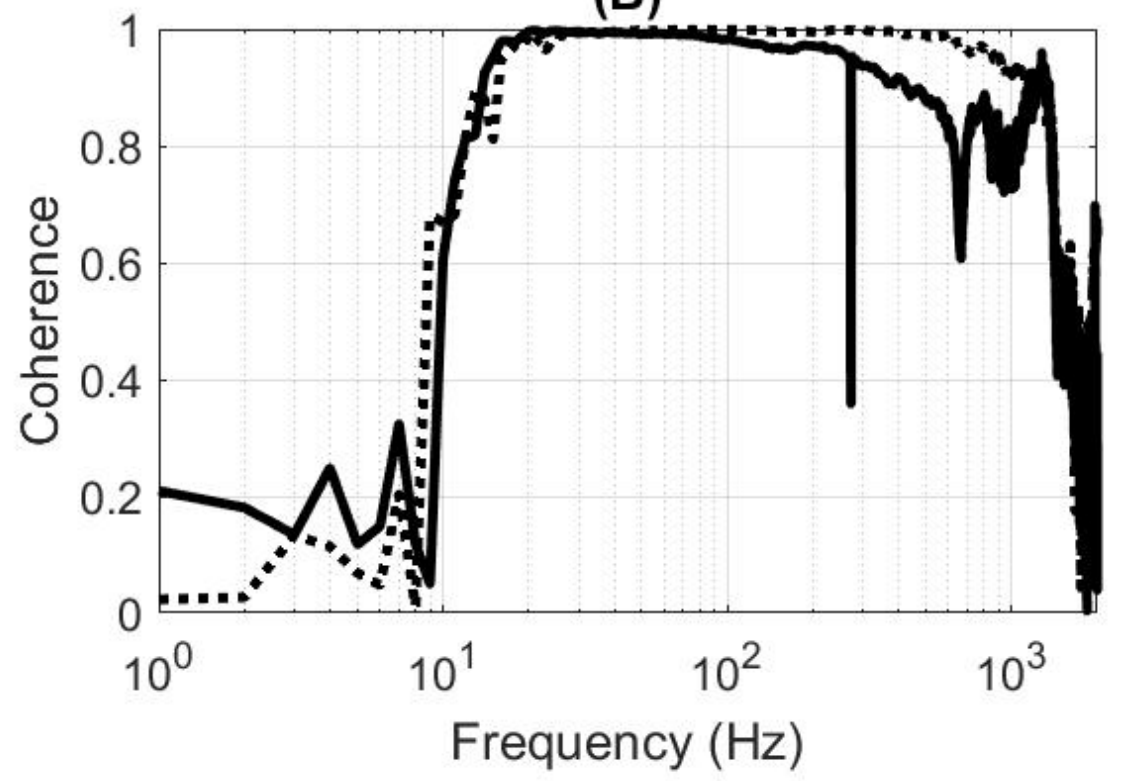

Figure 2.10 (A) Drive point FRFs and (B) $\mathrm{COH}$ measured between the hammer and the geophone (lefthand scale) or hydrophone (right-hand scale) at the source location. Theoretical infinite plate mobility for sea ice conditions in Barrow $(-66 \mathrm{~dB}$ re $1 \mathrm{~m} / \mathrm{s} / \mathrm{N})$ is indicated. 


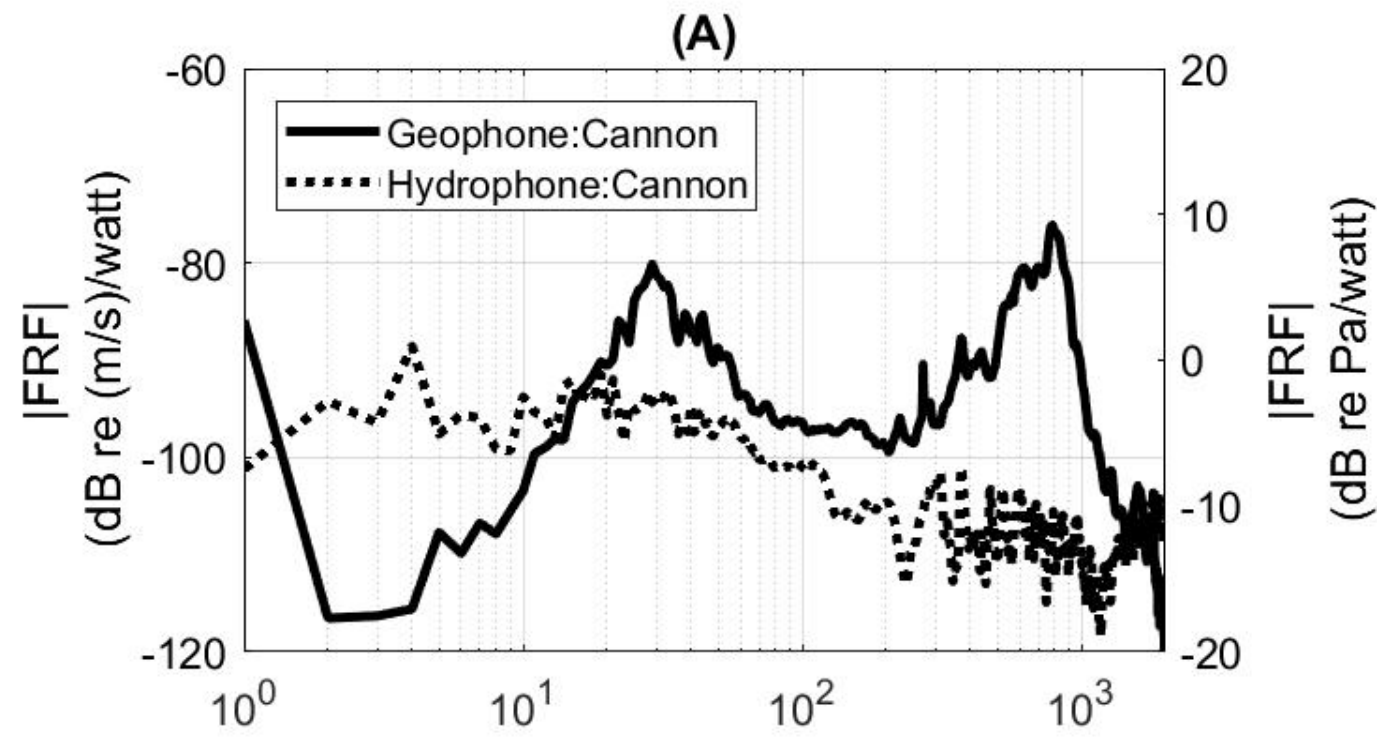

(B)

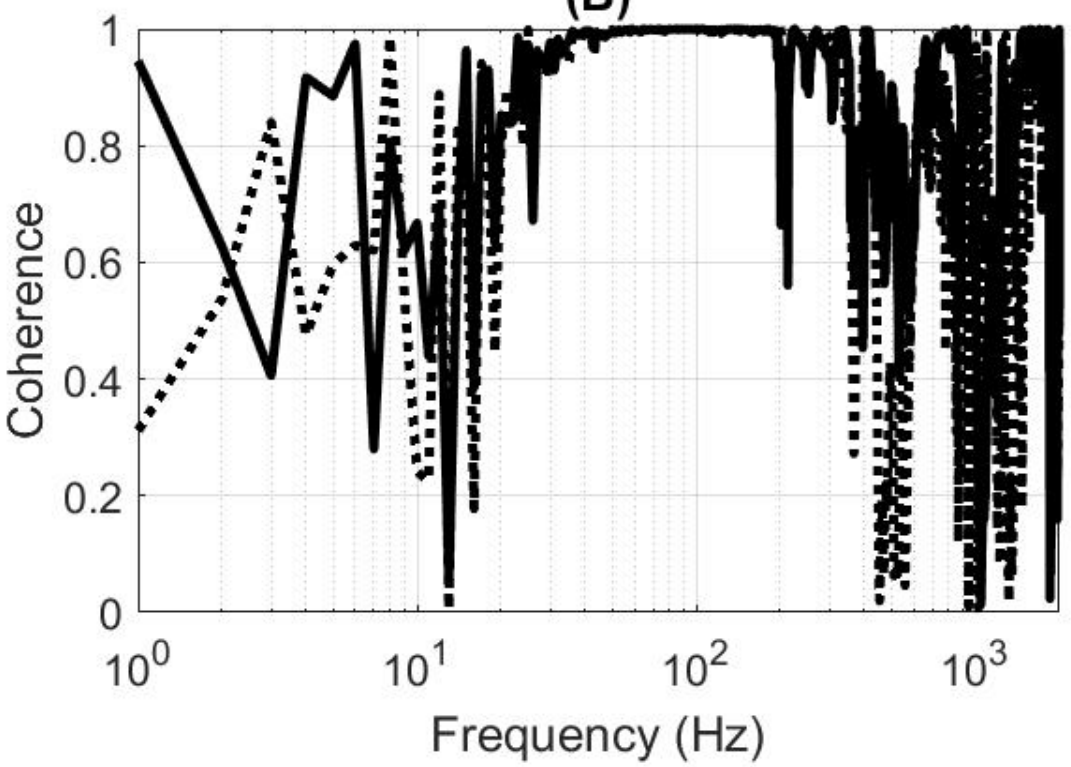

Figure 2.11 (a) Drive point FRFs and (b) $\mathrm{COH}$ between the cannon source and the geophone (left-hand scale) or hydrophone (right-hand scale) at the source location

\subsubsection{Cannon Experiment FRFs}

The input autopower spectra for the cannon source (Figure 2.9) was calculated by normalizing the microphone response at the source to $1 \mathrm{~m}$ distance. This was done assuming hemispherical spreading. There is input energy from the cannon in the frequency range of $1 \mathrm{~Hz}$ to $2 \mathrm{kHz}$. 
The cannon FRFs were normalized to the cannon sound power. The cannon sound power $(W)$ was computed with Equation $2.10^{34}$ assuming hemispherical spreading. The air density $\left(\rho_{0}=1.324 \frac{\mathrm{kg}}{\mathrm{m}^{3}}\right)$ and speed of sound $\left(c=327 \frac{\mathrm{m}}{\mathrm{s}}\right)$ were approximated based on the average air temperature, $-6.45^{\circ} \mathrm{C}$, and standard pressure, $101.3 \mathrm{kPa}$. The autopower spectra of the receiver was then divided by the sound power of the source to compute the FRF.

$$
W=\frac{p^{2}}{\rho_{0} c} * 2 \pi r^{2}
$$

In the drive point FRF between the cannon and the geophone in Figure 2.11a, two dominant peaks are seen: one at $29 \mathrm{~Hz}$ and one at $800 \mathrm{~Hz}$. The $800 \mathrm{~Hz}$ peak corresponds with the through-thickness mode previously identified in the hammer drive point mobility. In this instance, the through-thickness mode is excited by the cannon pressure wave.

The $29 \mathrm{~Hz}$ mode is due to an air-coupled flexural wave in the ice layer. Since air-coupled flexural waves are non-dispersive ${ }^{5,13}$, it is expected that the majority of its energy would appear at a single frequency. This is supported by Figure 2.11 a. Frank Press et al. ${ }^{5,13}$ describe air coupled flexural waves in detail. The dimensionless parameter $\gamma$ relates the ice thickness $(L)$ to the speed of sound in air and to the air-coupled wave frequency, $f$.

$$
\gamma=\frac{L}{\lambda}=\frac{L f}{c}
$$

It has been shown ${ }^{13}$ that $\gamma$ can also be expressed as a function of the compressional wave velocity in ice. Since the compressional wave velocity in the ice was previously determined, a $\gamma$ value of approximately 0.092 from Figure 6 of Frank Press et $\mathrm{al}^{13} \mathrm{can}$ also be determined. As previously mentioned, the sound speed in air (c) was $331 \mathrm{~m} / \mathrm{s}$ during the measurements and the ice thickness $(L)$ was $1.05 \mathrm{~m}$. By rearranging Equation 2.11, the air-coupled flexural wave frequency is computed to be $29 \mathrm{~Hz}$. This indicates that the observed $29 \mathrm{~Hz}$ peak is an air-coupled flexural wave in the ice.

As shown by Press et al. ${ }^{5,13}$ the air-coupled flexural wave can be observed in the time domain signals of the microphones and geophones in response to a cannon blast (Figure 2.12). Due to a higher group velocity in ice, the flexural wave in ice arrives before the pressure wave in air for an air-coupled flexural wave. Upon arrival of the atmospheric acoustic wavefront (i.e. when the speed of sound in air matches the phase velocity of the flexural wave), the flexural wave amplitude is immediately reduced and/or terminated ${ }^{5,13}$. This termination of amplitude is seen in the geophone data in Figure 2.12c. 


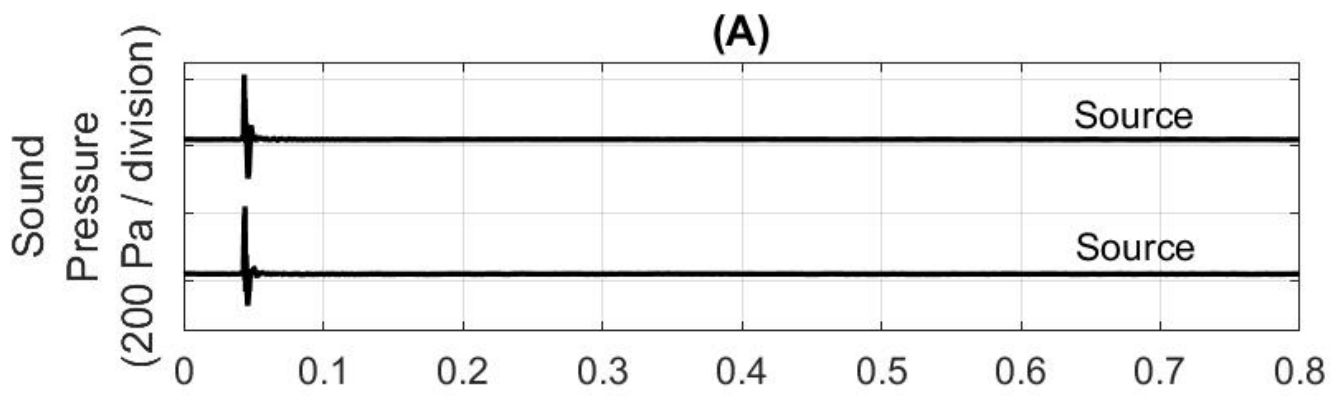

(B)
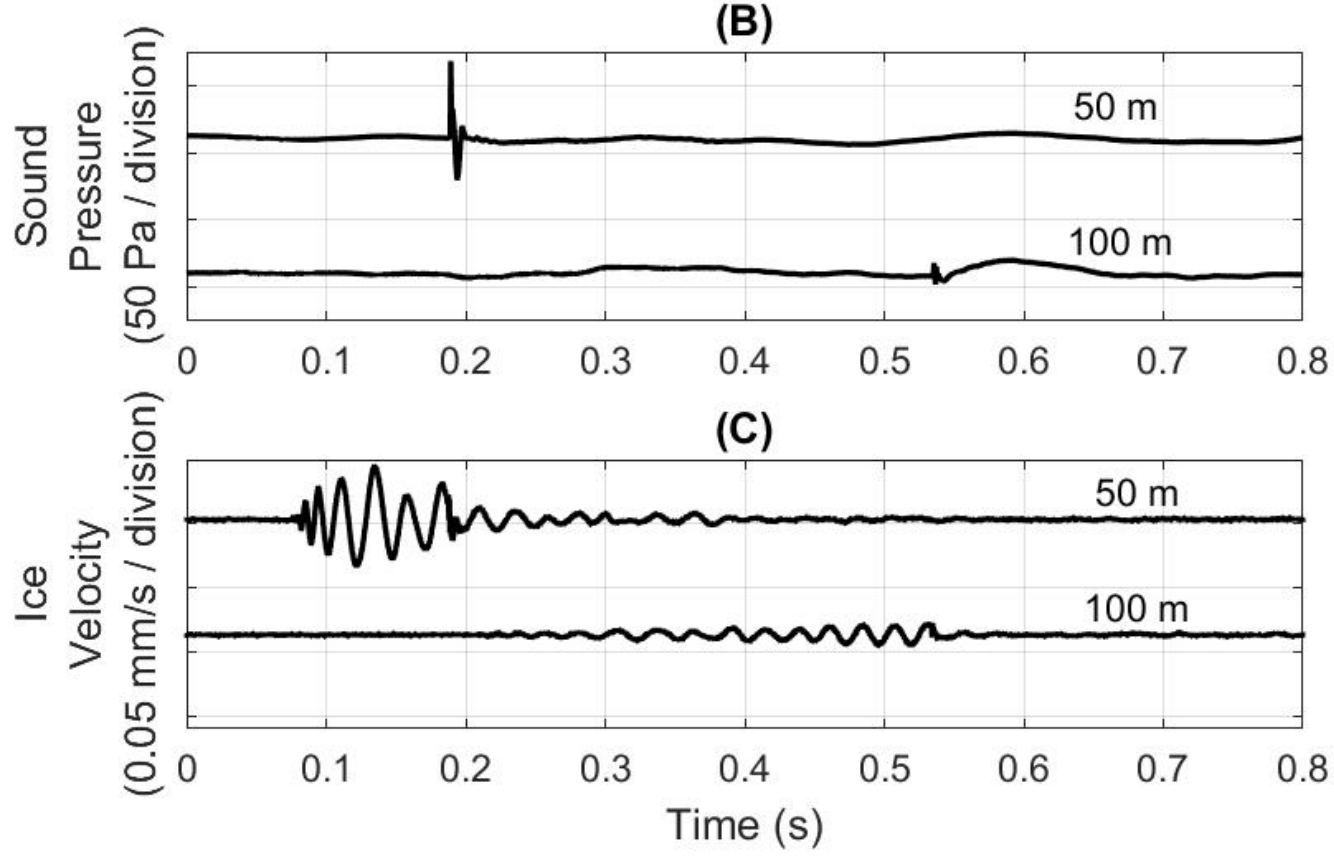

Figure 2.12 (A) Propane cannon blast measured at the source location microphone. (B) Microphone measurements at $50 \mathrm{~m}$ and $100 \mathrm{~m}$ receiver locations. (C) Geophone measurements at $50 \mathrm{~m}$ and $100 \mathrm{~m}$ locations. The flexural wave amplitude in the ice $(\mathrm{C})$ is significantly reduced upon arrival of the atmospheric acoustic wavefront $(B)$ indicating the detection of an air-coupled flexural wave at $29 \mathrm{~Hz}$.

Analysis of the hydrophone FRF in Figure 2.11 shows the air-ice-water path is behaving as a low-pass filter at the drivepoint location. This comes as no surprise when comparing to the previous evaluation of Figure 2.5 and Figure 2.7. The modal properties of the ice do not seem to have an effect on the hydrophone response. The coherence in Figure 2.11 show that between $20 \mathrm{~Hz}$ and $300 \mathrm{~Hz}$ and at specific frequencies of interest $(29 \mathrm{~Hz}$ and $800 \mathrm{~Hz}$ ) the system response and excitation are linearly related. Low coherence in frequency bands less than $20 \mathrm{~Hz}$ and greater than $300 \mathrm{~Hz}$ are likely due to low input signal amplitude from the cannon or non-linear stiffness and damping ice properties. 


\subsubsection{Transmission Loss}

\subsubsection{Tonal Underwater Transmission Loss}

To quantify the underwater transmission loss $\left(T L_{U W}\right)$ for the underwater tones, the meansquared pressure of each tone was determined at the source hydrophone, $\overline{p_{\text {SUW }}^{2}}$, and receiver hydrophone, $\overline{p_{\text {rUW }}^{2}}$, locations. For each tonal frequency, the source and receiver hydrophone data were time domain filtered with bandpass cutoffs at plus/minus $5 \%$ of the center frequency. The mean-squared pressure at the source and receiver was then determined in the filtered band. The ratio of receiver mean-squared pressure to source mean-squared pressure was then calculated for each experiment in every frequency band (Equation 2.12). Figure 2.13 shows $T L_{U W}$ as a function of distance.

$$
T L_{U W}(f)=\frac{\overline{p_{r U W}^{2}}(f)}{\overline{p_{s U W}^{2}}(f)}
$$

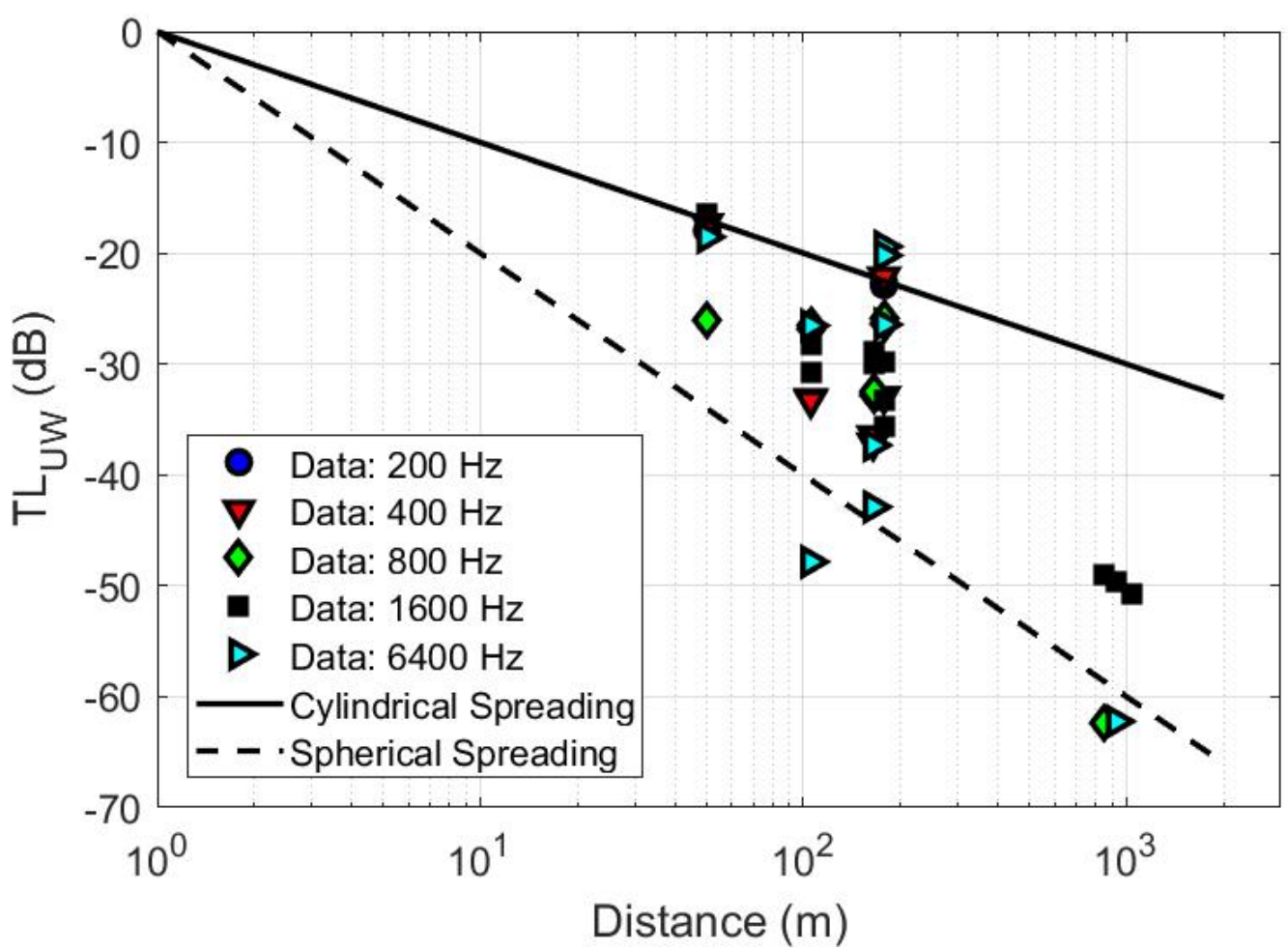

Figure 2.13 Measured underwater acoustic transmission loss data shown with theoretical cylindrical and spherical spreading curves for reference. 


\subsubsection{Atmospheric Transmission Loss}

To quantify the atmospheric transmission loss $\left(T L_{A A}\right)$, the ratio of autopower spectra between the source microphone and receiver microphones were computed for the cannon experiments (Figure 2.14). The source microphone autopower spectra was normalized to $1 \mathrm{~m}$ distance from the source. The ratio of autopower spectra between the receiver microphone and source microphone were then filtered into octave bands to determine the mean-squared pressure ratio in each respective band. The ratio of received meansquared pressure, $\overline{p_{r A}^{2}}$, to source mean-squared pressure, $\overline{p_{S A}^{2}}$, at $1 \mathrm{~m}$ in each frequency band was then converted to $\mathrm{dB}$ as shown in Equation 2.13.

$$
T L_{A A}(f)=10 \log _{10} \frac{\overline{p_{r A}^{2}}(f)}{\overline{p_{S A}^{2}}(f)}
$$

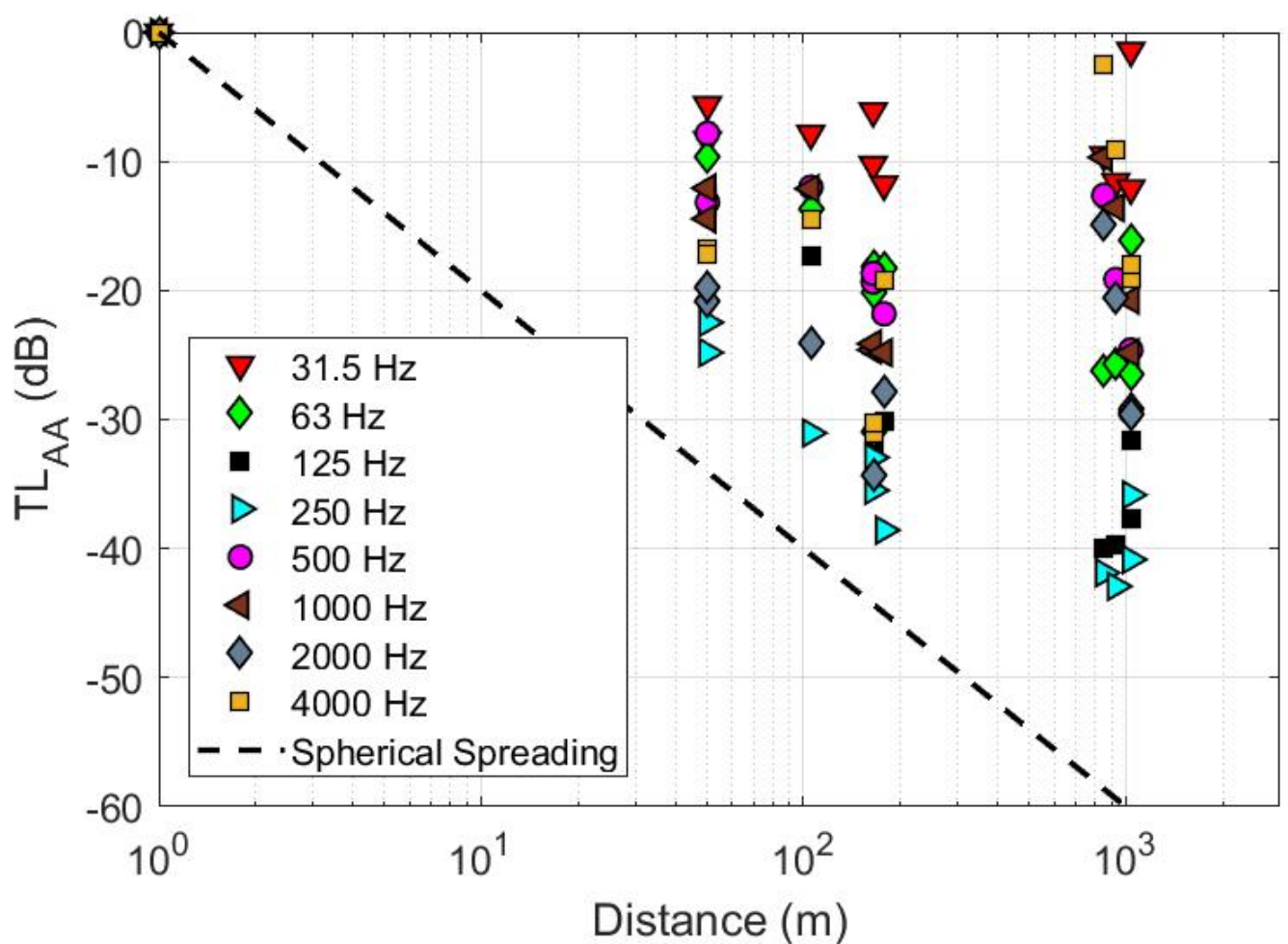

Figure 2.14 Measured atmospheric transmission loss data and theoretical spherical spreading curve shown for reference.

\subsubsection{Air - Ice - Water Transmission Loss}

The transmission loss between the cannon source and the down-range hydrophones was computed using an autopower spectra ratio. The source microphone autopower spectra was normalized to $1 \mathrm{~m}$. The ratio between the hydrophone sound pressure at the receiver location and the microphone sound pressure $1 \mathrm{~m}$ from the cannon was then filtered into 
octave bands to determine the transmission loss through the air, ice, and water $T L_{A I W}$ shown in Figure 2.15. It should be noted that the difference in reference pressures between air and water was not accounted for. Only a ratio of mean-squared pressure at the receiver hydrophone, $\overline{p_{r U W}^{2}}$, to mean-squared pressure of the source microphone, $\overline{p_{S A}^{2}}$, was computed. The TL in each frequency band was then converted to $\mathrm{dB}$ as shown in Equation 2.14.

$$
T L_{A I W}(f)=10 \log _{10} \frac{\overline{p_{r U W}^{2}}(f)}{\overline{p_{S A}^{2}}(f)}
$$

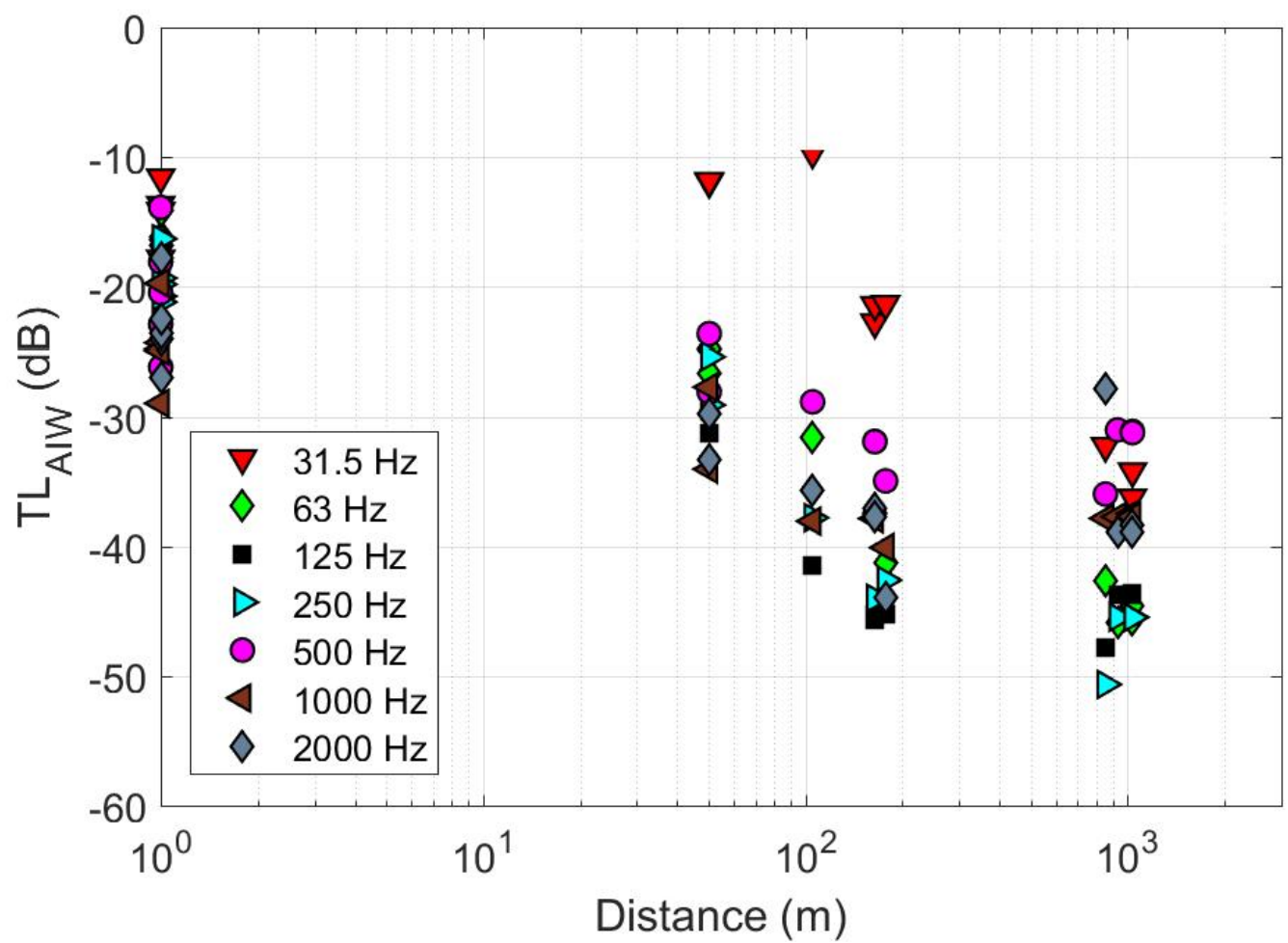

Figure 2.15 Measured transmission loss data through the combined air-ice-water path.

\subsection{Discussion and Future Works}

The frequency response functions in Figure 2.10 and Figure 2.11 are useful for determining the various acoustic path contributions between the air, ice, and the water. The through-thickness resonance in the ice at $800 \mathrm{~Hz}$ and the increase in attenuation at the same frequency are suspected to be related. This relationship should be proven with further investigation on the effect of various ice thicknesses and bottom reflection loss on the additional attenuation.

The majority of the underwater TL data (Figure 2.13) falls between the theoretical cylindrical and spherical spreading curves. This is similar to the transmission loss results 
determined by Pecknold et. al. for open water in Barrow Strait ${ }^{45}$. Granted, Pecknold's study was conducted over longer ranges, greater depths, and lower frequency than the data presented in this chapter.

The measured atmospheric transmission loss is less than theoretical spherical spreading (Figure 2.14) which indicates a downward refracting atmosphere over the course of this test. The propagation conditions (temperature and wind velocity profiles, humidity, and atmospheric turbulence) were not recorded with enough resolution to make any conclusion on the relative contributions of different path effects.

The offset of the $T L_{A I W}$ data (Figure 2.15) represents 11.3 to $24.8 \mathrm{~dB}$ of transmission loss through the air and ice at the source location. Upon coupling into the water, the $T L_{A I W}$ data appears to follow the trend of cylindrical spreading.

Due to the variation on the transmission loss data, there are loss mechanisms which are not accounted for in this data set. Underwater absorption, additional attenuation, and acoustic mode coupling are all factors which should be addressed in future studies.

The underwater absorption coefficient was not directly measured in Barrow but can be estimated from Equation 2.15 as shown by Urick $^{46}$. The underwater absorption coefficient was computed to be $1.8916 \mathrm{e}-5 \mathrm{~dB} / \mathrm{m}$ from Equation 2.15 where $\rho_{o}$ is the nominal density of sea water $1.029 \mathrm{~g} / \mathrm{cm}^{3}, c$ is the measured underwater sound speed $1.441 \mathrm{e} 5 \mathrm{~cm} . / \mathrm{s}$, and $f$ is the highest frequency of interest $6400 \mathrm{~Hz}$. At the longest range of interest, $1035 \mathrm{~m}$, the underwater absorption is less than $0.02 \mathrm{~dB}$ and therefore underwater absorption can be neglected for short range TL problems.

$$
\alpha=20 \log _{10}(e) \frac{16 \pi^{2}}{3 \rho_{o} c^{3}}(0.0311) f^{2} * \frac{100 \mathrm{~cm}}{1 \mathrm{~m}}
$$

Additional attenuation accounts for loss mechanisms which are not related to geometric spreading. In general, additional attenuation increases as propagation distance increases due to absorption in the sea bed and scattering from the complex under-ice surface. In this study, under-ice surveys of the seabed and ice layer were not carried out. Therefore, it was not possible to characterize the reflection losses of the bottom and undersurface of the ice as a function of frequency and grazing angle. In subsequent studies, it is recommended that all additional attenuation mechanisms be individually quantified in the under-ice environment to better understand loss mechanisms. Also, the effect of underwater acoustic modes on the transmission loss in the shore-fast, shallow water realm has not been considered. It is of interest to study acoustic modes in this unique environment and compare to the measured data presented here.

To build upon the results presented above, there are several additional areas where further investigation is necessary on first-year, shore-fast, thin ice sheets.

The transmission loss data is somewhat sparse in spatial resolution because weather and ice conditions on-site limited the number of receiver locations that were possible to 
measure. Due to the harsh environmental conditions encountered in the shore-fast Arctic region (high winds, large ice ridges, etc.), the quantity of experiments originally anticipated while in Barrow, AK were not possible to be conducted. The presence of ice ridges provided special challenges for deployment of hardware. The measurement methods for Arctic transmission loss, and similar Arctic measurements over long ranges, in the shore-fast region require significant improvement to provide more spatial resolution and a greater number of spectral averages.

At the test locations, holes were drilled in the ice to deploy the hydrophones. Ideally, none of the acoustic energy from the cannon would pass through the hole in the ice to the hydrophone, however, this was not likely the case because the sensors were not frozen in

the holes. It is unclear if the ice borehole acts as an acoustic short circuit for the sound to travel between the air and the water. Further investigation is required to determine how much of the acoustic energy is passing through the hole in the ice rather than directly through the ice sheet since this will have some effect on the hydrophone FRF measurement in Figure 2.11.

Finally, due to the changing Arctic environment, anthropogenic activity is expected to increase in the Arctic realm where this study has been conducted. Acoustic methods should be developed to detect, identify, and track anthropogenic sources in this new environment.

\subsection{Summary}

Acoustic data were collected $2 \mathrm{~km}$ offshore of Barrow, Alaska during April 2016. Experiments were conducted on first-year, shore-fast ice approximately $1.05 \mathrm{~m}$ thick and in shallow water between 8-12 m deep. At the test sites chosen, the surrounding ice was rough with many ice ridges $1-5 \mathrm{~m}$ in height. These conditions are typical of annuallyformed shore-fast ice and offshore first-year ice in the Arctic. Methods were developed to characterize the Arctic sound propagation in these shallow, thin, ice-covered waters including Frequency Response Functions and transmission loss. A propane cannon, instrumented force hammer, and underwater speaker were used as acoustic sources and microphones, geophones, and hydrophones were used as receivers.

Frequency Response Functions were computed between the various sources and receivers to further define the multi-modal response of the Arctic environment in the frequency domain. An air-coupled flexural wave at $29 \mathrm{~Hz}$ was identified in the FRF between the geophone and the cannon source. A through-thickness compressional mode was identified at $800 \mathrm{~Hz}$ in the geophone response to the hammer source. The $800 \mathrm{~Hz}$ mode was used to compute the compressional speed of sound in the ice which was $3360 \mathrm{~m} / \mathrm{s}$.

The transmission loss was determined through the air, the ice, and the water paths and combined multi-modal paths. Underwater, the transmission loss varied between the theoretical limits of cylindrical and spherical acoustic spreading ( -3 to $-6 \mathrm{~dB}$ per doubling of distance respectively). The variation in the data is suspected to be due to the complex 
ice-ridged environment causing reflections and scattering and bottom attenuation. In the air, the transmission loss was measured to be less than theoretical spherical spreading indicating the possibility of a downward refracting atmosphere. The computation of transmission loss through the combined air, ice, and water path in response to the cannon source led to the observation of 11.3 to $24.8 \mathrm{~dB}$ of TL through the air and $1.05 \mathrm{~m}$ of ice. 


\section{Acoustic Studies on an Arctic-Like Surrogate: Lake Superior}

\subsection{Relation to Previous Work}

Following the analysis of acoustic measurements in Barrow, AK (chapter 2), several queries arose prompting further investigation.

First, the data collected in Barrow lacked spatial resolution and did not allow for sufficient analysis of the transmission loss in the near-shore region. The effects of the bottom and ice reflection losses and other additional attenuation mechanisms were not individually quantified. Additionally, the local bathymetry was not measured below the shore-fast ice sheet. These considerations prevented comparative TL simulations to be developed with any accuracy and the data was only analyzed on gross comparison to spherical or cylindrical spreading. In order to validate TL data in shore-fast arctic environments, an increased number of test sites with a greater number of spectral averages is required as well as measurements of additional attenuation mechanisms and bathymetry.

Second, the effects of ice boreholes - which were drilled to deploy hydrophones under the ice - on frequency response function measurements was of concern. It was unclear if the ice borehole acts as an acoustic short circuit for the sound to travel between the air and the water. The quantity of the acoustic energy passing through the borehole (or lack thereof) is of interest.

Lastly, the modal behavior of the ice is of interest with respect to the impedance relationship between the ice and the water. Boundary condition assumptions were made to compare the measured dynamic mobility of the ice in AK. These assumptions may not hold for all environmental cases. Therefore, a thorough analytical derivation and investigation of the through-thickness ice resonance is necessary to compare to measured data. Furthermore, it is of interest to predict the ice thickness based on measurement of the through-thickness resonant frequencies.

In attempt to reconcile these queries, further data was collected on the Keweenaw Waterway which is part of Lake Superior. While Lake Superior is completely fresh water, the environment in the winter months is directly comparable to that of the Arctic. Ice thicknesses, water depths, wind speeds, and air temperature are all similar between the two environments and therefore data collected on Lake Superior is useful in predicting Arctic acoustics.

\subsection{Experimental Methods}

Acoustic propagation measurements were recorded on February $4^{\text {th }}$ and $8^{\text {th }}, 2018$. Measurements were made on Portage Lake which is part of the Keweenaw Waterway. 
The Keweenaw Waterway connects to Lake Superior at its north and south entries. The experiment site GPS coordinates are shown in Table 3.1 and are mapped in Figure 3.1. Site 1 was used as the source location and sites 2 through 11 were receiver locations. Table 3.1 shows the distance of the sites relative to the source location.

The experimental methods used during testing on Portage Lake were essentially the same as those described in chapter 2. Variations to those methods will be described in this section.

\subsubsection{Test Environment}

The ice covering Portage Lake during the experiments was nominally 30 to $43 \mathrm{~cm}$ thick with the top and bottom 5 to $7 \mathrm{~cm}$ being slushy and not completely frozen. Several $\mathrm{cm}$ of snow covered the top of the ice but was removed when placing transducers. The ice layer completely covered the entire area of Portage Lake and ice ridges were completely absent from the test area.
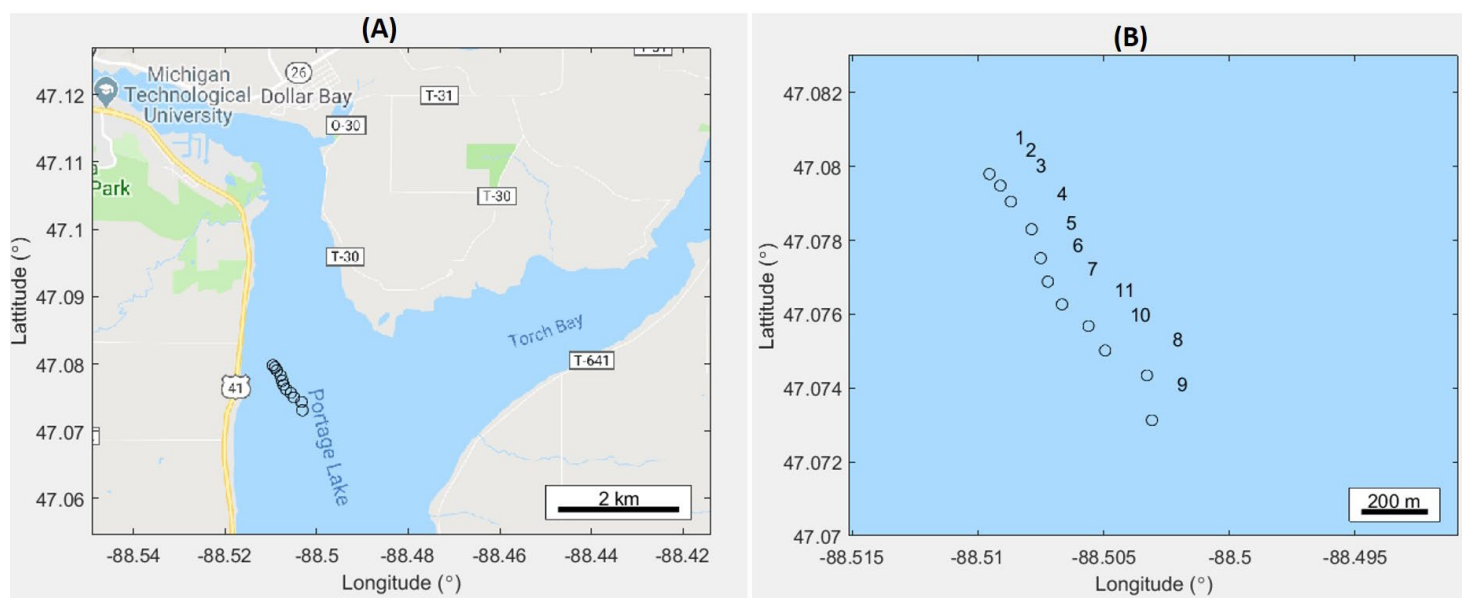

Figure 3.1 Acoustic experiment sites in the Keweenaw Waterway. (A) Keweenaw Waterway (Portage Lake). (B) Zoomed in view of test sites.

Comprehensive bathymetry of the Portage Lake test range was measured with a Lowrance Hook2 Sonar/GPS system on August $9^{\text {th }}, 2018$. Bathymetry plots may be seen in Appendix A.2. The water depth below the ice varied between 8.5 to $12.6 \mathrm{~m}$ over the test range. Underwater sound speed profiles were measured at the test sites with a CastAway CTD. The sound speed underwater ranged between 1404 to $1409 \mathrm{~m} / \mathrm{s}$ (Figure 3.2) with an average of $1406 \mathrm{~m} / \mathrm{s}$.

On the test days, air temperature ranged between -20 to $-12^{\circ} \mathrm{C}$ and wind speed ranged between 2 to $9.5 \mathrm{~m} / \mathrm{s}$ (Appendix A.1). Wind direction was out of the north-west for the majority of the test period with some $180^{\circ}$ direction shifts close to mid-day on February $8^{\text {th }}$. 
Table 3.1 Acoustic experiment site GPS position and distances on Portage Lake

\begin{tabular}{cccc} 
Site & N (GPS, d. $)$ & W (GPS, d. $)$ & Distance $(\mathrm{m})$ \\
\hline 1 & 47.079800 & -88.509533 & - \\
2 & 47.079483 & -88.509100 & 48 \\
3 & 47.079050 & -88.508683 & 110 \\
4 & 47.078300 & -88.507866 & 210 \\
5 & 47.077516 & -88.507500 & 300 \\
6 & 47.076883 & -88.507216 & 370 \\
7 & 47.076266 & -88.506650 & 450 \\
8 & 47.074350 & -88.503283 & 770 \\
9 & 47.073133 & -88.503083 & 890 \\
10 & 47.075016 & -88.504933 & 640 \\
11 & 47.075683 & -88.505600 & 550 \\
\hline
\end{tabular}
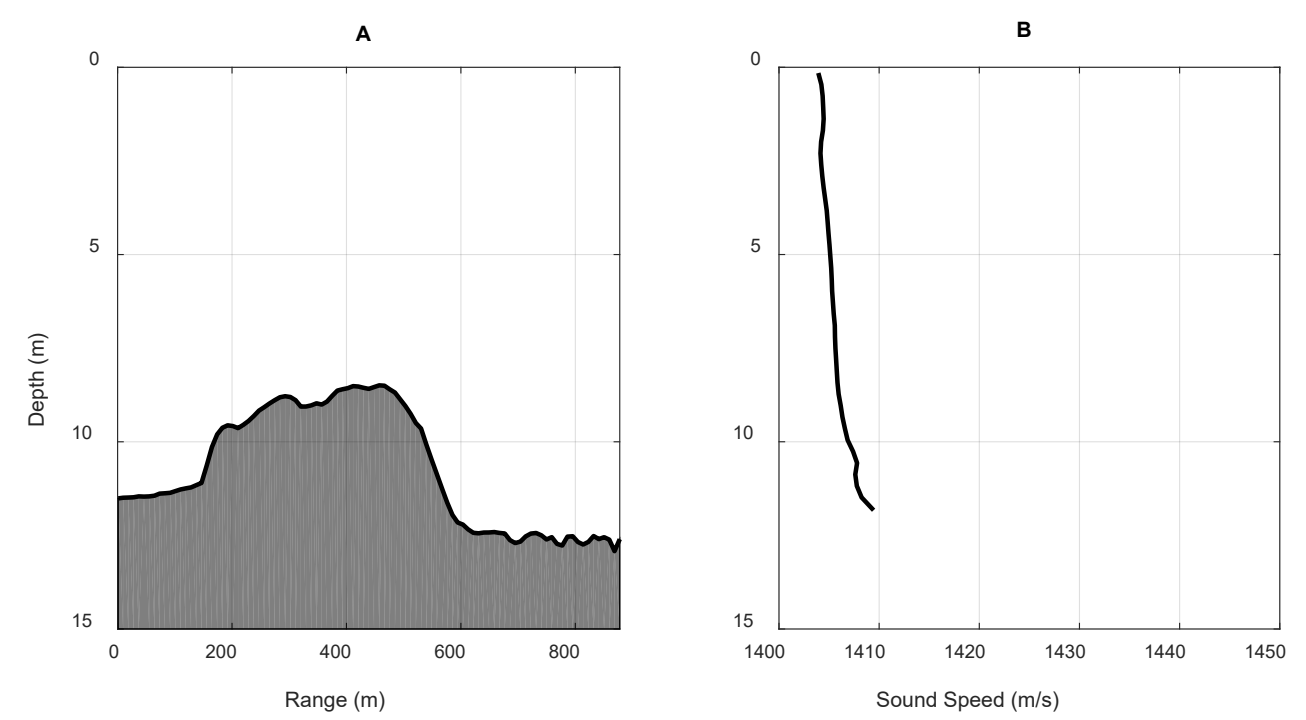

Figure 3.2 (A) Bathymetry and (B) underwater sound speed profile of Portage Lake in the measurement area 


\subsubsection{Sources and Receivers}

The experimental setup on Portage Lake is shown in Figure 3.3. The sources, receivers, and data collection systems used on Portage Lake were similar to those used during testing in Barrow, AK; described in section 2.2. At the source site (site 1), a propane cannon was used for atmospheric excitation, an instrumented impact hammer was used for ice excitation, and an underwater speaker was used for underwater excitation. Transducers were used at the source site to quantify the source levels. These included microphones, geophones, and hydrophones, in the air, on the ice, and underwater respectively. The transducer specifications are shown in Table 3.2. The underwater speaker was powered from a Boss R4002 audio amplifier. The data system and amplifier were powered by a 12 -volt car battery.

At the receiver sites, microphones, geophones, and hydrophones were similarly used to quantify the transmission loss downrange. The source and receiver data were time synchronized in post-processing via GPS timestamps from the data systems.

Table 3.2 Transducer model numbers and sensitivities used on Portage Lake

\begin{tabular}{c|c|c|c|c}
\hline $\begin{array}{c}\text { Transducer } \\
\text { Type }\end{array}$ & \multicolumn{2}{|c|}{ Source } & \multicolumn{2}{c}{ Receiver } \\
\hline & Model No. & Sensitivity & Model No. & Sensitivity \\
\hline Microphone & PCB 377C01 (426A05) & $2 \mathrm{mV} / \mathrm{Pa}$ & PCB 378B02 & $50 \mathrm{mV} / \mathrm{Pa}$ \\
Geophone & GS One 3C & $100 \mathrm{~V} / \mathrm{mm} / \mathrm{s}$ & GS One 3C & $100 \mathrm{~V} / \mathrm{mm} / \mathrm{s}$ \\
Accelerometer & PCB 302A07 & $10 \mathrm{mV} / \mathrm{g}$ & PCB 626A34 & $10.02 \mathrm{~V} / \mathrm{g}$ \\
Hydrophone & TC 4013 & $2.82 \mathrm{e}-05 \mathrm{~V} / \mathrm{Pa}$ & TC 4032 & $0.01 \mathrm{~V} / \mathrm{Pa}$ \\
Impact Hammer & PCB 0.086D20 & $0.23 \mathrm{mV} / \mathrm{N}$ & & \\
\hline
\end{tabular}




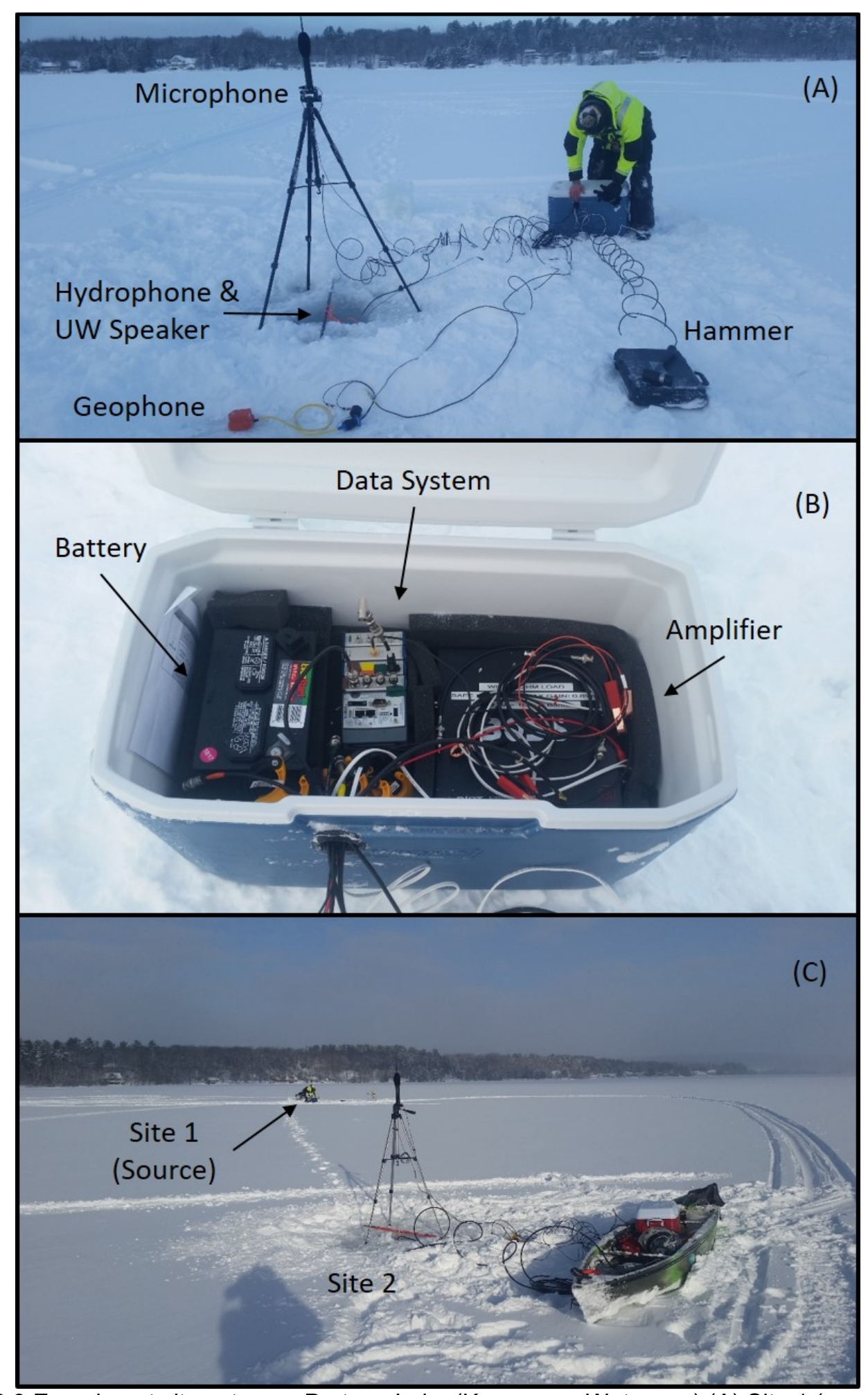

Figure 3.3 Experiment site setup on Portage Lake (Keweenaw Waterway) (A) Site 1 (source site). Propane cannon is not pictured. (B) Data system box. Data system and underwater speaker amplifier powered by $12 \mathrm{~V}$ car battery. (C) Site 2 looking north toward site 1. 


\subsection{Transmission Loss}

In acoustics, it is of interest to determine how sound energy propagates and decays through various environments. When regarding environmental acoustics, transmission loss (TL) is defined as the ratio of sound intensity at a selected receiver location $\left(I_{r}\right)$ to the sound intensity $1 \mathrm{~m}$ from an acoustic source $\left(I_{S}\right)^{47}$,

$$
T L=10 \log _{10} \frac{I_{r}}{I_{s}}=20 \log _{10} \frac{p_{r}}{p_{s}}
$$

The intensity ratio may also be considered as a mean squared pressure ratio $\frac{p_{r}}{p_{s}}$ shown above.

Classically speaking, TL is thought of in terms of geometrical spreading losses throughout the environment. An acoustic source provides sound energy to an environment, the sound energy spreads out geometrically, and reduces in amplitude as a function of that spreading. If cylindrical (2D) spreading geometry is considered the losses are proportional to $\frac{1}{2 \pi r}$, and if spherical (3D) spreading geometry is considered, the losses are proportional to $\frac{1}{4 \pi r^{2}}$. Transmission loss due to geometrical spreading may then be represented as,

$$
T L=20 \log _{10}(r)
$$

or,

$$
T L=10 \log _{10}(r)
$$

for spherical and cylindrical spreading losses respectively ${ }^{47}$.

In the majority of acoustic environments, spreading losses are not the only contributing factor to TL. Other contributing factors include:

- Absorption in the propagation medium

- Refraction due to environmental sound speed profiles

- Interaction with local boundary conditions including reflection losses and backscattering

- Bathymetry, altimetry, or topography of the environment

- Constructive and destructive interference within the propagation paths

all of which - depending on the environment - may play major roles in affecting the resultant acoustic energy which is present at a given receiver location. While previously 
referred to as additional attenuation, the above factors are increasingly lumped in with TL as a singular measurement to facilitate simulation and measurement alike.

As needs for more capable TL approximations become necessary, the above considerations must be included within TL models. With the number of factors in TL models increasing, analytical approximations of TL become less possible to develop. Therefore, the majority of TL simulations rely on numerical and computational approaches.

It is necessary to validate these models with experimental data. The below sections will discuss the relevant additional attenuation mechanisms in the Arctic environment, appropriate simulation techniques, and compare the TL data collected on Portage Lake.

\subsubsection{Additional Attenuation Mechanisms}

The most prominent additional attenuation mechanisms in the underwater Arctic environment are that which occur at the ice-water boundary and the water-bottom boundary. When an incident acoustic wave underwater impinges on the ice layer a portion of the wave is transmitted into the ice, a portion of the wave is scattered back towards the source, and a portion of the wave is reflected ${ }^{34,46-48}$. Likewise interaction takes place when an acoustic wave impinges on the sea floor (or lake floor as the case may be). The problem is complicated when the ice or bottom is layered. Each layer having different acoustic impedance causes an additional reflection. In general, backscattering effects are most significant when the boundary condition geometry is not smooth. For example, ice keels in the Arctic ice layers typically cause significant scattering ${ }^{46-53}$. Each of the reflected or scattered waves from the ice layer and the bottom layer contribute coherently to the overall TL in the water column, therefore; the additional attenuation mechanisms can have a significant effect on transmission loss.

A common characterization of the ice-water and water-bottom interaction is that of reflection loss (RL). Reflection loss represents the strength of the reflected wave in relation to the incident wave. Typically, RL is measured by outputting a short time duration signal such as a ping or blast and measuring the direct path and reflected path signals $^{48}$. The decibel ratio of the two signals then represents the RL,

$$
R L=10 * \log _{10}\left|\frac{H_{i}}{H_{r}}\right|
$$

where $H_{i}$ and $H_{r}$ represent incident and reflected the frequency response functions relative to the source signal. 


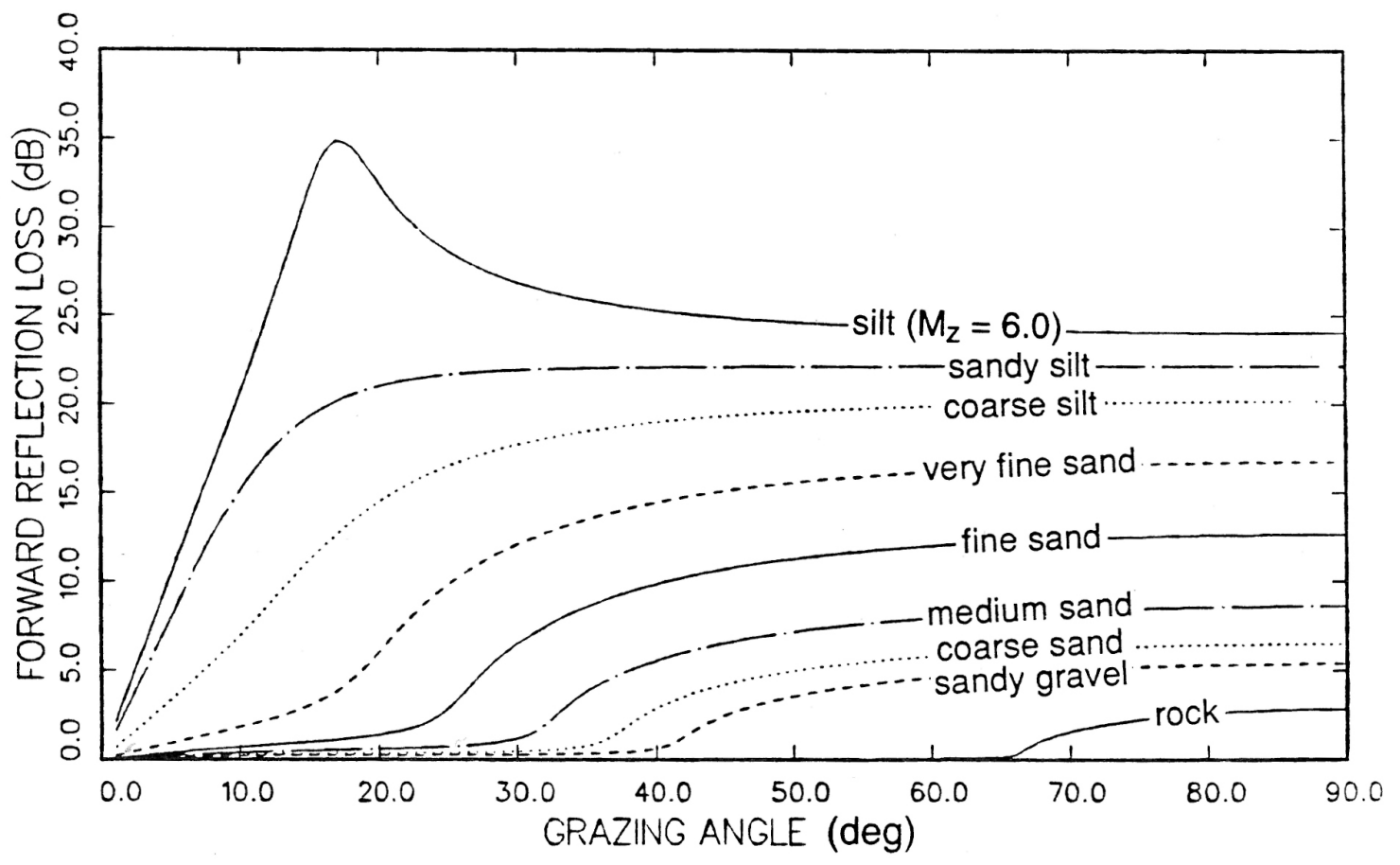

Figure 3.4 Modeled reflection loss for generic bottom types. Valid for 10-100 kHz. (Reproduced with permission from APL-UW High-Frequency Ocean Environmental Acoustic Models Handbook ${ }^{54}$ )

As Etter states 48 , "When narrowband pulses are used, measured losses are often irregular and variable, showing peaks and troughs due to the interference effects of the layering in the bottom." Therefore, RL is quite difficult to measure and leads to unreliable results. Furthermore, the acoustic properties of the ice and the bottom in shore-fast, first-year ice Arctic environments are not well known due to the difficulty in obtaining core samples in these regions ${ }^{48}$. This leads to inaccuracies in theoretical models of $\mathrm{RL}^{48}$.

$\mathrm{RL}$ is a function of grazing angle (incident angle). RL generally increases at low grazing angles between roughly 0 to 30 degrees. From roughly 30 degrees to normal incidence, RL flattens out to some constant value ${ }^{48,54}$. Many theoretical models of RL accounting for grazing angle and frequency have been developed, but the majority focus on high frequencies between 10 to $100 \mathrm{kHz}^{54,55}$. The frequency dependence of RL is usually not taken into account. Figure 3.4 shows an example of modeled RL for high frequencies from the Applied Physics Lab at the University of Washington. Generally speaking, higher impedance boundary conditions provide less reflection loss than low impedance boundary conditions. For example, a silty bottom will provide between 15 to $35 \mathrm{~dB}$ of $\mathrm{RL}$ for most grazing angles while a rock bottom provides less than $5 \mathrm{~dB}^{\mathrm{RL}}{ }^{54,55}$.

\subsubsection{Implementation of the MLS signal for RL measurement}

Barnard ${ }^{56}$ provided a novel method for characterizing the acoustical properties of multilayered media using a maximal length sequence (MLS) as an output signal and measuring 
the response with a single transducer. The MLS signal is created using a linear feedback shift register in which the coefficients of each tap are either one or zero. The MLS signal is then played through a speaker to excite the environment of interest. A response measurement is made at a transducer location to capture the entire MLS signal and the path effects from the environment. The global impulse response function (IRF) of the environment is determined using the Fast Hadamard Transform (FHT) of the MLS signal measured at the transducer location. From the global impulse response function, the direct path and reflected path sound waves are isolated using time domain windowing and then transformed to the frequency domain using the $\mathrm{FFT}^{57-59}$.

The direct path FRF is approximately equivalent to the incident FRF for small grazing angles and short path distances. If the difference in length between the incident path and the direct path becomes large, the amplitude difference may be corrected by subtracting $20 * \log _{10} r$ from the direct path amplitude, where $r$ is the difference in path length.

Effectively, this yields the frequency response function (FRF) for the incident sound wave as well as the reflected sound wave. The ratio of the two FRFs is the RL.

There are several advantages to using the MLS signal to determine RL ${ }^{60-62}$. The MLS signal is a deterministic pseudo-random binary signal of length,

$$
L=2^{m}-1
$$

where $m$ is any positive integer. The deterministic nature of the MLS signal allows it to be collected leakage free without windowing the signal and allowing for high SNR. Additionally, the auto-correlation of the MLS signal approaches the Dirac delta function. That is to say, the MLS signal allows for theoretically equal energy distribution across all frequencies in the output signal autopower spectrum; provided that the length of the signal is long enough to capture the entire impulse response of the environment.

Furthermore, the MLS signal will allow for the measurement of RL as a direct function of frequency which is not typically accounted for in the majority of RL studies.

\subsubsection{Portage Lake RL}

The theoretical arrival times of the direct path, ice reflection, and bottom reflection are shown in Figure 3.5 (a). The theoretical arrival times were computed assuming specular reflections from the bottom and ice surfaces and using geometric ray paths. Given the water depth, source depth, hydrophone depth, and site distances, the length of the ray path was computed. The time of flight along the ray path was computed as the length of the ray path divided by the speed of sound in water; taken to be constant $1406 \mathrm{~m} / \mathrm{s}$. These estimates did not account for refraction because the water was shallow and the sound speed profile did not vary greatly through the water column. 
(A)

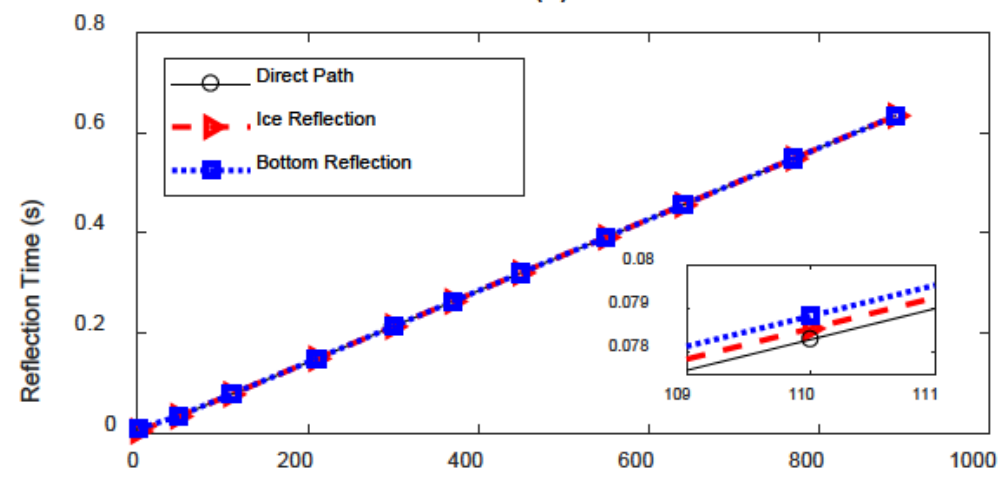

(B)

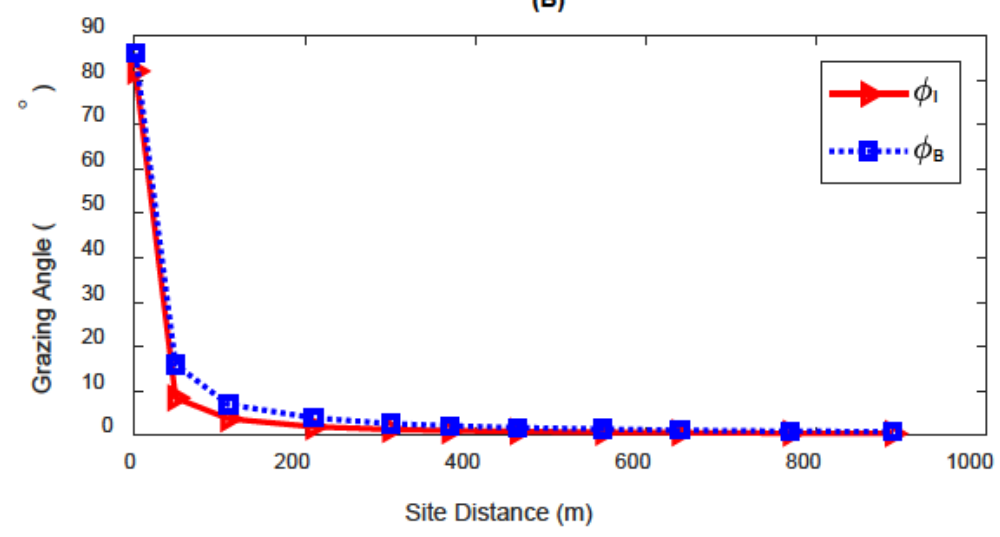

Figure 3.5 Theoretical (A) arrival times and (B) grazing angles for ice and bottom reflections at the measurement sites on Portage Lake.

It is observed that the theoretical time of arrival for the direct path, the ice reflection, and the bottom reflection are all very closely spaced. For example, at the $110 \mathrm{~m}$ site, there is less than 0.001 second spread between theoretical arrivals. The close spacing of arrivals makes it difficult to choose peaks within the global impulse response function which correspond to the particular arrival. Separating reflections, therefore, becomes difficult. The sites chosen on Portage Lake allow for measurement of reflection loss as a function of the theoretical grazing angles shown in Figure 3.5(b). The first four sites with ranges of $1 \mathrm{~m}, 48 \mathrm{~m}, 110 \mathrm{~m}$, and $210 \mathrm{~m}$, allow for measurement on the order of $86^{\circ}, 16^{\circ}, 7^{\circ}$, and $4^{\circ}$, respectively. Beyond $210 \mathrm{~m}$ the grazing angle continues decreasing, however the change in grazing between sites becomes very small. Therefore, RL will only be analyzed for the first four sites. 

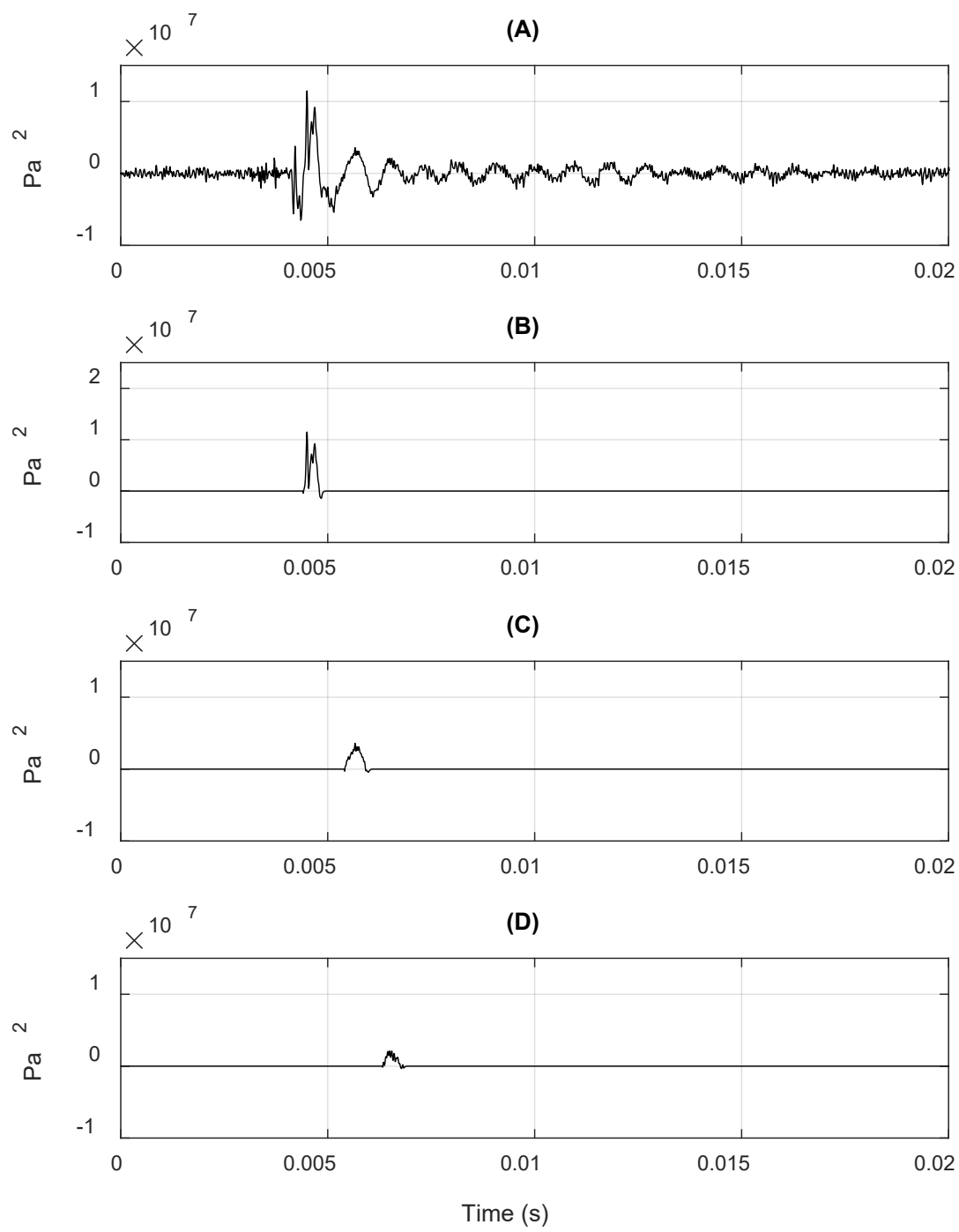

Figure 3.6 Impulse response function $1 \mathrm{~m}$ from the underwater source. (A) Full IRF from MLS signal. (B) Incident arrival. (C) Windowed ice reflection (D) Windowed bottom reflection.

The MLS signal was emitted by an underwater speaker at the source site. A hydrophone at the source site ( $1 \mathrm{~m}$ from the source) recorded the time series of the received signal. The fast Hadamard transform of the MLS signal was applied, yielding the global impulse response function. Distinct peaks can be seen in the global IRF in Figure 3.6(a). The first peak represents the direct path of the signal from the source to receiver. The direct arrival from source to receiver is assumed to be equal to the incident wave. The following peaks represent reflections from the ice layer and bottom layer respectively. The ice reflections arrive prior to the bottom reflection because the sound source and hydrophone were above the half-depth point in the water column. Multiple reflections are seen due to the signal reflecting back and forth between the ice and bottom layers. 
The reflections after the ice reflection and bottom reflection are termed parasitic reflections and are not considered ${ }^{56}$.

The direct arrival, ice reflection, and bottom reflection in the IRF were then separated by using a window function as shown in Figure 3.7. The window function is composed of a half-rectangular and half-blackman-harris window. The window was constructed such that the number of points of the rectangular window was the same number of points as the portion of the IRF to be extracted. The windowed IRF for the direct arrival, ice reflection, and bottom reflection are shown in Figure 3.6(b-d).

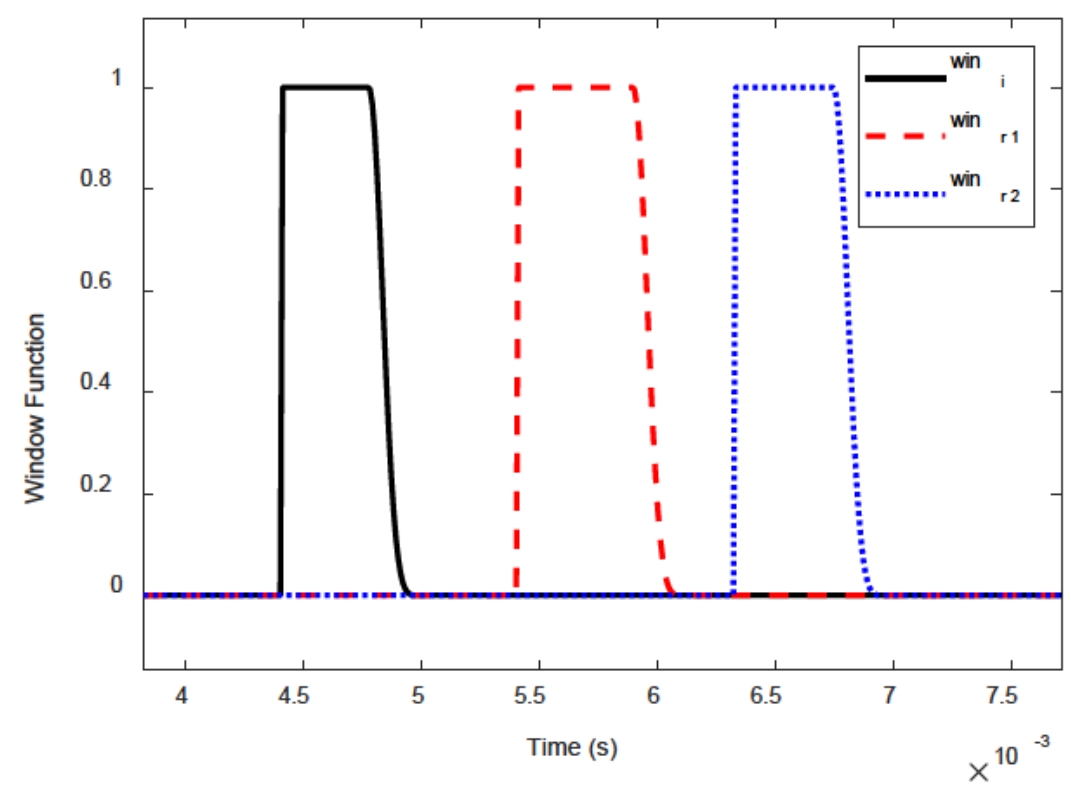

Figure 3.7 Example window functions used on the IRF to separate the incident, ice reflection, and bottom reflection components.

The frequency response functions are then computed from the windowed IRF via the FFT. The incident FRF, as well as the reflected FRFs from the ice and bottom, are shown in Figure 3.8(a). The difference between incident and reflected FRF yields the reflection loss of the ice and the bottom. RL is shown in Figure 3.8(b) with the ice grazing angle $\phi_{I}=89^{\circ}$ and the bottom grazing angle $\phi_{B}=86^{\circ}$. The RL is relatively constant at low frequencies (less than $2 \mathrm{kHz}$ ) with a value of $3 \mathrm{~dB}$ and $6 \mathrm{~dB}$ for the ice and bottom respectively. The $\mathrm{RL}$ increases as frequency increases beyond $2 \mathrm{kHz}$. Peaks are observed in the ice $\mathrm{RL}$ at 4.4, 6.4, 8.4, 12, 14, and $16 \mathrm{kHz}$ ranging from 7 to $20 \mathrm{~dB}$. Similar increase in RL is observed in the bottom layer with peaks at $6,9.5,13$, and 17 $\mathrm{kHz}$. 
(A)

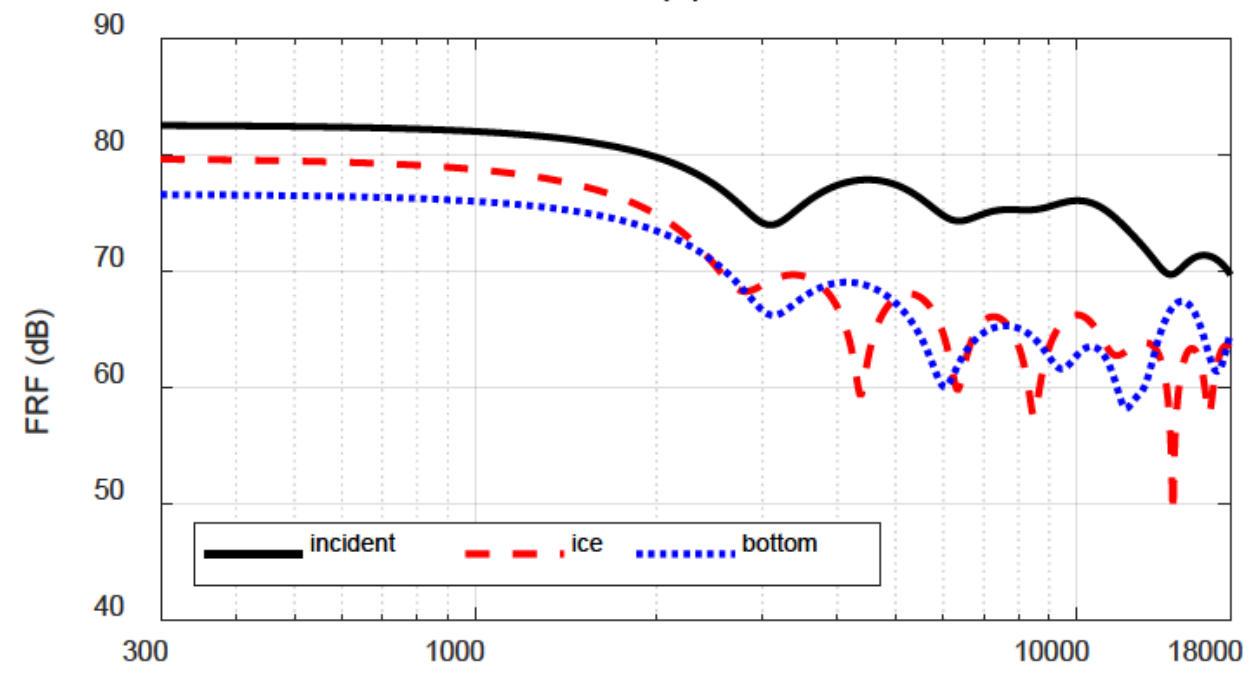

(B)

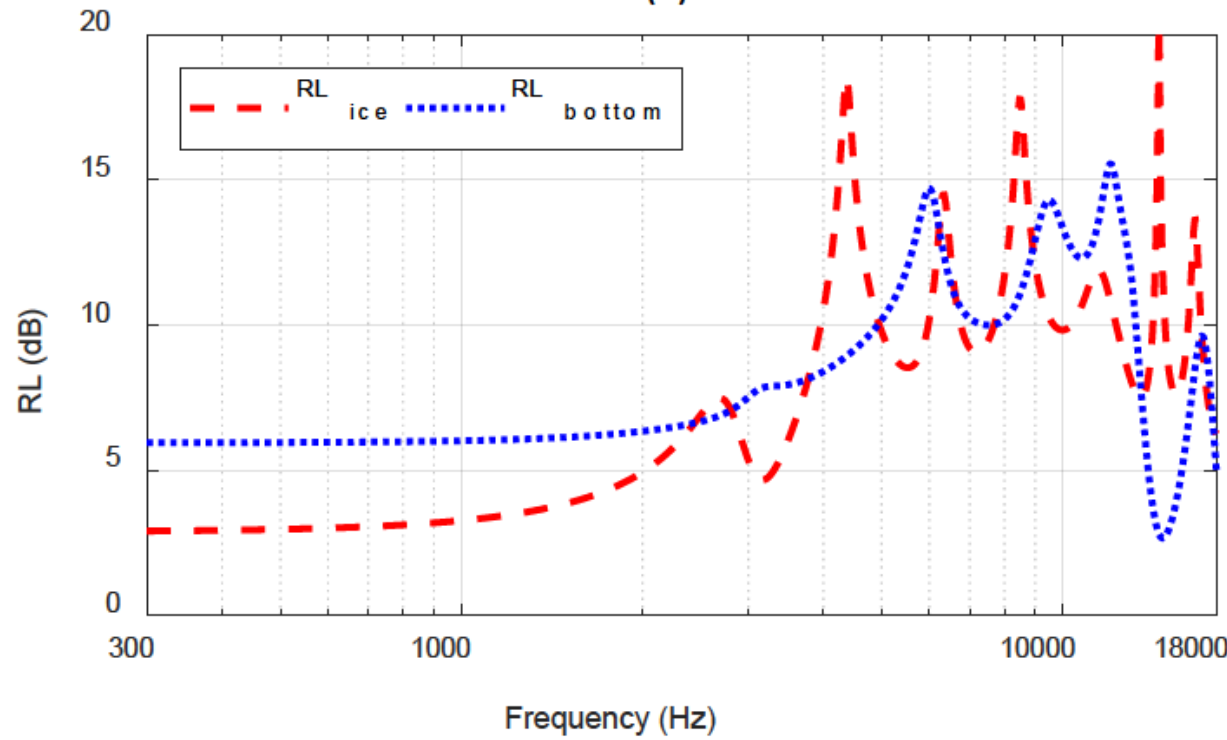

Figure $3.8(\mathrm{~A})$ Frequency response functions and (B) Reflection loss for $\phi_{I}=89^{\circ}$ and $\phi_{B}=86^{\circ}$. Source to receiver distance equal to $1 \mathrm{~m}$.

The reflection loss for shallower grazing angles is computed in a similar manner for sites at greater ranges. The global IRF for the receiver site at $110 \mathrm{~m}$ is shown in Figure 3.9(a) with windowed incident and reflected IRF signals in Figure 3.9(b-d). The corresponding FRFs and RL for the ice and bottom are shown in Figure 3.10 with grazing angles $\phi_{I}=$ $3.8^{\circ}$ and $\phi_{B}=7^{\circ}$ respectively. The RL for ice at the given incident angle is zero except in the range of 2,6 , and $15 \mathrm{kHz}$ on the order of 1 to $5 \mathrm{~dB}$. The bottom $\mathrm{RL}$ is on the order of $4 \mathrm{~dB}$ below $2 \mathrm{kHz}$ and has peaks at $6.2,8.4,12$, and $14 \mathrm{kHz}$ ranging from 7 to $15 \mathrm{~dB}$. 

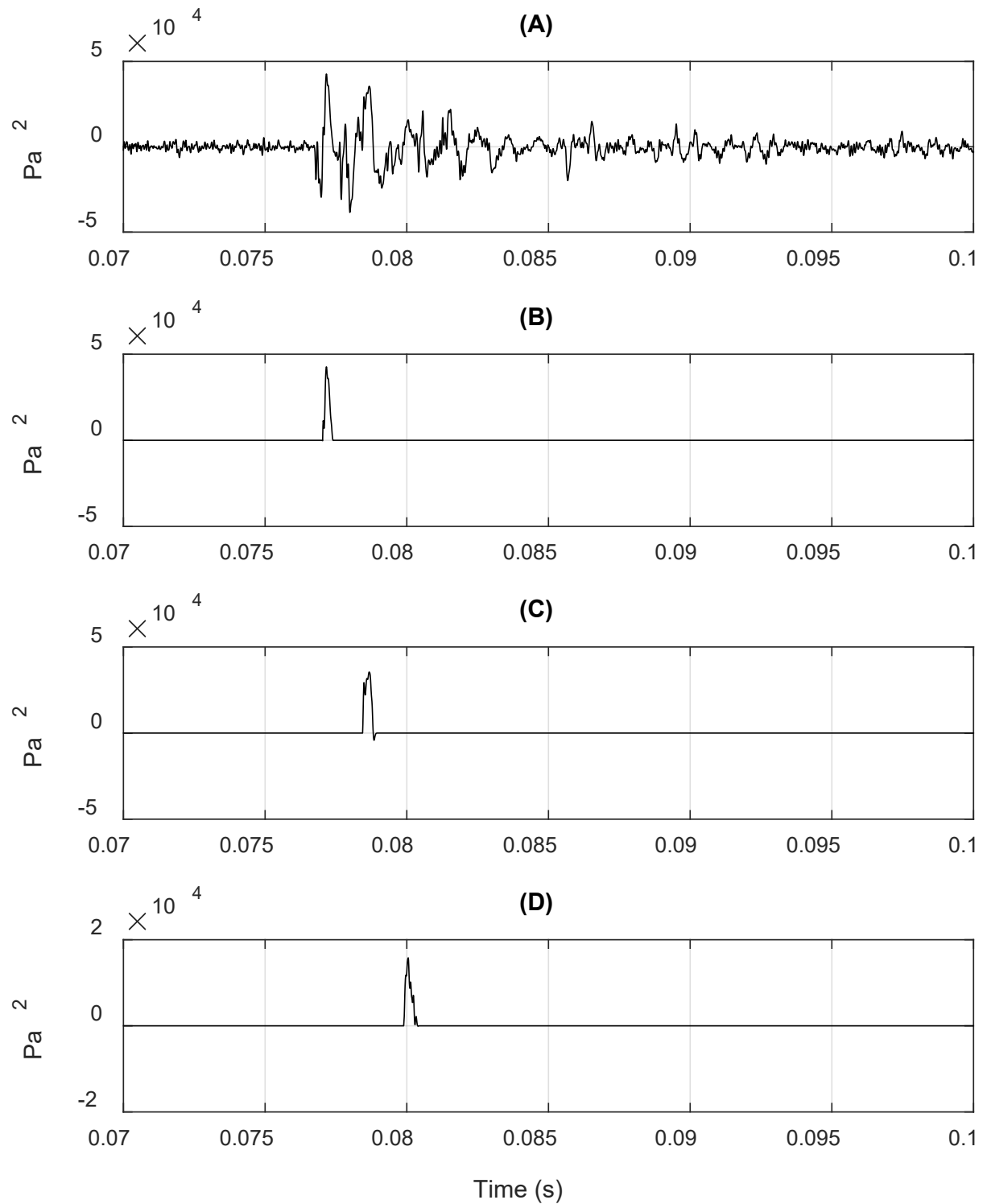

Figure 3.9 Impulse response function $110 \mathrm{~m}$ from the underwater source. (A) Full IRF from MLS signal. (B) Incident arrival. (C) Windowed ice reflection (D) Windowed bottom reflection. 
(A)

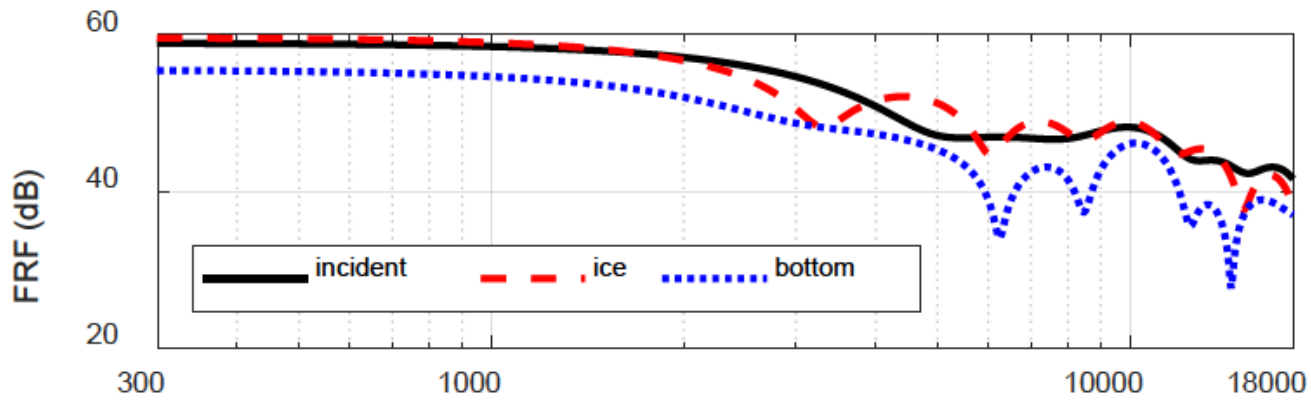

(B)

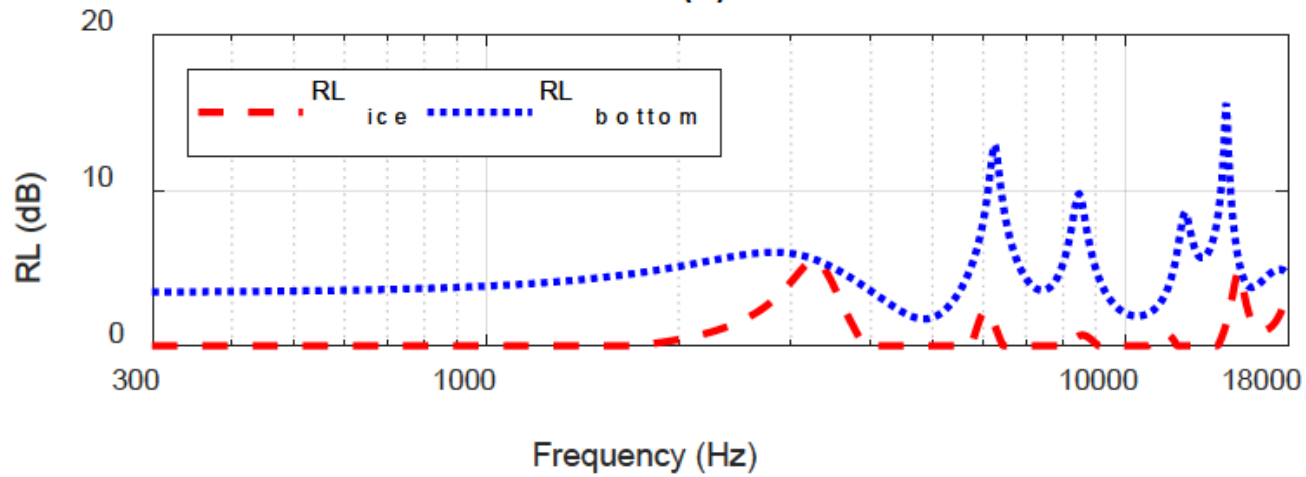

Figure 3.10 (A) Frequency response functions and (B) Reflection loss for $\phi_{I}=3.8^{\circ}$ and $\phi_{B}=7^{\circ}$. Source to receiver distance equal to $110 \mathrm{~m}$.

It is observed that the RL at shallower grazing angles is lower than that of grazing angles closer to normal incidence. This is supported by theoretical RL models such as the ones shown in Figure 3.4. While the theoretical models are presented as valid for frequencies greater than $10 \mathrm{kHz}$, they seem to be applicable for the data shown here. As expected, the RL generally increases as the frequency increases. As frequency decreases, the RL is observed to approach some (roughly) constant value, while at high frequencies the RL varies significantly. It is observed in the RL data shown here that there are critical frequencies which yield much greater losses. This phenomenon is not typically taken into account in RL models which usually claim to be valid across broadband frequency ranges.

The IRF, FRF, and RL for additional grazing angles may be seen in Appendix A.3.

\subsubsection{Simulation of TL}

The measurement of transmission loss is important for validation of simulated transmission loss models. This section will lay the groundwork for providing comparison between the collected TL data and currently available numerical models of TL. 
The theoretical basis for acoustic propagation simulation is the wave equation ${ }^{48}$

$$
\nabla^{2} \Phi=\frac{1}{c^{2}} \frac{\partial^{2} \Phi}{\partial t^{2}}
$$

where $\nabla$ represents partial derivatives with respect to all spatial variables, $c$ is the speed of sound, and $t$ is the time variable. $\Phi$ is assumed to be a function of both time and space (usually in two or three dimensions) as in,

$$
\Phi=\phi \mathrm{e}^{-j \omega t}
$$

where $\phi$ represents the spatial variables. Substituting and separating variables yields the Helmholtz equation

$$
\nabla^{2} \phi+k^{2} \phi=0
$$

which is only dependent on the spatial variations. The wavenumber $k=\frac{\omega}{c}=\frac{2 \pi}{\lambda}$, where $\lambda$ is the wavelength. The Helmholtz equation in cylindrical coordinates is represented as

$$
\frac{\partial^{2} \phi}{\partial r^{2}}+\frac{1}{r} \frac{\partial \phi}{\partial r}+\frac{\partial^{2} \phi}{\partial z^{2}}+k^{2}(z) \phi=0
$$

where $z$ is the depth coordinate and $r$ is the down-range coordinate. The Helmholtz equation is often implemented using various techniques to model transmission loss. Generally speaking, transmission loss may be defined as the ratio of acoustic pressure received at a down-range position $\left(z_{1}, r_{1}\right)$ to the acoustic pressure measured near the source $\left(z_{0}, r_{0}\right)$. This is expressed as,

$$
T L=-20 * \log _{10}\left|\frac{\phi\left(z_{1}, r_{1}\right)}{\phi\left(z_{0}, r_{0}\right)}\right| .
$$

Etter ${ }^{48}$ provides a very thorough comparison of the various types of TL simulation techniques. Jensen et al ${ }^{47}$ and Salomons ${ }^{63}$ provide a deep dive into the theory of computational acoustics. The most common numerical simulation techniques include, ray tracing (RT) algorithms, computation of normal modes (NM) of propagation, the Fast Field Program (FFP), and parabolic equation (PE) solving, among many others. These techniques have been developed over the course of several decades; each providing their own advantages and disadvantages. A brief explanation and comparison of these common methods is provided below. 


\subsubsection{Ray Tracing Algorithms}

Ray tracing methods assume a solution for $\phi$ to be a product of pressure amplitude function $A$ and phase function $P$ such that,

$$
\phi=A e^{j P} .
$$

The amplitude and phase functions are both dependent on all spatial coordinates considered. Substituting this form of $\phi$ into the Helmholtz equation yields a real component,

$$
\frac{1}{A} \nabla^{2} A-(\nabla P)^{2}+k^{2}=0
$$

which represents the geometry of the acoustic ray path as it travels through space, and an imaginary component,

$$
2(\nabla A * \nabla P)+A \nabla^{2} P=0
$$

which represents the acoustic wave amplitude along the ray path. A ray traveling through space is assumed to have some cross-sectional area $\alpha$. The cross-sectional area changes as steps through the spatial coordinates take place. By the conservation of energy, the amplitude at a new spatial position may be computed from a previous spatial position as in,

$$
A_{2}=\left(\frac{c_{2} \alpha_{2}}{c_{1} \alpha_{1}}\right)^{\frac{1}{2}} A_{1} .
$$

This facilitates stepping through discrete points to "trace" the amplitude of the ray along the geometrical path. Generally speaking, this method allows for range-dependent environment variables such as bathymetry, however, is limited to high frequencies; defined by,

$$
f>10 \frac{c}{d}
$$

where $d$ is the environment depth being considered.

\subsubsection{Normal Modes}

Starting with the non-homogeneous Helmholtz equation in two dimensions 


$$
\frac{1}{r} \frac{\partial}{\partial r}\left(r \frac{\partial p}{\partial r}\right)+\rho(z) \frac{\partial}{\partial z}\left(\frac{1}{\rho(z)} \frac{\partial p}{\partial z}\right)+\frac{\omega^{2}}{c^{2}(z)} p=-\frac{\delta(r) \delta\left(z-z_{s}\right)}{2 \pi r}
$$

where $\rho$ and $c$ are the density and sound speed profiles respectively. The right-hand side of the above equation represents a source impulse function at source depth $z_{s}$. The solution for acoustic pressure $p$ is sought in the form $p(r, z)=\Phi(r) \Psi(z)$. Substituting this form and separating variables yields two equations with the term $\Lambda$ introduced as the separation constant. One equation in terms of the $z$ coordinate,

$$
\rho(z) \frac{d}{d z}\left(\frac{1}{\rho(z)} \frac{d \Psi_{\mathrm{m}}(\mathrm{z})}{d z}\right)+\left(\frac{\omega^{2}}{c^{2}(z)}-\Lambda_{\mathrm{m}}^{2}\right) \Psi_{\mathrm{m}}(\mathrm{z})=0
$$

and one in terms of the $r$ coordinate,

$$
\frac{1}{r} \frac{d}{d r}\left(r \frac{d \Phi_{n}(r)}{d r}\right)+\Lambda_{n}^{2} \Phi_{n}(r)=-\frac{\delta(r) \Psi_{n}\left(z_{s}\right)}{2 \pi r \rho\left(z_{s}\right)}
$$

where

$$
\Phi_{n}(r)=\frac{j}{4 \rho\left(z_{s}\right)} \Psi_{n}\left(z_{s}\right) H_{0}^{1}\left(\Lambda_{n} r\right)
$$

and $H_{0}^{1}$ is a zeroth order Hankel function of the first kind. These are classic SturmLiouville (SL) eigenvalue problems for which solutions are well known ${ }^{47,64}$. The eigenfunctions of the SL problems are orthogonal such that,

$$
\int_{0}^{D} \frac{\Psi_{m}(z) \Psi_{n}(z)}{\rho(z)} d z=0, \text { for } m \neq n
$$

where $D$ is the upper bound of the $z$ domain. The value $m$ may take any integer value. The $m^{\text {th }}$ modeshape is characterized by the eigenfunction $\Psi_{m}(z)$ and the horizontal propagation constant is characterized by the eigenvalue $\Lambda_{m}$; for which there are infinite many orthogonal pairs. The infinite sum of the modeshapes provide the pressure as a function of $z$ and $r$,

$$
p(r, z) \cong \frac{j}{\rho\left(z_{s}\right) \sqrt{(8 \pi r)}} e^{-\frac{j \pi}{4}} \sum_{m=1}^{\infty} \frac{\Psi_{m}\left(z_{s}\right) \Psi_{m}(z) e^{j \Lambda_{m} r}}{\Lambda_{\mathrm{m}}}
$$


The transmission loss may then be computed as the ratio of pressure at some downrange position to pressure at the source position,

$$
T L(r, z) \cong-20 * \log _{10}\left|\frac{\sqrt{\frac{2 \pi}{r}}}{\rho\left(z_{S}\right)} \sum_{m=1}^{\infty} \frac{\Psi_{m}\left(z_{s}\right) \Psi_{m}(z) e^{j \Lambda_{m} r}}{\Lambda_{\mathrm{m}}}\right|
$$

\subsubsection{Fast Field Program}

Problems involving layered media have long been of interest within the acoustics community. When considering acoustic problems involving layered media, the classic approach has been to reduce the four-dimensional Helmholtz equation to several ordinary differential equations using integral transforms. Each ODE is then solved by matching the boundary conditions between each layer. This analytical approach is sufficiently effective for simple environments approximated with few numbers of layers. However, upon investigating more complex environments, numerical methods become necessary.

The fast field program falls into a larger category of numerical simulation techniques known as wavenumber integration which is used when studying layered media environments. The setup for this type of simulation typically involves a horizontally stratified environment with $m+1$ number of layers. Layer 1 being an upper halfspace, layer $m+1$ being a lower halfspace, and layers 2 to $m$ comprising different media layers within the propagation environment. Wavenumber integration at its core relies on solving the equation

$$
p(r, z)=\int_{0}^{\infty} g\left(k_{r}, z\right) J_{0}\left(k_{r}, z\right) k_{r} d k_{r},
$$

in each layer, where $J_{0}$ is the zeroth-order Bessel function and $g$ is the wavenumber kernel. It has been shown by the fast-field approximation, Equation 3.23 is equivalent to

$$
p(r, z) \cong \sqrt{\frac{1}{2 \pi r}} e^{-j\left(m+\frac{1}{2}\right) \frac{\pi}{2}} \int_{0}^{\infty} g\left(k_{r}, z\right) \sqrt{k_{r}} e^{j k_{r} r} d k_{r}
$$

for ranges which are greater than a few wavelengths. This may then be discretized to a form which may be solved numerically. The Fourier series summation is implemented such that,

$$
p\left(r_{i}, z\right)=\frac{\Delta k_{r}}{\sqrt{2 \pi r_{i}}} e^{j\left(k_{\min } r_{i}-\left(m+\frac{1}{2}\right) \frac{\pi}{2}\right)} \sum_{l=0}^{M-1}\left[g\left(k_{l}, z\right) e^{j r_{\min } l \Delta k_{r}} \sqrt{k_{l}}\right] e^{\frac{j 2 \pi l i}{M}}
$$


The indices $i$ and $l$ refer to the range steps for which there are $M$ total, such that,

$$
r_{i}=r_{\min }+i \Delta r, \quad i=0,1 \ldots(M-1)
$$

and

$$
k_{l}=k_{\min }+l \Delta k_{r}, \quad l=0,1 \ldots(M-1),
$$

and range steps are subject to the constraint

$$
\Delta r \Delta k_{r}=\frac{2 \pi}{M}
$$

The FFP is capable of handling range independent and horizontally stratified environments. That is to say, the properties of each layer (such as sound speed) may vary with depth but not range. Each layer interface is assumed to be planer and parallel.

\subsubsection{Parabolic Equation Solving with Finite Difference Approximation}

The previous sections have discussed typical methods of approximating solutions to the Helmholtz equation using methods such as amplitude and phase summations, separation of variables, and the Fourier transform. Perhaps the most direct method of solving the Helmholtz equation is through finite difference approximation of the partial derivatives within the parabolic equation.

Any second order, linear, partial differential equation of the general form

$$
A \frac{\partial^{2} u}{\partial x^{2}}+2 B \frac{\partial^{2} u}{\partial x \partial y}+C \frac{\partial^{2} u}{\partial y^{2}}+D=0
$$

may be said to be parabolic if $B^{2}-A C=0$ where $u, A, B$, and $C$ are functions of $x$ and $y$, and $D$ represents the lower order terms which must be a linear function of $u$. The Helmholtz equation may take many forms depending on the situation being considered, however is represented classically in this context as

$$
\frac{\partial^{2} \psi}{\partial r^{2}}+2 j k_{0} \frac{\partial \psi}{\partial r}+\frac{\partial^{2} \psi}{\partial z^{2}}+k_{0}^{2}\left(n^{2}-1\right) \psi=0
$$

Variation of the acoustic field is assumed to be slow with respect to range in comparison to depth ${ }^{63}$. Therefore, the term $\frac{\partial^{2} \psi}{\partial r^{2}}$ is typically neglected, leaving 


$$
2 j k_{0} \frac{\partial \psi}{\partial r}+\frac{\partial^{2} \psi}{\partial z^{2}}+k_{0}^{2}\left(n^{2}-1\right) \psi=0
$$

This is, by definition, a parabolic equation where $n(r, z)=c_{0} / c(r, z)$ is the index of refraction.

The acoustic environment being considered is discretized into a grid for which $l$ and $m$ are the depth and range indices, respectively, and $\psi_{l}^{m}$ is the field variable at a given point. For a given range index, the acoustic field is evaluated at every point along the depth grid. The solving process then steps through range, taking into account the acoustic field determined at the previous range (and depth) positions. Therefore, the key to evaluation of the acoustic field at all points is numerical implementation of the partial derivatives of $\psi$ with respect to the depth coordinate $z$. This is accomplished with the finite difference approximation of the derivative ${ }^{47,48,63,64}$

$$
\left.\frac{\partial \psi}{\partial z}\right|_{z_{l}} \cong \frac{\psi_{l+1}^{m}-\psi_{l}^{m}}{\Delta z}
$$

and

$$
\left.\frac{\partial^{2} \psi}{\partial z^{2}}\right|_{z_{l}} \cong \frac{\psi_{l+1}^{m}-2 \psi_{l}^{m}+\psi_{l-1}^{m}}{\Delta z^{2}}
$$

Essentially, these equations represent the definition of the first and second derivatives respectively without taking the limit as $\Delta z$ goes to zero; allowing for numerical evaluation in a discrete grid. Solving by this method produces a system of linear equations at each range step which may then be solved simultaneously to determine the acoustic field.

The finite difference approximation provides several advantages. The sound speed profile may vary as a function of both the depth dimension as well as range dimension ${ }^{63}$. The boundary conditions (i.e. impedance) and the terrain (or bathymetry) may vary along the range dimensions ${ }^{48,63}$. The variation of properties with depth and range make this method capable to handle layered media similar to that of the $\mathrm{FFP}^{47}$. Additionally, many explicit implementations of the finite difference approximation allow for unconditionally stable solutions.

\subsubsection{Comparison and Implementation}

To aid in selection of an appropriate propagation model, Etter provides comparison for the various available computational methods (Figure 3.11). The underwater environment considered in this chapter - Portage Lake - has range dependent characteristics; 
specifically bathymetry. The water depth is on the order of $10 \mathrm{~m}$ which is considered shallow water (less than $200 \mathrm{~m}$ ). The frequency range of the excitation is between 250 to $8000 \mathrm{~Hz}$.

For shallow water, high frequency (greater than $500 \mathrm{~Hz}$ ), and range dependent applications ray theory numerical models are both physically applicable and computationally efficient. For shallow water, low frequency (less than $500 \mathrm{~Hz}$ ), and range dependent applications parabolic equation numerical models are both physically applicable and computationally efficient. To cover the entire frequency range of interest, the parabolic equation and the ray tracing methods are therefore suitable for simulating the underwater TL in the Portage Lake environment.

\begin{tabular}{|c|c|c|c|c|c|c|c|c|}
\hline \multirow{4}{*}{ Model type } & \multicolumn{8}{|c|}{ Applications } \\
\hline & \multicolumn{4}{|c|}{ Shallow water } & \multicolumn{4}{|c|}{ Deep water } \\
\hline & \multicolumn{2}{|c|}{ Low frequency } & \multicolumn{2}{|c|}{ High frequency } & \multicolumn{2}{|c|}{ Low frequency } & \multicolumn{2}{|c|}{ High frequency } \\
\hline & RI & $\mathrm{RD}$ & RI & $\mathrm{RD}$ & RI & $R D$ & RI & $\mathrm{RD}$ \\
\hline Ray theory & & 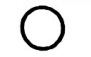 & & & & & & \\
\hline Normal mode & & & & & & & & \\
\hline Multipath expansio & & & & & & & & \\
\hline Fast field & & 0 & & 0 & & 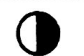 & & 0 \\
\hline Parabolic equation & & & & & & & & \\
\hline
\end{tabular}

Low frequency $(<500 \mathrm{~Hz})$

High frequency $(>500 \mathrm{~Hz})$
RI: Range-independent environment RD: Range-dependent environment

Modeling approach is both applicable (physically) and practical (computationally)

(1) Limitations in accuracy or in speed of execution

Neither applicable nor practical

Figure 3.11 Domains of applicability of underwater acoustic propagation models. (Reproduced with permission from P.C. Etter, Underwater Acoustic Modeling and Simulation ${ }^{48}$ )

While Etter focuses on mostly underwater applications for the PE, RT, FFP, and NM methods, they are by no means exclusive to underwater applications and are frequently implemented for atmospheric propagation, seismo-acoustic propagation, and other acoustic propagation problems with layered media. The atmospheric environment (above ice) on Portage Lake is assumed to be range independent and "deep" due to the large magnitude of atmosphere above the ice surface. Therefore, atmospheric TL may also be simulated reasonably using the PE and RT methods.

Maggi and Duncan ${ }^{65}$ combined several existing propagation models in a Matlab graphical user interface (GUI) entitled the Acoustic Toolbox User-interface and Post-processor 
(ACTUP). ACTUP includes models such as Bellhop (RT program with range dependent bathymetry), Kracken (NM propagation model), Scooter/Field (FFP and Green's function model), and RAM (PE solver using the Pade split-step algorithm ${ }^{66}$ ).

To compare the collected TL data on Portage Lake to computational models, the Bellhop and RAM models are implemented within the ACTUP program. Inputs to the models are discussed in the following sections and are shown in Appendix 0. The data collected and TL model outputs are compared in the following sections.

\subsubsection{Underwater TL}

This section will discuss the underwater transmission loss data collected on Portage Lake and provide comparison to the computational simulation.

\subsubsection{Simulation Inputs}

Underwater TL was simulated with the Bellhop and RAM models within the ACTUP software. While both Bellhop and RAM may be used for layered environments, the ACTUP software does not facilitate sources to be placed below stratified environmental layers. Therefore, the ice layer was not included within the environment model. While this is not preferable because it does not allow for implementation of ice reflection losses, the simulation outputs still provide adequate comparison for the TL data.

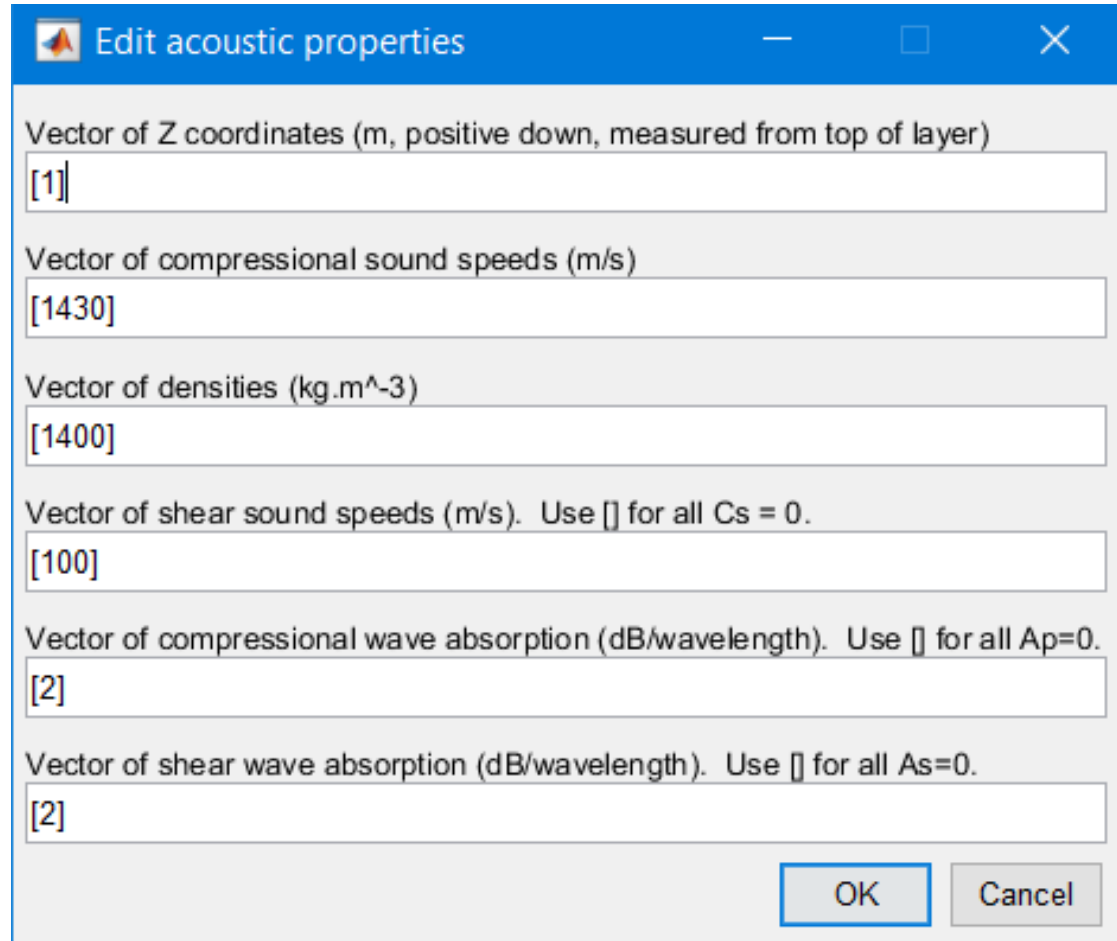

Figure 3.12 Acoustic Properties of the bottom half-space for simulating underwater TL. 
The underwater environment model was constructed within the ACTUP software with two layers; a water column layer and a bottom half-space. The water column was defined with the sound speed profile and bathymetry shown in Figure 3.2 and a density of 998 $\mathrm{kg} / \mathrm{m}^{3}$. Shear speed of sound, as well as absorption in the water column, was assumed to be zero.

The bottom was constructed as a bottom half-space with the properties set out in Figure 3.12. The goal in choosing the bottom's acoustic properties is to match the reflection loss data presented in section 3.3.1. To accurately simulate bottom reflection losses, it is imperative to accurately approximate the acoustic properties (ie. sound speeds, densities, etc.) in the layers below the water. Preceding studies on Portage Lake have not directly investigated its acoustic properties. However, from previous geological studies of the area, the bottom is expected to be a combination of silty sand and mud in a layer which is approximately 1 to $2 \mathrm{~m}$ thick ${ }^{67-69}$. Below the silt layer in Portage Lake is a sandstone basement $^{70}$.

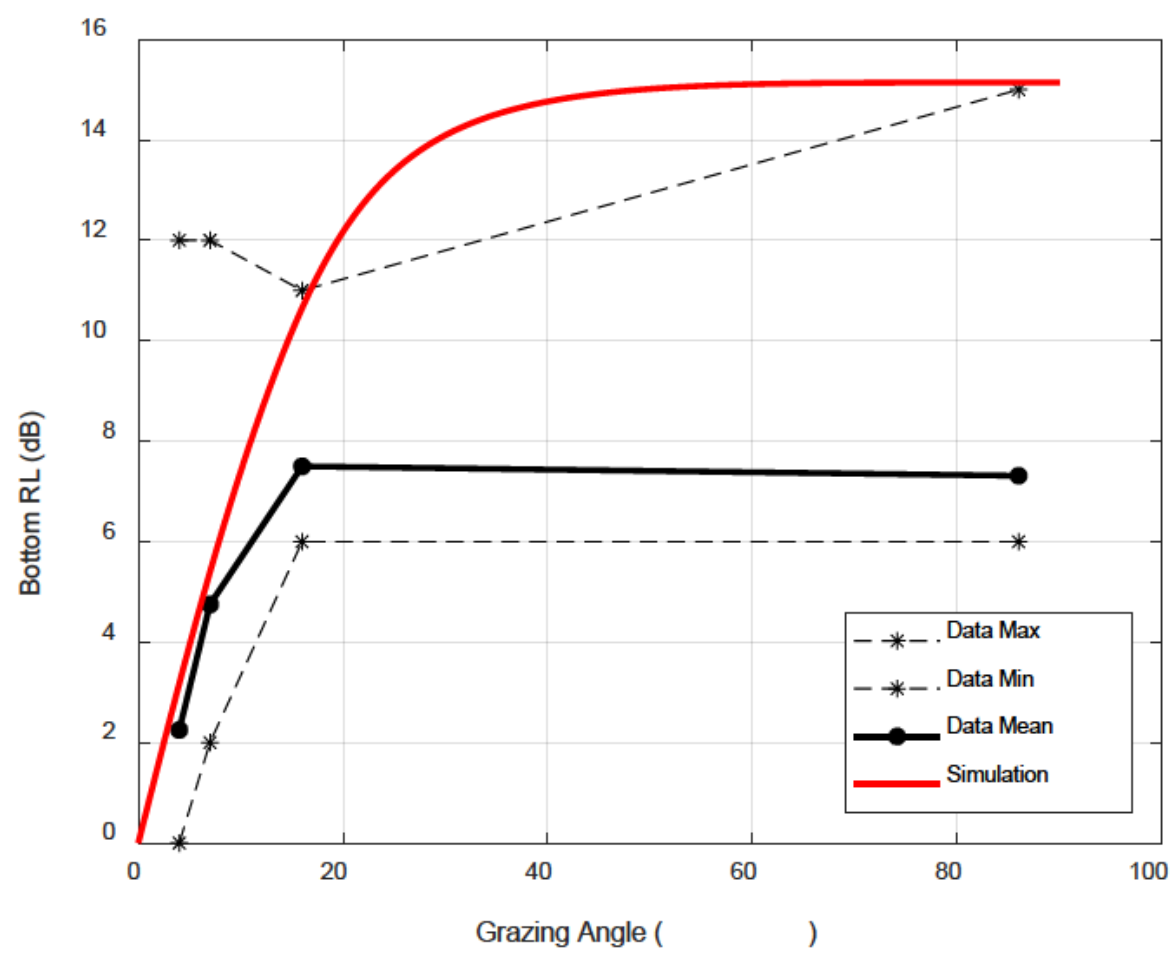

Figure 3.13 Simulated bottom RL from ACTUP environmental models.

Hamilton provides an overview of the elastic properties of marine sediments ${ }^{71}$ and Han et al. provide an overview of the properties of sandstone ${ }^{72}$. For various types of silt, sand, and mud the compressional speed, shear speed, and density range between 1400 to 1600 $\mathrm{m} / \mathrm{s}, 50$ to $240 \mathrm{~m} / \mathrm{s}$, and 1200 to $1800 \mathrm{~kg} / \mathrm{m}^{3}$, respectively ${ }^{71}$. Sandstone compressional speed and shear speed range between 3 to $5 \mathrm{~km} / \mathrm{s}$ and 1.5 to $3.5 \mathrm{~km} / \mathrm{s}$, respectively, and has a density on the order of $2650 \mathrm{~kg} / \mathrm{m}^{3} 72$. It is expected that the silt/mud layer is 
responsible for the majority of the bottom losses considering that the silt is likely highly saturated and has sound speeds close to that of the water column itself. The sandstone basement, having higher sound speeds and densities provides minor losses.

For the given environmental properties, the simulated bottom reflection loss is shown in Figure 3.13 and is compared to the measured RL from section 3.3.1. A range of RL values are shown for each grazing angle because the RL clearly varies dependent on the frequency considered. It is observed that the simulated RL matches the measured data closely. Furthermore, the simulated RL is consistent with the theoretical RL for a sandysilt (or silt) bottom presented in Figure 3.4. It is noted that there is no data for comparison of the grazing angles between 20 to 80 degrees, however, based on the theoretical models, the simulated RL is reasonable.

\subsubsection{TL vs. Range and Depth}

Both the Bellhop and RAM models utilize cylindrical coordinate assumptions. That is to say, the acoustic propagation is considered in two dimensions; range and depth.

Therefore, the primary output from the models consists of transmission loss as a function of range and depth.

An example of the Bellhop TL simulation for a frequency of $1000 \mathrm{~Hz}$ is shown in Figure 3.14. The source location for this simulation is at $3 \mathrm{~m}$ depth and $0 \mathrm{~m}$ range. The black line in this figure represents the bathymetry profile of Portage Lake. The color gradient represents the TL; amplitude with respect to the source. An analogous example for the RAM simulation is shown in Figure 3.15.

In both simulation types, the maximum TL is observed to be on the order of $60 \mathrm{~dB}$ over the depth and range domains. The propagation paths, bottom and surface reflections are observed clearly. Some acoustic energy is observed to propagate into the lake floor and become attenuated, which is expected based on the silty bottom type. A shadow zone is observed on the far side of the underwater ridge, beyond ranges and depths of $500 \mathrm{~m}$ and $8 \mathrm{~m}$ respectively.

In order to provide adequate comparison between the TL simulation and collected data, it is necessary to view the TL data as a function of range exclusively. The ACTUP software provides a method to extract a TL profile which is only a function of range. By selecting a receiver depth, TL information is determined at that depth over the entire range. 


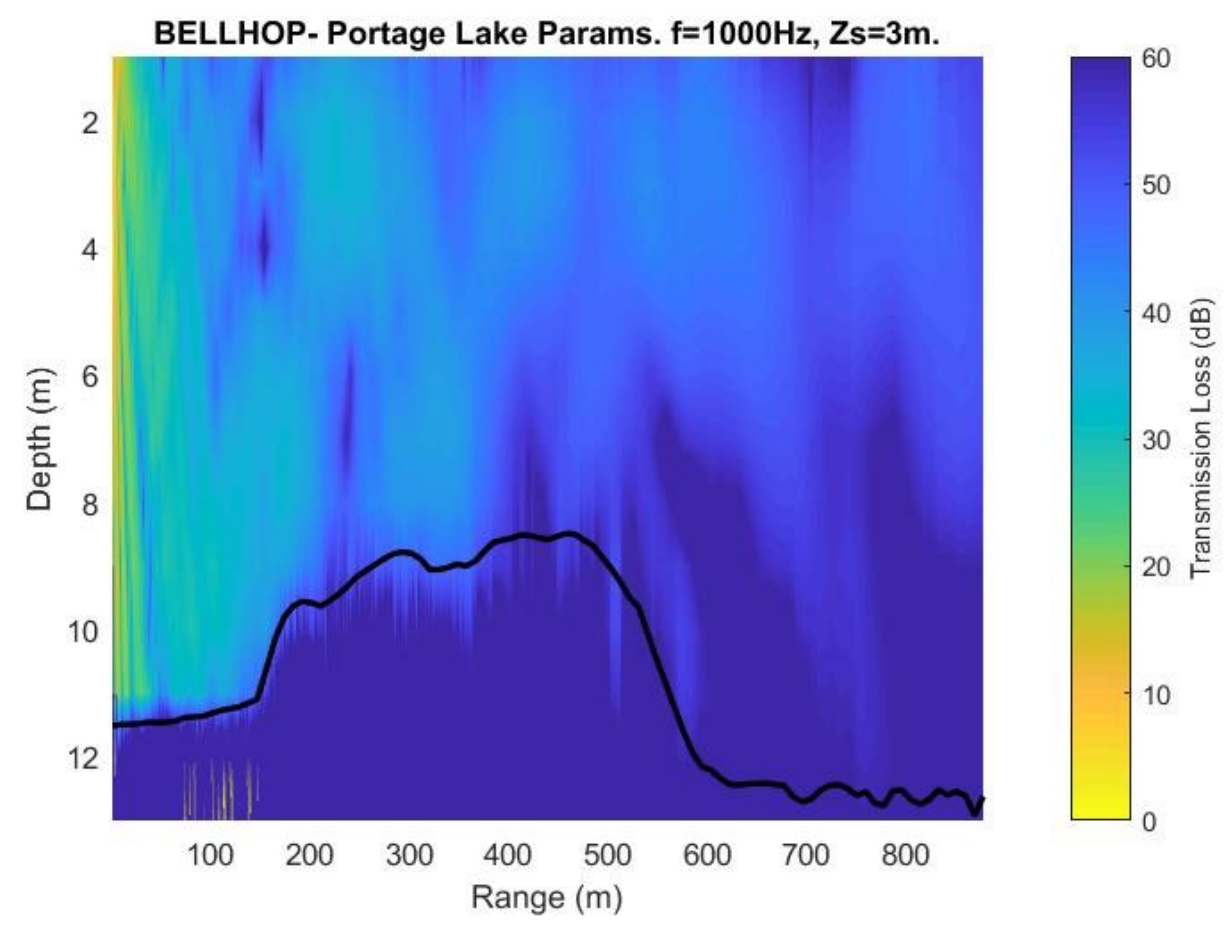

Figure 3.14 Simulated (Bellhop) underwater TL vs. range and depth at $1000 \mathrm{~Hz}$. Acoustic source depth at $3 \mathrm{~m}$. Portage Lake bathymetry shown in black.

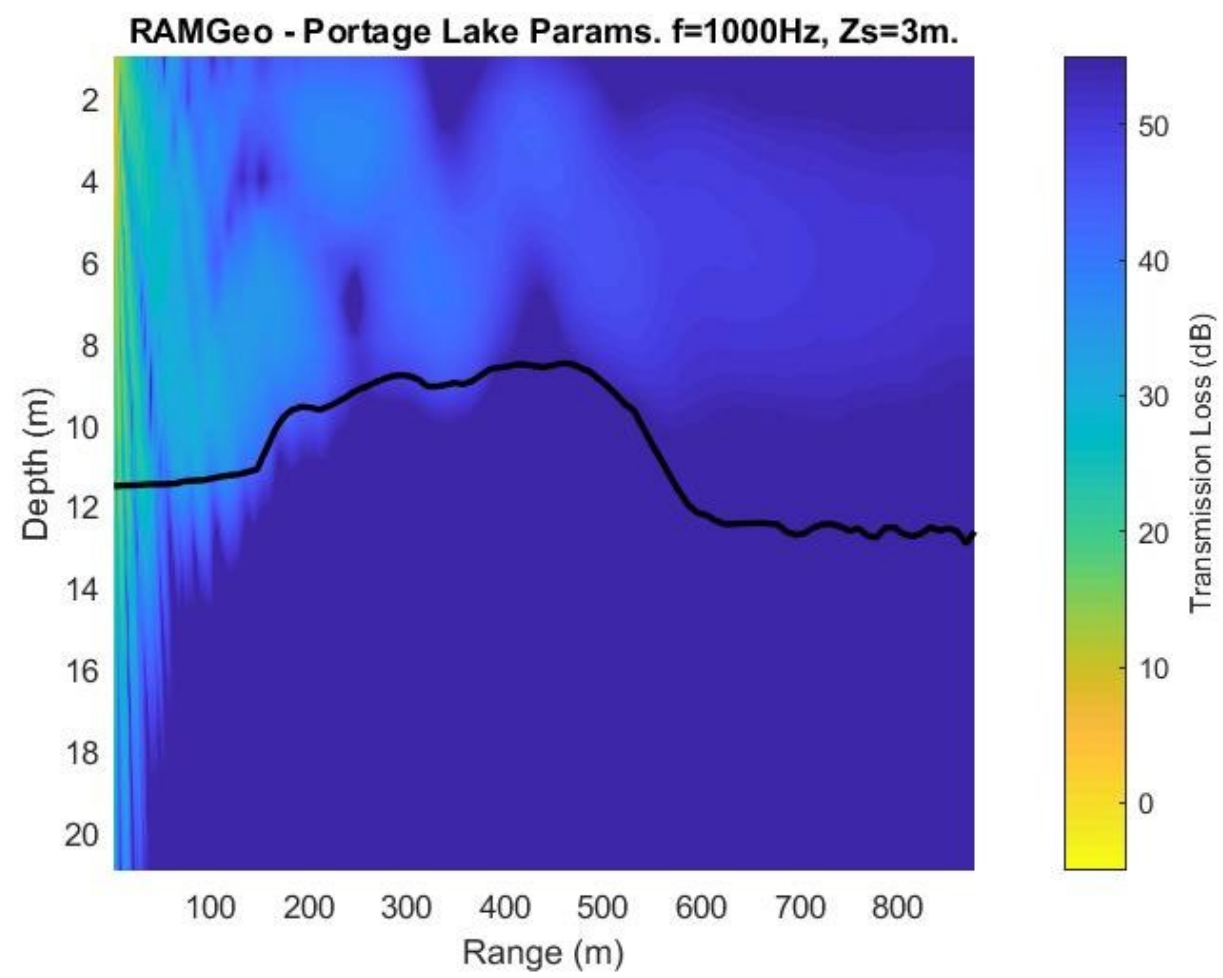

Figure 3.15 Simulated (RAM) underwater TL vs. range and depth at $1000 \mathrm{~Hz}$. Acoustic source depth at $3 \mathrm{~m}$. Portage Lake bathymetry shown in black. 

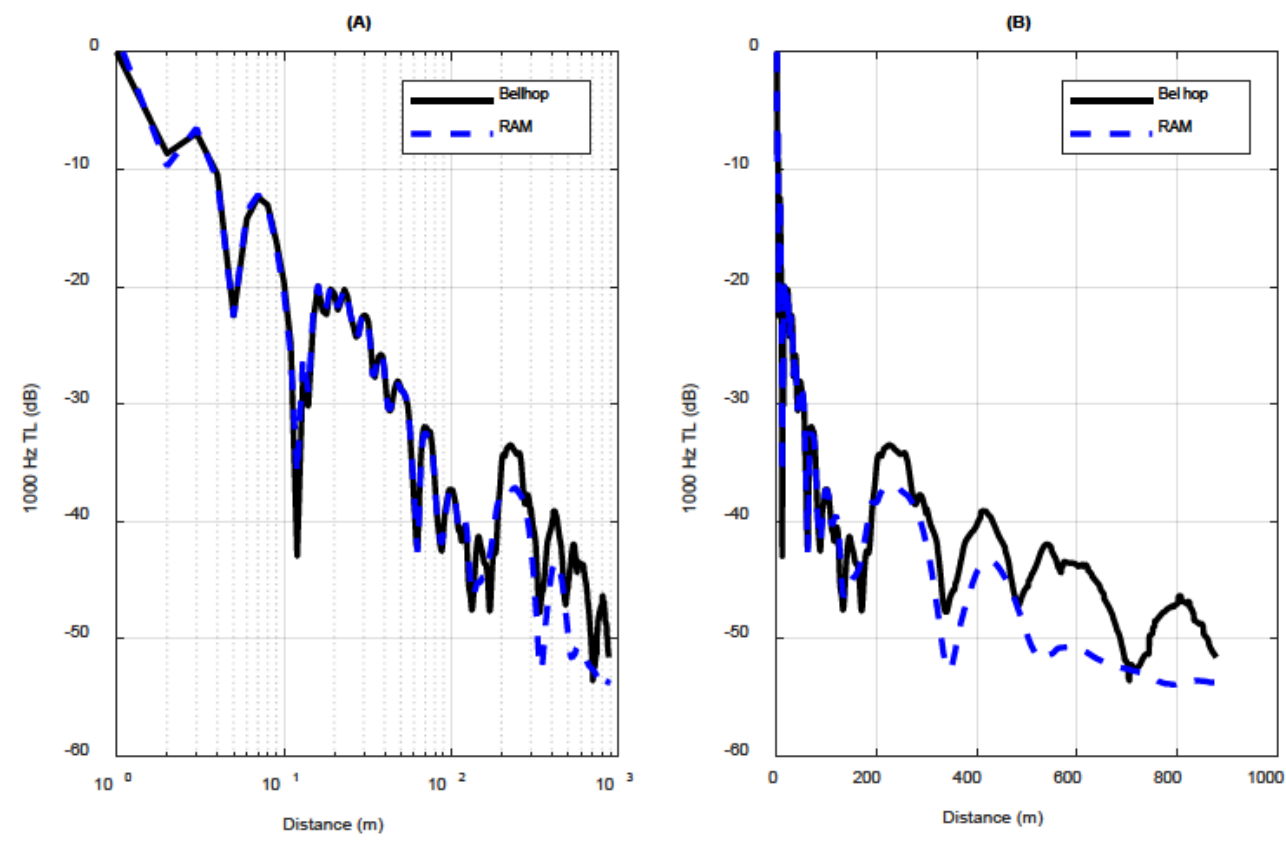

Figure 3.16 Underwater TL comparison for Bellhop and RAM simulations at $1000 \mathrm{~Hz}$, receiver depth $3 \mathrm{~m}$. Range detail shown with (A) Log and (B) Linear scales.

Figure 3.16 shows the TL as a function of range for both model types. The same plot is shown on both linear and logarithmic range scales to view the short-range and long-range detail more clearly. Good correlation between the Bellhop and RAM simulation is observed especially at short ranges (less than $100 \mathrm{~m}$ ). Beyond $100 \mathrm{~m}$, there is some separation between the models on the order of 5 to $10 \mathrm{~dB}$. This separation is likely due to the difference in method of accounting for boundary interaction as Bellhop directly accounts for RL at the boundary and RAM infers boundary losses based on the acoustic properties. Additionally, the stepping of RAM directly through the range dimension differs from that of Bellhop which steps along the propagation path of each ray.

\subsubsection{Data/Model Comparison}

The transmission loss data collected on Portage Lake are displayed in Table 3.3 and Figure 3.17. Each data point corresponds to a cyclically averaged acoustic measurement at one of the receiver locations in relation to the tonal excitation at the source site and were computed using Equation 2.12 / 3.1. The data are displayed as single data points in the figure and single values in the table because there was very little variation between the individual averages themselves.

Each subplot in Figure 3.17 and each row of Table 3.3 corresponds to a 1/3 - octave band frequency between $250 \mathrm{~Hz}$ and $8000 \mathrm{~Hz}$. Data which did not have sufficient SNR are not included. The mean broadband values of TL at each range step are also shown at the bottom of the table. These values are computed by averaging every row for a given site distance and will be compared in section 3.3.5. 
The results of the Bellhop (ray tracing theory model) and RAM (parabolic equation model using finite difference approximation) simulations are provided in comparison to the data. There is good correlation between the simulation and the data for the majority of frequencies and ranges observed in Figure 3.17, especially in frequency bands from $400 \mathrm{~Hz}$ to $3150 \mathrm{~Hz}$.

Table 3.3 Underwater Transmission Loss Data. Data measured on Portage Lake under ice. TL units in $\mathrm{dB}$

\begin{tabular}{|c|c|c|c|c|c|c|c|c|c|c|}
\hline Range (m) & 48 & 110 & 210 & 300 & 370 & 450 & 550 & 640 & 770 & 890 \\
\hline \multicolumn{11}{|l|}{$\begin{array}{c}\text { Frequency } \\
(\mathrm{Hz})\end{array}$} \\
\hline 250 & 35.4 & 35.2 & 39.0 & 50.6 & 53.2 & 50.6 & 48.8 & 51.8 & 55.7 & 69.9 \\
\hline 315 & 52.7 & 38.1 & 47.7 & 49.6 & 53.2 & 52.1 & 54.1 & 52.2 & 59.8 & 58.7 \\
\hline 400 & 28.7 & 39.9 & 43.5 & 42.7 & 50.4 & 53.9 & 56.3 & 59.1 & 54.5 & 68.1 \\
\hline 500 & 37.9 & 43.6 & 50.2 & 55.1 & 49.5 & 47.1 & 58.6 & 67.9 & 53.8 & 61.6 \\
\hline 630 & 32.3 & 39.1 & 45.4 & 60.0 & 56.5 & 67.4 & 48.2 & 39.9 & 63.9 & 69.8 \\
\hline 800 & 31.8 & 37.2 & 52.1 & 50.0 & 54.8 & 56.1 & 61.8 & 63.3 & 67.4 & 68.1 \\
\hline 1000 & 33.5 & 35.4 & 40.7 & 44.3 & 48.2 & 44.0 & 55.3 & 55.4 & 57.0 & 54.9 \\
\hline 1250 & 32.1 & 33.2 & 39.1 & 45.1 & 53.3 & 51.1 & 40.8 & 49.6 & 52.6 & 53.0 \\
\hline 1600 & 37.2 & 39.8 & 41.0 & 42.9 & 43.0 & 48.1 & 45.0 & 42.8 & 44.2 & 44.3 \\
\hline 2000 & 49.7 & 40.7 & 43.7 & 46.8 & 45.0 & 51.3 & 45.8 & 45.0 & 45.9 & 48.7 \\
\hline 2500 & 36.3 & 31.7 & 41.2 & 50.6 & 50.9 & 45.0 & 46.6 & 46.0 & 53.6 & 44.8 \\
\hline 3150 & 33.2 & 45.2 & 43.6 & 49.3 & 46.2 & 55.6 & 47.7 & 50.2 & 53.6 & 51.1 \\
\hline 4000 & 37.3 & 49.1 & 55.0 & 53.0 & 60.8 & 60.2 & 56.0 & 53.9 & 65.0 & 69.8 \\
\hline 5000 & 32.0 & 48.0 & 43.8 & 60.6 & 64.5 & 61.9 & 57.3 & 61.4 & 73.6 & 61.9 \\
\hline 6300 & 45.7 & 38.5 & 56.8 & 54.0 & 58.6 & 51.9 & 64.8 & 74.8 & 59.2 & 60.2 \\
\hline 8000 & 44.3 & 56.1 & 46.3 & 47.1 & 45.9 & 53.6 & 61.7 & 63.8 & 69.6 & 63.3 \\
\hline $\begin{array}{l}\text { Broadband } \\
\text { Mean: }\end{array}$ & 37.5 & 40.7 & 45.6 & 50.1 & 52.1 & 53.1 & 53.1 & 54.8 & 58.1 & 59.3 \\
\hline
\end{tabular}

For frequency bands of $4000 \mathrm{~Hz}$ and beyond, the measured TL appears to be 10 to $20 \mathrm{~dB}$ greater than the simulated TL particularly beyond ranges of $400 \mathrm{~m}$. As frequencies increase the TL becomes more difficult to predict when there are large interactions with the boundary conditions; as is the case in the shallow-water Arctic environment. At high frequencies, the wavelengths become shorter. In the frequency range of 4000 to 8000 $\mathrm{Hz}$, the wavelength underwater is on the order of $0.2 \mathrm{~m}$. The interaction with small variations in the bottom surface or ice layer (eg. small cracks or deformations in the ice or rocks and debris on the bottom) are increased. Variations such as this are not accounted for in the model and may explain the disparity in TL as frequency increases. 
Generally speaking, there is good correlation between the Bellhop and RAM simulations excluding the first two or three frequency bands considered in Figure 3.17. In the 250 and $315 \mathrm{~Hz}$ bands, the RAM simulation significantly overestimates the TL beyond 400 $\mathrm{m}$. However, the Bellhop simulation provides an accurate comparison to the measured TL data in these bands. 

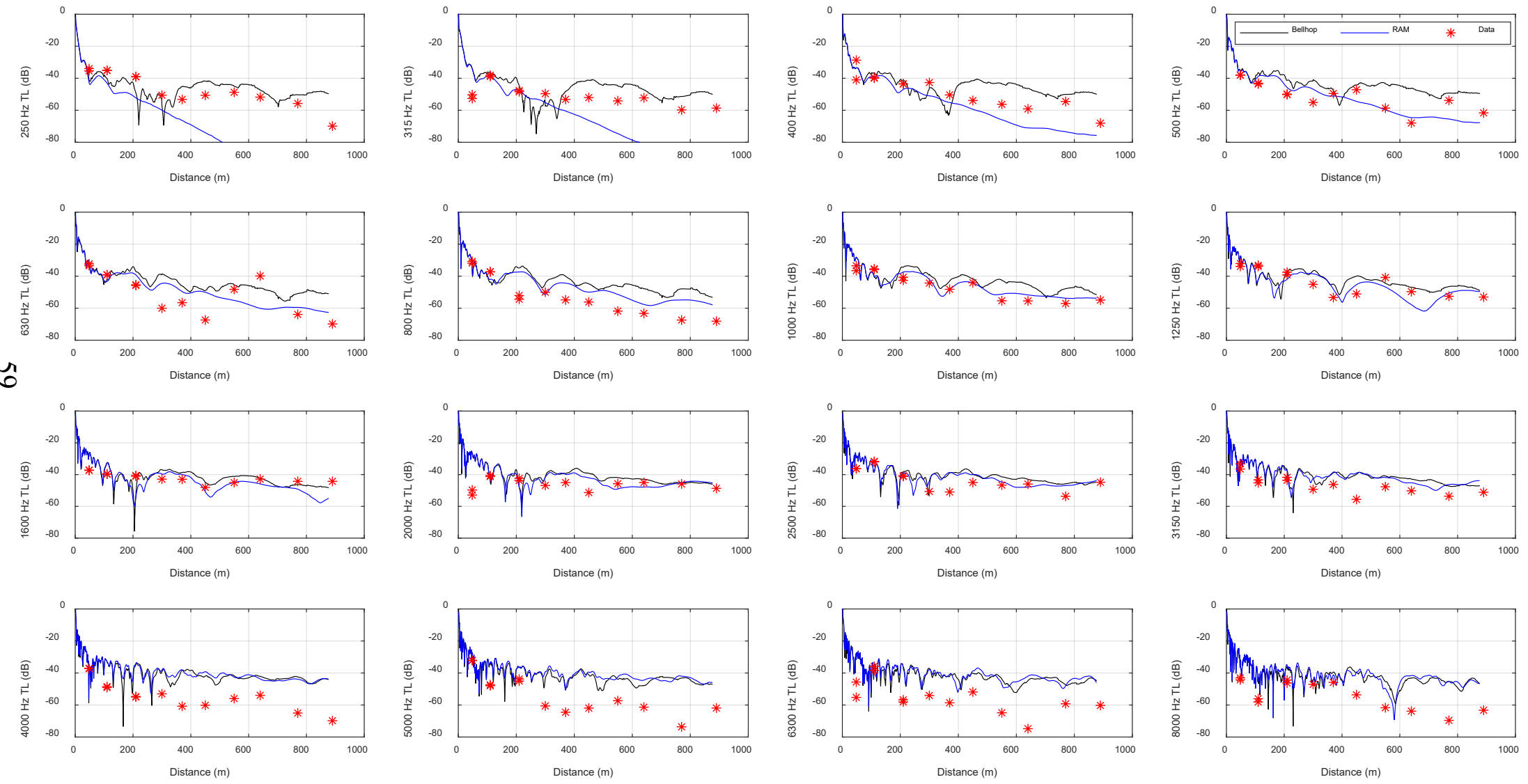

Figure 3.17 Underwater TL data and comparison to Bellhop and RAM simulations. 


\subsubsection{Atmospheric TL}

This section will discuss the collected atmospheric transmission loss data collected on Portage Lake and provide comparison to the computational simulation discussed above.

\subsubsection{Cannon Blast Signal}

The cannon blast provided by the propane cannon was very consistent in terms of signal level and frequency content. The narrowband, as well as $1 / 3$ - octave sound pressure level, is displayed in Figure 3.18. This data was measured at the source location microphone; $1 \mathrm{~m}$ from the cannon muzzle. The SPL data is averaged over all of the cannon blasts which were used in computing TL.

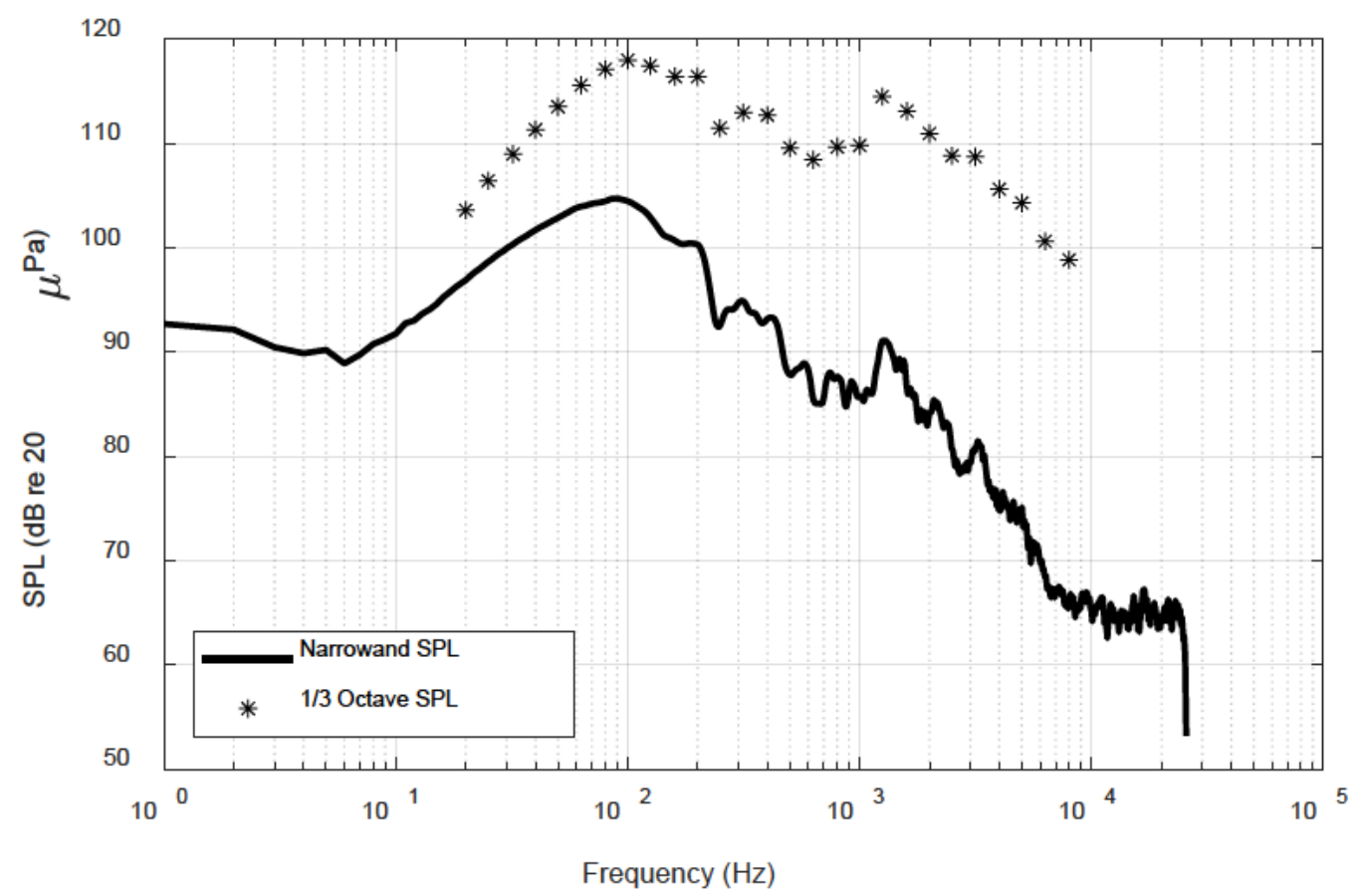

Figure 3.18 Cannon blast sound pressure level $(1 \mathrm{~m})$ averaged for all experiments.

\subsubsection{SNR}

During testing on Portage Lake, the wind speed ranged between 2 to $9.5 \mathrm{~m} / \mathrm{s}$. The high winds and variation in wind speed caused moment-to-moment changes in signal to noise ratio (SNR) at the receiver locations. Effectively, the background noise level changed between each cannon blast measured. Therefore, it was not sufficient to determine SNR in relation to one singular background noise measurement, because the background noise level may have changed significantly between any two sequential cannon blasts; let alone between experiments or throughout the course of a day's testing. 

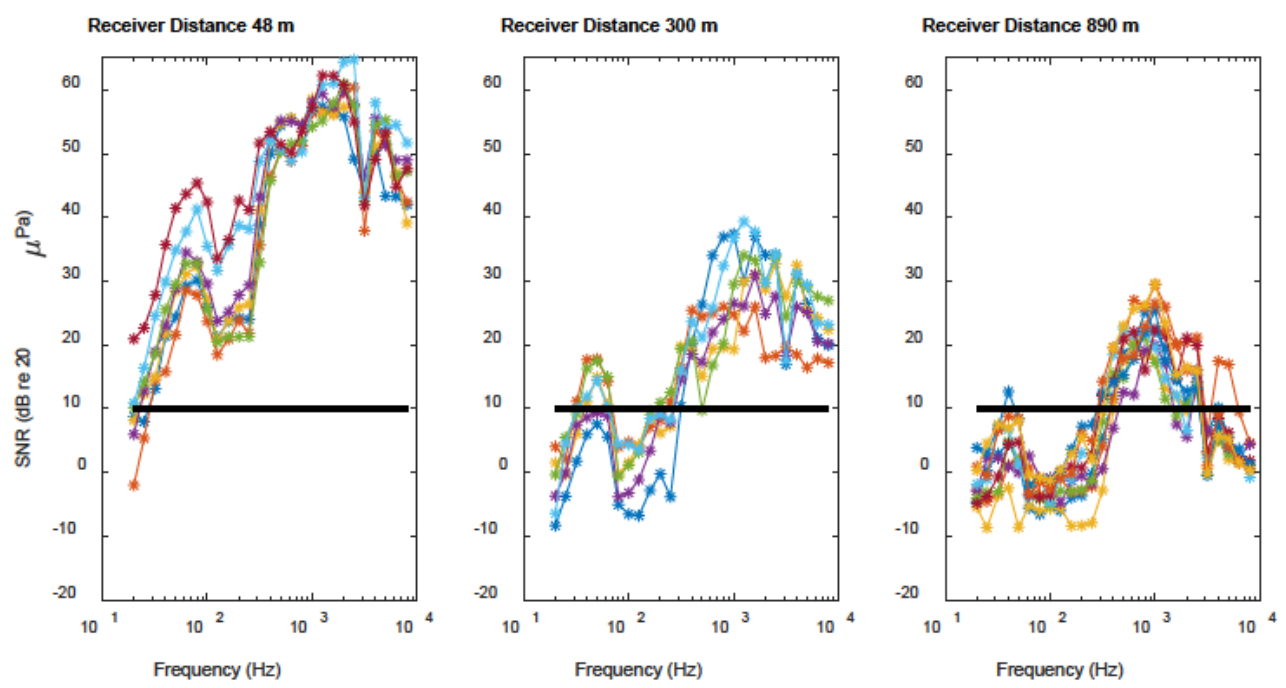

Figure 3.19 Signal-to-Noise Ratio for multiple cannon blasts at various ranges.

Still, to accurately measure TL, it was necessary to determine which down-range measurements had sufficient SNR and which did not. The background noise level was determined by measuring the SPL between each cannon blast. There was sufficient time between cannon blasts -2 to 4 seconds - that this was easily accomplished. The SNR was then determined by comparing the received cannon blast sound amplitude and the background noise level between each blast.

Figure 3.19 illustrates the variation in SNR between cannon blasts for three selected ranges. Each line plotted in Figure 3.19 represents the SNR for a single cannon blast as a function of frequency. The variation in SNR between cannon blasts at the same receiver location is observed to be 20 to $30 \mathrm{~dB}$. The overall SNR is observed to reduce as range increases with mid-frequencies (around $1000 \mathrm{~Hz}$ ) exhibiting higher SNR. Due to the large variation in the data between cannon blasts, it was necessary to treat each cannon blast individually in computing TL.

Received signals which had SNR of $10 \mathrm{~dB}$ were used straight away for averaging TL measurements. Some of the data which had SNR less than $10 \mathrm{~dB}$ was salvaged using the rules of coherent source addition.

By definition, when the SPL of two coherent sources of equal level are summed, the resulting SPL is $6 \mathrm{~dB}$ greater than the level of each individual source. Summing two incoherent sources of equal level yields an SPL of $3 \mathrm{~dB}$ greater than the level of each individual source ${ }^{38}$.

The total sound pressure level when summing two incoherent sources is defined as ${ }^{38}$, 


$$
L_{p_{\text {total }}}=10 * \log _{10} \frac{p_{1}^{2}+p_{2}^{2}}{p_{\text {ref }}^{2}}
$$

If $p_{1}$ is assumed to be the component of the total acoustic signal corresponding to the cannon blast and $p_{2}$ is assumed to be the component of the total acoustic signal corresponding to ambient noise, the cannon blast signal and the noise may be distinguished to within $3 \mathrm{~dB}$ difference. Effectively, a corrected signal level $p_{c}^{2}$ may be determined by subtracting the noise component from the total received signal,

$$
p_{c}^{2}=p_{\text {total }}^{2}-p_{\text {noise }}^{2} .
$$

Data with SNR of $3 \mathrm{~dB}$ or less was disregarded for analysis.

\subsubsection{Simulation Inputs}

Atmospheric TL was simulated with the Bellhop and RAM models within the ACTUP software. Given that the ACTUP software was originally developed for use in underwater environments, the coordinate systems in each layer are measured downward from the top of each layer. This does not affect the accuracy of the atmospheric simulation, because the individual models still solve the relative TL at each horizontal range coordinate and vertical height coordinate. It does, however, make the coordinate system and associated source and receiver locations less intuitive. The atmospheric simulation inputs are described as follows.

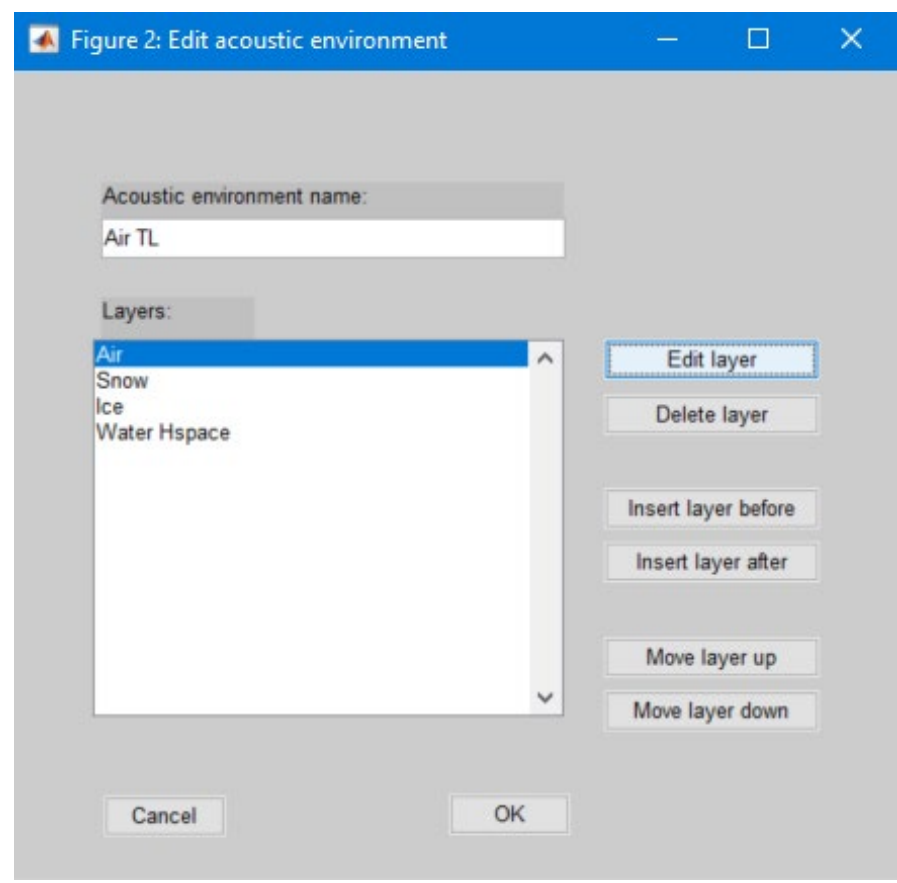

Figure 3.20 Atmospheric environment model in ACTUP. 
The atmospheric environment model was constructed within the ACTUP software with four layers; an air column, a snow layer, an ice layer, and a water half-space (Figure 3.20). The modeled air layer was $100 \mathrm{~m}$ thick with a varying sound speed profile and a constant density of $1.29 \mathrm{~kg} / \mathrm{m}^{3}$. The variation in sound speed profile was based on the measured wind speeds during testing. Salomons ${ }^{63}$ showed that the effective sound speed profile may be developed to represent the variations in wind speed throughout the air column with the equation,

$$
c_{e f f}(z)=c_{0}+b \ln \left(\frac{z}{z_{0}}+1\right)
$$

The first term, $c_{0}$, represents the nominal sound speed based on the mean air temperature,

$$
c_{0}=20.04 * \sqrt{\overline{T_{k}}} .
$$

The second term represents the variation in the sound speed profile based on the measured wind speed. The factor $b$ is positive 1 for a downward refracting atmosphere being that the air temperature was lowest near the surface of the ice this is the case in the Arctic environment - and negative 1 for an upward refracting atmosphere. The term,

$$
z_{0}=\frac{h}{\exp \left(\overline{V_{w}}\right)},
$$

is a function of the measured wind speed $V_{w}$ and the height $h$ where the wind speed was measured. Based on a nominal wind speed of $4.48 \mathrm{~m} / \mathrm{s}$ and air temperature $-16.4^{\circ} \mathrm{C}$ (see Appendix A.1), the sound speed profile in is used for the environmental model is shown in Figure 3.21 and Figure 3.22.

A modeled sound speed profile such as this assumes that the wind direction is not changing during the evaluation of the propagation. Furthermore, it only accounts for wind speed in the range dimension; assuming that the wind speed in the vertical and transverse directions are minimal in comparison. Turbulent effects in the atmosphere are also not taken into account.

The snow layer was developed with a thickness of $0.13 \mathrm{~m}$, a compressional sound speed of $500 \mathrm{~m} / \mathrm{s}$, density of $300 \mathrm{~kg} / \mathrm{m}^{3}$, shear sound speed of $300 \mathrm{~m} / \mathrm{s}$, and absorption coefficients of $0.5 \mathrm{~dB} / \lambda$ for both compression and shear waves. These properties are consistent with those measured by Capelli et al. ${ }^{73}$ on snow samples in laboratory studies

The ice layer was defined with a thickness of $0.3 \mathrm{~m}$, compressional speed of $2657 \mathrm{~m} / \mathrm{s}$, density of $915 \mathrm{~kg} / \mathrm{m}^{3}$, and shear speed of $1381 \mathrm{~m} / \mathrm{s}$. The attenuation coefficients for the ice layer were assumed to be zero. 


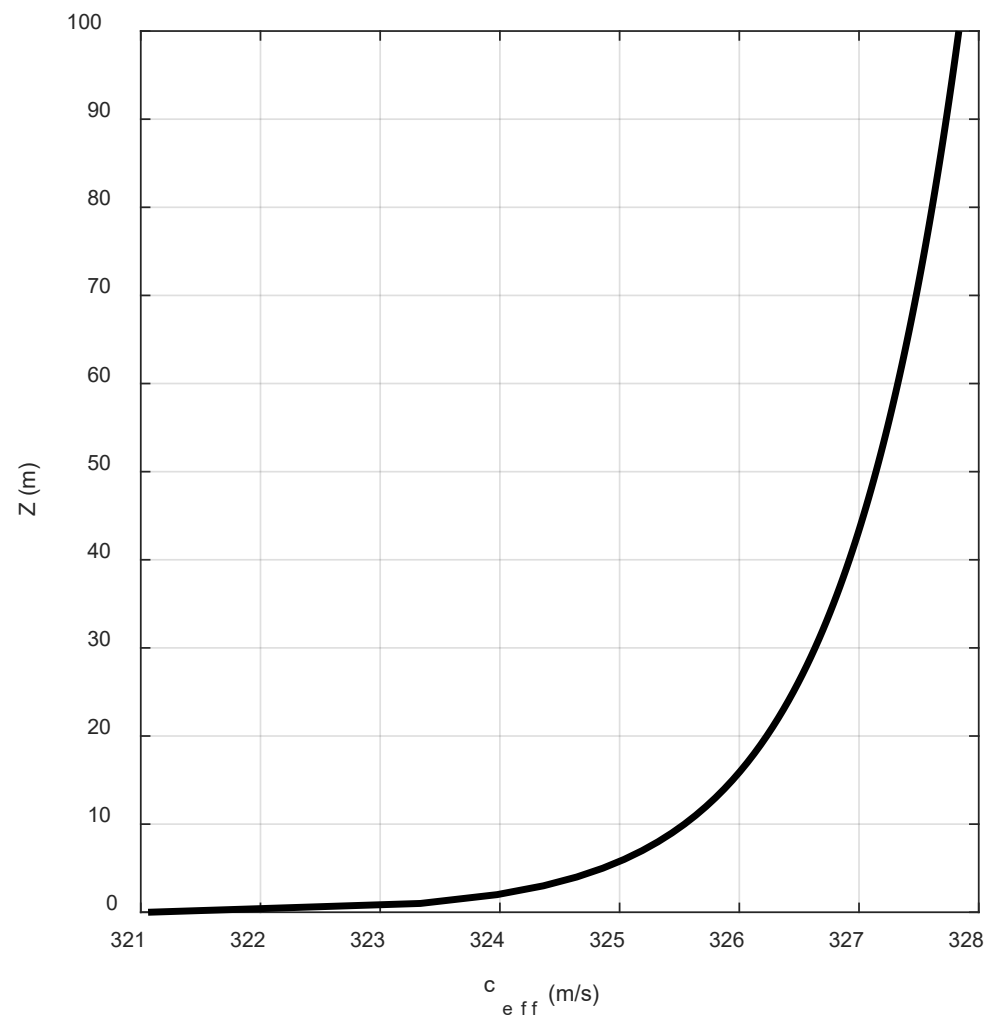

Figure 3.21 Atmospheric sound speed profile from wind speed and temperature data. For inputs: $T=-16.4^{\circ} \mathrm{C}, V_{w}=4.48 \mathrm{~m} / \mathrm{s}, h=10 \mathrm{~m}, b=1$.

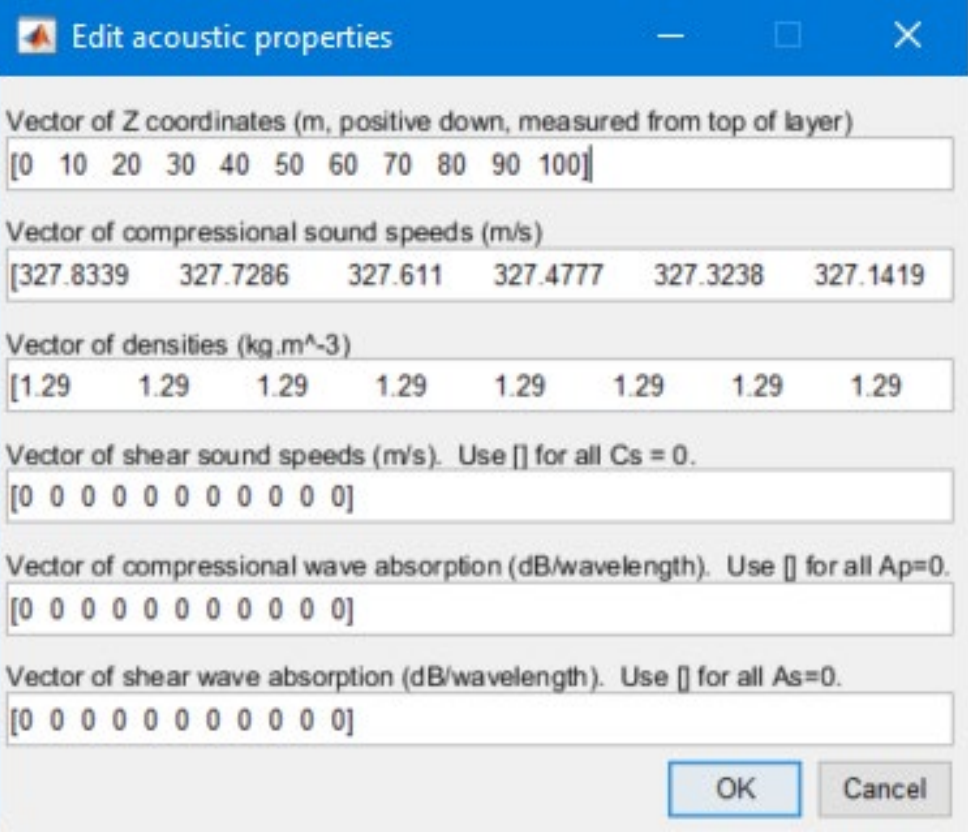

Figure 3.22 Air column properties within the atmospheric ACTUP model. 
These sound speeds used are slower than that of typical ice which is usually well above $3000 \mathrm{~m} / \mathrm{s}$ for compressional speed. Justification for the use of these properties will be developed further in section 3.5. For now, it will suffice to say that the ice layers were quite soft compared to that of normal ice. Additionally, the ice layer itself was stratified into layers; some of which were slush-like and some of which were solid. These variations in the ice layer were not rigorously measured and the assumption is made that the properties in the ice are roughly similar throughout. Therefore, the aggregate sound speeds through the ice layer are lower than normal.

The final layer is that of the water half-space. Half-space layers are not able to contain varying sound speed profiles. The nominal values for compressional sound speed and density in the water column were used. Those are, $1406 \mathrm{~m} / \mathrm{s}$ and $998 \mathrm{~kg} / \mathrm{m}^{3}$, respectively. The shear speed and attenuation coefficients were set to zero.

The total range considered within the simulation was $890 \mathrm{~m}$ and the simulation is evaluated at 1/3-octave bands between 20 to $8000 \mathrm{~Hz}$.

Additional details regarding the simulation setup are provided in Appendix 0 .

\subsubsection{TL vs. Range and Depth}

Examples of simulated atmospheric transmission loss for $500 \mathrm{~Hz}$ are shown in Figure 3.23 and Figure 3.24 for Bellhop and RAM models respectively. Both models show "depth" on the y-axis. The top of the atmosphere is located at $0 \mathrm{~m}$ depth and the top of the ice layer is located at $100 \mathrm{~m}$ depth, below which are the ice and water layers. The source depth in both models is positioned at $99 \mathrm{~m}$ "deep", which corresponds to $1 \mathrm{~m}$ above the ice layer.

The atmosphere in both models is observed to be downward refracting, as expected based on the input sound speed profile in the air column. The greatest amount of refraction is visible within $200 \mathrm{~m}$ in the Bellhop model, but are visible across the entire range in the RAM model.

Both models show TL on the order of $60 \mathrm{~dB}$ with variations in both spatial dimensions on the order of 10 to $20 \mathrm{~dB}$. Variations in TL within the atmosphere are expected due to the high wind speeds. While the models do not account for turbulence or scattering, the sound speed profile does account for the high wind speed measured in the range dimension and this contributes to the large variations in TL. The RAM model shows the transmission into the ice and water is attenuated quite rapidly within $100 \mathrm{~m}$ (note that Bellhop only provides TL outputs in the first layer but the ice and water effects are still accounted for in the model). Additionally, constructive interference and interactions with the boundary layer at the ice surface are observed to affect the TL in both models within the first 10 to $20 \mathrm{~m}$ above the ice layer. 


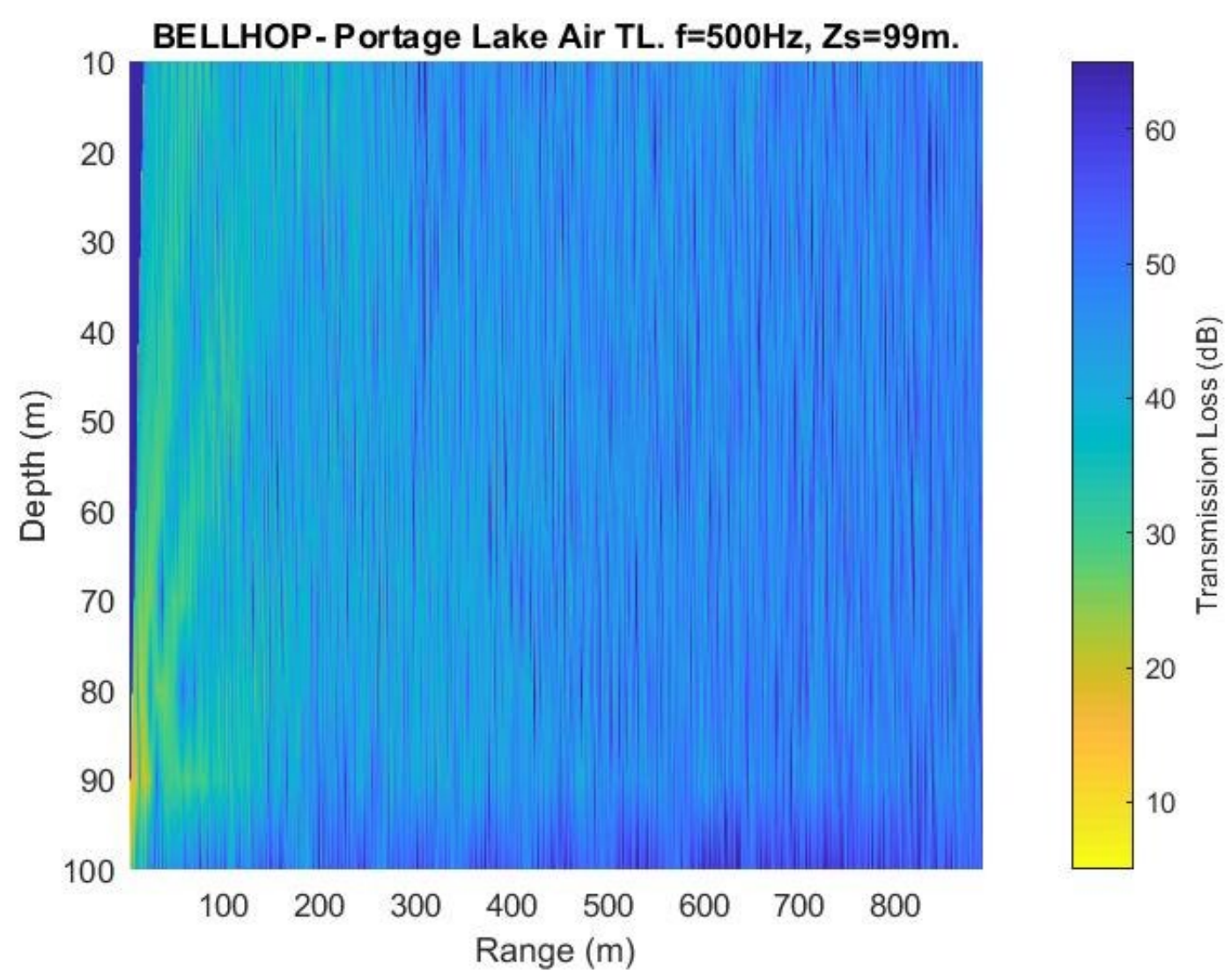

Figure 3.23 Simulated Bellhop atmospheric TL vs. range and depth at $500 \mathrm{~Hz}$. Acoustic source depth at $99 \mathrm{~m}$ (one meter above the ice).

Range-dependent atmospheric TL is shown in Figure 3.25 for both Bellhop and RAM models for a source and receiver depth $1 \mathrm{~m}$ above the ice surface. The same plot is shown on both linear and logarithmic range scales to view the short-range and long-range detail more clearly. Good correlation is observed between the models, however, the large variations in TL are again observed especially at ranges beyond $100 \mathrm{~m}$; causing some discrepancy on the order of \pm 10 to $\pm 20 \mathrm{~dB}$.

In the case of the TL in Figure 3.25 there is approximately an order of magnitude difference between the estimated TL at long ranges. As explained above in the case of TL vs. range and depth, this variation is also expected based on the high winds observed during testing and the wind-corrected sound speed profile used in the models.

As discussed in section 3.3.2.5 each model type has advantages and disadvantages leading to variations in model output and accuracy. Given that model accuracy is highly dependent on frequency, environment depth, spatial discretization, and other factors, the variation between the Bellhop and RAM simulations is reasonable. The comparison between the two is useful to cover a wide frequency range, and provides insight to the potential range of measured TL values. Simulated TL similar to that which is shown in 
Figure 3.25 will be used for comparison to the measured $1 / 3$ - octave TL data in the next section.

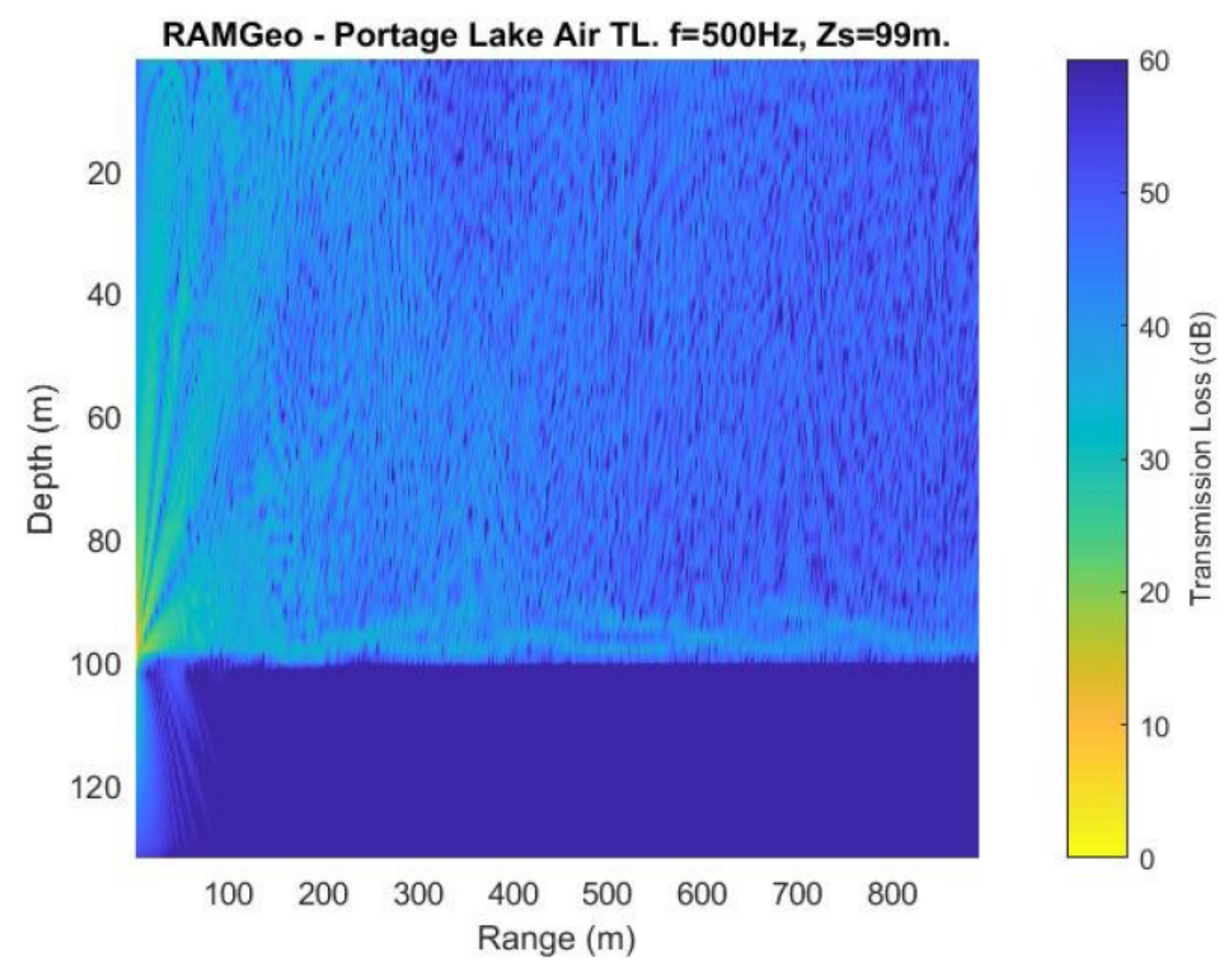

Figure 3.24 Simulated RAM atmospheric TL vs. range and depth at $500 \mathrm{~Hz}$. Acoustic source depth at $99 \mathrm{~m}$ (one meter above the ice).
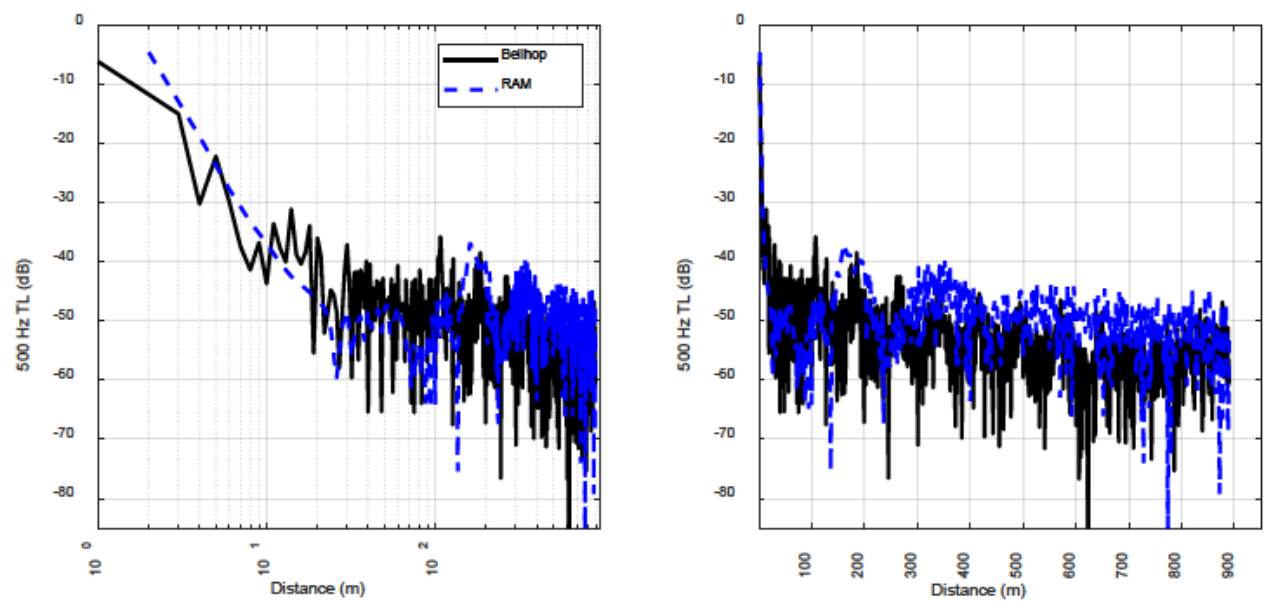

Figure 3.25 Atmospheric TL comparison for Bellhop and RAM simulations at $500 \mathrm{~Hz}$. Range detail shown with (A) Log and $(B)$ Linear scales. 


\subsubsection{Data/Model Comparison}

The measured atmospheric TL data are shown in Figure 3.26 through Figure 3.30 and are compared to the simulated Bellhop and RAM models. TL computation was done with Equation 2.13 / 3.1 for each cannon blast individually. Due to the large variation on atmospheric TL, the data at each receiver distance are represented as a range of values which show the minimum, maximum, and mean of all measured values. Data which did not have sufficient SNR are not included in the plots.

Table 3.4 Atmospheric Transmission Loss Data.

\begin{tabular}{|c|c|c|c|c|c|c|c|c|c|c|}
\hline Range $(\mathrm{m})$ & 48 & 110 & 210 & 300 & 370 & 450 & 550 & 640 & 770 & 890 \\
\hline \multicolumn{11}{|l|}{$\begin{array}{c}\text { Frequency } \\
(\mathrm{Hz})\end{array}$} \\
\hline 20 & 27.2 & 32.5 & 28.9 & & 42.8 & 15.7 & & & & 37.2 \\
\hline 25 & 28.0 & 31.5 & 35.6 & 42.0 & 44.4 & 17.4 & & & & \\
\hline 32 & 27.2 & 30.9 & 36.1 & 38.7 & 42.2 & 31.1 & & 49.6 & 38.6 & 45.7 \\
\hline 40 & 26.4 & 30.6 & 36.8 & 38.6 & 41.2 & 34.9 & & 54.0 & & 47.9 \\
\hline 50 & 26.8 & 31.5 & 38.9 & 41.9 & 43.3 & 35.6 & & & & 55.2 \\
\hline 63 & 28.3 & 35.1 & 46.1 & 51.6 & 51.3 & 28.2 & & & & \\
\hline 80 & 31.6 & 43.0 & 56.7 & & 70.9 & 28.8 & & & & \\
\hline 100 & 39.5 & 57.8 & 57.3 & & & 42.5 & & & & \\
\hline 125 & 45.7 & 58.3 & 57.4 & & & 32.2 & & & & \\
\hline 160 & 44.0 & 55.3 & 57.3 & 69.7 & 70.3 & 33.5 & & & & 70.2 \\
\hline 200 & 41.5 & 53.7 & 57.5 & 67.8 & 66.2 & 34.7 & & 78.8 & & 69.3 \\
\hline 250 & 32.4 & 44.4 & 48.3 & 53.7 & 59.2 & 40.6 & & 67.6 & 51.2 & 60.6 \\
\hline 315 & 31.0 & 42.5 & 49.4 & 57.2 & 56.1 & 55.1 & 52.5 & 63.0 & 61.0 & 60.9 \\
\hline 400 & 27.8 & 39.5 & 46.5 & 53.4 & 52.3 & 53.9 & 54.7 & 60.2 & 62.9 & 59.5 \\
\hline 500 & 23.4 & 35.0 & 41.9 & 48.7 & 48.2 & 50.6 & 52.5 & 57.7 & 61.2 & 54.5 \\
\hline 630 & 21.7 & 33.0 & 39.8 & 46.1 & 46.9 & 49.2 & 53.8 & 59.4 & 61.1 & 53.2 \\
\hline 800 & 23.3 & 33.6 & 40.1 & 46.4 & 49.3 & 50.8 & 60.2 & 61.1 & 63.1 & 55.4 \\
\hline 1000 & 22.8 & 32.6 & 39.4 & 45.3 & 47.8 & 52.4 & 65.7 & 66.8 & 65.8 & 58.1 \\
\hline 1250 & 27.0 & 36.7 & 44.2 & 50.5 & 52.0 & 58.3 & 71.3 & 77.0 & 73.7 & 67.4 \\
\hline 1600 & 26.1 & 33.8 & 45.1 & 50.5 & 54.0 & 58.8 & 73.4 & 76.5 & 75.1 & 69.3 \\
\hline 2000 & 24.7 & 31.3 & 46.1 & 53.5 & 56.7 & 59.2 & 73.6 & 80.9 & & 71.1 \\
\hline 2500 & 25.6 & 31.9 & 44.0 & 50.9 & 60.3 & 61.8 & 77.0 & 80.1 & & 69.9 \\
\hline 3150 & 30.1 & 35.0 & 48.4 & 52.7 & 64.4 & 58.2 & & & & 68.5 \\
\hline 4000 & 27.3 & 34.0 & 46.9 & 52.8 & 64.2 & 66.4 & & 77.3 & & 74.6 \\
\hline 5000 & 27.5 & 35.6 & 48.0 & 54.4 & 67.4 & 59.9 & & 75.7 & & 72.8 \\
\hline 6300 & 25.7 & 32.8 & 45.8 & 52.3 & 65.7 & 27.6 & & & & 67.6 \\
\hline 8000 & 25.2 & 31.4 & 45.5 & 52.1 & 63.7 & 29.6 & & & & \\
\hline Mean: & 29.2 & 37.9 & 45.5 & 50.9 & 55.2 & 43.2 & 63.5 & 67.9 & 61.4 & 61.4 \\
\hline
\end{tabular}


Table 3.4 shows the measured atmospheric TL data (mean values only) in terms of receiver distance and frequency bands. Data which did not have sufficient SNR are not included in the table. The mean broadband values of TL at each range step are also shown at the bottom of the table. These values are computed by averaging every row for a given site distance and will be compared in section 3.3.5.

Generally speaking, the measured data correlate well with the simulated Bellhop and RAM models. Excellent correlation between the model and the data is observed for frequency bands between 315 to $4000 \mathrm{~Hz}$. Variation in the measured data matches the variation of the simulated data very closely.

There are, however, several points observed in the figures which seem to be unaccounted for by the simulations. There is significant variance on some of the atmospheric TL depending on the frequency and site distance. Spread in the data of \pm 6 to $\pm 12 \mathrm{~dB}$ in the data at any particular site are common with several sites having $\pm 0 \mathrm{~dB}$ of spread. The most extreme spread on any of the data is in the $5000 \mathrm{~Hz}$ data at the $450 \mathrm{~m}$ site which has $\pm 24 \mathrm{~dB}$ of variation.

For all frequencies considered, the $450 \mathrm{~m}$ site appears to have the largest spread and/or the largest mean difference from the simulations. The measured data at $450 \mathrm{~m}$ also is observed to deviate from the trend of the other measured data. These data points are known to have good SNR based on the analysis conducted in section 3.3.4.2. Therefore, this large discrepancy between the model and the data may potentially be due to atmospheric turbulence - which is not accounted for in the models - or some other unknown environmental effects. It is also possible that some of these points are statistical outliers; however, it is not possible to determine this in cases where there were only a few samples which had reasonable SNR.

In terms of mean values, it is also observed that the simulations appear to overestimate TL at site distances less than $100 \mathrm{~m}$ and underestimate TL at site distances $600 \mathrm{~m}$ and beyond. However, there are several sites beyond $600 \mathrm{~m}$ where the maximum values of measured data fall within reasonable ranges in comparison to the models.

At low frequencies, especially below $250 \mathrm{~Hz}$, the separation between the RAM and Bellhop models is significant; greater than $20 \mathrm{~dB}$ in some cases. The RAM model generally provides better correlation with the data at low frequencies except for a few points at long distances. The RAM and Bellhop models appear to converge slightly as frequency increases, and both provide acceptable correlation with the data. These effects are expected based on the differences in applicability of models (Figure 3.11). 

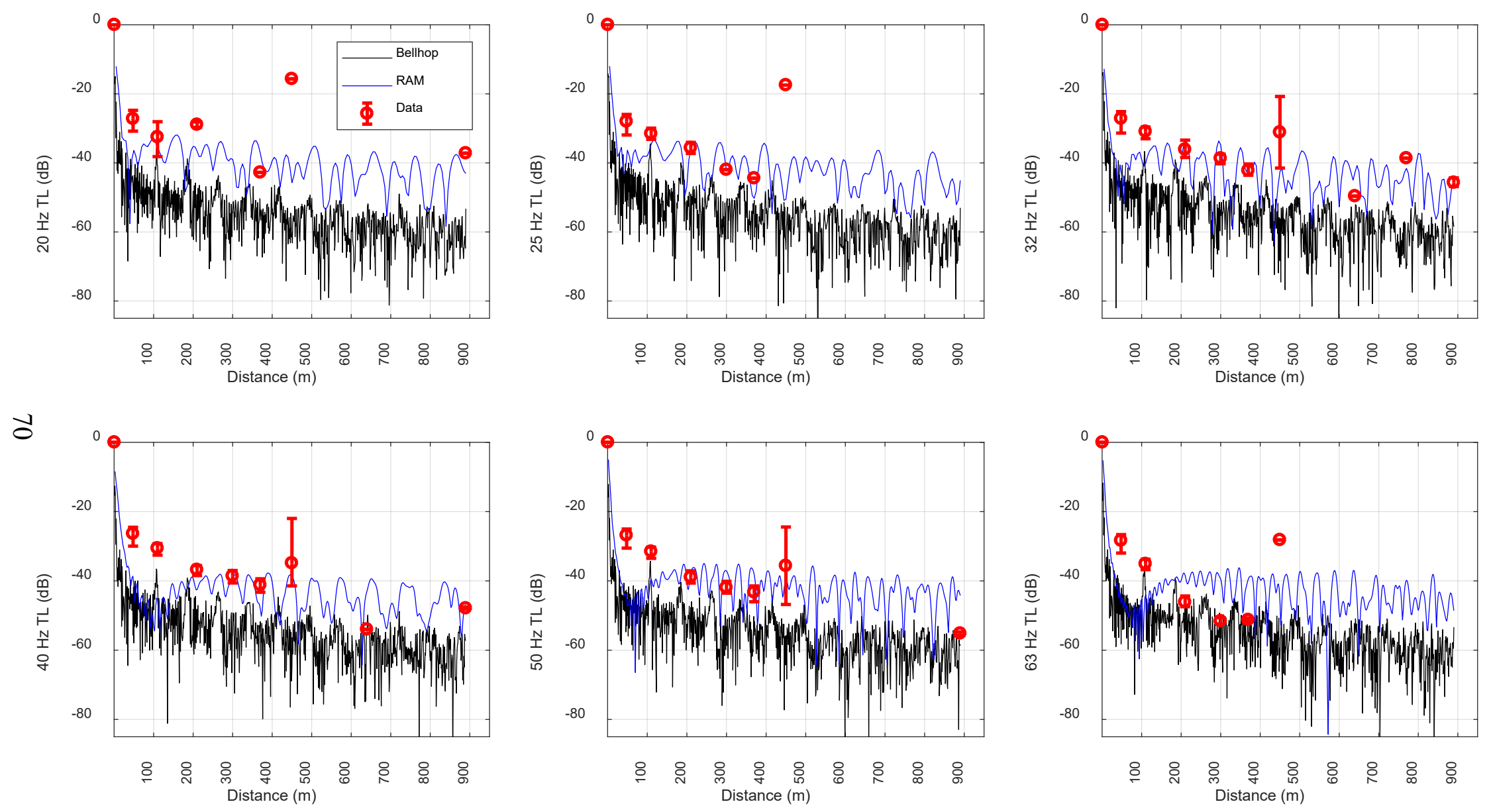

Figure 3.26 Atmospheric TL for $1 / 3$ - Octave bands $20 \mathrm{~Hz}$ to $63 \mathrm{~Hz}$. 

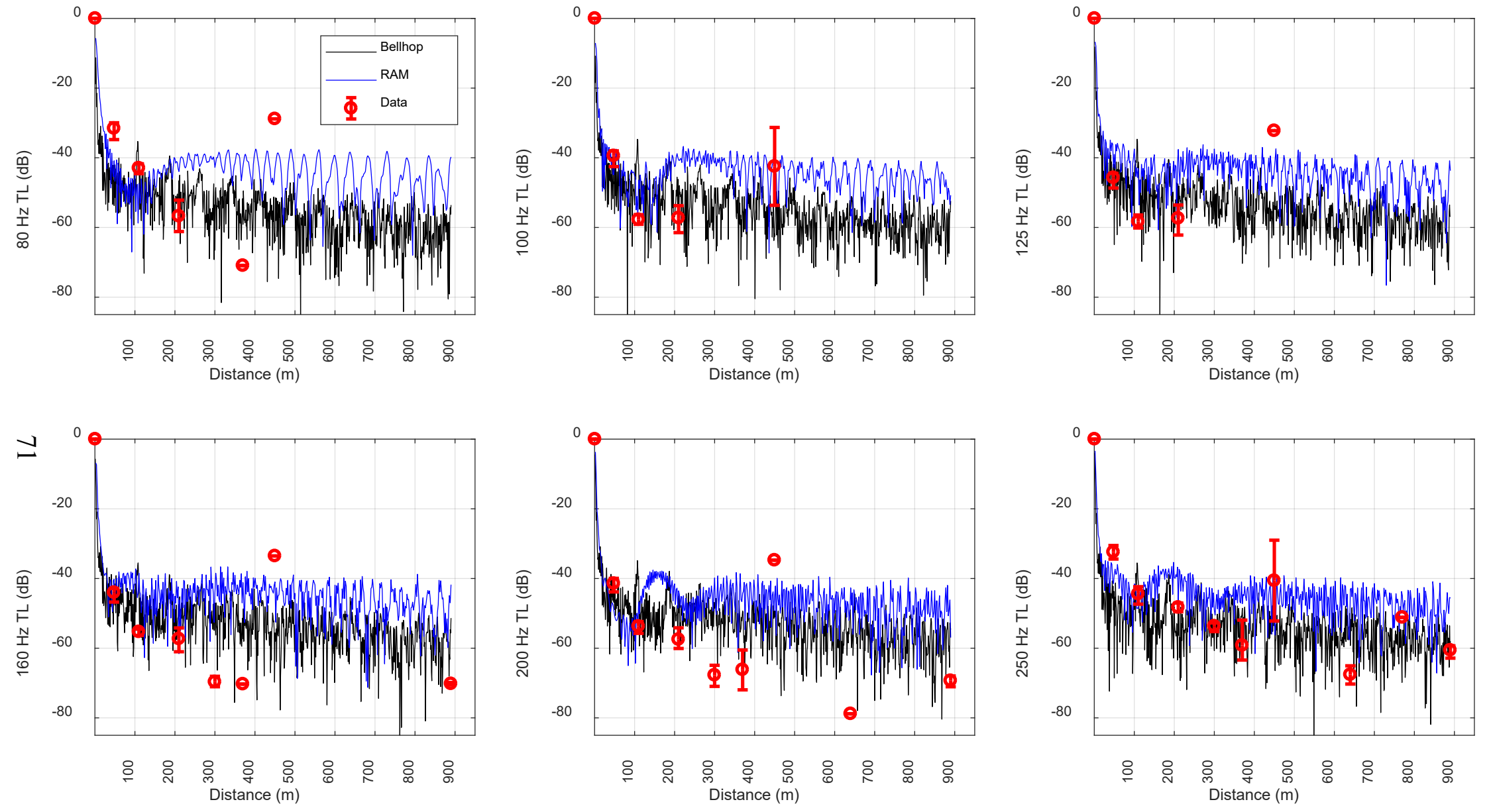

Figure 3.27 Atmospheric TL for $1 / 3$ - Octave bands $80 \mathrm{~Hz}$ to $250 \mathrm{~Hz}$. 

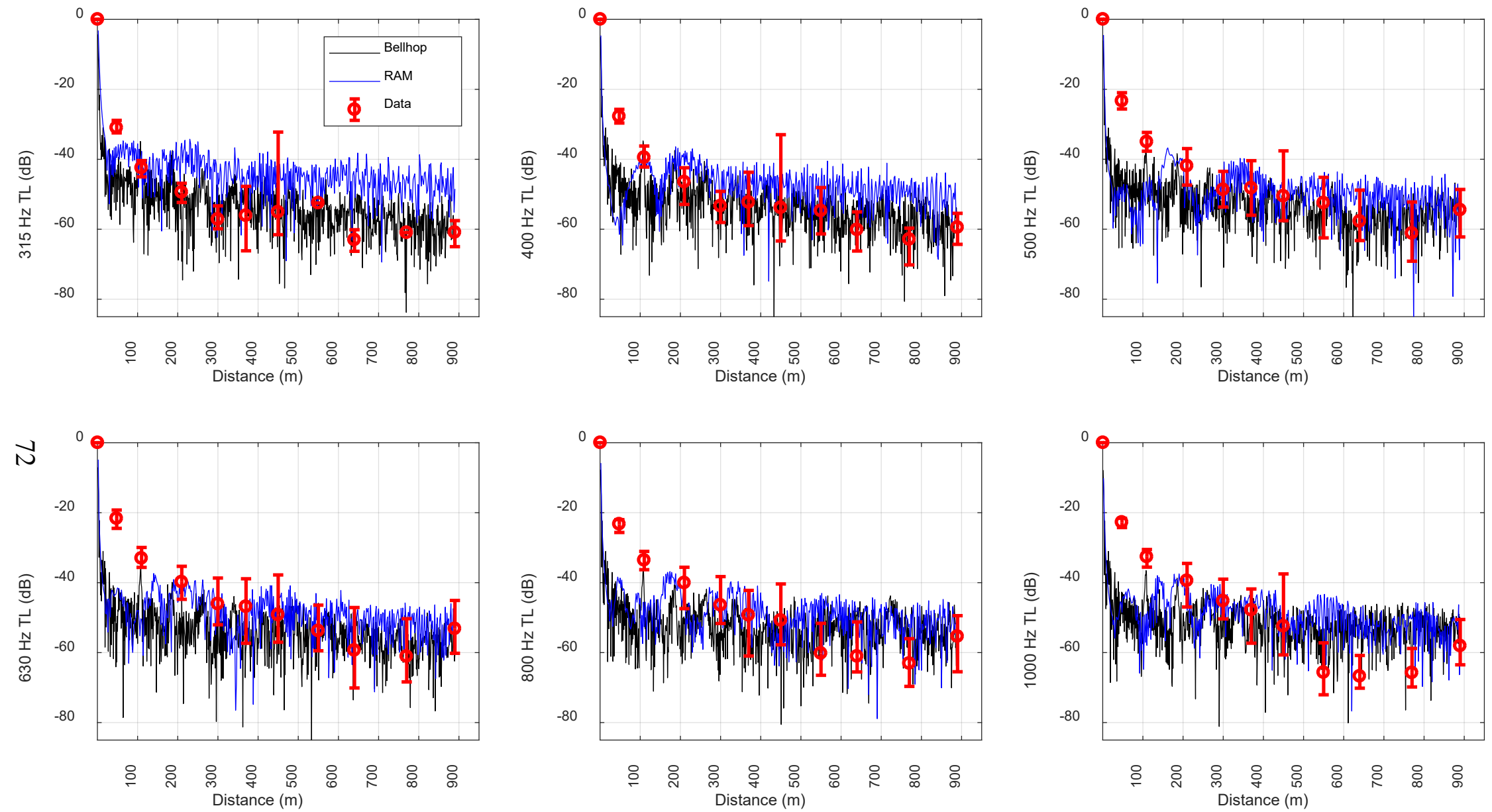

Figure 3.28 Atmospheric TL for $1 / 3$ - Octave bands $315 \mathrm{~Hz}$ to $1000 \mathrm{~Hz}$. 

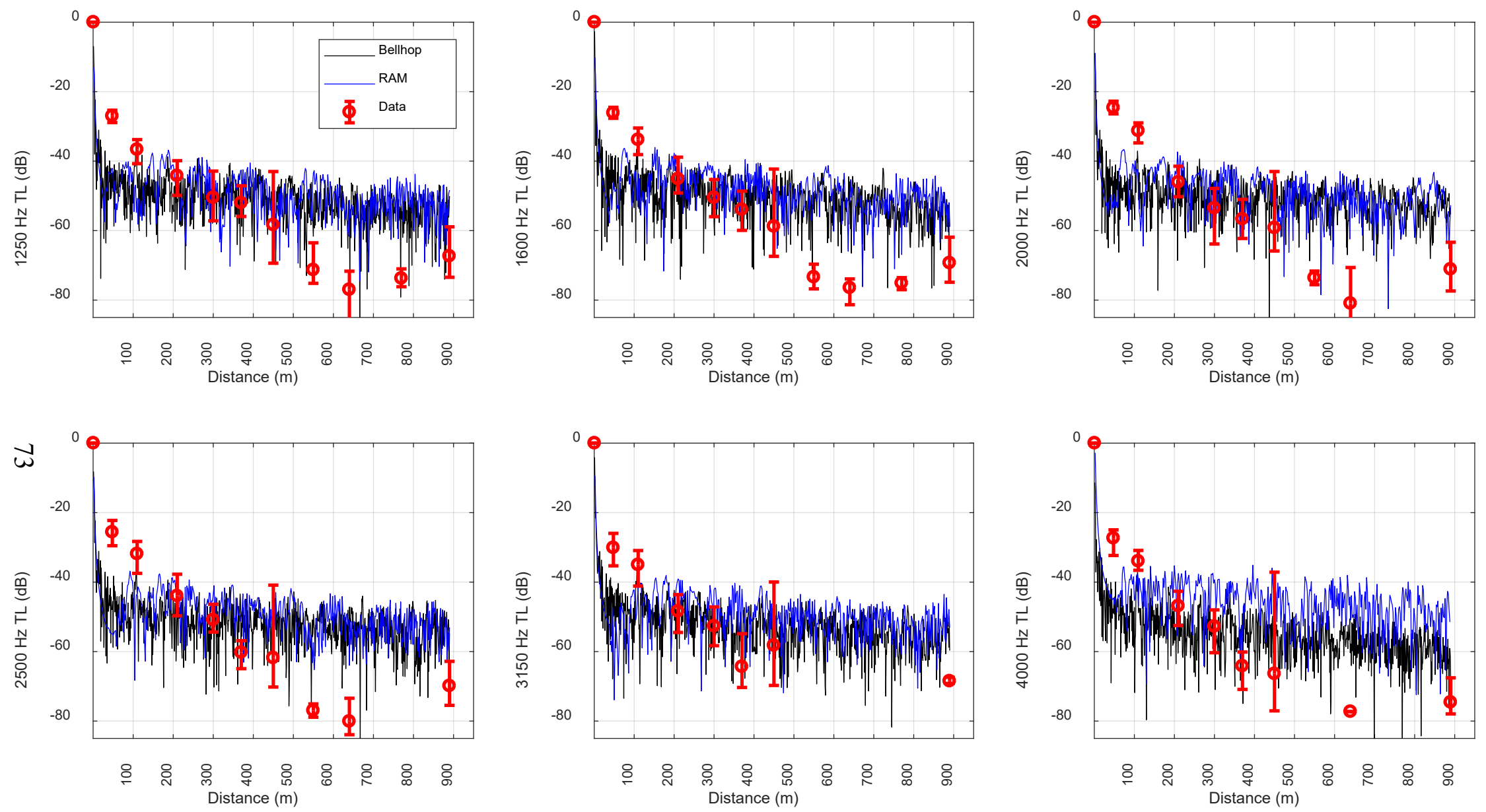

Figure 3.29 Atmospheric TL for $1 / 3$ - Octave bands $1250 \mathrm{~Hz}$ to $4000 \mathrm{~Hz}$. 

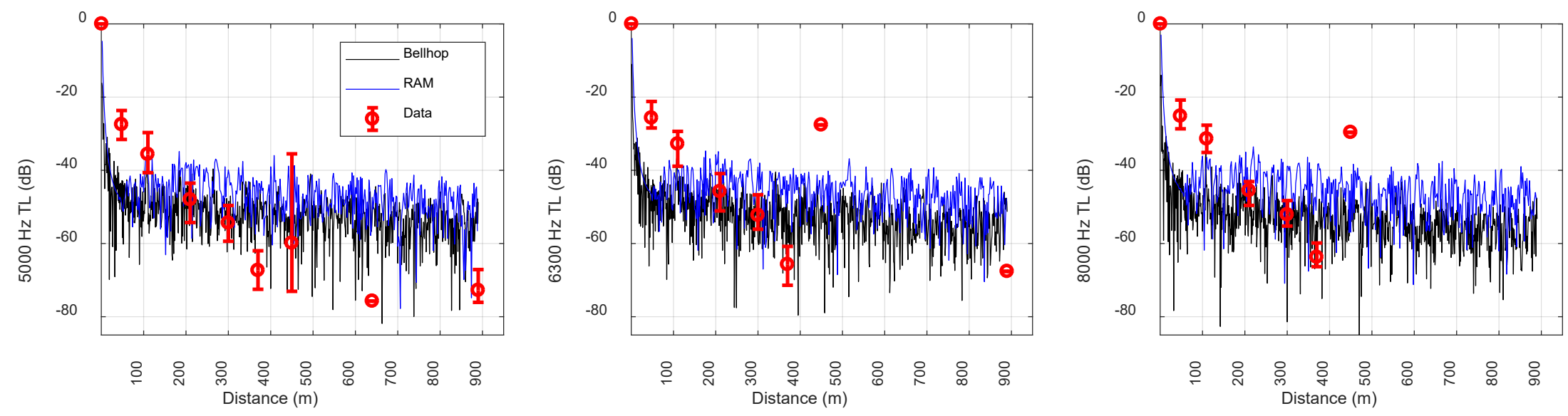

$\stackrel{\square}{\perp} \quad$ Figure 3.30 Atmospheric TL for $1 / 3$ - Octave bands $5000 \mathrm{~Hz}$ to $8000 \mathrm{~Hz}$. 


\subsubsection{Broadband TL}

To gain a comprehensive understanding of the propagation underwater and in the atmosphere, it is of interest to compute the broadband TL; independent of frequency output by the source. The broadband TL at each receiver distance is determined by averaging the transmission loss across all $1 / 3$ - octave bands at each measured range. The broadband TL is the mean of the rows in each column of Table 3.3 and Table 3.4; the result of which is shown in the last row in the tables.

The broadband TL is also shown in Figure 3.31. The theoretical cylindrical spreading curve as well as the theoretical spherical spreading curve are show for comparison per Equations 3.2 and 3.3. It is observed that the underwater broadband TL data closely matches the spherical spreading contour. The atmospheric TL data also roughly follows the spherical spreading contour, but with much more variation especially at ranges of 450,550 , and $640 \mathrm{~m}$. Variations from spherical spreading at these site distances is on the order of $10 \mathrm{~dB}$, while the rest of the data points only vary on the order of 1 to $4 \mathrm{~dB}$.

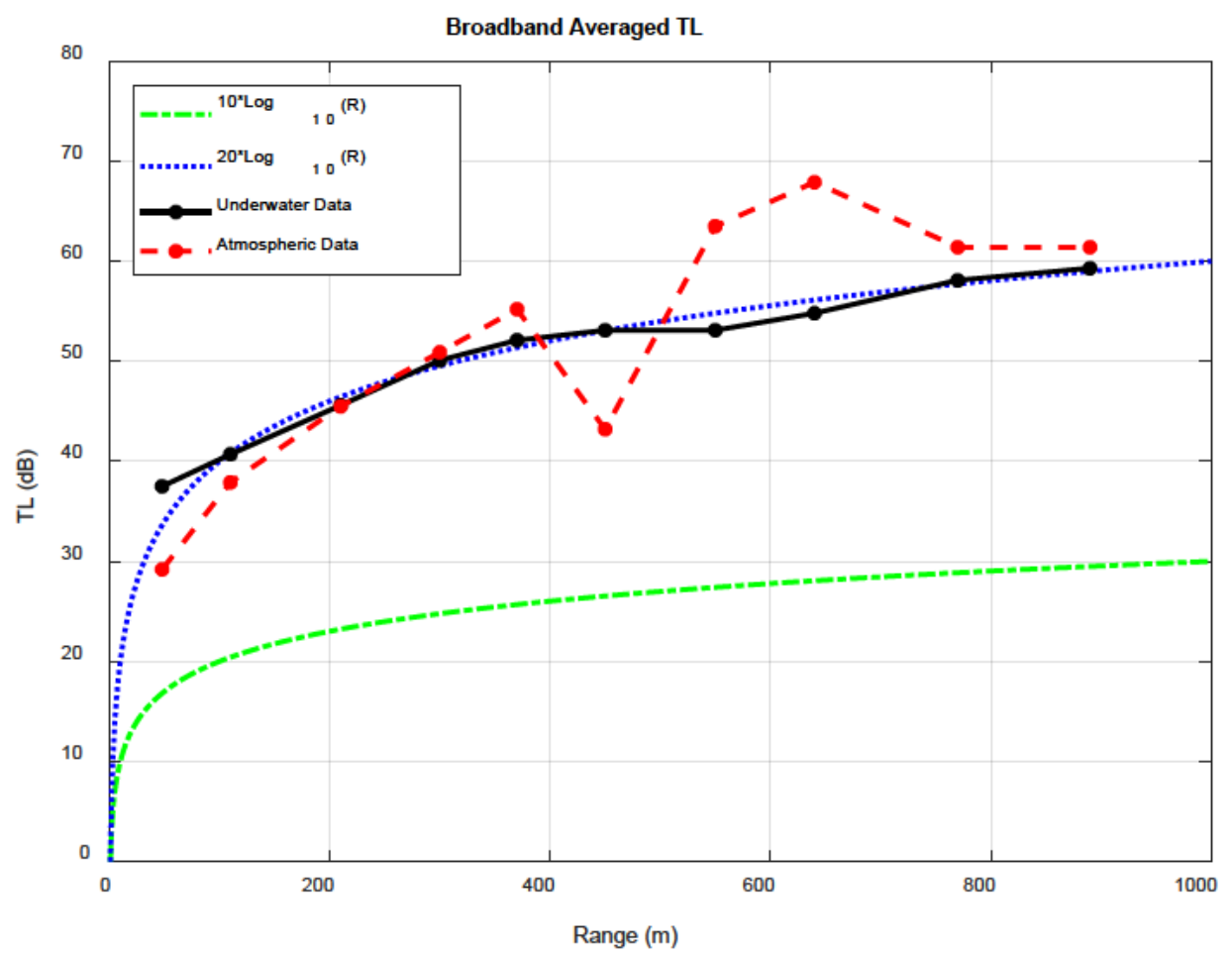

Figure 3.31 Underwater and atmospheric broadband averaged TL comparison.

The greater variation on atmospheric broadband TL is expected based on the larger variation on the $1 / 3$ - octave band data from the previous section. This comparison is not useful for considering propagation of a specific source, at a given frequency, through a specific environmental path. However, it provides confidence that the gross collected 
data is valid based on a long-known physical representation of geometric spreading and propagation.

Additionally, it is of interest to note that the broadband spreading type in both the atmosphere and the underwater environment is spherical in type; not cylindrical. Some of the error induced in the underwater TL simulations may be induced based on the 2D approximation of the Helmholtz equation. A 3D approximation or using a source type with specific directivity in each $1 / 3$ - octave band could potentially reduce these errors. However, this cannot be confirmed without using software which contains these capabilities.

\subsection{Effects of Ice Boreholes on FRFs between Air and Water}

For the Arctic experiments discussed in chapter 2, hydrophones were deployed underwater through bored holes in the ice. This method raised questions regarding the amount of acoustic energy which would be allowed to transmit directly between the air and the water without passing through the ice; in effect creating an acoustic short circuit. This was of particular interest for the cannon experiments during analysis of the FRF between the microphone and the hydrophone at the source site.

\subsubsection{Explanation of the Test}

During testing on Portage Lake, two experiments were conducted to investigate the effect of the ice boreholes on the FRF between the microphone in air and the hydrophone underwater. For the first experiment, the hydrophone was deployed, as normal, through the hole in the ice. For the second experiment, the hydrophone was frozen into the ice to simulate a continuous ice sheet without a hole. The propane cannon was used as the acoustic source in air for both experiments. Multiple cannon blasts were measured for each experiment to facilitate spectral averaging of the FRF.

In order to freeze the hydrophone into the ice without damaging equipment, the hydrophone cable was passed through a small PVC tube. The PVC tube was frozen into a cylinder of ice in a laboratory freezer before the test date (Figure 3.32). The hydrophone and ice cylinder assembly was then transported to the test location on the test date. The cylinder of ice was placed into the borehole on the Portage Lake ice sheet and allowed 2 hours to freeze solidly in place before the second experiment was carried out. The dimensions of the ice cylinder were roughly equal to the borehole diameter and the Portage Lake ice thickness. Therefore, only 2 hours were necessary because the ice cylinder was already completely frozen. 


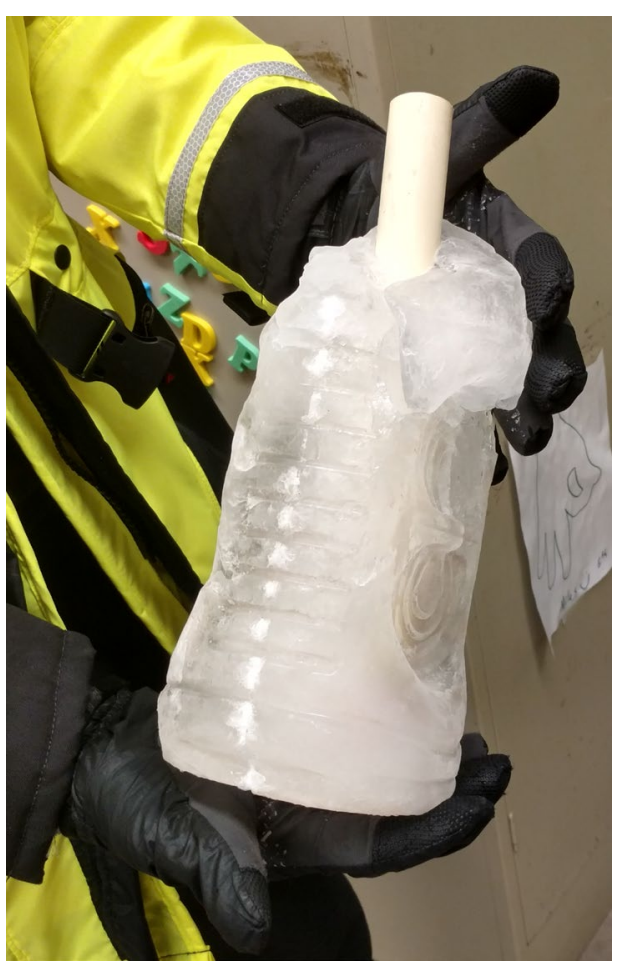

Figure 3.32 Ice cylinder with frozen-in PVC for ice borehole experiment.

\subsubsection{FRF Comparison}

Figure 3.33 shows the FRF magnitude, phase, and coherence for the open-hole and the frozen-hole experiments between the microphone in air and the hydrophone underwater.

The FRFs are almost identical at low to middle frequency ranges $(<400 \mathrm{~Hz})$. Above 400 $\mathrm{Hz}$, there appears to be broadband attenuation on the order of 2 to $8 \mathrm{~dB}$, however coherence also drops off significantly in this frequency range. This coherence drop-off indicates that the received signal at the hydrophone is not linearly related to the source signal (microphone) in this band. The low coherence is most probably explained by lower received signal at the hydrophones in this band for both the open-hole and the frozen-hole cases. Therefore, the apparent broadband attenuation above $400 \mathrm{~Hz}$ may be caused by higher variance on the FRF magnitude and phase in this band. At $183 \mathrm{~Hz}$, there is $4 \mathrm{~dB}$ difference between the open-hole and frozen-hole FRFs, however, this may be attributed to measurement error.

Overall, there is very little difference in path variation between the open-hole and frozenhole scenarios. In most cases where hydrophones are used for under-ice data collection, there is probably little benefit from freezing the hydrophones into the ice. 

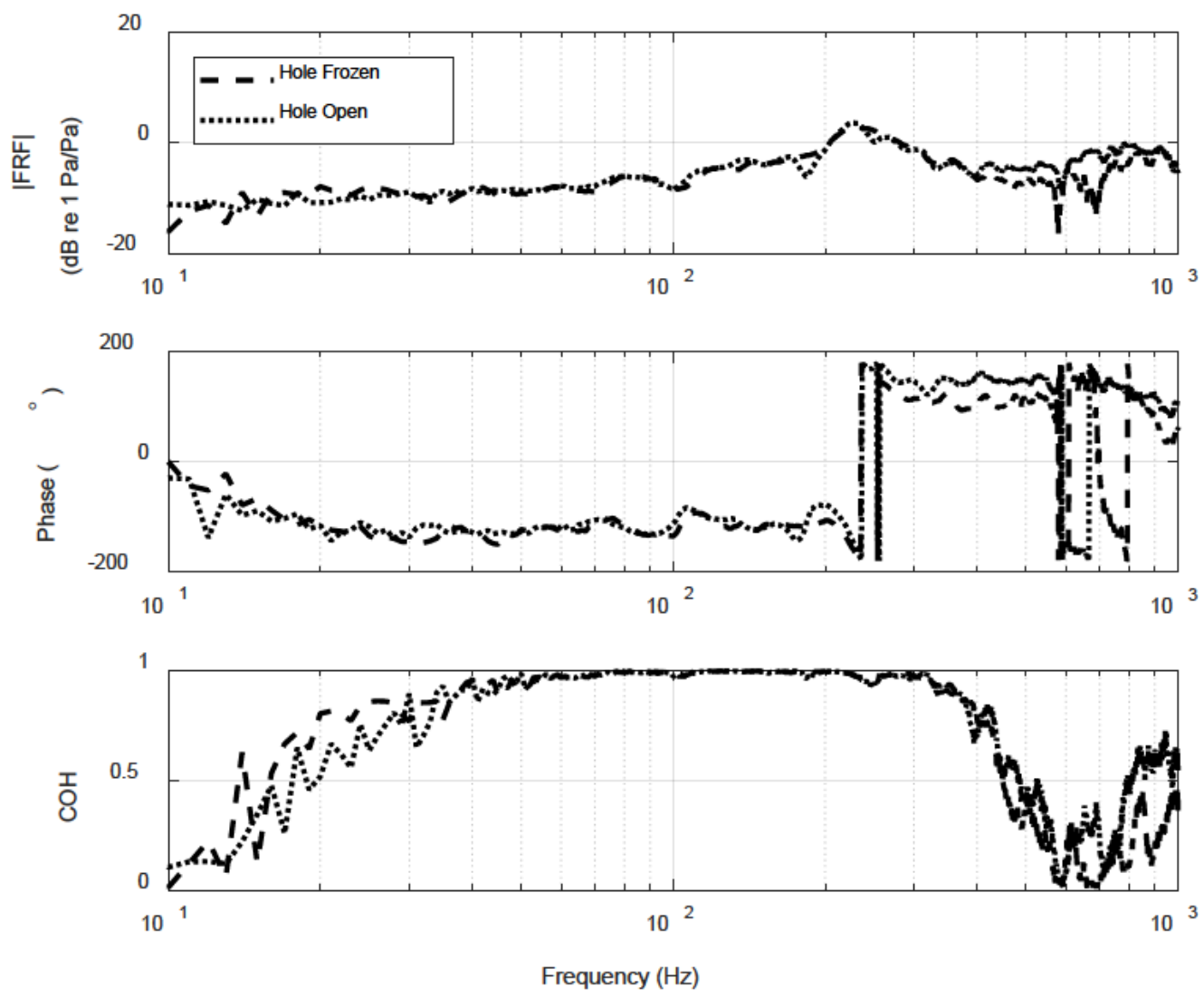

Figure 3.33 Air to Water FRF comparison with open and closed ice borehole.

\subsection{Impedance Relationship between Ice and Water}

The through-thickness mode identified in the Barrow, AK ice layer was validated upon quarter-wavelength resonance assumptions. That is to say, the boundary condition at the top of the ice sheet was assumed to be a pressure release (pressure equal to zero) and the bottom of the ice sheet was assumed to be a perfect reflector (particle velocity equal to zero). While this is a good first-pass approximation, the through-thickness behavior of the ice sheet most probably is not precisely a quarter-wavelength resonator due to the fluid-loaded condition on the bottom of the ice sheet (i.e. it is not perfectly reflective). The boundary condition at the ice-water interface, therefore, becomes of importance.

To make accurate predictions of the through-thickness modal behavior of the ice, it is necessary to understand the properties of the ice and water such as sound speed and density profiles. As will be described, these properties are fundamental in estimating the impedance relationship between the ice and water layers. For the environment considered, the sound speed in water was directly measured with the CTD probe. The sound speed in ice depends on the type of wave generated. For example, compressional waves travel faster than shear waves which in turn travel faster than flexural waves. 
Furthermore, dispersion of some wave-types in non-homogeneous environments causes higher frequencies travel faster than low frequencies. The sound speeds in the ice depend largely on elastic properties such as elastic modulus, shear modulus, density, and Poisson's ratio.

A discussion of the elastic properties and sound speeds in the ice will be followed by evaluation of the measured through-thickness modes in the ice layer

\subsubsection{Elastic Properties and Sound Speeds of the Ice}

The ice layer is assumed to be a two-dimensional solid which is surrounded by air on the top of the layer and water on the bottom (Figure 3.34). Particle motion is restricted to the vertical dimension, $z$, and the range (longitudinal) dimension, $r$. The ice layer is excited with a vertical impulse $F$ in the $z$-direction; perpendicular to the top boundary. There are four types of waves which may be excited in this scenario; compressional waves, vertically polarized shear waves, Rayleigh waves, and flexural waves.

Compressional waves are the fastest to propagate through a medium followed by that of shear waves; both of which are non-dispersive. The compressional wave is observed when the particle displacement of the medium is in the same dimension as the direction of the wave propagation. In the 2D case, a compressional wave may be observed in either the vertical or longitudinal dimensions as the energy from the impulse spreads through the solid. The theoretical compressional wave speed in a plate (2D solid) is known to be $e^{4,6,44}$,

$$
c_{p}=\sqrt{\frac{E}{\rho\left(1-v^{2}\right)}},
$$

where $E, \rho$, and $v$ are the elastic modulus, density, and Poisson's ratio of the plate material respectively.

Comparatively, a shear wave is observed when the particle motion is perpendicular to the direction of travel of the wave propagation. The theoretical shear speed is defined as ${ }^{10,44}$,

$$
c_{s}=\left[\frac{G}{\rho}\right]^{\frac{1}{2}}=\left[\frac{E}{2 \rho(1+v)}\right]^{\frac{1}{2}},
$$

where $G$ is the shear modulus. Relating the shear speed to the compressional speed yields the equation,

$$
c_{s}=c_{p}\left[\frac{(1-2 v)}{2(1-v)}\right]^{\frac{1}{2}} .
$$




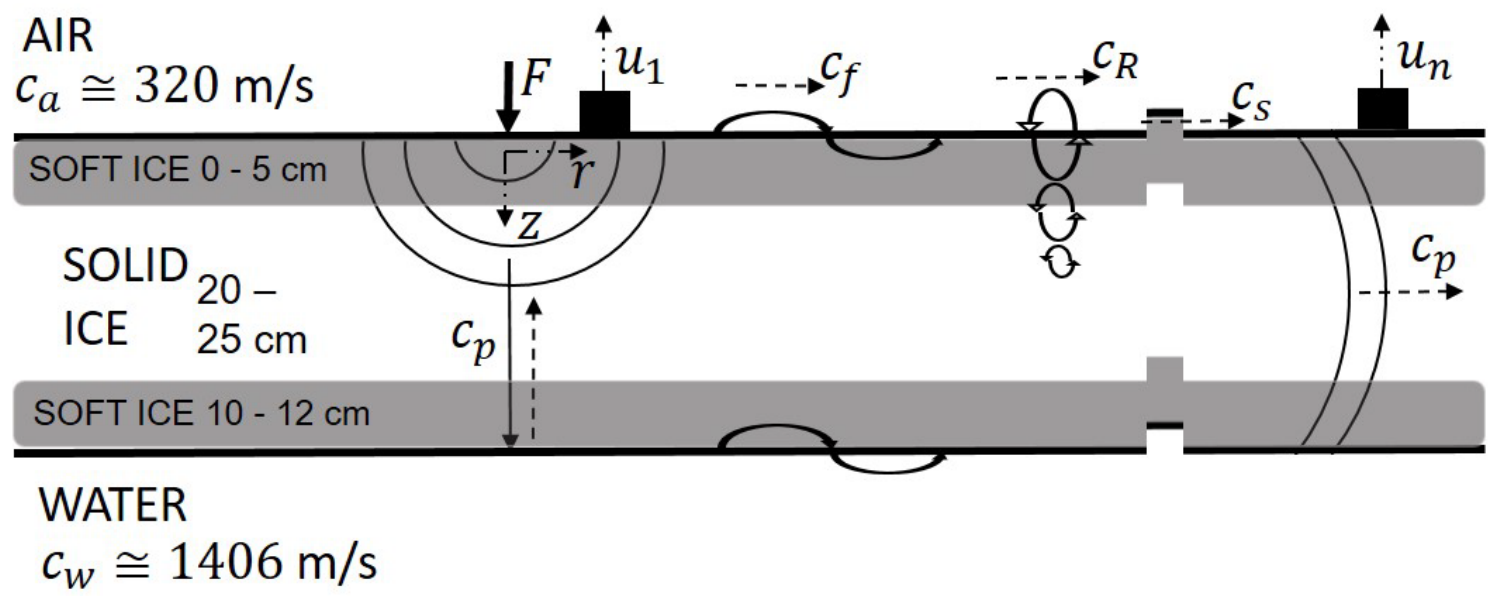

Figure 3.34 Ice layer wave-speed diagram. Wave types considered for the ice layer are compressional, shear, Rayleigh, and flexural. Air and water boundary conditions effect the through-thickness resonant frequency in drivepoint mobility. Force input $F$ and geophone responses $u_{i}$ are measured quantities.

Rayleigh waves travel near the surface of the layer and have retrograde elliptical particle motion in plane with the layer itself ${ }^{10}$. As depth in the layer increases, the amplitude of the Rayleigh wave rapidly decreases. Rayleigh waves propagate in the $r$-dimension and have wave speeds which are known to be non-dispersive in isotropic layers and are proportional to the shear velocity,

$$
c_{R}=0.9 c_{s}
$$

but are dispersive in non-isotropic layers ${ }^{10,14}$. Rayleigh waves contrast flexural waves in that they only exist very close to the surface of the layer in question while flexural waves represent bending motion of the full thickness of the layer. Flexural waves are generally slower than shear waves, but due to the dispersive nature of flexural waves, they may approach or exceed the shear speed at high frequencies (greater than $\sim 5000 \mathrm{~Hz}$ ) ${ }^{44}$.

The theoretical flexural wave speed in a thin plate is defined as ${ }^{44}$

$$
c_{f}=\left[\frac{\omega^{2} D}{\rho L}\right]^{\frac{1}{4}},
$$

where,

$$
D=\frac{E L^{3}}{12\left(1-v^{2}\right)}
$$

is the flexural rigidity and $L$ is the plate thickness. The dependence on $\omega$ indicates the dispersive nature of the flexural wave speed.

The particle velocity of the ice was measured at vertically oriented geophone locations $u_{i}$ indicated in Figure 3.34 in response to instrumented impact hammer excitation at $F$. The 
measured hammer and geophone data provides opportunity for direct measurement of the various wave speeds (Figure 3.35). A hammer impact is observed at 0.017 seconds. In the geophone data measured at $48 \mathrm{~m}$ from the source, a compressional wave arrival is observed at 0.035 seconds, a shear wave arrival is observed at 0.052 seconds, and a Rayleigh wave arrival is observed at 0.059 seconds. The resulting measured wave speeds are reported in Table 3.5. In addition, flexural waves are observed at the $48 \mathrm{~m}$ site between 0.1 to 0.3 seconds after the hammer impact event. These arrivals are observed to be dispersive and correspond to speeds ranging between roughly 100 to $500 \mathrm{~m} / \mathrm{s}$ and frequencies 15 to $350 \mathrm{~Hz}$, respectively.
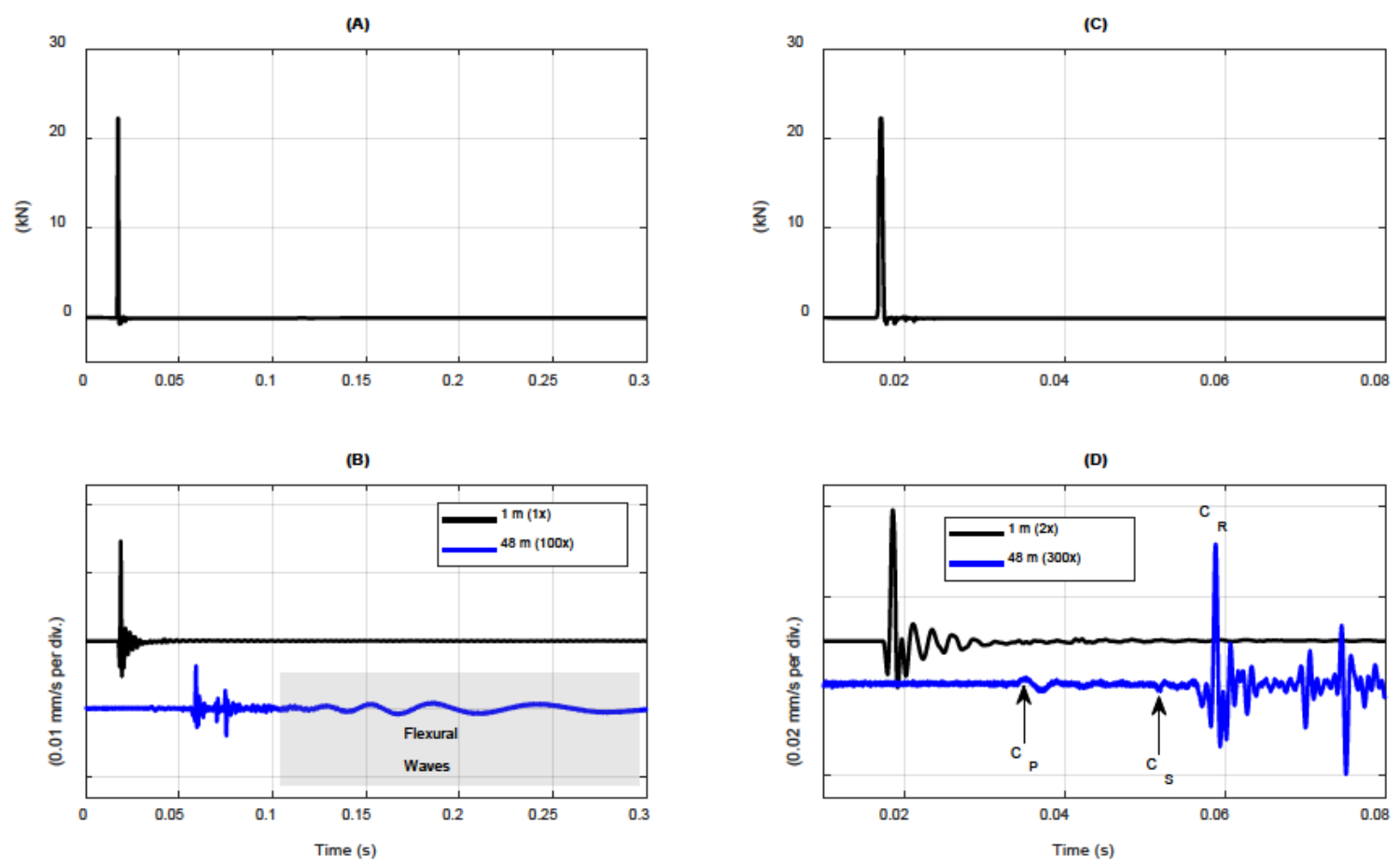

Figure 3.35 Hammer impulse and geophone response of ice layer. (A) Hammer impact. (B) Geophone traces. (C) Hammer impact zoomed in. (D) Geophone traces zoomed in with higher gain.

It is important to recall at this point, the overall ice layer thickness was measured between 30 to $43 \mathrm{~cm}$ at various locations. However, these measurements are not indicative of completely solid ice. When drilling holes through the ice, several $\mathrm{cm}$ on the top and bottom of the ice layer were observed to be soft and slushy (Figure 3.34). This consideration is certain to affect the measured wave speeds and elastic properties of the ice layer as well as attenuation of wave amplitude.

The particle velocity in the ice measured at the $48 \mathrm{~m}$ site in comparison to that measured at the $1 \mathrm{~m}$ site is between 2 to 4 orders of magnitude lower in amplitude. This is observed given the relative scale and gains applied to view the data in Figure 3.35. Given the case of the high-velocity layer (ice) between two low-velocity layers (air and water), the ice forms an inefficient waveguide and it is expected that wave energy will rapidly 
dissipate to the surrounding environment ${ }^{14,74}$. Also, due to the soft ice on the top and bottom of the ice layer, attenuation of wave energy is expected. Therefore, the downrange reduction in amplitude is reasonable in this scenario. At sites beyond $48 \mathrm{~m}$, geophone amplitudes were too low to be analyzed reliably.

Table 3.5 Ice layer sound speeds and elastic properties. Comparison of theoretical and measured speeds of in the ice and estimation of ice layer elastic properties. Theoretical sound speeds computed with the elastic properties shown.

\begin{tabular}{c|c|c|c}
\hline Measured & $c_{P}$ & $c_{S}$ & $c_{R}$ \\
\hline$\Delta \mathrm{t}(\mathrm{s})$ & 0.018065 & 0.03477 & 0.041779 \\
\hline \hline Speed $(\mathrm{m} / \mathrm{s})$ & 2657 & 1381 & 1149 \\
\hline Theoretical & $c_{P}$ & $c_{S}$ & $c_{R}$ \\
\hline Speed $(\mathrm{m} / \mathrm{s})$ & 2653 & 1379 & 1267 \\
\hline Equation No. & 3.39 & 3.41 & 3.42 \\
\hline Elastic Properties & \multicolumn{3}{|c}{$5.8 \mathrm{GPa}$} \\
\hline$E$ & \multicolumn{3}{|c}{$0.315 \mathrm{~kg} / \mathrm{m}^{3}$} \\
\hline$\rho$ & \multicolumn{3}{|c}{}
\end{tabular}

The Rayleigh wave is clearly the highest in amplitude of the waves considered in the ice layer. It is observed predominantly in both the $1 \mathrm{~m}$ and $48 \mathrm{~m}$ geophone data. The high amplitude is expected due to the ease of creating Rayleigh waves with impacts to the surface of a solid ${ }^{10}$.

The compressional and shear wave arrivals are an order of magnitude lower in amplitude than that of the Rayleigh wave. Near the source, the compressional wave propagates in both the vertical and longitudinal dimensions. As the compressional wave propagates farther downrange, it predominately travels longitudinally (in the $r$-dimension) through the ice. The vertical geophone detects the compressional wave at $48 \mathrm{~m}$ due to Poisson's effect; that is to say change of length in the $r$-dimension effects change of length in the $z$ dimension, and vice versa. Given that this is the mechanism for detection for the compressional wave, the amplitude is lower than it might be if a horizontally-oriented geophone were used. The measured shear wave is even lower in amplitude because the hammer is not a strong source for shear waves and due to the high dissipation within the ice.

The elastic properties of freshwater ice are discussed in several references ${ }^{75-79}$. Elastic modulus and Poisson's ratio both vary significantly based upon the environment and the type of ice considered $25,39,42,76$. Generally speaking, the elastic modulus of ice is on the order of 8 to $12 \mathrm{GPa}$, density of is on the order of 850 to $950 \mathrm{~kg} / \mathrm{m}^{3}$, and Poisson's ratio 
varies between 0.2 to 0.49 . However, reports of ice with Poisson's ratio of 0.8 to 1.2 have been found ${ }^{40}$.

It is of interest to compare the measured wave speeds with the theoretical values and estimated elastic properties of the ice layer. Determining the elastic properties essentially requires tuning the values of elastic modulus, Poisson's ratio, and density such that the theoretical speeds match the measured speeds based on the equations in Table 3.5.

The theoretical compressional speed (Equation 3.39) is shown in Figure 3.36(a) and the theoretical shear speed (Equation 3.41) is shown in Figure 3.36(b). Both compressional and shear speed are plotted as a function of Poison's ratio with the assumption of the ice density being $915 \mathrm{~kg} / \mathrm{m}^{3}$. Higher elastic moduli imply higher compressional sound speed. As Poisson's ratio approaches \pm 1 , the compressional speed asymptotically approaches infinity. The shear speed approaches zero as Poisson's ratio approaches 0.5 . The measured compressional speed $2657 \mathrm{~m} / \mathrm{s}$ and measured shear speed $1381 \mathrm{~m} / \mathrm{s}$ are plotted as horizontal lines across Figure 3.36.
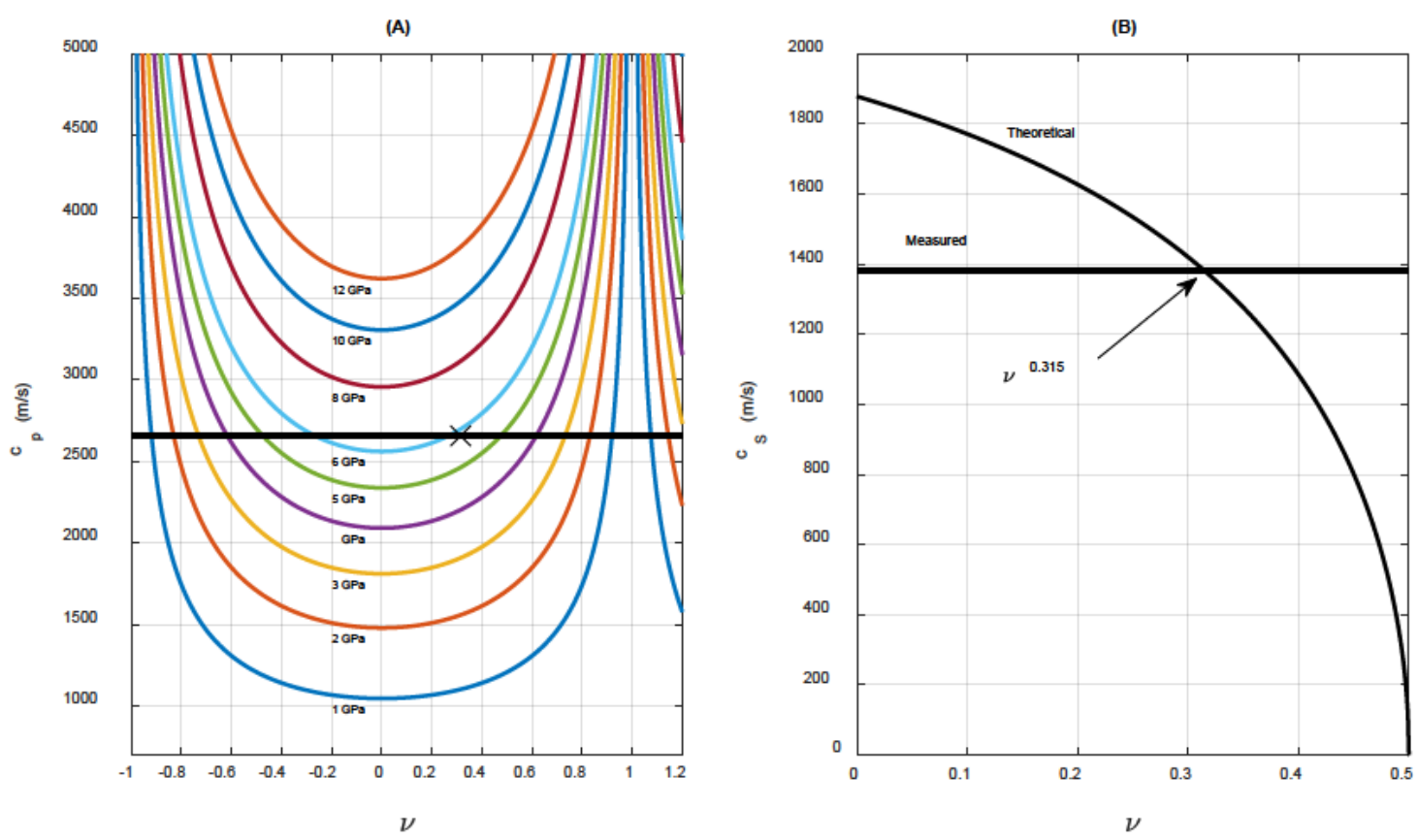

Figure 3.36 Theoretical compressional and shear wave speed. Ice density $(\rho)$ assumed 915 $\mathrm{kg} / \mathrm{m}^{3}$. A) Compressional speed for multiple $E$. Measured compressional wave speed $2657 \mathrm{~m} / \mathrm{s}$ shown as horizontal line. B) Shear wave speed

A range of elastic moduli ( 5 to $7 \mathrm{GPa}$ ) are reasonable for the measured compressional speed. Solving Equation 3.41 for $v$ and inputting the measured values for compressional and shear speeds yields a value of Poisson's ratio of 0.315 . Given this value for Poisson's ratio and the assumed ice density, the elastic modulus is $5.8 \mathrm{GPa}$. The elastic modulus being lower than that of nominal freshwater ice makes sense based on the soft and slush ice observed at the top and the bottom of the layer. 
Some disparity is noted in the comparison between theoretical and measured values for the shear wave speeds. However, this difference is very small and may simply be attributed to variability in the ice properties as a function of position in the environment. Larger disparity is observed for the Rayleigh wave. The theoretical Rayleigh wave speed based on Equation 3.42 is roughly $100 \mathrm{~m} / \mathrm{s}$ faster than the measured Rayleigh wave. This is not surprising due to the non-homogeneity of the ice layer. Not only is the Rayleigh wave likely dispersive in this environment, but it will travel slower due to the soft ice within the layer.

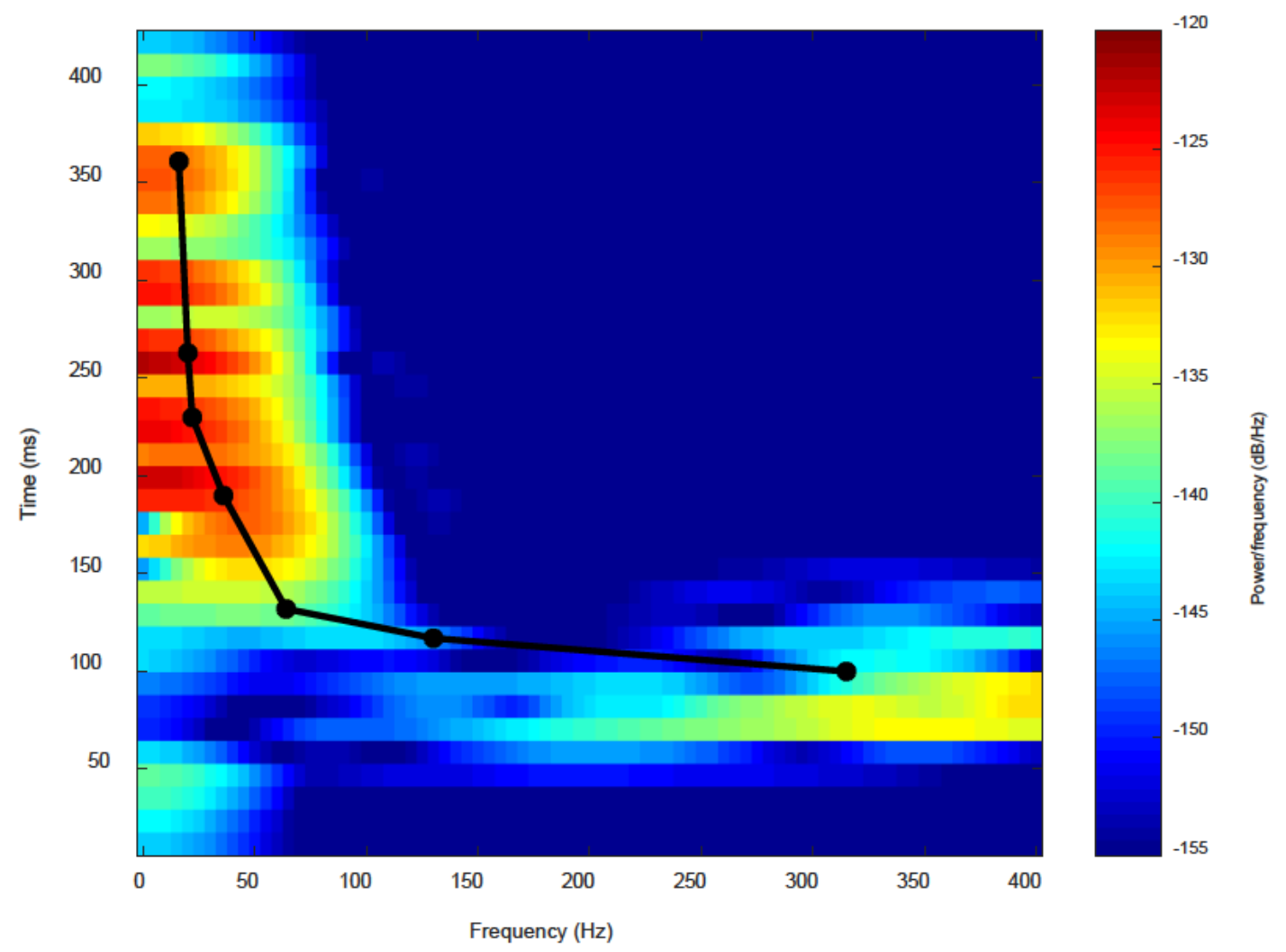

Figure 3.37 Flexural wave dispersion curve from geophone data $48 \mathrm{~m}$ from source. Flexural waves begin at approximately $100 \mathrm{~ms}$. Wave arrivals preceding $100 \mathrm{~ms}$ are compressional, shear, or Rayleigh waves.

Given the elastic properties reported in Table 3.5, the theoretical flexural dispersion curves are plotted in Figure 3.38 for several layer thicknesses. The measured flexural wave speeds and frequencies are shown as discrete points on the figure. Given that the flexural wave is highly dispersive - that is to say the rate of change of frequency and speed is large - it is difficult to determine the precise frequency and speed at any given moment in the data. The measured dispersion data were determined by observing the spectrogram of the geophone data (Figure 3.37). The selected points are shown both on the spectrogram and the theoretical dispersion curves. 


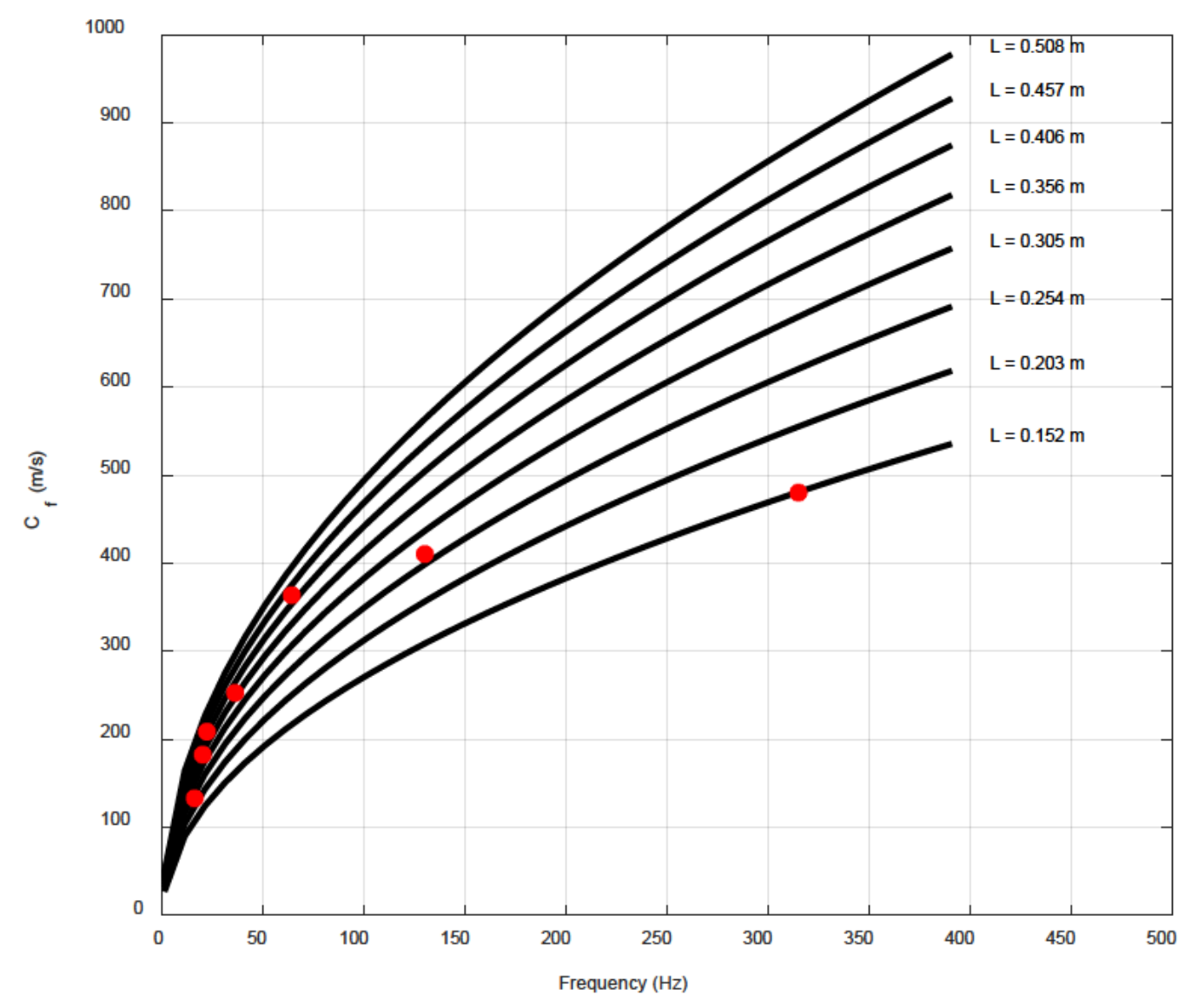

Figure 3.38 Theoretical flexural wave speed for thin plates per Equation 3.43. Measured flexural speed and frequency indicated with red data points.

The flexural waves observed in the geophone data do not match up precisely with a particular layer thickness. However, the data does stay within a reasonable range of layer thicknesses compared to the measured ice thickness. It makes sense that different frequencies may propagate through various thickness of the ice layer especially given the non-homogeneity. That is to say a low-frequency flexural wave may propagate through a thicker portion of the layer compared to that of a high-frequency wave.

Overall, the theoretical sound speeds are generally in agreement with those measured and the resulting elastic properties are reasonable given values published within the literature.

\subsubsection{Solving the Wave Equation with Impedance Matched Boundary Conditions}

The through-thickness mode in the ice layer is expected to be caused by a compressional wave traveling perpendicular to the plane of the ice layer. 
A more rigorous analysis of the ice-water interaction can be formed by starting with the wave equation, applying proper boundary conditions to it, and solving for the throughthickness modal frequencies.

To formulate the problem mathematically, it is advantageous to begin with the wave equation in one dimension ${ }^{64}$,

$$
\frac{\partial^{2} p}{\partial z^{2}}=\frac{1}{c^{2}} \frac{\partial^{2} p}{\partial t^{2}}
$$

where the acoustic pressure in the ice $p=p(z, t)$ and $z$ is the spatial coordinate in the through-thickness direction (the origin of $z$ is at the air-ice interface and is positive down towards the ice-water interface), and $c=c_{P}$ which is the compressional speed of sound in the ice. The equation may then be simplified as follows. Assuming that the solution for $p(t)$ will take the form,

$$
p(t)=A e^{j \omega t}
$$

the full solution may then be represented,

$$
p(z, t)=p(z) e^{j \omega t}
$$

The second partial derivative with respect to time is,

$$
\frac{\partial^{2} p}{\partial t^{2}}=-\omega^{2} p(z)
$$

and the second partial derivative with respect to the spatial coordinate is,

$$
\frac{\partial^{2} p}{\partial z^{2}}=-k^{2} p(z)
$$

where the wavenumber

$$
k=\frac{\omega}{c}=\frac{2 \pi}{\lambda} .
$$

At the air-ice interface, a pressure release boundary condition is assumed. That is to say, $p(z=0)=0$. At the ice-water boundary and impedance match boundary condition is assumed. Impedance in this section is denoted as $\mathbf{Z}$ to distinguish from the spatial coordinate. The impedance is known to be equal to the ratio of pressure and particle 
velocity which is also equal to the medium density multiplied by the compressional sound speed. In this case, the impedance being matched is that of the water. Therefore,

$$
\mathbf{Z}_{w}(z=L)=\frac{p(L)}{u(L)}=\rho_{0_{w}} c_{w},
$$

where $L$ is the ice thickness and the subscript $w$ denotes the density and sound speed of the water. The boundary value problem may then be represented as the Helmholtz equation in one dimension with the boundary conditions,

$$
\left\{\begin{array}{ll} 
& \frac{\partial^{2} p}{\partial z^{2}}+k^{2} p(z)=0 \\
\text { B.C. } 1: & p(z=0)=0 \\
\text { B.C. } 2: & \frac{p(L)}{u(L)}=\rho_{0_{w}} c_{w}
\end{array} .\right.
$$

The total solution of the wave equation takes the form,

$$
p(z, t)=\left[A \sin \left(k_{I} z\right)+B \cos \left(k_{I} z\right)\right] e^{j \omega t}
$$

however, may be simplified by considering only the spatial variation and ignoring variation with respect to time

$$
p(z)=A \sin \left(k_{I} z\right)+B \cos \left(k_{I} z\right) .
$$

Applying the first boundary condition yields $B=0$, therefore $p(z)=A \sin \left(k_{I} z\right)$. In order to apply the second boundary condition, the relationship between pressure and particle velocity must be established. This is well defined via Euler's equation,

$$
-\nabla p=\rho_{0_{I}} \frac{\partial u}{\partial t}
$$

where the subscript $I$ represents the ice density. Understanding that the solution $u(t)$ is oscillatory, we may then represent Euler's equation as,

$$
-\frac{\partial p}{\partial z}=j \omega \rho_{0_{I}} u
$$

Therefore,

$$
u(z)=\frac{j}{\omega \rho_{0_{I}}} \frac{\partial p}{\partial z}
$$


Taking the partial derivative of Equation 3.57 with respect to $z$ gives

$$
u(z)=\frac{A k_{I} j}{\omega \rho_{0_{I}}} \cos \left(k_{I} z\right) .
$$

The second boundary condition may now be applied,

$$
\frac{p(L)}{u(L)}=\rho_{0_{w}} c_{w}=\frac{\sin \left(k_{I} L\right)}{\frac{k_{I} j}{\omega \rho_{0_{I}}} \cos \left(k_{I} L\right)},
$$

which reduces to the transcendental equation,

$$
\tan \left(k_{I} L\right)=\frac{j \rho_{0_{w}} c_{w} k_{I}}{\omega \rho_{0_{I}}} *\left(\frac{L}{L}\right)
$$

Solving the transcendental equation requires plotting the left and right-hand sides of the equation as a function of $k_{I} L$ and determining where the functions cross. The term $\frac{L}{L}$ is multiplied through so the right hand side of 3.60 is dependent on $k_{I} L$. The modal frequencies are determined by,

$$
f_{n}=\frac{\left(k_{I} L\right)_{n} c_{I}}{2 \pi L}
$$

where $\left(k_{I} L\right)_{n}$ is the value of the independent axis where the transcendental equation is solved.

\subsubsection{Ice layer drivepoint FRF (dynamic mobility)}

From the collected data, the FRF between the geophone signal at the source site and the hammer signal may be computed. The frequency domain ratio of particle velocity in $\mathrm{m} / \mathrm{s}$ and input force in $\mathrm{N}$ is often referred to as dynamic mobility ${ }^{80}$. The inverse of mobility is known as dynamic impedance.

The theoretical FRF (mobility) can be estimated from the pole-residue theorem,

$$
h(j \omega)=j \omega \sum_{n=1}^{m} \frac{A_{n}}{j \omega-\lambda_{n}}+\frac{A_{n}^{*}}{j \omega-\lambda_{n}^{*}}
$$

where $m$ is the number of modes of the system, $\lambda_{n}$ is the $n^{\text {th }}$ pole of the system and $A_{n}$ is the $n^{\text {th }}$ residue $\mathrm{e}^{32,80}$. These are defined by, 


$$
\begin{gathered}
A=\frac{1}{2 j M \omega_{d}}, \\
\lambda=-\zeta \omega_{n}+j \omega_{d}, \\
\omega_{d}=\omega_{n} * \sqrt{1-\zeta^{2}}, \\
\omega_{n}=2 \pi f_{n},
\end{gathered}
$$

where $M$ is the modal mass, $\zeta$ is the modal damping ratio, $\omega_{n}$ is the natural frequency, and $\omega_{d}$ is the damped natural frequency.

Comparison of the data to a theoretical FRF requires knowledge of the frequency of the mode being synthesized. The modal frequency $f_{n}$ is computed from Equation 3.61 after solving the transcendental equation in Equation 3.60. Inputs to Equation 3.60 are the environmental properties shown in Table 3.6 which were measured and discussed in the previous sections. The ice thickness was chosen to be $20 \mathrm{~cm}$, which is representative of the solid ice layer only; not including the soft and slushy ice on the top and bottom of the layer. The left and right-hand side of Equation 3.60 are plotted in Figure 3.39. The value of the $k_{I} L$-axis at the intersection of the two lines represent the modal frequency information per Equation 3.61.

Additional inputs to the theoretical FRF are modal damping ratio and modal mass. Damping ratio was assumed to be 0.045 and modal mass was scaled to unity.

Table 3.6 Input properties for theoretical FRF

\begin{tabular}{clcc}
\hline Symbol & Description & Value & Unit \\
\hline \hline$\rho_{w}$ & Water Density & 1000 & $\mathrm{~kg} / \mathrm{m}^{3}$ \\
$c_{w}$ & Water Sound Speed & 1406 & $\mathrm{~m} / \mathrm{s}$ \\
$\rho_{I}$ & $\begin{array}{l}\text { Ice Density } \\
\text { Ice Sound Speed } \\
\text { (Compressional) }\end{array}$ & 915 & $\mathrm{~kg} / \mathrm{m}^{3}$ \\
$c_{I}$ & Ice Thickness & 2657 & $\mathrm{~m} / \mathrm{s}$ \\
$L$ & & 20 & $\mathrm{~cm}$ \\
\hline \hline
\end{tabular}

The value of $k_{I} L$ at the first intersection in Figure 3.39 is 0.694 . This corresponds to a theoretical modal frequency of $1445 \mathrm{~Hz}$. It is worth noting that the modes beyond this are not considered because the theoretical frequency is far beyond the maximum frequency of excitation by the hammer impulse. The resulting theoretical FRF is shown 
overlaid with the experimental FRF in Figure 3.40. The primary simulated mode coincides directly with a $10 \mathrm{~dB}$ peak in the measured data near $1445 \mathrm{~Hz}$. This is indicative of a resonance in the ice layer in the $z$-dimension. A mode at this frequency is also confirmed by the phase of the dynamic mobility crossing through zero degrees in the vicinity of $1445 \mathrm{~Hz}$.

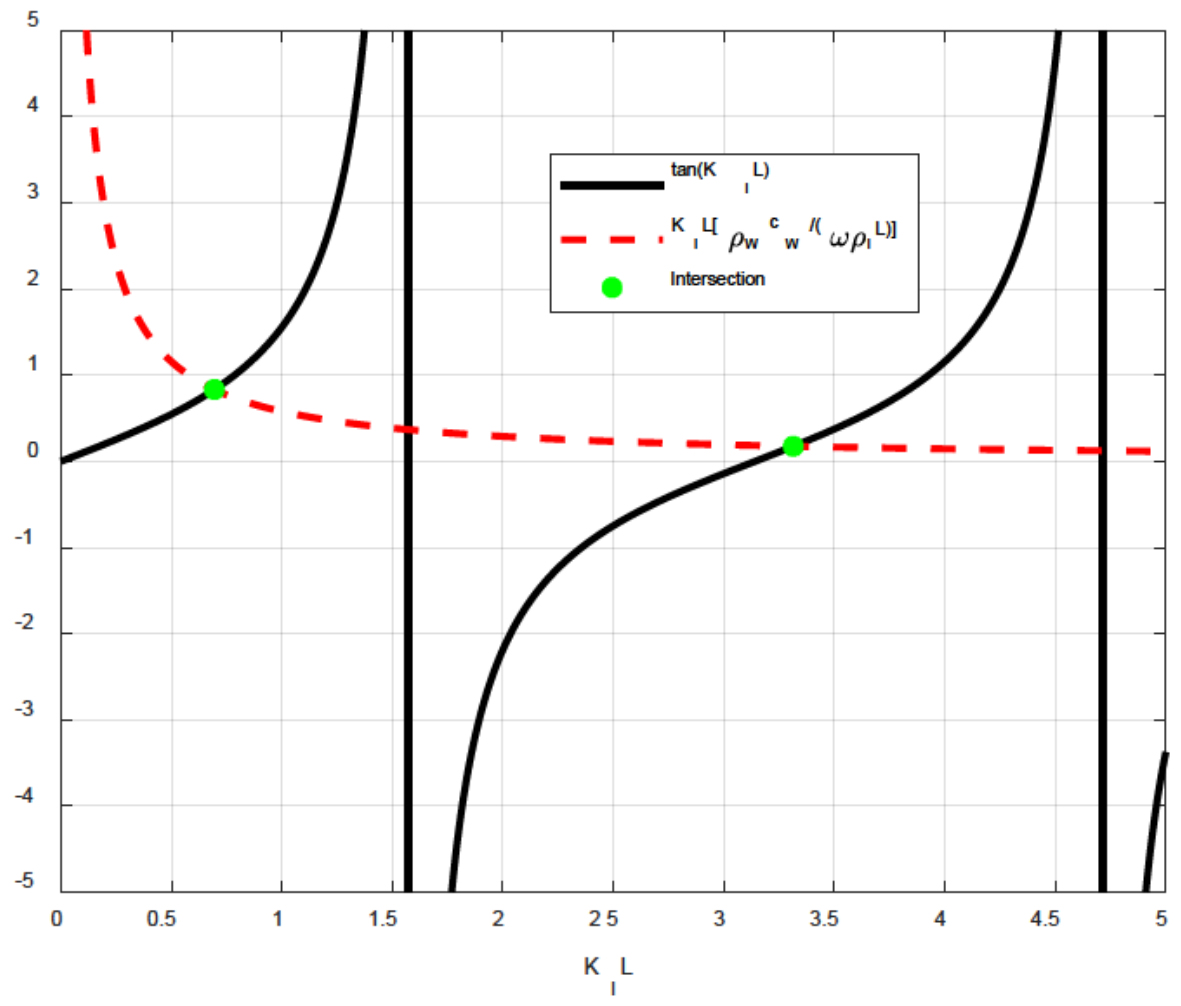

Figure 3.39 Transcendental equation for determining modal frequencies. Through-thickness modal frequencies in the ice layer are determined at the intersections.

The peak in the measured data is quite broad; spanning roughly $100 \mathrm{~Hz}$ on either side of $1445 \mathrm{~Hz}$. For the assumed modal damping ratio of 0.045 , the magnitude and phase of the theoretical FRF match closely to the measured data. This relatively high value for damping is reasonable based on the soft ice observed at the top and bottom of the ice layer as well as the expected losses to the surrounding environment. Slight differences in frequency are reasonable based on the high value of damping causing frequency shifts and potentially non-linear effects. 
(A)

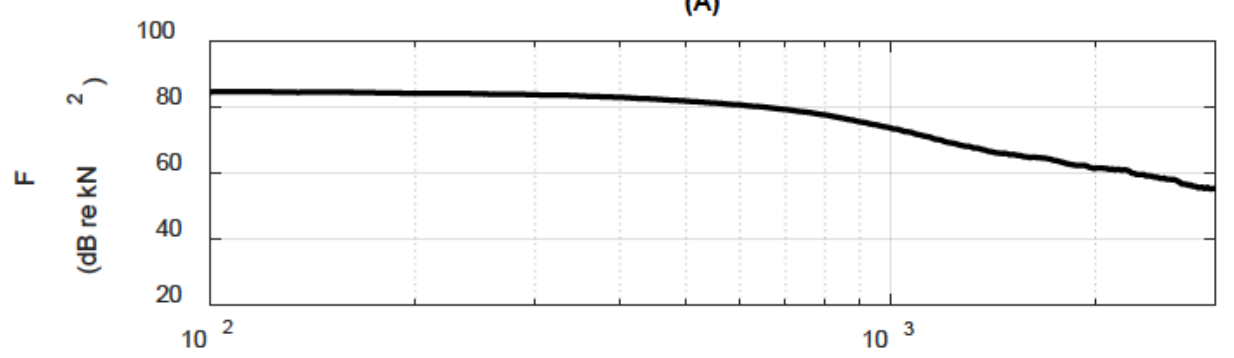

(B)

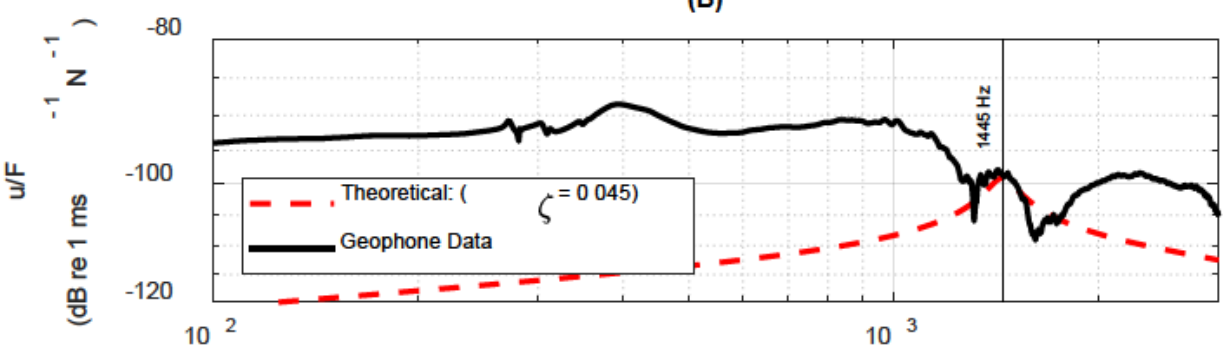

(C)

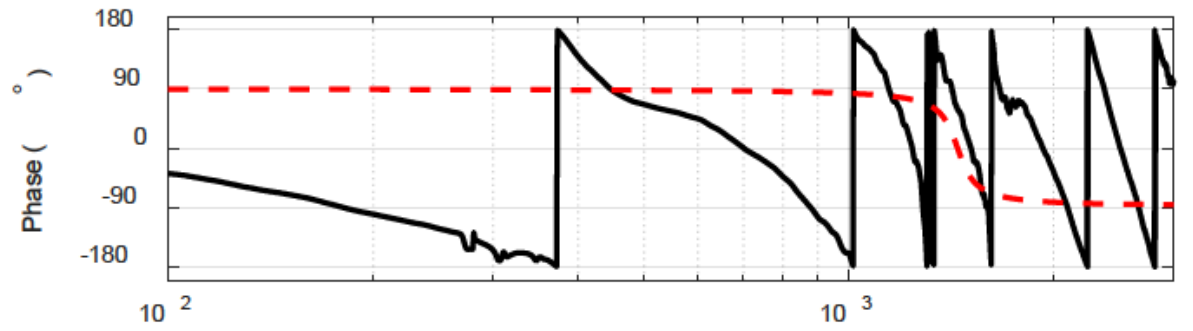

(D)

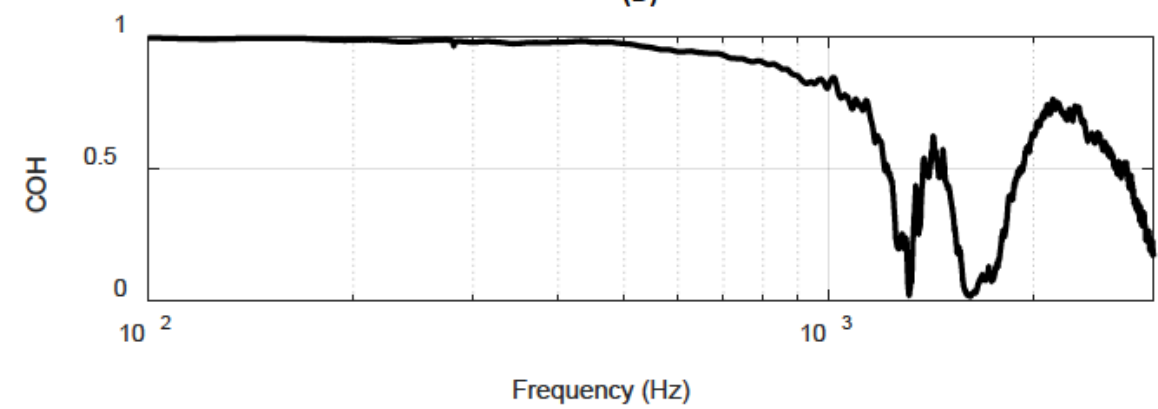

Figure 3.40 Drivepoint FRF (mobility) for hammer input and geophone response. (A) Hammer input autopower spectra (B) Drivepoint mobility (C) Phase of FRF (D) Coherence

The measured coherence of the FRF is close to one for the majority of the frequency range. Coherence drop-off begins near $600 \mathrm{~Hz}$ and continues to roughly $1200 \mathrm{~Hz}$. A sharp increase in coherence is observed at the through-thickness resonance; indicating good relationship between the hammer and geophone signals. While the coherence is 
lower than normally expected (less than one), this is reasonable given the drop-off in excitation in the frequency range greater than $1 \mathrm{kHz}$.

In the experimental FRF, lower frequency modes observed in the 250 to $400 \mathrm{~Hz}$ frequency range are likely due to the Rayleigh and flexural waves in the ice layer. This is confirmed by again viewing the data in Figure 3.35. The primary ring-down in the $1 \mathrm{~m}$ geophone data corresponds to roughly $400 \mathrm{~Hz}$; which is also observed as a broad peak in the drivepoint mobility. The lower frequencies present in the drivepoint mobility are also observed in the time-domain data, but to a lesser extent.

It is of interest to determine the ice thickness from the through-thickness resonant frequency observed in the drivepoint mobility. This may be accomplished by rearranging Equation 3.60 to the form,

$$
L=\frac{c_{I}}{2 \pi f} \tan ^{-1}\left[\frac{j \rho_{w} c_{w}}{\rho_{I} c_{I}}\right] .
$$

Utilizing this equation for practical measurement of the ice thickness also requires knowledge of the ice and water impedance; that is to say the density and speed of sound in the ice and water. The resonant frequency determined from the drivepoint mobility then may be used to estimate ice thickness.

\subsection{Summary}

As follow-up to the acoustic studies carried out in Barrow, AK in 2016, several experiments were conducted on Lake Superior's Keweenaw Waterway during February 2018.

The atmospheric (above-ice) and underwater (under-ice) transmission loss (TL) were measured at 11 sites which were identified in the Keweenaw Waterway. The greatest separation between sites was $890 \mathrm{~m}$. A propane cannon blast was the acoustic source for atmospheric TL and an underwater speaker with tonal output was the source for underwater TL. TL was processed for 1/3-octave bands of 20 to $8000 \mathrm{~Hz}$ for atmospheric and 250 to $8000 \mathrm{~Hz}$ for underwater TL. Comparative TL simulations were developed with parabolic equation and ray tracing algorithms.

The underwater reflection loss (RL) for the lake bottom and the ice layers were directly measured with pseudo-random maximal length sequence (MLS) signals. Considering the silty bottom type at the experiment sites, the measured bottom RL as a function of grazing angle matched closely with theoretical RL from previous studies. The measured RL ranged from 0 to $5 \mathrm{~dB}$ at frequencies less than $3000 \mathrm{~Hz}$ and 10 to $20 \mathrm{~dB}$ at frequencies 3 to $18 \mathrm{kHz}$. Large variability in RL as a function of frequency was observed in the latter frequency range. 
The TL simulations included bathymetry, sound speed profiles, and reflection loss inputs where supported by the software program. Generally, good agreement was observed between the measured and simulated TL. Due to the high wind speeds during testing, the atmospheric TL was observed to have some large variances between the measurements (6 to $24 \mathrm{~dB}$ in some cases) even when there was high signal-to-noise ratio. By comparison, the underwater TL had much lower variance (less than $3 \mathrm{~dB}$ between measurements).

In terms of broadband comparison, both the atmospheric and the underwater TL closely matched theoretical spherical spreading curves. While this does not provide information for narrowband sources or specific source-to-receiver paths, the broadband comparison does provide confidence that the measured data is valid.

The effect of ice boreholes was tested in relation to the amount of acoustic energy which passes into the water. The propane cannon source was fired near the opening of a borehole and the FRF between the microphone and the hydrophone was measured. The borehole was then allowed to freeze with the hydrophone in place underwater. Frequency response functions were compared for the open borehole case and the frozen borehole case. Little difference was observed in the FRF. Some broadband mid-to-high frequency $(>500 \mathrm{~Hz})$ attenuation was observed on the order of 2 to $8 \mathrm{~dB}$. However, the coherence for both compared measurements (open and frozen-over holes) was also low in this frequency band. Therefore, it is not possible to rule out that this attenuation may be due to measurement error. Overall, there is little benefit from freezing the hydrophones into the ice for the majority of measurement scenarios.

Compressional, shear, and Rayleigh wave speeds in the ice were measured to be 2657 $\mathrm{m} / \mathrm{s}, 1379 \mathrm{~m} / \mathrm{s}$, and $1267 \mathrm{~m} / \mathrm{s}$ respectively. Utilizing the theoretical equations for the respective wave speeds, elastic properties of the ice in the Keweenaw Waterway were determined as follows. The elastic modulus, density, and Poisson's ratio were estimated to be $5.8 \mathrm{GPa}, 915 \mathrm{~kg} / \mathrm{m} 3$, and 0.315 respectively.

A through-thickness resonance in the ice at $1445 \mathrm{~Hz}$ was predicted from the wave equation using impedance-match boundary conditions at the ice-water interface and pressure releases at the ice-air interface. The predicted resonance matched up closely in the measured drive-point mobility between the instrumented impact hammer and the vertically-oriented geophone. It was demonstrated that the ice thickness may be determined from the resonant frequency measured in the drivepoint mobility if the sound speed and density (or impedance) of the water and ice are also known. This finding is useful for practical applications when non-penetrative methods of estimating ice thickness are necessary.

The data presented in this chapter are quite possibly the first ever investigation into the acoustic propagation in the Keweenaw Waterway. The presented information regarding transmission loss, reflection loss, sound speed profiles, elastic properties of the ice, and ice wave speeds provide a baseline for further acoustic studies to be conducted in the 
Great Lakes and in the Arctic. Furthermore, given the similarities to the Arctic environment, these data provide insight to refine the developing Arctic-acoustic models. 


\section{Acoustic Vector Sensor (AVS) Tracking}

\subsection{Introduction}

The increase in open water in the Arctic for longer periods during the year is projected to invite civilian, commercial, and military activity $24,26-28,49,50,81,82$. From a military defense perspective, it is of interest to detect, identify, and track all anthropogenic sources to increase battlespace awareness. Multi-domain acoustic platforms - that is, underwater, onice, and, atmospheric sensors - are good candidates to provide source localization due to their ease of deployment and implementation in defense systems. ${ }^{83}$

It has been shown that using acoustic vector sensors (AVS) provide one approach to achieve direction of arrival (DOA) estimation and acoustic tracking ${ }^{84-88}$. Vector sensors provide a method to determine instantaneous magnitude and direction to an acoustic sound source. By combining two or more AVS, the source position can be instantaneously localized. The common types of AVS that have been researched include pressure-particle velocity $(p u)$, pressure-particle acceleration $(p a)$, pressure-pressure $(p p)$, and particle velocity-particle velocity $(u u)^{89}$. Of these types, $p p$ and $p a$ vector sensors are by far the most prevalent because they utilize common equipment such as microphones and accelerometers. ${ }^{83}$

Many other methods of source localization have been studied. The two most prevalent include acoustical holography and beamforming. Both of these methods have benefits within their respective fields.

Acoustic holography has been extensively studied for industrial source localization and directivity measurement applications. Implementation of acoustic holography requires measurement of the sound pressure on a holographic plane and the use of spatial Fourier transforms and inverse Green's functions to back-propagate and reconstruct the surface velocity at the source ${ }^{90}$. This procedure relies on measuring evanescent waves which decay exponentially with distance from the source ${ }^{90}$. Therefore, acoustic holography may only be used in the nearfield of the source being measured (giving rise to the familiar term nearfield acoustic holography, or NAH). This essentially disqualifies acoustic holography from any long-range tracking applications.

Beamforming, by comparison, is another method of localization which has been widely studied for DOA, long-range localization, and SONAR applications ${ }^{38,46,48,91-93}$. However, in order to facilitate accurate beamforming calculations, extensive numbers of transducers (even in so-called "sparse" applications) are required as well as large, expensive data systems to facilitate measurements on each transducer channel ${ }^{94-98}$. Generally speaking, beamforming applications typically rely on minimizing the width of the spatial filter response ${ }^{91}$, 


$$
\psi_{B}=\sin ^{-1} \frac{\lambda}{M d}
$$

for an array of transducers where $M$ is the number of sensors and $d$ is the transducer spacing. For beamforming in a uniform linear array (ULA), transducer spacing is required to be less than $\lambda / 2$ to prevent spatial aliasing ${ }^{99-102}$. Therefore, depending on signal frequency, array size and transducer quantity can become large in order to minimize $\psi_{B}$ and provide adequate angular resolution (Figure 4.1).

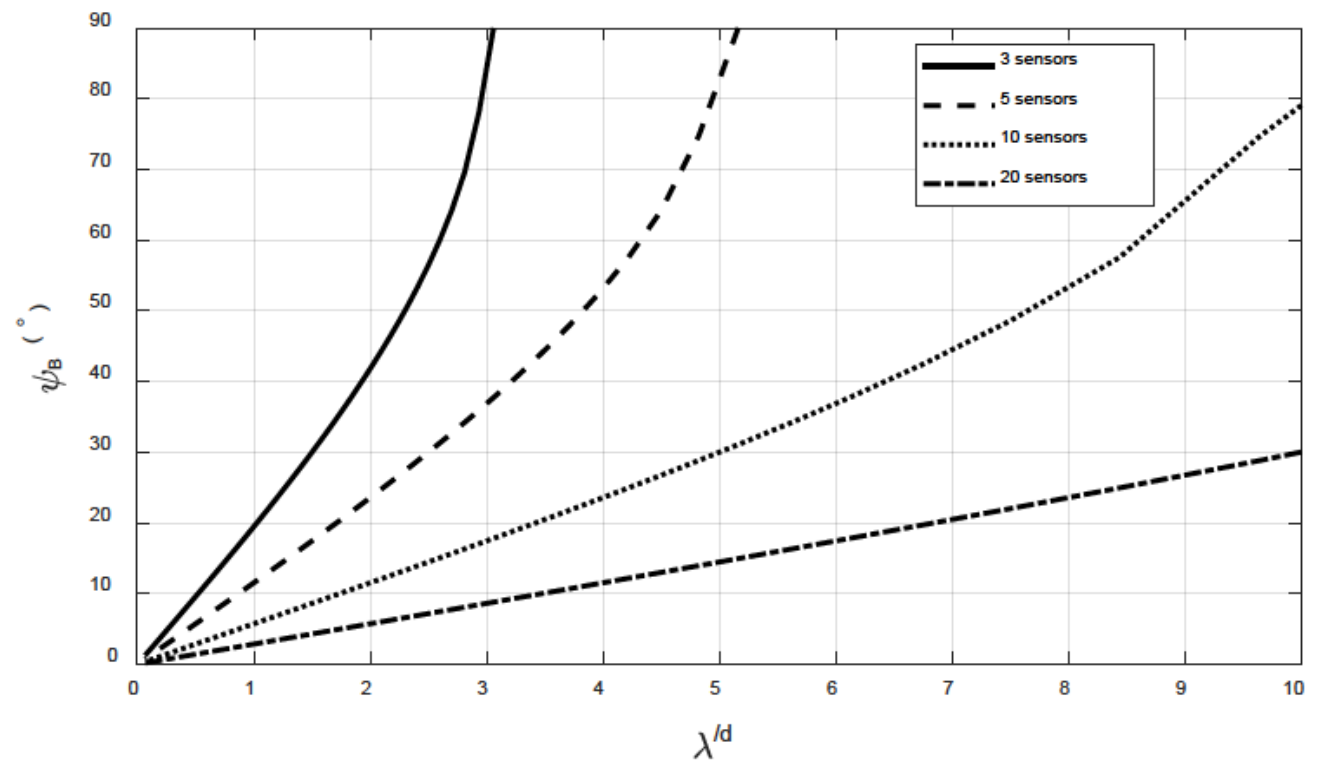

Figure 4.1 Spatial filter width for classic beamforming in ULA with various number of sensors.

To reduce the number of channels in beamforming applications, so-called virtual phased arrays have been implemented ${ }^{103}$. Virtual phased arrays utilize one stationary sensor node and one moving sensor node. When post-processed, the moving sensor provides blocks of data at multiple spatial positions. While the virtual phased array method has been shown to be effective for stationary sources, it has not been proven effective for moving sources and therefore may not be applicable in all DOA and localization applications.

The derivation of algorithms for arrays of sensors has allowed for the estimation of DOA of sources in various environments and applications. One such algorithm known as multiple signal classification (MUSIC) was developed by Schmidt in 1986 and has become one of the most widely adapted processing routines for vector sensors and array processing ${ }^{104-108}$. Accuracy of MUSIC is dependent on the number of sensors in an array, sensor spacing, signal wavelength, signal incident angle, and data blocksize. A simulation of MUSIC implemented for a theoretical ULA is shown in Figure 4.2. For the simulation, two $2000 \mathrm{~Hz}$ sources are located at $-18^{\circ}$ and $52^{\circ}$. It is observed that the algorithm is unable to reliably estimate the DOA with less than 5 sensors. More sensors 
are beneficial to reduce the width of the peak in the spatial spectrum and improve the accuracy of the DOA estimation.

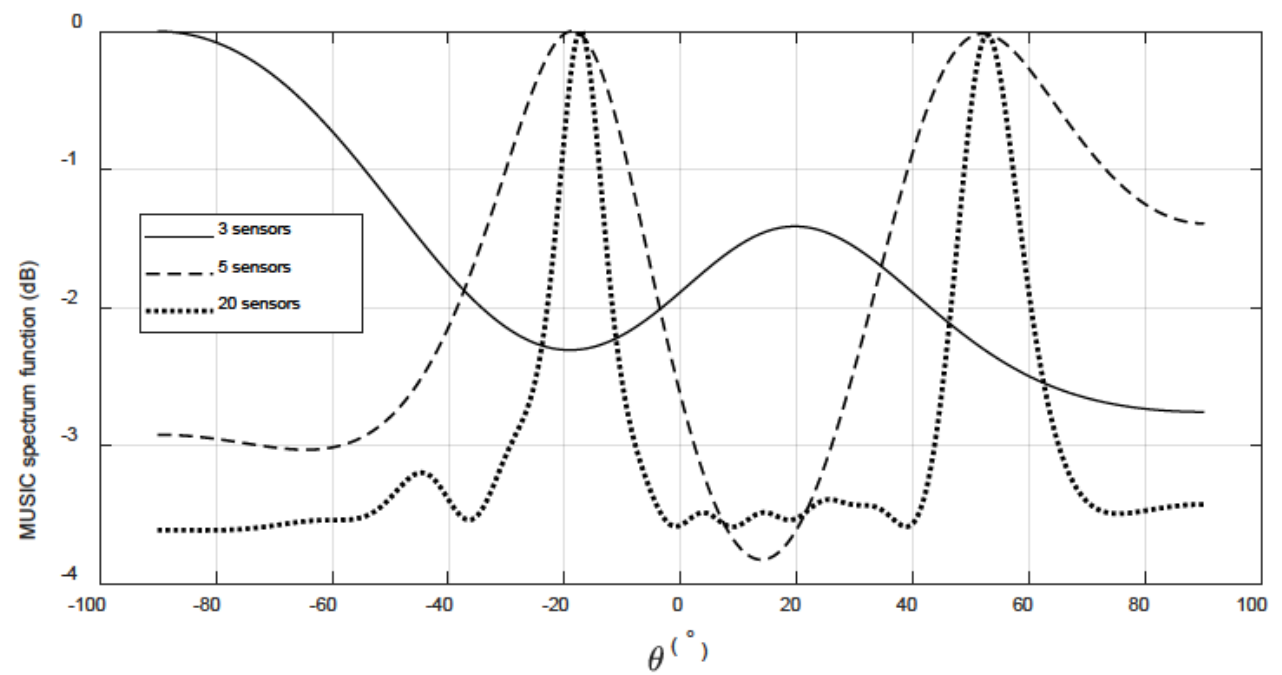

Figure 4.2 Simulated MUSIC DOA estimation for various number of sensors in ULA. Two sources of $2000 \mathrm{~Hz}$ at $-18^{\circ}$ and $52^{\circ}, \mathrm{N}=1024$ samples, $\mathrm{d}=0.0508 \mathrm{~m}$.

Generally speaking, classic arrays are more accurate with greater numbers of sensors, closer spacing of sensors, and larger blocksizes. One advantage of AVS is their capability to localize acoustic sources in the absence of great numbers of transducers and large transducer arrays. Vector sensors provide a localization option which relies on fewer sensor nodes, fewer transducers per node and overall smaller array size. In addition, these sparse sensor nodes may be stationary when tracking non-stationary sources, or the sensor nodes may be moving as long as their relative position is known. It has also been shown that AVS are capable to provide accurate DOA estimation with misaligned principal axis orientations ${ }^{109}$. Therefore, AVS benefit from reduced cost and complexity of hardware and reduced data computation in comparison to other DOA methods.

AVS have been used for many various localization applications including industrial noise sources, battlespace targets and transients, human speech sources, mobile communication, signal separation, and many others ${ }^{110-118}$. A large portion of AVS research has been conducted for underwater applications ${ }^{84,85,88,119-121}$. Studies of atmospheric AVS have also been conducted ${ }^{99,122-125}$. Zhao et al. conducted experiments to track aerial targets with underwater vectors sensors ${ }^{124}$. Shujau et al. studied using inair AVS for tracking moving speakers ${ }^{126,127}$. De Bree was the first to present implementation of the $\mu$-flown (microflown) 3D pu AVS ${ }^{122,123}$. The LMS SoundBrush utilizes microphones and $p p$-intensity methods to localize acoustic sources for industrial components, systems, and machinery ${ }^{128,129}$. Similar to the microflown, the SoundBrush is designed to be used in close proximity to the acoustic source with high SNR but is not intended for long-range tracking or DOA problems. 
The majority of AVS research studies stationary sources in a laboratory environment. An exceptional number of theoretical AVS simulations and tracking algorithms have been developed with minimal real-world experimental validation. A few studies explore DOA estimation of moving sources. In these cases, key parameters, such as source frequency, number of sources, range from source to receiver, and environmental parameters are typically known and controlled.

It is of interest to examine the localization and tracking capabilities of both underwater $(p a)$ and atmospheric $(p p)$ vector sensors for practical anthropogenic tracking applications. This chapter will examine the implementation and effectiveness of AVS when sensing non-stationary mechanical noise sources in a realistic non-laboratory environment where ambient noise is present. The DOA measurements at multiple AVS locations are used to test the acoustic source localization potential for these methods. Specifically, the feasibility of tracking on-ice vehicles with underwater AVS as well as tracking ground vehicles with atmospheric AVS are of primary interest.

\subsection{Theory}

Acoustic vector sensors largely rely on acoustic intensity theory. Acoustic intensity is most simply defined as the product of acoustic pressure and particle velocity,

$$
I=p u
$$

which represents the amount of acoustic power passing through a unit area of a medium that is perpendicular to the direction of sound propagation. In other words, the intensity vector represents the energy flow of sound.

Acoustic intensity was first discussed by Rayleigh ${ }^{130}$ in the late 1800 s and was then expanded upon greatly throughout the 1900 s and through present day ${ }^{89}$. The first patent for a sound intensity measurement device was submitted by Olson in 1931 at the Radio Corporation of America ${ }^{89}$. Olson's design relied on direct measurement of pressure and particle velocity. However, until the 1960s, practical hardware and data collection limitations caused performance issues including limited frequency range, high noise levels, and overall inaccuracy. In 1956, Schultz made the first intensity predictions based upon the finite difference approximation; allowing the particle velocity - and therefore the sound intensity - to be determined from the pressure gradient between two pressure transducers separated by a small known distance ${ }^{131}$. In the 1970s and 1980s, sound intensity research increased significantly due to the improvement of hardware and signal processing capabilities. The first commercially available $p p$ sound intensity probes were created by Metravib and Bruel \& Kajer in the early $1980 \mathrm{~s}^{89,132}$. This was followed by the microflown $p u$ probe originally developed by de Bree in $1994^{122,123}$.

Subsequently, sound intensity measurements have become increasingly useful in applications in which source localization is of critical importance, such as industrial noise control and sensor array signal processing. Therefore, special care is taken in this 
document to develop the theory of sound intensity and its application to AVS as currently is understood. Much of the theory in the following sections have been well documented by Fahy ${ }^{89}$, but it is reproduced here for completeness and understanding by the reader.

\subsubsection{Derivation of the acoustic intensity vector}

Sound is defined as the oscillation of fluid particles in response to a pressure gradient which disturbs the particles. The particle velocity,

$$
u=\frac{d x}{d t},
$$

is defined as the rate of change of position $x$ of the particles, and pressure,

$$
p=\frac{F}{\delta S}
$$

is defined as the force $F$ acting on a differential area $\delta S$ of the fluid. From classical mechanics, the work done on the particle is defined as,

$$
W=\int_{A}^{B} F d x
$$

when the particle moves from point $A$ to point $B$ in the $x$-direction. The acoustic power is the time rate of change of work (or energy), which is thereby equivalent to the product of force and particle velocity,

$$
\frac{d W}{d t}=F \frac{d x}{d t}=F u .
$$

By substituting $p \delta S$ for the force, the acoustic power per unit area may be then described by,

$$
\frac{\left(\frac{d W}{d t}\right)}{\delta S}=p u_{x} \equiv I_{x}
$$

which is the definition of sound intensity $I$ in the $x$-direction.

Particle velocity, as well as the force acting on the particle, are both vector quantities; that is to say, they possess a magnitude and a direction. Therefore, the sound intensity is also a vector quantity. The pressure and particle velocity are commonly represented by complex exponential functions (or sinusoids) which are functions of space and time such as in,

$$
p(x, t)=P e^{j \phi_{p}} e^{j \omega t}
$$


and

$$
u(x, t)=U e^{j \phi u} e^{j \omega t}
$$

where $\phi_{p}$ and $\phi_{u}$ are the spatially dependent phase and $P$ and $U$ are the amplitudes, respectively. Complex intensity can therefore be represented as,

$$
I(x, t)=P U \cos \left(\omega t+\phi_{p}\right) \cos \left(\omega t+\phi_{u}\right) .
$$

It is of interest to understand the phase relationship between $p$ and $u$. Introducing,

$$
\phi_{p u}=\phi_{p}-\phi_{u}
$$

the active intensity,

$$
I_{a}=\frac{1}{2} P U \cos \left(\phi_{p u}\right)
$$

is defined as the component of the particle velocity which is in phase with the acoustic pressure, while reactive intensity,

$$
I_{r}=\frac{1}{2} P U \sin \left(\phi_{p u}\right)
$$

is defined as the component of the particle velocity which is in quadrature with acoustic pressure. Active and reactive intensity are then the real and imaginary components of complex intensity,

$$
I=I_{a}+j I_{r}
$$

The active intensity represents the net transport of acoustic energy while the reactive component represents the oscillatory transport of energy. Time averaging of the components will yield a non-zero result for active intensity and zero for reactive intensity. ${ }^{89}$

In order to facilitate the measurement of acoustic intensity, the phase of the crosspower spectra between a pressure transducer and a particle velocity transducer $G_{u_{x} p}$ may be used to represent the active or reactive components of intensity. In most cases, active intensity is measured to allow for spectral averaging which does not result in a zero value. Therefore, the relation,

$$
I_{x}(\omega)=\operatorname{Re}\left\{G_{u_{x} p}(\omega)\right\}
$$

is useful for $p u$ intensity probes and AVS. Adapting this equation for use with a $p a$ AVS is done readily by dividing the crosspower spectra between a pressure transducer and a particle acceleration transducer by $j \omega$. 


$$
I_{x}(\omega)=\operatorname{Re}\left\{\frac{G_{a_{x} p}(\omega)}{j \omega}\right\}
$$

\subsubsection{Intensity from Finite Difference Methods}

The complication with measurement of acoustic intensity is the reliance on accurate particle velocity measurement. Acoustic particle velocity is difficult to measure directly ${ }^{89}$ (although it has become feasible thanks to the development of the microflown sensor). Particle acceleration is more readily measured with accelerometers - especially for underwater applications - giving rise to $p a$ AVS being more common than the $p u$ type. In order to measure acoustic intensity using pressure transducers only ( $p p$ method), an approximation of the particle velocity must be made exclusively from the pressure gradient between two points. That approximation is developed below.

From Euler's equation in one dimension, the pressure gradient is described mathematically by,

$$
\frac{\partial p(r, t)}{\partial x}=-\rho_{0} \frac{\partial u_{x}(r, t)}{\partial t}
$$

where $\rho_{0}$ is the density of the medium, $p$ is the complex sound pressure and $u_{x}$ is the $x$ component of the complex particle velocity. Both pressure and particle velocity are functions of space $r$ and time $t$. The finite difference approximated particle velocity is then determined by ${ }^{89}$,

$$
u_{x}(t) \cong-\frac{1}{\rho_{0}} \int_{-\infty}^{t} \frac{p_{2}(\tau)-p_{1}(\tau)}{d} d \tau
$$

and pressure is,

$$
p(t)=\frac{1}{2}\left[p_{2}(t)-p_{1}(t)\right]
$$

The averaged intensity in the $x$-direction can be written as ${ }^{89}$,

$$
\begin{gathered}
I_{x}=\lim _{T \rightarrow \infty} \frac{1}{T} \int_{0}^{T} p(r, t) u_{x}(r, t) d t \\
=-\left(\frac{1}{\rho_{0} d}\right) \lim _{T \rightarrow \infty} \frac{1}{T} \int_{0}^{T}\left[p_{1}(t) \int_{-\infty}^{t} p_{2}(\tau) d \tau\right] d t
\end{gathered}
$$

which is equivalent to ${ }^{89}$, 


$$
I_{x}(\omega)=\frac{1}{\rho_{0} \omega d} \operatorname{Im}\left(G_{p_{2} p_{1}}(\omega)\right)
$$

in the frequency domain. The above form utilizes the crosspower spectra $G_{p_{2}} p_{1}$ between two pressure transducers and is therefore useful for $p p$ intensity probes and AVS.

\subsubsection{Phase Calibration for $p p$ Methods}

Pressure-pressure methods of sound intensity measurement rely on accurate quantification of the phase delay of a sound wave as it passes between two microphone locations separated by a known distance. Inherent phase delay in the microphone, cabling, and data system can cause inaccuracies in the measurement of the sound wave's phase. Therefore, it is important to implement microphones, cabling, and data systems which have zero delay (or very small delay) between the measurements of pressure at one position relative to the other. While theoretically straightforward, this is almost always not practical for most microphone measurement systems. Therefore, it is necessary to correct for inherent phase mismatch between the microphones and measurement channels. This is possible via phase calibration techniques.

In order to phase calibrate, both microphones are inserted into a random white noise generator such as a pistonphone or a small speaker enclosure. The microphones are held at the same constant distance from the face of the white noise generator. A measurement is made with both microphones simultaneously and the crosspower spectra between the microphones is computed. The phase of the crosspower spectra between the microphones represents the phase mismatch, $\Delta \phi$, between the two measurement channels. This phase mismatch can then be removed from subsequent intensity measurements; effectively reducing measurement error.

A given crosspower spectra measurement can be represented as,

$$
G_{p_{1} p_{2}}=A_{1}(\omega) e^{-j \phi_{1}} A_{2}(\omega) e^{-j \phi_{2}} e^{j(\Delta \phi)}
$$

where $A$ represents the amplitude for the indicated microphone channel and $\phi$ represents the phase for the indicated microphone channel. The latter factor of Equation 4.22 represents the inherent phase delay in the measurement system between the two microphone channels. By subtracting $e^{j(\Delta \phi)}$ from the measured crosspower spectra, the measurement is corrected for inherent phase delays.

An example of the relative phase mismatch and the acoustic measurement hardware used in this report may be seen in Appendix A.4.1. 


\subsubsection{Underwater pa Vector Sensors}

The underwater AVS considered in this chapter measure underwater sound pressure, particle acceleration in the local $x, y$, and $z$-axes, the gravitational field relative to the $x, y$, and $z$-axes, and the magnetic heading ${ }^{133}$. The gravity vector,

$$
g=[\operatorname{Pr} X \operatorname{Pr} Y \operatorname{Pr} Z]_{(1 \times 3),}
$$

contains the pitch-roll information about each axis and the magnetic field vector,

$$
m=[H d X H d Y H d Z]_{(1 \times 3),}
$$

contains the heading information with components in each local coordinate direction. The quantities $\operatorname{Pr} X, \operatorname{Pr} Y$, and $\operatorname{Pr} Z$ represent the $x, y$, and $z$ components of the gravitational vector, respectively, and the quantities $H d X, H d Y$, and $H d Z$ represent the $x, y$, and $z$ components of the magnetic field vector, respectively. These quantities are read directly from the vector sensor via serial communications. ${ }^{83}$

The $3 \times 3$ transformation matrix,

$$
Q_{\text {fixed }}^{T}=\left[\begin{array}{l}
n_{a c} \\
w_{a c} \\
u_{a c}
\end{array}\right](3 \times 3),
$$

is used to rotate the gravity and magnetic field vectors from local coordinates to global magnetic north, magnetic west, and up coordinates. In the formulation of the transformation matrix, $n_{a c}, w_{a c}$, and $u_{a c}$ are the magnetic north, west, and up rotation vectors, respectively. The rotation vectors are determined using the gravity and magnetic field vectors by,

$$
\begin{aligned}
& n_{a c}=\frac{(g \times m) \times g}{|(g \times m) \times g|}(1 \times 3), \\
& w_{a c}=-\frac{g \times m}{|g \times m|}(1 \times 3), \text { and } \\
& u_{a c}=-\frac{g}{|g|}(1 \times 3),
\end{aligned}
$$

where the $\times$ represents the cross-product operator. The acoustic intensity, $I$, is determined in the local $x, y$, and $z$ directions with respect to the vector sensor by

$$
I_{x}=\operatorname{Re}\left[\frac{G_{a_{x} p}}{j \omega}\right], I_{y}=\operatorname{Re}\left[\frac{G_{a_{y} p}}{j \omega}\right], I_{z}=\operatorname{Re}\left[\frac{G_{a_{z} p}}{j \omega}\right],
$$


where $G_{a_{i} p}$ is the crosspower spectrum between the particle acceleration in the $i^{t h}$ direction and the sound pressure, $j$ is the imaginary unit, $\sqrt{-1}$, and $\omega$ is the radial frequency in $\mathrm{rad} / \mathrm{s} .{ }^{83}$

The local $x, y$, and $z$ components of intensity are then transformed to global coordinates magnetic north, west, and up by multiplying the intensity in local coordinates by the transformation matrix, Equation 4.25,

$$
\left[\begin{array}{c}
I_{\text {North }} \\
I_{\text {West }} \\
I_{U p}
\end{array}\right]=Q_{\text {fixed }}^{T}\left[\begin{array}{c}
I_{x} \\
I_{y} \\
I_{z}
\end{array}\right] .
$$

The intensity magnitude, azimuth angle, and elevation angle are computed as,

$$
\begin{gathered}
|I|=\sqrt{I_{\text {North }}^{2}+I_{\text {West }}^{2}+I_{U p}^{2}} \\
\theta_{V S}=\tan ^{-1}\left(\frac{I_{\text {West }}}{I_{\text {North }}}\right), \text { and } \\
\Phi_{V S}=\tan ^{-1}\left(\frac{I_{U p}}{\sqrt{I_{\text {North }}^{2}+I_{\text {West }}^{2}}}\right) \text {, respectively. }{ }^{83}
\end{gathered}
$$

The azimuth angle and the elevation angle represent the direction of arrival of the acoustic energy from the sound source at the measurement location. The positive azimuth angle is defined as degrees from true east towards true north. For example, an azimuth angle of +90 represents a DOA from due north and an azimuth angle of -90 degrees represents a DOA from due south. The positive elevation angle is defined as degrees from horizontal towards the earth. For example, an elevation angle of +90 degrees represents a DOA vertically downward (toward the sea floor) and an elevation angle of -90 degrees is pointing vertically up. ${ }^{83}$

The range between the measurement location and the source are computed as,

$$
R_{V S}=\left|\frac{D_{V S}}{\tan \left(\Phi_{V S}\right)}\right|
$$

where $D_{V S}$ is the vector sensor depth, which was $5 \mathrm{~m}$ for all experiments. ${ }^{83}$

\subsubsection{Atmospheric pp Vector Sensors}

Each $p p$ vector sensor is comprised of 3 microphones which measure the acoustic pressure as a function of time. The microphones are arranged in a configuration such that two principle axes are formed (Figure 4.3). The line intersecting microphone 1 and 2 create the $x$-axis, and the line intersecting microphone 1 and 3 create the $y$-axis; 
orthogonal to the $x$-axis. The separation distance $d$ between the microphones allows for the relative phase between microphones on a given axis to be accounted for when measuring in an acoustic environment.

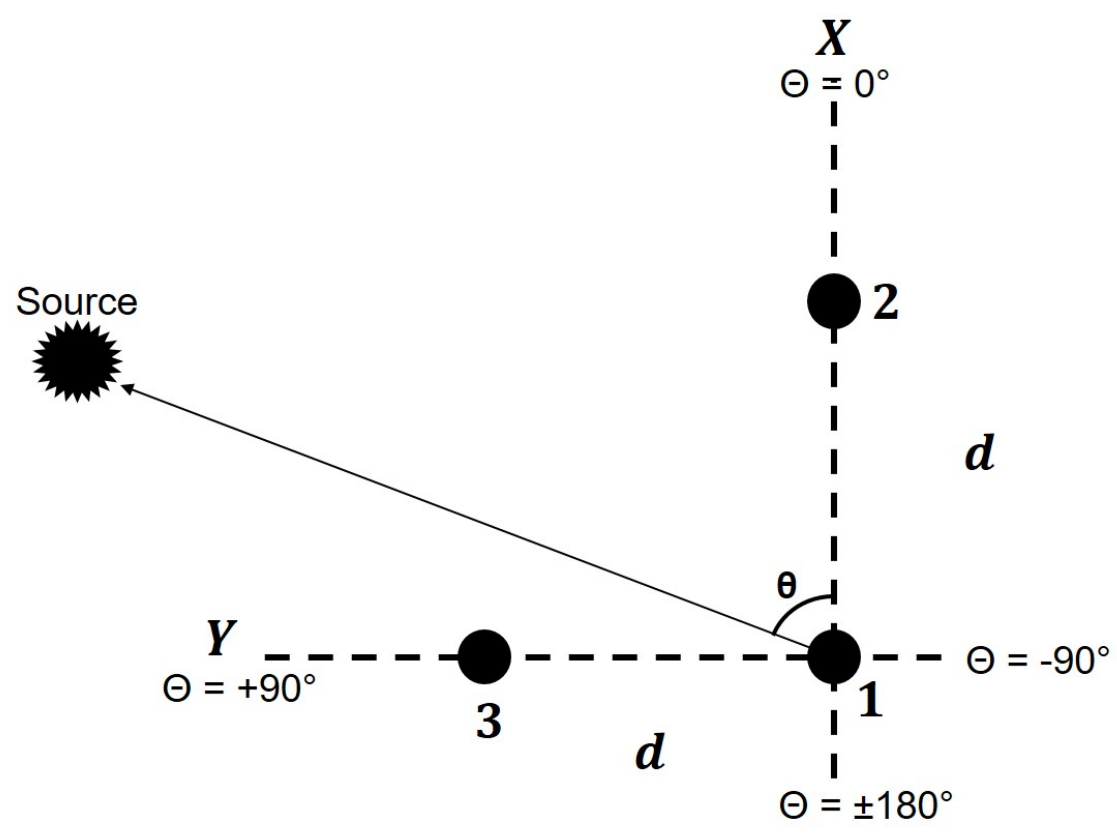

Figure 4.3 Microphone configuration and coordinate system for atmospheric $(p p)$ vector sensor.

Utilizing the $p p$ method, sound intensity between any two microphones $m$ and $n$ can be computed,

$$
I_{m n}(\omega)=\frac{1}{\rho_{0} \omega d_{m n}} \operatorname{Im}\left[G_{p_{m} p_{n}}(\omega)\right]
$$

where $G_{p_{m} p_{n}}$ is the crosspower spectrum between the microphones, $\rho_{0}$ is the air density in $\mathrm{kg} / \mathrm{m}^{3}$, and $\omega$ is the radial frequency in $\mathrm{rad} / \mathrm{s}$. Effectively, the sound intensity along the $x$-axis and $y$-axis are determined by $I_{12}$ and $I_{13}$, respectively. Alignment of the $x$-axis with magnetic north and the $y$-axis with magnetic west yields intensity magnitude,

$$
|I|=\sqrt{I_{\text {North }}^{2}+I_{\text {West }}^{2}}
$$

and azimuth,

$$
\theta_{V S}=\tan ^{-1}\left(\frac{I_{\text {West }}}{I_{\text {North }}}\right)
$$

which are similar in form to Equations 4.31 and 4.32. Computation of an elevation angle, in this case, would require a fourth microphone measurement at a different $z$-position, 
which is not included. The azimuth angle represents the estimated direction of arrival from the sound source and is measured counterclockwise from the positive $x$-axis.

Wajid et al. provided DOA estimation equation and performance analysis of various 2D pp AVS designs ${ }^{99}$. In their formulations, the intensity in a given principal axis may be determined by averaging the component projections of intensity between sensors onto the principal axis. For the type of $p p$ AVS considered here, the components in the $x$ and $y$ directions would respectively be,

$$
I_{x}=\frac{1}{3 \rho d}\left[\int_{0}^{\infty} \frac{\operatorname{Im}\left(G_{13}\right)}{\omega} d \omega+\cos \left(45^{\circ}\right) \int_{0}^{\infty} \frac{\operatorname{Im}\left(G_{23}\right)}{\omega} d \omega\right]
$$

and

$$
I_{y}=\frac{1}{3 \rho d}\left[\int_{0}^{\infty} \frac{\operatorname{Im}\left(G_{12}\right)}{\omega} d \omega+\sin \left(45^{\circ}\right) \int_{0}^{\infty} \frac{\operatorname{Im}\left(G_{23}\right)}{\omega} d \omega\right]
$$

where

$$
\overline{I_{m n}}=\int_{0}^{\infty} I_{m n}(\omega) d \omega
$$

is the spectral averaged intensity between two sensors. The estimated DOA may then be determined by,

$$
\theta_{V S}=\tan ^{-1}\left(\frac{I_{x}}{I_{y}}\right)
$$

This integral formulation for DOA estimation is essentially similar to the method applied in the previous sections. That is to say, the arctangent of intensity ratio is used to determine an approximation of angle. However, the integration with respect to frequency accounts for all spectral energy within the frequency band. This is sufficient for cases in which sources emit high SNR frequency content, but may result in errors when multitonal sources are analyzed.

\subsubsection{Moving Standard Deviation (MSD) Processing}

The acoustic intensity is a spectral measurement and as such the DOA is also a spectral measurement (Equation 4.35). It is important to determine the frequency range over which these measurements should be analyzed based on the spectral energy emitted from 
the acoustic source. The mean $(\bar{\theta})$ and standard deviation $(\sigma(\theta))$ of these measurements may be computed over this frequency range at a selected time interval to determine the DOA from the acoustic source as a function of time.

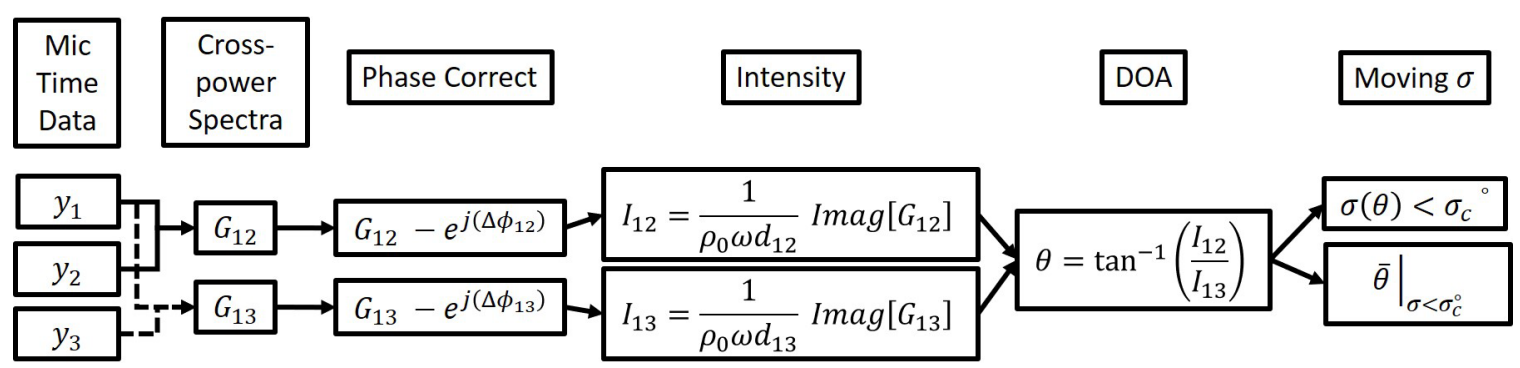

Figure 4.4 Data processing flow for atmospheric $p p$ AVS.

By Shannon's sampling theorem, the time interval $(T)$ is specified by the sample rate $\left(F_{S}\right)$ and blocksize $(N)$ such that,

$$
\begin{gathered}
F_{s}=\frac{1}{\Delta t}, \\
T=N \Delta t,
\end{gathered}
$$

where $\Delta t$ is the time between acoustic samples, and

$$
\Delta f=\frac{1}{T}
$$

is the frequency resolution of the spectral measurements.

At each time interval of interest, the DOA may be computed at each AVS site. The processing flow for each time interval is shown in Figure 4.4. Iterative computation of this process will result in time domain DOA. The time data block measured from microphone 1 and 2 are used to compute the crosspower spectra $G_{12}$. Likewise, the time data from microphone 1 and 3 are used to compute the crosspower spectra $G_{13}$. The crosspower spectra are then phase corrected to account for inherent phase delay in the measurement system by subtracting $e^{j(\Delta \phi)}$. The phase corrected crosspower spectra are then implemented in Equation 4.35 to determine the sound intensity spectra for each principal axis. The direction of arrival spectra is then computed with Equation 4.37.

To determine a single value for the DOA at a given time interval, the DOA spectra must be averaged across specified frequencies. As Fahy shows ${ }^{89}$, the microphone spacing of the AVS determines the valid frequency range across which the AVS data has small error. However, averaging across this entire frequency range may still not provide an accurate DOA estimate if the source being considered does not have acoustic energy at all frequencies within the specified range. If the SNR is low in a portion of the valid frequency range, large errors may still be present. 


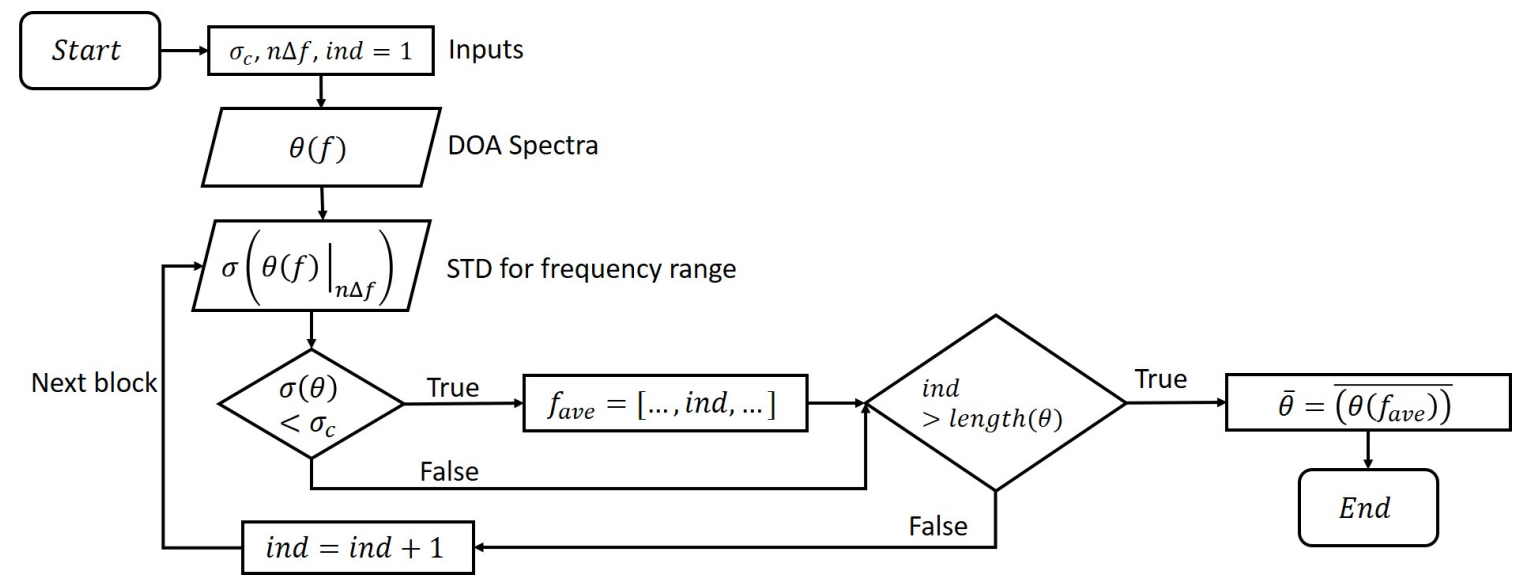

Figure 4.5 Moving Standard Deviation (MSD) processing routine block diagram.

It is therefore advantageous to carry out a processing routine to check for the DOA variation within the valid frequency range and only average across frequencies which have low variation. This is accomplished with a moving standard deviation (MSD) routine (Figure 4.5). A data block consisting of the first $n$ frequency lines (that is, $n \Delta f$ within the valid frequency range) of the DOA spectra is selected. The standard deviation of the DOA spectra for the block is computed. This represents the standard deviation at the center frequency of the block (index $\frac{n}{2}$ ). If the standard deviation within the frequency block is less than some pre-selected cutoff standard deviation $\sigma_{c}$, then the freqnency range index is stored for later. The frequency start and end indices for the block are increased by one. Therefore, the start and end indices for the next block would be 2 and $n+1$ respectively. The process is iterated until the standard deviation is computed across the entire valid frequency range using blocks of $n$ frequency lines. The averaged DOA $(\bar{\theta})$ may then be determined by averaging across frequencies which have a standard deviation which is below the cutoff standard deviation. This process has the potential to increase the accuracy of DOA estimation.

The assignment of $\sigma_{c}, n \Delta f$, and $N$, effect the resulting DOA estimate. Therefore, careful optimization of these parameters within the MSD routine is necessary for accurate results.

\subsubsection{GPS Ground Truth DOA and Error}

To compare the computed vector sensor DOA to the actual GPS position of the acoustic source, the azimuth angles, elevation angles, and ranges between the experiment sites GPS coordinates and the target vehicle GPS coordinates are computed. The difference in latitude,

$$
\triangle L A T=L A T_{\text {Source }}-L A T_{\text {Site }}
$$

and longitude, 


$$
\Delta L O N=L O N_{\text {Source }}-L O N_{\text {Site }},
$$

are determined between the experiment site and the acoustic source at each interval in time. The ground truth GPS azimuth angle with respect to true east,

$$
\theta_{G P S}=\tan ^{-1}\left(\frac{\Delta L A T}{\Delta L O N}\right),
$$

are then computed with the inverse tangent of the difference in latitude divided by the difference in longitude. The ground truth GPS range between the measurement site and the target vehicle are computed with the distance formula,

$$
R_{G P S}=\sqrt{(\triangle L A T)^{2}+(\triangle L O N)^{2}},
$$

where the difference in latitude and longitude are converted from degrees to meters. In the case of underwater AVS, the ground truth GPS elevation angle,

$$
\Phi_{G P S}=\tan ^{-1}\left(\frac{D_{V S}}{R_{G P S}}\right),
$$

is computed with the inverse tangent of the sensor depth divided by the ground truth GPS range. ${ }^{83}$

To assess the accuracy of the AVS, the error may be computed as

$$
\begin{gathered}
E(\theta)=\left|\theta_{G P S}-\theta_{V S}\right|, \\
E(\Phi)=\left|\Phi_{G P S}-\Phi_{V S}\right|,
\end{gathered}
$$

and

$$
E(R)=\left|R_{G P S}-R_{V S}\right|,
$$

which is simply the absolute value of the difference between the ground truth DOA and the DOA measured by the AVS.

\subsubsection{Analytical DOA}

The experimentally determined DOA may be analyzed in comparison to the ground truth DOA using error functions as explained in the previous section. While this provides a metric for assessing the performance of the AVS, it does not necessarily explain the physical behavior of the vector sensor. Analytical models of the acoustic propagation between source and receiver positions aid in understanding the physical meaning of AVS behavior. 


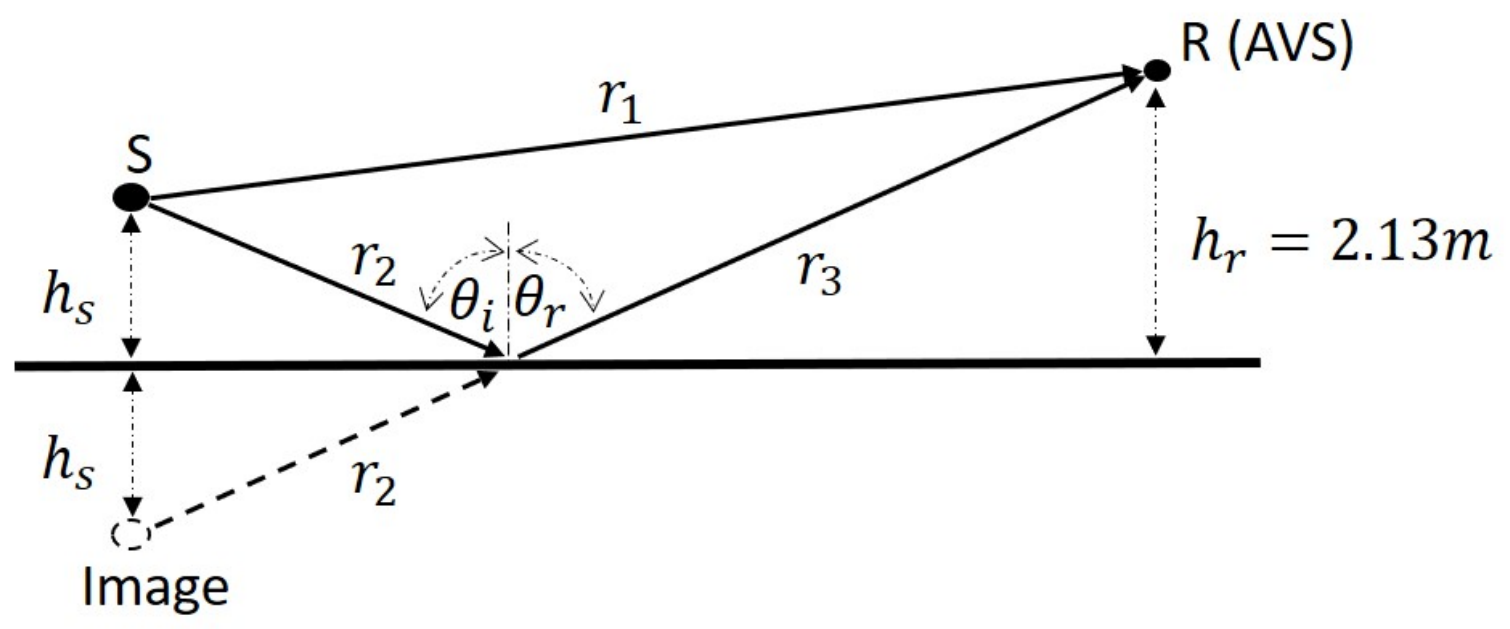

Figure 4.6 Image Source-Path-Receiver diagram for atmospheric AVS

It is known that AVS are sensitive to both the direct and reflected paths from acoustic emitters when the AVS is not in a diffuse field ${ }^{99,127,134}$. An analytical model of the atmospheric AVS in a free filed (which is the case for applications herein) may be developed using the method of images ${ }^{38,63}$. A source at a height $h_{s}$ above the ground and of a given frequency $\omega$ radiates sound to the receiver at a height $h_{r}$ via a direct path $r_{1}$ and a reflected path $r_{2}+r_{3}$ (Figure 4.6). The reflected path impinges on the ground plane at an incident angle $\theta_{i}$ and is reflected at angle $\theta_{r}$. For specular reflection $\theta_{i}=\theta_{r}$, however, this does not necessarily hold true for ground conditions with non-negligible impedance. Using the method of images, the reflected path can be represented as a second source in a position mirrored across the ground plane. The signal at the receiver location may be determined as a superposition of the direct and reflected waves with the equation,

$$
P(x, t)=\frac{j \omega \rho_{0} Q}{4 \pi}\left[\frac{e^{-j k r_{1}}}{r_{1}}+\frac{e^{-j k\left(r_{2}+r_{3}\right)}}{r_{2}+r_{3}}\right] e^{j \omega t}
$$

The source amplitude is effectively a function of frequency, air density $\left(\rho_{0}\right)$, and volume velocity $(Q)$ and the received amplitude is inversely proportional to the distance traveled by the sound wave. The amplitude and phase of the analytical signals at each AVS microphone are shifted due to the relative spacing between the sensors; allowing for DOA processing similar to the experimental data.

The direct and reflected path distances may be determined readily if the source height, receiver height, and horizontal distance between the source and receiver are known. For the case of the vector sensor experiments considered here, the source height was fixed at $2.13 \mathrm{~m}$ and the horizontal distance is known via relative GPS coordinates of the source and receiver (Equation 4.48). Assuming a source height and source frequency, analytical signals for each AVS microphone may then be synthesized. The analytical signals are 
then processed in the exact same manner as experimental data to determine analytical DOA and analytical error.

\subsubsection{Multi-Sensor Localization}

By combining the DOA from multiple sites an acoustic source can be localized as a function of time. Due to statistical variation on the computed DOA at each site, it is not possible to localize directly using the mean value alone (even if the MSD methods discussed in 4.2.5 are implemented). Therefore, the standard deviation of the DOA can be added or subtracted from the mean DOA creating a beam of localization area corresponding to the instant in time and the site of interest. ${ }^{83}$

Extending this to two sites allows the two overlapping beams to create a polygonal area which represents the localized area predicting where the acoustic source is located. The localized area can be computed as,

$$
A_{\text {poly }}=\frac{1}{2} \sum_{i=0}^{n-1} x_{i} y_{i+1}-x_{i+1} y_{i}
$$

where $\left(x_{i}, y_{i}\right)$ are the vertices of the $n$-sided polygon and $\left(x_{n}, y_{n}\right)=\left(x_{0}, y_{0}\right) .{ }^{83}$

In some cases, specifically for underwater AVS, the elevation angle may also be used to aid in localization and reduce the predicted localization area. The elevation effectively represents the range to the acoustic source from the measurement sites as shown by Equation 4.34. For an underwater vector sensor tracking a source on the ice, this simplification is valid because the sound source is taken to be at a constant height ( $z$ position) during the entire measurement period and the vector sensor is at a constant depth. The computed range, $R_{V S}$, represents the radius of a circle centered at the measurement location. If this circle intersects the localization area determined with the azimuth angle, the localization area may then be reduced by the bound of the circle. In this case, the arc of the circle creates one side of the localization area. For small azimuth angles, the localization area created with the arc of the circle may still be assumed to be polygonal because the arc is very close to a straight line. The error in this assumption is proportional to the area of the segment of the circle,

$$
A_{\text {segment }}=\frac{R^{2}}{2}(\theta-\sin (\theta))
$$

which is not included in the localization area. This error becomes large as $\theta$ becomes large for a given $R .^{83}$

\subsection{Implementation}

This section will discuss the implementation of underwater $(p a)$ and atmospheric ( $p p)$ acoustic vector sensors for direction of arrival and localization measurements in practical 
acoustic environments. Much of the test setup for the underwater AVS is discussed previously in chapter 2 and will not be replicated in this section.

\subsubsection{Underwater pa Vector Sensors}

During testing in Barrow, AK (refer to chapter 2), underwater $p a$ vector sensors (Meggitt VS-209, Figure 4.7) were placed at the central location of sites 1, 2, and 3 (for site layout, refer to Figure 2.1) and were used to measure the underwater SPL and particle acceleration during experiments. Experiments were carried out to localize a stationary source and a moving source.
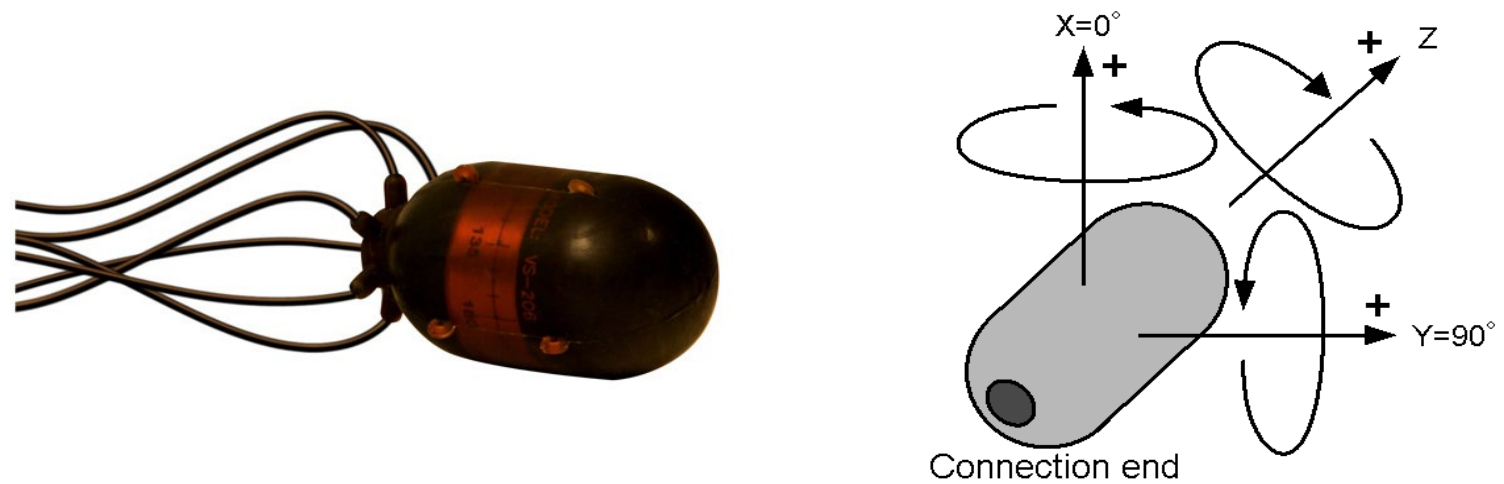

Figure 4.7 Vector sensor (Meggitt VS-209) with local Cartesian axes identified. Pitch, Roll, and Heading are defined as rotation about the $\mathrm{Y}, \mathrm{Z}$, and $\mathrm{X}$-axes respectfully. ${ }^{133}$

\subsubsection{Stationary Source Experiment}

To test the $p a$ vector sensors in a stationary source environment, an underwater sound source (Lubell Labs Model LL916) was deployed through a drilled hole in the ice at site 4. The sound source played broadband pings in the frequency ranges of 40-2500 Hz and 4000$25000 \mathrm{~Hz}$. The pings were 0.01 seconds in duration. The vector-sensor data were then post processed to determine the average acoustic intensity magnitude and direction to the sound source over the 120 -second data collect. ${ }^{83}$

\subsubsection{Non-Stationary Source Experiment}

To test the tracking capabilities of the underwater $p a$ vector sensors with respect to a moving on-ice source, a snowmobile (Figure 4.8) was driven around the perimeter of sites 2, 3, and 6 (for site layout, refer to Figure 2.1) while acoustic measurements were taken at the sites. The driver of the snowmobile carried a handheld GPS which recorded position and coordinated universal time (UTC) during their ride around the course (Figure 4.9). While the snowmobile was traveling around the perimeter of the sites, the microphone, 
geophone, hydrophone, and vector sensor data were simultaneously collected at each site. The vector sensor data were then post processed to determine the acoustic intensity magnitude and direction to the snowmobile at each instant in time. For validation of the vector sensor fidelity, the calculated intensity data were then compared to the directly acquired GPS position in relation to the test sites. ${ }^{83}$

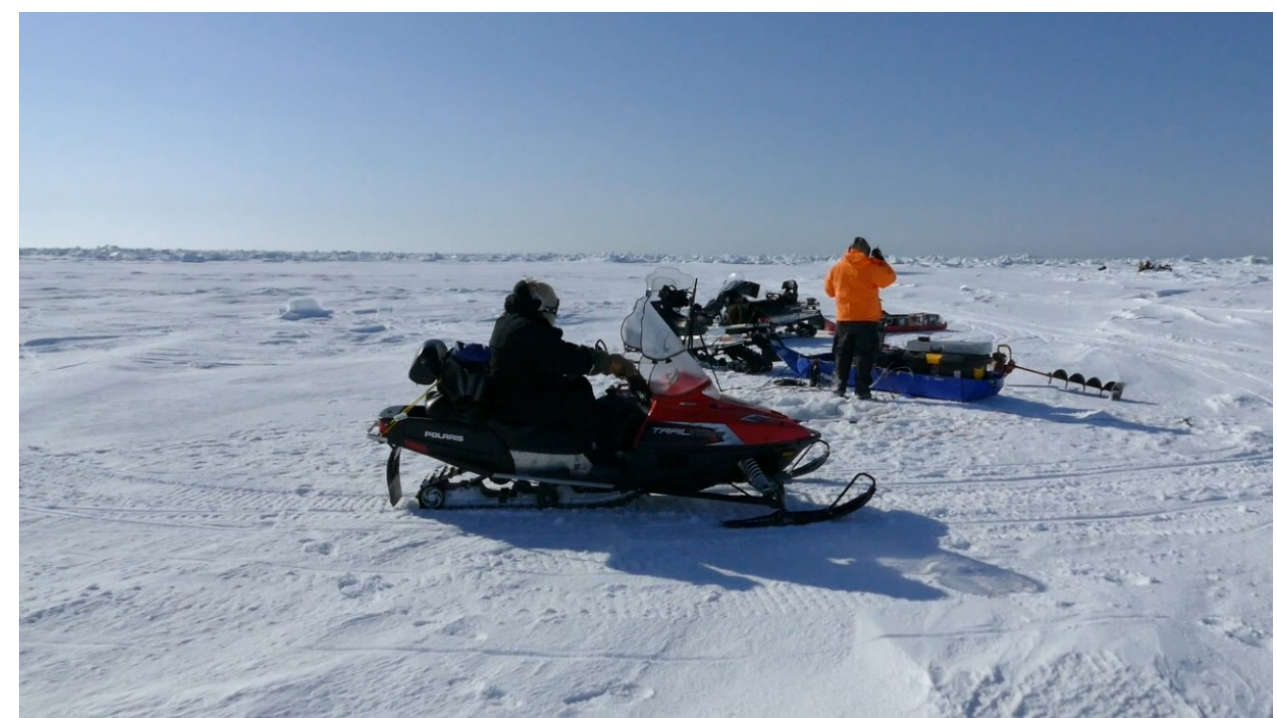

Figure 4.8 Snowmobile used for underwater vector sensor tracking experiment shown at site 6 near the start of the snowmobile track.

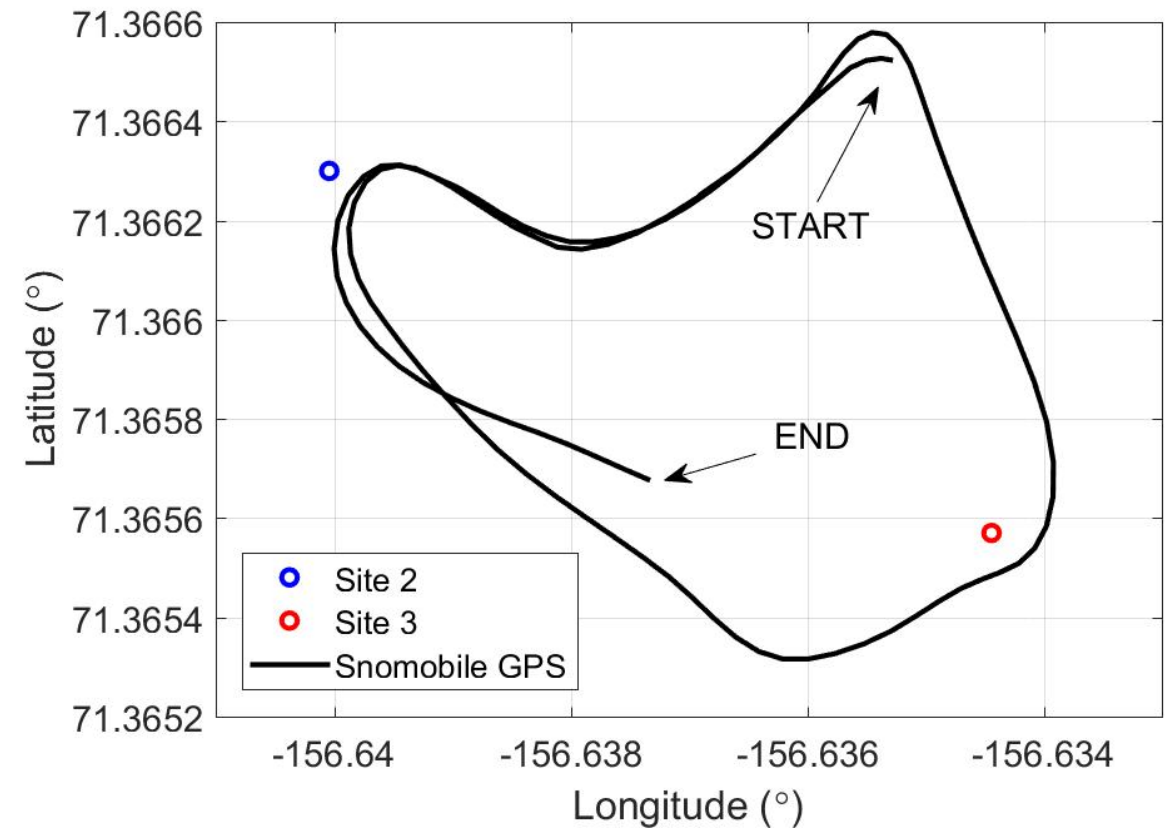

Figure 4.9 Snowmobile position measured over 120 seconds by handheld GPS onboard the snowmobile. Underwater vector sensor measurements were made at sites 2 and 3 . GPS coordinates of sites 2 and 3 are: N 71.36630, W 156.64004 and N 71.36557, W 156.63444 respectively. 


\subsubsection{Atmospheric pp Vector Sensors}

To determine the effectiveness of $p p$ vector sensors, experiments were carried out on a test range (Figure 4.10) at the Keweenaw Research Center (KRC) in Calumet, MI on September 18, 2018. The test range was approximately $550 \mathrm{~m}$ long by $95 \mathrm{~m}$ wide and was a mixture of grass-covered and dirt-covered area. The test area was surrounded by lightly-wooded area. The KRC is used extensively for military vehicle testing such as tanks, troop transports, and other combat vehicles. Test tracks adjacent to the test range were in use by such vehicles during AVS testing. Additionally, the KRC is directly adjacent to the Houghton County Memorial Airport. During testing several small propeller and jet aircraft were taking off and landing. The combination of military vehicle and small aircraft activity created significant intermittent background noise. During AVS testing, efforts were made to make measurements when aircraft and military ground vehicles were either not active or as far away as possible. However, this was not always possible and both contaminating noise sources created significant low-frequency background noise even when far away.

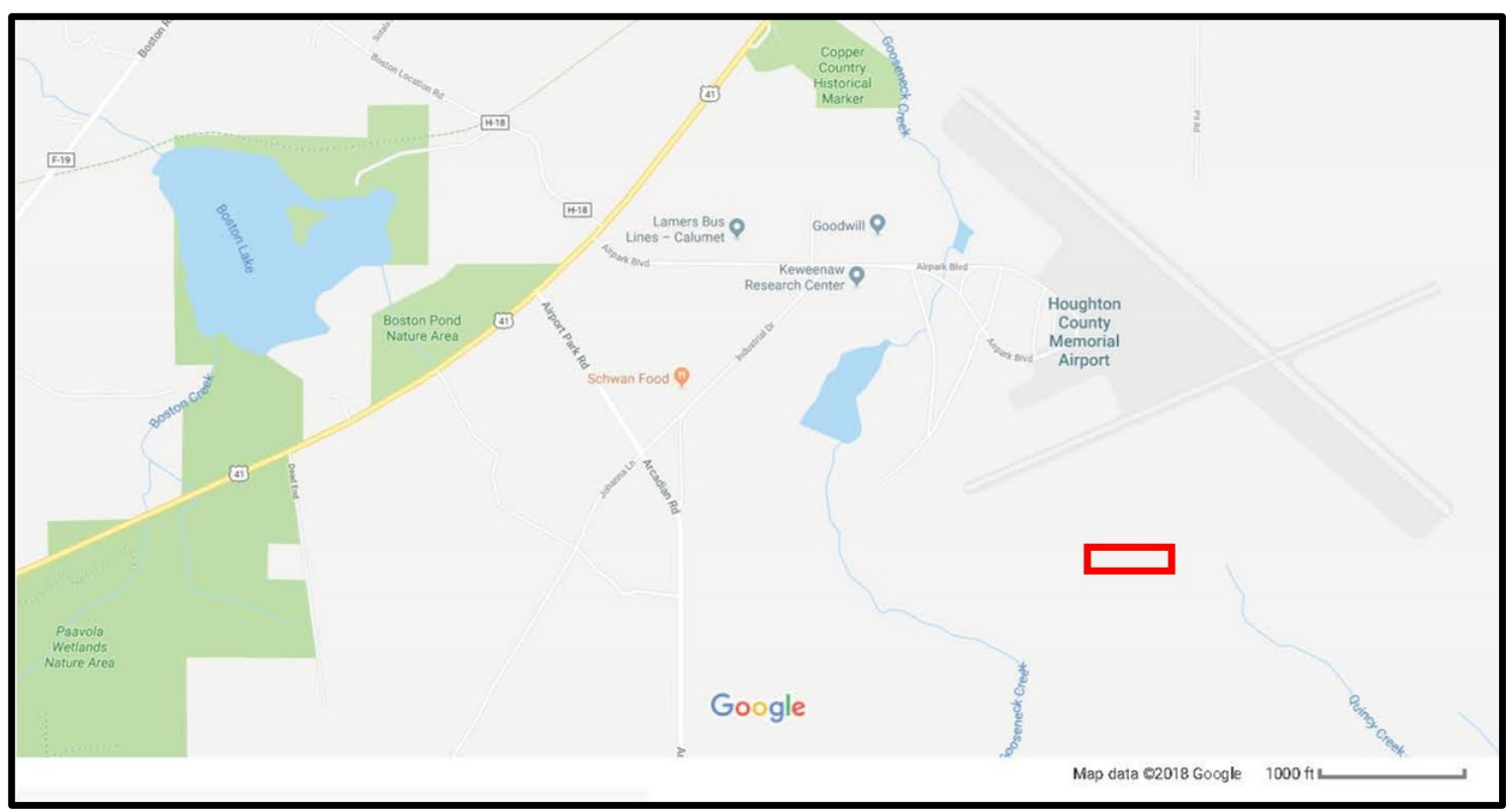

Figure 4.10 Map of approximate vector sensor test range location (red box) at KRC.

Two vector sensor sites were identified on the test range. Site 100 was located at GPS coordinates N 47.1578220, W 88.4843412 and site 200 was located at GPS coordinates N 47.1577581, W 88.4852868. Separation distance between the AVS sites was $72 \mathrm{~m}$. One of the AVS sites is shown in Figure 4.11.

The vector sensors consisted of three microphones each (PCB 130D21). The microphones were vertically held in a 3D-printed fixture which aligned their measurement points along two principal axes (Figure 4.11d). Microphone spacing along the axes was $5.08 \mathrm{~cm}(2 \mathrm{in})$. The measurement points formed a plane which was parallel to the ground plane during testing. The 3D-printed fixture was mounted to a tripod with 
a piece of threaded rod and the microphones were fitted with a foam windscreen to reduce environmental wind noise. The principal axes of the vector sensors were aligned to magnetic north and west using a magnetic compass during test setup (Figure 4.11b). The height of the vector sensor microphones at each site was $2.13 \mathrm{~m}$ above ground level.

At each vector sensor site, a HEAD Acoustics SQobold data system was used to collect the AVS microphone data (Figure 4.11c). The SQobold systems were also equipped with GPS antennas to collect position of the AVS sites and timing of the AVS data. The acoustic data was sampled at $51200 \mathrm{~Hz}$.

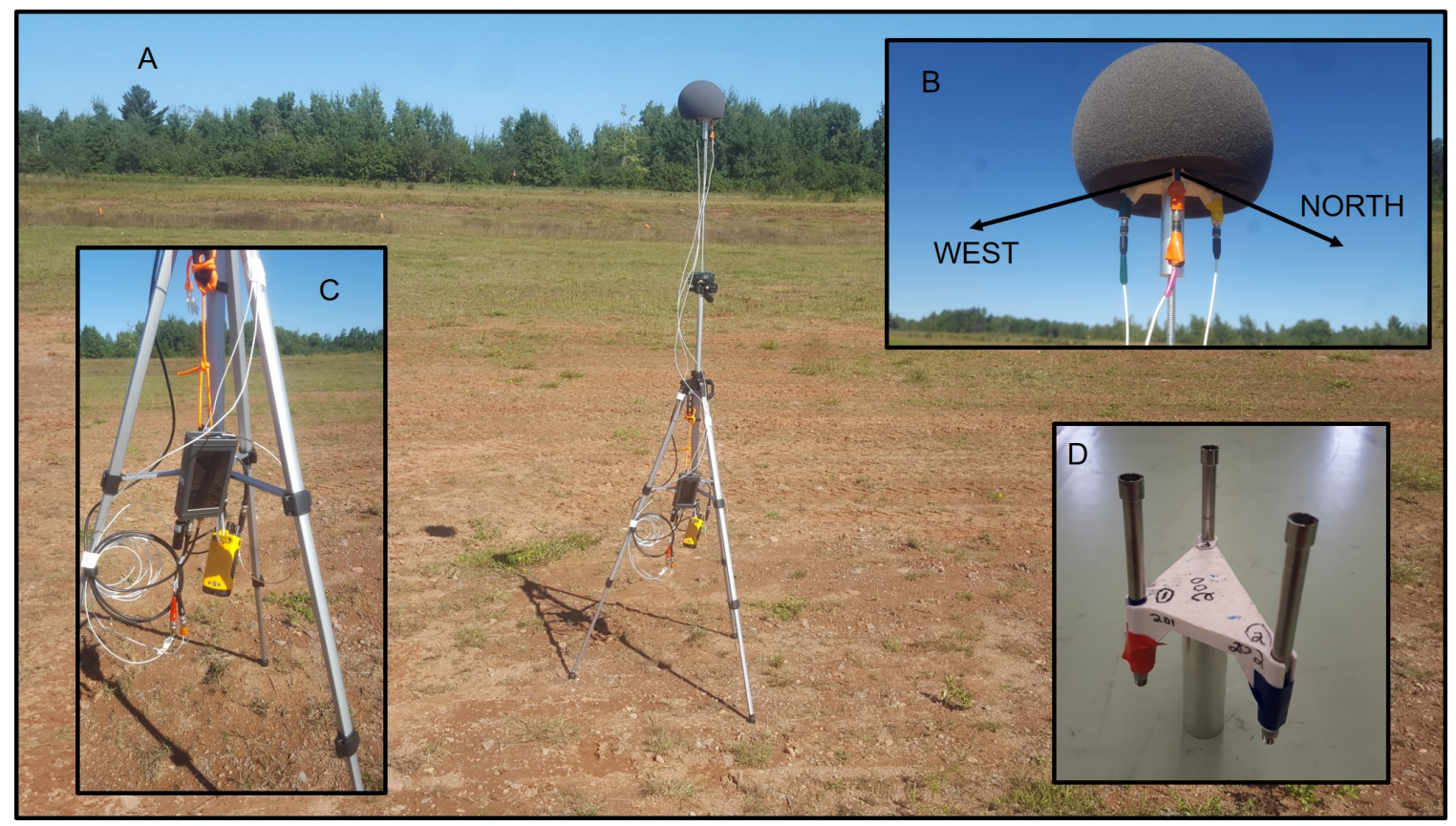

Figure 4.11 (A) pp vector sensor setup at KRC test site. (B) AVS orientation with respect to north and west. (C) SQobold data system and Motorola two way-radio for triggering data collects. (D) Microphones in the 3D printed fixture without the windscreen.

A Polaris Ranger utility task vehicle (UTV) was driven around the AVS sites in various routes and maneuvers on the test range (Figure 4.12). The UTV was equipped with a Racelogic VBOX 3i dual antenna GPS and inertial measurement unit (IMU) which was capable of real-time kinematic (RTK) computations ${ }^{135}$. The VBOX acquired signals from two antennas. The first acquired GPS and GLONASS signals and the second acquired differential correction from the Michigan Department of Transportation (MDOT) continuously operating reference stations (CORS). These signals combined with computation from the IMU allowed for position measurement of the UTV to within $\pm 2 \mathrm{~cm}$ sampled at $100 \mathrm{~Hz}^{135}$, thereby providing a ground truth position to compare with the AVS estimations. The VBOX/IMU also provided vehicle heading and speed information. 


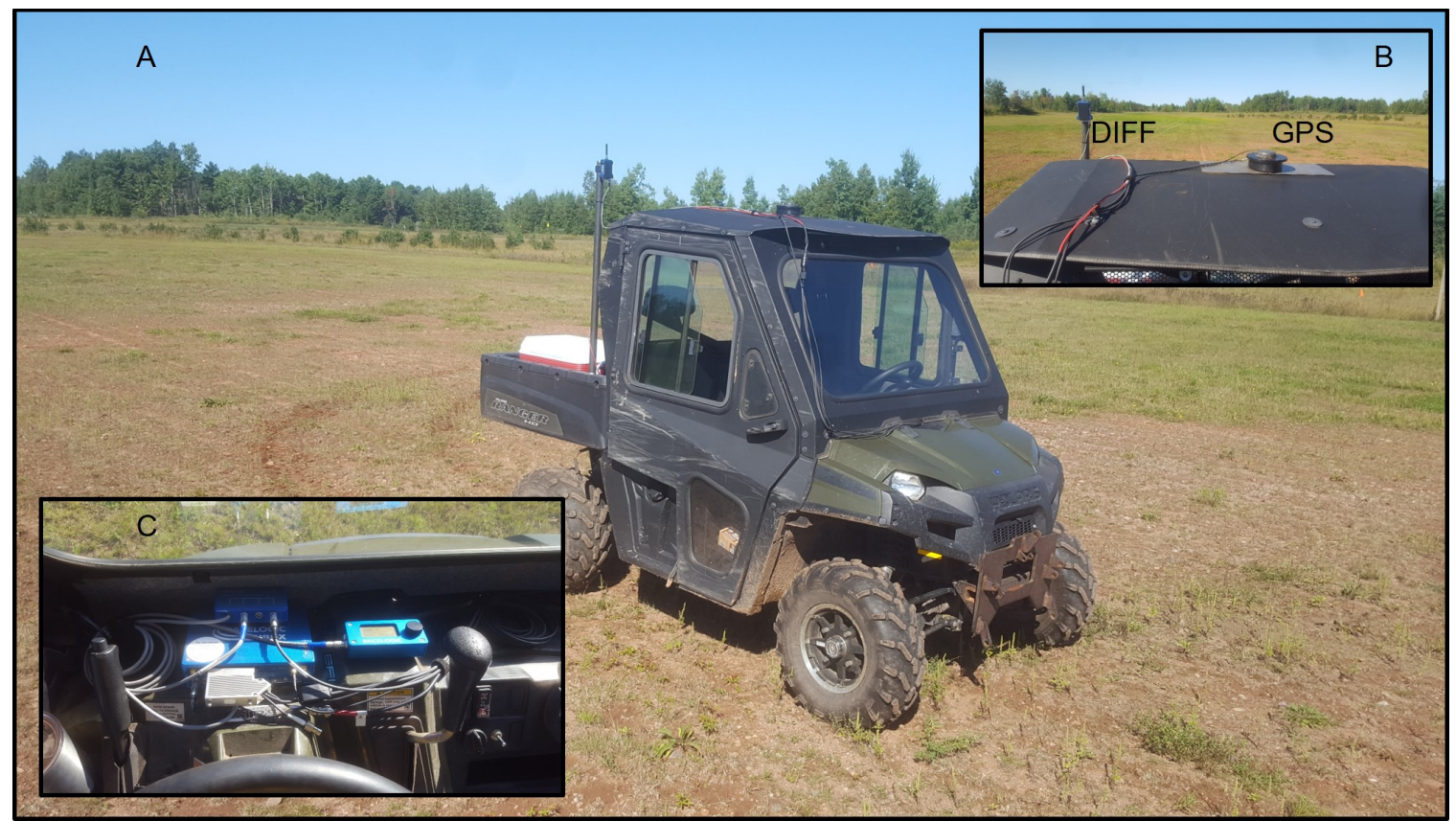

Figure 4.12 (A) Polaris Ranger driven around the test sites at KRC. (B) GPS/GLONASS antenna and differential correction antenna (C) VBOX GPS System in Ranger cabin.

In order to measure synchronized time domain data, it was necessary to trigger the SQobold systems and the VBOX to begin each data collect simultaneously. To facilitate this, a Motorola two-way radio was mounted at each AVS site (Figure 4.11c) as well as inside the UTV. The two-way radio headphone output channel was connected to the trigger channel of the SQobold systems and one of the auxiliary channels on the VBOX. During testing, an additional two-way radio was used to begin the collect by pressing the "call" button. The call signal provided a sufficient voltage spike to trigger the SQobold systems. The voltage spike was also recorded by the VBOX system, allowing for the GPS and AVS data to be time aligned in post-processing.

A total of 31 experiments are considered for this analysis. Experiments are numbered 62 through 92 and are indexed 1 to 31 . Table 4.1 lists the experiments considered, the experiment index, a description of the vehicle maneuvers executed during the experiment, and vehicle speed. Latitude vs longitude plots for all experiments are shown in Appendix A.4.2. 
Table 4.1 Atmospheric pp AVS experiments description of target vehicle maneuvers

\begin{tabular}{|c|c|c|c|}
\hline Index & Number & Description & $\begin{array}{c}\text { Vehicle } \\
\text { speed } \\
\text { (kmph) }\end{array}$ \\
\hline 1 & 62 & CCW loop around site 100 and 200 & 20 \\
\hline 2 & 63 & CCW loop around site 100 and 200 & 20 \\
\hline 3 & 64 & CCW loop around site 100 and 200 & 15 to 40 \\
\hline 4 & 65 & CW loop around site 100 and 200 & 20 \\
\hline 5 & 66 & CW loop around site 100 and 200 & 15 to 40 \\
\hline 6 & 67 & CW circular pattern site 100, Nominal Radius $=6 \mathrm{~m}$ & 10 \\
\hline 7 & 68 & CW circular pattern site 100, Nominal Radius $=7 \mathrm{~m}$ & 20 \\
\hline 8 & 69 & CW circular pattern site 100, Nominal Radius $=17 \mathrm{~m}$ & 15 to 20 \\
\hline 9 & 70 & CW circular pattern site 100, Nominal Radius $=18 \mathrm{~m}$ & 20 to 30 \\
\hline 10 & 71 & CW circular pattern site 100, Nominal Radius $=32 \mathrm{~m}$ & 10 to 20 \\
\hline 11 & 72 & CW circular pattern site 100, Nominal Radius $=37 \mathrm{~m}$ & 30 to 40 \\
\hline 12 & 73 & North/South pattern between sites & 10 to 20 \\
\hline 13 & 74 & North/South pattern between sites & 10 to 40 \\
\hline 14 & 75 & North/South line East of site 100 & 10 to 40 \\
\hline 15 & 76 & North/South line West of site 200 & 20 to 40 \\
\hline 16 & 77 & Figure-8 pattern 1 CW site 100, CCW site 200 & 20 to 40 \\
\hline 17 & 78 & Figure- 8 pattern 2 CCW site $100, C W$ site 200 & 10 \\
\hline 18 & 79 & CCW circular pattern site 200 , Nominal Radius $=7 \mathrm{~m}$ & 20 to 30 \\
\hline 19 & 80 & CCW circular pattern site 200, Nominal Radius $=10 \mathrm{~m}$ & 10 \\
\hline 20 & 81 & CCW circular pattern site 200, Nominal Radius $=22 \mathrm{~m}$ & 30 \\
\hline 21 & 82 & CCW circular pattern site 200, Nominal Radius $=25 \mathrm{~m}$ & 20,35 \\
\hline 22 & 83 & CCW circular pattern site 200, Nominal Radius $=39 \mathrm{~m}$ & 20,35 \\
\hline 23 & 84 & Circular pattern between sites & 20,40 \\
\hline 24 & 85 & Circular pattern between sites & 20,30 \\
\hline 25 & 86 & Long range pattern 1 , site exterior & 20 to 55 \\
\hline 26 & 87 & Long range pattern 2 , site exterior & 5 to 50 \\
\hline 27 & 88 & Long range pattern South/West to North/East & 10 to 60 \\
\hline 28 & 89 & Long range pattern North/West to South/East & 10 to 60 \\
\hline 29 & 90 & Random pattern 1 & 20 to 40 \\
\hline 30 & 91 & Random pattern 2 & 15 to 40 \\
\hline 31 & 92 & North/South line between sites & 10 to 40 \\
\hline
\end{tabular}




\subsection{Results}

This section will present the results for underwater $(p a)$ and atmospheric $(p p)$ AVS.

\subsubsection{Underwater pa Vector Sensors}

Underwater AVS data was collected in Barrow, AK in 2016. That data is analyzed here.

\subsubsection{Pitch, Roll, and Heading Variation of pa AVS}

The gravity sensor measures the $x, y$, and $z$ components of the earth's gravitational field with a resolution of 1 milli-g $(0.001 \mathrm{~g})$. A positive reading on the gravity sensor indicates that the respective axis of the sensor is pointing vertically down toward earth. A zero reading on the gravity sensor indicates that the respective axis of the sensor is horizontal with respect to earth. In a normal gravitational field, the magnitude of the $x, y$, and $z$ components is equal to 1000 milli-g. ${ }^{83}$

Table 4.2 Gravity and magnetic field sensor data over the course of one 120-second data collect.

\begin{tabular}{l|c|c|c|c|c|c}
\hline & \multicolumn{3}{|c|}{ Gravity Sensor Data (milli-g) } & \multicolumn{2}{c}{ Magnetic Field Sensor Data (nT) } \\
\hline & PrX & PrY & PrZ & HdX & HdY & HdZ \\
\hline Max & 4 & 74 & 1019 & -6440 & 10160 & -8900 \\
\hline Mean & -9 & 55 & 1017 & -7580 & 9220 & -9060 \\
\hline Min & -21 & 35 & 1014 & -8820 & 8400 & -9280 \\
\hline St. Dev. & 5 & 7 & 1 & 520 & 320 & 80 \\
\hline \hline & \multicolumn{6}{|c|}{ Orientation of the Vector Sensor Given Data Above $\left(^{\circ}\right)$} \\
\hline Mean & -86.8 & \multicolumn{3}{c}{ Roll } & \multicolumn{2}{c}{ Heading } \\
\hline
\end{tabular}

The magnetic sensor measures the $x, y$, and $z$ components of the earth's magnetic field with a resolution of $20 \mathrm{nT}$ (nano-Tesla). A positive reading on the magnetic sensor indicates that the respective axis of the sensor is pointing away from the earth's north pole. $^{83}$

It is noted that the rotational orientations of the vector sensor, pitch, roll, and heading, are measured about the $\mathrm{y}$-axis, $\mathrm{z}$-axis, and $\mathrm{x}$-axis respectively. Positive rotation is defined by the right-hand rule. ${ }^{83}$

During the vector sensor experiments, there was little change in the orientation of the vector sensor. An example data set's statistics for the gravity sensor and magnetic field sensor data is shown in Table 4.2 along with corresponding pitch, roll, and heading angles. The data indicate that the $z$-axis of the vector sensor was pointing vertically downward in the water column, as intended, with little variation in tilt during the 120second measurement. In addition, the vector sensor may have been slightly rotating or drifting due to currents over the course of the data collect, represented by small variations 
in the magnetic sensor data. However, these changes are small, with a standard deviation of less than $520 \mathrm{nT}$ (less than $10 \%$ of the mean sensor reading), resulting in little variation in the computed pitch, roll, or heading of the vector sensor during the measurement. ${ }^{83}$

\subsubsection{DOA of the Stationary Source}

The DOA of the stationary underwater sound source at site 4 is determined with the vector sensor at site 1 . The AVS data collected is only analyzed at frequencies where there is greater than $10 \mathrm{~dB}$ signal-to-noise ratio (SNR) for both the hydrophone and accelerometer. This represents $99.30 \%$ of the hydrophone data and $99.96 \%$ of the accelerometer data in the frequency ranges selected for analysis, 1000 to $2000 \mathrm{~Hz}$ and 5000 to $9000 \mathrm{~Hz}$ (Figure 4.13). The acoustic intensity, azimuth, and elevation are computed with Equations 4.31 to 4.33 (Figure 4.14 and Figure 4.15). It is observed that the azimuth angle is consistent across the frequency bands. The average azimuth angle from the vector sensor to the sound source is -65.63 degrees and -70.5 degrees for the low frequency and high-frequency pings respectively. This is compared to the actual azimuth angle of -69.45 degrees. The actual azimuth angle,

$$
\theta_{\text {actual }}=90^{\circ}-15.51^{\circ}-\tan ^{-1}\left(\frac{\Delta L A T_{1,4}}{\Delta L O N_{1,4}}\right)
$$

was determined by computing the complementary angle between the magnetic heading vector and the vector between sites 1 and 4 based on GPS coordinates. The magnetic heading vector was 15.51 degrees east of true north at the test sites according to the NOAA magnetic declination calculator ${ }^{83,136}$. 

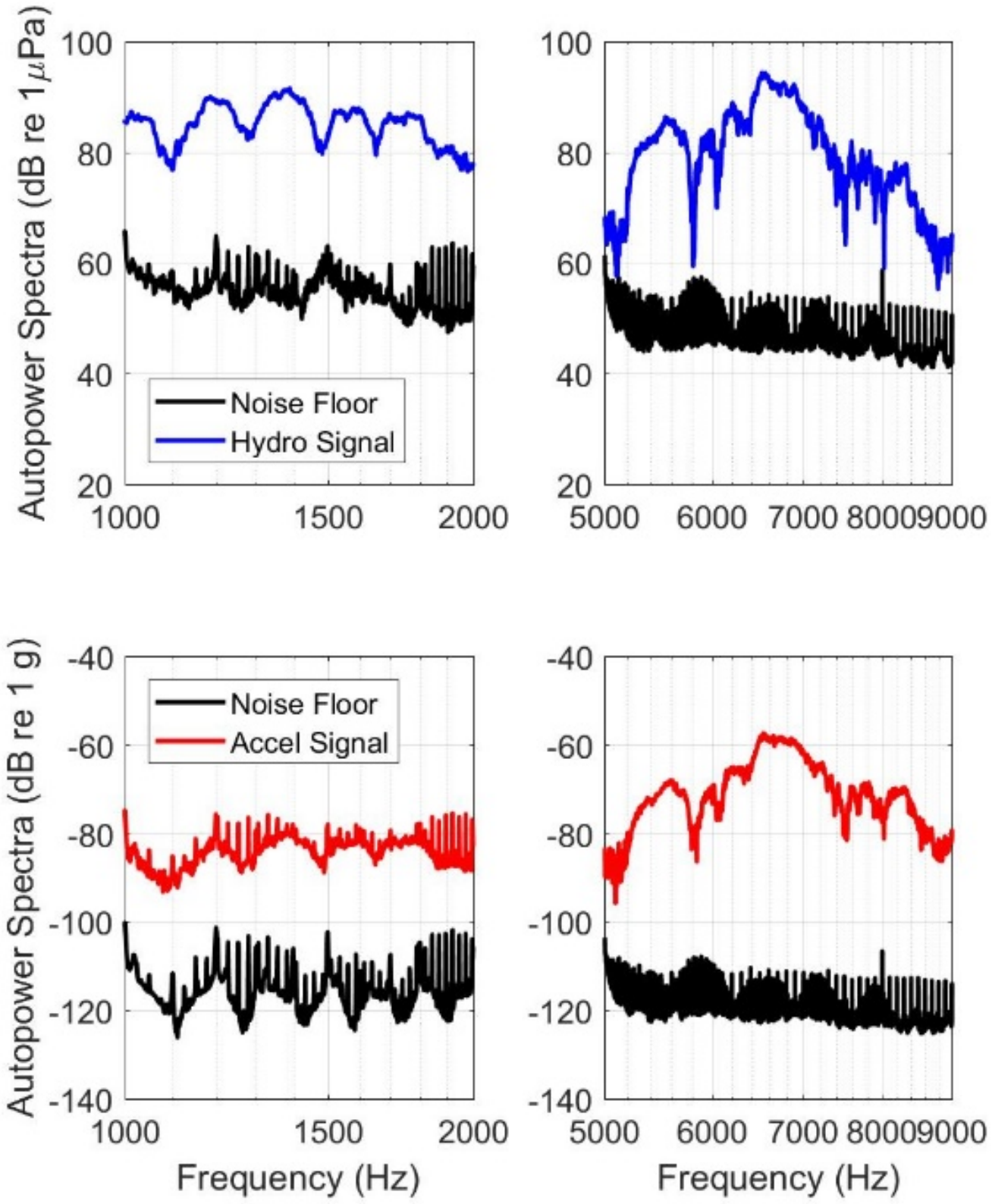

Figure 4.13 Underwater AVS measurement for stationary pings. Signal and background noise level in selected frequency bands. Low-frequency data (left) and high-frequency data (right) are shown for the vector sensor hydrophone (upper) and accelerometer (lower). 

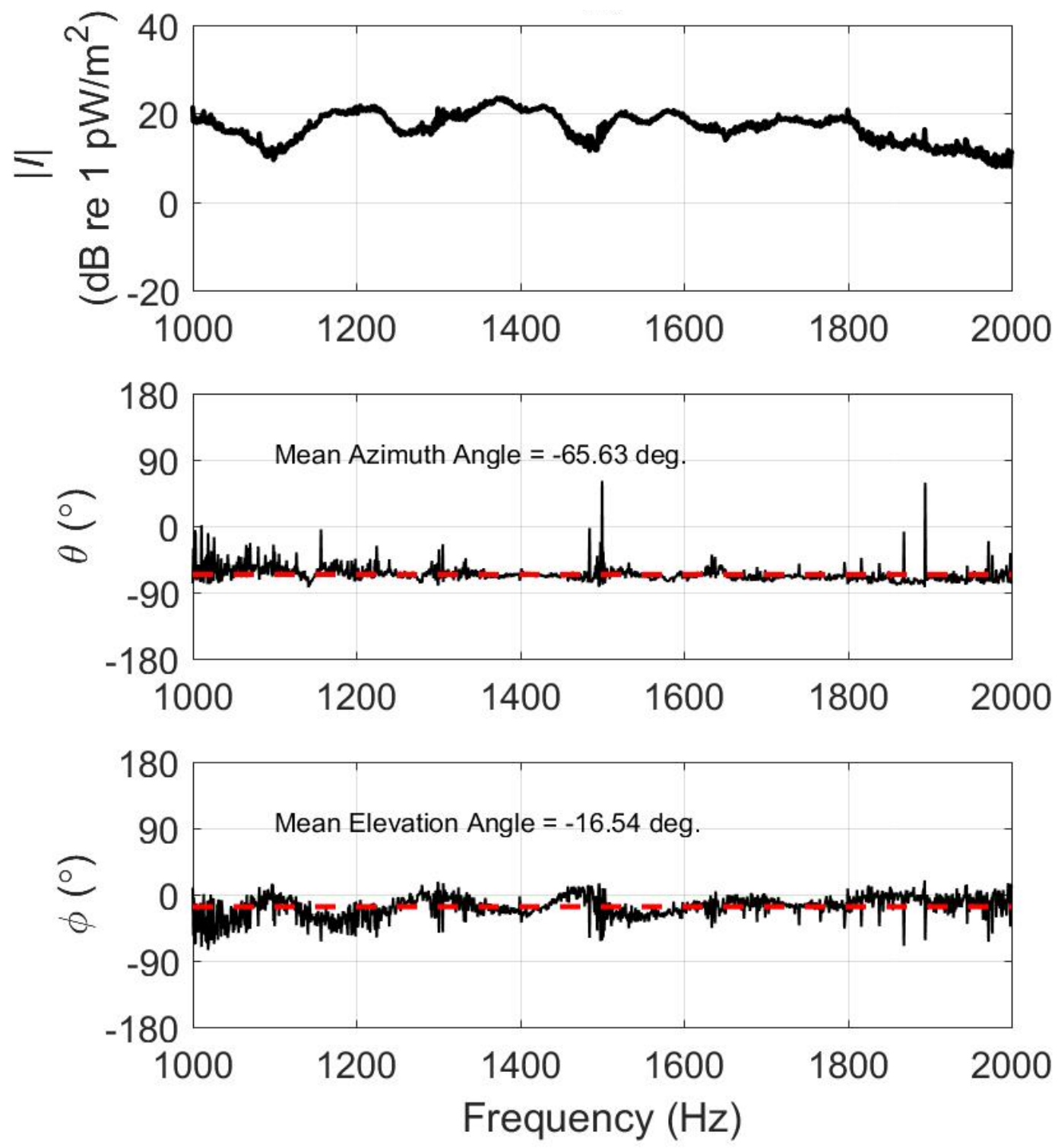

Figure 4.14 Underwater AVS data at site 1 for stationary underwater sound source at site 4 Lowfrequency ping range. Mean azimuth and elevation angles are shown with red dashed lines. 

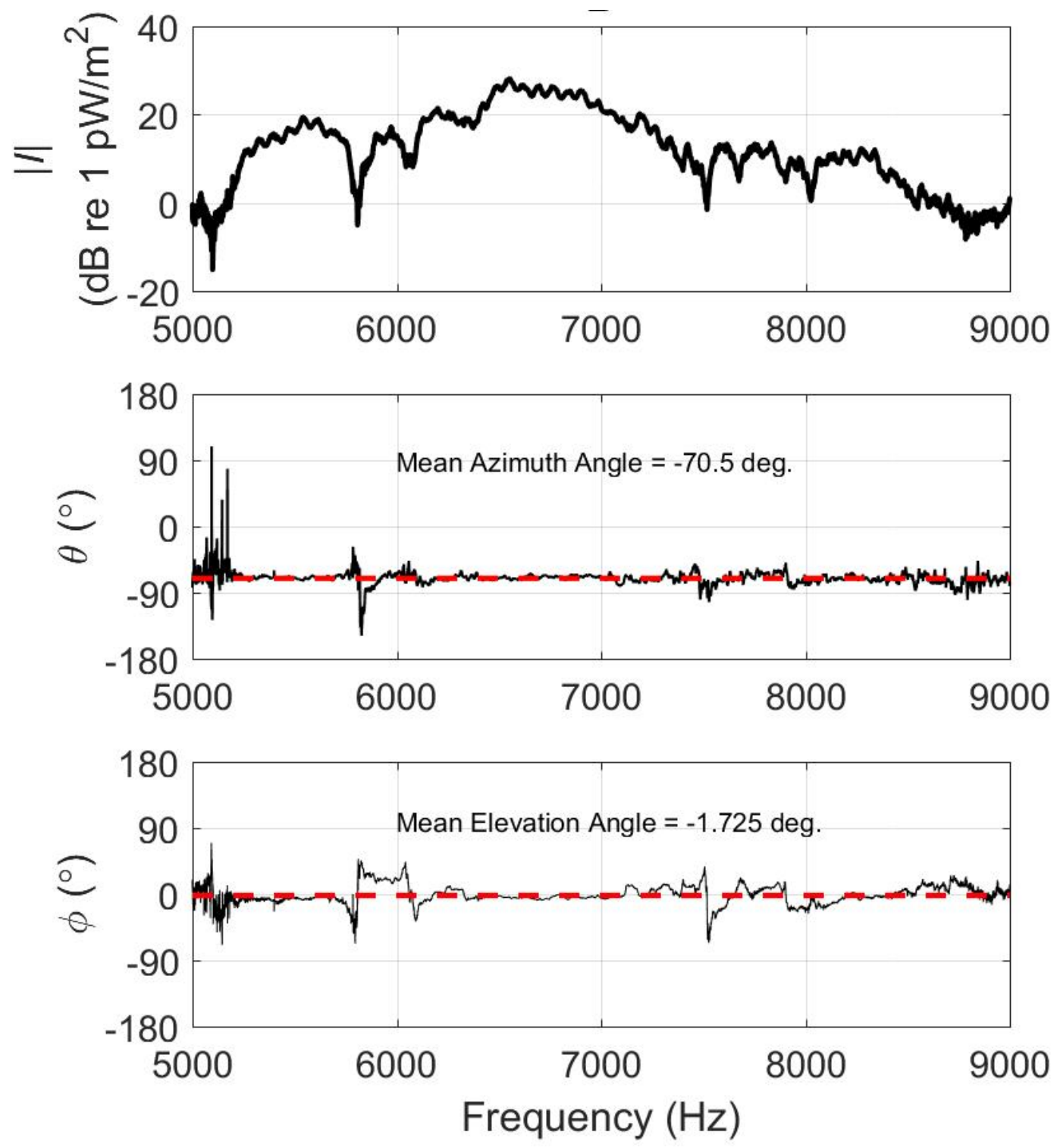

Figure 4.15 Underwater AVS data at site 1 for stationary underwater sound source at site 4 Highfrequency ping range. Mean azimuth and elevation angles are shown with red dashed lines.

The average elevation angle from the vector sensor to the sound source is -16.54 degrees and -1.73 degrees for the low-frequency and high-frequency pings respectively. This is compared to the actual elevation angle of -1.18 degrees. The actual elevation angle is defined as,

$$
\Phi_{\text {actual }}=\tan ^{-1}\left(\frac{D_{V S}-D_{S}}{\sqrt{\left(\Delta L A T_{1,4}\right)^{2}+\left(\Delta L O N_{1,4}\right)^{2}}}\right),
$$


where $D_{S}$ is the depth of the underwater source which was $1.9 \mathrm{~m}$ for all stationary source experiments. The variation in the elevation angle is expected due to the complex ice ridging in the shore-fast environment (see Figure 2.2) and is expected to vary with frequency. ${ }^{83}$

\subsubsection{DOA of the Non-Stationary Source}

The vector sensors at sites 2 and 3 were used to determine DOA from the snowmobile as a function of time over the 120 -second data collect with 1 -second time resolution. The azimuth and elevation computation at each time step were made with 100 spectral averages, each with a frequency resolution of $100 \mathrm{~Hz}$. A coarse frequency resolution was acceptable in favor of having more spectral averages per time step; reducing statistical variance on the averaged azimuth and elevation angles. The averaged azimuth and elevation spectra for a selected time instant, $\mathrm{T}=38$ seconds, are shown in Figure 4.17 and Figure $4.16 .^{83}$
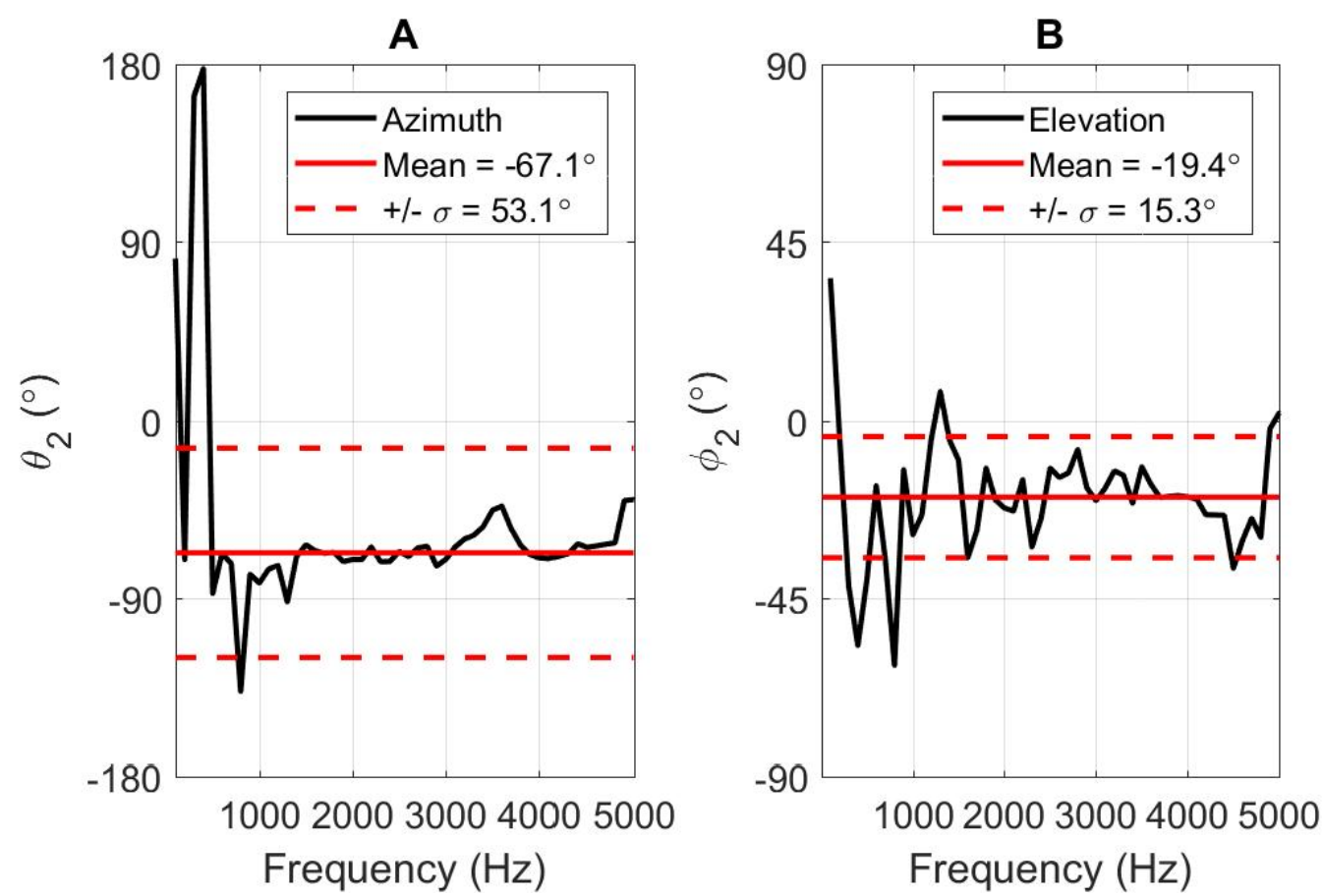

Figure 4.16 (A) Azimuth and (B) elevation spectra measured at site 2. Mean and standard deviation indicated for the selected time instant $\mathrm{T}=38$ seconds.

The mean and standard deviation of the azimuth and elevation spectra were computed at each time step over the frequency range 10 to $5000 \mathrm{~Hz}$ which contains the majority of the acoustic frequency content produced by the snowmobile. The mean azimuth angle and the standard deviation are shown as a function of time in Figure 4.17. Likewise, the mean elevation angle and standard deviation are shown as a function of time in Figure 4.18. The azimuth and elevation angles from the vector sensor are compared to the ground truth GPS. There is good correlation between the vector sensor and the GPS at 
both sites. The true GPS azimuth angle falls within plus or minus 1 standard deviation of the mean vector sensor azimuth $92 \%$ of the time for site 2 and $78 \%$ of the time for site 3 . The true GPS elevation angle falls within plus or minus 1 standard deviation of the mean vector sensor elevation angle $85 \%$ of the time for site 2 and $91 \%$ of the time for site $3 .{ }^{83}$

The range between the measurement site and the snowmobile was computed with Equation 4.34 and is compared to the ground truth GPS range in Figure 4.19. It is observed that the general trend of the measured range from the vector sensor follows the ground truth range with several large deviations in the measured data and high variance for several time intervals. The measured range most closely approximates the ground truth range when the snowmobile was closest to the measurement locations. By definition, the tangent function of the vector sensor elevation goes to zero when the elevation angle is zero degrees and the tangent goes to infinity when the elevation angle is \pm 90 degrees. When the sound source is far away from the measurement location, the elevation angle gets closer to zero, as does the tangent of the elevation. This, in turn, causes $R_{V S}$ to approach a very high value (infinity if the tangent is zero) and become very sensitive to small errors in elevation angle. The mean value of the elevation measurement in Figure 4.18 crosses zero frequently when the snowmobile is far from the measurement site. This explains the large deviations in range, towards infinity, in Figure 4.19. Therefore, the elevation and range measurements are only useful for localization when the snowmobile is close to the site; that is 21 to 42 seconds and 100 to 119 seconds for site 2 and 48 to 75 seconds for site $3 .{ }^{83}$ 


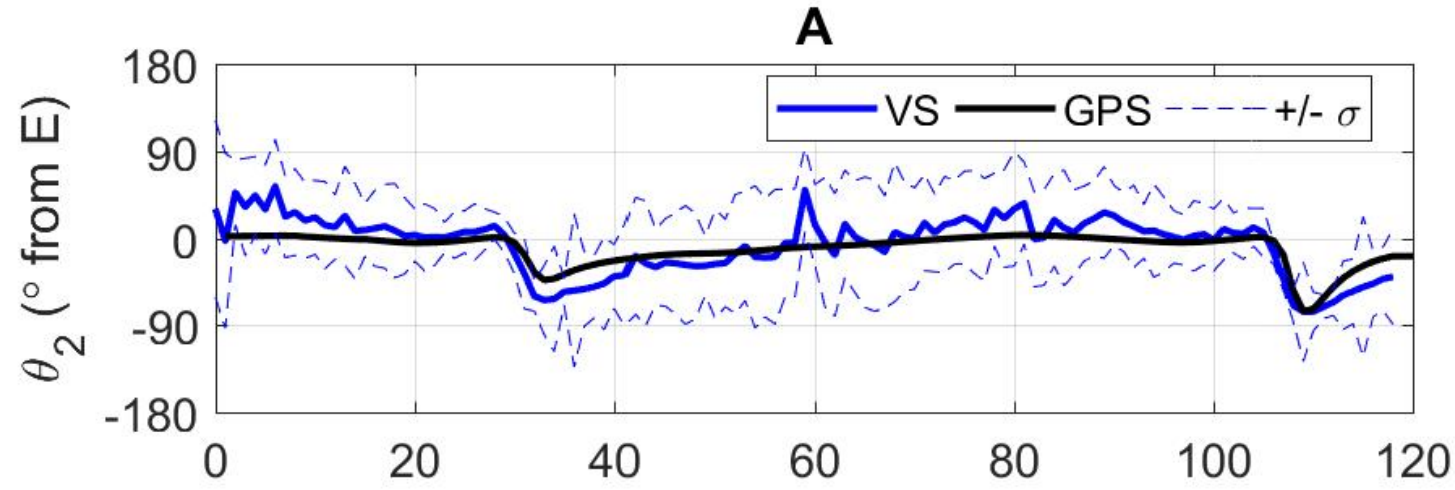

B

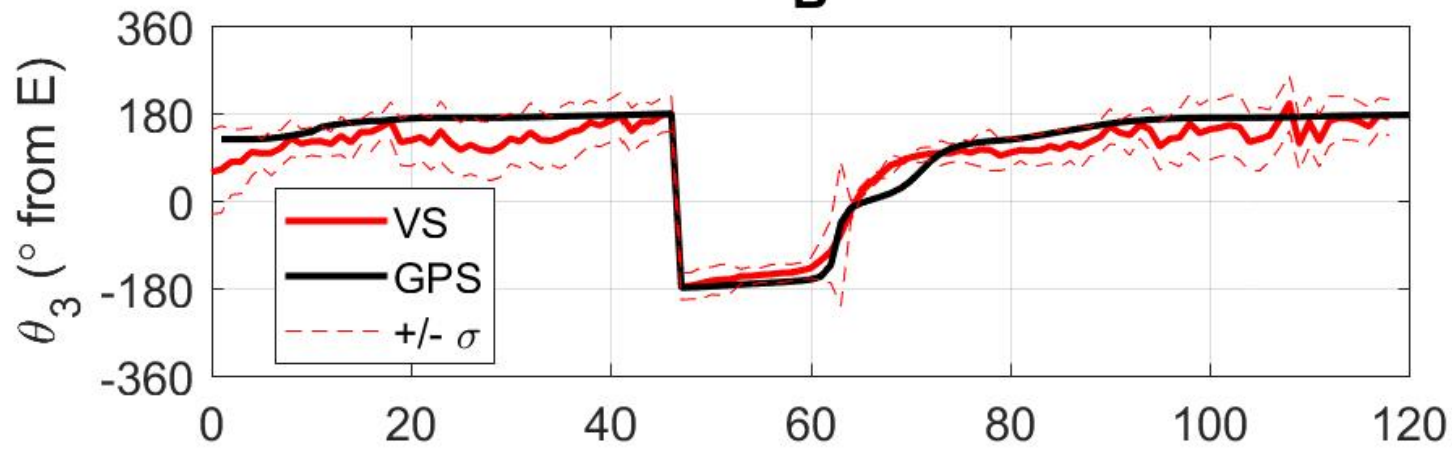

C

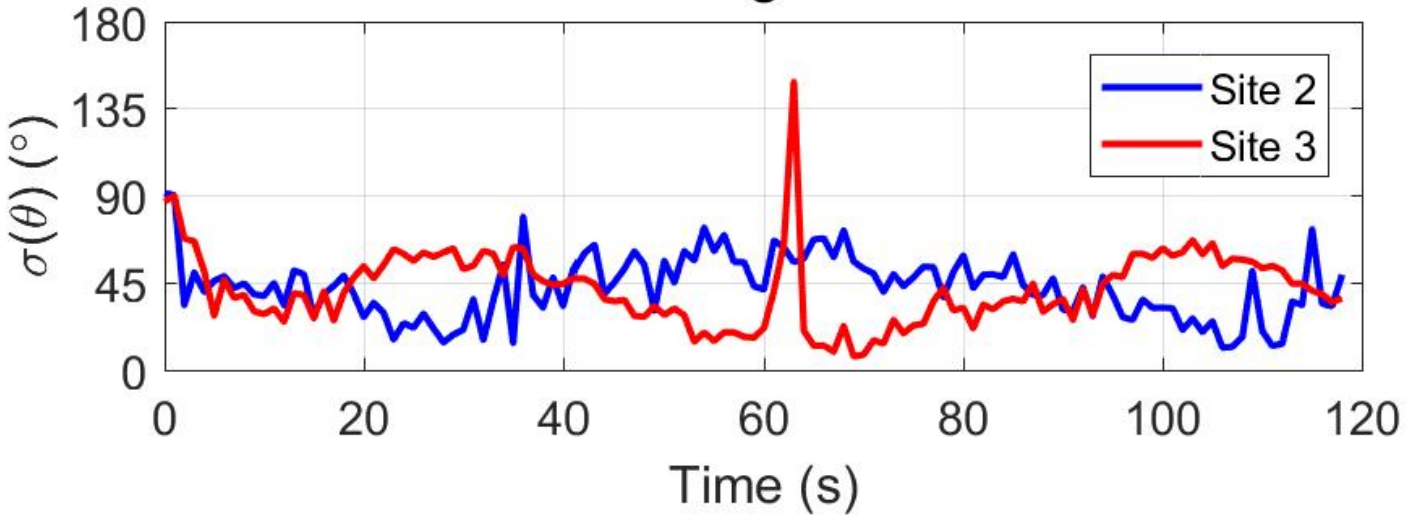

Figure 4.17 Mean azimuth angle computed from the underwater AVS(A) site 2, and (B) site 3, compared to ground truth GPS DOA. AVS DOA averaged between 10 to $5000 \mathrm{~Hz}$ at each time step. (C) Standard deviation of the DOA computed at each time step. 

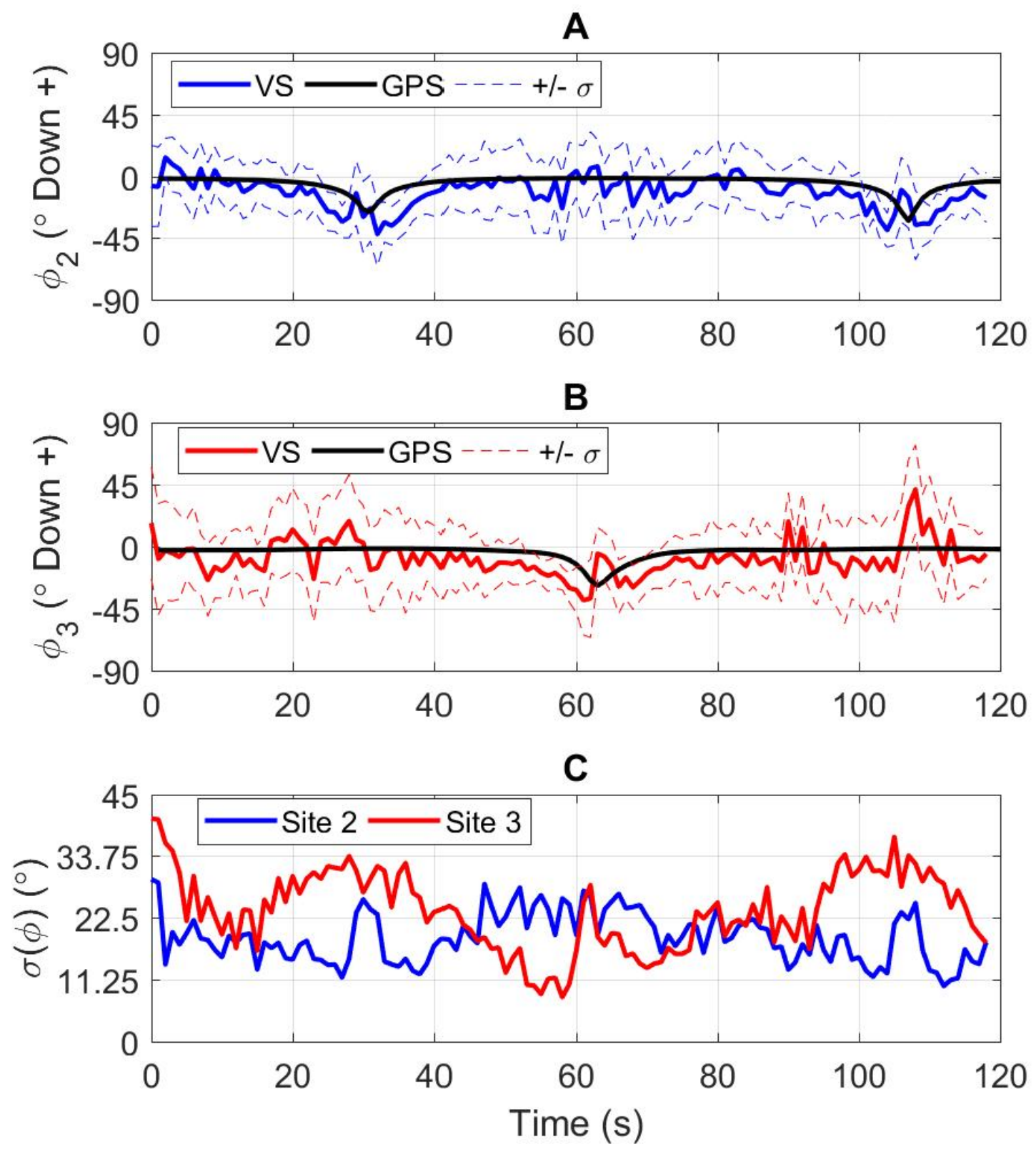

Figure 4.18 Mean elevation angle computed from the underwater AVS(A) site 2, and (B) site 3, compared to ground truth GPS elevation. AVS DOA averaged between 10 to $5000 \mathrm{~Hz}$ at each time step. (C) Standard deviation of the elevation computed at each time step

The vector sensor SNR is computed for both sites as a function of time (Figure 4.20). When the snowmobile is closest to the measurement location, there is between 30 to 35 $\mathrm{dB}$ SNR at the vector sensor hydrophone and 33 to $36 \mathrm{~dB}$ SNR at the vector sensor accelerometer. When the snowmobile is furthest away from the measurement location there is 3 to $8 \mathrm{~dB}$ SNR at the hydrophone and $23 \mathrm{~dB}$ SNR at the accelerometer. ${ }^{83}$ 

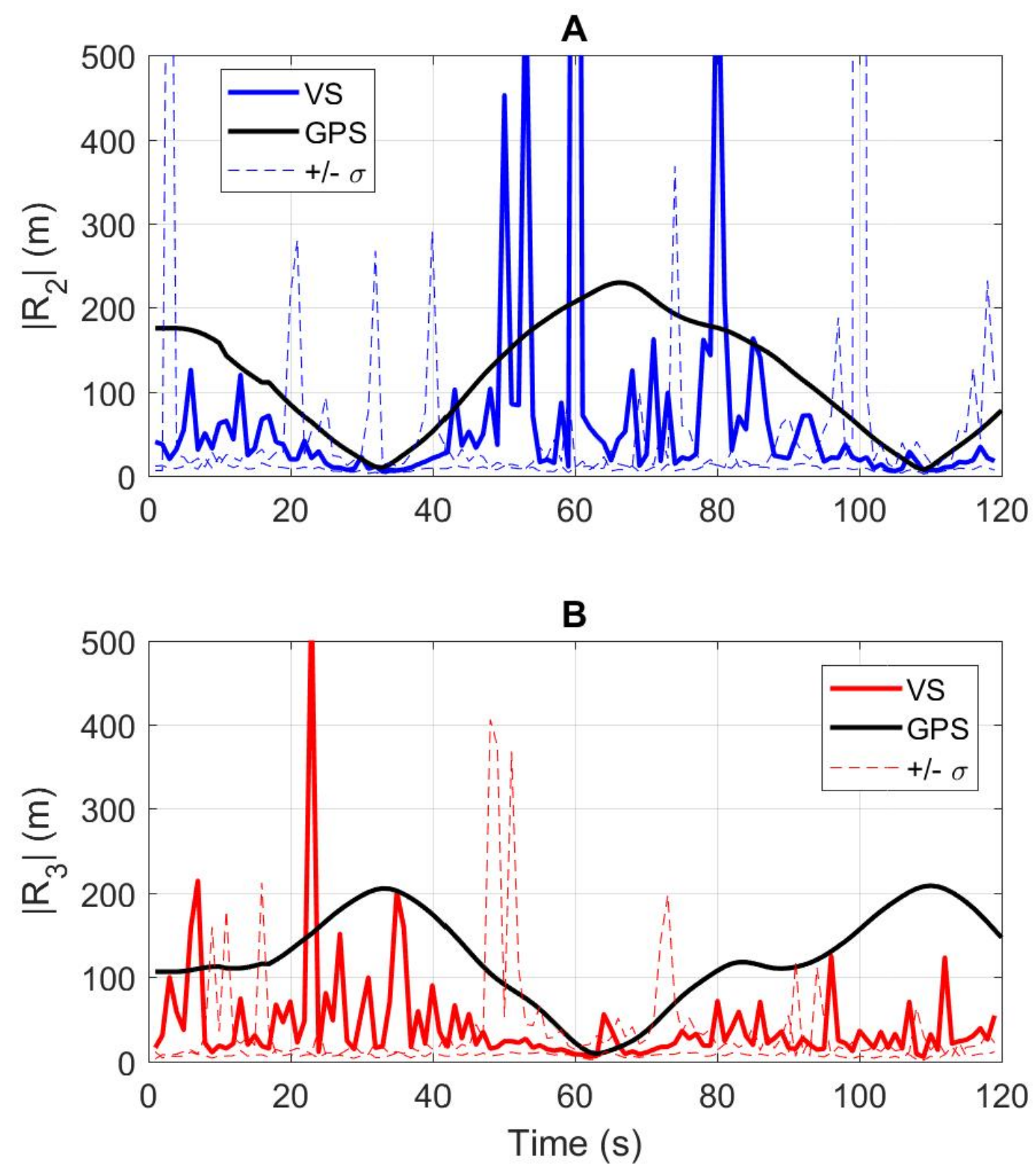

Figure 4.19 Mean range from measurement site to the snowmobile computed from the underwater vector sensor $(A)$ site 2 and $(B)$ site 3 compared to ground truth GPS range. 

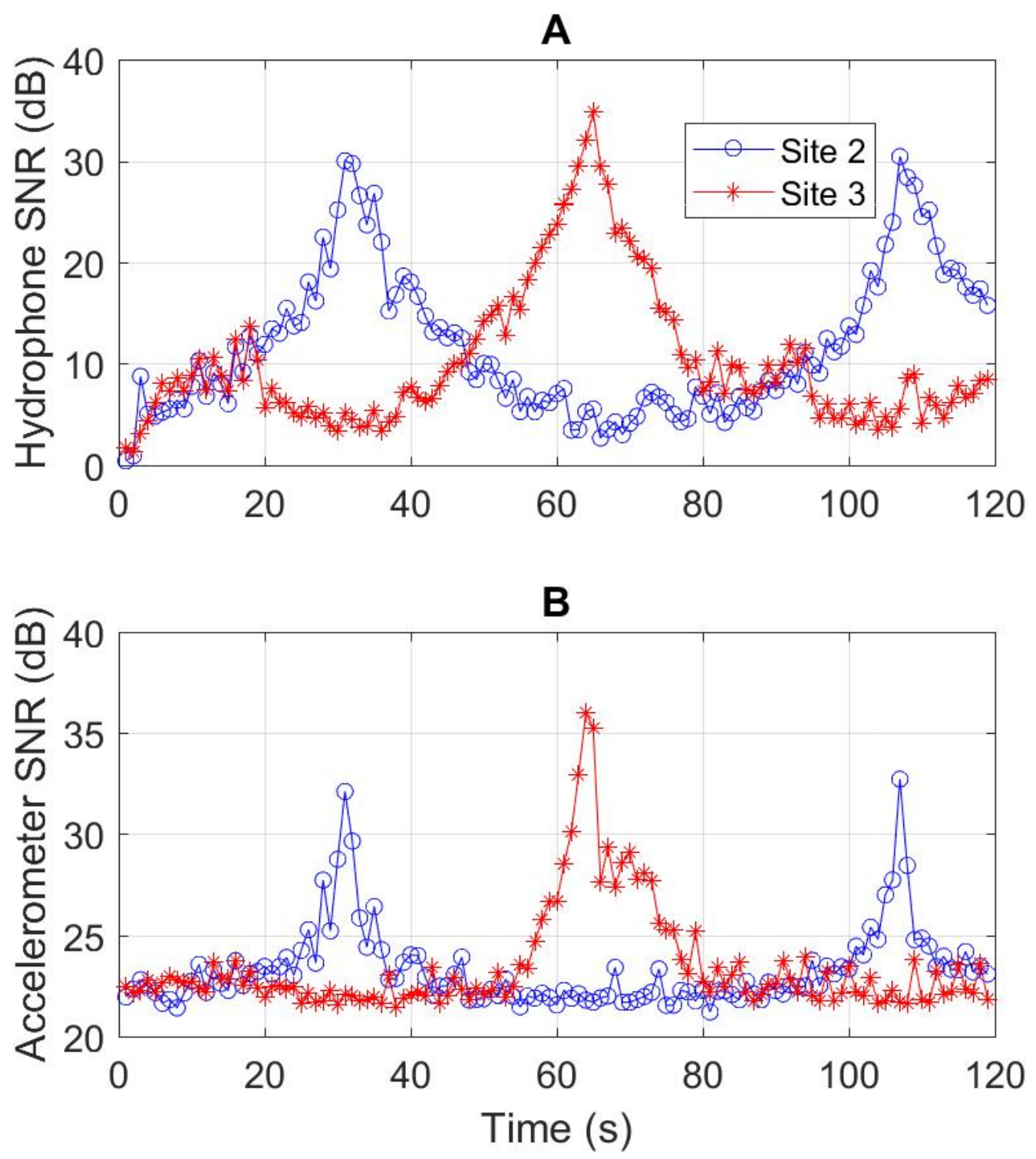

Figure 4.20 Underwater AVS signal to noise ratio during the 120-second measurement period (A) hydrophone and (B) accelerometer.

It is observed that the standard deviation of the azimuth and elevation increase sharply when the snowmobile is closest to the measurement site (same time intervals stated above). This increase in standard deviation is unexpected because Figure 4.20 shows that there is high SNR when the snowmobile is closest to the measurement site. The increase in standard deviation is explained by the snowmobile being positioned vertically overhead of the vector sensor. Theoretically, in this position, the azimuth angle becomes undefined and the elevation angle becomes -90 degrees (However, this was not seen in the figures presented here because the snowmobile was not perfectly overhead). Additionally, it becomes very difficult to measure these quantities in this position due to the acoustic reflection off the seafloor. The reflection off of the seafloor causes an 
increase in variance of both DOA measurements during the computation of each spectral average due to constructive and destructive interference. ${ }^{83}$

\subsubsection{Multi-Sensor Localization of the Non-Stationary Source}

Combining the DOA data from sites 2 and 3 , the snowmobile is localized as a function of time. Plus and minus $1 \sigma$ are added to the mean azimuth, creating a beam used for localization at each site. The overlap of the standard deviation beams from each site creates an intersecting patch of area which contains the estimated snowmobile position. Due to the large localization beam width (nominally around 45 degrees), using more than one standard deviation for localization was not possible. Figure 4.21 shows selected time instances when the snowmobile was localized. The included time instances are representative of the type of localization that is typical for this method. The blue and red dashed lines represent the standard deviation beams for sites 2 and 3 respectively, the shaded area represents the localization area, and the black $\times$ represents the true GPS location of the snowmobile. The time instant and the localization area are noted at the top of each plot. In order for the standard deviation beams of each site to successfully localize the snowmobile, they must both encompass the true snowmobile location and intersect to form a closed and bounded area. This is evident in all subplots in Figure $4.21 .^{83}$

Figure 4.22 shows selected time instances when it was not possible to localize the snowmobile. The common reasons for not localizing fall into three categories: 1) neither of the sites standard deviation beams encompass the snowmobile location, 2) only one of the sites standard deviation beams encompass the snowmobile location, or 3 ) the standard deviation beams from both sites encompass the snowmobile location, but do not create a closed, bounded, intersecting area. These three categories are illustrated in subplots A through $\mathrm{C}$ in Figure 4.22. The solid lines in this figure represent the mean azimuth angle computed for the time instant and the dashed lines represent the plus or minus standard deviation lines. ${ }^{83}$

As mentioned, when the snowmobile is close to the measurement site, it is possible for the elevation (and therefore range) measurements to assist in reducing the localization area. The range measurement effectively represents a circle which is centered at the measurement location. This effect is shown in Figure 4.23 for a selected time instant when the snowmobile was close to site 2 . The reduction in localization area for this specific time instant is $0.0081 \mathrm{~km}^{2} .{ }^{83}$ 

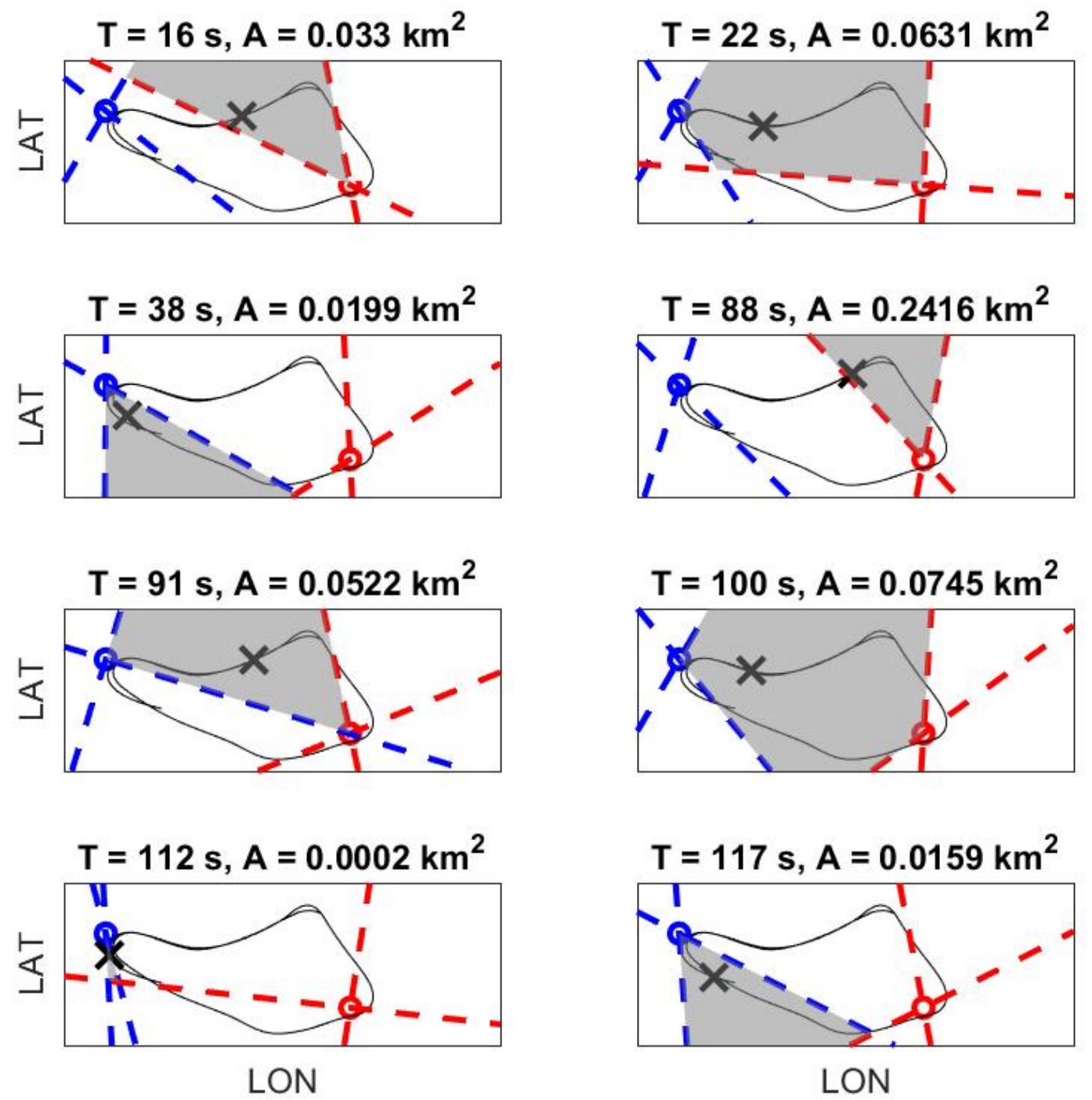

Figure 4.21 Selected example time instances and localization areas. Dashed lines indicate the standard deviation beams for sites 2 (blue) and 3 (red). 

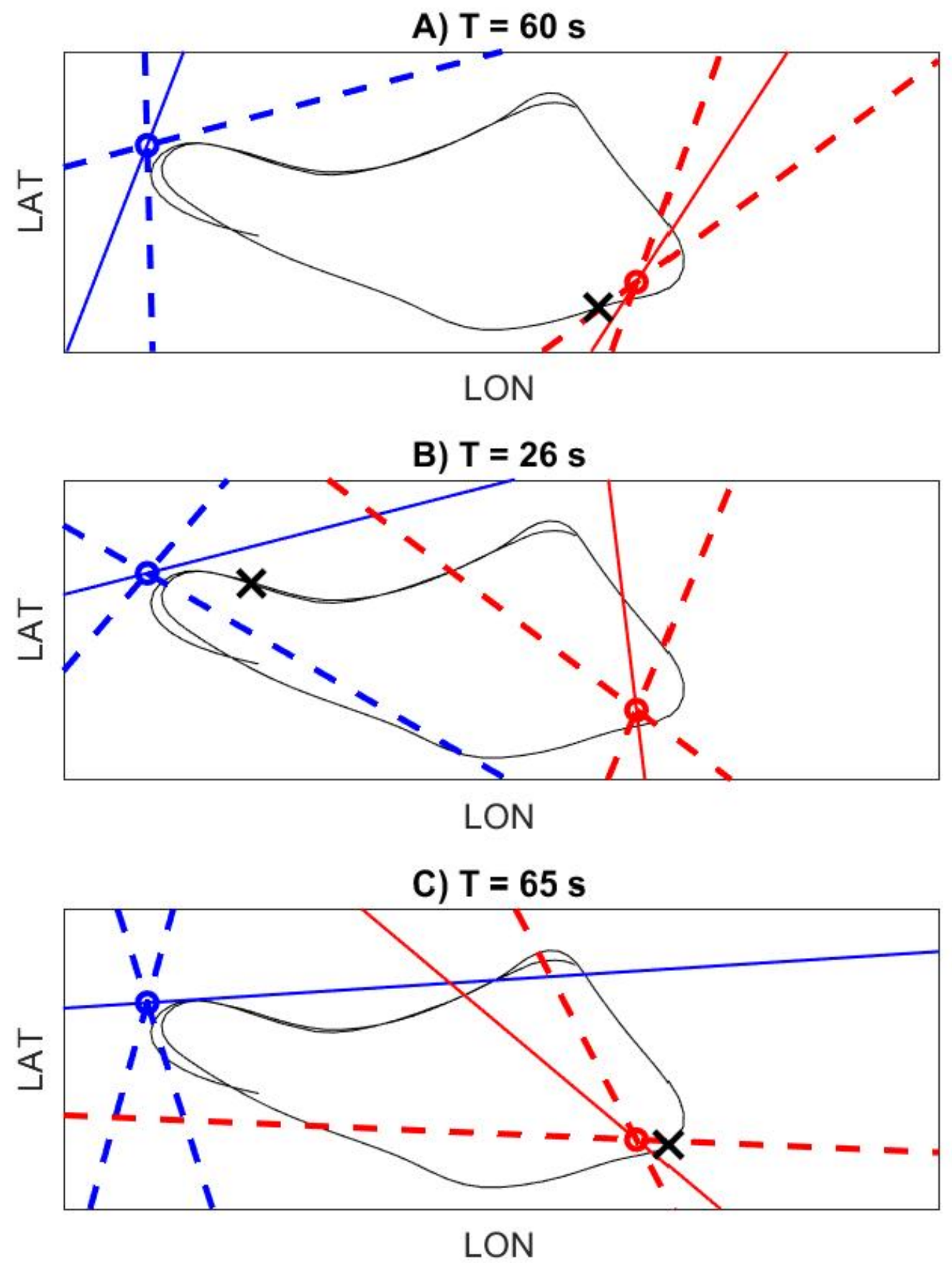

Figure 4.22 Selected example time instances when it was not possible to localize the snowmobile. 

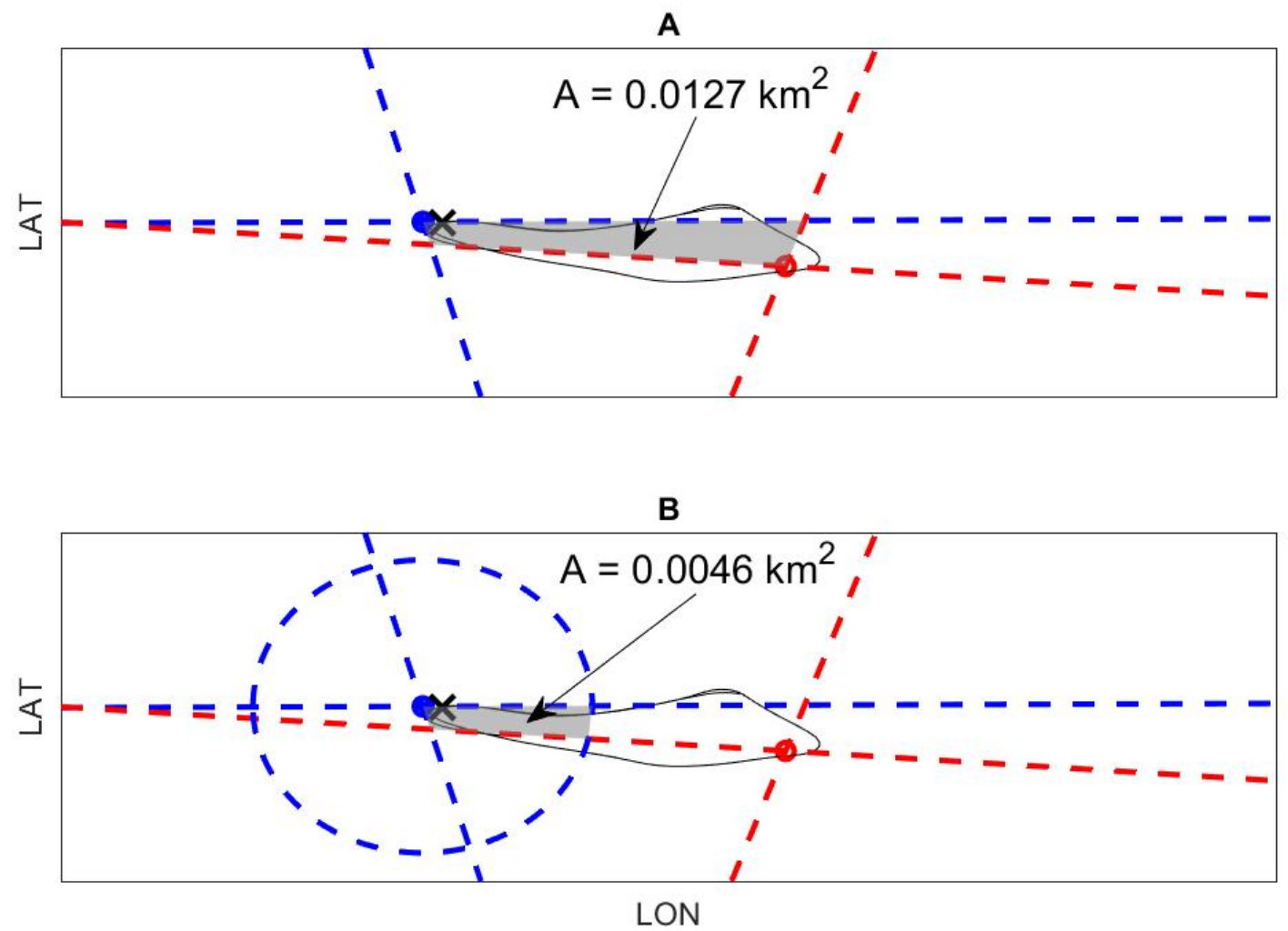

Figure 4.23 Selected time instance $(T=32 \mathrm{~s})$ with localization area computed $(\mathrm{A})$ without elevation circle and (B) with elevation circle.

Figure 4.24 and Figure 4.25 show the localized area as a function of time and azimuth angle respectively. The localization area is shown using the azimuth localization only as well as the combined azimuth and elevation localization for instances when the elevation data was useful. During the 120 -second measurement, localization was achieved $66 \%$ of the time using the azimuth only. Of these instances, reduction of the localization area by including the elevation data was achieved $15 \%$ of the time. ${ }^{83}$

It is observed in Figure 4.25 that the majority of the localization instances occur between 0 to -90 degrees for site 2 and between +90 to 180 degrees for site 3 , with a few exceptions. This makes sense based on the positioning of the snowmobile track in relation to the measurement sites. With respect to site 2, the snowmobile traveled in the south-east quadrant during the majority of its path. With respect to site 3 , the snowmobile traveled in the north-west quadrant for the majority of its path. This can be visualized in Figure 4.9, Figure 4.21, and Figure $4.23 .^{83}$ 


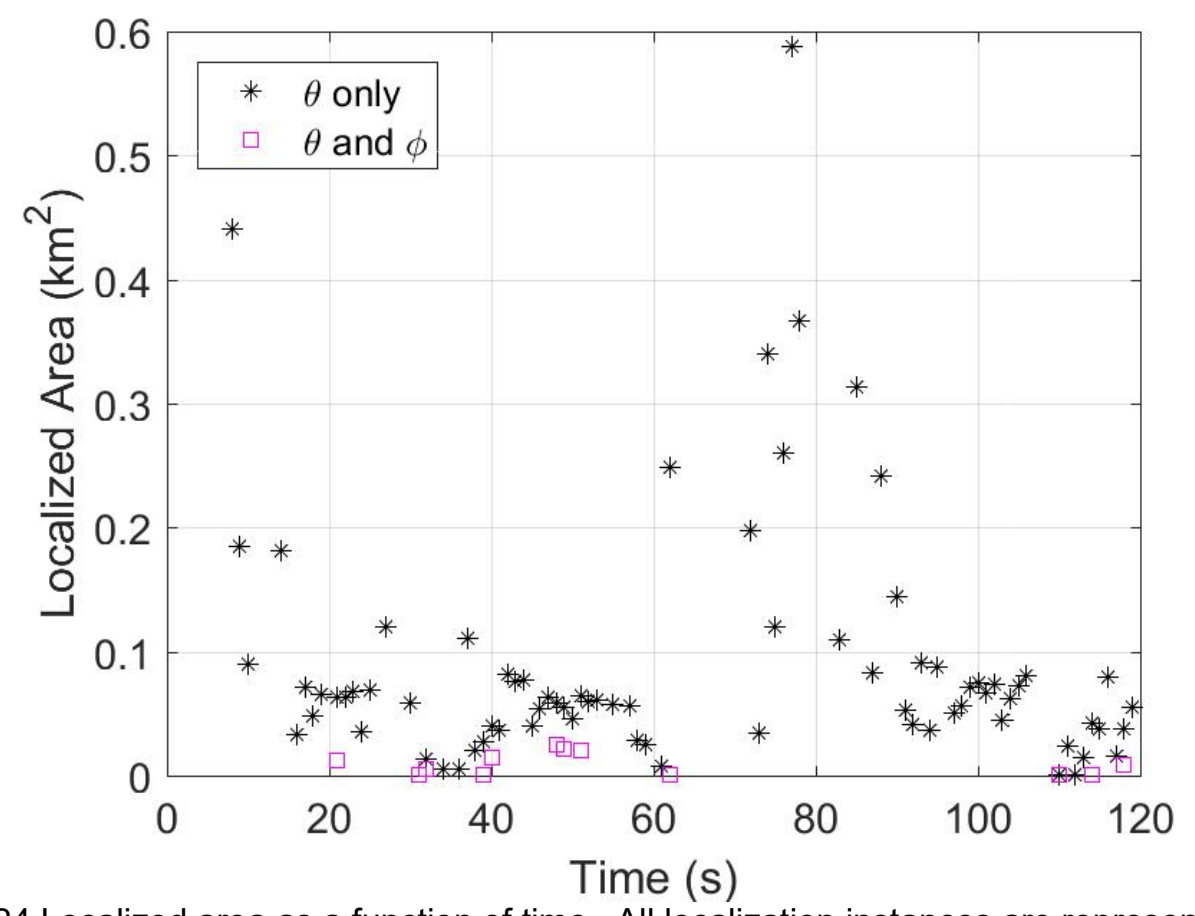

Figure 4.24 Localized area as a function of time. All localization instances are represented using azimuth data alone as well as azimuth plus elevation data when possible

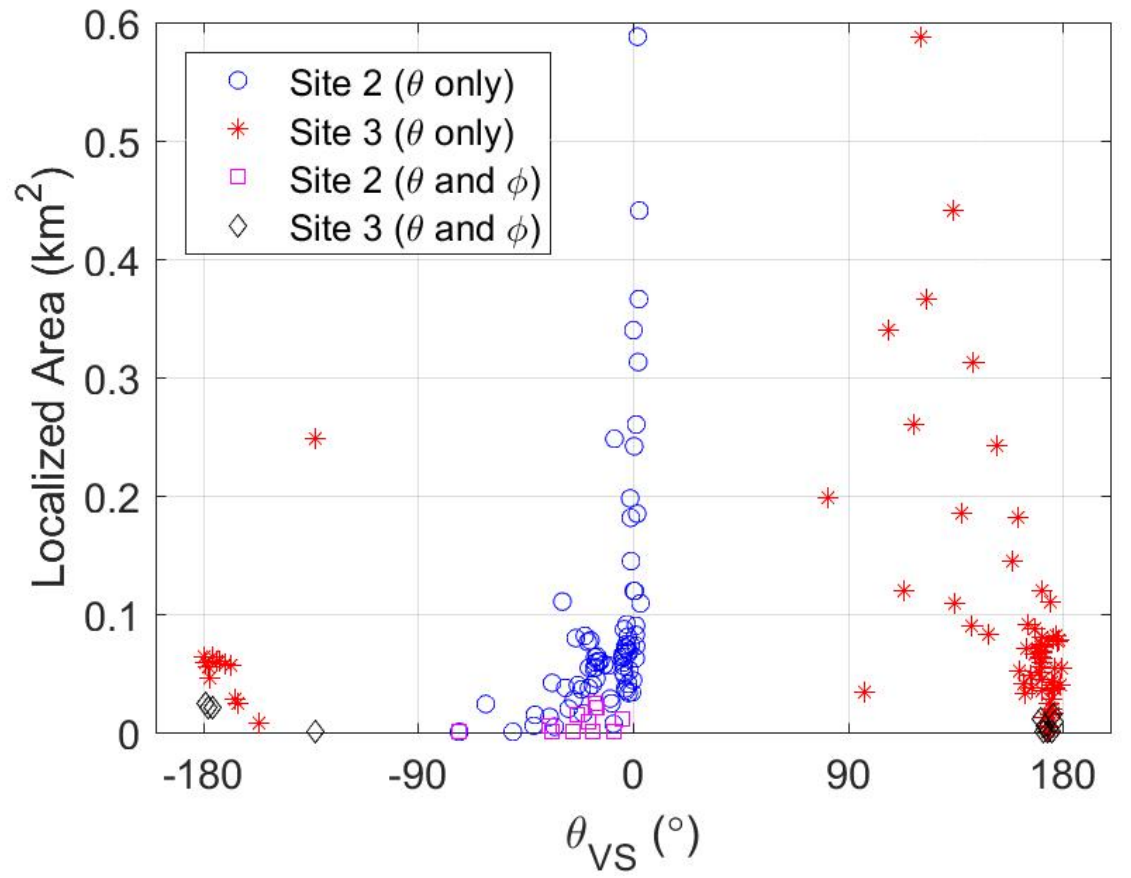

Figure 4.25 Localized area as a function of azimuth angle. All localization instances are represented for both site 2 and site 3 using azimuth data alone as well as azimuth plus elevation data when possible. 
Table 4.3 Underwater AVS localized area statistics for selected time intervals. Time intervals represent instances when there was low SNR (row 2) or high SNR (row 3) at the vector sensor measurement location.

\begin{tabular}{l|c|c|c|c|c}
\hline \hline & $\begin{array}{l}\text { Max } \\
\left(\mathrm{km}^{2}\right)\end{array}$ & $\begin{array}{l}\text { Min } \\
\left(\mathrm{km}^{2}\right)\end{array}$ & $\begin{array}{l}\text { Mean } \\
\left(\mathrm{km}^{2}\right)\end{array}$ & $\begin{array}{l}\text { Median } \\
\left(\mathrm{km}^{2}\right)\end{array}$ & $\begin{array}{l}\text { Stdev. } \\
\left(\mathrm{km}^{2}\right)\end{array}$ \\
\hline $\begin{array}{l}\text { Localized area [All Time] } \\
\begin{array}{l}\text { Localized area } \\
{[\mathrm{T}<20 \mathrm{~s}, 75 \mathrm{~s}<\mathrm{T}<95 \mathrm{~s}]}\end{array}\end{array}$ & 0.588 & $1.55 \mathrm{E}-04$ & 0.091 & 0.061 & 0.102 \\
\hline $\begin{array}{l}\text { Localized area } \\
{[20 \mathrm{~s}<\mathrm{T}<60 \mathrm{~s}, 96 \mathrm{~s}<\mathrm{T}<120 \mathrm{~s}]}\end{array}$ & 0.588 & 0.033 & 0.173 & 0.109 & 0.149 \\
\hline $\begin{array}{l}\text { Area reduction } \\
\text { using } \theta \text { and } \Phi \text { together }\end{array}$ & 0.119 & $1.55 \mathrm{E}-04$ & 0.051 & 0.056 & 0.026 \\
\hline
\end{tabular}

Table 4.3 shows the statistics of the localization area at times when it was possible to localize. As seen in row 1 of Table 4.3, the mean localized area during the entire measurement is $0.091 \mathrm{~km}^{2}$ with a standard deviation of $0.102 \mathrm{~km}^{2}$. When the hydrophone SNR is low at both sites, less than $10 \mathrm{~dB}$ and in the time ranges listed in row 2 of Table 4.3 , it is observed that the mean localization area is $0.173 \mathrm{~km}^{2}$ with a standard deviation of $0.149 \mathrm{~km}^{2}$. During these times, it is observed in Figure 4.24 that the snowmobile is localized much less frequently. It is also not possible to garner any localization advantage from the elevation data because the snowmobile is not near to either site. ${ }^{83}$

When there is strong SNR at one or both sites, greater than $10 \mathrm{~dB}$ and in the time ranges listed in row 3 of Table 4.3 , the mean localization area is observed to be $0.051 \mathrm{~km}^{2}$ with a standard deviation of $0.026 \mathrm{~km}^{2} .83$

The fourth row of Table 4.3 represents the reduction in area when using both the elevation and the azimuth data compared to using the azimuth data alone. The mean reduction in localization area when including the elevation data is $0.049 \mathrm{~km}^{2}$ with a standard deviation of $0.068 \mathrm{~km}^{2} .83$

\subsubsection{Atmospheric pp Vector Sensors}

The following sections will assess the performance and feasibility of $p p$ acoustic vector sensors for practical tracking applications.

\subsubsection{DOA Spectral Variation}

As explained in section 4.3.2 a total of 31 total experiments were carried out with the $p p$ vector sensors at the KRC. Each experiment featured different vehicle maneuver patterns and speeds. Experiment 90 is chosen for close analysis because it features vehicle maneuvers which cover a large area of the test course and thereby a large range of 
azimuth angles for both sites (Figure 4.26). Also, this experiment provides good potential to examine the localization capabilities of the $p p$ AVS pair at sites 100 and 200.

Due to the variation of the intensity and direction of arrival (DOA) spectra, the time domain DOA for the $p p$ AVS is computed using the MSD process shown in Figure 4.4. This chapter will illustrate the variation in the DOA spectra and the improvement to $p p$ AVS accuracy garnered by implementing the MSD routine as well as comparison to the integration method (Equation 4.38).

Figure 4.27 shows the SPL and DOA spectra for experiment 90 during the selected time instant $\mathrm{t}=25$ seconds. The actual DOA for the time instant is $88^{\circ}$ given by the relative GPS coordinates of the measurement sites and the vehicle position. The estimated DOA by averaging across the entire spectra from 60 to $2000 \mathrm{~Hz}$ is $48^{\circ}$; an error of $40^{\circ}$. A large standard deviation $\left(72^{\circ}\right)$ is measured over the frequency range and is visually evident in the frequency ranges of 60 to $110 \mathrm{~Hz}$ and 800 to $2000 \mathrm{~Hz}$. A lower standard deviation is observed between 110 to $800 \mathrm{~Hz}$ and the DOA spectra better represents the actual DOA within this frequency range.

In order to reduce the standard deviation and error associated with this time interval, the MSD routine is employed. Blocks of 50 frequency lines $(n \Delta f)$ are used with a cutoff standard deviation $\left(\sigma_{c}\right)$ of $20^{\circ}$. The results of the MSD for this time instance is shown in Figure 4.28. The majority of the frequencies used for DOA averaging fall between 150 to $650 \mathrm{~Hz}$ with a small group of frequencies around $180 \mathrm{~Hz}$. These frequencies have a much lower standard deviation between $8^{\circ}$ to $20^{\circ}$. Computing the average DOA across these frequencies yields $79^{\circ}$; an error of $9^{\circ}$. 


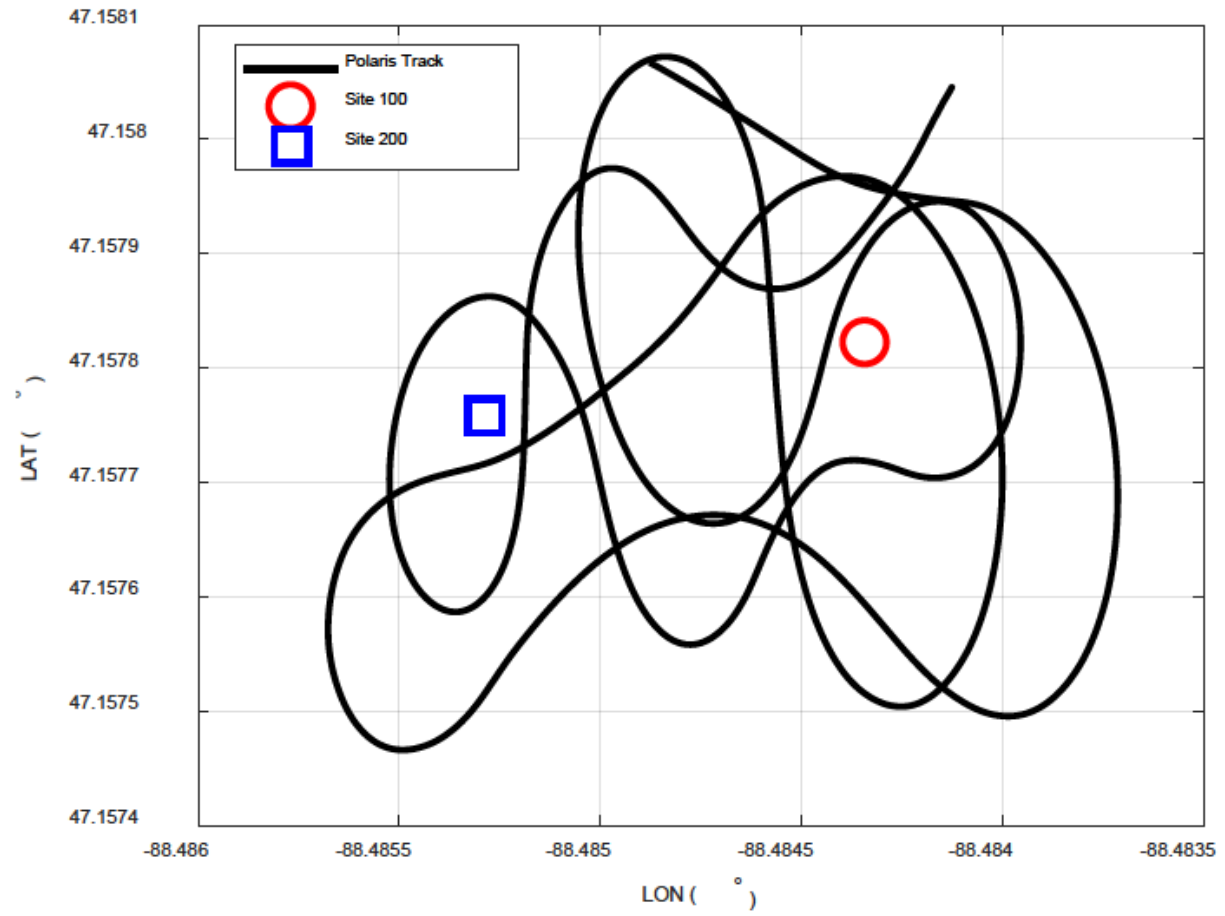

Figure 4.26 GPS track of the Polaris vehicle maneuvers for experiment 90 . 

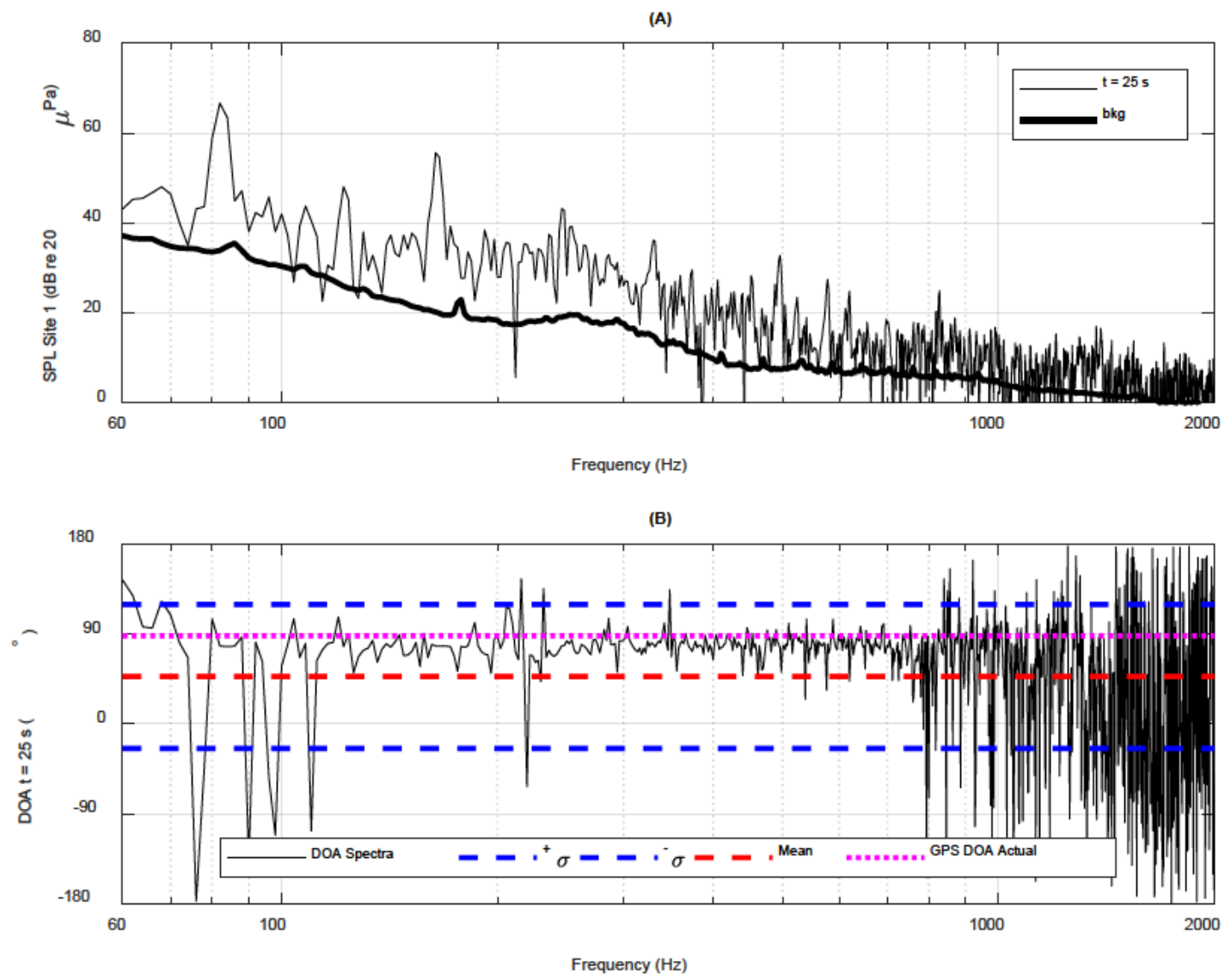

Figure 4.27 (A) SPL and (B) DOA for site 100 experiment 90 at $t=25$ seconds. Mean and standard deviation computed across the entire spectra. Actual DOA $=90^{\circ}$.

Clearly, from this analysis, the MSD routine produces an improvement in the accuracy of the AVS data by reducing the error between measured vs. true DOA. The extent of the improvement realized by the MSD routine is affected by its inputs: blocksize for spectral processing, number of frequency lines used for MSD processing, and cutoff standard deviation. To assess the effect of each of these input parameters, the time domain error (computed with Equation 4.50) is analyzed while changing one input while holding the others constant.

Figure 4.29 shows the DOA error for various number of frequency lines used for MSD processing while holding constant the standard deviation cutoff to $20^{\circ}$ and a blocksize of 51200 samples. It is observed that the error generally decreases as the number of frequency lines decreases. Decreasing the number of frequency lines below approximately 10 lines has detrimental effects on the accuracy for the given data. For example, it is observed that using $3 \Delta f$ for processing increases the error at several time intervals by approximately $10^{\circ}$ in comparison to that of a $10 \Delta f$ process. 


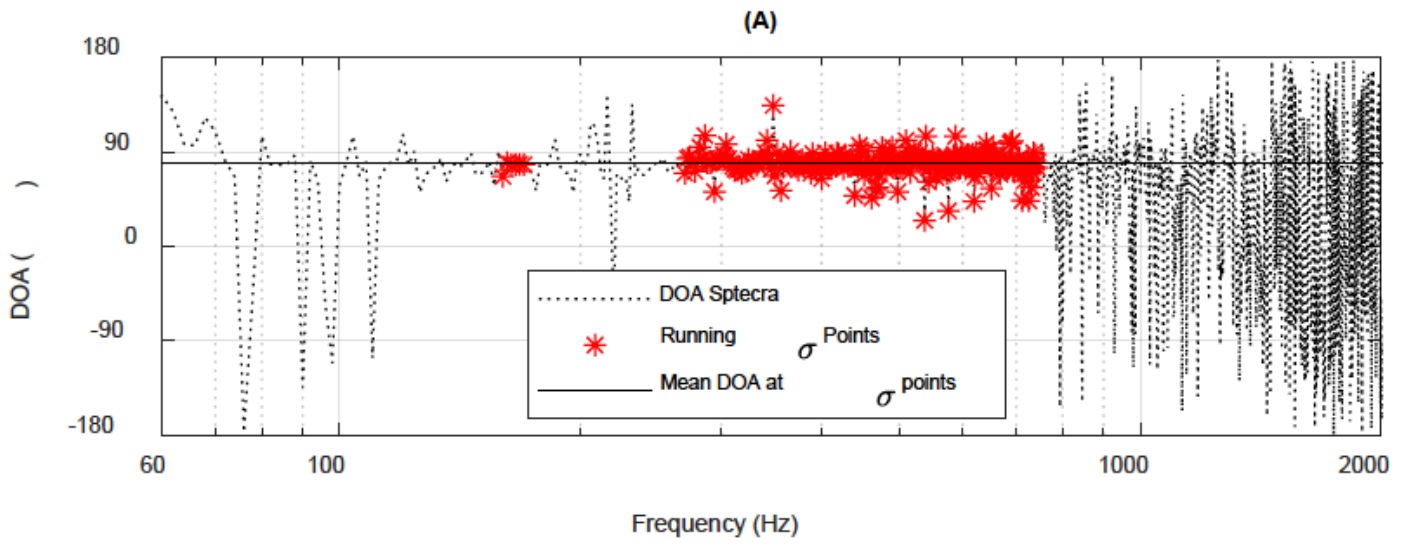

(B)

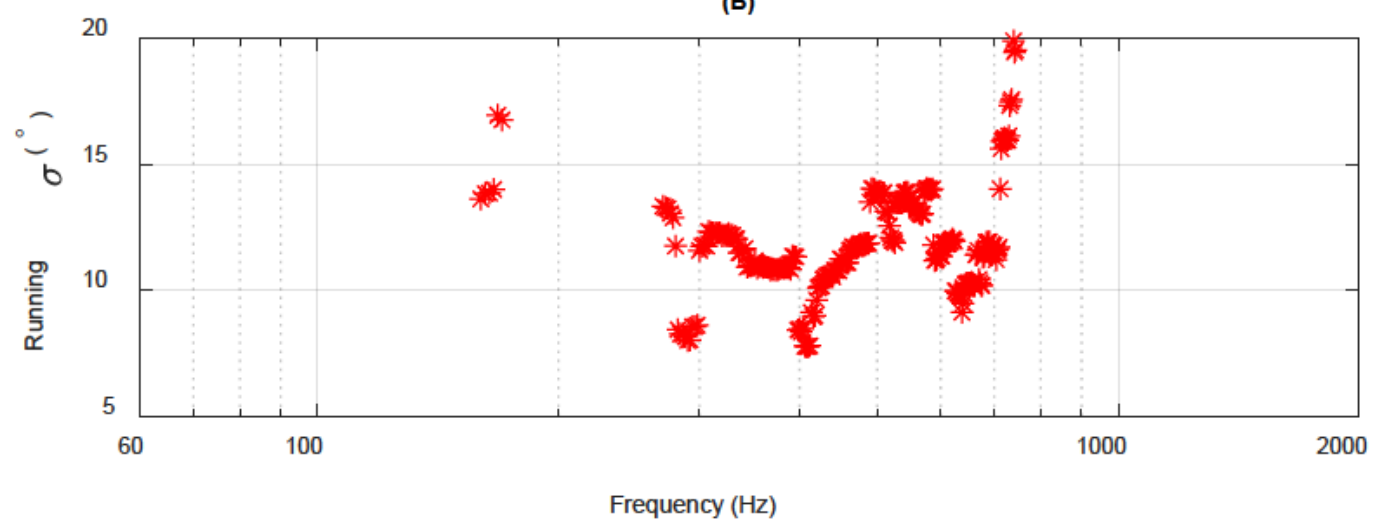

Figure 4.28 DOA spectra and MSD for time instant $t=25$ seconds. MSD computed across 50 frequency lines. Frequencies included for averaging when standard deviation less than $20^{\circ}$. Actual $\mathrm{DOA}=90^{\circ}$

Figure 4.30 shows the DOA error for several standard deviation cutoff values while holding constant the number of frequency lines to $10 \Delta f$ and a blocksize of 51200 samples. A cutoff value not being used indicates the entire spectrum is used for averaging during the time interval. That is to say, when the whole spectrum is used, the standard deviation routine is not implemented. Implementing the MSD with cutoffs at $30^{\circ}$ and $20^{\circ}$ significantly reduce the DOA error; on the order of $50^{\circ}$ improvement in the most extreme cases. However, further restriction of the cutoff to $5^{\circ}$ is detrimental; increasing the error. 


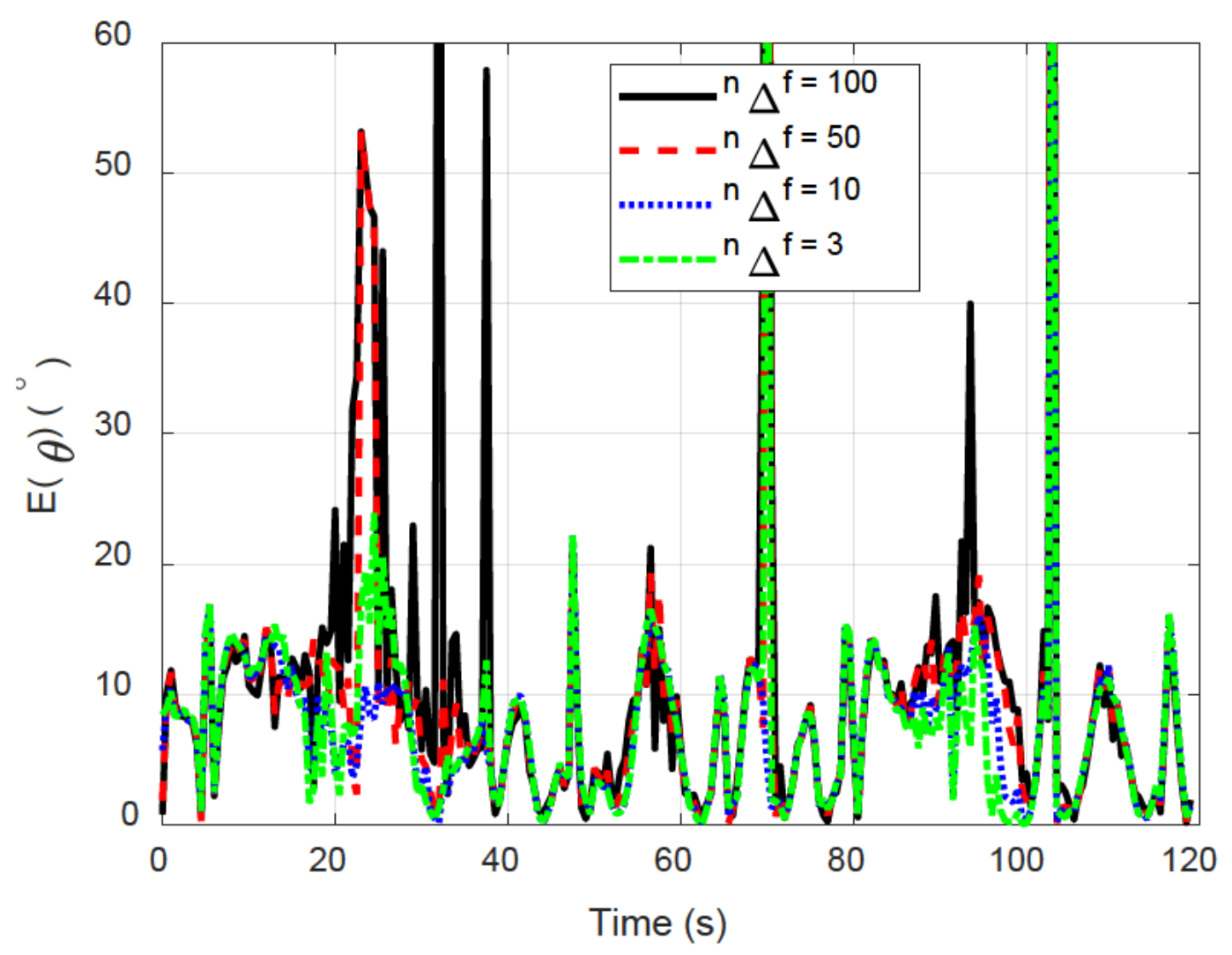

Figure 4.29 Site 100 AVS DOA error for various number of frequency lines in $\sigma$ processing. Experiment $90 . \mathrm{N}=25600$ samples, $\sigma_{c}=20^{\circ}$

Figure 4.31 shows the DOA error for several blocksizes while holding constant the number of frequency lines to $10 \Delta f$ and the standard deviation cutoff to $20^{\circ}$. The blocksize effects both the time resolution and the frequency resolution of the processed data per Equations 4.42 through 4.44. For a blocksize which is equal to the sample rate of the data, the frequency resolution of the DOA spectra is $1 \mathrm{~Hz}$ and the time-domain resolution is 1 second. Decreasing the blocksize results in a coarser frequency resolution and finer time resolution. Smaller blocksizes, for example 2560 and 10240 samples, are observed to induce larger errors for most time instances. While some time instances do have lower errors with larger blocksize, the benefit at these time instances does not compensate for the large errors on the order of $60^{\circ}$. A blocksize of 51200 sample is observed to produce the optimal performance for the data shown. 


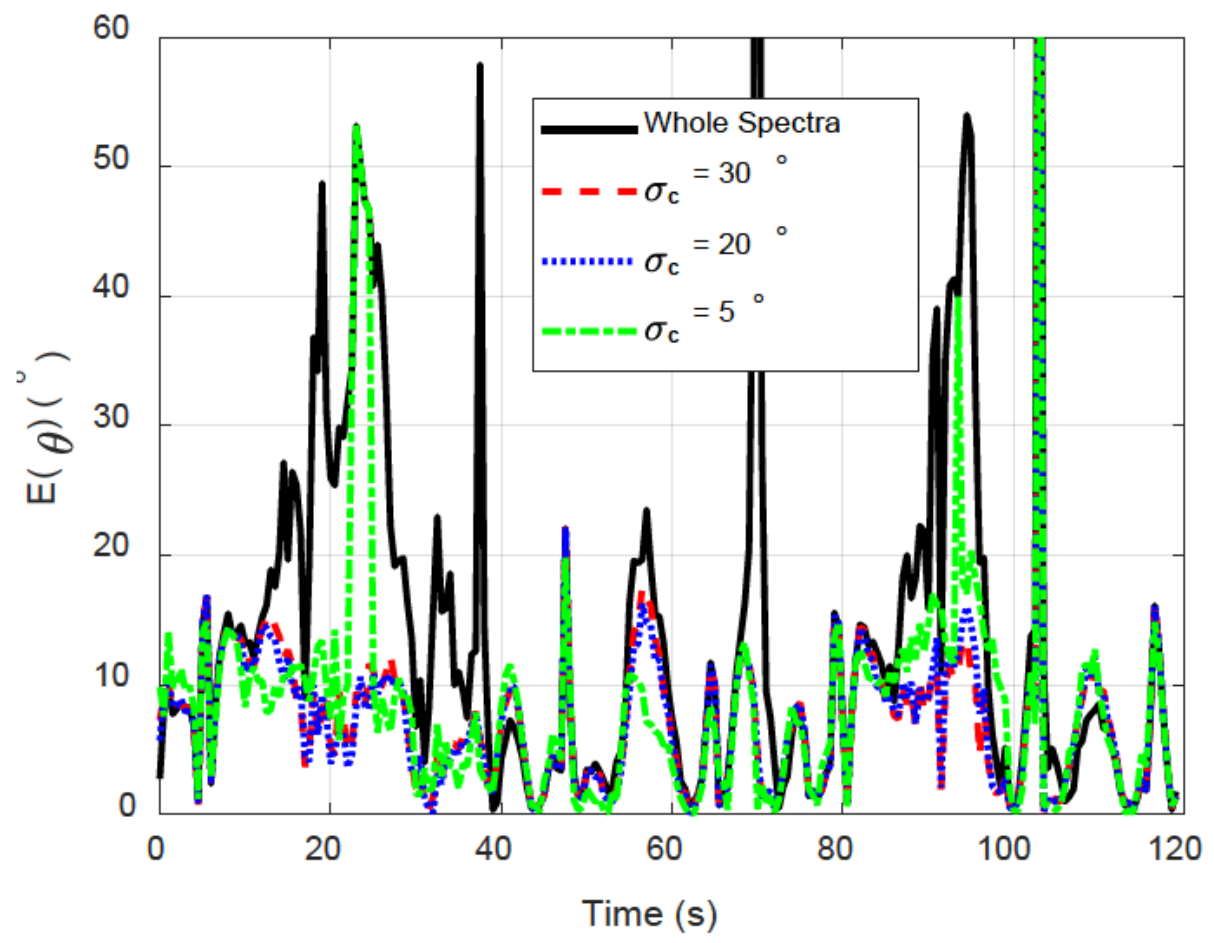

Figure 4.30 Site 100 AVS DOA error for various standard deviation cutoffs. Experiment 90 , $\mathrm{N}=25600$ samples, $n \Delta f=10$ frequency lines.

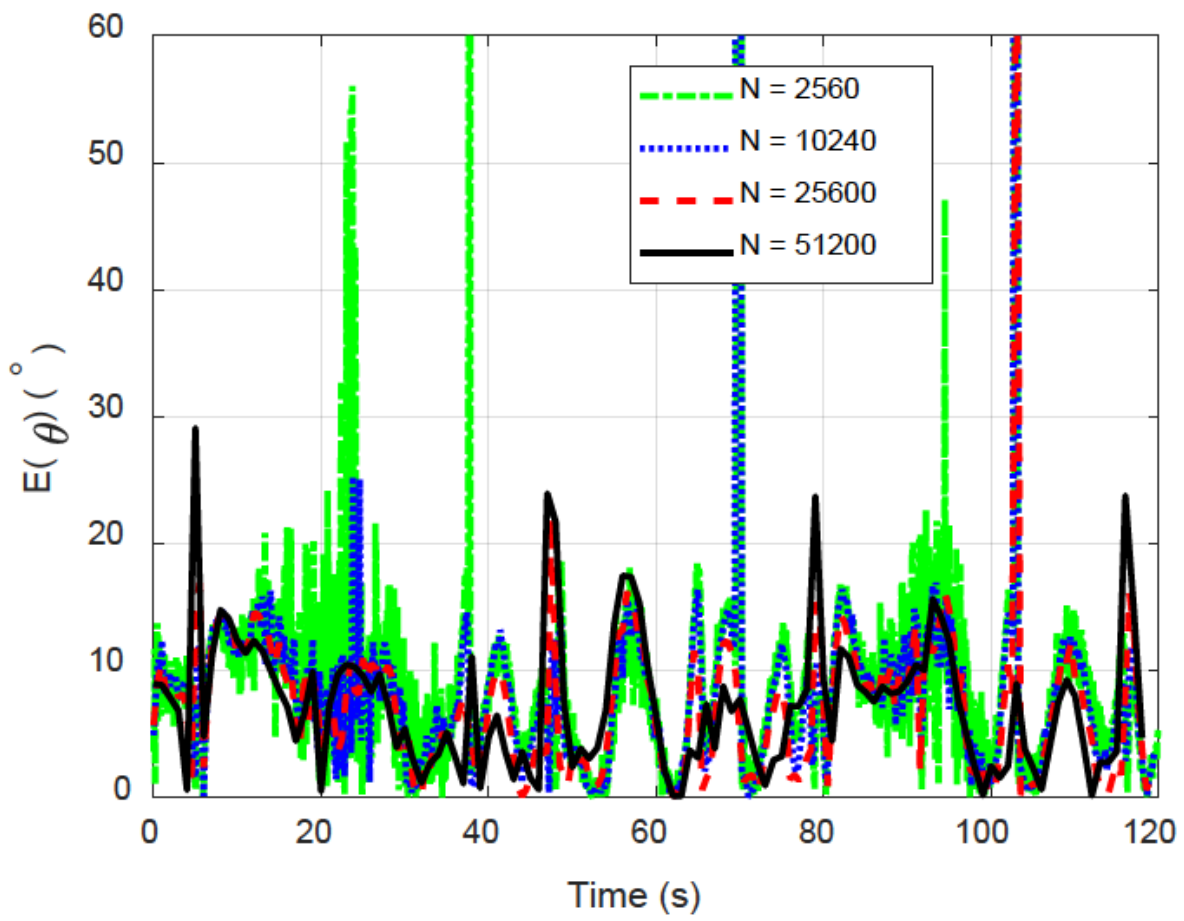

Figure 4.31 Site 100 AVS DOA error for various blocksizes. Experiment $90, n \Delta f=10$ frequency lines, and $\sigma_{c}=20^{\circ}$. 
Also observed in the figures is a large error at 103 seconds which is not affected by the parameters discussed. This error is expected to be due to $180^{\circ}$ phase wrapping. Given that $\pm 180^{\circ}$ is really a single value, the error may appear to be large in the proximity to this value when in reality the error is only a few degrees. For example, an error between a measured DOA of $+179^{\circ}$ and an actual DOA of $-179^{\circ}$ yields an absolute error of $2^{\circ}$. However, when the DOA is wrapped to $180^{\circ}$ this may be represented as an error of $358^{\circ}$. This issue is exacerbated by difference in $\Delta t$ between the DOA estimates from the vector sensor and the GPS. Processing of the AVS data with a blocksize of 51200 samples yields a $\Delta t$ of 1 second between DOA estimates. The GPS sampling at $100 \mathrm{~Hz}$ yields a $\Delta t$ of 0.01 second between DOA estimates. Down-sampling the GPS data by a factor of 100 provides the correct number of data points as well as the correct $\Delta t$. However, the inherent difference in absolute time between the GPS and acoustic data system introduces error when down-sampling and time-aligning. In the proximity of wrap-around points, these small differences are magnified. Furthermore, this error not mitigated by simply wrapping the DOA to $360^{\circ}$ because the increased error will simply be visible at any wrap-around value selected. While this effect is observed in the error plots, it is generally not an issue with the accuracy of the AVS, but rather an artifact of phasewrapping and is not a problem for determination of DOA.

Given this analysis, a blocksize of 51200 samples, a cutoff standard deviation of $20^{\circ}$, and 10 frequency lines per processing block are selected to be the parameters which are best suited for processing the collected datasets. While each dataset is expected to possess some variability, these parameters provide a good starting point for accurate processing.

This type of MSD processing relies on some frequencies which having a low standard deviation and providing an accurate representation of the true DOA. Therefore, this processing routine may significantly increase the AVS accuracy when a portion of the DOA spectra has high standard deviation and a portion has low standard deviation. However, when the standard deviation of the DOA spectra is large across all frequencies for a given time instant, there is essentially no benefit to using the MSD processing and the entire spectra must be used to provide a DOA estimate at the given time in question. This also explains why the DOA standard deviation at a given time instant may exceed the standard deviation cutoff specified for processing.

\subsubsection{DOA of the Non-Stationary Source}

The computed direction of arrival (DOA) from the AVS using the MSD method is shown for sites 100 and 200 respectively in Figure 4.32(a) and (b) for experiment 90 (note that a zoomed in view of the data may be seen in Figure 4.33 for closer analysis and comparison of the data between 20 to 60 seconds). The DOA at each time step represents the mean of selected DOA spectral lines which were determined via the MSD processing routine. The number of frequency lines used for each block in standard deviation processing was selected to be $10 \Delta f$, the standard deviation cutoff was selected to be $20^{\circ}$, and the blocksize for spectral processing was selected to be 25600 samples. Justification for these choices is explained in the previous section. 
Good agreement is observed between the GPS ground truth DOA and the DOA computed from the vector sensors. The standard deviation of the DOA spectra at each time instant (Figure 4.32 (d)) is observed to be between $2^{\circ}$ to $40^{\circ}$. Instances when the standard deviation is above $20^{\circ}$ indicate that the MSD routine was not able to reduce the error, and the full spectra was used for averaging.

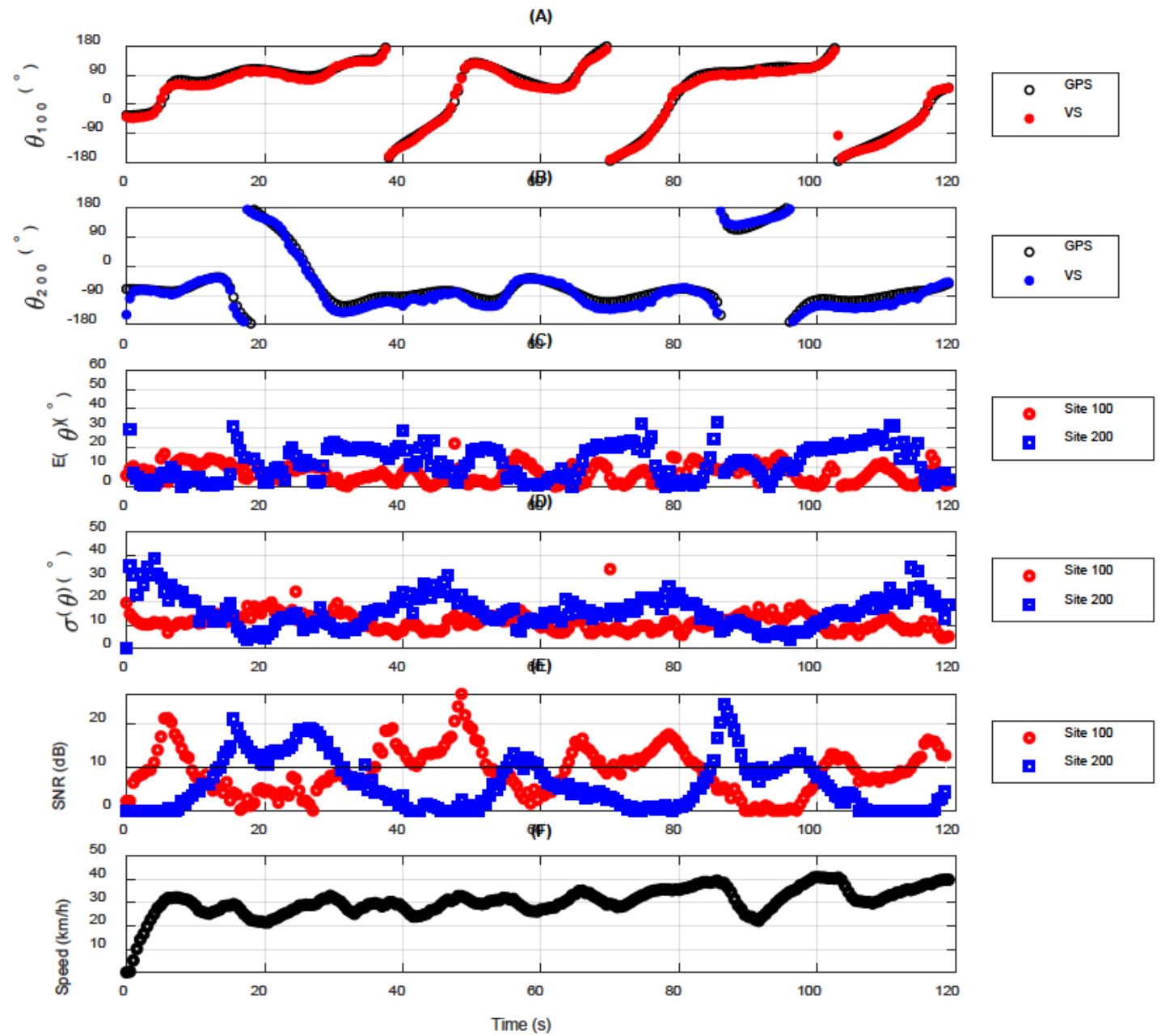

Figure $4.32 p p$ vector sensor data for experiment 90. (A) Site 100 DOA. (B) Site 200 DOA. (C) Absolute angular error between DOA measured with AVS and ground truth. (D) Standard deviation of site 100 and site 200 DOA. (E) SNR at measurement locations. (F) Vehicle speed. DOA data in this figure are computed with MSD processing.

The absolute error between the vector sensor DOA in comparison to the ground truth DOA determined with Equation 4.50 is observed to be between $0^{\circ}$ to $30^{\circ}$ in Figure 4.32 (c). Generally speaking, decreases in error are observed when SNR (Figure 4.32 (e)) increases, and increases in error are observed when the SNR decreases. Some spikes in azimuthal error are observed due to $\pm 180^{\circ}$ wrap-around, however, these spikes are 
artifacts; not actual representations of the error. It is noteworthy that even when SNR is very low, the AVS still reasonably approximates the true DOA. While the error in lowSNR scenarios is greater than high-SNR scenarios by $10^{\circ}$ to $20^{\circ}$, the AVS is still feasible for short-range tracking with low SNR.

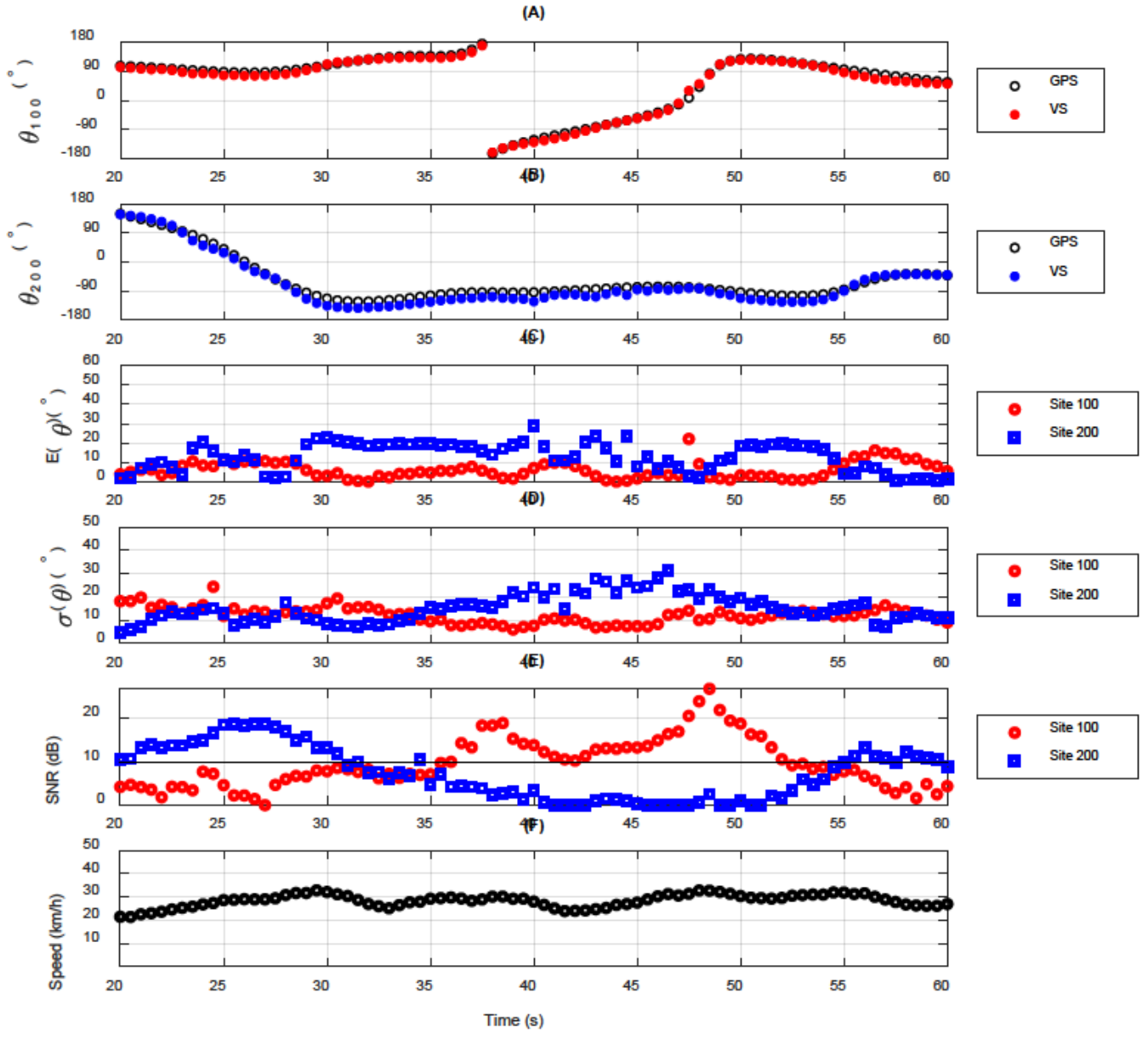

Figure 4.33 Zoomed in view of Figure 4.32 from 20 to 60 seconds.

The linear vehicle speed is observed to be between 20 to $40 \mathrm{kmph}$ for the majority of the 120 -second data collect (Figure 4.32 (f)). Increases in vehicle speed appear to be correlated with increases in SNR which is likely due to louder exhaust output during heavy acceleration. These increases in SNR correspond to reduced error and better tracking with the AVS.

Appendix A.4 contains plots for all experiments including time domain DOA, error, standard deviation, SNR, and vehicle speed. 


\subsubsection{MSD vs. Integration DOA Techniques}

The direct integration technique which is described by Wajid et al. is implemented via Equations 4.38 through $4.41^{99}$. Utilizing this method, the entire frequency spectra is used to determine the DOA estimation. This approach is reasonable when there is spectral energy across the entire bandwidth. However, for tonal sources and signals with various SNR across the frequency range, the integration technique may induce errors in DOA estimation.

The MSD method presented in previous sections has been developed to mitigate this issue. For signals such as the one produced by the UTV, the MSD approach allows for adaptation to the specific frequency content and SNR possessed by the signal. Once again, this is accomplished by selecting particular indices to average for which the standard deviation of the DOA spectra is low.
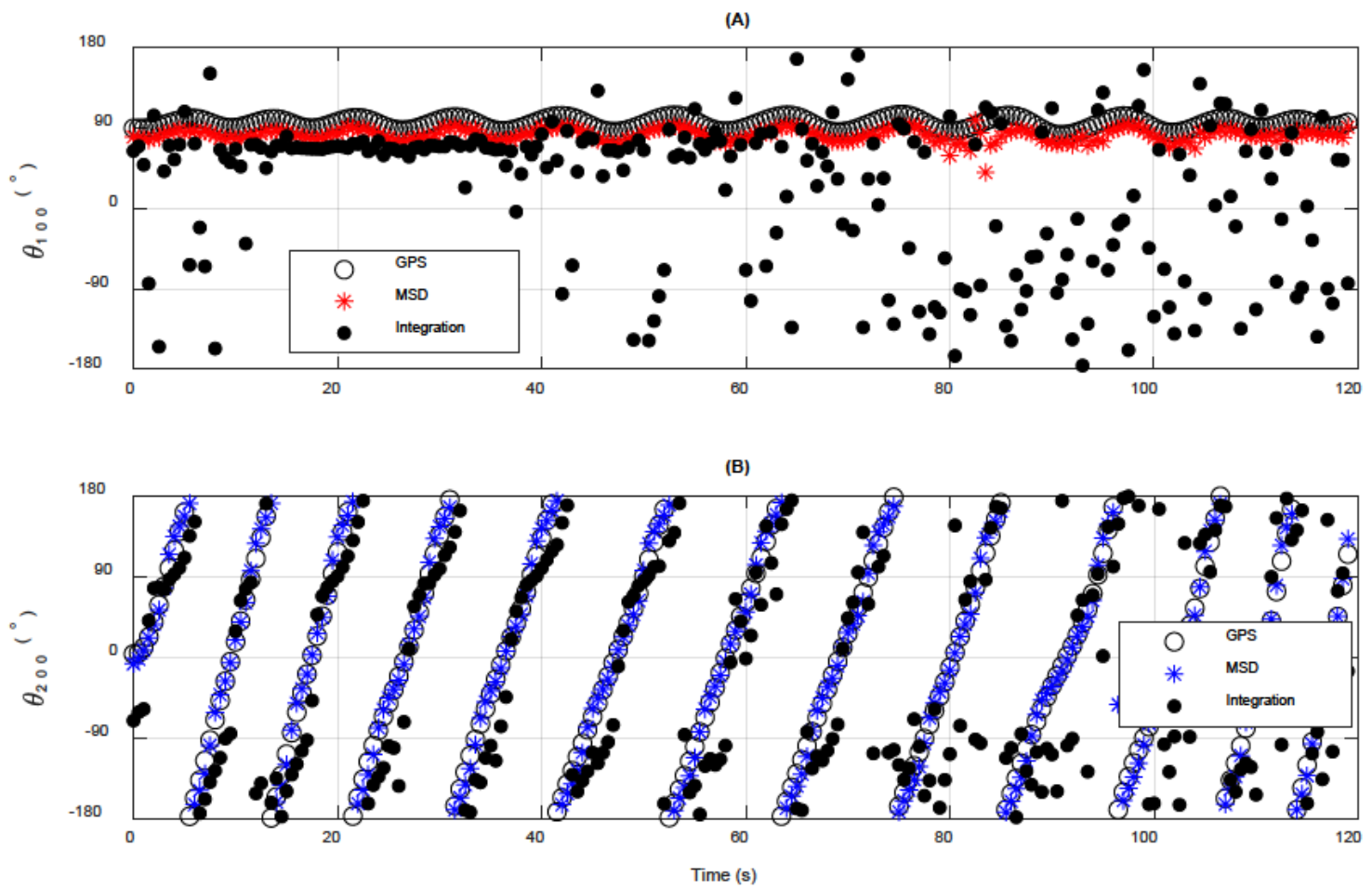

Figure 4.34 Comparison of integration vs MSD method for DOA estimation, experiment 80 .

It is observed in Figure 4.34 that the integration method provides some reasonable correlation with the ground truth GPS data for some given time instances, but also results in very large errors for a majority of the time instances. The MSD method is observed to provide better DOA estimation and smaller errors across the entirety of the experiment. This exemplifies the utility of the MSD routine to provide tracking and DOA estimation in situations where source frequency content and SNR is variable. 


\subsubsection{Azimuthal Error of pp AVS}

It is of interest to investigate the error in the DOA measurements with respect to the azimuthal direction of the vector sensor. This is best assessed when the vehicle was traveling around the vector sensor site in a circular pattern. This corresponds to experiments 67 to 71 for site 100 and experiments 79 to 83 for site 200. Experiment 80 is chosen for closer inspection (Figure 4.35). DOA azimuthal error plots for all experiments are shown in Appendix A.4.8.

For the coordinate system defined in Figure 4.3, the Cartesian quadrants are defined as Q1: $0^{\circ}$ to $+90^{\circ}, \mathrm{Q} 2:+90^{\circ}$ to $+180^{\circ}$, Q3: $-90^{\circ}$ to $-180^{\circ}$, and Q4: $0^{\circ}$ to $-90^{\circ}$. Based on finite element simulations and stationary lab experiments carried out by Wajid et al. ${ }^{99}$ for AVS similar to the one shown in Figure 4.3, the lowest DOA error should be observed in the quadrant formed by the positive principle axes of the AVS (Q1). In general, the observed error should be least in Q1, followed by the opposite quadrant; Q3. The error in Q2 and Q4 are of roughly equal magnitude and are higher than Q3 (note that the data presented by Wajid et al. are transposed to the same coordinate system used here by multiplying by -1 and subtracting $90^{\circ}$ ). Furthermore, minimums in error, indicating higher AVS accuracy, should be observed at azimuth angles which are at roughly integer multiples of 45 degrees in Q1 and Q3, but multiples of 90 degrees in Q2 and Q4.

Similar results are observed in Figure 4.35 from measured data at the KRC experiments. The minimums approach zero degrees of error while the maximums are on the order of 10 to 20 degrees of error. The absolute value of the error measured is approximately an order of magnitude higher than that reported by Wajid ${ }^{99}$. However, this is reasonable considering simulations by Wajid were conducted with several advantages: 1) The sources were at discrete stationary DOA angular positions, 2) The sources were singlefrequency and the source frequency was known, 3) The source-to-receiver distance was 1 $\mathrm{m}, 4)$ In the case of simulations, the SNR was infinite, and 5) 10,000 averages were used for estimating the DOA.

The data in Figure 4.35 are for vehicle speeds on the order of $20 \mathrm{kmph}$. Higher vehicle speeds result in higher DOA errors. Signal-to-noise-ratio for experiment 80 was on the order of $20 \mathrm{~dB}$ measured as the average SNR across the DOA spectra. That is to say, this doesn't account for variations in SNR with respect to frequency. Furthermore, the source vehicle contained unknown and complex frequency content and only one average per time instant was used for estimating DOA. 
(A)

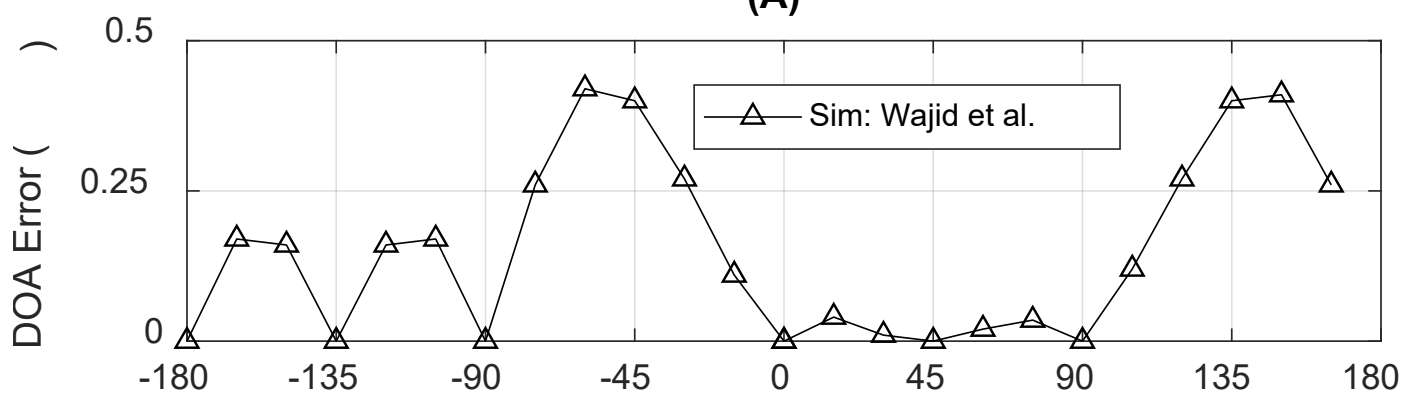

(B)

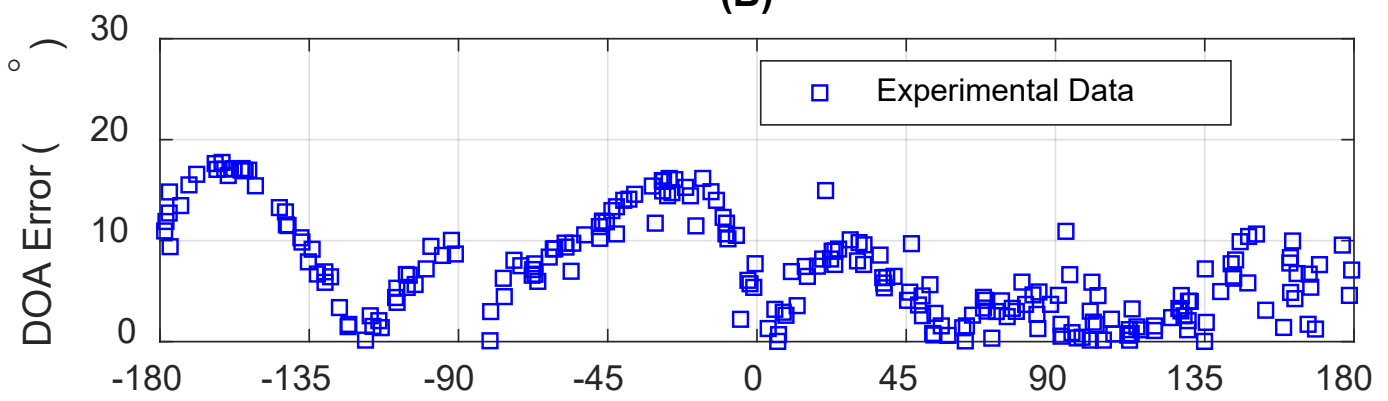

(C)

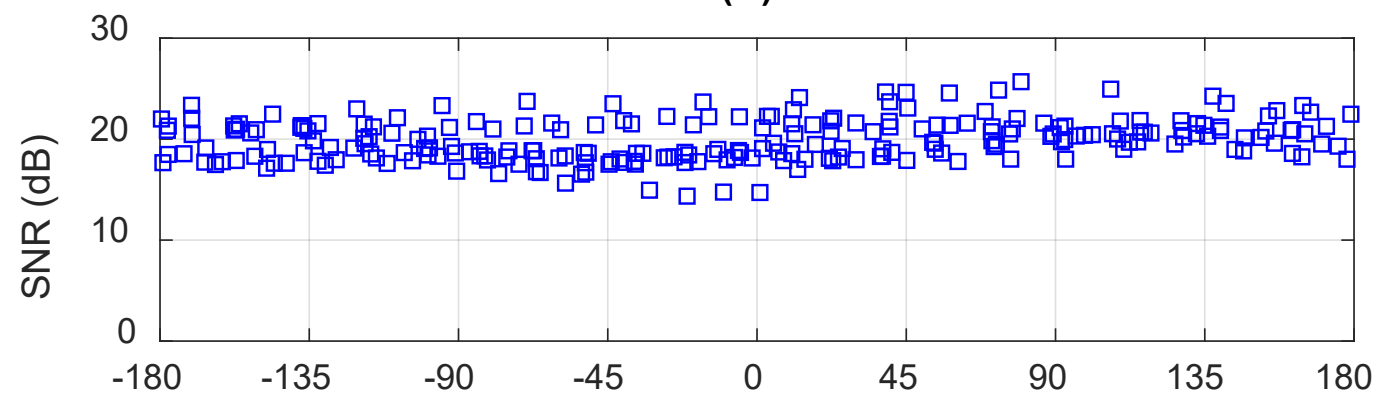

(D)

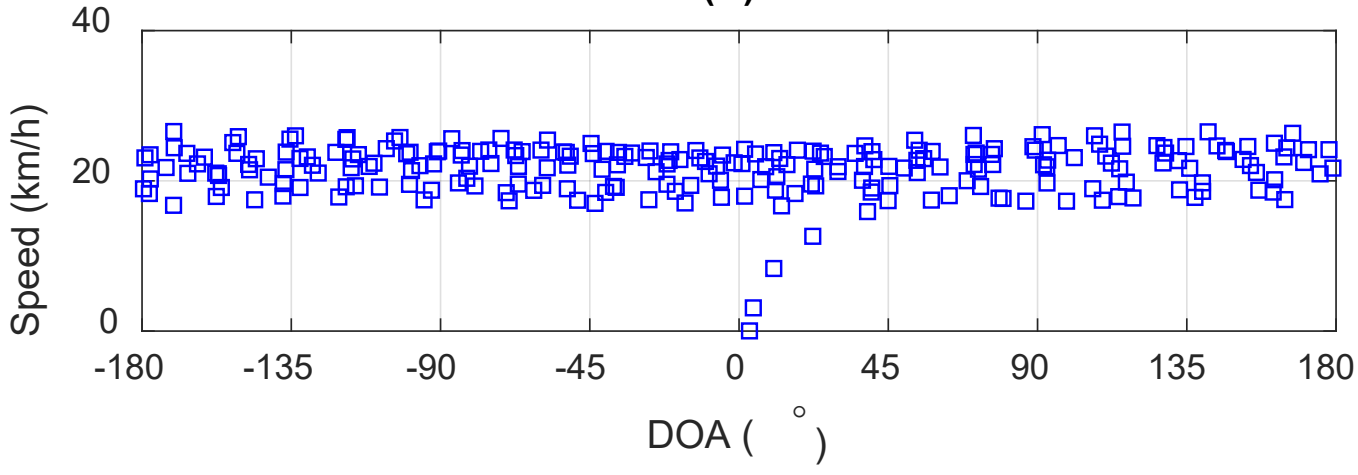

Figure 4.35 Azimuthal AVS error site 200, experiment 80. $\mathrm{N}=25600, n \Delta f=10, \sigma_{c}=20^{\circ}$. (A) FEA error for stationary source with infinite SNR from Wajid et al. (B) Measured DOA error, (C) SNR, (D) Vehicle speed. 
(A)

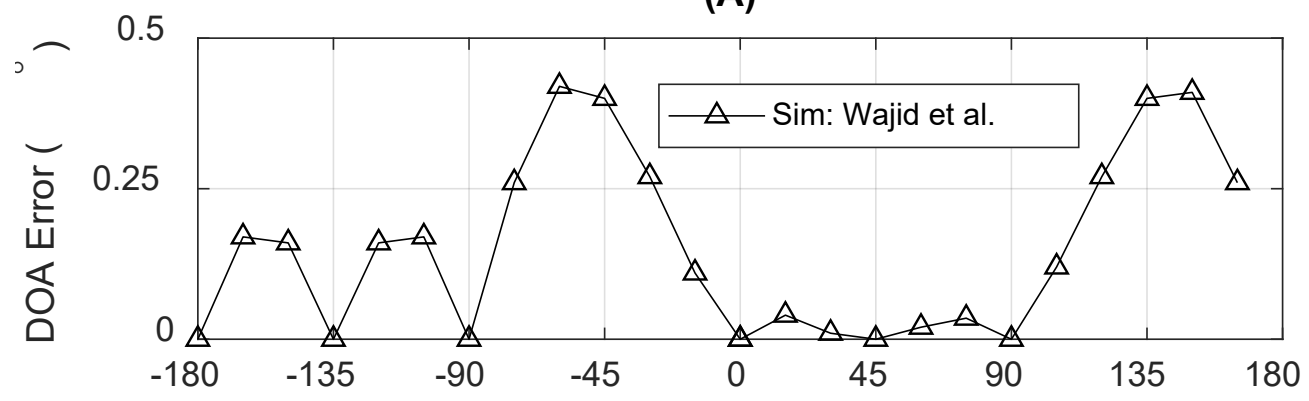

(B)

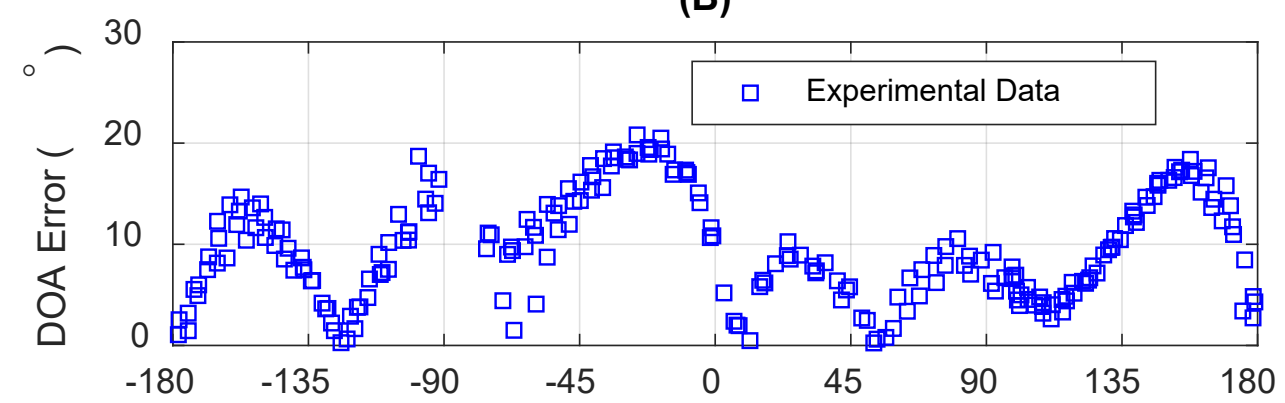

(C)

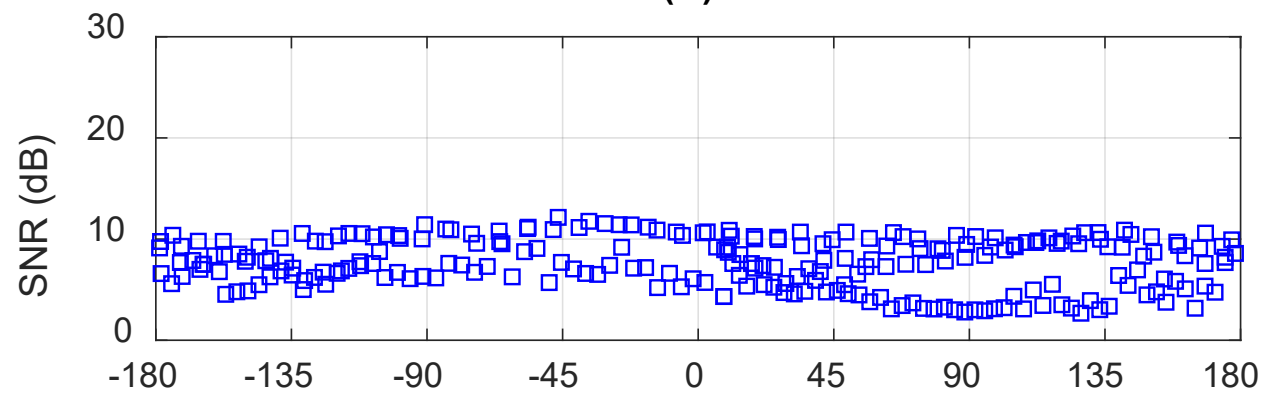

(D)

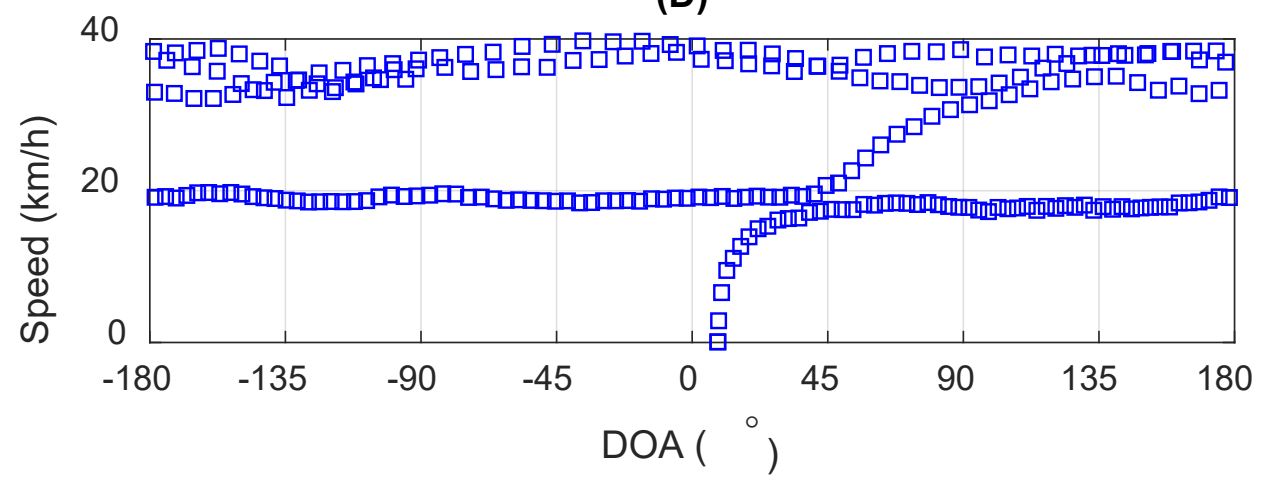

Figure 4.36 Azimuthal AVS error site 200, experiment 83. $\mathrm{N}=25600, n \Delta f=10, \sigma_{c}=20^{\circ}$.(A) FEA error for stationary source with infinite SNR from Wajid et al. (B) Measured DOA error, (C) SNR, (D) Vehicle speed. 
(A)

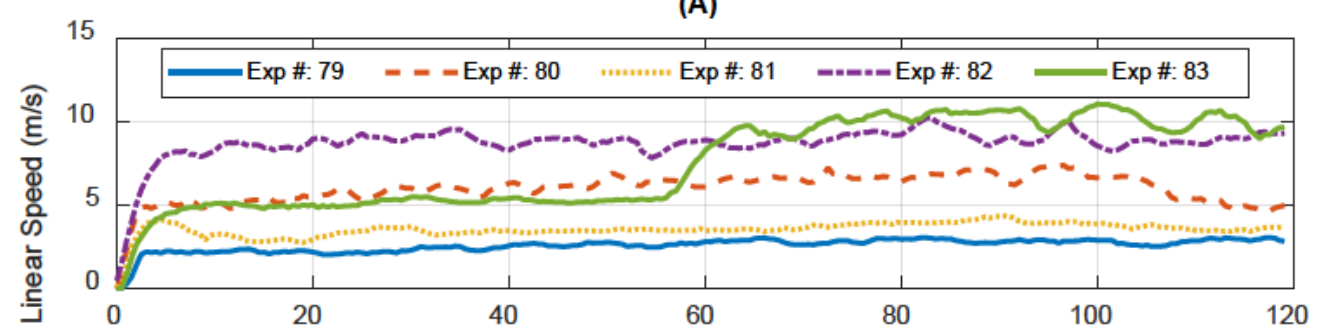

(B)

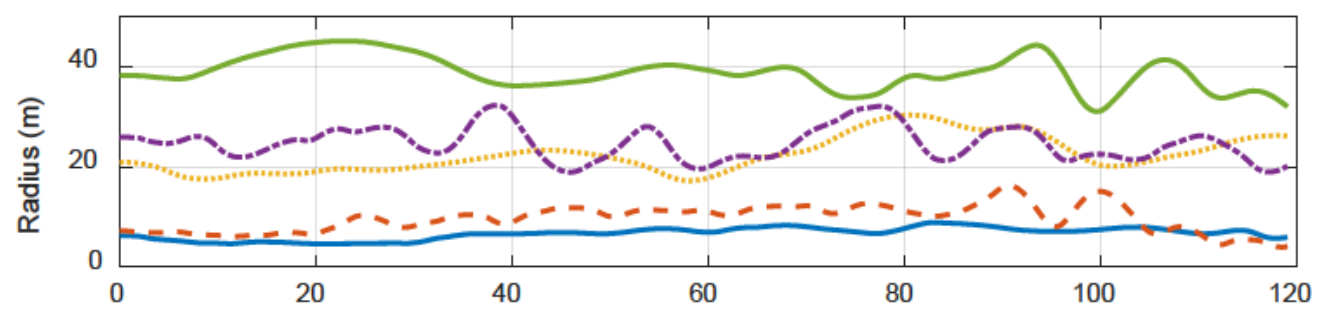

(C)

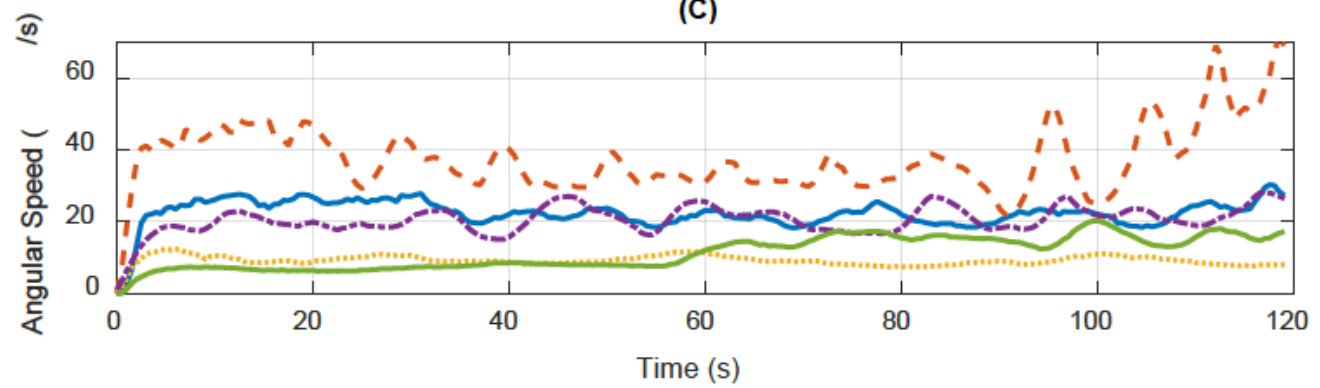

Figure 4.37 Vehicle speed characteristics for experiments 79 to 83 relative to site 200 . (A) Vehicle Speed. (B) Distance between site 200 and vehicle position. (C) Angular Speed around site 200.

Figure 4.37 shows the linear vehicle speed, distance between the measurement site and the vehicle (ie. radius), and angular speed around site 200 for experiments 79 to 83 . Based on the nominal radius of $10 \mathrm{~m}$ for experiment 80 and the vehicle speed of $5.56 \mathrm{~m} / \mathrm{s}$ $(20 \mathrm{kmph})$, the angular speed of the vehicle around site 200 is approximated to be $32 \% \mathrm{~s}$. Given that the sample rate of time data acquisition was $51200 \mathrm{~Hz}$ and the block size of each processed time interval was 25600 samples, the time duration of each processed block was 0.5 seconds. That is to say, the time resolution on the plots in Figure 4.32 is 0.5 seconds. During this time the vehicle traveled through $16^{\circ}$ of arc around the site. This explains the maximum DOA errors on the order of 10 to 20 degrees.

By comparison, experiment 83 (Figure 4.36) has a nominal linear speed of $7.5 \mathrm{~m} / \mathrm{s}$ and nominal radius of $39 \mathrm{~m}$, resulting in a nominal angular speed of $11.3 \%$ s around site 200 . Therefore, during one 0.5 second processing block duration, the arc traveled by the vehicle is approximately $5.7^{\circ}$. It is expected that this would in turn lower the angular error realized for experiment 83 in comparison to experiment 80 . However, the error observed is still on the order of 10 to 20 degrees. This is likely explained because the SNR in experiment 83 is lower than $10 \mathrm{~dB}$ for many of the measured data points. 
Generally speaking, the SNR is higher when the linear vehicle speed increases and when the distance between the vehicle and the AVS is small. When the vehicle is traveling in a circular path around the measurement site, these two factors are inversely proportional based on the equation,

$$
v=R \dot{\theta}
$$

where $v$ is the linear vehicle speed, $R$ is the radius of the circular path, and $\dot{\theta}$ is the angular speed of the vehicle around the measurement site. This relationship ensures that as the angular speed decreases, the radius must increase to maintain the same linear vehicle speed. Additionally, it is impossible to decrease both the radius and the angular speed while increasing the vehicles linear speed. Therefore, if the vehicle is traveling in a circular path around the measurement site, it is very difficult to make a measurement where there is high SNR and low angular speed around the measurement site. This effectively explains why the DOA error remains on the order of 10 to 20 degrees no matter the angular vehicle speed considered, due to the drop in SNR as the radius decreases.

\subsubsection{Analytical DOA comparison}

To better understand the lobed behavior of the DOA error, an analytical model was created as explained in Section 4.2.8. Within this model, two cases are considered. First, the direct path propagation only is considered between the acoustic source and the receiver (the AVS in this case), and second, the direct path plus the ground-reflected paths between source and receiver are considered. Also of importance are the effects of various source heights, receiver heights, and frequencies (or wavelengths) of the signal.

For this analysis, the relative source and receiver positions were determined with the GPS data from experiment 81 . The pressures at the AVS microphones were then simulated via Equation 4.53 based on the relative positions at each time interval considered during the experiment.

Figure 4.38 and Figure 4.39 show direct comparison of the analytical DOA error for a direct-path-only and a direct-plus-reflected-path situations respectively. For both models the source height and receiver height are constant at $1 \mathrm{~m}$ and $2.13 \mathrm{~m}$ respectively and the source frequency is $1000 \mathrm{~Hz}$. It is observed that there is on the order of 3 to $8^{\circ}$ of error with some spatial oscillation with the direct-only simulation. With the direct-plusreflected simulation, lobed behavior is observed which is very similar to that which is experimentally observed in Figure 4.35. The peaks of the lobes are on the order of $20^{\circ}$ of error which is consistent with experimental results. Furthermore, the dips in the error are roughly at integer multiples of $45^{\circ}$, which is once again consistent with the experimental results. This finding indicates that the reflected path has some significant contributions to the error observed at the AVS locations; at least for this given situation. 


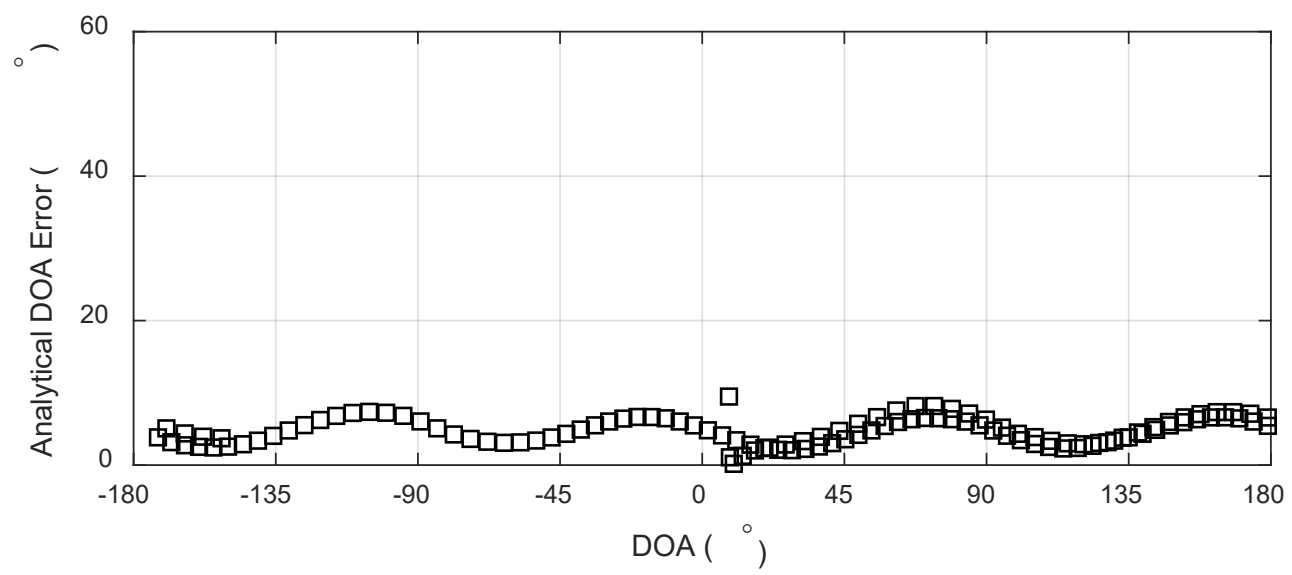

Figure 4.38 Analytical AVS DOA error considering direct path propagation only. $h_{s}=1 \mathrm{~m}, f=$ $1000 \mathrm{~Hz}$.

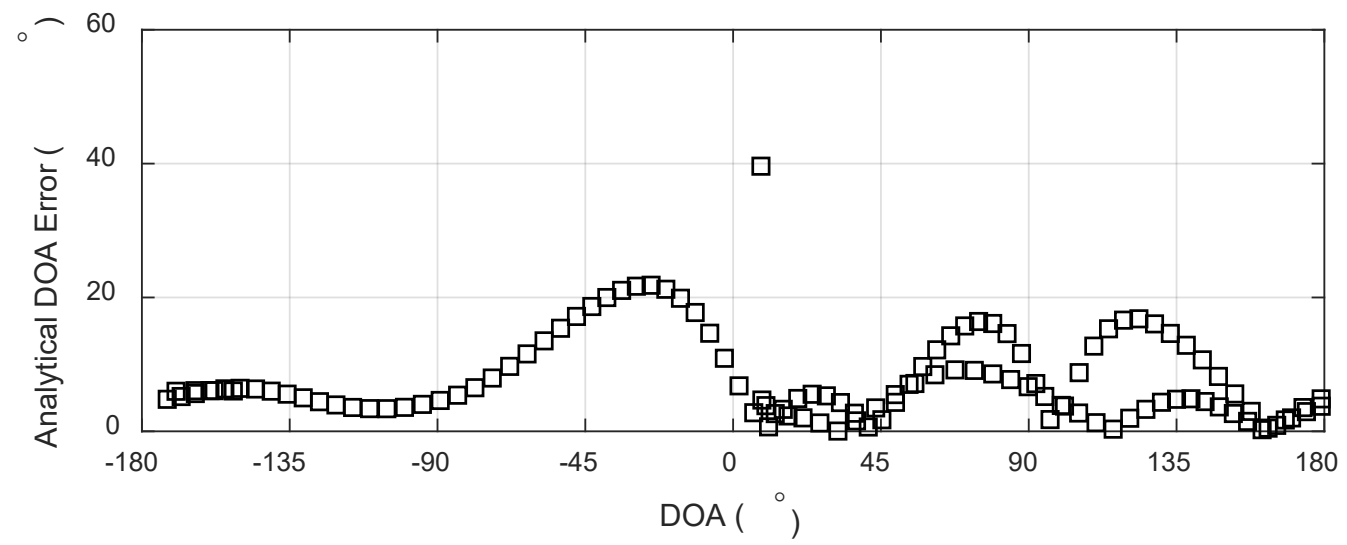

Figure 4.39 Analytical AVS DOA error considering direct and reflected paths. $h_{s}=1 \mathrm{~m}, f=1000$ $\mathrm{Hz}$.

Changing the source height, receiver height, or source frequency may also have significant impact on the DOA error estimations. For this analysis, the receiver height was chosen to remain constant because it was constant for all of the experiments considered. However, the true source height as well as source frequency are unknown. The source height and source frequency depend on underlying acoustics of the target vehicle which cause different portions of the vehicle to radiate sound. In fact, multiple frequencies are radiated from the vehicle simultaneously at different frequencies and intensities. For example, the engine, the exhaust tip, the transmission, and the wheel interaction with the ground all contribute complex frequency content which radiate from different parts of the vehicle. It is for this reason a wide range of frequencies and source heights are analyzed here.

The dimensionless ratio of source height $\left(h_{s}\right)$ to wavelength $(\lambda)$ is defined in order to provide an understanding of the variables being compared. Figure 4.40 shows the increase of $h_{S} / \lambda$ as frequency increases for a given source height. 
The DOA error for a constant frequency and variable source height is analyzed in Figure 4.41. This corresponds to a column of data in Figure 4.40 at $1000 \mathrm{~Hz}$. It is observed that for $h_{s} / \lambda$ less than or equal to 0.73 , the error is on the order of $5^{0}$ with little spatial lobing. As $h_{s} / \lambda$ increases beyond this threshold, the error becomes larger and spatial lobing is observed.

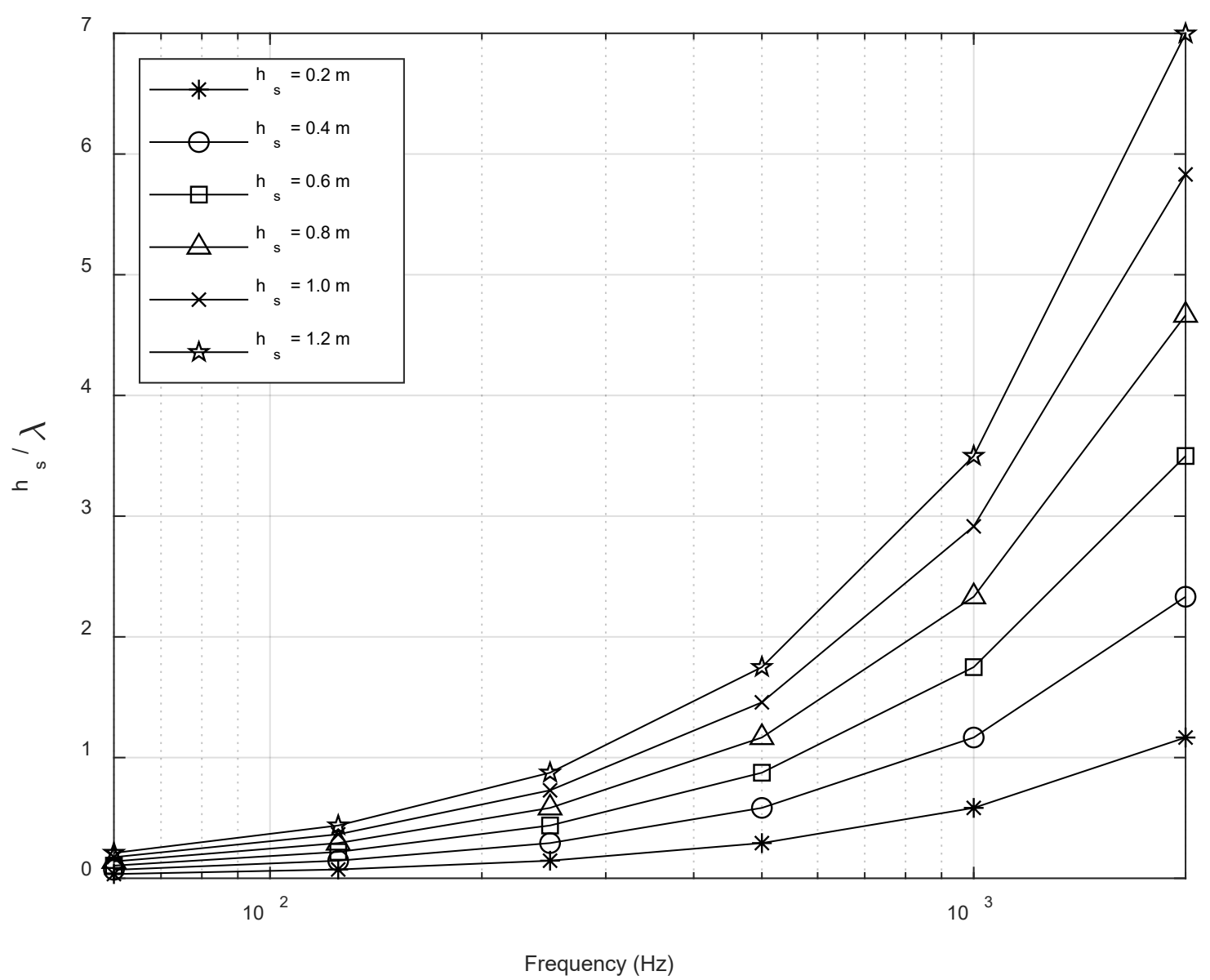

Figure 4.40 Source height to wavelength ratio for various frequencies.

It is also noted that the particular magnitude of error is also dependent on the source location relative to the receiver. For example, in Figure 4.41 with $h_{s} / \lambda$ of 2.92, the lobes between 0 to $180^{\circ}$ have some variation in error amplitude is observed between 18 to $40^{\circ}$. This is due to the relative position between the source and receiver changing during the experiment. For experiment 81 , the vehicle is moving in a circular pattern around the AVS site, however, the circle is not perfect and the effective radius of the circle varies between passes by the site. The difference in relative position accounts for different incident angle, and therefore different DOA error. 

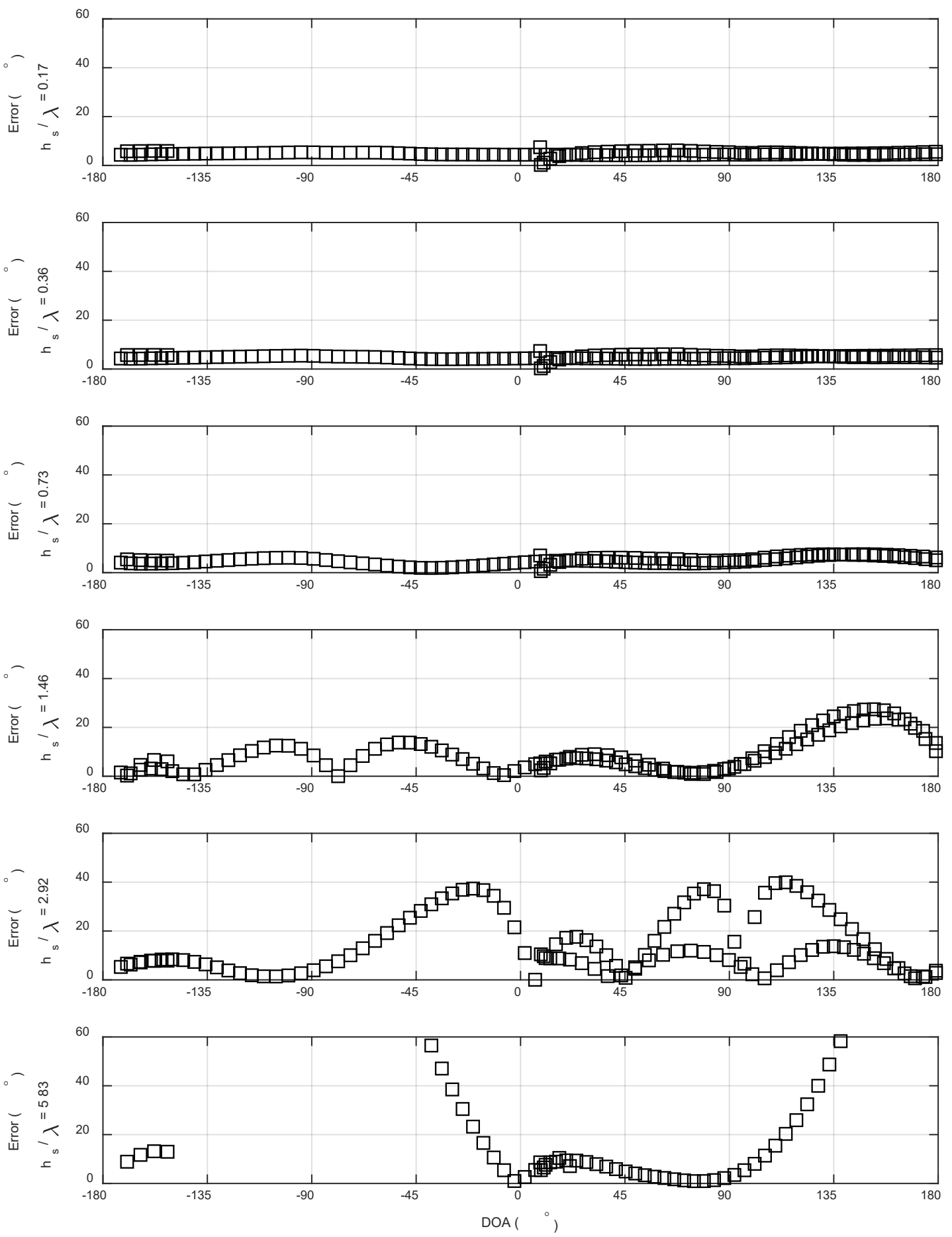

Figure 4.41 DOA error for various $h_{s} / \lambda$ with increasing source height and constant frequency $f=$ $1000 \mathrm{~Hz}$. 

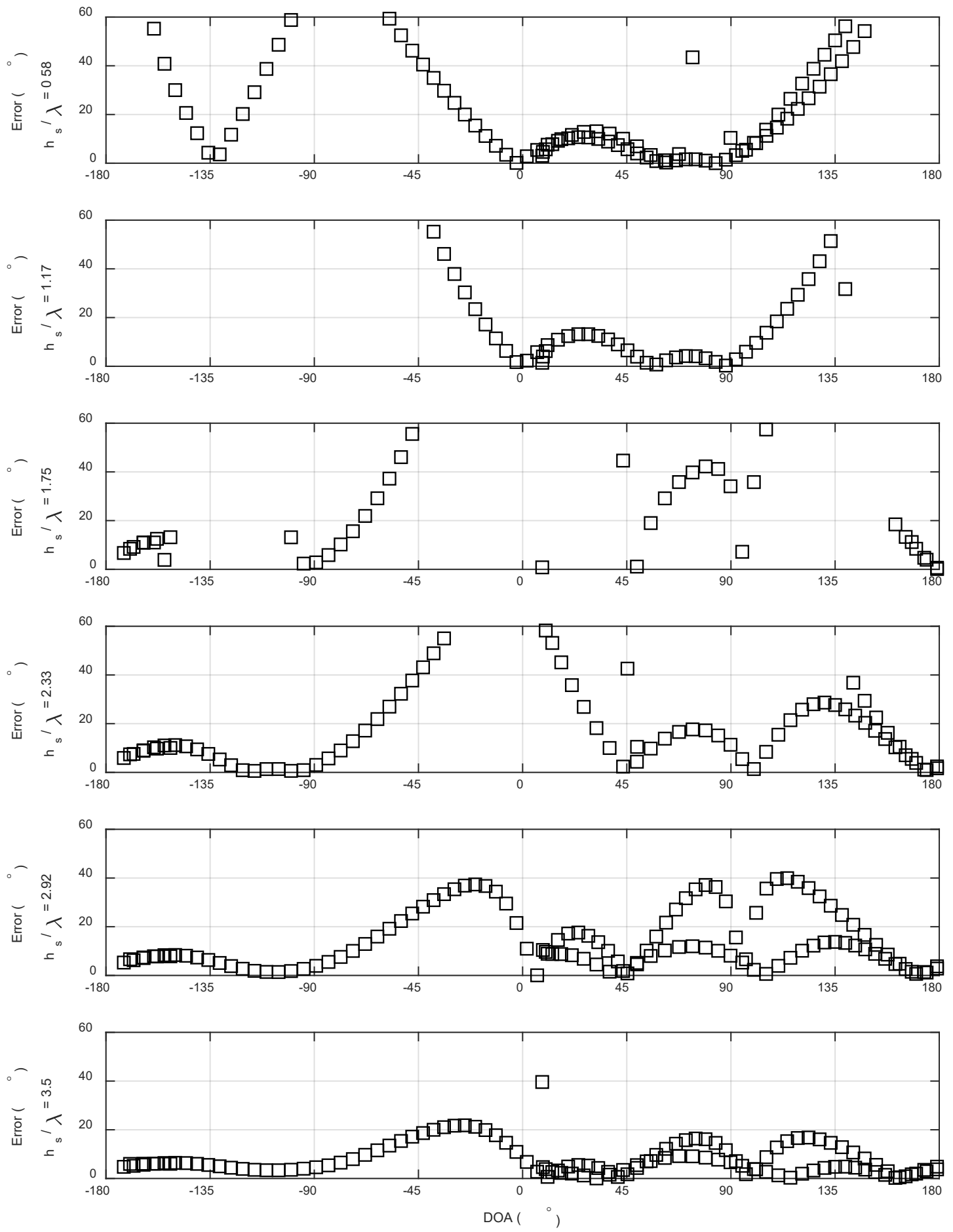

Figure 4.42 DOA error for various $h_{s} / \lambda$ with increasing frequency and constant source height $h_{s}=$ $1 \mathrm{~m}$.

The DOA error for a constant source height and variable frequency (wavelength) is analyzed in Figure 4.42. This corresponds to the line in Figure 4.40 marked with $\times$ and 
corresponding to $1 \mathrm{~m}$ source height. For $h_{s} / \lambda$ less than or equal to 2.33 a divergent behavior of the DOA error is observed. Effectively, very high errors are realized in the regions where the error diverges. As the wavelength decreases for the given source height, the DOA error becomes more stable and demonstrates similar behavior to that of

From this analysis, the lobing behavior within the experimental DOA error is explained. It is clear that the AVS are sensitive to the relative source height and source wavelength. Sources which are very close to a reflective surface and of low frequency (long wavelength) are less susceptible to the errors explicitly shown here. In practical applications when a source is near a reflective surface, the source height and source frequency are often unknown, therefore increasing the challenge of estimating direction of arrival and localization.

\subsubsection{Multi-Sensor Localization}

Combining the DOA data from sites 100 and 200 facilitates localization of the vehicle as a function of time. Plus and minus $1 \sigma$ are added to the mean azimuth, creating a beam used for localization at each site. The overlap of the standard deviation beams from each site creates an intersecting patch of area which contains the estimated vehicle position. Figure 4.43 shows an example of the localization area (LA) intersection for a given time instance of experiment 90 . Factors contributing to time instances when localization was not possible are the same as those discussed in section 4.4.1.4.

The performance of the combined AVS is assessed in terms of the localization with respect to the azimuth angle (DOA) of each sensor. This is represented in Figure 4.44 for experiments when the target vehicle was traveling in a circular pattern around either site (See Table 4.1, experiments 69 through 72 and experiments 79 through 83). The square root of the localization area (SLA) is plotted to better visualize the spread of the data and intuitively grasp the magnitude of a "square" area; as opposed to a polygonal area which is difficult to intuit. Essentially the y-axis of this figure represents the length of one side of a square which corresponds to the localized area. Squaring the values of the plot gives the true localization in $\mathrm{m}^{2}$. 


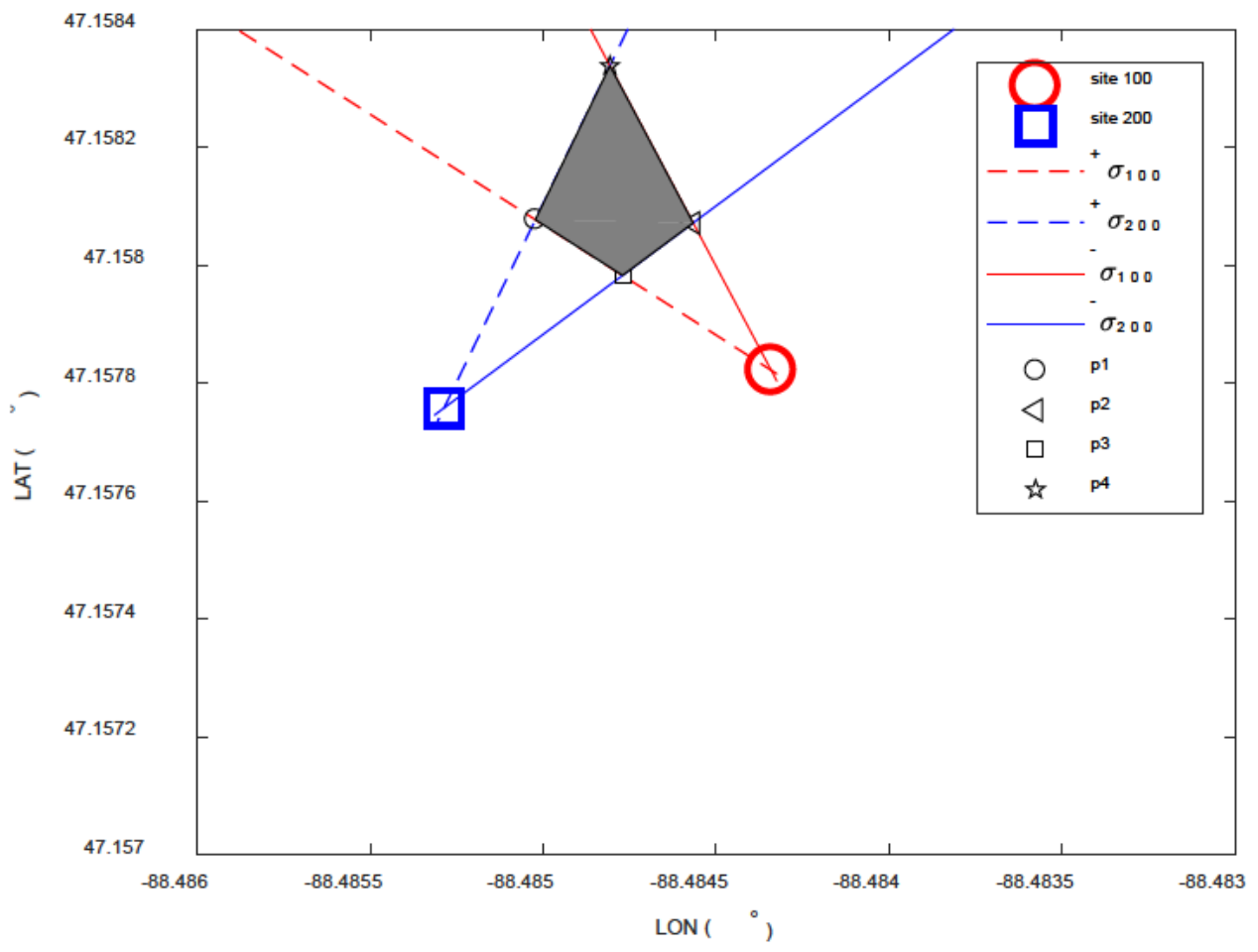

Figure 4.43 Example localization estimation utilizing $\mathrm{DOA} \pm \sigma$ lines for $\mathrm{t}=9.5$ seconds.

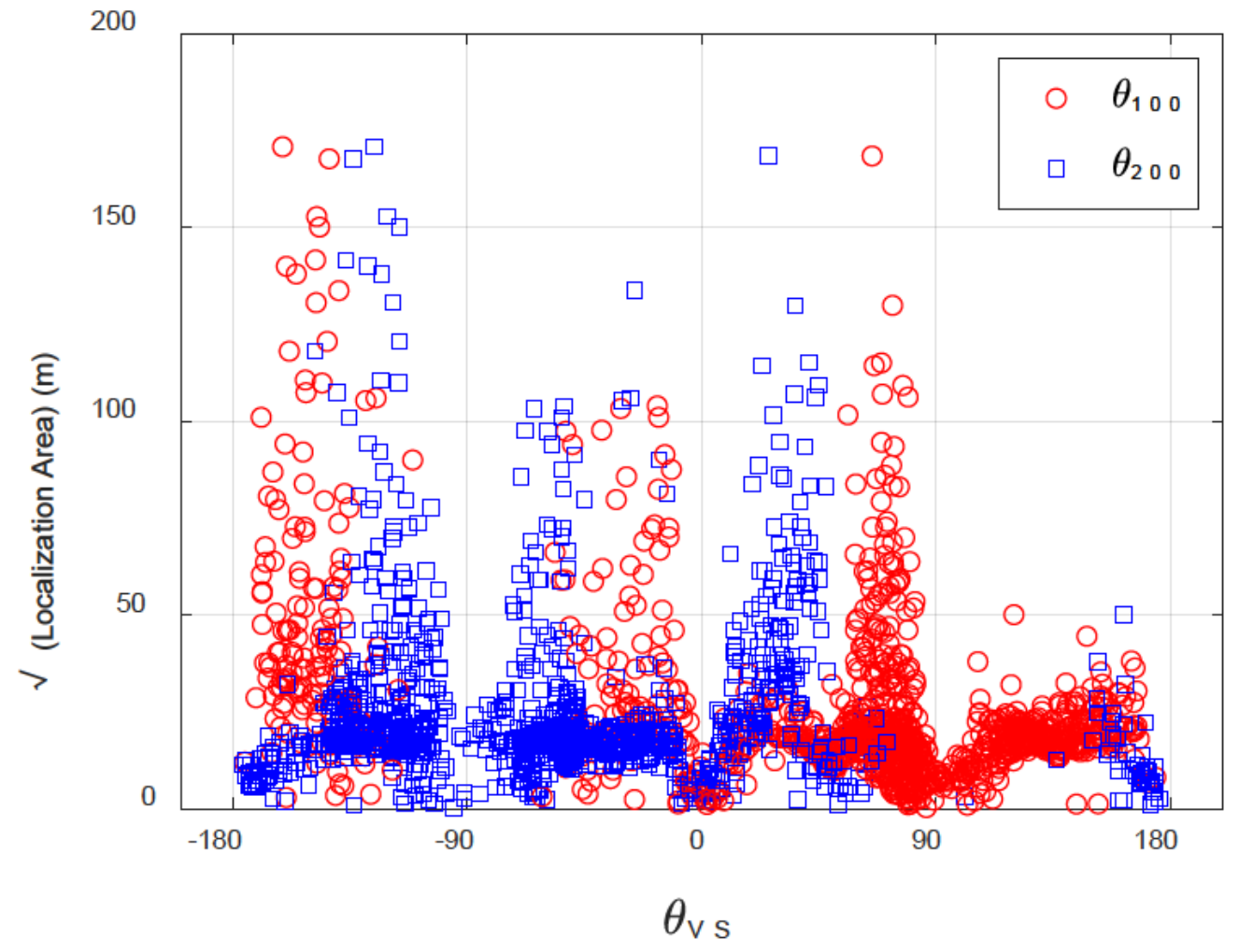

Figure 4.44 Square root localization area for experiments with vehicle traveling in circular pattern. 


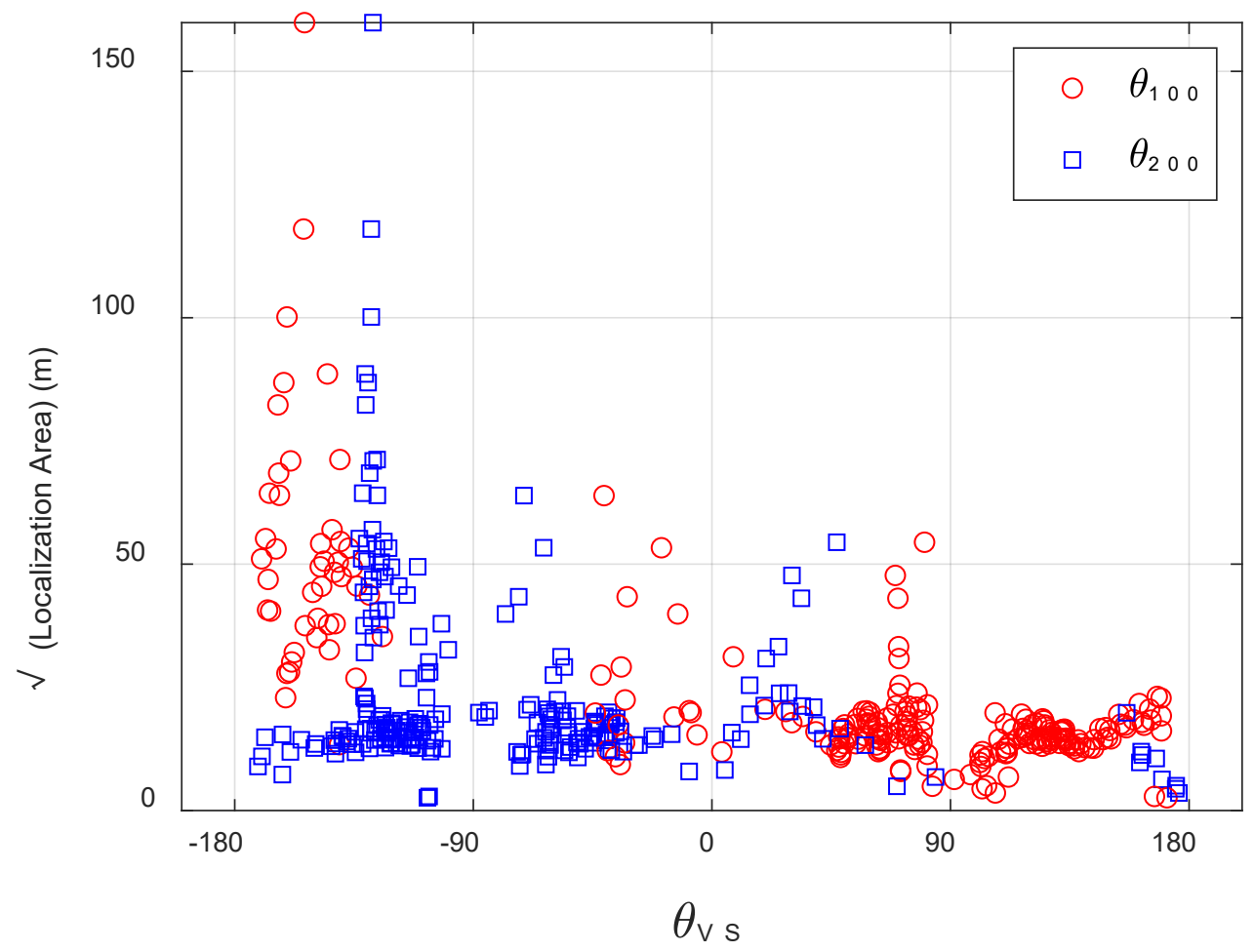

Figure 4.45 Square root localization area for experiments 90 and 91 (irregular pattern).

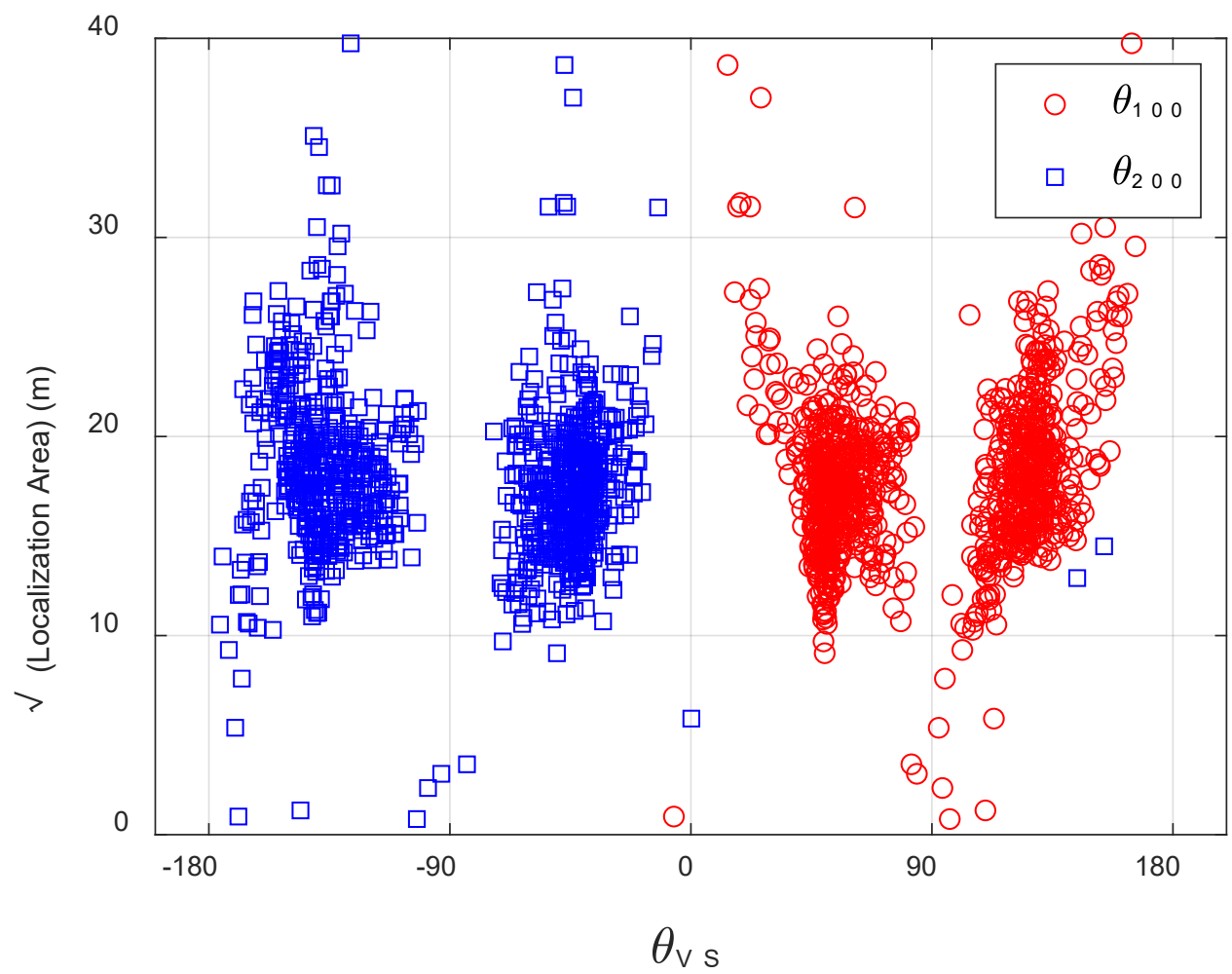

Figure 4.46 Square root localization area for experiments in between AVS sties. 


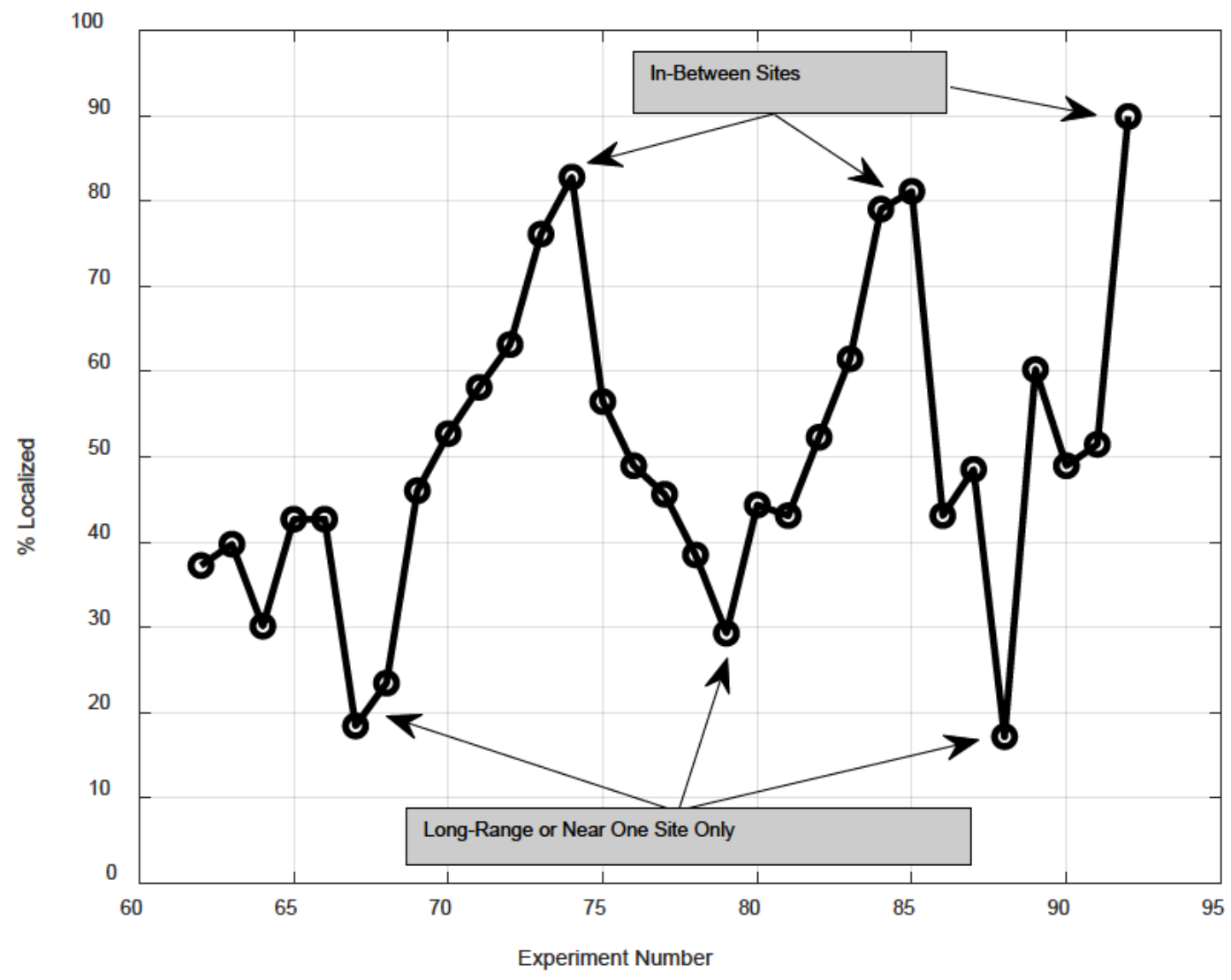

Figure 4.47 Percent localization for all experiments.

To evaluate the characteristics of the localization area for all different travel patterns, the statistics of SLA are shown in Table 4.4. For all experiments conducted, the percentage of time instances when it was possible to localize are reported, as well as the minimum, mean, maximum, and standard deviation of the SLA.

It is observed that the behavior of the data in Figure 4.44 follows a similar contour to that of the DOA error presented in Figure 4.35, Figure 4.41, etc. This is expected due to the lower error in DOA estimation close to integer multiples of 45 degrees. A mean SLA value of $33 \mathrm{~m}$ (LA of $1089 \mathrm{~m}^{2}$ ) is observed for the data shown in Figure 4.44, and an aggregate SLA standard deviation of $49 \mathrm{~m}$ for all experiments considered in this figure.

By comparison, experiments 90 and 91 have an irregular vehicle pattern. The SLA for these experiments is shown in Figure 4.45. For these experiments, a mean SLA value of $31 \mathrm{~m}$ (LA of $961 \mathrm{~m}^{2}$ ) is observed with standard deviations of 54 and $38 \mathrm{~m}$ respectively. This represents similar mean SLA when compared with the experiments when the vehicle was traveling in a circular pattern around AVS sites as well as similar spread on the data.

It is observed that localization is more common for experiments when the vehicle traveled in patterns between the two sites as opposed to staying close to one site only. This makes sense because both AVS are able to be utilized in coordination with one- 
another to localize. When the vehicle is near one sensor only, the ability to localize is frequently lost because only one sensor is capable of providing a DOA estimate.

Generally speaking, the majority of experiments conducted possess a coefficient of variation (ratio of standard deviation to mean) which is greater than one. It is observed that the experiments which take place between the AVS sites (experiments 73, 74, 84, 85, and 92) have coefficients of variation which are less than one. This indicates that there is far less variation on the SLA in comparison with the mean during these experiments. This effect is visible in Figure 4.46. Additionally, these experiments correspond with the greatest percent localizations of all experiments conducted. The lowest percent localizations were experiments $67,68,79$, and 88 , which correspond to either long-range tracking (and consequently poor SNR) or traveling around one of the AVS sites in a circle which disallows for both AVS to provide reasonable DOA estimation. An illustration of the percent localization for each experiment is shown in Figure 4.47. 
Table 4.4 Square root localization area statistics for all vehicle tracking experiments. The values are represented as the square root of the area for improved intuition.

\begin{tabular}{|c|c|c|c|c|c|c|c|}
\hline Index & Exp. No. & Desc. & $\begin{array}{c}\% \\
\text { Localized }\end{array}$ & $\frac{\operatorname{Min}}{\sqrt{m^{2}}}$ & $\begin{array}{l}\text { Mean } \\
\sqrt{m^{2}}\end{array}$ & $\begin{array}{l}\text { Max } \\
\sqrt{m^{2}}\end{array}$ & $\begin{array}{l}\text { Std } \\
\sqrt{m^{2}}\end{array}$ \\
\hline 1 & 62 & CCW Loop & 37.2 & 1.04 & 29.98 & 132.24 & 52.35 \\
\hline 2 & 63 & “ & 39.7 & 0.13 & 21.97 & 92.09 & 30.61 \\
\hline 3 & 64 & “ & 30.1 & 0.37 & 24.13 & 66.94 & 29.23 \\
\hline 4 & 65 & CW Loop & 42.7 & 1.34 & 22.06 & 81.66 & 28.84 \\
\hline 5 & 66 & “ & 42.7 & 0.42 & 20.86 & 78.26 & 28.47 \\
\hline 6 & 67 & Circle@100 & 18.4 & 1.30 & 36.05 & 150.15 & 59.11 \\
\hline 7 & 68 & " & 23.4 & 1.25 & 27.97 & 101.05 & 37.88 \\
\hline 8 & 69 & “ & 46.0 & 0.33 & 35.16 & 138.05 & 52.52 \\
\hline 9 & 70 & “ & 52.7 & 3.19 & 37.91 & 170.88 & 57.91 \\
\hline 10 & 71 & “ & 58.2 & 2.94 & 42.81 & 141.69 & 57.59 \\
\hline 11 & 72 & " & 63.2 & 3.80 & 39.96 & 167.78 & 61.99 \\
\hline 12 & 73 & N/S Between & 76.2 & 0.78 & 20.03 & 39.75 & 14.32 \\
\hline 13 & 74 & “ & 82.8 & 0.91 & 19.61 & 38.66 & 14.43 \\
\hline 14 & 75 & N/S@ 100 & 56.5 & 2.71 & 58.95 & 193.95 & 80.38 \\
\hline 15 & 76 & N/S@ 200 & 49.0 & 1.29 & 32.57 & 84.51 & 35.76 \\
\hline 16 & 77 & Figure-8 & 45.6 & 0.56 & 28.95 & 126.86 & 46.40 \\
\hline 17 & 78 & Figure-8 & 38.5 & 0.99 & 28.78 & 138.29 & 46.51 \\
\hline 18 & 79 & Circle@200 & 29.3 & 1.90 & 21.61 & 88.68 & 32.74 \\
\hline 19 & 80 & “ & 44.4 & 1.05 & 23.89 & 106.28 & 36.34 \\
\hline 20 & 81 & " & 43.1 & 0.96 & 30.14 & 114.38 & 46.08 \\
\hline 21 & 82 & “ & 52.3 & 4.11 & 32.38 & 129.94 & 48.12 \\
\hline 22 & 83 & " & 61.5 & 2.58 & 35.36 & 168.50 & 53.67 \\
\hline 23 & 84 & Circle Between & 79.1 & 3.06 & 16.74 & 26.37 & 9.78 \\
\hline 24 & 85 & “ & 81.2 & 11.21 & 16.98 & 24.62 & 9.24 \\
\hline 25 & 86 & Long-Range & 43.1 & 5.32 & 31.22 & 133.11 & 48.20 \\
\hline 26 & 87 & “ & 48.5 & 2.85 & 36.72 & 129.97 & 51.94 \\
\hline 27 & 88 & “ & 17.2 & 1.90 & 43.35 & 129.89 & 62.98 \\
\hline 28 & 89 & “ & 60.3 & 2.71 & 47.03 & 147.57 & 61.69 \\
\hline 29 & 90 & Irregular & 49.0 & 3.56 & 31.03 & 159.88 & 53.77 \\
\hline 30 & 91 & Irregular & 51.5 & 2.59 & 30.20 & 88.59 & 37.60 \\
\hline 31 & 92 & N/S Between & 90.0 & 2.34 & 18.36 & 27.31 & 11.62 \\
\hline
\end{tabular}




\subsection{Summary}

Experiments to assess the performance of underwater $p a$ and atmospheric $p p$ acoustic vector sensors (AVS) for direction of arrival (DOA) estimation and localization were carried out. These experiments largely focused on tracking moving mechanical noise sources which radiate complex and unknown frequency content in real-world

environments where ambient noise was present. DOA was estimated with the arctangentof-intensity method.

Underwater AVS localization experiments were carried out in Barrow, AK in April 2016 on shore-fast ice. Underwater AVS were deployed through holes in the ice and were used to localize both stationary underwater acoustic sources and a moving acoustic source on the ice. ${ }^{83}$

When localizing a stationary underwater sound source, the AVS was able to determine the azimuth DOA of the source to within $3.8^{\circ}$ and $1.1^{\circ}$ for low-frequency and highfrequency pings, respectively. The elevation DOA was determined to within $15.36^{\circ}$ and $0.55^{\circ}$ for low-frequency and high-frequency pings, respectively. ${ }^{83}$

During a 120-second data collect, a snowmobile on the ice was localized as a function of time by combing DOA data (azimuth and elevation) from sensors at two sites. Using azimuthal data alone, the vector sensors were able to localize $66 \%$ of the time with a mean localization area of $0.091 \mathrm{~km}^{2}$. During $15 \%$ of these localized instances, when the snowmobile was in the vicinity of the measurement location, combining elevation data further reduced the mean localization area by $0.049 \mathrm{~km}^{2} .{ }^{83}$

Atmospheric AVS localization experiments were carried out in August 2018 at the Keweenaw Research Center in Calumet, MI. A total of 31 experiments, each consisting of a 120-second data collect, were conducted to track a non-stationary utility vehicle with 2 AVS sites. A variety of vehicle patterns were driven around the AVS sites during the experiments. The test range was directly adjacent to a county airport and military vehicle test tracks. This proximity resulted in intermittent background noise during AVS testing.

A moving standard deviation (MSD) processing routine was developed to determine the DOA for a given time interval. It is shown that the MSD method is superior to direct integration (or averaging) of the DOA spectra across all frequencies. This was shown to be due to the source radiating various frequency content and at various SNR for successive time intervals. The MSD routine was shown to manage this variation and provide improvement over the traditional integration method.

The atmospheric DOA data was assessed in terms of absolute error from the ground truth GPS DOA. The DOA error ranged between 0 to $20^{\circ}$ dependant on the azimuth angle of the AVS. It was experimentally observed that the smallest DOA errors occurred at roughly integer multiples of $45^{\circ}$. This observation was consistent with FEA models from Wajid et al. as well as an analytical simulation which was developed. 
The analytical simulation also showed that the DOA estimation was sensitive to the ground-reflected paths from the source to the receiver. The analytical simulation was assessed in terms of the source height and wavelength. The particular source height and wavelength were unknown for the real-world tracking scenario because different parts of the vehicle radiate different frequencies for different vehicle speeds. This particular fact further justifies the implementation of the MSD processing.

The localization area is reported for all experiments as well as the percentage of time intervals when it was possible to localize. It was observed that high percentages of localization ( 80 to $90 \%$ ) were realized when the target vehicle remained between the two AVS sites for the entire experiment. The lowest percent localization (15 to 30\%) was realized when the target vehicle was near one site only, or was very far away from the sites. This intuitively makes sense, because both AVS sites are needed to localize and both have good received signal when the source is between the sites.

While there are some significant fluctuations in the localization capabilities with underwater and atmospheric AVS presented here, the trend tracking capability is still good. For the underwater AVS, the largest deviations appear when the snowmobile is nearly overhead of the vector sensor due to constructive and destructive acoustic reflections from the seafloor. The largest deviations for atmospheric AVS appear when the test vehicle is far away from the AVS sites. It should also be noted that these experiments were conducted at relatively short ranges from source to receiver (less than $200 \mathrm{~m}$ ). Conclusions regarding longer range tracking of anthropogenic sources cannot be drawn from these data. It has been shown that tracking an on-ice source using underwater AVS and tracking ground vehicles with atmospheric AVS is feasible. It is expected that with improved processing algorithms, improved AVS design, and improved understanding of the environment where AVS are being used, better DOA estimation and localization accuracy may be expected. ${ }^{83}$ 


\section{Conclusions and Future Work}

In recent years, the Arctic has changed from a primarily multi-year ice to a primarily first-year ice environment. This change coupled with the increased activity in the Arctic has provided a need for improved military awareness in the Arctic. To provide this awareness, knowledge regarding acoustic transmission loss is necessary to determine the decay of sound between acoustic sources and sensors. Additionally, localization methods with small, rapidly deployable sensor packages are necessary to provide estimation of acoustic source positions over time.

Acoustic propagation studies were carried out in Barrow, AK in 2016 on first-year, shore-fast ice. The experiments conducted provided insight to the relative propagation of various frequencies in the air, ice, and underwater. The data lacked spatial resolution and spectral averaging due to weather conditions limiting the test window while in AK.

Follow-up experiments were conducted on Lake Superior in 2018 to collect more propagation data on an Arctic-like surrogate ice layer. This surrogate environment provided many of the same first-year ice characteristics when compared to the Arctic; excluding salinity. Atmospheric and underwater transmission loss data has been provided for the first-year ice surrogate environment.

Experimental TL data were compared to numerical simulations of two types; ray tracing (Bellhop) and parabolic equation (RAM). These simulations were selected based upon the environmental depth, range-dependent characteristics, and frequency ranges of interest. Inputs to the simulations included sound speed profiles of the air, ice, water, and lake-floor as well as bathymetry profile and reflection loss of the bottom and under-ice surface.

The effective atmospheric sound speed was estimated based on nominal air temperature and measured windspeed. The ice sound speeds were measured via time delays between hammer impact and geophone responses at downrange sites. Underwater sound speed profiles were measured directly with a CTD probe. The bathymetry profile was measured via summer-time depth sounding measurements. Reflection loss data of the bottom and the under-ice surface were measured directly using pseudo-random maximal length sequence processing techniques. The lake-floor sound speed was estimated based upon the known bottom type and bottom reflection loss information.

Good agreement is observed between the TL data and the simulations with some discrepancies which are noted. It is stressed that the comparative numerical models presented do not consider backscattering, turbulence, 3D propagation effects, under-ice altimetry, and many other environmental factors. These factors along with numerical modeling limitations can contribute significantly discrepancies between the models and the data, especially at the low and high-frequency extremes. Variation in windspeed is observed to cause large variability on the SNR of atmospheric acoustic measurements. In turn, this results in large variability on atmospheric TL; especially at high frequencies. 
The experimental TL data reported here is provided with the intention that it be used in the future for validation for newly developed models of the Arctic acoustic propagation. The models used for comparison in this document are lacking in the areas mentioned above. With improved derivations for models which include more environmental factors, better understanding may be garnered of the first-year Arctic TL.

A through-thickness resonance was observed in the drivepoint mobility measured between the instrumented impact hammer and geophone response in the Alaskan firstyear ice layer. The through-thickness resonance was confirmed during follow-up experiments on Lake Superior. An equation has been derived to determine the ice thickness from the drivepoint mobility measurement. To utilize this equation the ice impedance and the water impedance must be known.

Underwater $(p a)$ and atmospheric $(p p)$ acoustic vector sensors have been shown to be feasible for tracking in practical applications. During testing in Barrow, AK, underwater AVS experiments were conducted to localize both a stationary underwater sound source and a non-stationary snowmobile on the ice surface. A total of 31 experiments were conducted at Keweenaw Research Center in Calumet, MI in 2018 to localize a nonstationary ground utility vehicle with atmospheric AVS. Various vehicle driving patterns around AVS sites were analyzed.

A moving standard deviation (MSD) processing routine was developed to improve the DOA estimation for sources which have complex frequency content and various emitted signal amplitude. The MSD processing has been shown to be superior to direct integration or averaging of the entire acoustic intensity spectra. The adaptive nature of the MSD processing allows for diverse application in situations where SNR may be various and the source frequency is unknown.

Analytical models of atmospheric AVS were developed and show good agreement with the experimental DOA estimation. The analytical DOA error matches closely with the experimental DOA error. It was observed that the DOA error is dependent on the groundreflected path between the acoustic source and receiver as well as source height and wavelength.

Continued research is necessary to refine DOA estimation and localization for real-world scenarios. Rigorous investigation of the effects of sensor design and spacing, number of sensors, number of AVS sites, and spacing of AVS sites need to be developed in nonlaboratory environments, with complex frequency content, and various SNR. As discussed by Wajid et al., better AVS performance (ie. lower DOA estimation error) may be realized by using different $p p$ microphone configurations such as a star or delta configuration ${ }^{99}$. These various configurations must also be investigated for future design applications.

Going forward, AVS processing may be adapted for real-time applications. Traditional processing algorithms may be implemented and MSD processing may be improved for 
greater speed and accuracy of DOA estimation. AVS show potential for implementation with machine learning algorithms due to the ease of collecting training datasets. Machine learning may also result in improved DOA estimation accuracy beyond that of traditional algorithms. 


\section{References}

[1] C. Starr. (2016). Weekly Animation of Arctic Sea Ice Age with Graph of Ice Age By Area: 1984 - 2016. Available: https://svs.gsfc.nasa.gov/4510

[2] M. B. Penhale, A. R. Barnard, and R. Shuchman, "Multi-Modal and Short-Range Transmission Loss in Thin, Ice-Covered, Near-Shore Arctic Waters," J. Acoust. Soc. Am., vol. 143, no. 5, pp. 3126-3137, 2018.

[3] M. Ewing and A. P. Crary, "Propagation of Elastic Waves in Ice. Part II," Journal of Applied Physics, vol. 5, pp. 181-184, 1934.

[4] M. Ewing, A. P. Crary, and A. M. Thorne, Jr., "Propagation of Elastic Waves in Ice. Part I," Journal of Applied Physics, vol. 5, pp. 165-168, 1934.

[5] F. Press and M. Ewing, "Theory of Air-Coupled Flexural Waves," Journal of Applied Physics, vol. 22, no. 892, 1951.

[6] M. Ewing, W. S. Jardetzky, and F. Press, Elastic Waves in Layered Media. McGraw-Hill, 1957.

[7] A. Langley, "The sound fields of an infinite, fluid-loaded plate excited by a point force," J. Acoust. Soc. Am., vol. 83, no. 4, 1987.

[8] T. C. Yang and T. W. Yates, "Flexural waves in a floating ice sheet: Modeling and Comparison with Data," J. Acoust. Soc. Am., vol. 97, no. 2, 1994.

[9] W. S. Jardetzky and F. Press, "Rayleigh Wave Coupling to Atmospheric Compression Waves," Seismological So. of Am., vol. 42, no. 2, pp. 135-144, 1952.

[10] R. E. Sherrif, "Introduction to Seismic Methods," in Geophysical MethodsEnglewood Cliffs, NJ: Prentice Hall, 1989, pp. 209-227.

[11] W. I. Futterman, "Dispersive Body Waves," J. of Geophysical Research, vol. 67, no. 13, pp. 5279-5291, 1962.

[12] F. E. Francios and T. Wen, "Propagation of Sound Generated on the Ice Surface Into Water," in Oceans, Seattle, 1989.

[13] F. Press, A. P. Crary, J. Oliver, and S. Katz, "Air-Coupled Flexural Waves In Floating Ice," American Geophysical Union, vol. 32, no. 2, 1951.

[14] F. Press and M. Ewing, "Propagation of Elastic Waves in a Floating Ice Sheet," American Geophysical Union, vol. 32, no. 5, 1951. 
[15] A. R. Milne, "Shallow Water Under-Ice Acoustics in Barrow Strait," J. Acoust. Soc. Am., vol. 32, no. 8, 1960.

[16] K. Hunkins and K. Henry, "Shallow-Water Propagation in the Arctic Ocean," $J$. Acoust. Soc. Am., vol. 35, no. 542, 1963.

[17] A. R. Milne, "A $90 \mathrm{~km}$ Sound Transmission Test in the Arctic," J. Acoust. Soc. Am., vol. 35, no. 1459, 1963.

[18] T. C. Yang and G. R. Giellis, "Experimental characterization of elastic waves in a floating ice sheet,," J. Acoust. Soc. Am., vol. 95, no. 5, 1994.

[19] B. E. Miller and H. Schmidt, "Observation and Inversion of Seismo-Acoustic Waves in a Complex Artic Ice Enviroment," J. Acoust. Soc. Am., vol. 84, no. 4, 1990.

[20] R. W. Knapp, "Observations of the Air-Coupled Wave as a Function of Depth," Geophysics, vol. 51, no. 9, pp. 1853-1857, 1986.

[21] J. Maslanik, J. Stroeve, C. Fowler, and W. Emery, "Distribution and Trends in Arctic Sea Ice Age Through Spring 2011," Geophysical Research Letters, vol. 38, 2011.

[22] J. C. Stroeve, T. Markus, L. Boisvert, J. Miller, and A. Barrett, "Changes in Arctic Melt Season and Implications for Sea Ice Loss," Geophysical Research Letters, vol. 41, pp. 1216-1225, 2014.

[23] J. Rodrigues, "The Rapid Decline of the Sea Ice in the Russian Arctic," Cold Regions in Sci. and Technology, vol. 54, pp. 124-142, 2008.

[24] R. Lei, H. Xie, J. Wang, M. Lepparanta, I. Jonsdottir, and Z. Zhang, "Changes in Sea Ice Conditions along the Arctic Northeast Passage from 1979-2012," Cold Regions in Sci. and Technology, vol. 119, pp. 132-144, 2015.

[25] G. W. Timco and W. F. Weeks, "A Review of the Engineering Properties of Sea Ice," Cold Regions in Sci. and Technology, vol. 60, 2010.

[26] S. Somanathan, P. C. Flynn, and J. Szymanski, "The Northwest Passage: A Simulation," in Winter Simulation Confrence, 2006.

[27] L. C. Smith and S. R. Stephenson, "New Trans-Arctic Shipping Routes Navigable by Midcentury," Proc. of the Nat. Academy of Sci. of the United States of America, vol. 110, no. 13, pp. 1191-1195, 2013. 
[28] S. R. Stephenson, L. W. Brigham, and L. C. Smith, "Marine Accessibility Along Russia's Northern Sea Route," Polar Geography, vol. 37, no. 2, pp. 111-133, 2014.

[29] G. J. Heard, M. McDonald, N. R. Chapman, and L. Jaschke, " Underwater Lightbulb Implosions: A Useful Acoustic Source," in IEEE/Oceans, 1997.

[30] S. E. Dosso, G. J. Heard, and M. Vinnins, "Seismo-Acoustic Propagation in an Ice-covered Arctic Ocean Environment," in MTS/IEEE Oceans Honolulu, 2001.

[31] D. L. Brown and R. J. Allemang, "Review of Spatial Domain Modal Parameter Estimation Procedures and Testing Methods," in IMAC 2009.

[32] W. Heylen, S. Lammens, and P. Sas, Modal Analysis Theory and Testing. K.U. Leuven, 2013.

[33] C. V. Karsen. (1984) Averaging for Improved Frequency Response Functions. S.V. Sound and Vibration. 18-24.

[34] H. W. Lord, W. S. Gatley, and H. A. Evensen, Noise Control for Engineers. Malabar, CA.: Krieger Publishing Company, 1980.

[35] "CastAway User Manual - CTD Principles of Operation," ed, 2010, p. 74.

[36] N. P. Fofonoff and R. C. Millard, "Algorithims for computation of fundamental properties of seawater," Unesco technical papers in marine science, no. 44, 1983.

[37] J. S. Bendat and A. G. Piersol, Random Data: Analysis and Measurement Procedures, 3 ed. Wiley, 2000.

[38] L. E. Kinsler, A. R. Frey, A. B. Coppens, and J. V. Sanders, Fundamentals of Acoustics. Wiley, 1999.

[39] G. W. Timco and R. M. W. Frederking, "A Review of Sea Ice Density," Cold Regions in Sci. and Technology, vol. 24, 1995.

[40] N. K. Sinha, "Effective Poisson's Ratio of Isotropic Ice," in International Offshore Mechanics and Arctic Engineering Symposium, Huston, TX, 1987, vol. IV, pp. 189-195.

[41] J. R. Murat and L. M. Lainey, "Some Experimental Observations on the Poisson's Ratio of Sea Ice," Cold Regions in Science and Technology, vol. 6, pp. 105-113, 1982. 
[42] A. Traetteberg, L. W. Gold, and R. Frederking, "The Strain Rate and Temperature Dependence of Young's Modulus of Ice," in IAHR International Symposium on ICE Problems, Hanover, New Hampshire, 1975, vol. 3.

[43] M. P. Langleben, "Young's Modulus for Sea Ice,", Canadian J. of Physics, vol. 40, no. $1,1962$.

[44] S. A. Hambric, S. H. Sung, and D. J. Nefske, Engineering Vibroacoustic Analysis: Methods and Applications, 1 ed. Wiley, 2016.

[45] S. P. Pecknold, N. Pelavas, and G. J. Heard, "Measurements and modeling of transmission loss variability in Barrow Strait," in ICA, Montreal, 2013, vol. 19: Acoustical Society of America.

[46] R. J. Urick, "Propagation of Sound in the Sea: Transmission Loss, I and II," in Principles of Underwater Sound3 ed.: Peninsula Publishing, 1996.

[47] F. B. Jensen, W. A. Kuperman, M. B. Porter, and H. Schmidt, Computational Ocean Acoustics. Springer, 2011.

[48] P. C. Etter, Underwater Acoustic Modeling and Simulation, 5 ed. Boca Raton, FL: CRC Press, 2018.

[49] M. S. Ballard and J. D. Sagers, "Three-Dimensional Propagation Induced by the Rough Sea Ice Interface," in International Conference on Theoretical and Computational Acoustics, Vienna, Austria, 2017.

[50] M. Isakson and M. Ballard, "Finite Element Modeling of Acoustics Scattering from First-Year and Multi-Year Ice Keels in the Arctic," in International Conference on Theoretical and Computational Acoustics, Vienna, Austria, 2017.

[51] D. F. McCammon and S. T. McDaniel, "The influence of the physical properties of ice on reflectivity," J. Acoust. Soc. Am., vol. 77, pp. 499-507, 1985.

[52] O. I. Diachok, "Effects of sea-ice ridges on sound propagation in the Arctic Ocean," J. Acoust. Soc. Am., vol. 59, pp. 1110-1120, 1976.

[53] O. I. Diachok, "Effects of sea-ice ridge characteristics on under-ice reflection loss," J. Acoust. Soc. Am., vol. 55, 1974.

[54] B. Blumenthal, APL-UW High-Frequency Ocean Environmental Acoustic Models Handbook. Applied Physics Laboratory University of Washington, 1994.

[55] N. P. Chotiros and V. Pallayil, "Seabed Characterization Using Acoustic Communication Signals on an Autonomous Underwater Vehicle With a Thin- 
Line Towed Array," IEEE JOURNAL OF OCEANIC ENGINEERING, vol. 38, no. 3, pp. 410-418, 2013.

[56] A. R. Barnard, "Evaluation of Measurement Techniques to Determine the Acoustical Properties of Porous and Multi-Layered Acoustic Treatments," Mechanical Engineering, Michigan Technological University, 2004.

[57] J. Borish and J. B. Angell, "An Efficient Algorithm for Measuring the Impulse Response Using Pseudorandom Noise," J. Audio Engineering Soc., vol. 31, no. 7, pp. 478-488, 1983.

[58] W. T. Chu, "Impulse-Response and Reverberation-Decay Measurements Made by Using a Periodic Pseudorandom Sequence," Applied Acoustics, vol. 29, pp. 193205, 1990.

[59] M. Garai, "Measurement of the Sound-Absorption Coefficient In Situ: The Reflection Method Using Periodic Pseudo- random Sequences of Maximum Length," Applied Acoustics, vol. 39, pp. 119-139, 1993.

[60] C. Bleakley and R. Scaife, "New formulas for predicting the accuracy of acoustical measurements made in noisy environments using the averaged $\mathrm{m}$ sequence correlation technique," The Journal of the Acoustical Society of America, vol. 97, no. 2, pp. 1329-1332, 1995/02/01 1995.

[61] M. Vorlander and M. Kob, "Practical Aspects of MLS Measurements in Building Acoustics," Applied Acoustics, vol. 52, pp. 239-258, 1997.

[62] J. Vanderkooy, "Aspects of MLS Measuring Systems," J. Audio Engineering Soc., vol. 42, no. 4, pp. 219-231, 1994.

[63] E. M. Salomons, Computational Atmospheric Acoustics. Kluwer Academic Publishers, 2001.

[64] R. Haberman, Applied Partial Differential Equations: with fourier series and boundary value problems, 5th ed. Pearson, 2013.

[65] A. L. Maggi and A. J. Duncan, "ACTUP v2.2L Acoustic Toolbox User-Interface and Post-Processor: Installation and User Guide," Available: http://cmst.curtin.edu.au/products/underwater/

[66] M. D. Collins, "A split-step Padé solution for the parabolic equation method," $J$. Acoust. Soc. Am., vol. 93, pp. 1736-1742, 1993.

[67] J. Budd, W. C. Kerfoot, A. Pilant, and L. M. Jipping, "The Keweenaw Current and Ice Rafting: Use of Satellite Imagery to Investigate Copper-rich Particle Dispersal," J. Great Lakes Res., vol. 25, no. 4, pp. 642-662, 1999. 
[68] W. C. Kerfoot, S. Harting, R. Rossmann, and J. A. Robbins, "Anthropogenic Copper Inventories and Mercury Profiles from Lake Superior: Evidence for Mining Impacts," J. Great Lakes Res., vol. 25, no. 4, pp. 663-682, 1999.

[69] W. C. Kerfoot and L. J. Weider, "Experimental paleoecology (resurrection ecology): Chasing Van Valen's Red Queen hypothesis," Limnol. Oceanogr., vol. 49, no. 4, pp. 1300-1316, 2004.

[70] R. Gillespie, W. B. Harrison, and G. M. Grammer, "Geology of Michigan and the Great Lakes," in Michigan Geological Repository for Research and Education, ed. Western Michigan University, 1987.

[71] E. L. Hamilton, "Elastic Properties of Marine Sediments," J. Geophysical Research, vol. 76, no. 2, pp. 579-604, 1971.

[72] D. Han, A. Nur, and D. Morgan, "Effects of porosity and clay content on wave velocities in sandstones," J. Soc. Geophysics, vol. 51, pp. 2093-2107, 1989.

[73] A. Capelli, J. C. Kapil, I. Reiweger, D. Or, and J. Schweizer, "Speed and attenuation of acoustic waves in snow: Laboratory experiments and modeling with Biot's theory," Cold Regions in Sci. and Technology, vol. 125, pp. 1-11, 2016.

[74] C. B. Park, J. Ivanov, R. D. Miller, and J. Xia, "SEISMIC INVESTIGATION OF PAVEMENTS BY MASW METHOD —GEOPHONE APPROACH," Soc. Exploration Geophysicists, 2001.

[75] B. Michel, "The strength of polycrystalline ice," Canadian J. Civil Engineering, vol. 5, no. 3, pp. 285-300, 1978.

[76] L. W. Gold, "Engineering Properties of Fresh-Water Ice," J. of Glaciology, vol. 19, no. 81, pp. 197-212, 1977.

[77] H. Han, Q. Jia, W. Huang, and Z. Li, "Flexural Strength and Effective Modulus of Large Columnar-Grained Freshwater Ice," J. Cold Reg. Eng., vol. 30, no. 2: $04015005,2016$.

[78] D. M. Masterson, "Interpretation of in situ borehole ice strength measurement tests," Canadian J. Civil Engineering, vol. 23, pp. 165-179, 1996.

[79] K. F. Voitkovskii, "The Mechanical Properties of Sea Ice," The American Meteorological Society1960.

[80] R. R. Craig and A. J. Kurdila, Fundamentals of Structural Dynamics, 2nd ed. 2006. 
[81] F. Lasserre and S. Pelletier, "Polar Super Seways? Maritime Transport in the Arctic: an Analysis of Shipowners' Intentions," J. of Transport Geography, vol. 19, pp. 1465-1473, 2001.

[82] M. Roston, "The Northwest Passage's Emergence as an International Highway," Southwestern J. of International Law, vol. 15, 2009.

[83] M. B. Penhale and A. R. Barnard, "(U) Localization of On-ice Mechanical Noise Sources in the Shore-fast Arctic Ice Zone using Underwater Vector Sensors," (Unclassified) JUR(USN), vol. 68, pp. 235-255, 2018.

[84] A. R. Barnard and S. A. Hambric, "Design and implementation of a shielded underwater vector sensor for laboratory environments," J. Acoust. Soc. Am., JASA Express Letters vol. 130, no. 6, 2011.

[85] K. Kim, T. B. Gabrielson, and G. C. Lauchle, "Development of an accelerometerbased underwater acoustic intensity sensor," J. Acoust. Soc. Am., vol. 116, no. 6, 2004.

[86] Y. Song and K. T. Wong, "Azimuth-elevation direction finding using a microphone and three orthogonal velocity sensors as a non-collocated subarray," J. Acoust. Soc. Am., vol. 133, pp. 1987-1995, 2013.

[87] D. Levin, E. A. P. Habets, and S. Gannot, "Maximum likelihood estimation of direction of arrival using an acoustic vector-sensor," J. Acoust. Soc. Am., vol. 131, pp. 1240-1248, 2012.

[88] A. Bereketli, M. B. Guldogan, T. Kolcak, T. Gudu, and A. L. Avsar, "Experimental Results for Direction of Arrival Estimation with a Single Acoustic Vector Sensor in Shallow Water," J. Sensors, vol. 2015, no. 1, 2015, Art. no. 401353.

[89] F. J. Fahy, Sound Intensity, 2nd ed. 1995.

[90] E. G. Williams, Fourier Acoustics: Sound Radiation and Nearfield Acoustical Holography. Academic Press, 1999.

[91] W. S. Burdic, Underwater Acoustic System Analysis, 2 ed. Peninsula Publishing, 2002 .

[92] A. S. Douglass, H. C. Song, and D. R. Dowling, "Performance comparisons of frequency-difference and conventional beamforming," J. Acoust. Soc. Am., vol. 142, pp. 1663-1673, 2017.

[93] B. D. VanVeen and K. M. Buckley. (1988) Beamforming: A Versatile Approach to Spatial Filtering. IEEE ASSP Magazine. 4-24. 
[94] M. J. Earwicker, "Signal-to-noise ratio gain of sparse array processors," J. Acoust. Soc. Am., vol. 68, pp. 1129-1134, 1980.

[95] C. R. Greene and R. C. Wood, "Sparse Array Performance," J. Acoust. Soc. Am., vol. 63, pp. 1866-1978, 1978.

[96] M. V. Greening and J. E. Perkins, "Adaptive Beamforming for Nonstationary Arrays," J. Acoust. Soc. Am., vol. 112, pp. 2872-2881, 2002.

[97] M. B. Hawes and W. Liu, "Sparse Array Design for Wideband Beamforming With Reduced Complexity in Tapped Delay-Lines," IEEE, vol. 22, no. 8, pp. 1236-1247, 2014.

[98] H. C. Pumphrey, "Design of sparse arrays in one, two, and three dimensions," $J$. Acoust. Soc. Am., vol. 93, pp. 1620-1628, 1993.

[99] M. Wajid, A. Kumar, and R. Bahl, "Design and analysis of air acoustic vectorsensor configurations for two-dimensional geometry," J. Acoust. Soc. Am., vol. 139, no. 5, pp. 2815-2832, 2016.

[100] P. S. Naidu, Sensor Array Signal Processing. Boca Raton, FL: CRC Press LLC, 2001.

[101] D. H. Johnson and D. E. Dudgeon, Array Signal Processing: Concepts and Techniques. Englewood Cliffs, NJ: PTR Prentice Hall, 1993.

[102] D. G. Manolakis, V. K. Ingle, and S. M. Kogon, Statistical and Adaptive Signal Processing: Spectral Estimation, Signal Modeling, Adaptive Filtering, and Array Processing. 2005.

[103] D. F. Comesana, K. R. Holland, D. G. Escribano, and H. E. deBree, "An introduction to virtual phased arrays for beamforming applications," Archives of Acoustics, vol. 39, no. 1, pp. 81-88, 2014.

[104] R. O. Schmidt, "Multiple Emitter Location and Signal Parameter Estimation," IEEE Transactions on Antennas and Propagation, vol. AP-34, no. 3, 1986.

[105] A. Anand and M. K. Mukul, "Comparative analysis of different direction of arrival estimation techniques," in 2016 IEEE International Conference on Recent Trends in Electronics, Information \& Communication Technology (RTEICT), 2016, pp. 343-347.

[106] A. Nehorai and E. Paldi, "Acoustic Vector-Sensor Array Processing," IEEE, vol. 42, no. 9, pp. 2481-2491, 1994. 
[107] P. Stoica and A. Nehorai, "MUSIC, maximum likelihood, and Cramer-Rao bound," IEEE Transactions on Acoustics, Speech, and Signal Processing, vol. 37, no. 5, pp. 720-741, 1989.

[108] M. Hawkes and A. Nehorai, "Wideband Source Localization Using a Distributed Acoustic Vector-Sensor Array," IEEE Transactions on signal processing, vol. 51, no. 6, pp. 1479-1491, 2003.

[109] X. Yuan, Direction-Finding with a Misoriented Acoustic Vector Sensor. 2012, pp. 1809-1815.

[110] S. L. Collier et al., "Atmospheric Turbulence Effects on Acoustic Vector Sensing," presented at the J. Acoust. Soc. Am. , 2017.

[111] D. Fernandez Comesaña, J. Wind, A. Grosso, and K. R. Holland, "Performance of P-P and P-U intensity probes using Scan \& Paint," in International Congress on Sound and Vibration, Rio de Janeiro, Brazil, 2011.

[112] M. T. Silvia and R. T. Richards, "A theoretical and experimental investigation of low-frequency acoustic vector sensors," in OCEANS '02 MTS/IEEE, 2002, vol. 3, pp. 1886-1897 vol.3.

[113] G. Moschioni, B. Saggin, and M. Tarabini, "Sound source identification using coherence and intensity based methods," in Proceedings of the 21st IEEE Instrumentation and Measurement Technology Conference (IEEE Cat. No.04CH37510), 2004, vol. 3, pp. 1959-1964 Vol.3.

[114] B. Gunel, H. Hacihabiboglu, and A. M. Kondoz, "Intensity vector direction exploitation for exhaustive blind source separation of convolutive mixtures," in 2009 IEEE International Conference on Acoustics, Speech and Signal Processing, 2009, pp. 41-44.

[115] Y. X. Zou, W. Shi, B. Li, C. H. Ritz, M. Shujau, and J. Xi, "Multisource DOA estimation based on time-frequency sparsity and joint inter-sensor data ratio with single acoustic vector sensor," in 2013 IEEE International Conference on Acoustics, Speech and Signal Processing, 2013, pp. 4011-4015.

[116] X. Zhong, C. Xiaoyi, W. Wang, A. Alinaghi, and A. B. Premkumar, "Acoustic vector sensor based reverberant speech separation with probabilistic timefrequency masking," in 21st European Signal Processing Conference (EUSIPCO 2013), 2013, pp. 1-5.

[117] Y. Song and K. T. Wong, "Three-Dimensional Localization of a Near-Field Emitter of Unknown Spectrum, using an Acoustic Vector Sensor Corrupted by Additive Noise of Unknown Spectrum," IEEE Transactions on Aerospace and Electronic Systems, vol. 49, no. 2, pp. 1035-1041, 2013. 
[118] K. Wu, V. G. Reju, and A. W. H. Khong, "Multisource DOA Estimation in a Reverberant Environment Using a Single Acoustic Vector Sensor," IEEE/ACM Transactions on Audio, Speech, and Language Processing, vol. 26, no. 10, pp. 1848-1859, 2018.

[119] P. Felisberto, O. Rodriguez, and S. M. Jesus, "Estimating the multipath structure of an underwater channel using a single vector sensor," in Proceedings of Meetings on Acoustics, 2012, vol. 17: Acoustical Soc. of Am.

[120] P. J. M. Santos, P. Felisberto, F. Zabel, S. Jesus, and L. Sebastiao, "Dual accelerometer vector sensor mounted on an autonomous underwater vehicle (AUV) - Experimental results," in J. Acoust. Soc. Am., 2017, vol. 30: Acoustical Soc. Am.

[121] J. C. Shipps and B. M. Abraham, "The Use of Vector Sensors for Underwater Port and Waterway Security," in IEEE Sensors for Industry Confrence, 2004, pp. 4144.

[122] H.-E. deBree, W. F. Druyvesteyn, E. Berenschot, and M. Elwenspoek, "THREEDIMENSIONSOAULN D INTENSITY MEASUREMENTS USING MICROFLOWN PARTICLE VELOCITY SENSORS," IEEE, pp. 124-129, 1999.

[123] H.-E. deBree, P. Leussink, T. Korthorst, H. Jansen, T. Lammerink, and M. Elwenspoek, "The Micro-Flown: A Novel Device Mearuring Acoustical Flows," presented at the The 8th International Conference on Solid-state Sensors and Actuators, and Eurosensors IX, Stockholm, 1995.

[124] A. Zhao, X. Bi, J. Hui, C. Zeng, and L. Ma, "An Improved Aerial Target Localization Method with a Single Vector Sensor," J. Sensors, vol. 17, 2017.

[125] T. Padois, "Acoustic source localization based on the generalized crosscorrelation and the generalized mean with few microphones," J. Acoust. Soc. Am. Express Letters, vol. 143, pp. EL393-398, 2018.

[126] M. Shujau, C. H. Ritz, and I. S. Burnett, "Designing Acoustic Vector Sensors for localisation of sound sources in air," in 2009 17th European Signal Processing Conference, 2009, pp. 849-853.

[127] M. Shujau, C. H. Ritz, and I. S. Burnett, "Using in-air Acoustic Vector Sensors for tracking moving speakers," in 2010 4th International Conference on Signal Processing and Communication Systems, 2010, pp. 1-5.

[128] P. Rasmussen. (1989) Source Location Using Vector Intensity Measurements. S.V. Sound and Vibration. 28-33. 
[129] K. Janssens, D. DeWeer, and F. Bianciardi, "On-Line Sound Brush Measurement Technique for 3D Noise Emission Studies," presented at the SAE International, 2013.

[130] J. W. S. Rayleigh, The Theory of Sound. London: MacMillan and Co., Ltd., 1896.

[131] T. J. Schultz, "Acoustic Wattmeter," J. Acoust. Soc. Am., vol. 28, pp. 693-699, 1956.

[132] O. Roth, "A sound intensity real-time analyser," in Recent developments in acoustic intensity measurement, Senlis, France, 1981, pp. 69-74.

[133] Meggitt, "Vector Sensor Operating Guide," vol. Rev E,

[134] D. P. Jarrett, E. A. P. Habets, and P. A. Naylor, "Eigenbeam-based acoustic source tracking in noisy reverberant environments," in 2010 Conference Record of the Forty Fourth Asilomar Conference on Signals, Systems and Computers, 2010, pp. 576-580.

[135] RaceLogic, "VBOX 3i Dual Antenna | 100Hz Vehicle Dynamics Measurement," Available: https://www.vboxautomotive.co.uk/index.php/en/products/dataloggers/vbox-3i-dual-antenna

[136] NOAA. (2017). Geomagnetism Magnetic Field Calculators. Available: https://www.ngdc.noaa.gov/geomag-web/ 


\section{A Additional Plots}

\section{A.1 Weather station data}
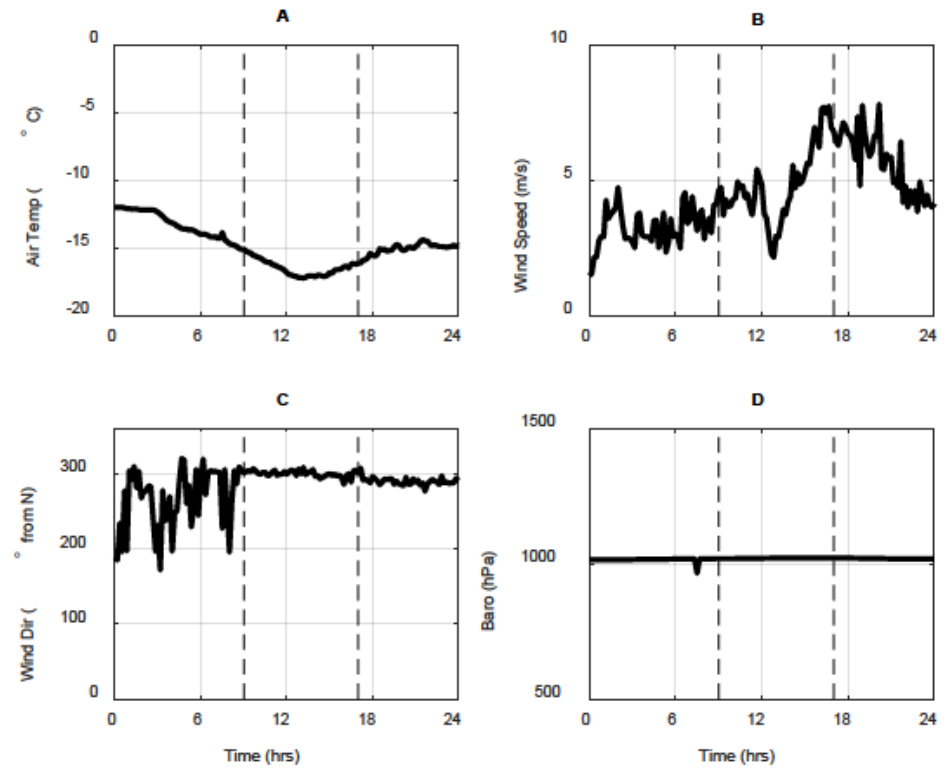

Figure A.1 Weather station data for February $4^{\text {th }}$ 2018. Measured at Great Lakes Research Center in Houghton, MI. Experiments on Portage Lake took place during times between the vertical dashed lines.
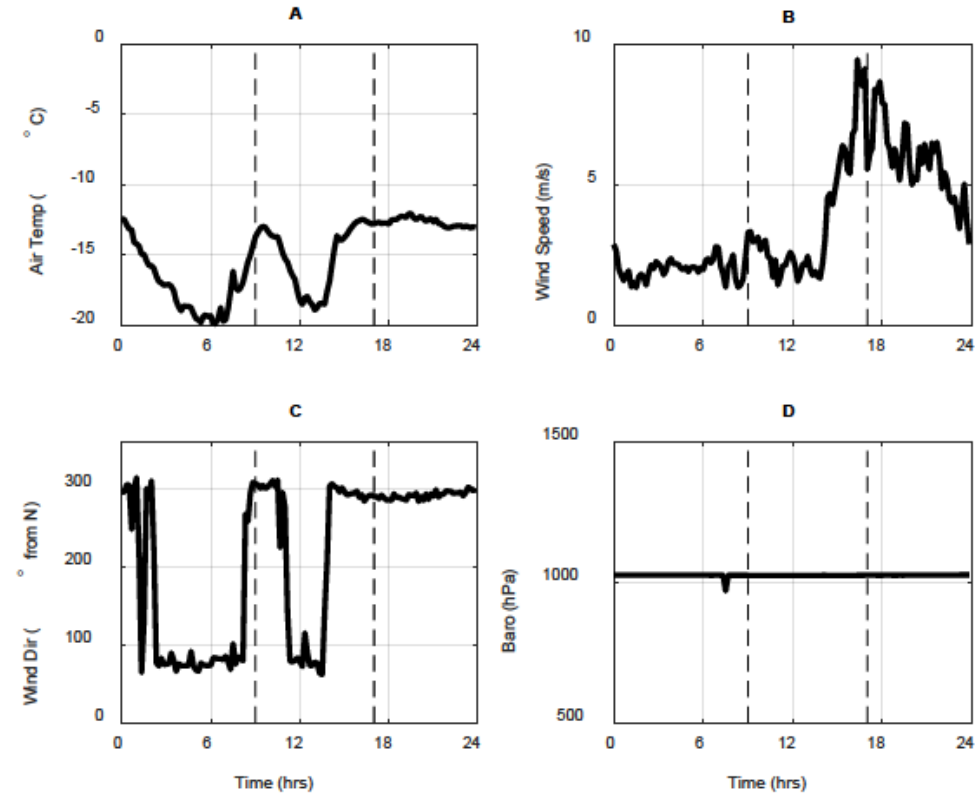

Figure A.2 Weather station data for February $8^{\text {th }}$ 2018. Measured at Great Lakes Research Center in Houghton, MI. Experiments on Portage Lake took place during times between the vertical dashed lines. 


\section{A.2 Portage Lake Bathymetry}

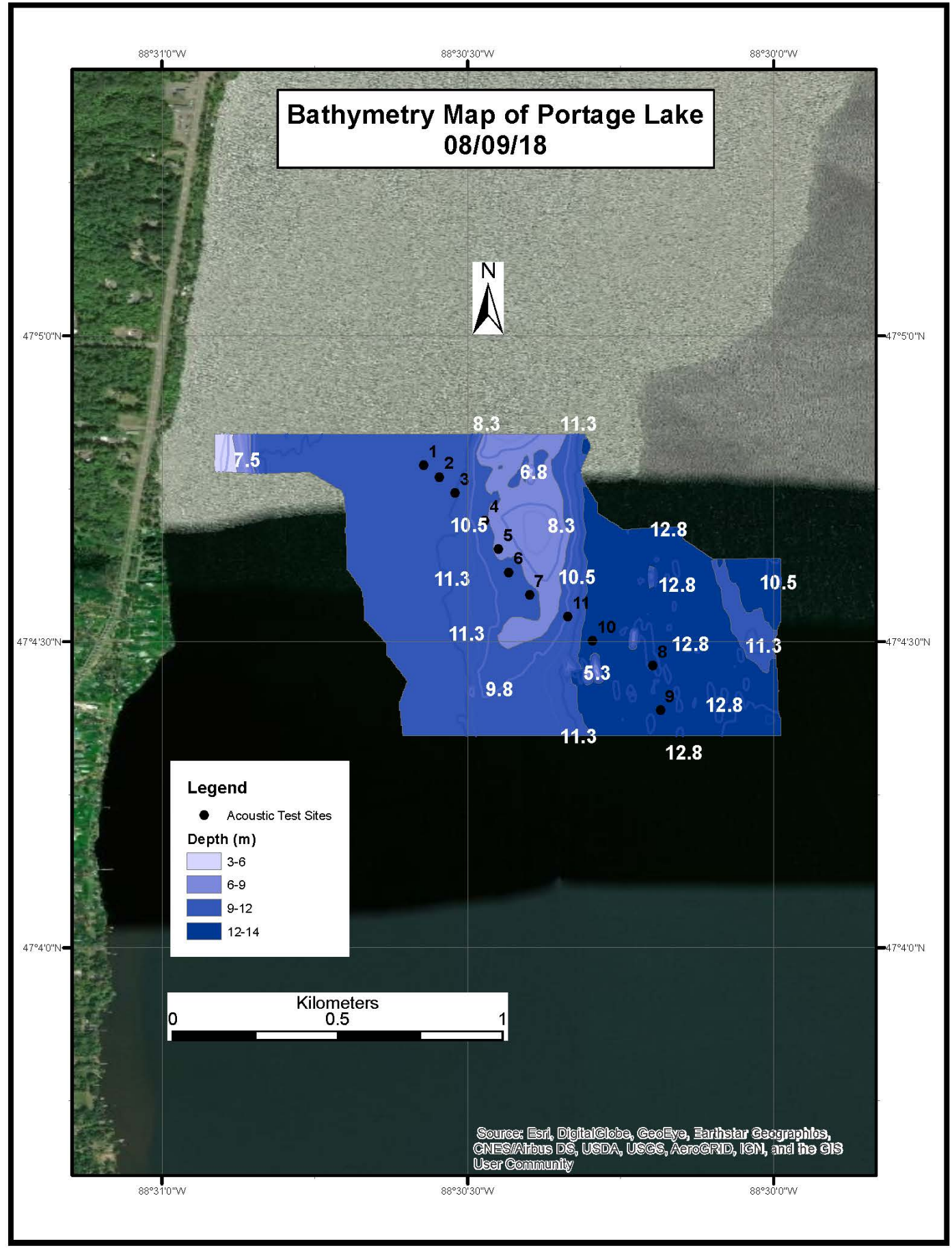

Figure A.3 Portage Lake bathymetry map for test site area. 


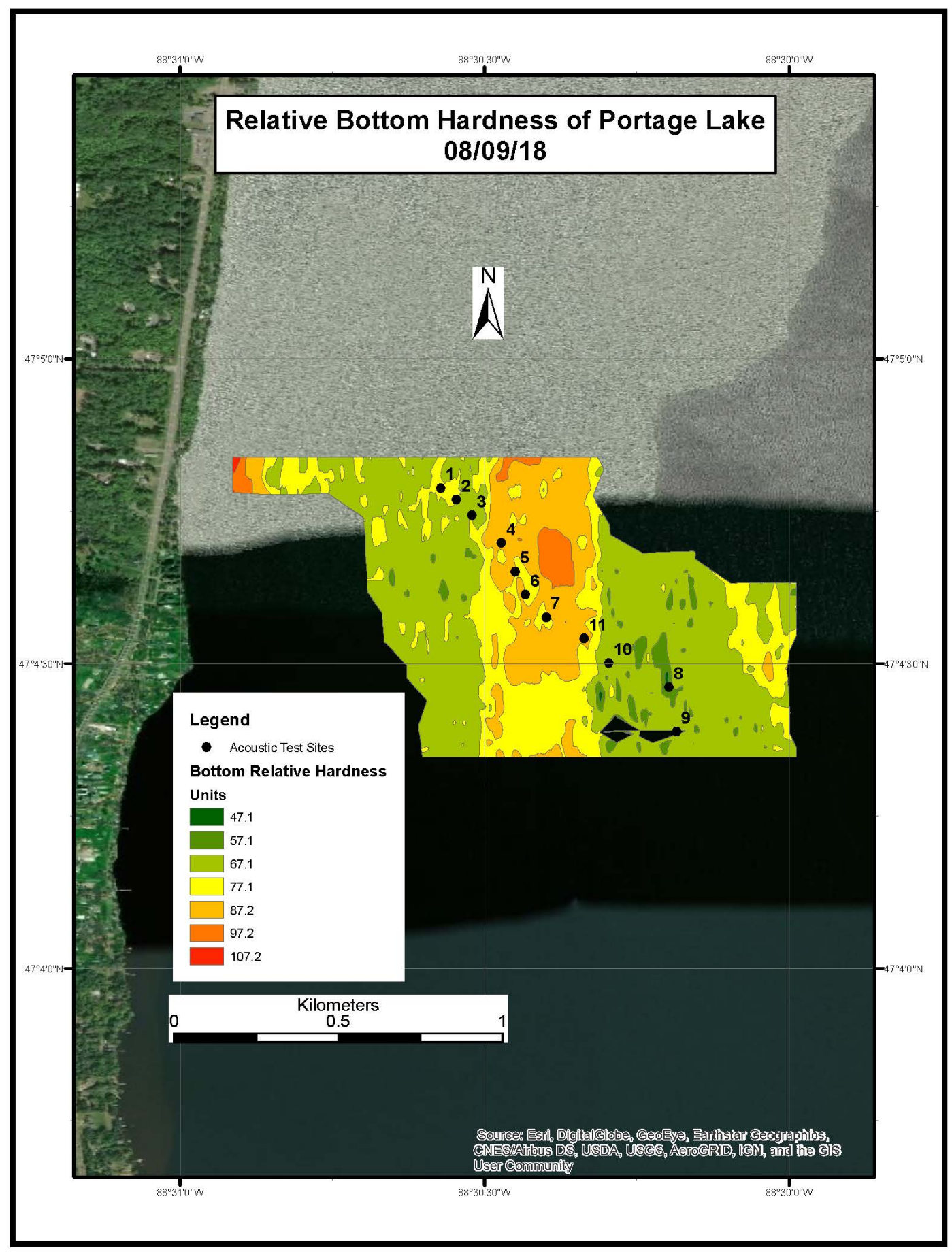

Figure A.4 Portage Lake relative bottom hardness for test site area. Absolute units are not reported. 


\section{A.3 Portage Lake IRF, FRF, and RL}
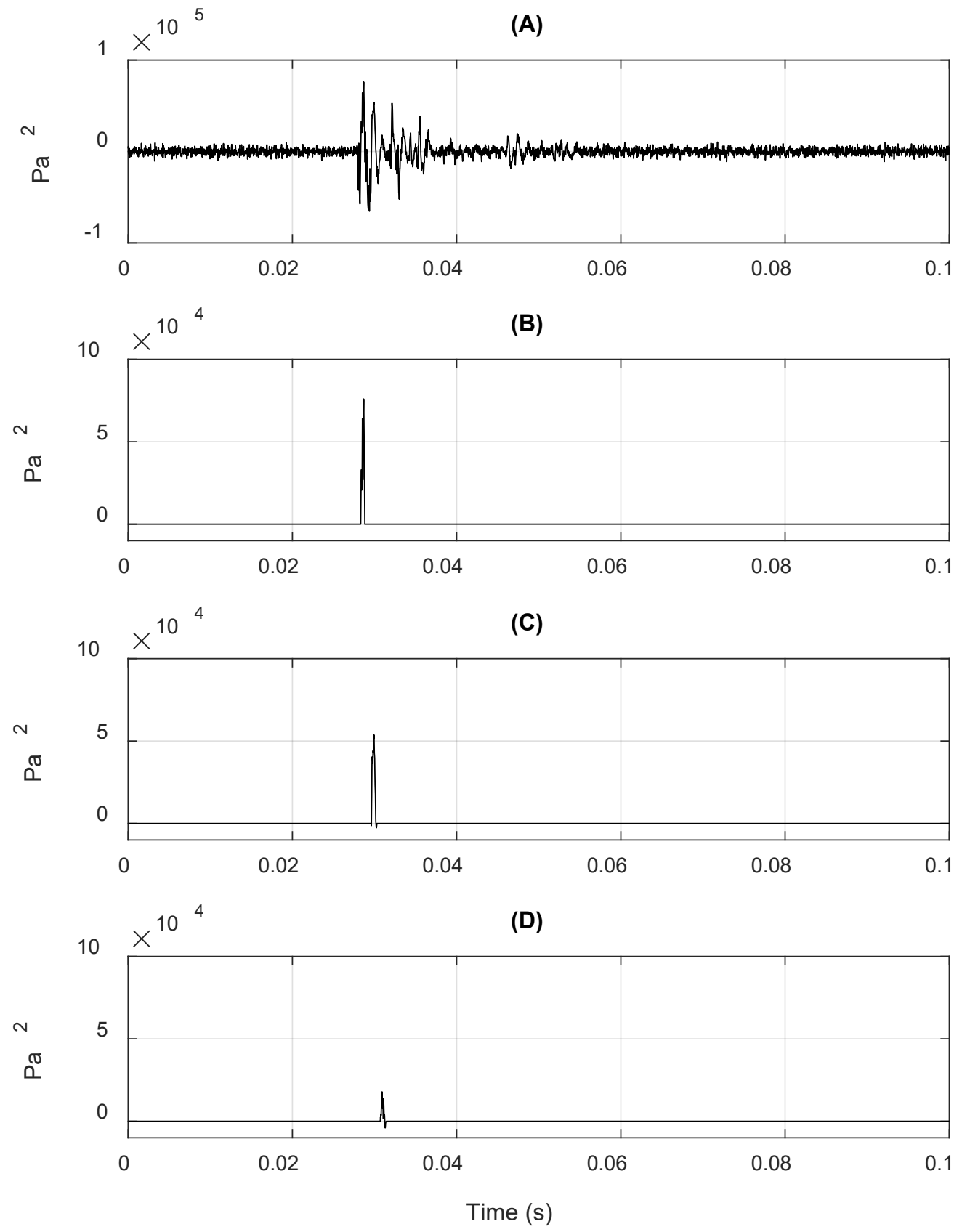

Figure A.5 Impulse response function $48 \mathrm{~m}$ from the underwater source. (A) Full IRF from MLS signal. (B) Incident arrival. (C) Windowed ice reflection (D) Windowed bottom reflection. 
(A)

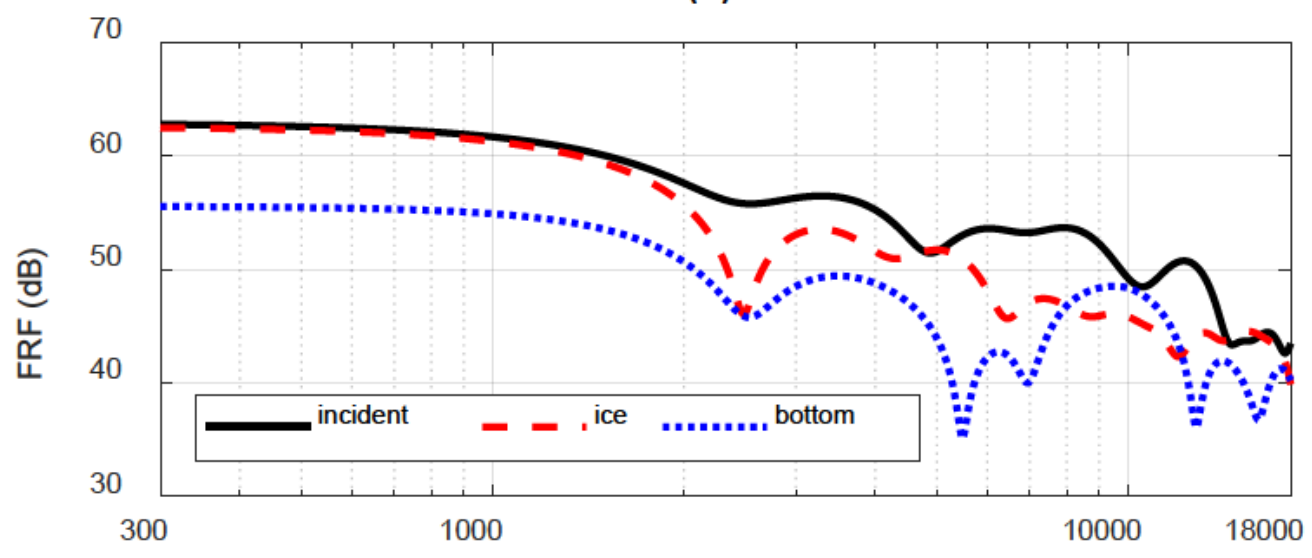

(B)

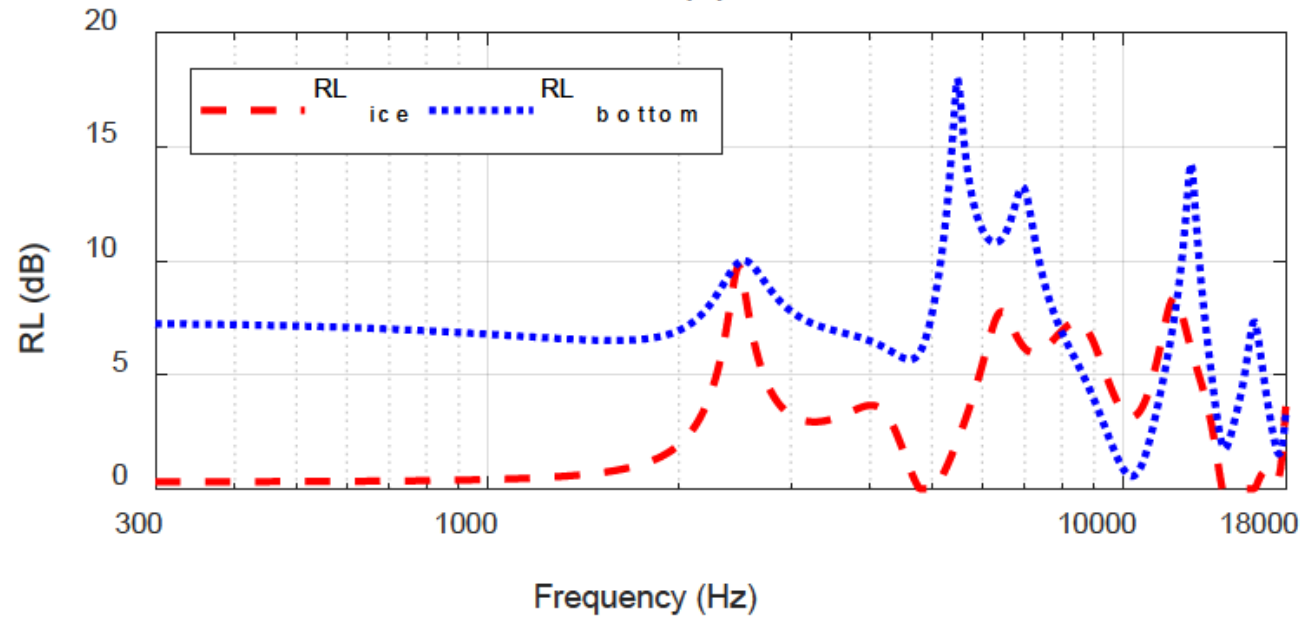

Figure A.6 (A) Frequency response functions and (B) Reflection loss for $\phi_{I}=8.5^{\circ}$ and $\phi_{B}=16^{\circ}$. Source to receiver distance equal to $48 \mathrm{~m}$. 

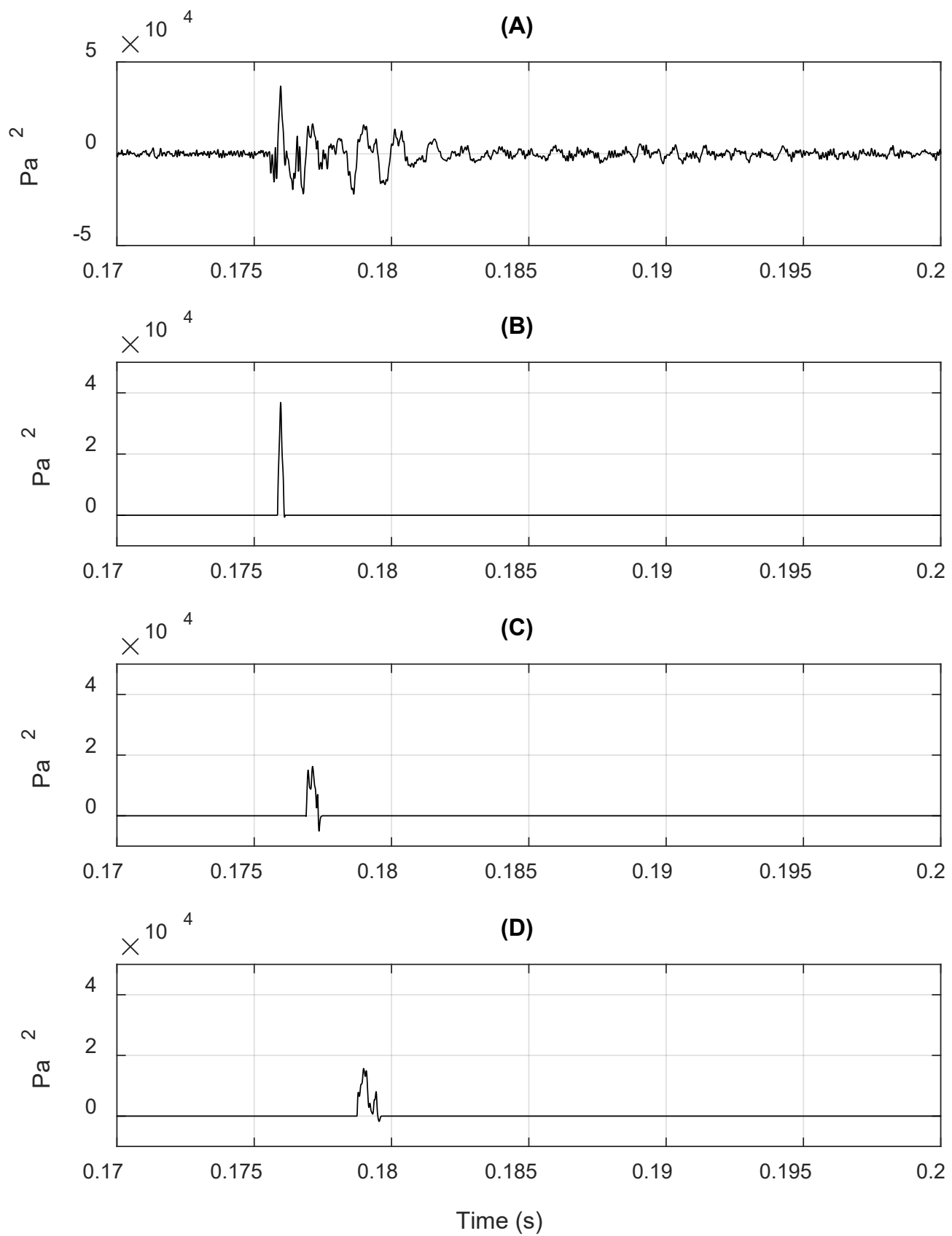

Figure A.7 Impulse response function $210 \mathrm{~m}$ from the underwater source. (A) Full IRF from MLS signal. (B) Incident arrival. (C) Windowed ice reflection (D) Windowed bottom reflection. 
(A)

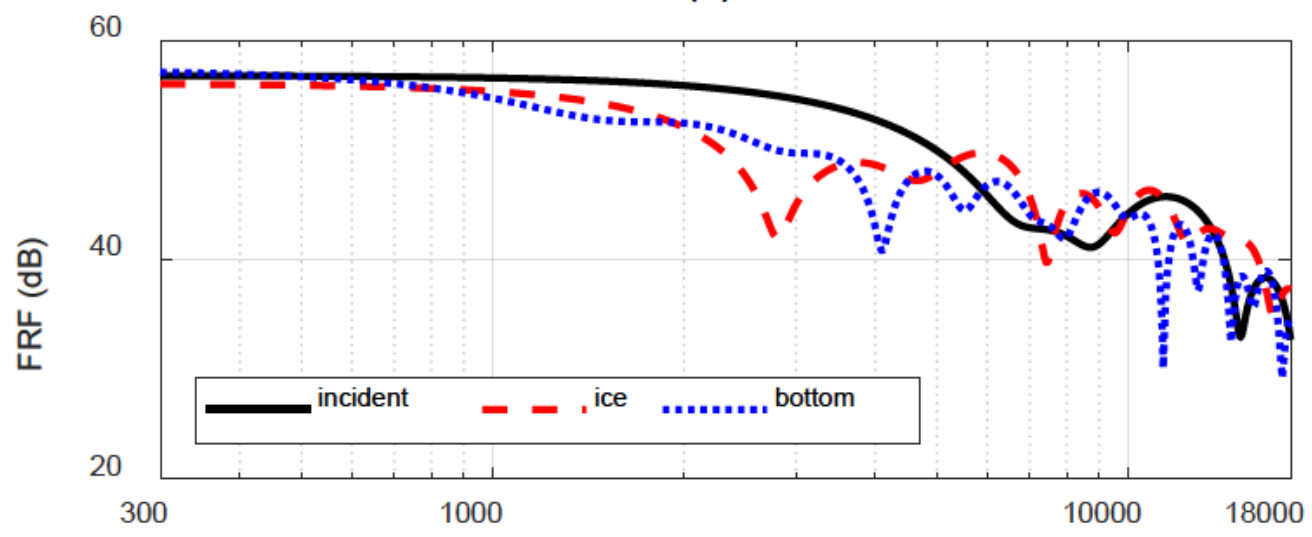

(B)

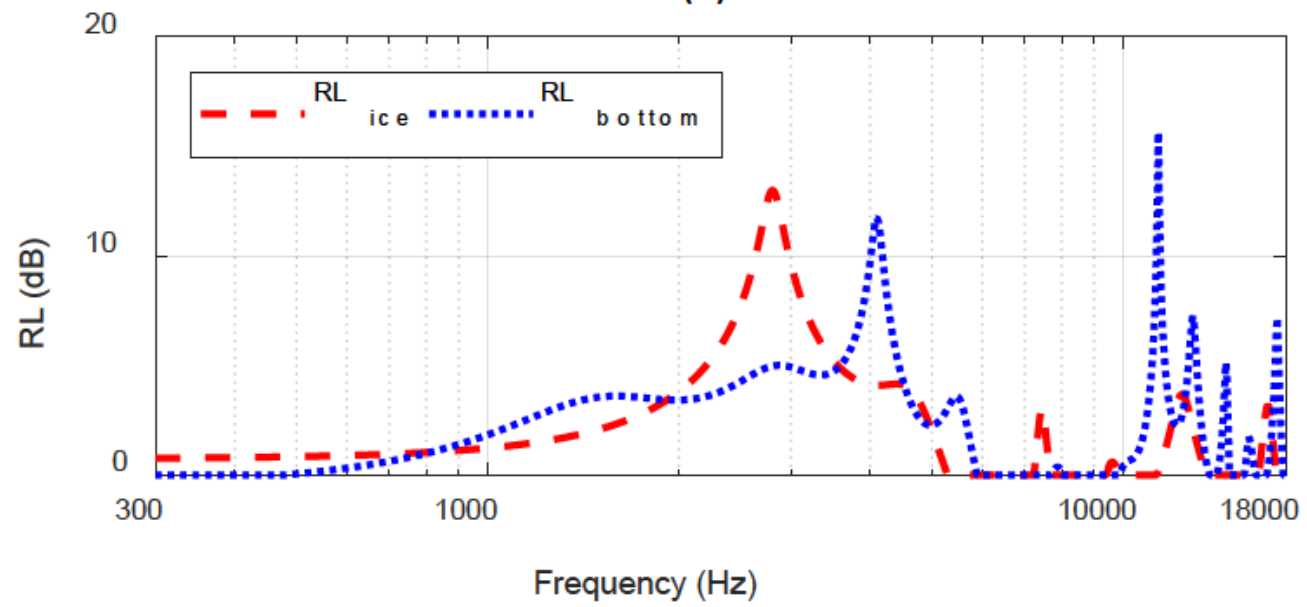

Figure A.8 (A) Frequency response functions and (B) Reflection loss for $\phi_{I}=2^{\circ}$ and $\phi_{B}=4^{\circ}$. Source to receiver distance equal to $210 \mathrm{~m}$. 


\section{A.4 AVS Data}

\section{A.4.1 Acoustic Hardware Phase Mismatch}

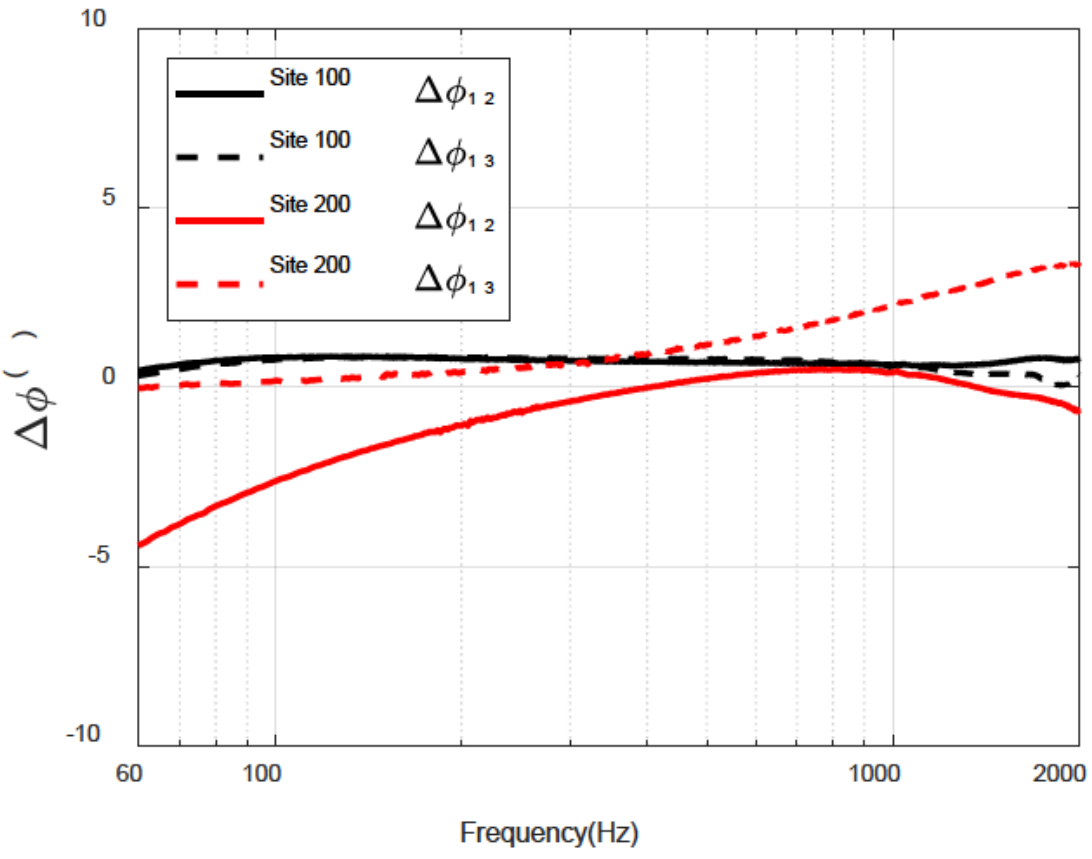

Figure A.9 AVS measurement channel phase mismatch for site 100 and site 200 .

\section{A.4.2 Target Vehicle Path (GPS Position)}

These plots show the path driven by the target vehicle (Polaris Ranger) in terms of latitude and longitude coordinates collected with the VBOX differential GPS system. The red circle represent the coordinates of site 100 and the blue square represents the coordinates of site 200 . 


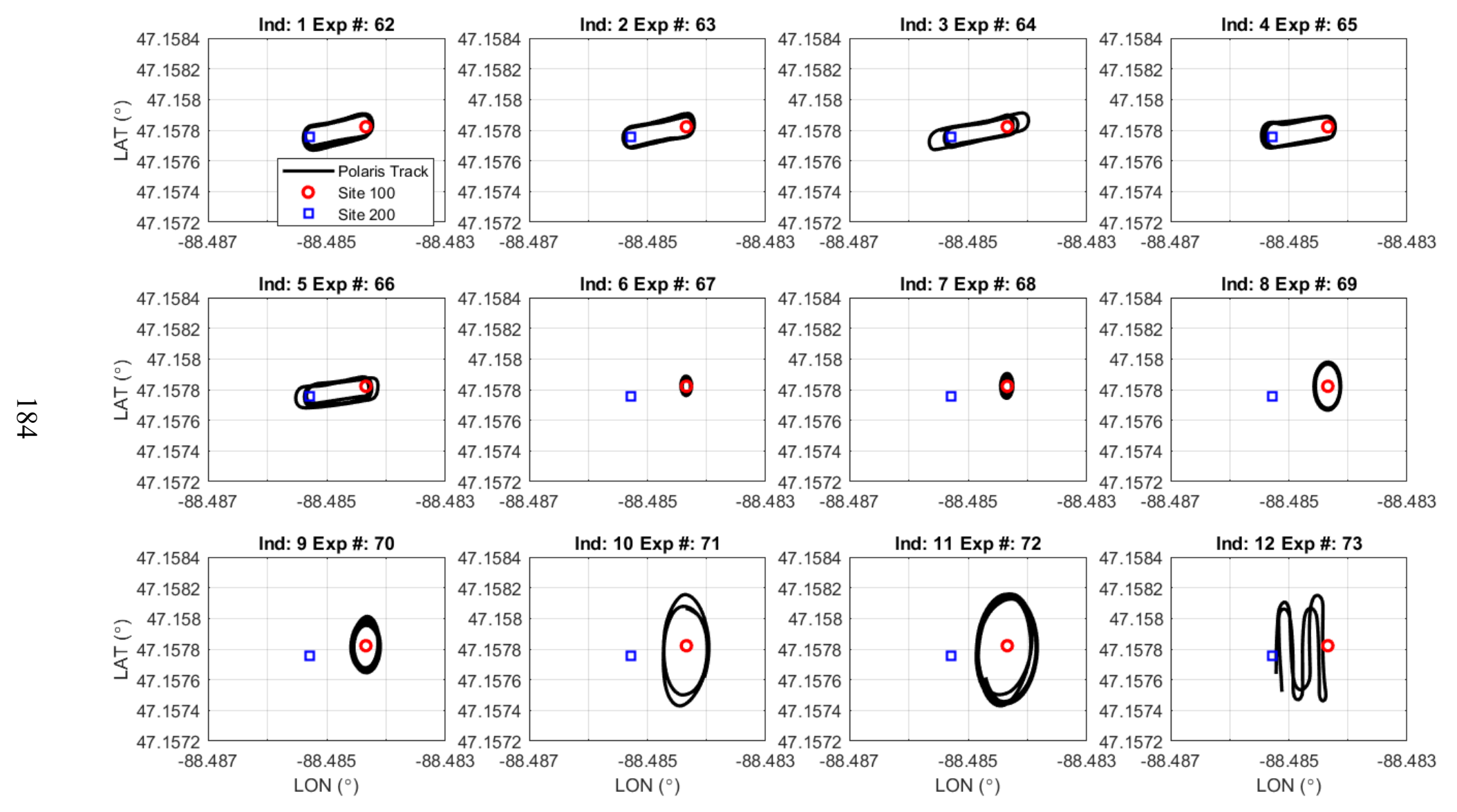

Figure A.10 Vehcile GPS tracks for AVS experiments 62 through 73 

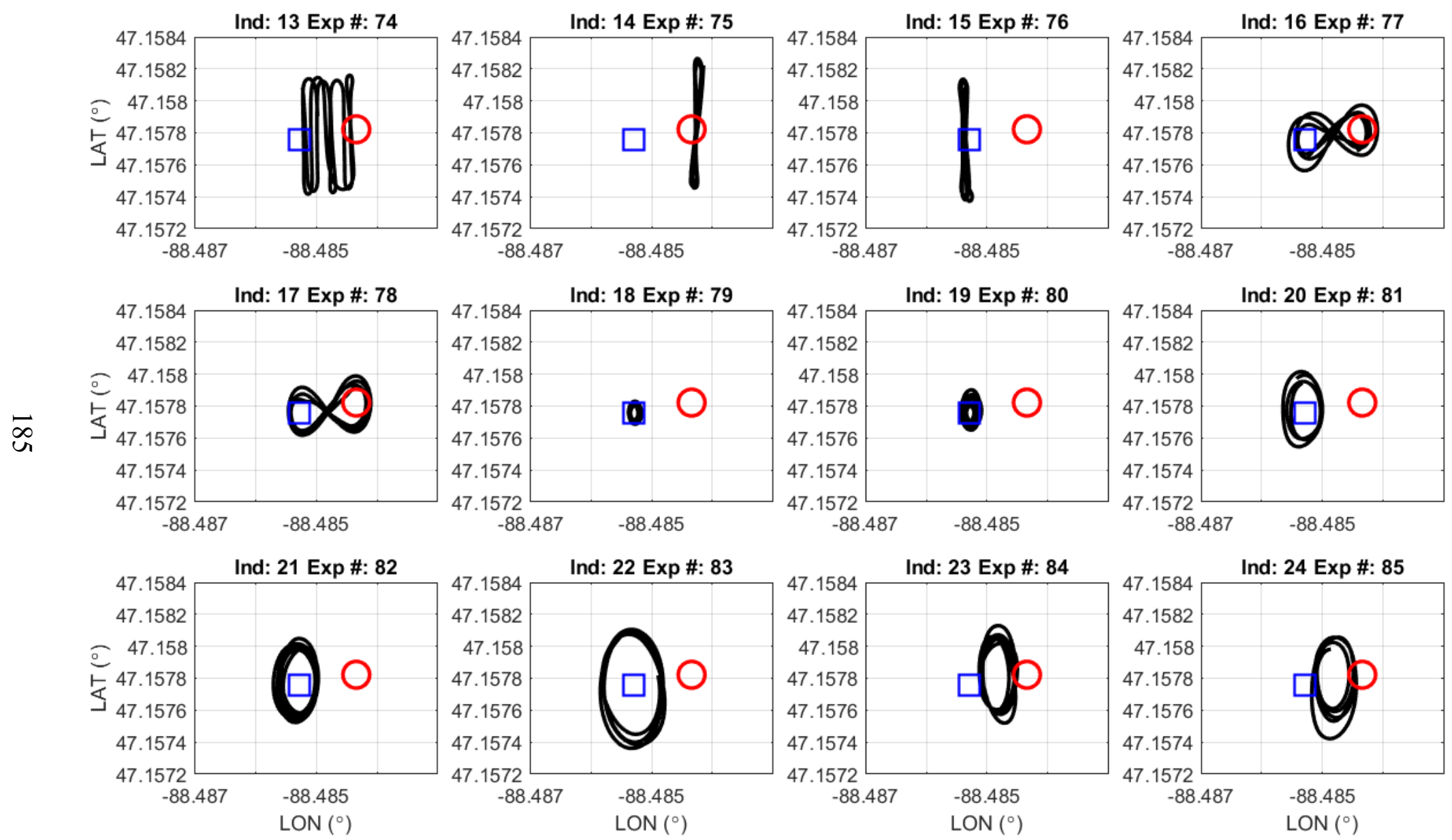

Figure A.11 Vehcile GPS tracks for AVS experiments 74 through 85 

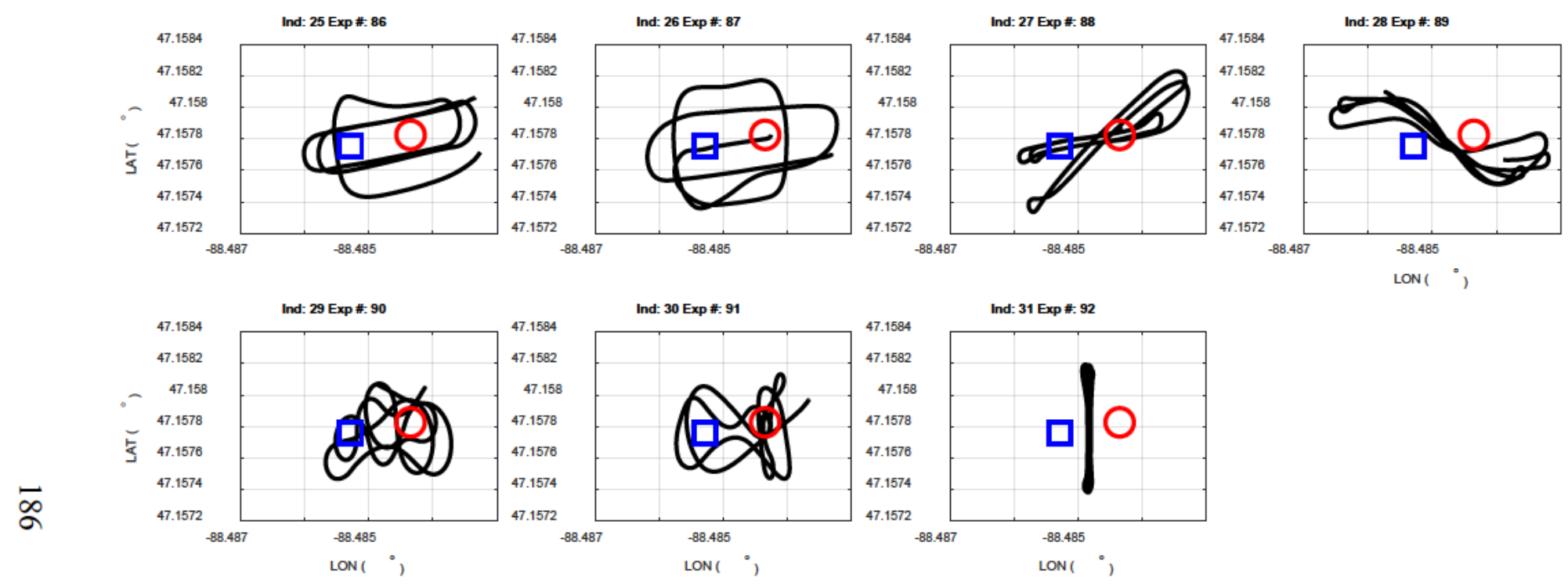

Figure A.12 Vehcile GPS tracks for AVS experiments 86 through 92 


\section{A.4.3 Site $100 \mathrm{DOA}$}

These plots show the direction of arrival computed from site 100 AVS for all experiments.
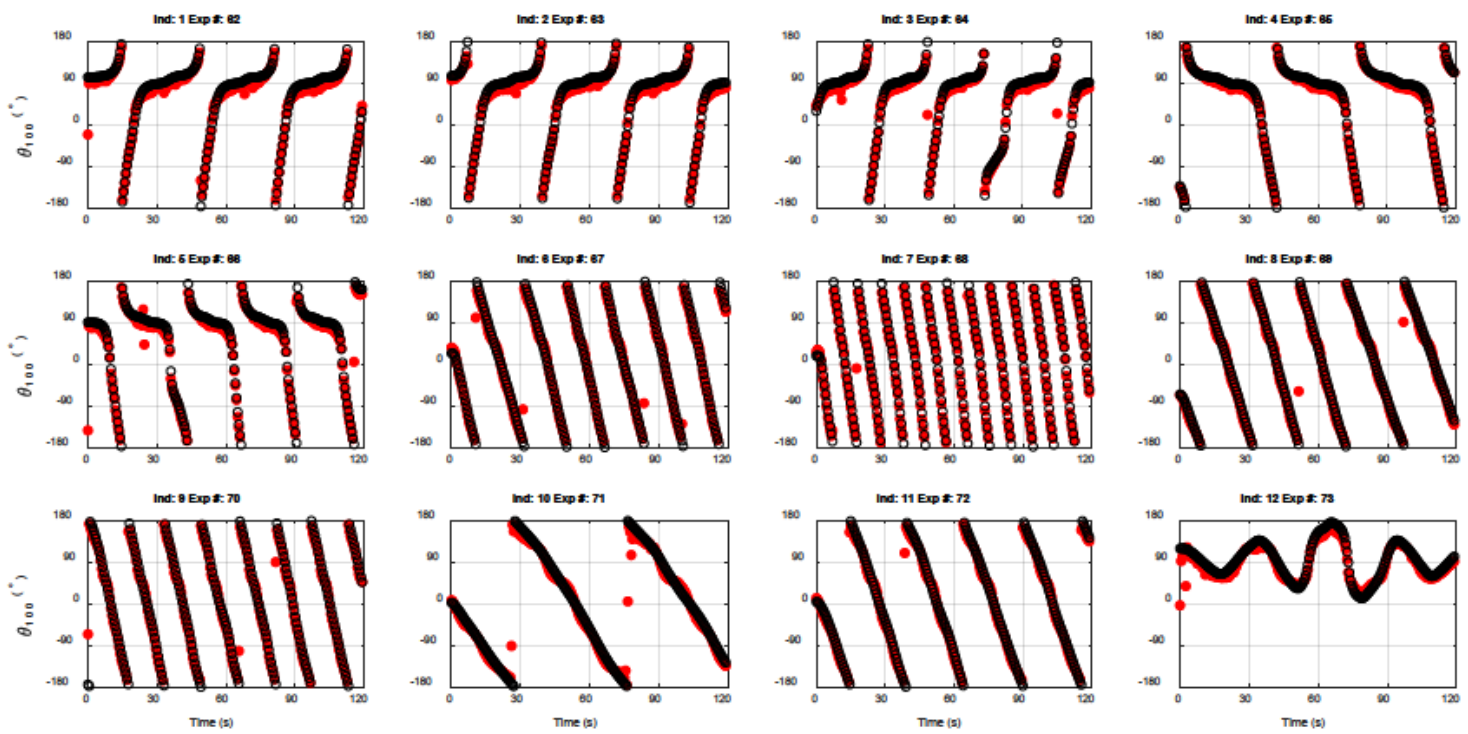

Figure A.13 DOA from site 100 (red dot) and GPS ground truth (black). AVS experiments 62 to 73.
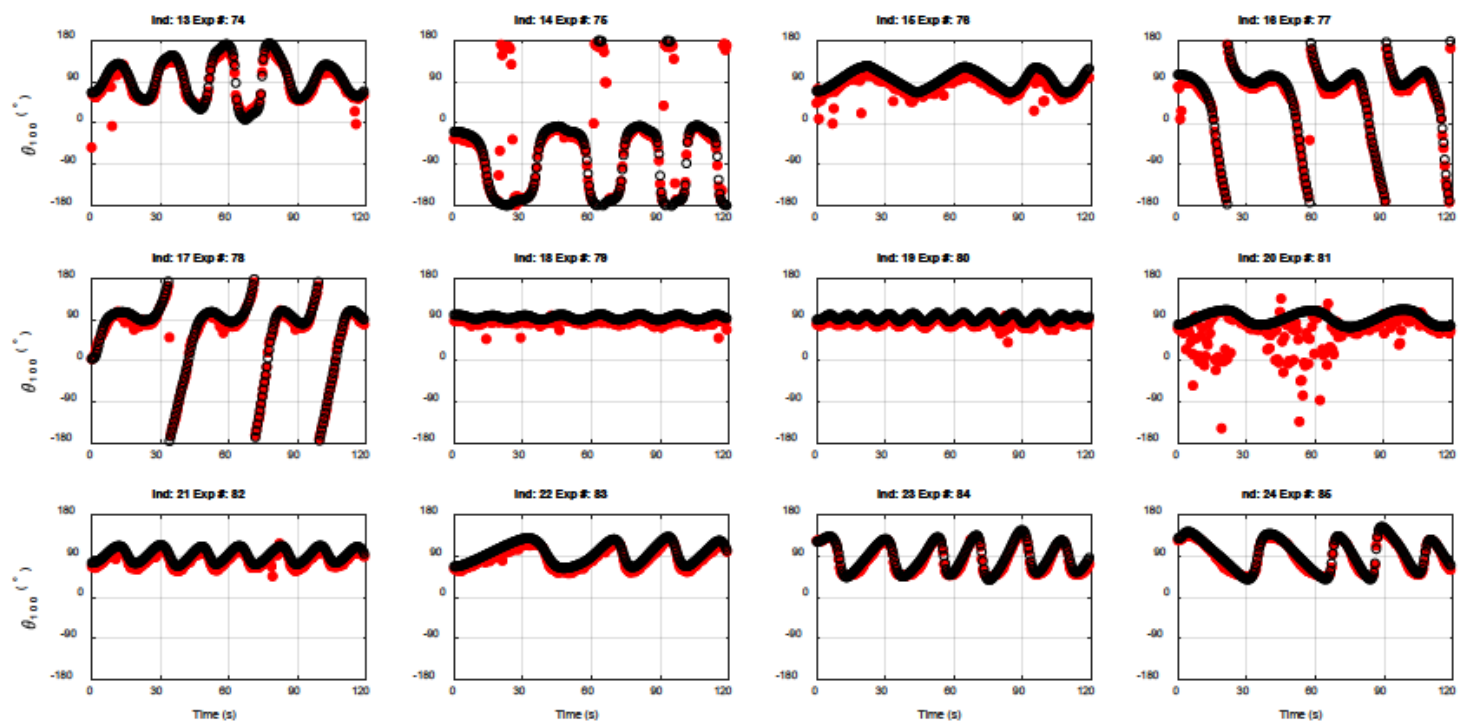

Figure A.14 DOA from site 100 (red dot) and GPS ground truth (black). AVS experiments 74 to 85. 

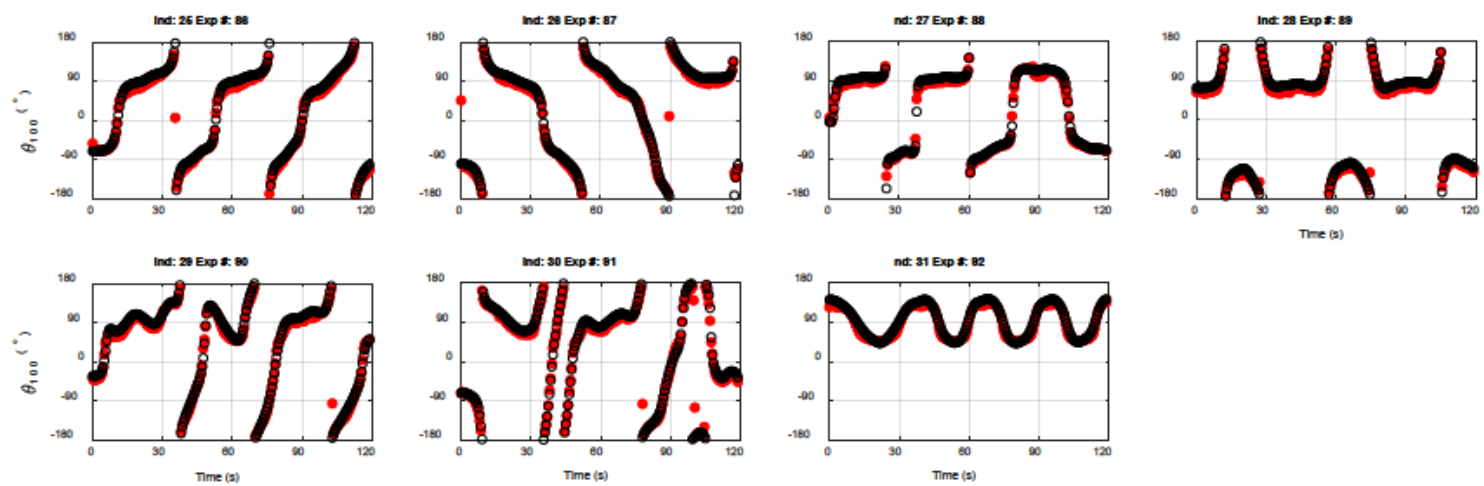

Figure A.15 DOA from site 100 (red dot) and GPS ground truth (black). AVS experiments 86 to 92.

\section{A.4.4 Site 200 DOA}

These plots show the direction of arrival computed from site 200 AVS for all experiments.
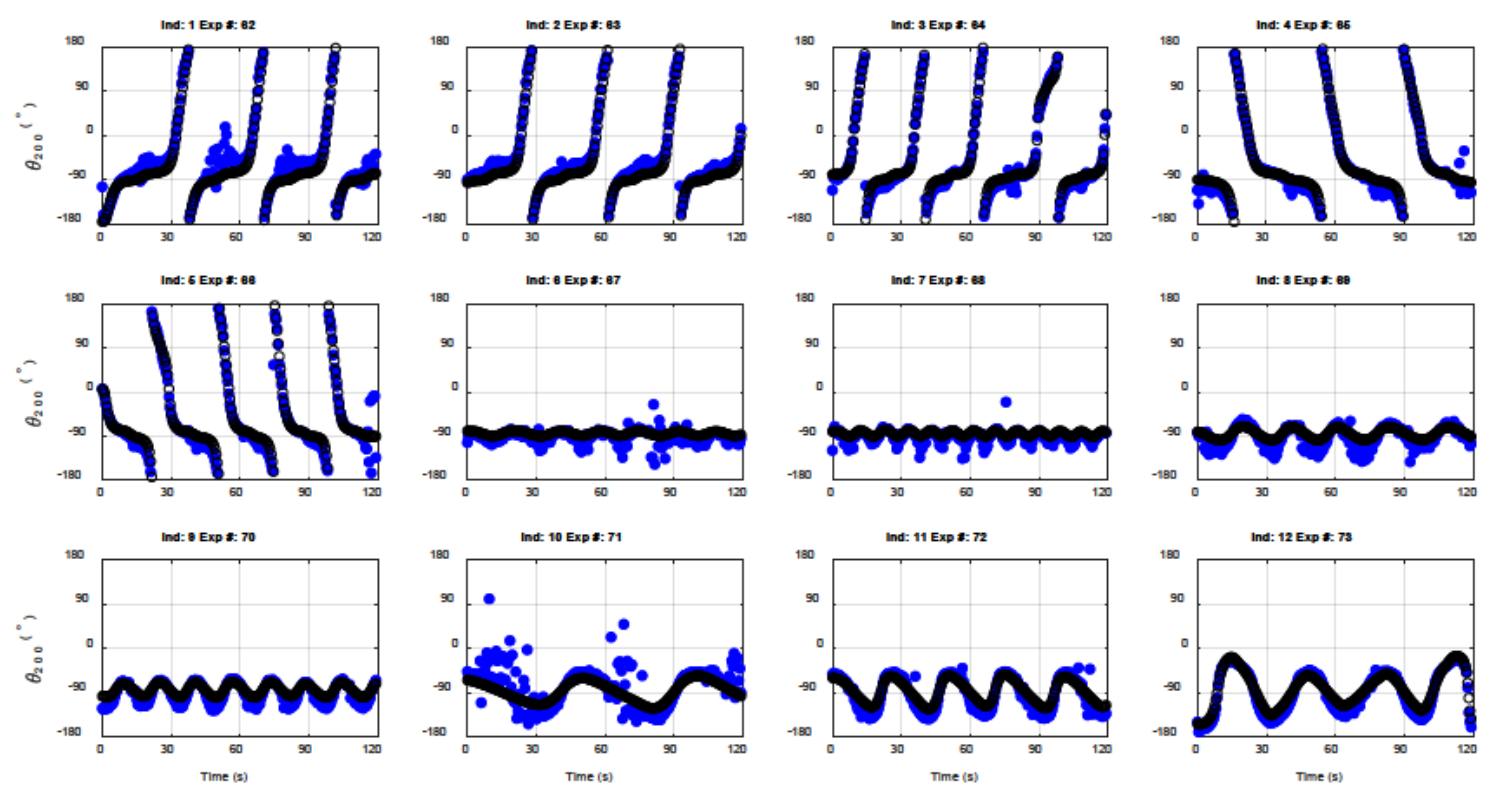

Figure A.16 DOA from site 200 (blue dash) and GPS ground truth (black). AVS experiments 62 to 73. 

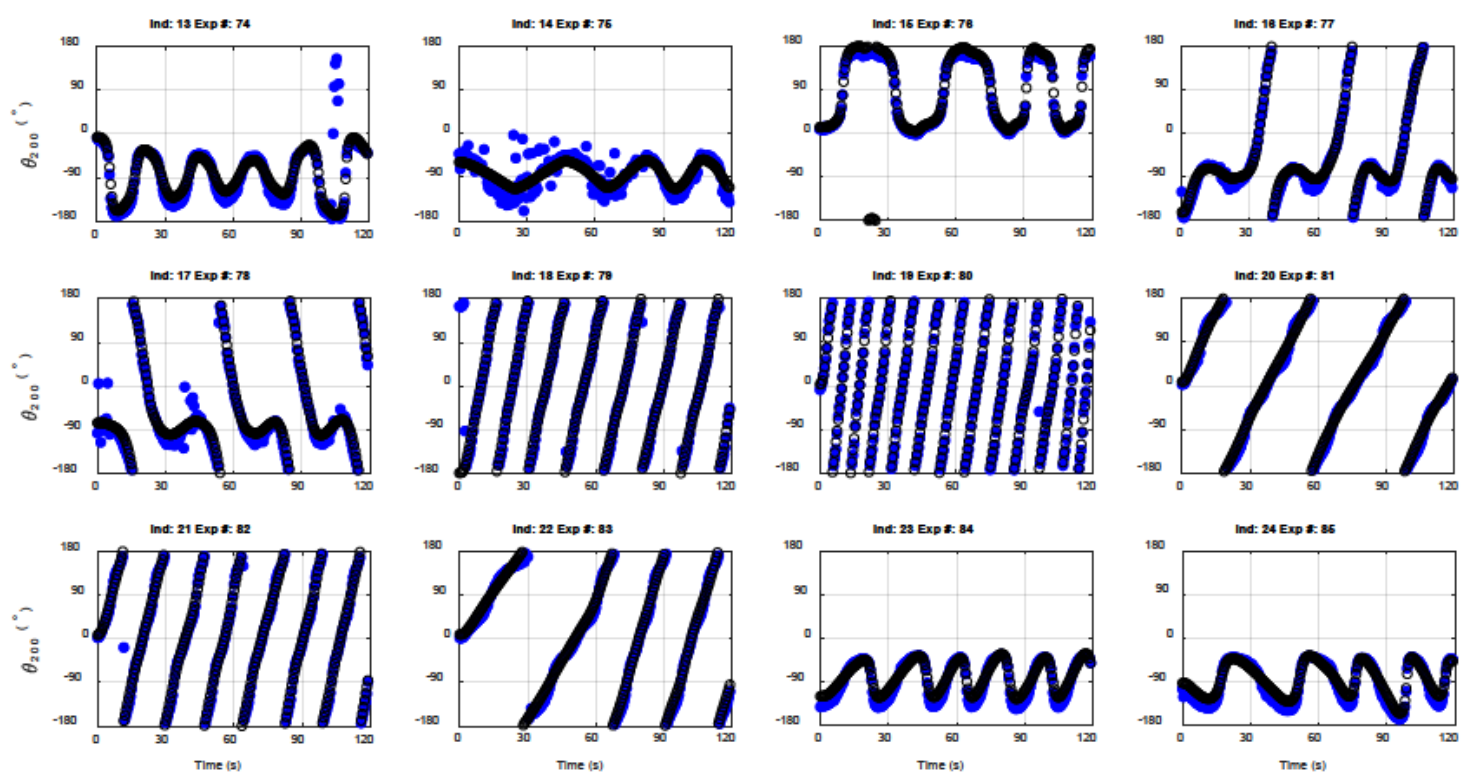

Figure A.17 DOA from site 200 (blue dash) and GPS ground truth (black). AVS experiments 74 to 85.
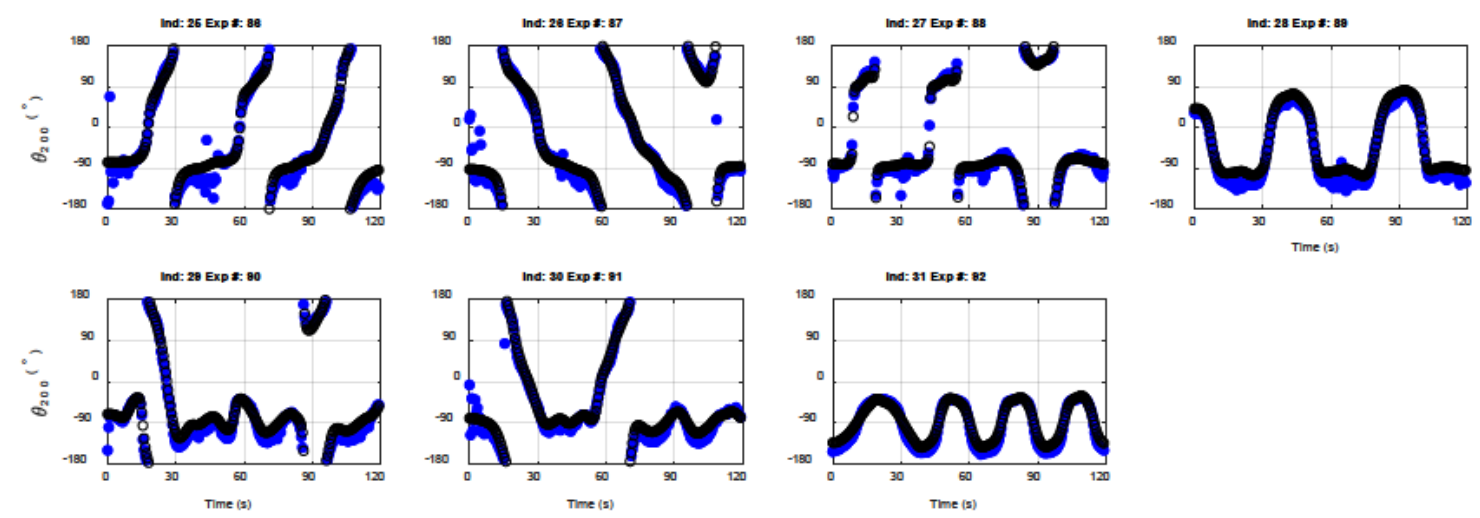

Figure A.18 DOA from site 200 (blue dash) and GPS ground truth (black). AVS experiments 86 to 92.

\section{A.4.5 Standard Deviation of DOA}

These plots show the standard deviation of the direction of arrival measured at sites 100 and 200 . 

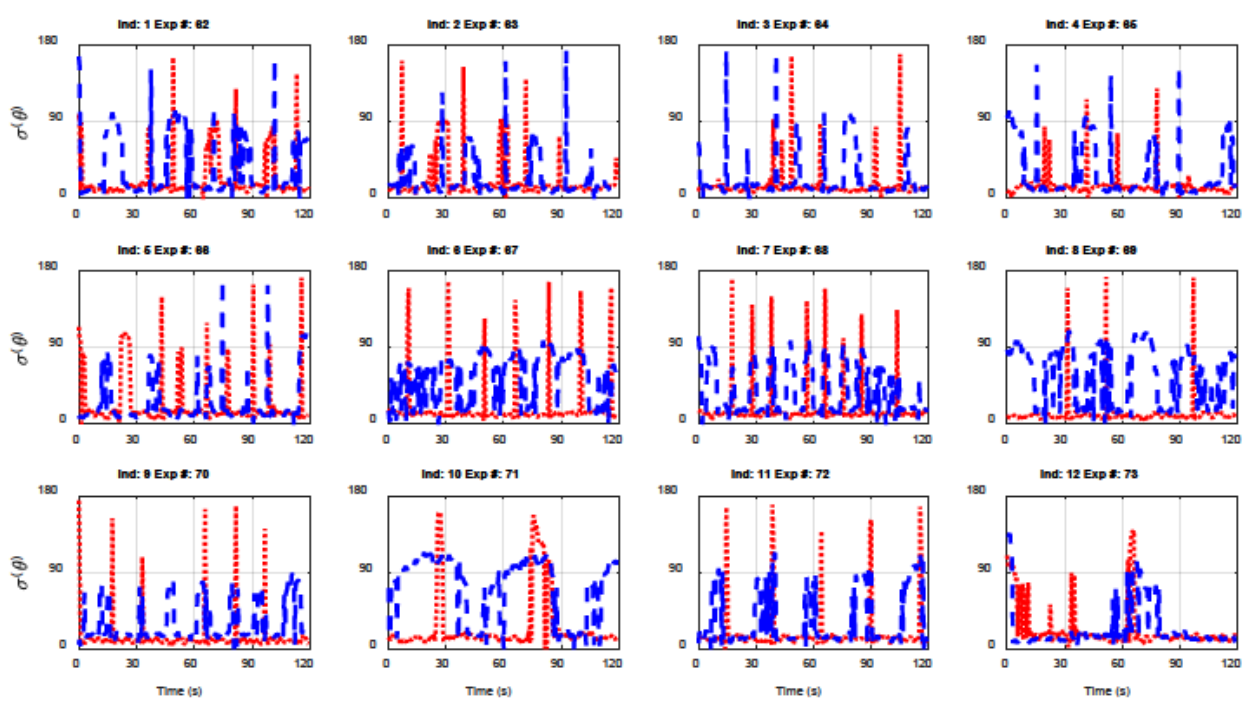

Figure A.19 DOA standard deviation for sites 100 (red dot) and 200 (blue dash). AVS experiments 62 to 73 .
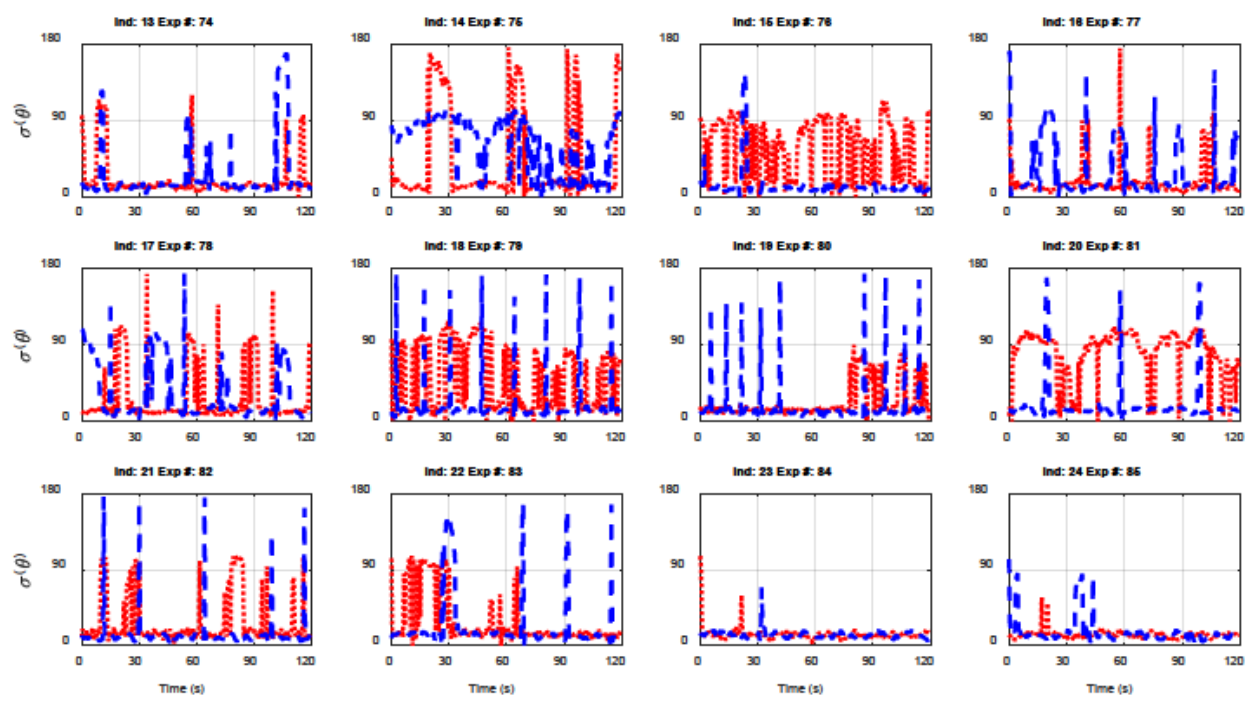

Figure A.20 DOA standard deviation for sites 100 (red dot) and 200 (blue dash). AVS experiments 74 to 85 . 

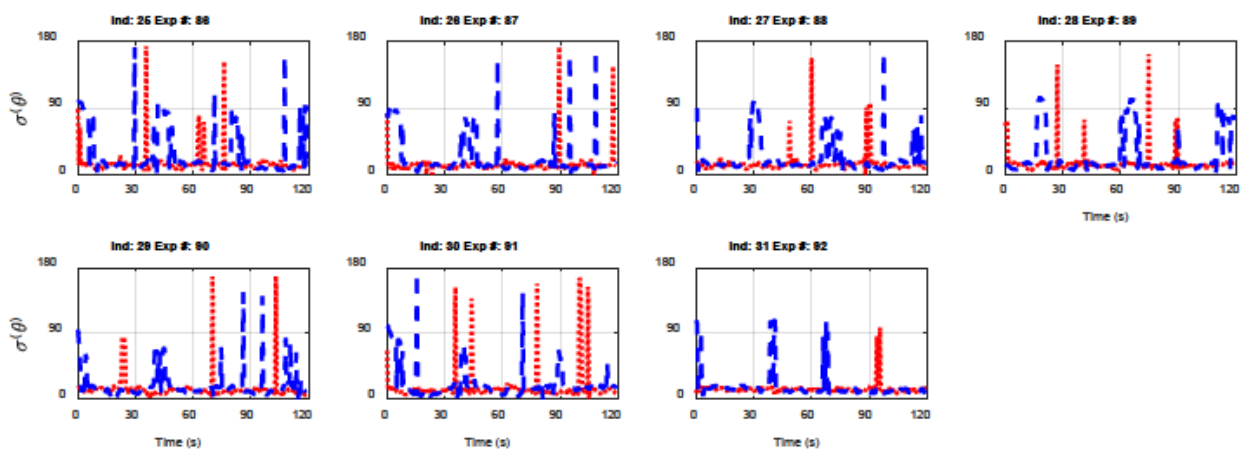

Figure A.21 DOA standard deviation for sites 100 (red dot) and 200 (blue dash). AVS experiments 86 to 92 .

\section{A.4.6 AVS SNR}

These plots show the signal-to-noise ratio at the AVS sites (100 and 200) for all experiments.
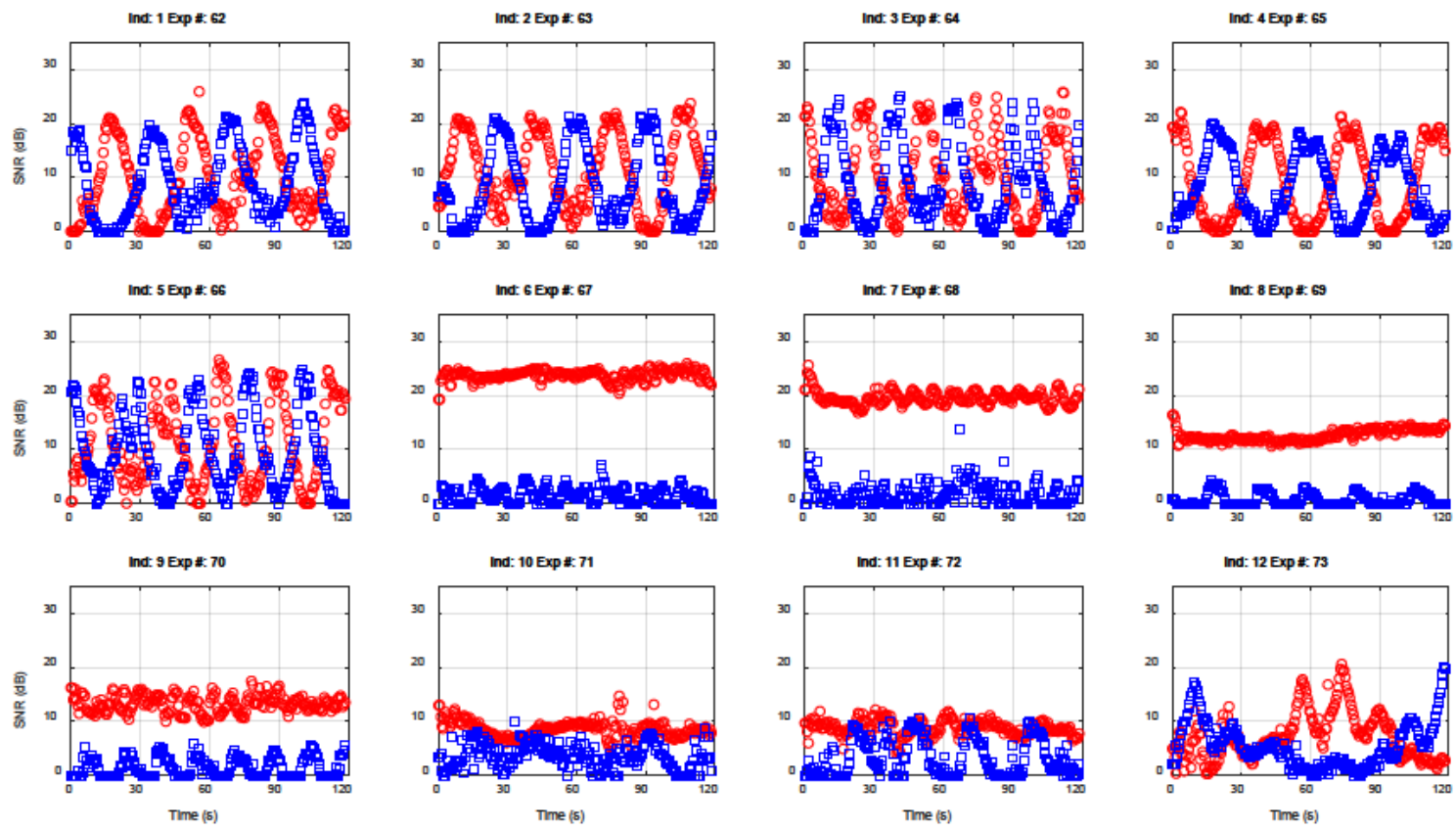

Figure A.22 SNR for sites 100 and 200. AVS experiments 62 to 73. 

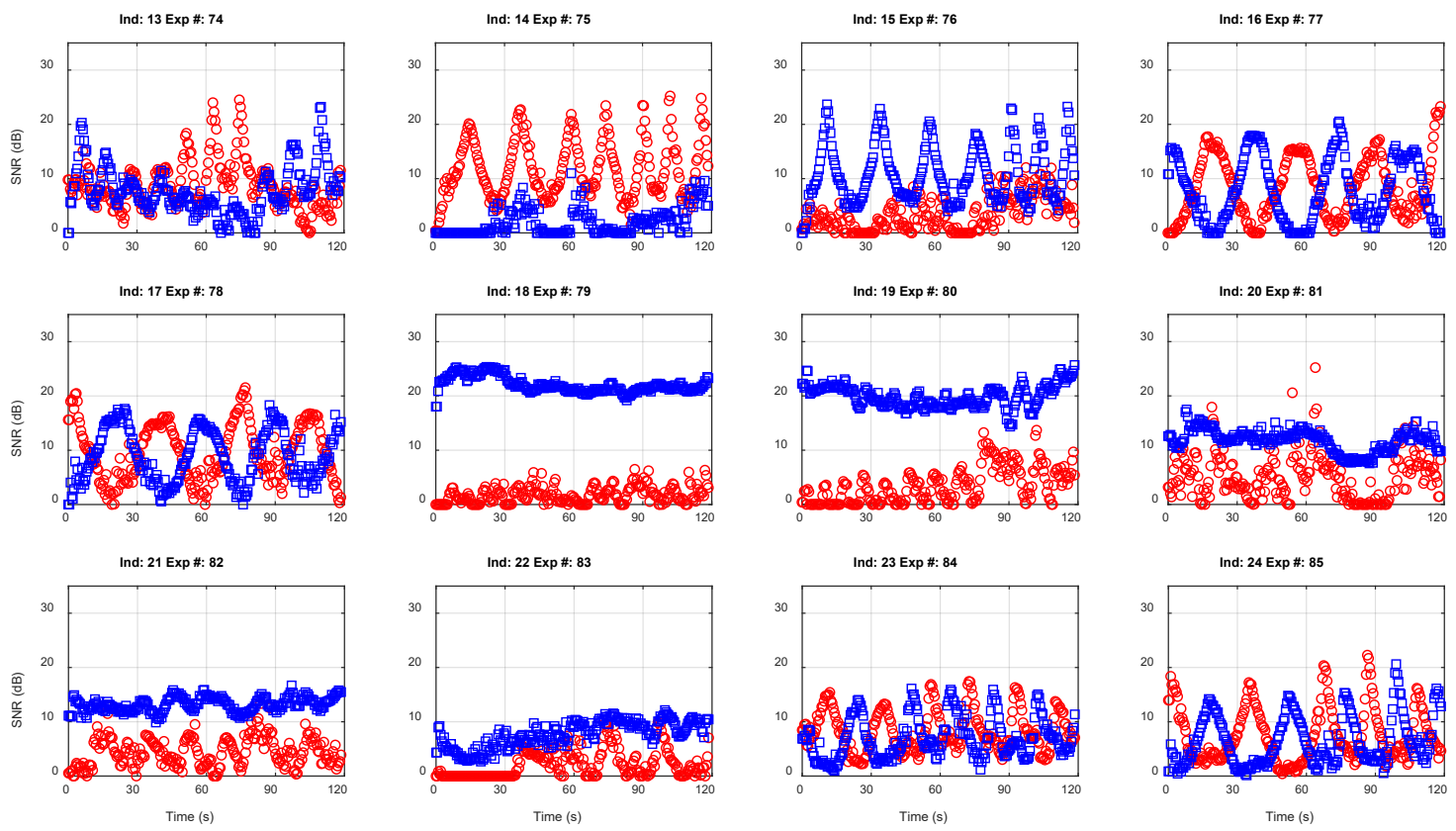

Figure A.23 SNR for sites 100 and 200. AVS experiments 74 to 85 .
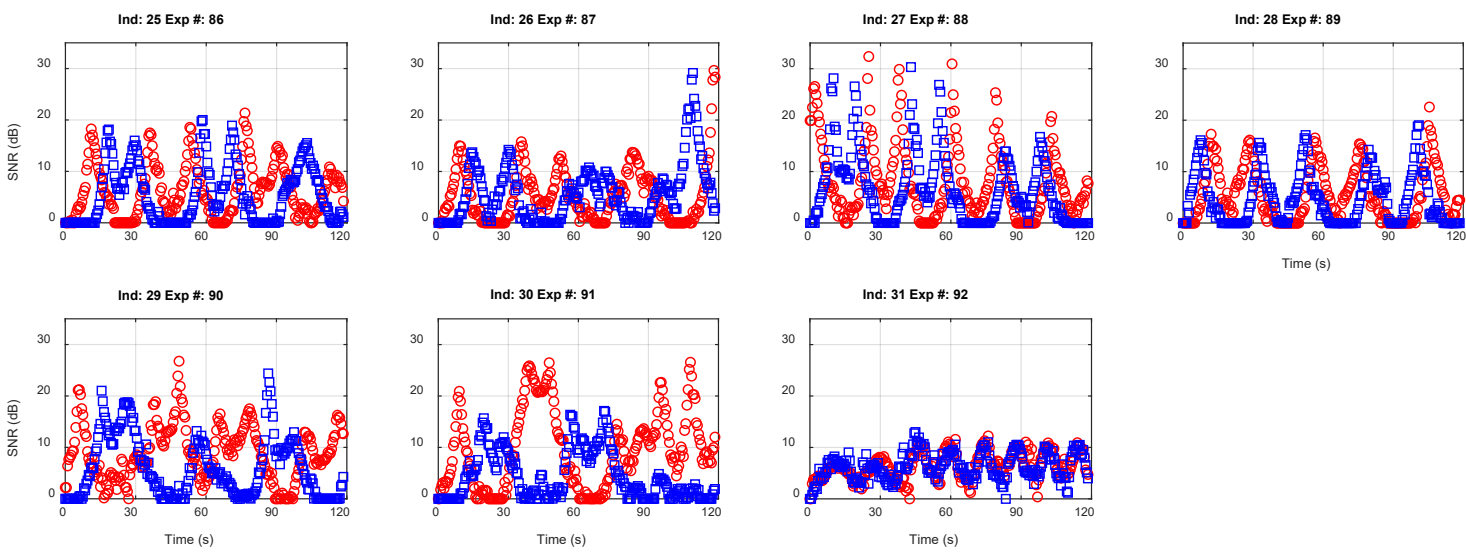

Figure A.24 SNR for sites 100 and 200. AVS experiments 86 to 92.

\section{A.4.7 Vehicle Speed}

These plots show the target vehicle (Polaris Ranger) speed for all AVS experiments measured with the VBOX differential GPS system. 

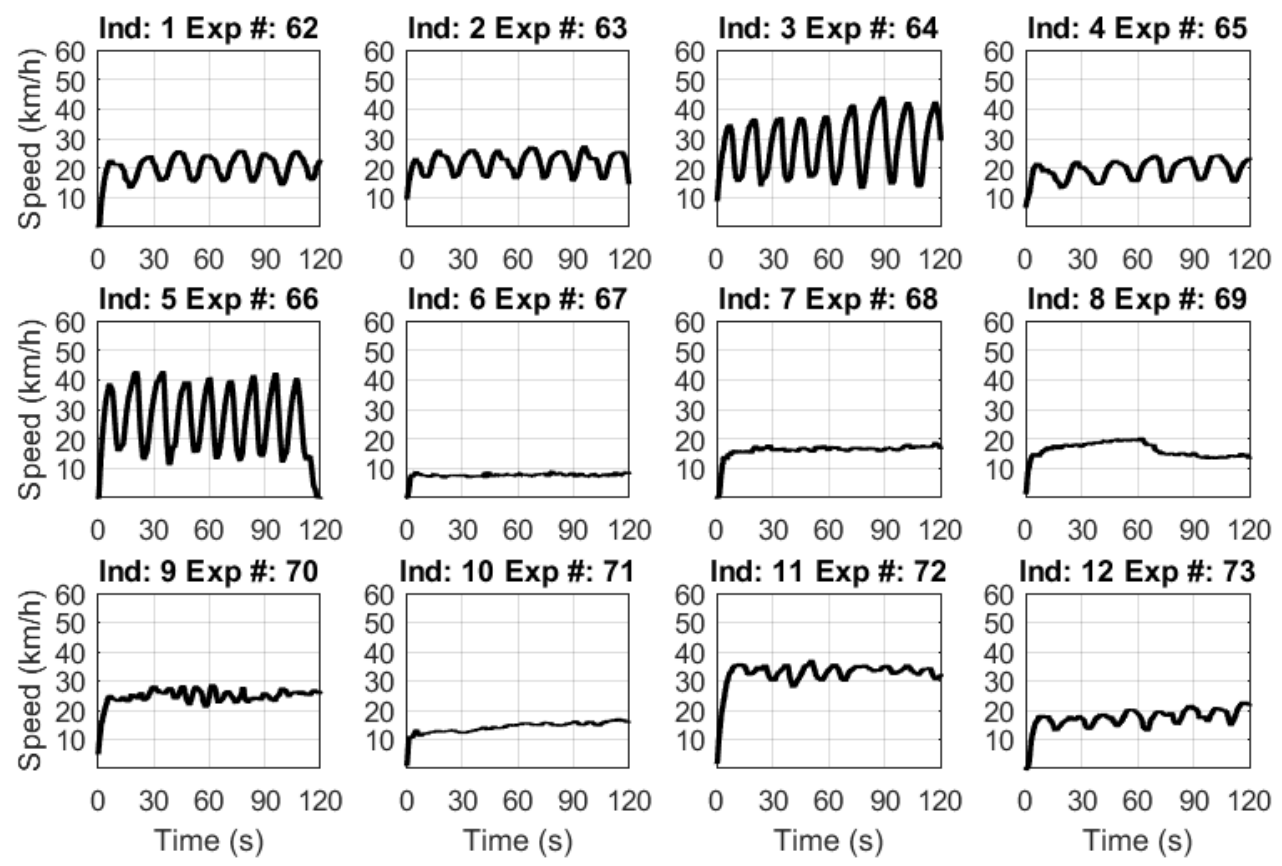

Figure A.25 Target Vehicle Speed. AVS experiments 62 to 73.
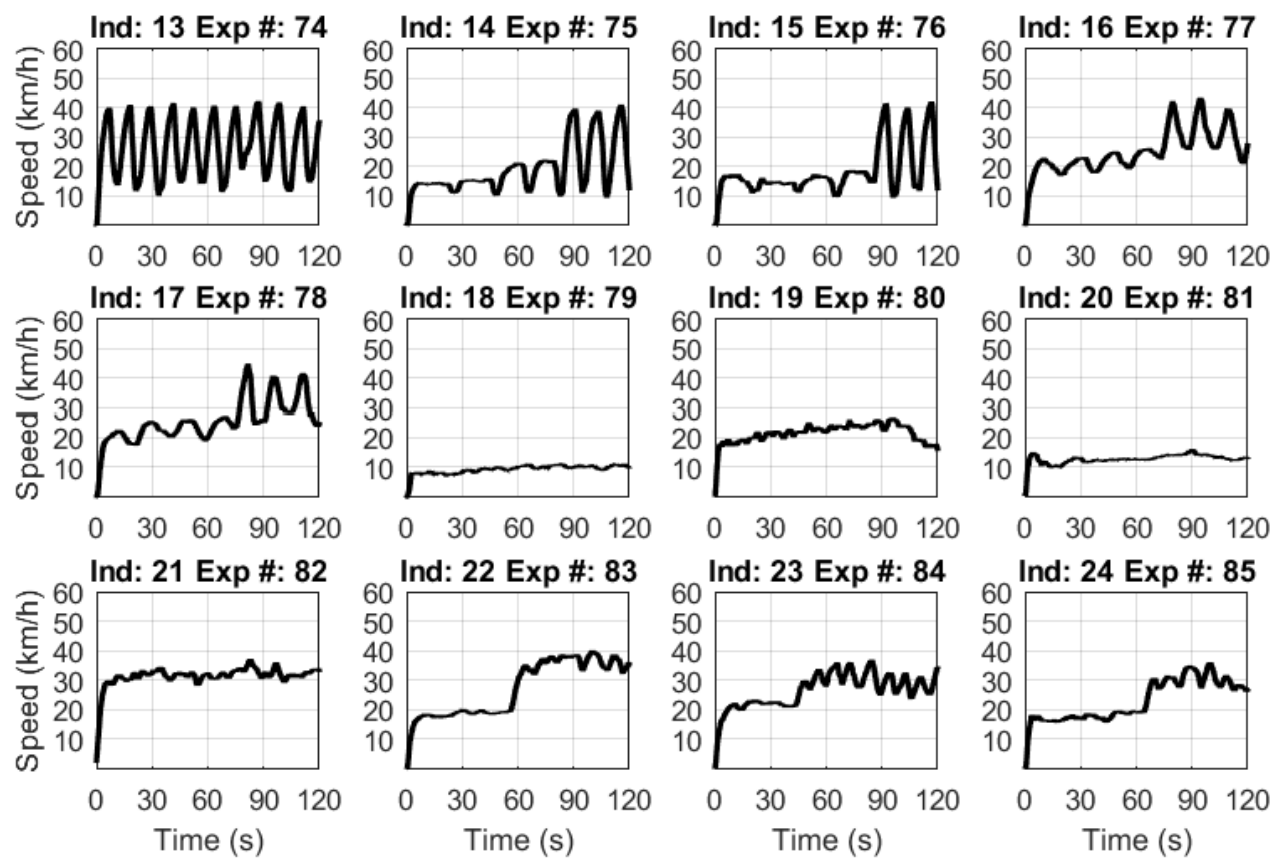

Figure A.26 Target Vehicle Speed. AVS experiments 74 to 85 .

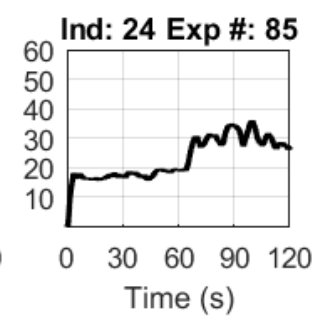



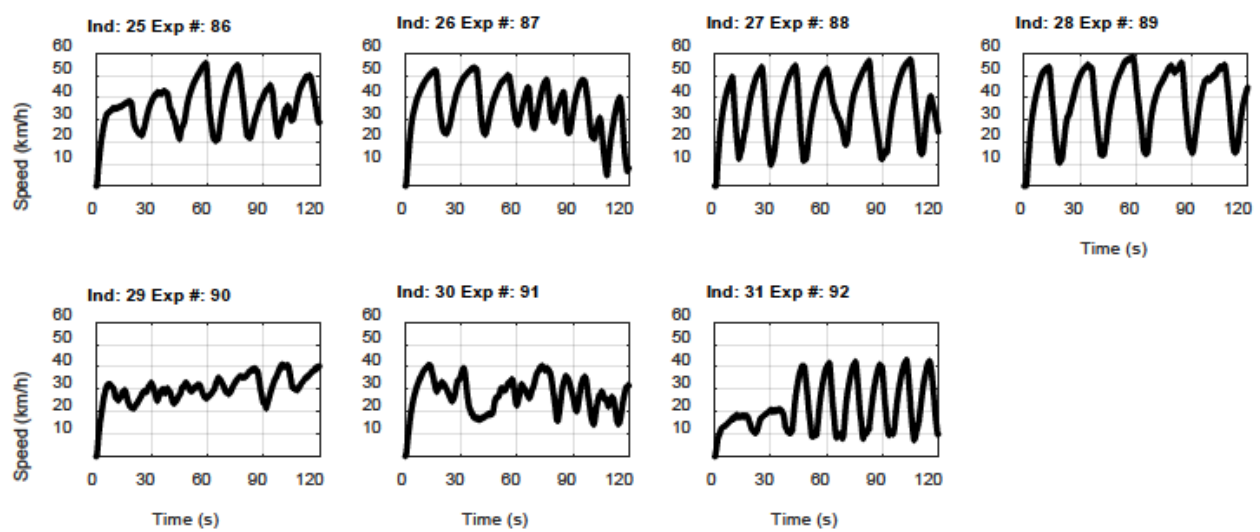

Figure A.27 Target Vehicle Speed. AVS experiments 86 to 92.

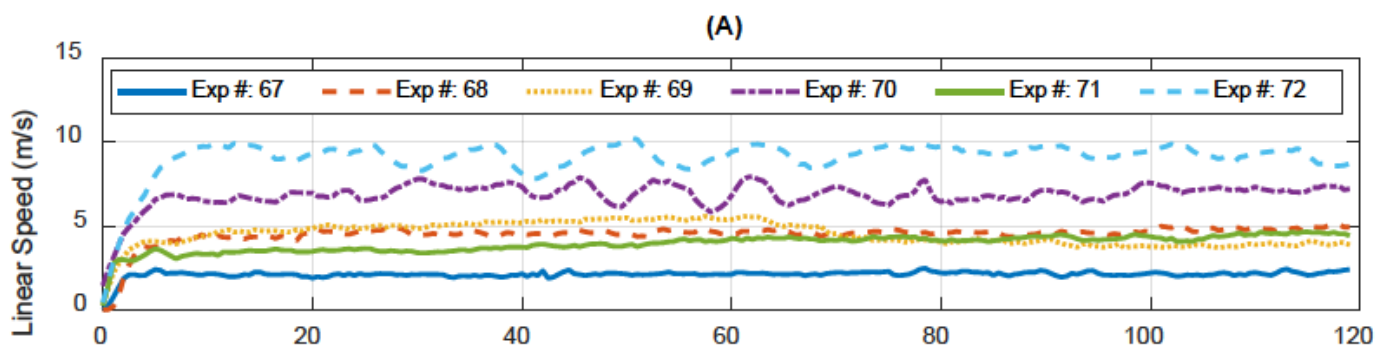

(B)

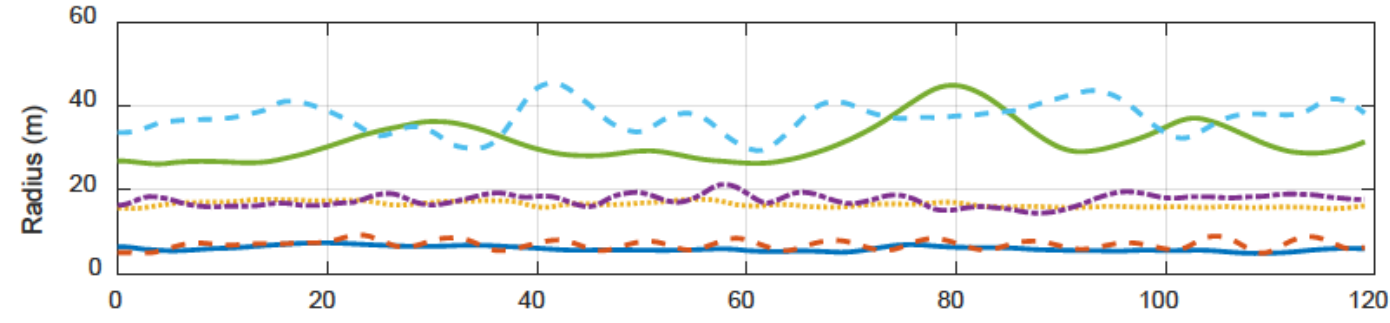

(C)

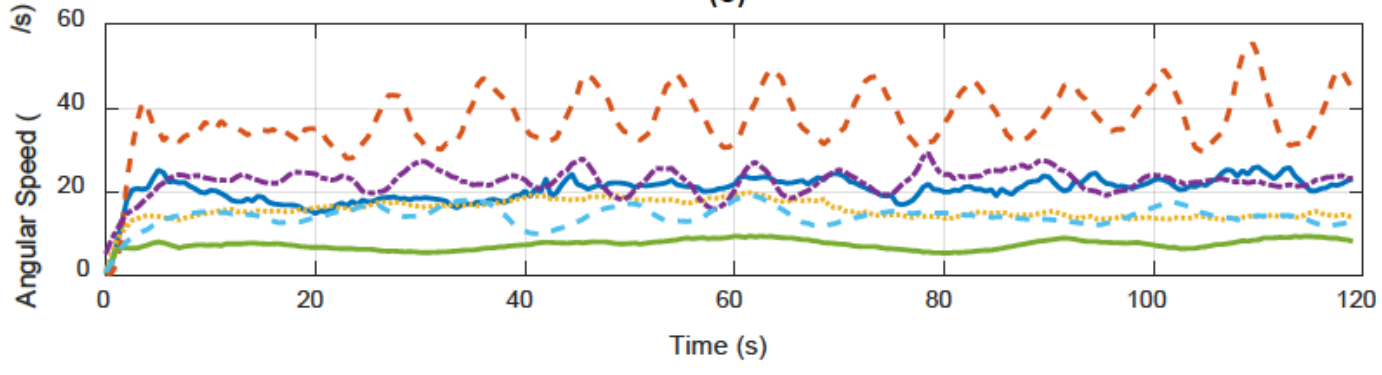

Figure A.28 Vehicle speed characteristics for experiments 67 to 72 relative to site 100 . (A) Vehicle Speed. (B) Distance between site 100 and vehicle position. (C) Angular Speed around site 100. 


\section{A.4.8 DOA Error}

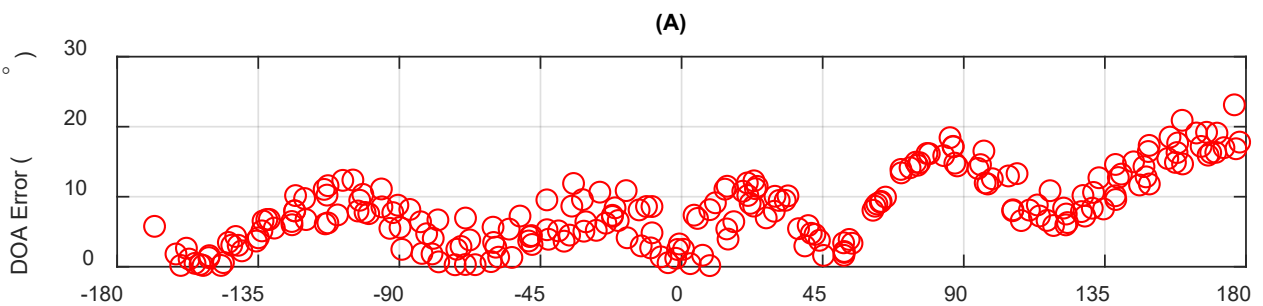

(B)

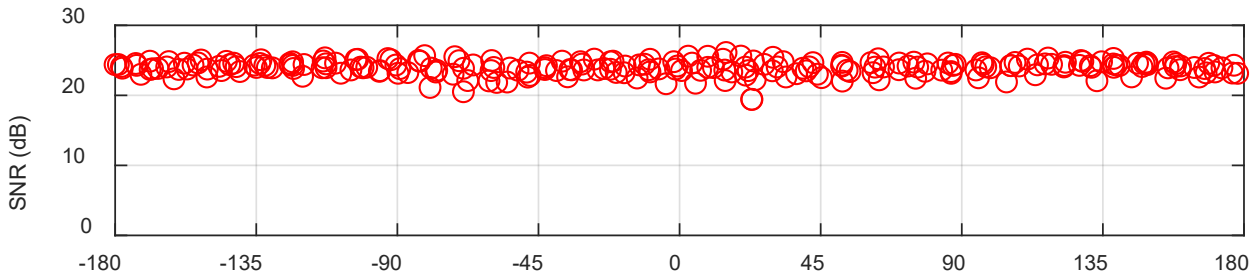

(C)

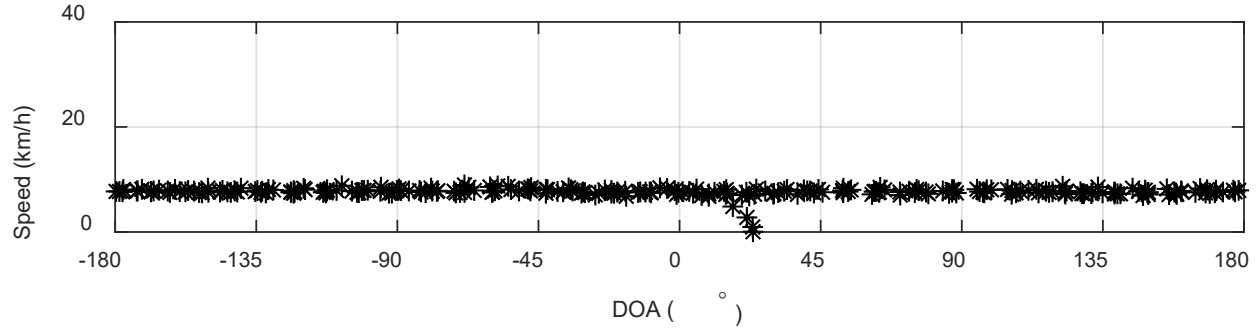

Figure A.29 pp AVS error at site 100, experiment 67. (A) DOA error and (B) SNR (C) Vehicle speed. 


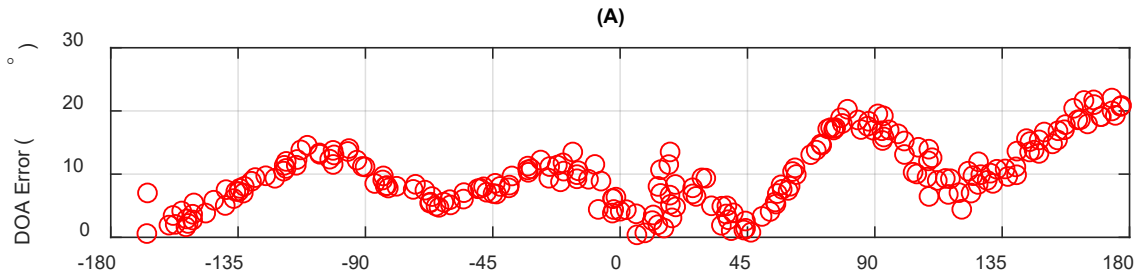

(B)

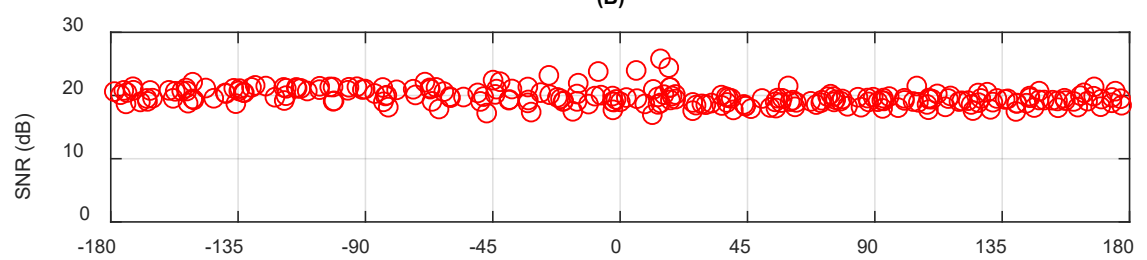

(C)

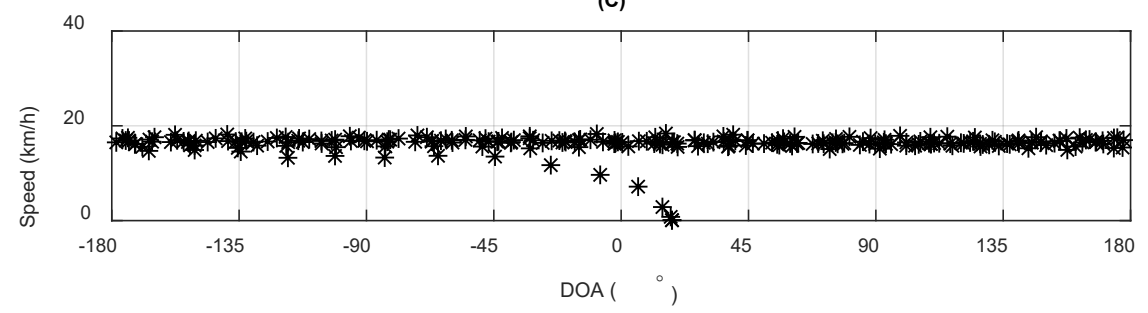

Figure A.30 pp AVS error at site 100, experiment 68. (A) DOA error and (B) SNR (C) Vehicle speed.

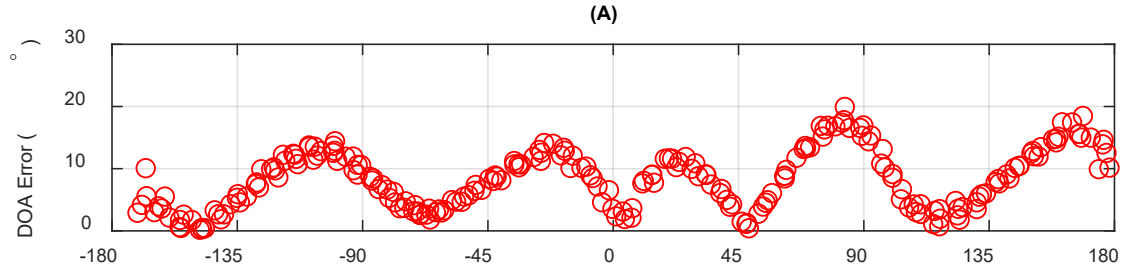

(B)

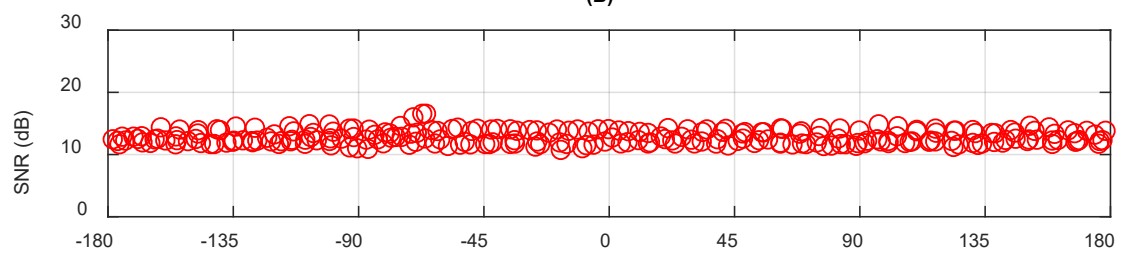

(C)

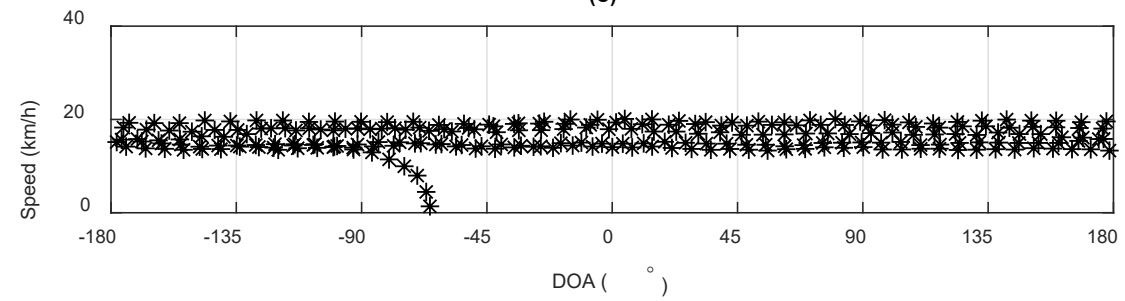

Figure A.31 pp AVS at site 100, experiment 69. (A) DOA error and (B) SNR (C) Vehicle speed. 


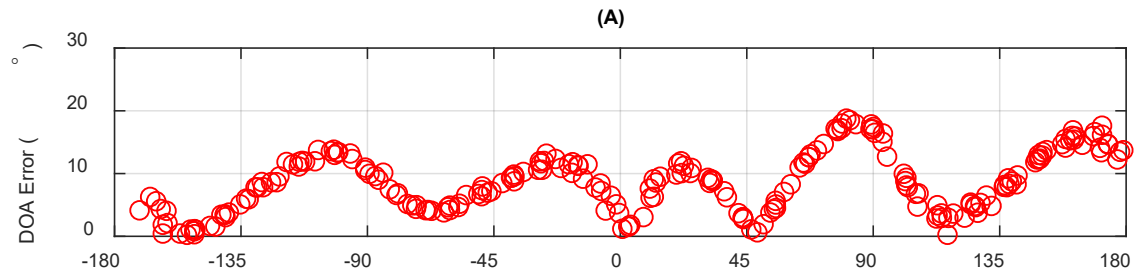

(B)

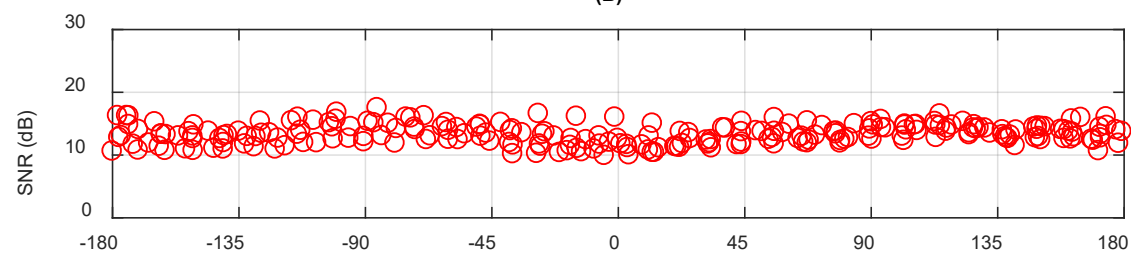

(C)

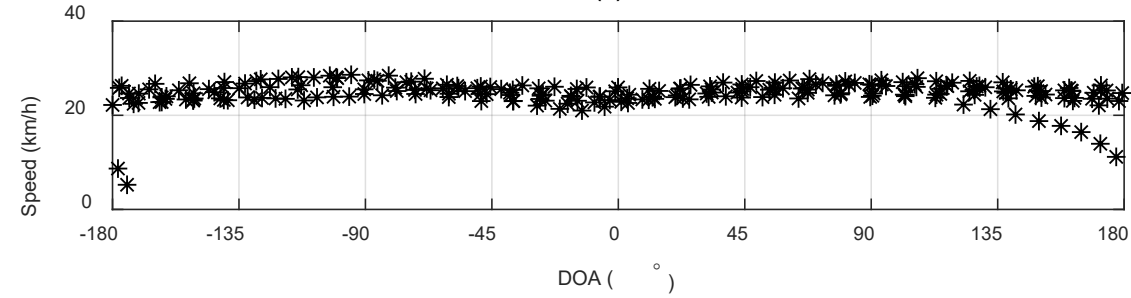

Figure A.32 pp AVS error at site 100, experiment 70. (A) DOA error and (B) SNR (C) Vehicle speed.

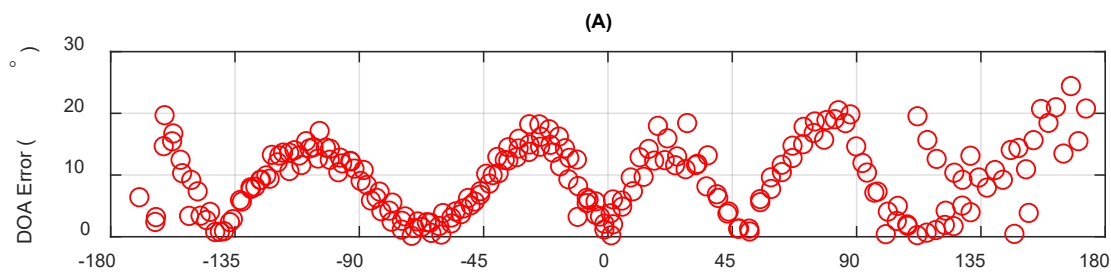

(B)

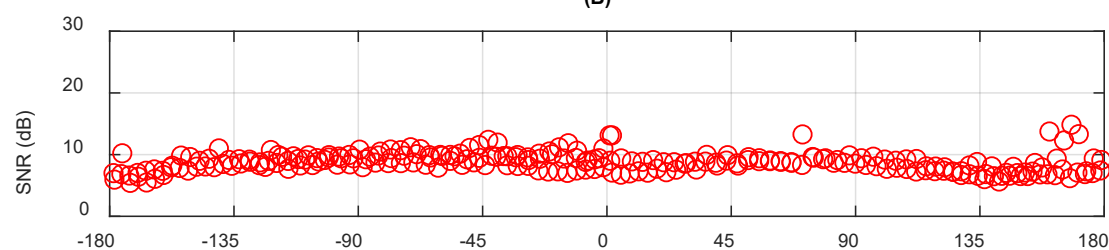

(C)

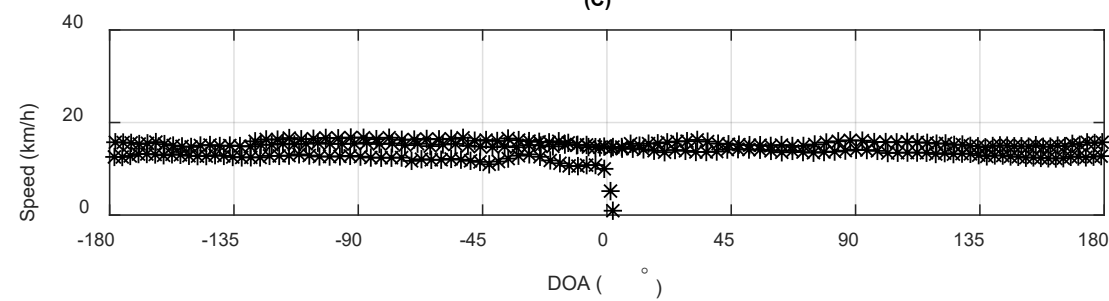

Figure A.33 pp AVS error at site 100, experiment 72. (A) DOA error and (B) SNR (C) Vehicle speed. 
(A)

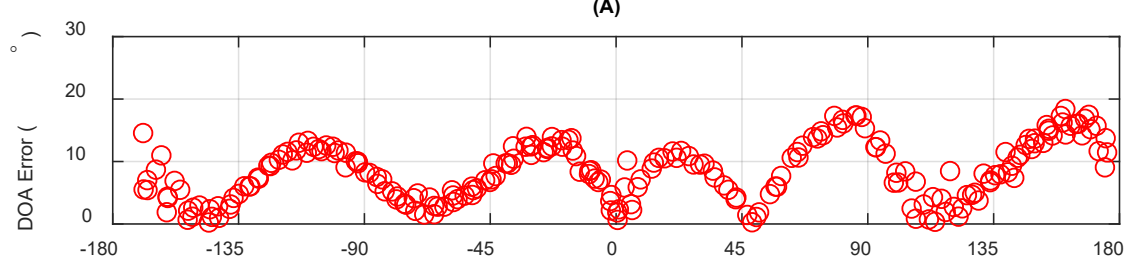

(B)

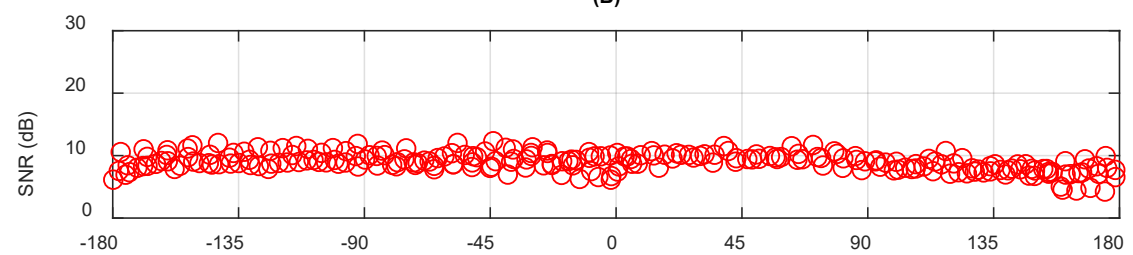

(C)

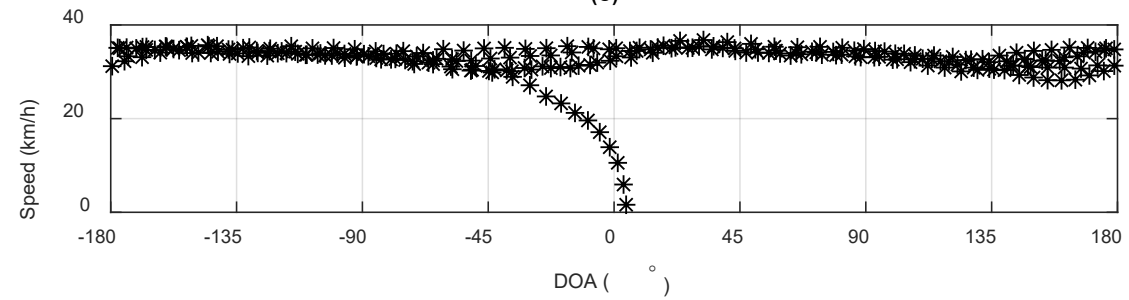

Figure A.34 pp AVS error at site 100, experiment 72. (A) DOA error and (B) SNR (C) Vehicle speed.

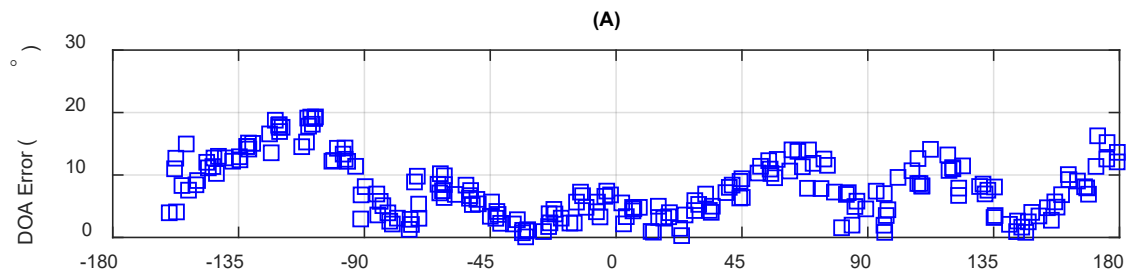

(B)

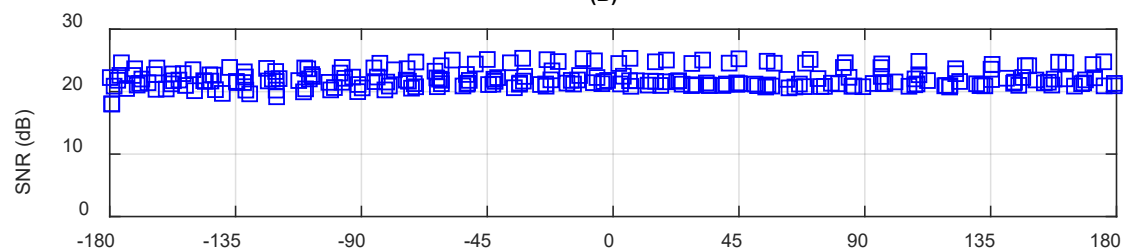

(C)

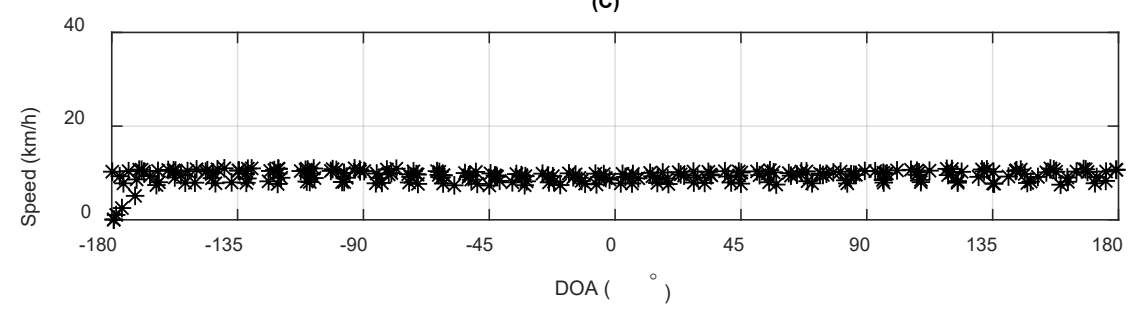

Figure A.35 pp AVS error at site 200, experiment 79. (A) DOA error and (B) SNR (C) Vehicle speed. 


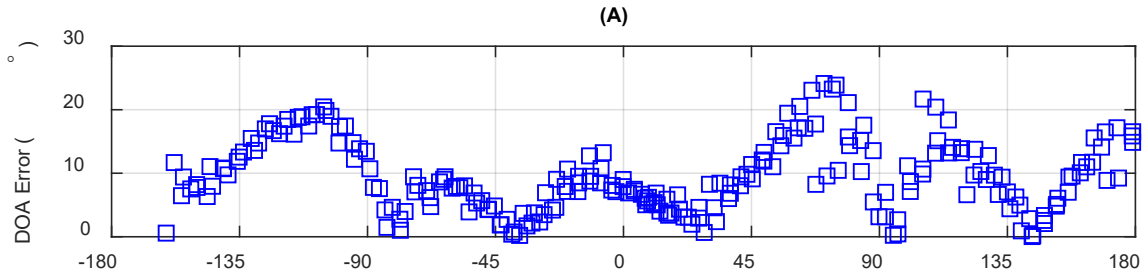

(B)

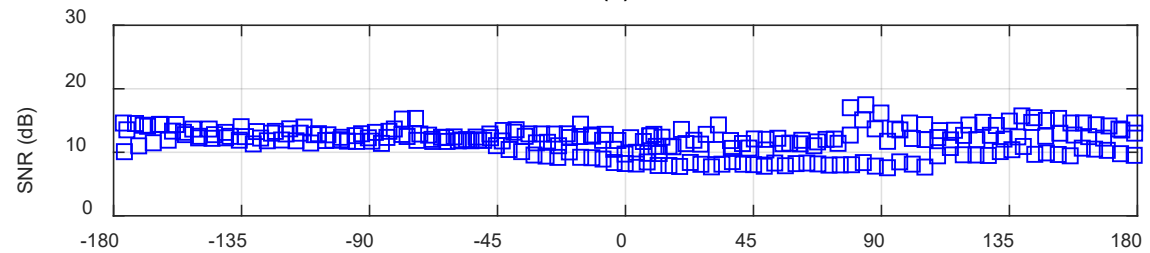

(C)

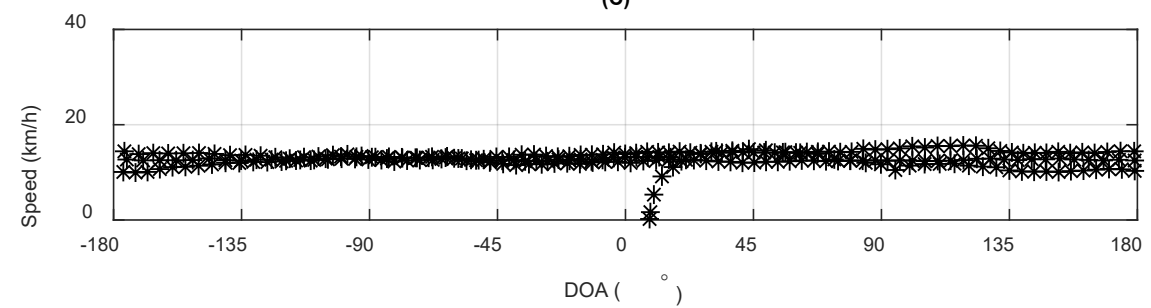

Figure A.36 pp AVS error at site 200, experiment 81. (A) DOA error and (B) SNR (C) Vehicle speed.

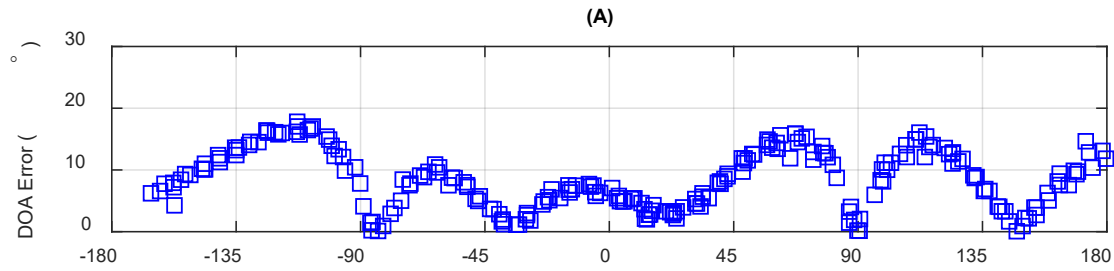

(B)

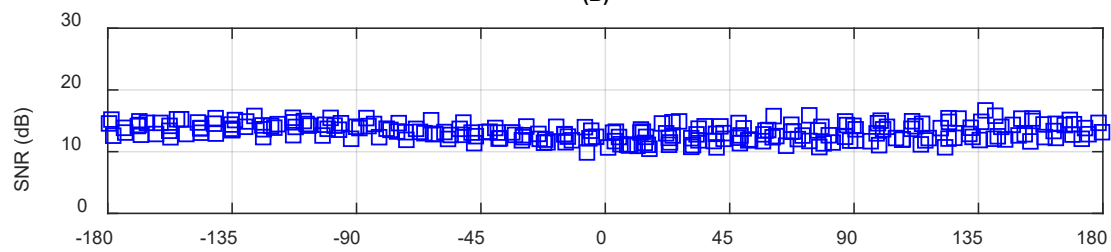

(C)

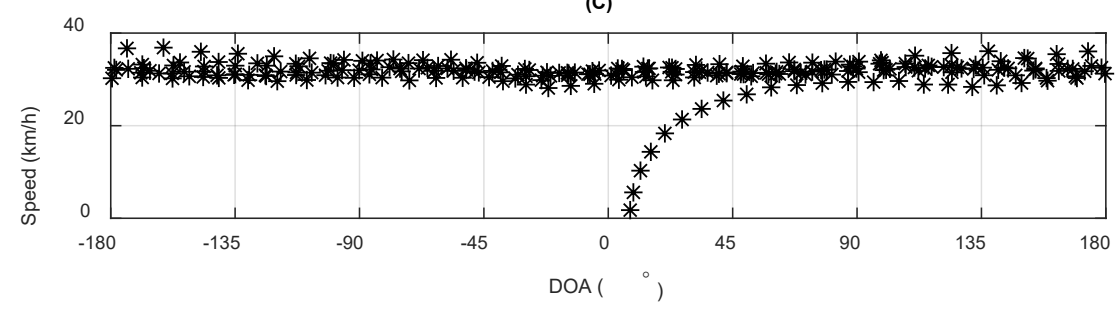

Figure A.37 pp AVS error at site 200, experiment 82. (A) DOA error and (B) SNR (C) Vehicle speed. 


\section{B TL Simulation Inputs}

Acoustic Toolbox User-interface and Post-processor (ACTUP) inputs for Bellhop and RAM.

\section{B.1 Model-Independent Parameters}

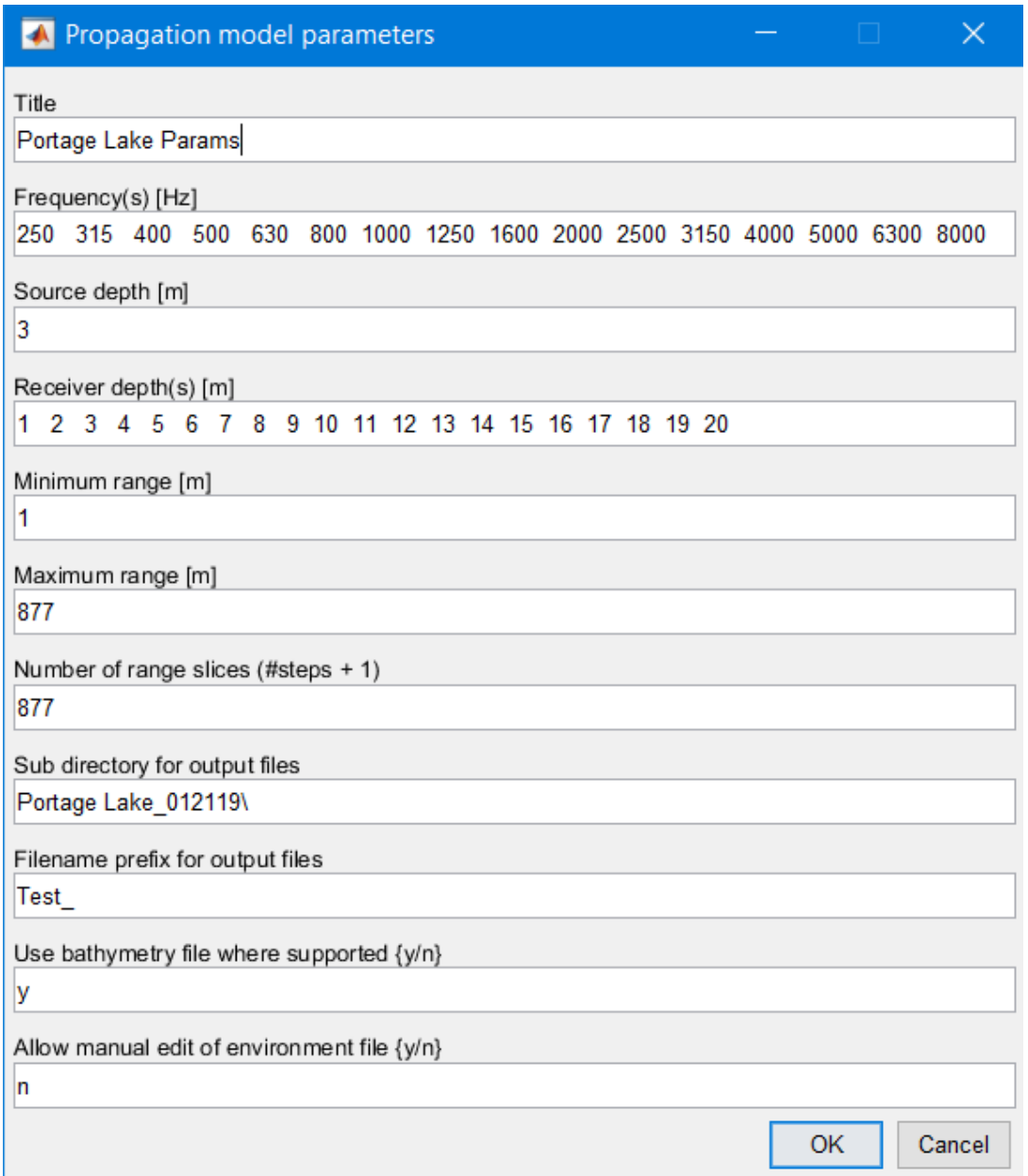




\section{B.2 Ray Tracing (Bellhop)}

Bellhop specific parameters

Run type: $\mathrm{R}=$ ray, $\mathrm{C}=$ coherent $\mathrm{TL}, \mathrm{I}=$ incoherent $\mathrm{TL}, \mathrm{S}=$ semicoherent $\mathrm{TL}, \mathrm{A}=$ amplitude-delay c)

Beam type: $\mathrm{B}$ = Gaussian bundles, $\mathrm{G}=$ geometric

B

Beam displacement: $0 / 1=$ off/on

1

Number of beams

1000

Launch angle of 1st ray (degrees from horizontal, -ve towards surface)

$-80$

Launch angle of last ray (degrees from horizontal, -ve towards surface)

80

Step size along ray for raytrace (wavelengths, 0 to let Bellhop choose)

\section{B.3 Parabolic Equation (RAM)}

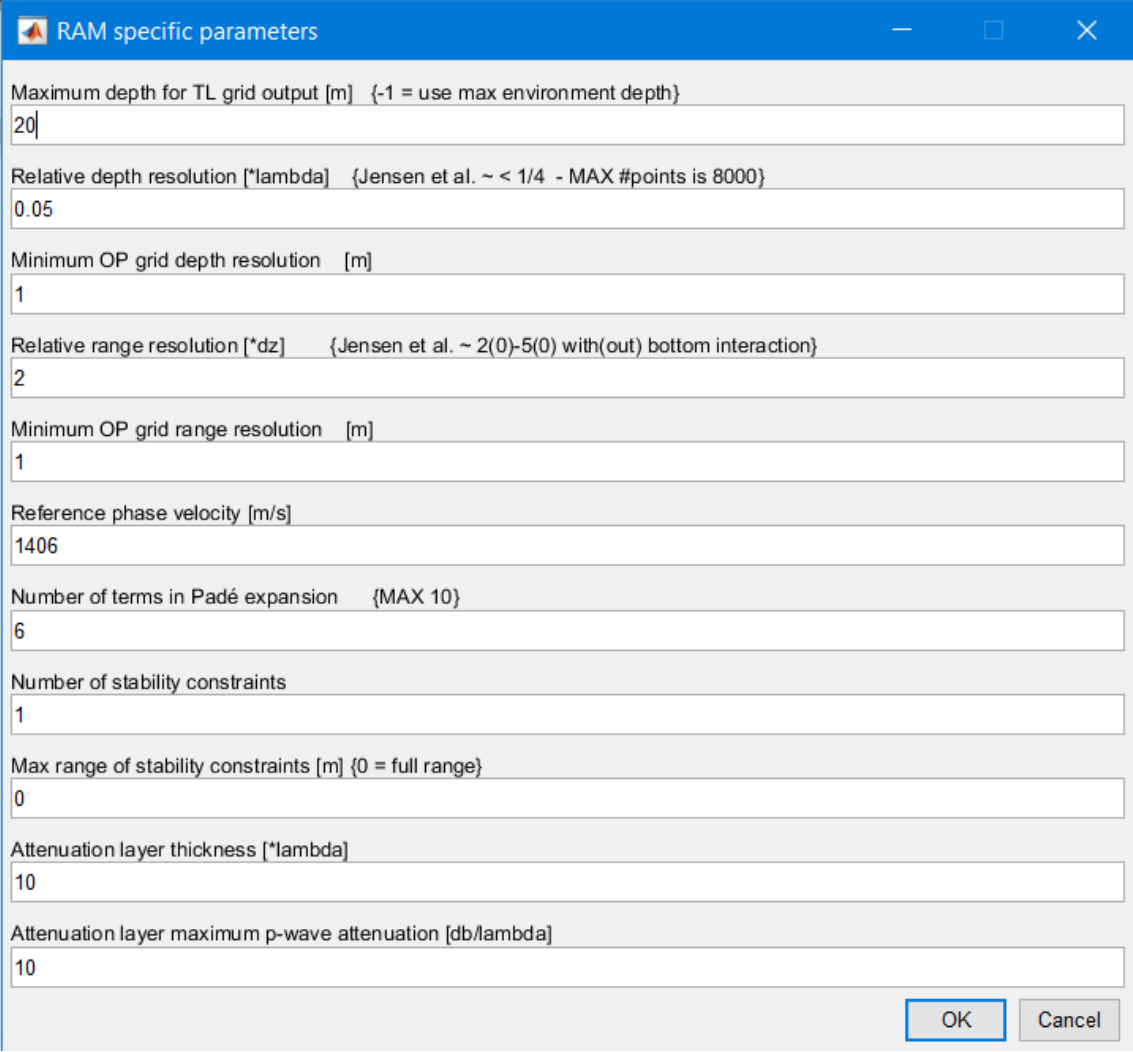




\section{B.4 Portage Lake Bathymetry File (.bty)}

$\begin{array}{ll}98 & \\ 0 & 11.51169682 \\ 0.001234577 & 11.51169682 \\ 0.009607628 & 11.49400043 \\ 0.018711022 & 11.48900032 \\ 0.027836816 & 11.48222637 \\ 0.03696898 & 11.457798 \\ 0.046103557 & 11.46385765 \\ 0.055238872 & 11.45824432 \\ 0.064375359 & 11.43990231 \\ 0.073512714 & 11.3843708 \\ 0.082649016 & 11.37520409 \\ 0.091786439 & 11.36346912 \\ 0.100924439 & 11.3178587 \\ 0.110061206 & 11.2728138 \\ 0.119199404 & 11.24389553 \\ 0.128337244 & 11.21861362 \\ 0.137474218 & 11.15863419 \\ 0.146612582 & 11.09911728 \\ 0.155750557 & 10.63537693 \\ 0.164888067 & 10.14019871 \\ 0.174026098 & 9.803134918 \\ 0.18316323 & 9.626106262 \\ 0.192301726 & 9.560513496 \\ 0.201439814 & 9.580441475 \\ 0.210577419 & 9.634553909 \\ 0.219715533 & 9.548071861 \\ 0.228853658 & 9.444518089 \\ 0.237991295 & 9.313165665 \\ 0.247129439 & 9.167505264 \\ 0.256268013 & 9.076000214 \\ 0.265405249 & 8.977999687 \\ 0.274543413 & 8.890057564 \\ 0.283682005 & 8.81126976 \\ 0.292819258 & 8.783388138 \\ 0.30195786 & 8.804340363 \\ 0.311096044 & 8.890045166 \\ 0.320233309 & 9.060214043 \\ 0.329371922 & 9.058606148 \\ 0.338510117 & 9.027797699 \\ 0.347647813 & 8.972409248 \\ 0.356786013 & 9.006404877 \\ 0.365924216 & 8.926953316\end{array}$




$\begin{array}{ll}0.37506192 & 8.773525238 \\ 0.384200127 & 8.632223129 \\ 0.393338759 & 8.596690178 \\ 0.402476047 & 8.567383766 \\ 0.41161426 & 8.513753891 \\ 0.420752896 & 8.524401665 \\ 0.429890189 & 8.558389664 \\ 0.439028828 & 8.587491035 \\ 0.448167047 & 8.539205551 \\ 0.457304344 & 8.492765427 \\ 0.466442988 & 8.503125191 \\ 0.47558121 & 8.599064827 \\ 0.484718932 & 8.685012817 \\ 0.493857157 & 8.865412712 \\ 0.502995383 & 9.045190811 \\ 0.512133107 & 9.253832817 \\ 0.521271335 & 9.49844265 \\ 0.530409985 & 9.647134781 \\ 0.53954729 & 10.07200909 \\ 0.54868552 & 10.47205448 \\ 0.557824172 & 10.85817623 \\ 0.56696148 & 11.24592209 \\ 0.576100133 & 11.62465477 \\ 0.585237442 & 11.95421028 \\ 0.594375675 & 12.15538597 \\ 0.60351433 & 12.21103382 \\ 0.612651641 & 12.33931637 \\ 0.621790296 & 12.43135643 \\ 0.630928532 & 12.44408703 \\ 0.640065844 & 12.4234724 \\ 0.649204501 & 12.42181683 \\ 0.658342737 & 12.4109993 \\ 0.667480472 & 12.43426895 \\ 0.676618709 & 12.44814777 \\ 0.685756947 & 12.62619877 \\ 0.694894682 & 12.70486355 \\ 0.70403292 & 12.6658268 \\ 0.71317158 & 12.52720642 \\ 0.722308895 & 12.45100021 \\ 0.731447135 & 12.43609142 \\ 0.740585796 & 12.49737549 \\ 0.749723112 & 12.60787773 \\ 0.758861773 & 12.54709625 \\ 0.768000013 & 12.72699928 \\ 0.77713733 & 12.77081013\end{array}$




$\begin{array}{ll}0.786275992 & 12.5326004 \\ 0.795414234 & 12.52263546 \\ 0.804551972 & 12.67670918 \\ 0.813690214 & 12.7427969 \\ 0.822828456 & 12.67430115 \\ 0.831966195 & 12.51699543 \\ 0.841104437 & 12.59902096 \\ 0.850243101 & 12.54768562 \\ 0.85938042 & 12.61296368 \\ 0.868518663 & 12.92075157 \\ 0.877657327 & 12.6004467\end{array}$




\section{Copyright documentation}

\section{C.1 Permission 1}

\section{RE: Permission Letter for Use of Previously Copyrighted Material}

October 11,2018

Susann Brailey

AIP Publisling

1305 Walt Whitman Rd., Suite 300

Mclille, NY 11747-4300. USA

1.516 .576 .2268

Dear Susann Brailey:

I am completing a doctornl disscrtation at Michigan Technological University entilled "Acoustic localization Techniques for Application in Near-Shore Arctic Environments." I would like your permission to reprint in my dissertation the following:

Miles Penhale, Andrew Barnard and Robert Shuchunan, "Multi-Modal and Short-Range Transmission Loss in Thin, Ice-Covered. Near-Shore Aretic Waters", J. Acoust. Soc. Am. 143(5), 3126-3137 (2018).

The requested permission extends to any future revisions and editions of iny dissertation, including nonexclusive world rights in all languages, and to the prospective publication of my dissertation by ProQuest through its ProQuest $\mathbb{R}$ Dissertation Publishing business. ProQuest may produce and sell copies of my dissertation on demand and may' make my dissertation available for free internet download at my request. These riglyts will in no way restrict republication of the material in any other form by you or by others autherized by yon. Your signing of this letter will also confirm that you own [or your company owns] the copyright to the above-described naterial.

If these arrangements mect with your approval, please sign this letter where indicated below and return it to me via email. Thank you very much.

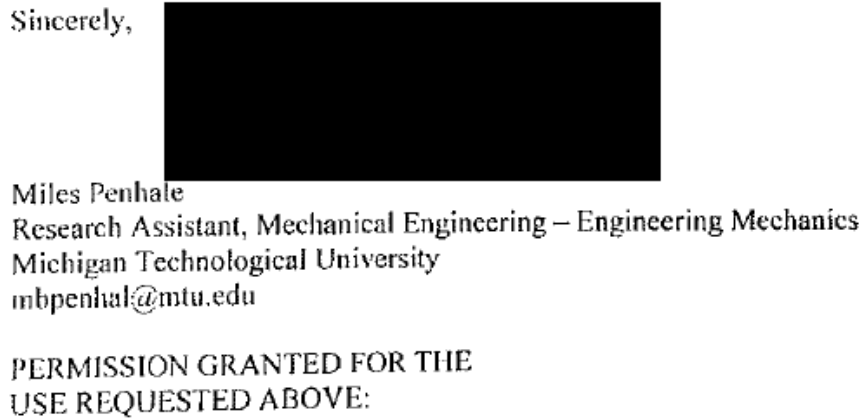

Research Assistant, Mechanical Engineering - Engineering Mechanics

Michigan Technological University

mbpeniulamtu.edu

PERMISSION GRANTED FOR THE
USE REQUESTED ABOVE:

Susann Brailey

AlP Publishing

Manager, Rights \& Permissions

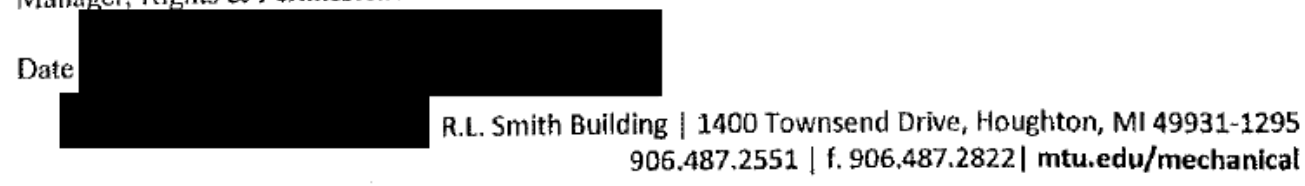




\section{C.2 Permission 2}

\section{RE: Permission Letter for Use of Previously Copyrighted Material}

November 6, 2018

University of Washington - Applied Physics Lab

1013 NE $40^{\text {th }}$ Street

Seattle, WA 98105

T: 2065431300

F: 2065436785

To whom it may concern:

I am completing a doctoral dissertation at Michigan Technological University entitled "Acoustic Localization Techniques for Application in Near-Shore Arctic Environments." I would like your permission to reprint in my dissertation the following:

Figure 1 (Curves predicted by the forward loss model for generic bottom types...) on page IV-19 of the APL-UW High-Frequency Ocean Environmental Acoustic Models Handbook. ADB199453.

The requested permission extends to any future revisions and editions of my dissertation, including nonexclusive world rights in all languages, and to the prospective publication of my dissertation by ProQuest through its ProQuest $®$ Dissertation Publishing business. ProQuest may produce and sell copies of my dissertation on demand and may make my dissertation available for free internet download at my request. These rights will in no way restrict republication of the material in any other form by you or by others authorized by you. Your signing of this letter will also confirm that you own [or your company owns] the copyright to the above- described material.

If these arrangements meet with your approval, please sign this letter where indicated below and return it to me via email. Thank you very much.

Sincerely,

Miles Penhale

Research Assistant, Mechanical Engineering - Engineering Mechanics

Michigan Technological University

mbpenhal@mtu.edu

PERMISSION GRANTED FOR THE

USE REQUESTED ABOVE:

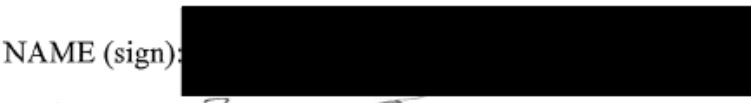

NAME (print): Brian S. Rasmusson

Date: 7 Nasmbor zol 8 


\section{C.3 Permission 3}

\section{Taylor and Francis Group LLC Books LICENSE TERMS AND CONDITIONS}

Nov 26, 2018

This is a License Agreement between Miles Penhale ("You") and Taylor and Francis Group LLC Books ("Taylor and Francis Group LLC Books") provided by Copyright Clearance Center ("CCC"). The license consists of your order details, the terms and conditions provided by Taylor and Francis Group LLC Books, and the payment terms and conditions.

All payments must be made in full to CCC. For payment instructions, please see information listed at the bottom of this form.

License Number

License date

Licensed content publisher

Licensed content title

Licensed content date

Type of Use

Requestor type

Format

Portion

Number of charts/graphs/tables/figures

The requesting person/organization is:

Title or numeric reference of the portion(s)
4464960668157

Nov 09, 2018

Taylor and Francis Group LLC Books

Underwater Acoustic Modeling and Simulation

Apr 6, 2018

Thesis/Dissertation

Publisher, for-profit

Print, Electronic

chart/graph/table/figure

1

Miles Penhale

Figure 4.18 Domains of applicability of underwater acoustic propagation models. 
Title of the article or chapter the portion is from

Editor of portion(s)

Author of portion(s)

Volume of serial or monograph.

Page range of the portion

Publication date of portion

Rights for

Duration of use

Creation of copies for the disabled

With minor editing privileges

For distribution to

In the following language(s)

With incidental promotional use

The lifetime unit quantity of new product

Title

Institution name

Expected presentation date

Billing Type

Billing Address
Chapter 4: Propagation II: Mathematical Models

N/A

Paul C. Etter

N/A

155

2018

Main product

Life of current edition

no

no

Worldwide

Original language of publication

no

Up to 499

ACOUSTIC LOCALIZATION TECHNIQUES FOR APPLICATION IN NEAR-SHORE ARCTIC ENVIRONMENTS

Michigan Technological University

Jul 2019

Invoice

Miles Penhale

1400 Townsend Dr.

R.L. Smith MEEM Building

Houghton, MI 49931 
United States

Attn: Miles Penhale

Total (may include CCC user fee)

0.00 USD

Terms and Conditions

\section{TERMS AND CONDITIONS}

The following terms are individual to this publisher:

Taylor and Francis Group and Informa healthcare are division of Informa plc. Permission will be void if material exceeds $10 \%$ of all the total pages in your publication and over $20 \%$ of the original publication. This includes permission granted by Informa plc and all of its subsidaries.

Other Terms and Conditions:

\section{STANDARD TERMS AND CONDITIONS}

1. Description of Service; Defined Terms. This Republication License enables the User to obtain licenses for republication of one or more copyrighted works as described in detail on the relevant Order Confirmation (the "Work(s)"). Copyright Clearance Center, Inc. ("CCC") grants licenses through the Service on behalf of the rightsholder identified on the Order Confirmation (the "Rightsholder"). "Republication", as used herein, generally means the inclusion of a Work, in whole or in part, in a new work or works, also as described on the Order Confirmation. "User", as used herein, means the person or entity making such republication.

2. The terms set forth in the relevant Order Confirmation, and any terms set by the Rightsholder with respect to a particular Work, govern the terms of use of Works in connection with the Service. By using the Service, the person transacting for a republication license on behalf of the User represents and warrants that he/she/it (a) has been duly authorized by the User to accept, and hereby does accept, all such terms and conditions on behalf of User, and (b) shall inform User of all such terms and conditions. In the event such person is a "freelancer" or other third party independent of User and CCC, such party shall be deemed jointly a "User" for purposes of these terms and conditions. In any event, User shall be deemed to have accepted and agreed to all such terms and conditions if User republishes the Work in any fashion.

3. Scope of License; Limitations and Obligations.

3.1 All Works and all rights therein, including copyright rights, remain the sole and exclusive property of the Rightsholder. The license created by the exchange of an Order Confirmation (and/or any invoice) and payment by User of the full amount set forth on 
that document includes only those rights expressly set forth in the Order Confirmation and in these terms and conditions, and conveys no other rights in the Work(s) to User. All rights not expressly granted are hereby reserved.

3.2 General Payment Terms: You may pay by credit card or through an account with us payable at the end of the month. If you and we agree that you may establish a standing account with CCC, then the following terms apply: Remit Payment to: Copyright Clearance Center, 29118 Network Place, Chicago, IL 60673-1291. Payments Due: Invoices are payable upon their delivery to you (or upon our notice to you that they are available to you for downloading). After 30 days, outstanding amounts will be subject to a service charge of $1-1 / 2 \%$ per month or, if less, the maximum rate allowed by applicable law. Unless otherwise specifically set forth in the Order Confirmation or in a separate written agreement signed by CCC, invoices are due and payable on "net 30" terms. While User may exercise the rights licensed immediately upon issuance of the Order Confirmation, the license is automatically revoked and is null and void, as if it had never been issued, if complete payment for the license is not received on a timely basis either from User directly or through a payment agent, such as a credit card company.

3.3 Unless otherwise provided in the Order Confirmation, any grant of rights to User (i) is "one-time" (including the editions and product family specified in the license), (ii) is non-exclusive and non-transferable and (iii) is subject to any and all limitations and restrictions (such as, but not limited to, limitations on duration of use or circulation) included in the Order Confirmation or invoice and/or in these terms and conditions. Upon completion of the licensed use, User shall either secure a new permission for further use of the Work(s) or immediately cease any new use of the Work(s) and shall render inaccessible (such as by deleting or by removing or severing links or other locators) any further copies of the Work (except for copies printed on paper in accordance with this license and still in User's stock at the end of such period).

3.4 In the event that the material for which a republication license is sought includes third party materials (such as photographs, illustrations, graphs, inserts and similar materials) which are identified in such material as having been used by permission, User is responsible for identifying, and seeking separate licenses (under this Service or otherwise) for, any of such third party materials; without a separate license, such third party materials may not be used.

3.5 Use of proper copyright notice for a Work is required as a condition of any license granted under the Service. Unless otherwise provided in the Order Confirmation, a proper copyright notice will read substantially as follows: "Republished with permission of [Rightsholder's name], from [Work's title, author, volume, edition number and year of copyright]; permission conveyed through Copyright Clearance Center, Inc. " Such notice must be provided in a reasonably legible font size and must be placed either immediately adjacent to the Work as used (for example, as part of a by-line or footnote but not as a separate electronic link) or in the place where substantially all other credits 
or notices for the new work containing the republished Work are located. Failure to include the required notice results in loss to the Rightsholder and CCC, and the User shall be liable to pay liquidated damages for each such failure equal to twice the use fee specified in the Order Confirmation, in addition to the use fee itself and any other fees and charges specified.

3.6 User may only make alterations to the Work if and as expressly set forth in the Order Confirmation. No Work may be used in any way that is defamatory, violates the rights of third parties (including such third parties' rights of copyright, privacy, publicity, or other tangible or intangible property), or is otherwise illegal, sexually explicit or obscene. In addition, User may not conjoin a Work with any other material that may result in damage to the reputation of the Rightsholder. User agrees to inform CCC if it becomes aware of any infringement of any rights in a Work and to cooperate with any reasonable request of CCC or the Rightsholder in connection therewith.

4. Indemnity. User hereby indemnifies and agrees to defend the Rightsholder and CCC, and their respective employees and directors, against all claims, liability, damages, costs and expenses, including legal fees and expenses, arising out of any use of a Work beyond the scope of the rights granted herein, or any use of a Work which has been altered in any unauthorized way by User, including claims of defamation or infringement of rights of copyright, publicity, privacy or other tangible or intangible property.

5. Limitation of Liability. UNDER NO CIRCUMSTANCES WILL CCC OR THE RIGHTSHOLDER BE LIABLE FOR ANY DIRECT, INDIRECT, CONSEQUENTIAL OR INCIDENTAL DAMAGES (INCLUDING WITHOUT LIMITATION DAMAGES FOR LOSS OF BUSINESS PROFITS OR INFORMATION, OR FOR BUSINESS INTERRUPTION) ARISING OUT OF THE USE OR INABILITY TO USE A WORK, EVEN IF ONE OF THEM HAS BEEN ADVISED OF THE POSSIBILITY OF SUCH DAMAGES. In any event, the total liability of the Rightsholder and CCC (including their respective employees and directors) shall not exceed the total amount actually paid by User for this license. User assumes full liability for the actions and omissions of its principals, employees, agents, affiliates, successors and assigns.

6. Limited Warranties. THE WORK(S) AND RIGHT(S) ARE PROVIDED “AS IS". CCC HAS THE RIGHT TO GRANT TO USER THE RIGHTS GRANTED IN THE ORDER CONFIRMATION DOCUMENT. CCC AND THE RIGHTSHOLDER DISCLAIM ALL OTHER WARRANTIES RELATING TO THE WORK(S) AND RIGHT(S), EITHER EXPRESS OR IMPLIED, INCLUDING WITHOUT LIMITATION IMPLIED WARRANTIES OF MERCHANTABILITY OR FITNESS FOR A PARTICULAR PURPOSE. ADDITIONAL RIGHTS MAY BE REQUIRED TO USE ILLUSTRATIONS, GRAPHS, PHOTOGRAPHS, ABSTRACTS, INSERTS OR OTHER PORTIONS OF THE WORK (AS OPPOSED TO THE ENTIRE WORK) IN A MANNER CONTEMPLATED BY USER; USER UNDERSTANDS AND 


\section{AGREES THAT NEITHER CCC NOR THE RIGHTSHOLDER MAY HAVE SUCH ADDITIONAL RIGHTS TO GRANT.}

7. Effect of Breach. Any failure by User to pay any amount when due, or any use by User of a Work beyond the scope of the license set forth in the Order Confirmation and/or these terms and conditions, shall be a material breach of the license created by the Order Confirmation and these terms and conditions. Any breach not cured within 30 days of written notice thereof shall result in immediate termination of such license without further notice. Any unauthorized (but licensable) use of a Work that is terminated immediately upon notice thereof may be liquidated by payment of the Rightsholder's ordinary license price therefor; any unauthorized (and unlicensable) use that is not terminated immediately for any reason (including, for example, because materials containing the Work cannot reasonably be recalled) will be subject to all remedies available at law or in equity, but in no event to a payment of less than three times the Rightsholder's ordinary license price for the most closely analogous licensable use plus Rightsholder's and/or CCC's costs and expenses incurred in collecting such payment.

\section{Miscellaneous.}

8.1 User acknowledges that CCC may, from time to time, make changes or additions to the Service or to these terms and conditions, and CCC reserves the right to send notice to the User by electronic mail or otherwise for the purposes of notifying User of such changes or additions; provided that any such changes or additions shall not apply to permissions already secured and paid for.

8.2 Use of User-related information collected through the Service is governed by CCC's privacy policy, available online here:http:/www.copyright.com/content/cc3/en/tools/footer/privacypolicy.html.

8.3 The licensing transaction described in the Order Confirmation is personal to User. Therefore, User may not assign or transfer to any other person (whether a natural person or an organization of any kind) the license created by the Order Confirmation and these terms and conditions or any rights granted hereunder; provided, however, that User may assign such license in its entirety on written notice to CCC in the event of a transfer of all or substantially all of User's rights in the new material which includes the Work(s) licensed under this Service.

8.4 No amendment or waiver of any terms is binding unless set forth in writing and signed by the parties. The Rightsholder and CCC hereby object to any terms contained in any writing prepared by the User or its principals, employees, agents or affiliates and purporting to govern or otherwise relate to the licensing transaction described in the Order Confirmation, which terms are in any way inconsistent with any terms set forth in the Order Confirmation and/or in these terms and conditions or CCC's standard operating procedures, whether such writing is prepared prior to, simultaneously with or 
subsequent to the Order Confirmation, and whether such writing appears on a copy of the Order Confirmation or in a separate instrument.

8.5 The licensing transaction described in the Order Confirmation document shall be governed by and construed under the law of the State of New York, USA, without regard to the principles thereof of conflicts of law. Any case, controversy, suit, action, or proceeding arising out of, in connection with, or related to such licensing transaction shall be brought, at CCC's sole discretion, in any federal or state court located in the County of New York, State of New York, USA, or in any federal or state court whose geographical jurisdiction covers the location of the Rightsholder set forth in the Order Confirmation. The parties expressly submit to the personal jurisdiction and venue of each such federal or state court.If you have any comments or questions about the Service or Copyright Clearance Center, please contact us at 978-750-8400 or send an email toinfo@copyright.com.

V 1.1

Questions? customercare@copyright.com or +1-855-239-3415 (toll free in the US) or +1-978-646-2777. 